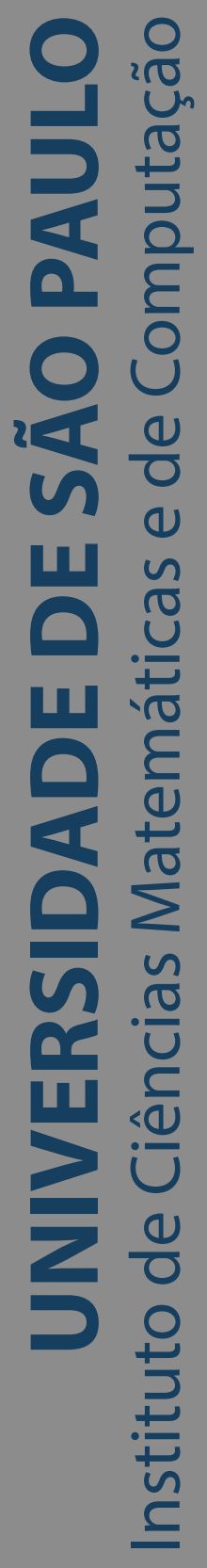

\title{
Modeling spreading processes in complex networks
}

\section{Guilherme Ferraz de Arruda}

Tese de Doutorado do Programa de Pós-Graduação em Ciências de Computação e Matemática Computacional (PPG-CCMC) 

SERVIÇO DE PÓS-GRADUAÇÃO DO ICMC-USP

Data de Depósito:

Assinatura:

Guilherme Ferraz de Arruda

\section{Modeling spreading processes in complex networks}

Doctoral dissertation submitted to the Institute of Mathematics and Computer Sciences - ICMC-USP, in partial fulfillment of the requirements for the degree of the Doctorate Program in Computer Science and Computational Mathematics. FINAL VERSION

Concentration Area: Computer Science and Computational Mathematics

Advisor: Prof. Dr. Francisco Aparecido Rodrigues 
Ficha catalográfica elaborada pela Biblioteca Prof. Achille Bassi e Seção Técnica de Informática, ICMC/USP, com os dados inseridos pelo(a) autor(a)

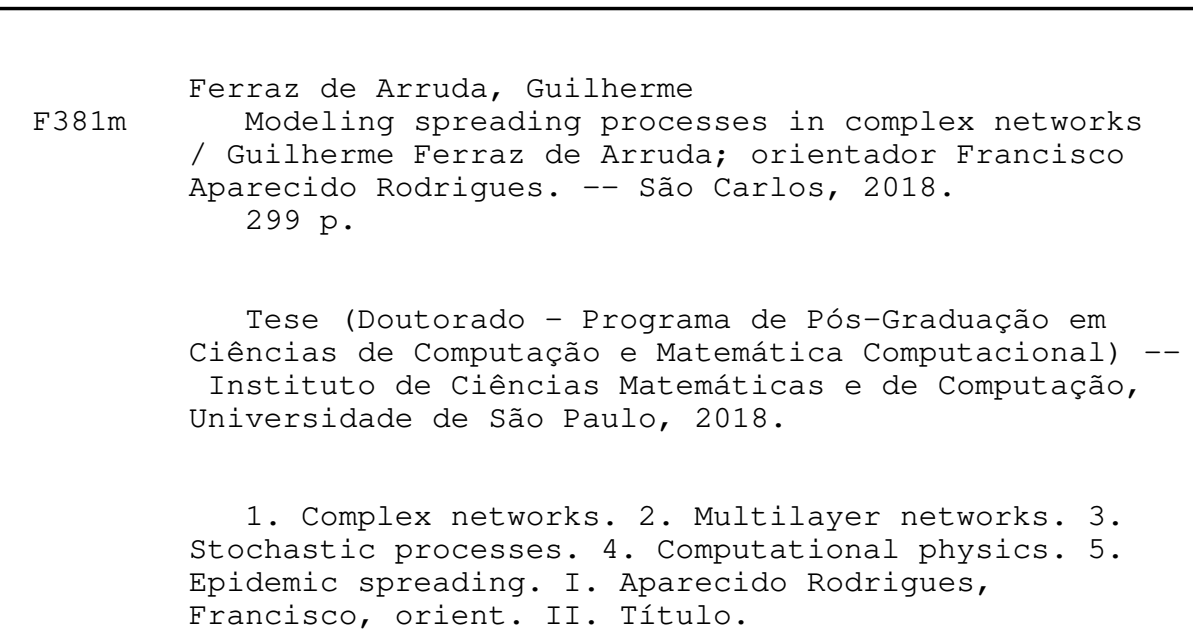

1. Complex networks. 2. Multilayer networks. 3. Stochastic processes. 4. Computational physics. 5. Epidemic spreading. I. Aparecido Rodrigues, Francisco, orient. II. Título.

Bibliotecários responsáveis pela estrutura de catalogação da publicação de acordo com a AACR2: Gláucia Maria Saia Cristianini - CRB - 8/4938

Juliana de Souza Moraes - CRB - 8/6176 


\title{
Guilherme Ferraz de Arruda
}

\section{Modelagem de processos de propagação em redes complexas}

\author{
Tese apresentada ao Instituto de Ciências \\ Matemáticas e de Computação - ICMC-USP, \\ como parte dos requisitos para obtenção do título \\ de Doutor em Ciências - Ciências de Computação e \\ Matemática Computacional. VERSÃO REVISADA \\ Área de Concentração: Ciências de Computação e \\ Matemática Computacional \\ Orientador: Prof. Dr. Francisco Aparecido Rodrigues
}

USP - São Carlos

Fevereiro de 2018 

To my family and friends. 

I would like to express my special appreciation and thanks to my advisor Professor Dr. Francisco Rodrigues and co-advisor Professor Dr. Yamir Moreno, you both have been tremendous mentors for me. I would like to thank you for encouraging my research and for allowing me to grow as a research scientist. Your advice on both research and career as well as on my personal life have been priceless. I would also like to thank my committee members, Angélica Sousa da Mata, Jose Fernando Fontanari, and, Silvio Ferreira, for serving as my committee members and for your comments, which improved the final version of this thesis. I might also specially thank Professor Dr. Pablo Martin Rodriguez, who motivated me to study further stochastic processes and professor Dr. José Antonio Méndez Bermúdez, who motivated me to study random matrix theory. I am also grateful for Professor Dr. Luciano da Fontoura Costa, who was the first to present me to the network theory. Besides, in a general comment, I would like to extend my thanks to all the researchers on complex systems and correlated areas, pushing our field to its current stage and enabling my research. I am also thankful to everyone that support open science since I believe that this is the only manner we can improve our field, and it is the only way to grow as a society.

Special thanks to my family. Words cannot express how grateful I am to my mother Ana Sylvia Batissaco de Arruda, father Juarez Antonio Ferraz de Arruda, and brother Henrique Ferraz de Arruda for all of the sacrifices that you have made on my behalf. I might not forget my brother's girlfriend, Caroline Ceribeli. Specially, I would also like to express appreciation to my beloved girlfriend Gabriela de Oliveira Alberini who supported me in the moments when there was no one to answer my queries.

In the end, I would like to thank all of my friends who supported me in this process, researching, writing, and encouraging me to strive towards my goal. Fortunately, this list is quite extensive. To begin with my friends and collaborators in Brazil, I would like to thank Thomaz Peron and Andre Luiz Barbieri. I also would like to thank my friends at my institute (in alphabetical order) Adriano Takata, Alfredo Jaramillo, Bianca Oe, Carol Viezel, Didier Vega-Oliveros, Fabiano Berardo, Hugo Checo, Irineu Lopes Palhares Junior, Josuel Kruppa Rogenski, Larissa Petri, Matheus Tozo, Marilaine Colnago, Roberto Gueleri, Stevens Paz and Wallace Casaca. Next, I cannot forget to thank my friends in Zaragoza, (in alphabetical order) Alberto Aleta, Carlos Gracia, Felipe Cardoso, Pablo Piedrahita, Sandro Meloni and Sergio Arregui Remón. I am especially grateful 
to my collaborator and friend Emanuele Cozzo, who helped and motivated me to study multilayer networks. I am also especially thankful to Alexis El Chechi Hernandez, for all his academic comments and opinions and, most of all I am grateful for his friendship and support during my stay in Zaragoza.

Throughout my Ph.D., I had the opportunity to meet a lot of people and make many friends that directly or indirectly contributed to my work. Since I will probably forget many names, I would like to express my gratitude to everyone that helped me during this period. From my friends in Brazil, who always supported me, to my Friends in Zaragoza, who welcomed me in Spain and made me feel at home.

As could not be different, I would like to thank all the professors and teachers, which helped me and motivated to pursue this Ph.D. Moreover, I would like to thank Leonardo Martinussi and Marcelo Tadeu Mirabelli Marchesoni, laboratory technicians, and friends for all your help. Last but not least, I am also thankful to all the employees in our institute (ICMC-USP), special thanks to the employees that take care of our coffee machine. Without you, none of this would be possible.

Finally, I acknowledge FAPESP for the financial support under the grants 2012/252192 and 2015/07463-1 (BEPE scholarship), without this support this thesis would not be possible. 
"Nullius in verba"

(Royal Society) 



\section{ABSTRACT}

DE ARRUDA, G. F. Modeling spreading processes in complex networks. 2018. 299 p. Tese (Doutorado em Ciências - Ciências de Computação e Matemática Computacional) - Instituto de Ciências Matemáticas e de Computação, Universidade de São Paulo, São Carlos - SP, 2018.

Mathematical modeling of spreading processes have been largely studied in the literature, and its presented a boom in the past few years. This is a fundamental task on the understanding and prediction of real spreading processes on top of a population and are subject to many structural and dynamical constraints. Aiming at a better understanding of this processes, we focused in two task: the modeling and the analysis of both dynamical and structural aspects of these processes. Initially, we proposed a new and general model that unifies epidemic and rumor spreading. Besides, regarding the analysis of these processes, we extended the classical formalism to multilayer networks, in which the theory was lacking. Interestingly, this study opened up new challenges concerning the understanding of multilayer networks. More specifically, regarding their spectral properties. In this thesis, we analyzed such processes on top of single and multilayer networks. Thus, throughout our analysis, we followed three complementary approaches: (i) analytical, (ii) numerical and (iii) simulations, mainly Monte Carlo simulations. Our main results are: (i) a new unifying model, enabling us to model and understand spreading processes on large systems, (ii) characterization of new phenomena on multilayer networks, such as layer-wise localization and the barrier effect and (iii) an spectral analysis of multilayer systems, suggesting a universal parameter and proposing a new analytical tool for its analysis. Our contributions enable further research on modeling of spreading processes, also emphasizing the importance of considering the complete multilayer structure instead of any coarse-graining. Additionally, it can be directly applied on the prediction and modeling real processes. Thus, aside from the theoretical interest and its mathematical implications, it also presents important social impact.

Keywords: Complex networks, Multilayer networks, Stochastic processes, Computational physics, Epidemic spreading. 



\section{RESUMO}

DE ARRUDA, G. F. Modelagem de processos de propagação em redes complexas. 2018. 299 p. Tese (Doutorado em Ciências - Ciências de Computação e Matemática Computacional) - Instituto de Ciências Matemáticas e de Computação, Universidade de São Paulo, São Carlos - SP, 2018.

A modelagem matemática dos processos de disseminação tem sido amplamente estudada na literatura, sendo que o seu estudo apresentou um "boom" nos últimos anos. Esta é uma tarefa fundamental na compreensão e previsão de epidemias reais e propagação de rumores numa população, ademais, estas estão sujeitas a muitas restrições estruturais e dinâmicas. Com o objetivo de entender melhor esses processos, nos concentramos em duas tarefas: a de modelagem e a de análise de aspectos dinâmicos e estruturais. No primeiro, propomos um modelo novo e geral que une a epidemia e propagação de rumores. Também, no que diz respeito à análise desses processos, estendemos o formalismo clássico às redes multicamadas, onde tal teoria era inexistente. Curiosamente, este estudo abriu novos desafios relacionados à compreensão de redes multicamadas, mais especificamente em relação às suas propriedades espectrais. Nessa tese, analisamos esses processos em redes de uma e múltiplas camadas. Ao longo de nossas análises seguimos três abordagens complementares: (i) análises analíticas, (ii) experimentos numéricos e (iii) simulações de Monte Carlo. Assim, nossos principais resultados são: (i) um novo modelo que unifica as dinâmicas de rumor e epidemias, nos permitindo modelar e entender tais processos em grandes sistemas, (ii) caracterização de novos fenômenos em redes multicamadas, como a localização em camadas e o efeito barreira e (iii) uma análise espectral de sistemas multicamadas, sugerindo um parâmetro de escala universal e propondo uma nova ferramenta analítica para sua análise. Nossas contribuições permitem que novas pesquisas sobre modelagem de processos de propagação, enfatizando também a importância de se considerar a estrutura multicamada. Dessa forma, as nossas contribuições podem ser diretamente aplicadas à predição e modelagem de processos reais. Além do interesse teórico e matemático, nosso trabalho também apresenta implicações sociais importantes.

Palavras-chave: Redes complexas, Redes de múltiplas camadas, Processos estocásticos, Física Computacional, Propagação de epidemias. 

Figure 1 - The undirected network $G=([N],[L])$, where $[N]=\{0,1,2,3,4,5\}$ and $[L]=\left\{l_{0 \rightarrow 1}, l_{0 \rightarrow 2}, l_{0 \rightarrow 3}, l_{0 \rightarrow 4}, l_{0 \rightarrow 5}, l_{5 \rightarrow 1}, l_{4 \rightarrow 5}, \ldots\right\}$, where the reciprocal edges were omitted. Its graphical representation is shown in (a), while its adjacency matrix is shown in (b). . . . . . . . . . . . . 54

Figure 2 - An example of clustering coefficient. Three network configurations that result in different values of the clustering coefficient.

Figure 3 - Comparison of three different centrality measures: degree in (a), betweenness centrality in (b) and Page Rank in (c). The size of the nodes are proportional to the centrality of the node. The network used is the São Carlos road map, extracted using the Python library OSMNX (BOEING, 2017) (Version: 0.5.1 - https://github.com/gboeing/osmnx). 58

Figure 4 - Illustration of the concept of accessibility (values calculated from Eq.2.12) in the Zachary Karate-club network (ZACHARY, 1977). Node size is also proportional to their accessibility values. Nodes at the center of the network present the highest accessibility. . . . . . . . . . . .

Figure 5 - In (a) we present the degree distribution of an Erdös and Rényi network with $N=10^{5}$ and $\mathbb{E}(k)=20$, while in the inset, a small ER network is shown for visualization purposes. In (b) we present the degree distribution of a Barabási - Albert network with $N=5 \times 10^{5}$ and $\mathbb{E}(k)=10$, where the estimated $\zeta \approx 3.0$. In the inset, a small BA network is shown for visualization purposes. This figure and its approximation of $\zeta$ were generated using the code provided in http://tuvalu.santafe.edu/ aaronc/powerlaws/ (Accessed on 4th September, 2017). . . . . . . . . . 6

Figure 6 - In (a) we present a network visualization of the Waxman model (homogeneous), while in (b) the spatial scale free model proposed by Barthélemy (heterogeneous). Each networks have $N=200$. Observe that (a) does not present hubs and long-range connections are rare, while in (b) those features are more often observed. . . . . . . . . . .

Figure 7 - Spectrum of the adjacency matrix of an Erdös and Rényi network with $n=3000$ and $\mathbb{E}(k)=10$. The red line represents the spectral distribution given by the Wigner's semicircle law. . . . . . . . . . . . . . . 68

Figure 8 - Spectrum of the Laplacian matrix of an Erdös and Rényi network with $n=3000$ and $\mathbb{E}(k)=10$. 
Figure 9 - Spectrum of the Non-backtracking matrix of an Erdös and Rényi network with $n=3000$ and $\mathbb{E}(k)=10 \ldots \ldots$. . . . . . . . . 71

Figure 10 - Schematic Illustration of the 3 multilayer networks cases considered as examples. Left panels represent the original networks which give rise to three distinct configurations for the networks of layers. See the text for more details.

Figure 11 - In (a) the graph representation of the exact Markov chain, where each node is a state, defined by its binary and integer representation. In (b) the numerical solution of the exact model for a small Erdös and Rényi network with $N=10, \mathbb{E}(k)=5, \delta=1$ and $\lambda=2$. Time is measured in

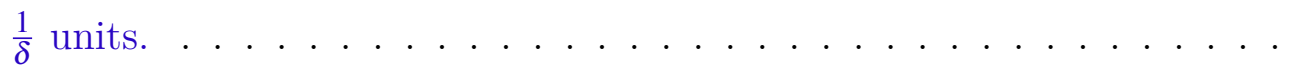

Figure 12 - Multiplex network composed by two Erdös and Rényi layers, $m=2$, and $n=10^{4}$ nodes on each layer, where the first with $\mathbb{E}(k)=16$, while the second with, $\mathbb{E}(k)=12$. The effective spreading rate $\tau=0.15$ and $\eta=0.01$. The continuous lines represents the first (QMF) and second (PQMF) order approximations on the whole population, the order parameter $\rho$, while the dot-dashed and dashed lines represents the average of infected individuals on the first and second layers, respectively. Each colored line represents one of the 50 independent Monte Carlo simulations. . . . . . . . . . . . . . . . . . . . . 123

Figure 13 - Distribution of 500 simulations over a complete graph with $N=2 \times 10^{3}$ nodes in order to validate the simulation. Comparing with the results shown in Section 3.5.1 (Equations 3.18 and 3.19) we obtained an error of 0.00014 on the average and 0.0166 on the variance of the simulations. Additionally, we performed the Kolmogorov - Smirnov test obtaining $D=0.0408$, suggesting that our data is normally distributed. . . . . . . 124

Figure 14 - Finite size analysis for a random regular graph with $\mathbb{E}(k)=10$ from $N=$ $10^{3}$ to $N=3 \times 10^{4}$ considering the fraction of infected individuals (left panel) and susceptibility (right panel) as a function of the spreading rate $\lambda$ and recovering rate $\delta=1$. The dashed lines represent the QMF predictions for the critical point.

Figure 15 - Finite size analysis for a star from $N=10^{3}$ to $N=10^{5}$ considering the fraction of infected individuals (left panel) and susceptibility (right panel) as a function of the spreading rate $\lambda$ and recovering rate $\delta=1$. The dashed lines represent the PQMF predictions for the critical point. 128 
Figure 16 - Example of discrete Monte Carlo simulations. Phase diagrams for epidemic spreading considering the $\mathrm{CA}(\mathrm{RP})$ formalism. In (a) the SIS model, considering two cases, with or without reinfections at the same time step. In (b) the SIR model. The continuous lines are the analytical solutions, while the symbols are an average of $10^{2}$ the Monte Carlo simulation, $\eta=0.5$ fixed in all simulations. The multiplex considered is composed by two scale-free networks, $P(k) \sim k^{\zeta}$, the first with $\zeta \approx 2.8$, while the second with $\zeta \approx 3.0$. The variance is the size of the symbols.

Figure 17 - Estimated errors in the bars and their standard error in the deviations. From left to right, we present the error of the SIS, SIR and MT processes with respect to the QMF and PQMF mean filed approaches in four different structures, uncorrelated, assortative, disassortative and a clustered network. The networks have $N=10^{4}$, see text for more details on their structural properties. . . . . . . . . . . . . . . . 131

Figure 18 - Estimated errors in the bars and their standard errors in the deviations. From top to bottom, we present the error of the SIS, SIR and MT processes with respect to the QMF and PQMF mean filed approaches in four different structures: ARR (Assortative+RRN - randomly coupled), AAA (Assortative+Assortative - Assortativelly coupled), ACA (Assortative+Clustered - Assortativelly coupled) and ACR (Assortative+Clustered - randomly coupled). From left to right, we change the coupling parameter $\eta=10^{-3}, \eta=10^{-2}, \eta=10^{-1}$. The networks have $n=10^{4}$ and $m=2$, see text for more details on their structural properties. 134

Figure 19 - Estimated errors in the bars and their standard errors in the deviations. From above to bottom, we present the error of the SIS with reinfections, SIS and SIR processes with respect to the CA approach in four different structures: ARR (Assortative+RRN - randomly coupled), AAA (Assortative+Assortative - Assortativelly coupled), ACA (Assortative+Clustered - Assortativelly coupled) and ACR (Assortative+Clustered - randomly coupled). From left to right, we change the coupling parameter $\eta=10^{-3}, \eta=10^{-2}, \eta=10^{-1}$. The networks have $n=10^{4}$ and $m=2$, see text for more details on their structural properties. 135

Figure 20 - Finite size scaling for a power-law network with $\mathbb{P}(k) \sim k^{-2.25}$. In (a) the susceptibility as a function of the spreading rate $\lambda$ with $\delta=1$, while in (b) the scaling of the the susceptibility peak and its respective fraction of infected individuals, both obtained using the QS algorithm. 136 
Figure 21 - Finite size scaling for a power-law network with $\mathbb{P}(k) \sim k^{-2.75}$. In (a) the susceptibility as a function of the spreading rate $\lambda$ with $\delta=1$, while in (b) the scaling of the the susceptibility peak and its respective fraction of infected individuals, both obtained using the QS algorithm.

Figure 22 - Finite size scaling for a power-law network with $\mathbb{P}(k) \sim k^{-3.5}$. In (a) the susceptibility as a function of the spreading rate $\lambda$ with $\delta=1$, while in (b) the scaling of the the susceptibility peak and its respective fraction of infected individuals, both obtained using the QS algorithm. . . . . . 137

Figure 23 - Evaluation of the HMF, QMF and PQMF mean field predictions for the critical point considering different system sizes. The critical points were estimated using the QS algorithm on top of three different uncorrelated power-law networks with: $\zeta=2.25$ in (a), $\zeta=2.75$ in (b) and $\zeta=3.5$ (rigid cutoff) in (c) . . . . . . . . . . . . . . 140

Figure 24 - A simplified diagram of all possible transitions between the three different dynamical states in our model. $X, Y$ and $Z$ stand for ignorant or susceptible, spreader and stifler or recovered, respectively. Most of the transitions involve interactions between two individuals, however, spontaneous ones are also allowed (represented by dashed lines). . . . . 147

Figure 25 - Phase diagram of the steady state $(t \rightarrow \infty)$ considering the complete infinity graph. The dynamical parameters have been set to $\delta_{1}=\delta_{2}=$ $\gamma=0.1, \lambda=1, \beta=0.5$ and $\eta=1 \ldots \ldots \ldots . \ldots \ldots$. . . . . . . . . . . .

Figure 26 - Numerical evaluation of equation (4.6) considering the RP for $\beta=$ $0, \delta_{1}=\gamma=0.25, \delta_{2}=0.2$ and varying the spreading probability $\lambda$. The network considered is a scale-free network with $\zeta \approx 2.7, N=10^{4}$ and $\mathbb{E}(k) \approx 10$. The dashed line indicates the critical point (Equation $((4.18)))$ for $\eta=1$, whereas the continuous line corresponds to the critical value for the case in which $\eta=0.5 . \ldots . . . . .157$

Figure 27 - Numerical evaluation of Equation (4.6) considering the CP with parameters $\delta_{1}=\gamma=0.15, \delta_{2}=0.1$ and varying the spreading probability $\lambda$. The network considered is a scale-free network with $\zeta \approx 2.7, N=10^{4}$ and $\mathbb{E}(k) \approx 100$. In panel (a) $\eta=0.5$ and $\alpha=1$, whereas in (b) we have set $\eta=1$ and also explored the limiting cases of $\alpha=0$ and $\alpha=1$. The vertical lines correspond to the critical point Equation(4.18) evaluated for the parameters used here. . . . . . . . . . . . . . . . . 158

Figure 28 - Numerical evaluation of Equation (4.6) considering the CP and, without loss of generality, fixing the parameters $\delta_{1}=\gamma=\delta_{2}=0.2, \lambda=1$ and varying the parameter $\beta$. The network considered is a scale-free network with $\zeta \approx 2.7, N=10^{4}$ and $\mathbb{E}(k) \approx 100$. . . . . . . . . . 159 
Figure $29-(\boldsymbol{\lambda}, \boldsymbol{\beta})$ phase diagrams at the stationary state for the CP case on a scale-free network with degree distribution $P(k) \sim k^{-\zeta}$ and $\zeta \approx 2.7$. The network is made up of $N=10^{4}$ nodes and has an average degree $\mathbb{E}(k) \approx 100$. The rest of the dynamical parameters have been set to $\eta=0.01, \delta_{1}=\delta_{2}=\gamma=0.1$ and $\alpha=1.0$. The intensity of the color (as given by the legend) represents the final fraction of ignorants (panel (a)), spreaders (panel (b)) and stiflers (panel (c)). The continuos lines are the analytical values for the critical point.

Figure $30-(\boldsymbol{\lambda}, \boldsymbol{\beta})$ phase diagrams as in Figure 29 but for the RP. The underlying network of contacts has the same features except for the average degree that is $\mathbb{E}(k) \approx 10$. The rest of the parameters are $\eta=0.01, \delta_{1}=\delta_{2}=$ $\gamma=0.25$ and $\alpha=1.0$. The intensity of the color (as given by the legend) represents the final fraction of ignorants (panel (a)), spreaders (panel

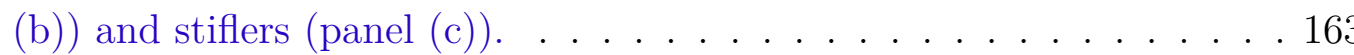

Figure 31 - Phase diagram at the steady state for (a) a CP considering a scale-free network with $\zeta \approx 2.7, N=10^{4}$ and $\mathbb{E}(k) \approx 100$, and $(\mathrm{b})$ a RP simulated on top of a scale-free network with $\zeta \approx 2.7, N=10^{4}$ and $\mathbb{E}(k) \approx 10$. The simulations were carried out using the following dynamical parameters: $\delta_{1}=\delta_{2}=\gamma=0.1, \lambda=1, \beta=0.5$ and $\eta=1$. The continuous lines are the theoretical predictions, whereas the symbols are the results of $\mathrm{MC}$ simulations. The standard deviation has approximately the size of the symbols.

Figure 32 - Probability of finding a node $i$ in any of the dynamical states of the model ( $X, Y$ or $Z$ ) obtained by solving analytically the system of Equations (4.6) as compared with Monte Carlo simulations. The probabilities are calculated by averaging $10^{3}$ simulations. Panel (a) corresponds to the CP, whereas the RP is represented in panel (b). . . . . . . . 165 
Figure 33 - Phase diagram of the system's dynamics at the steady state as a function of $\alpha$. In all panels, the rest of the parameters were fixed to $\delta_{1}=\delta_{2}=\gamma=0.1, \lambda=1, \beta=0.5$ and $\eta=1$ and the continuous lines are the theoretical predictions, while the symbols correspond to the MC simulations. Panels (a) and (b) show results obtained varying the exponent $\zeta$ of the power law degree distribution, $P(k) \sim k^{-\zeta}$, of the underlying networks of contacts of size is $N=10^{4}$ and $\mathbb{E}(k) \approx 100$ $(\mathrm{CP}$, panel $(\mathrm{a}))$ and $\mathbb{E}(k) \approx 10(\mathrm{RP}$, panel $(\mathrm{b}))$. We considered the following exponents $\zeta$ : $2.3(\bullet), 2.5(\times), 2.7(\square), 3.3(\nabla)$ and $3.5(\diamond)$. The effects of the network size are shown in Panel (c) for scale-free networks $\left(P(k) \sim k^{-\zeta}\right.$ with $\left.\zeta \approx 2.7\right)$ with $\mathbb{E}(k) \approx 100$ (main plot) and $\mathbb{E}(k) \approx 10$ (inset) and the following sizes: $N=5 \times 10^{2}(\bullet), N=10^{3}(\square)$, $N=5 \times 10^{3}(\diamond)$ and $N=10^{4}(\nabla)$. Finally, in panel (d), we represent results obtained for the $\mathrm{RP}$ and different average degrees: $\mathbb{E}(k) \approx 10$ $(\bullet), \mathbb{E}(k) \approx 20(\times), \mathbb{E}(k) \approx 35(\square), \mathbb{E}(k) \approx 45(\nabla)$ and $\mathbb{E}(k) \approx 60(\diamond)$. The rest of the network's parameters are the same as in panel (c). . . . 166

Figure 34 - Time evolution of the average probabilities for the (a) CP and (b) RP. The continuous lines are the theoretical predictions, while the symbols are obtained by Monte Carlo simulations. . . . . . . . . . . . . . . . 168

Figure 35 - Phase diagram at the steady state for the CP considering a scale-free network with $\zeta \approx 2.7, N=10^{4}$ and the dynamical parameters $\delta_{1}=$ $\delta_{2}=\gamma=0.1$ and $\lambda=1$. The continuous lines are obtained by the least squares method. . . . . . . . . . . . . . . . . 169 
Figure 36 - Application of the proposed discrete time Markov chain formulation to the analysis of spreading processes that take place on top of social networks. Panels (a) and (b) show the probability of node $i$ belonging to state $X, Y$ or $Z$ obtained by solving numerically the system of Equations (4.6)) and by Monte Carlo simulations. Panel (a) corresponds to a RP that takes place over the Twitter network, where as panel (b) has been obtained simulating a $\mathrm{CP}$ on the email network. The model parameters: $\lambda=1.0, \eta=0.01, \delta_{1}=\delta_{2}=\gamma=0.01, \beta=1.0$ and $\alpha=1.0$ for the RP; and $\lambda=1.0, \eta=0.01, \delta_{1}=\delta_{2}=\gamma=0.1, \beta=1.0$ and $\alpha=1.0$. Results for MC simulations are averages over $10^{3}$ different simulations. Panel (c) depicts the time evolution of the Higgs boson rumor spreading, taking into account tweets of the dataset shown in (De Domenico et al., 2013). The dashed lines represent the real data, while the continuous lines are the numerical solutions of our model. The vertical lines mark the three time windows used, with the following parameters: (i) $0 \leq t \leq 77, \lambda=0.00025, \alpha=0.0002, \delta_{2}=0.0001$ and $\eta=0.85$; (ii) $77<t \leq 106, \lambda=0.021, \alpha=0.00075, \delta_{2}=0.0015$ and $\eta=0.17$; (iii) $t>106, \lambda=0.065, \alpha=0.002, \delta_{2}=0.002$ and $\eta=0.01 .170$

Figure 37 - Correlation between spreading capacity and the degree, $k$, k-core index, $k_{c}$, and accessibility, $\alpha$, covering all possible combinations of parameters $((\boldsymbol{\lambda}, \boldsymbol{\delta})$ for epidemic spreading (SIR model) and $(\boldsymbol{\lambda}, \alpha)$ for rumor propagation (MT-TP model), for the road networks of US and Germany. 176

Figure 38 - Correlation between spreading capacity and the degree, $k$, k-core index, $k_{c}$, and accessibility, $\alpha$, covering all possible combinations of parameters $((\boldsymbol{\lambda}, \boldsymbol{\delta})$ for epidemic spreading (SIR model) and $(\boldsymbol{\lambda}, \alpha)$ for rumor propagation (MT-TP model), for the email contact network and political blogs (polblogs). . . . . . . . . . . . . . . . . . . . 177

Figure 39 - The percentage of recovered individuals in the SIR epidemic spreading model $(\lambda=0.3, \delta=1.0)$ according to the local measures for the US road network: (a) degree; (b) clustering coefficient; (c) average degree of the nearest neighbors; (d) betweenness centrality; (e) PageRank; (f) k-core index; (g) eigenvector centrality and (h) closeness centrality. . . 178

Figure 40 - The percentage of stiflers on the MT (TP) rumor model $(\lambda=0.3, \alpha=$ 1.0) according to the local measures for the United States network: (a) degree; (b) clustering coefficient; (c) average degree of the nearest neighbors; (d) betweenness centrality; (e) PageRank; (f) k-core index; (g) eigenvector centrality and $(\mathrm{h})$ closeness centrality. . . . . . . . . . 179 
Figure 41 - The percentage of recovered individuals on the SIR epidemic spreading model $(\lambda=0.3, \delta=1.0)$ according to the accessibility measure for the road networks of (a) Japan, (b) England, (c) United States and (d) Germany

Figure 42 - The percentage of stiflers on the MT (TP) rumor model $(\lambda=0.3, \alpha=$ 1.0) according to the accessibility measure for the road networks of (a) Japan, (b) England, (c) United States and (d) Germany. . . . . . . . . 180

Figure 43 - Network visualization of the real road networks of (a) Japan, (b) England, (c) United States and (d) Germany. The colors represent the values of the accessibility. . . . . . . . . . . . . . . . . . 182

Figure 44 - Probability distribution of the accessibility in the road networks of Japan, Germany, US and England. . . . . . . . . . . . . . . 183

Figure 45 - Probability distribution of the accessibility in the social networks of advogato, email, political blogs and Google+. . . . . . . . . . . . . 183

Figure 46 - Scatter plots of the accessibility measure and the final percentage of recovered individuals on the SIR epidemic spreading model $(\lambda=0.3, \delta=$ 1.0) for the networks of (a) advogato, (b) political blogs, (c) e-mail, (d) Google+. . . . . . . . . . . . . . . . . . . . . . 185

Figure 47 - Scatter plots of the accessibility measure and the final percentage of stiflers on the MT (TP) rumor model $(\lambda=0.3, \alpha=1.0)$ for the networks of (a) advogato, (b) political blogs, (c) e-mail, (d) Google+. . . . . . . 185

Figure 48 - Phase diagrams over a 2-Layer multiplex system, where each layer is a scale-free network with $n=10^{4}$ nodes, for a fixed value of $\delta=1$. (a) Density of spreaders as a function of the parameters $\eta$ and $\lambda$. (b) Density of recovered individuals as a function of the parameters $\eta$ and $\lambda$. Colors represent the fraction of spreaders and the white line is the threshold calculated using Equation 3.47. . . . . . . . . . . . . . . . . . 194

Figure 49 - Individual layer behavior over a 2-Layer multiplex system. Each layer has $n=10^{4}$ for a fixed value of $\delta=1$. The results considering both layers are shown in (a), while the dynamics in the individual layers are shown in (b) $\left(P(k) \sim k^{-2.5}\right)$ and $(\mathrm{c})\left(P(k) \sim k^{-4.5}\right)$. The arrows indicate the leading eigenvalues of the layers.

Figure 50 - Spectral properties of the tensor $\mathscr{R}(\lambda, \eta)$ as a function of the ratio $\frac{\eta}{\lambda}$ for a multiplex with two layers, the first with $\zeta \approx 2.2$, while the second $\zeta \approx 2.8$. Both have $\mathbb{E}(k) \approx 8$. In the left panel, we present the inverse participation ratio $(\operatorname{IPR}(\Lambda))$ of the two larger eigenvalues and the individual layer contributions, while in the right panel, we show the leading eigenvalues. Every curve is composed by $10^{3} \log$ spaced points, in order to have enough resolution. . . . . . . . . . . . 196 
Figure 51 - Diagram of the contribution of each layer to the $\operatorname{IPR}(\Lambda)$ for different values of the spreading ratio $\frac{\eta}{\lambda}$. The dashed line represents the case where both layers have the same contribution, i.e. a line with a slope one. In the inset we show the angle $\theta$ between the vector composed by the contributions of each layer to the $\operatorname{IPR}(\Lambda), v=\left[\operatorname{IPR}\left(\Lambda_{1}^{1}\right), \operatorname{IPR}\left(\Lambda_{1}^{2}\right)\right]^{T}$, and the $x$-axis. The multiplex network used here is composed of two Erdös-Rényi networks, both with $n=5 \times 10^{4}$, the first layer $\mathbb{E}(k)=16$ $\left(\left(\Lambda_{1}^{1}\right)^{-1} \approx 0.0625\right)$, while the second $\mathbb{E}(k)=12\left(\left(\Lambda_{1}^{2}\right)^{-1} \approx 0.0833\right) . .$.

Figure 52 - Susceptibility, $\chi$, as a function of the spreading rate $\lambda$ for different ratios of inter and intra-layer spreading ratings, $\frac{\eta}{\lambda}$ for a fixed value of $\delta=1$ over a 2-Layer multiplex system, where each layer has $n=10^{3}$, the first with $\zeta \approx 2.2$, while the second $\zeta \approx 2.8$. Both have $\mathbb{E}(k) \approx 8$. The simulated values are $\frac{\eta}{\lambda}=0.1,0.2,0.3,0.4,0.5,0.6,0.7,0.8,0.9$, $1.0,1.1,1.2,1.3,1.4,1.5,1.6,2,3,4,5,6,7,8,9,10,20,30 \ldots 200$

Figure 53 - Time evolution of the fraction of infected nodes in the second layer for $\delta=1$, different values of $\eta=10^{-4}, 10^{-3}, 10^{-2}, 10^{-1}$ and different values of spreading rate: (a) $\lambda=0.078$, (b) $\lambda=0.083$, (c) $\lambda=0.085$ and (d) $\lambda=0.088$. The multiplex network used is composed of two Erdös-Rényi networks, both with $n=5 \times 10^{4}$, the first layer $\mathbb{E}(k)=16$ $\left(\left(\Lambda_{1}^{1}\right)^{-1} \approx 0.0625\right)$, while the second $\mathbb{E}(k)=12\left(\left(\Lambda_{1}^{2}\right)^{-1} \approx 0.0833\right) . .201$

Figure 54 - Final number of infected nodes in the second layer (with lowest individual eigenvalue) as a function of the size of the layers on the main panels, while in the insets we present the fraction of infected nodes on the left and the standard deviation in the steady state on the right. The parameters used in the simulations are shown on the tile of each panel. They are a combination of the parameters $\lambda=0.078,0.083,0.085,0.088$ and $\eta=10^{-4}, 10^{-3}, 10^{-2}, 10^{-1}$. Furthermore, the layer sizes are $n=$ $2 \times 10^{3}, 3 \times 10^{3}, 4 \times 10^{3}, 5 \times 10^{3}, 6 \times 10^{3}, 7 \times 10^{3}, 8 \times 10^{3}, 9 \times 10^{3}, 10^{4}, 2 \times$ $10^{4}, 3 \times 10^{4}, 4 \times 10^{4}$ and $5 \times 10^{4}$ and $m=2$ in all cases.

Figure 55 - Final fraction of infected nodes on the layer with lowest individual eigenvalue as a function of the the size of the layers. The colors represent different values of $\eta$, while on we have $\lambda=0.078$ in (a), $\lambda=0.083$ in (b) $\lambda=0.085$ in (c) and $\lambda=0.088$ in (d). Furthermore, the layer sizes are $n=2 \times 10^{3}, 3 \times 10^{3}, 4 \times 10^{3}, 5 \times 10^{3}, 6 \times 10^{3}, 7 \times 10^{3}, 8 \times$ $10^{3}, 9 \times 10^{3}, 10^{4}, 2 \times 10^{4}, 3 \times 10^{4}, 4 \times 10^{4}$ and $5 \times 10^{4}$ and $m=2$ in all cases. Each curve is the result of a parameter $\eta$, from bottom to top $\eta=10^{-4}, 10^{-3}, 10^{-2}, 10^{-1} \ldots \ldots \ldots \ldots$. . . . . . . . . . . . . . . . 
Figure 56 - Finite size analysis of the susceptibility. In the main panel, we have the susceptibility as a function of $\lambda$ for different sizes of 2 layer multiplex network, where the first layer have $\mathbb{E}(k)=16$ and the second $\mathbb{E}(k)=12$. In this experiment, we fixed the ratio $\frac{\eta}{\lambda}=0.01$. In the inset, we show the susceptibility of the two peaks as a function of the layer size, where the blue symbols refer to the first peaks, while the green symbols refer to the second peak. Besides, the red lines are a linear fitting of these points. The layer sizes evaluated are $n=3 \times 10^{3}, 4 \times 10^{3}, 5 \times 10^{3}, 6 \times$ $10^{3}, 7 \times 10^{3}, 8 \times 10^{3}, 9 \times 10^{3}, 10^{4}, 2 \times 10^{4}, 3 \times 10^{4}, 4 \times 10^{4}, 5 \times 10^{4}, 10^{5}$. . 205

Figure 57 - Spectral properties of the tensor $\mathscr{R}(\lambda, \eta)$ as a function of the ratio $\frac{\eta}{\lambda}$ for a multiplex with two layers with the same degree distribution (different random realizations of the configuration model) and connected to its counterpart on the other layer. In the left panel, we present the inverse participation ratio $(\operatorname{IPR}(\Lambda))$ of the two larger eigenvalues and the individual layer contributions, while in right panel, we show the leading eigenvalues. Every curve is composed by $10^{3} \log$ spaced points, in order to have enough resolution. In (a) we have the line $(2.3+2.9+2.6)$, while in (b) the multiplex case. . . . . . . . . . 207

Figure 58 - Susceptibility $\chi$ as a function of $\lambda$ considering all three layer configurations and many different ratios $\frac{\eta}{\lambda}$, which is represented by the color of the lines. The recovering rate is $\delta=1$. The simulated values are $\frac{\eta}{\lambda}=$ $0.05,0.06,0.07,0.08,0.09,0.1,0.2,0.3,0.4,0.5,0.6,0.7,0.8,0.9,1.0$, $2,3,4,5,6,7,8,9,10,20$. In (a) we have the line $(2.3+2.9+2.6)$, while in (b) the multiplex case. . . . . . . . . . . . . . . 208

Figure 59 - Example of an airport transportation multilayer network. Each layer represents an airline, in which each node represents an airport and the edges are flights between two airports. This visualization was generated using MuxViz (De Domenico; PORTER; ARENAS, 2014) . . . . . . . . 212

Figure 60 - Pair-wise assortativity coefficient using Spearman rank correlation, $\rho^{S}\left(\mathbf{S}_{\beta}^{\alpha}\right)$. Observe that the main diagonal presents the same coefficient considering the layer extraction operation, $\rho^{S}\left(C_{\beta}^{\alpha}(\tilde{r} \tilde{r})\right) \ldots \ldots . \ldots . . \ldots 215$

Figure 61 - Pair-wise assortativity coefficient using Spearman rank correlation, $\rho^{S}\left(\mathbf{Z}_{\beta}^{\alpha}\right)$. Observe that the main diagonal presents the same coefficient considering the layer extraction operation, $\rho^{S}\left(C_{\beta}^{\alpha}(\tilde{r} \tilde{r})\right) \ldots \ldots . \ldots . . \ldots 216$ 
Figure 62 - Phase diagram for epidemic spreading on different levels of assortativity. (a) First layer with $\zeta \approx 3$ and $\mathbb{E}(k) \approx 17$, and second with $\zeta \approx 2.8$ and $\mathbb{E}(k) \approx 12$. (b) First layer with $\zeta \approx 3$ and $\mathbb{E}(k) \approx 17$, and second with $\zeta \approx 4.5$ and $\mathbb{E}(k) \approx 13$. All networks are composed by $n=10^{4}$ nodes. We adopt $\frac{\eta}{\lambda}=2$ and $\delta=1$. The continuous lines are the analytical solution (Equation 3.82), while the symbols are obtained from Monte Carlo simulations, averaging over $10^{2}$ runs. The standard deviation is of the size of the symbols.

Figure 63 - Spectral properties of the tensor $\mathscr{R}(\lambda, \eta)$ as a function of the ratio $\frac{\eta}{\lambda}$ for a multiplex with two layers with the exact same degree distribution and connected to its counterpart on the other layer. In the right panel, we present the inverse participation ratio $(\operatorname{IPR}(\Lambda))$ of the three larger eigenvalues, while in the left panel, we show the leading eigenvalues. Every curve is composed by $10^{3} \log$ spaced points, in order to have enough resolution.

Figure 64 - Spectral properties of the tensor $\mathscr{R}(\lambda, \eta)$ as a function of the ratio $\frac{\eta}{\lambda}$ for a multiplex with two layers with the same degree distribution (different random realizations of the configuration model) and connected to its counterpart on the other layer. In the right panel, we present the inverse participation ratio $(\operatorname{IPR}(\Lambda))$ of the two larger eigenvalues and the individual layer contributions, while in the left panel, we show the leading eigenvalues. Every curve is composed by $10^{3} \mathrm{log}$ spaced points, in order to have enough resolution. . . . . . . . . . . . . . . .

Figure 65 - Distribution of the eigenvalues. On the rows, from top to bottom, for the interconnected networks of Lines $2.3+2.6+2.9,2.3+2.9+2.6$, $2.6+2.3+2.9$ and the multiplex. On the columns, from left to right, we varied the ratios $\frac{\eta}{\lambda}=1,10,100$ and 1000 respectively. All histograms were built with 100 bins.

Figure 66 - (a) Scaled localization length $\beta$ as a function of $x=b_{\text {eff }}^{2} / N$ for ensembles of multilayer networks characterized by the sparsity $\tilde{\alpha}$. The dashed line close to the data for $\tilde{\alpha}=0.8$ is Equation (6.6) with $\tilde{\gamma}=1.4$. Horizontal dot-dashed lines at $\beta \approx 0.12$ and 0.88 are shown as a reference, see the text. (b) Logarithm of $\beta /(1-\beta)$ as a function of $\ln (x)$. Upper inset: Power $\delta$, from the fittings of the data with Equation (6.10), as a function of $\tilde{\alpha}$. Lower inset: Enlargement in the range $\ln [\beta /(1-\beta)]=$ $[-2,4]$ including data for $\tilde{\alpha}=0.6,0.8$, and 1 . Lines are fittings of the data using Equation (6.10). 
Figure $67-$ (a) $\beta$ as a function of $x^{*}$ [as defined in Equation (6.12)] for ensembles of multilayer networks with $\tilde{\alpha} \in[0.2,1]$ in steps of 0.05 . Inset: Data for $\tilde{\alpha} \in[0.5,1]$ in steps of 0.05. Dashed lines are Equation (6.13). (b) Logarithm of $\beta /(1-\beta)$ as a function of $\ln \left(x^{*}\right)$ for $\tilde{\alpha} \in[0.2,1]$ in steps of 0.05. Inset: Enlargement in the range $\ln [\beta /(1-\beta)]=[-2,2]$ including curves for $\tilde{\alpha} \in[0.5,1]$ in steps of 0.05. Dashed lines are Equation (6.11). 232

Figure 68 - (a) Scaled localization length $\beta$ as a function of $x=b_{\text {eff }}^{2} / N$ for ensembles of weighted multiplex networks characterized by the sparsity $\tilde{\alpha}$. The black dashed line corresponds to Equation 6.6 with $\tilde{\gamma}=1.4$. Horizontal black dot-dashed lines at $\beta \approx 0.12$ and 0.88 are shown as a reference, see the text. (b) Logarithm of $\beta /(1-\beta)$ as a function of $\ln (x)$. Upper inset: Power $\delta$, from the fittings of the data with Equation 6.8, as a function of $\tilde{\alpha}$. Lower inset: Enlargement in the range $\ln [\beta /(1-\beta)]=[-2,2]$ including data for $\tilde{\alpha}=0.6,0.8$, and 1 . Lines are fittings of the data with Equation 6.8. . . . . . . . . . . . . . . . 234

Figure $69-$ (a) $\beta$ as a function of $x^{*}$ [as defined in Equation 6.10] for ensembles of weighted multiplex networks with $\tilde{\alpha} \in[0.2,1]$ in steps of 0.05 . Inset: Data for $\tilde{\alpha} \in[0.5,1]$ in steps of 0.05 . Dashed lines in main panel and inset are Equation 6.11. (b) Logarithm of $\beta /(1-\beta)$ as a function of $\ln \left(x^{*}\right)$ for $\tilde{\alpha} \in[0.2,1]$ in steps of 0.05 . Inset: Enlargement in the range $\ln [\beta /(1-\beta)]=[-2,2]$ including curves for $\tilde{\alpha} \in[0.5,1]$ in steps of 0.05 . Dashed lines in main panel and inset are Equation 6.9 . . . . . . . . . 235

Figure 70 - (a) Scaled localization length $\beta$ as a function of $x=b_{\text {eff }}^{2} / N$ for ensembles of unweighted multiplex networks characterized by the sparsity $\tilde{\alpha}$. The black dashed line corresponds to Equation 6.6 with $\tilde{\gamma}=1.4$. Horizontal black dot-dashed lines at $\beta \approx 0.5$ and 0.98 are shown as a reference, see the text. (b) Logarithm of $\beta /(1-\beta)$ as a function of $\ln (x)$. Lower inset: Enlargement in the range $\ln [\beta /(1-\beta)]=[-2,4]$ including data for $\tilde{\alpha}=0.6,0.7$, and 0.85. Lines are fittings of the data with Equation 6.9.236

Figure $71-$ (a) $\beta$ as a function of $x^{*}$ [as defined in Equation 6.10] for ensembles of unweighted multiplex networks with $\tilde{\alpha} \in[0.2,1]$ in steps of 0.05 . Inset: Data for $\tilde{\alpha} \in[0.5,1]$ in steps of 0.05 . Dashed lines in main panel and inset are Equation 6.11. (b) Logarithm of $\beta /(1-\beta)$ as a function of $\ln \left(x^{*}\right)$ for $\tilde{\alpha} \in[0.2,1]$ in steps of 0.05 . Inset: Enlargement in the range $\ln [\beta /(1-\beta)]=[-1,4]$ including curves for $\tilde{\alpha} \in[0.5,1]$ in steps of 0.05 . Dashed lines in main panel and inset are Equation 6.9 . . . . . . . . 237

Figure $72-(\mathrm{a}) \delta$ and (b) $\tilde{\gamma}$ as a function of $\tilde{\alpha}$ for ensembles of unweighted multiplex networks (symbols). Continuous lines are cubic spline interpolations of the data. . . . . . . . . . . . . . . . . . . . . . 2 . . . . . . . . . . . . . . . . 
Figure $73-\ln [\beta /(1-\beta)]$ as a function of $\ln \left(x^{*}\right)$ for several real-world multiplex networks, see symbol code in Table 8. Dashed line is Equation (6.11).

Figure 74 - Reproduction of a similar experiment as presented in (RADICCHI; ARENAS, 2013) for the algebraic connectivity and the Fiedler vector, here divided as $v=\left[v_{a}, v_{b}\right]$. Additionally, we also show our prediction of the structural transition.

Figure 75 - Evaluation of the eigenvalues $\lambda(\mathbf{L})$ as a function of the coupling parameter $p$ of a multiplex network composed by two Erdös Renyi layers with $n=10^{3}$ nodes and the first layer have average degree $\mathbb{E}(k)=12$, while the second with $\mathbb{E}(k)=16$. The continuous lines are the upper bounds, while the dashed lines the lower bounds. . . . . . . . . . . 245

Figure 76 - Evaluation of the eigenvalues $\lambda(\mathbf{L})$ as a function of the coupling parameter $p$ of a multiplex network composed by two Erdös Renyi layers with $n=10^{3}$ nodes and the first layer have average degree $\mathbb{E}(k)=$ 12, while the second with $\mathbb{E}(k)=16$. The coupling matrix is $\mathbf{D}=$ $\frac{n}{\sum_{i}^{n}{ }^{n}} \operatorname{diag}(1,2, \ldots, n)$. The continuous lines is the adapted upper bound, while the dashed line is the adapted lower bound.

Figure 77 - Evaluation of the eigenvalues $\lambda_{i}(\mathbf{A})$ as a function of the coupling parameter $p$ of a multiplex network composed by two Erdös Renyi layers with $n=10^{3}$ nodes and the first layer have average degree $\mathbb{E}(k)=12$, while the second with $\mathbb{E}(k)=16$. The continuous lines are the upper bounds, while the dashed lines the lower bounds. . . . . . . . . . . . 249

Figure 78 - Evaluation of the eigenvalues $\lambda_{i}(\mathbf{L})$ as a function of the coupling parameter $p$ of a multiplex network composed by two Erdös Renyi layers with $n=10^{3}$ nodes and the first layer have average degree $\mathbb{E}(k)=$ 12, while the second with $\mathbb{E}(k)=16$. The coupling matrix is $\mathbf{D}=$ $\frac{n}{\sum_{i}^{n} i} \operatorname{diag}(1,2, \ldots, n)$. The continuous lines is the adapted upper bound, while the dashed line is the adapted lower bound. . . . . . . . . . . . 250

Figure 79 - Evaluation of the eigenvalues $\lambda_{i}(\mathbf{A})$ as a function of the coupling parameter $p$ of a multiplex network composed by two Erdös Renyi layers with $n=10^{3}$ nodes and the first layer have average degree $\mathbb{E}(k)=12$, while the second with $\mathbb{E}(k)=16$. The coupling matrix is sparse. The continuous lines is the adapted upper bound, the dashed line is the adapted lower bound and the dotted line was obtained experimentally at the largest value of $p$ and extended for reference. . . . . . . . . . . 251 
Figure 80 - (Color online) Accessibility calculated in star (from Eqs. A.7 and A.8), complete, ring and line graphs (extreme nodes) compared to the maximum value obtained in Erdös-Rényi (ER) random graphs, scale-free networks of Barabási-Albert (BA), and spatial scale-free networks (SSF). $N$ is the network size. For complex networks, each point is an average over 50 networks with $\langle k\rangle \approx 4 \ldots \ldots . \ldots . \ldots . . \ldots 275$

Figure 81 - Evaluation of the 8 first eigenvalues of $\mathscr{R}(\lambda, \eta)$ for the multiplex configuration as a function of of the ratio $\frac{\eta}{\lambda}$. It is noteworthy that such plot is visually equivalent for all the layer topologies composed by 3 layers. The dashed lines represents the individual layer leading eigenvalues. . . 288

Figure 82 - Spectral properties of the tensor $\mathscr{R}(\lambda, \eta)$ as a function of the ratio $\frac{\eta}{\lambda}$ for a multiplex with two layers with the same degree distribution (different random realizations of the configuration model) and connected to its counterpart on the other layer. In the left panel we present the inverse participation ratio $(\operatorname{IPR}(\Lambda))$ of the two larger eigenvalues and the individual layer contributions, while in the right panel we show the leading eigenvalues. Every curve is composed by $10^{3} \log$ spaced points, in order to have enough resolution. . . . . . . . . . . . . . . . . 289

Figure 83 - Susceptibility $\chi$ as a function of $\lambda$ considering all three layer configurations and many different ratios $\frac{\eta}{\lambda}$, which is represented by the color of the lines. The recovering rate is $\mu=1$. The simulated values are $\frac{\eta}{\lambda}=$ $0.05,0.06,0.07,0.08,0.09,0.1,0.2,0.3,0.4,0.5,0.6,0.7,0.8,0.9,1.0$, $2,3,4,5,6,7,8,9,10,20 \ldots \ldots \ldots \ldots \ldots \ldots \ldots \ldots \ldots \ldots \ldots$

Figure 84 - Phase diagrams comparing the accuracy of QMF and PQMF mean field approaches considering different levels of inter-layer coupling and the AAA (Assortative+Assortative - Assortativelly coupled) network. For more see Section 3.7.6.2 . . . . . . . . . . . . . . . . . . . . 296

Figure 85 - Phase diagrams comparing the accuracy of QMF and PQMF mean field approaches considering different levels of inter-layer coupling and the ACA (Assortative+Clustered - Assortativelly coupled) network. For more see Section 3.7.6.2 . . . . . . . . . . . . . . . . . . 297

Figure 86 - Phase diagrams comparing the accuracy of QMF and PQMF mean field approaches considering different levels of inter-layer coupling and the ACR (Assortative+Clustered - randomly coupled) network. For more see Section 3.7.6.2 . . . . . . . . . . . . . . . . . . . . . . . 298

Figure 87 - Phase diagrams comparing the accuracy of QMF and PQMF mean field approaches considering different levels of inter-layer coupling and the ARR (Assortative+RRN - randomly coupled) network. For more see Section 3.7.6.2. . . . . . . . . . . . . . . . . . . . . . 299 
Table 1 - Structure and spectra of the normalized network of layers $\Phi_{\tilde{\delta}}^{\tilde{\gamma}}(\lambda, \eta)$. The eigenvalues assume that the average degree of each layer, $\mathbb{E}\left(k^{l}\right)$, is the same, i.e. $\mathbb{E}\left(k^{l}\right)=\mathbb{E}(k), \forall l$. . . . . . . . . . . . . . 80

Table 2 - A brief literature review: A summary of previous epidemic and rumor spreading models. The states are (i) susceptible or ignorant $(X)$, (ii) infected or spreader $(Y)$ and (iii) recovered or stifler $(Z)$. . . . . . . . 89

Table 3 - Structural properties of the complex networks. . . . . . . . . . . 175

Table 4 - Correlation between centrality measures and the final fraction of recovered individuals (SIR model) or the final fraction of stiflers (MT model for the contact (CP) or truncated (TP) cases). The measures are the degree $(k)$, clustering coefficient $(c c)$, betweenness centrality $(B)$, average neighborhood degree $(r)$, PageRank $(\pi)$, eigenvector centrality $(x)$, k-core index $\left(k_{c}\right)$, closeness centrality $(C)$ and accessibility $(\alpha)$. The highest correlations are in bold. . . . . . . . . . . . . . . 181

Table 5 - Correlation between centrality measures and the final fraction of stiflers (MT model for the contact (CP) or truncated (TP) cases) or recovered individuals (SIR model). The measures are the degree $(k)$, clustering coefficient $(c c)$, betweenness centrality $(B$, average neighborhood degree $(r)$, PageRank $(\pi)$, eigenvector centrality $(x)$, k-core index $\left(k_{c}\right)$, closeness centrality $(C)$ and accessibility $(\alpha)$. The highest correlations are in bold. 184

Table 6 - Structural properties of the airport transportation multilayer networks. 213

Table 7 - Structural properties of each layer of the airport transportation multilayer networks. . . . . . . . . . . . . . . . . . . . . . . . . . 214

Table 8 - Parameter values of the networks reported in Figure 73. . . . . . . . 238

Table 9 - Part I of the summary of mean field approximations for the SIS, SIR and MT processes. . . . . . . . . . . . . . . . . . . . . . . . 284

Table 10 - Part II of the summary of mean field approximations for the SIS, SIR and MT processes. . . . . . . . . . . . . . . . . 285 



\section{LIST OF ABBREVIATIONS AND ACRONYMS}

\begin{tabular}{|c|c|}
\hline ARW & Accessibility Random Walk \\
\hline BA & Barabási - Albert model \\
\hline $\mathrm{BRM}$ & Banded random matrix \\
\hline $\mathrm{CA}$ & Cellular Automaton \\
\hline $\mathrm{CP}$ & Contact Process \\
\hline DK & Daley and Kendal (model) \\
\hline DTMC & Discrete-time Markov Chain \\
\hline ER & Erdös - Rényi model \\
\hline GOE & Gaussian Orthogonal Ensemble \\
\hline HMF & Heterogeneous Mean Field \\
\hline IBMF & Individual-based mean field \\
\hline IPR & Inverse Participation ratio \\
\hline MF & Mean Field \\
\hline MT & Maki Thompson (model) \\
\hline NIMFA & N-Intertwined mean field approximation \\
\hline PQMF & Pair Quenched Mean Field \\
\hline QEP & Quadratic Eigenvalue Problem \\
\hline QMF & Quenched Mean Field \\
\hline QS & Quasi-stationary algorithm \\
\hline RMT & Random Matrix Theory \\
\hline $\mathrm{RP}$ & Reactive Process \\
\hline RRN & Random regular networks \\
\hline SI & Susceptible-Infected \\
\hline SIR & Susceptible-Infected-Recovered/Removed \\
\hline SIRS & Susceptible-Infected-Recovered/Removed-Susceptible \\
\hline SIS & Susceptible-Infected-Susceptible \\
\hline SSF & Spatial Scale free networks \\
\hline $\mathrm{TP}$ & Truncated Process \\
\hline
\end{tabular}



$G$ - Graph or network

$[N]$ - Set of nodes in the network

$[L]$ - Set of edges in the network

$l_{i \rightarrow j}$ - Directed edge from node $i$ to node $j$

A - Adjacency or supra-adjacency matrix

W - Weighted adjacency matrix

$k_{i}$ - Degree of node $i$

$s_{i}$ - Strength of node $i$

$r_{i}$ - Average degree of the nearest neighbors of node $i$

$C_{i}$ - Closeness centrality of node $i$

$B_{i}$ - Betweenness centrality of node $i$

$\sigma(a, i, b)$ - The number of the shortest paths connecting nodes $a$ and $b$ that pass through node $i$

$\sigma(a, b)$ - The total number of the shortest paths between $a$ and $b$

$c c(i)$ - Local clustering coefficient of node $i$

$N_{\triangle}(i)$ - Number of triangles involving the node $i$

$N_{3}(i)$ - Number of triples centered around $i$

$k_{c}(i)-k$-core of node $i$

$x_{i}$ - eigenvector centrality of node $i$

$\pi_{i}$ - Google PageRank of node $i$

$\mathbf{P}$ - Probability transition matrix

$\mathbf{P}^{(h)}(i, j)$ - Probability of reaching node $j$ by performing random walks of length $h$ departing from $i$

$\mathbf{W}$ - Exponential of $\mathbf{P}$

$\mathbf{P}$ - Probability of transition between any pair of nodes through ARW

$\alpha(i)$ - Generalized random walk accessibility 
$\mathbb{E}\left(X^{m}\right)-m$-th moment

$\mathbb{V}(X)$ - Variance

$\rho^{P}$ - Assortativity coeeficient or degree correlation can be calculated considering the Pearson correlation coefficient

$\rho^{S}$ - Degree correlation can be calculated considering the Spearman correlation coefficient $d_{i j}$ - Euclidean distance between nodes $i$ and $j$

D - Ussually denotes a diagonal matrix

L - Laplacian or supra-Laplacian matrix

$\mathbf{B}_{(i \rightarrow j, k \rightarrow l)}$ - Non-backtracking matrix

$\mathscr{M}=(V, L, P, M)-$ Multiplex network

$\mathfrak{M}$ - Layer-graphs

$G_{P}=(V, L, P)$ - where $P \subseteq V \times L$ : a binary relation that indicate which node of a multiplex participates in which layers. $G_{P}=(V, L, P)$ can be interpreted as a (bipartite) graph where $P$ is the edge set

$(u, \alpha) \in P$ - An ordered tuple called node-layer pair; it is the representative of node $u$ in layer $\alpha$

$\left|V_{\beta}\right|=n_{\beta}$ - The number of node-layer pairs in layer $\beta$

$G_{\beta}\left(V_{\beta}, E_{\beta}\right)$ - where $V_{\beta}=\{(u, \alpha) \in P \mid \alpha=\beta\}$ : a layer-graph

$M=\left\{G_{\alpha}\right\}_{\alpha \in L}$ - The set of all layer-graph

$G_{C}\left(P, E_{C}\right)$ - The coupling graph in which there is an edge between two node-layer pairs $(u, \alpha)$ and $(v, \beta)$ if and only if $u=v$

$G_{l}=\bigcup_{\alpha} G_{\alpha}$ - the intra-layer graph. The union of all the layer-graphs, i.e. $G_{l}=\bigcup_{\alpha} G_{\alpha}$

$G_{\mathscr{M}}=G_{l} \cup G_{C}$ - the supra-graph

$Q_{l}(\mathbf{A})$ - the left quotient of $\mathbf{A}$

$\lambda_{i}(\mathbf{M})-i$-th eigenvalue of $\mathbf{M}$ (sometimes $\mathbf{M}$ is suppressed)

$\mu_{i}(\mathbf{M})-i$-th eigenvalue of $\mathbf{M}$ (sometimes $\mathbf{M}$ is suppressed)

$n$ - Number of nodes on each layer (multiplex)

$m$ - Number of layers

$N=n \times m$ - Total number of nodes (multiplex)

$M_{\beta \tilde{\gamma}}^{\alpha \tilde{\delta}} \in \mathbb{R}^{n \times n \times m \times m}$ - Adjacency tensor

$\mathscr{R}_{\beta \tilde{\delta}}^{\alpha \tilde{\gamma}}(\lambda, \eta) \in \mathbb{R}^{n \times n \times m \times m}$ - Supra contact tensor 
$A_{\beta}^{\alpha}(\tilde{r})$ - Selected layer - adjacency matrix of layer $\tilde{r}$

$\Psi_{\tilde{\delta}}^{\tilde{\gamma}} \in \mathbb{R}^{m \times m}$ - network of layers

$P_{\beta}^{\alpha} \in \mathbb{R}^{n \times n}$ - Projected layer

$O_{\beta}^{\alpha} \in \mathbb{R}^{n \times n}$ - Overlay network

$f_{\beta \tilde{\delta}}(\Lambda)$ - Eigentensor associated to the eigenvalue $\Lambda$

$\Phi_{\tilde{\delta}}^{\tilde{\gamma}}(\lambda, \eta)-$ Normalized network of layers

$\mathbf{P}_{\beta}^{\alpha}-$ Normalized projection

$\Lambda_{i}-i$-th eigenvalue (normally used in tensor notation)

$\Lambda_{i}^{l}$ - Individual $i$-th eigenvalue of the $l$-th layer (normally used in tensor notation)

$X$ - Susceptible/Ignorant

$Y$ - Spreader/Informant

$Z$ - Recovered or Removed/Stifler

$\lambda$ - Spreading rate/probability

$\delta$ - Curing rate/probability

$\alpha$ - Stifling rate/probability

$\mathbb{P}(X=1)$ - Probability that the random variable $X$ is equal to 1

$\mathbb{E}(X)$ - Expectation of the random variable $X$

$\tau=\frac{\lambda}{\delta}-$ Control parameter for SIS

$\rho$ - Order parameter: fraction of infected individuals

$Q_{i j}$ - Infinitesimal generator

$\mathscr{N}\left(\mu, \sigma^{2}\right)$ - Normal distribution with average $\mu$ and variance $\sigma^{2}$

$W_{0}$ - First branch of the Lambert function

$\Theta(\tau)$ - Probability of finding an infected node following a randomly chosen edge (HMF)

$y_{i \infty}$ - Probability of node $i$ being infected when $t \rightarrow \infty$ (QMF)

$\operatorname{IPR}(f(\Lambda))$ - Inverse Participation ratio of the eigenvector $f(\Lambda)$

$\eta$ - Rate of spreading between layers

$\frac{\eta}{\lambda}$ - Spreading ratio: ratio between inter and intra layer spreading rates

$p_{n^{*}}(t)$ - Probability of having $n^{*}$ infected individuals (or occupied sites in a more general context) at time $t$

$P(t)$ - Survival probabilities 
$W_{S_{i}, S_{j}}$ - Transition rates (Used on the context of QS algorithm)

$\chi$ - Susceptibility

$\operatorname{Err}_{i}$ - Error between theoretical predictions and Monte Carlo runs for a single set of parameters

$S E$ - Standard Error

$\delta_{1}$ - Forgetting probability for a spreader (in the context of Section 4.1)

$\delta_{2}$ - Spontaneous loss of interest probability (in the context of Section 4.1)

$\gamma$ - Forgetting probability for a stifler (in the context of Section 4.1)

$\eta$ - Probability that after informed an individual turns into a spreader (in the context of Section 4.1)

$\beta$ - Probability of a stifler recovering interest on spreading the information (in the context of Section 4.1)

$U_{i}(0,1)$ - A random variable uniformly distributed in $(0,1)$

$p_{i}^{X}(t)$ - Probability that node $i$ is in state $X$ at time $t$

$p_{i}^{Y}(t)$ - Probability that node $i$ is in state $Y$ at time $t$

$p_{i}^{Z}(t)$ - Probability that node $i$ is in state $Z$ at time $t$

$a_{i}(t)$ - The contact based probabilities. Probability that node $i$ remains at state $X$ due to contacts

$b_{i}(t)$ - The contact based probabilities. Probability that node $i$ remains at state $Y$ due to contacts

$c_{i}(t)$ - The contact based probabilities. Probability that node $i$ remains at state $Z$ due to contacts

$\rho\left(\mathscr{W}_{\beta}^{\alpha}\right)$ - Assortativity coefficient of the tensor $\mathscr{W}_{\beta}^{\alpha}$

$\mathbf{S}$ - P-assortativity matrix

$\mathbf{Z}$ - O-assortativity matrix

$A^{(m)}-m$-th layer adjacency matrix

$C^{\left(m, m^{\prime}\right)}$ - Coupling matrix between layers $m$ and $m^{\prime}$

$S$ - The Shannon entropy

$\beta$ - Scaled localization length

$\mathbf{Q}(\lambda)-\lambda$-matrix of degree 2 - quadratic eigenvalue problem, where $\lambda$ is an eigenvalue J - Jordan matrix 
||'|| - A matrix metric

$\|\mathbf{A}\|_{2}$ - The spectral norm of the matrix $\mathbf{A}$ 

INTRODUCTION ...................... 45

$1.1 \quad$ Objectives . . . . . . . . . . . . . . . . 48

1.2 Thesis organization .................... 50

$1.3 \quad$ Contributions: list of publications . . . . . . . . . . . . 51

2 STRUCTURAL ASPECTS OF COMPLEX NETWORKS . . . . . 53

$2.1 \quad$ Classical network theory . . . . . . . . . . . . 53

2.1.1 Network representation . . . . . . . . . . . . . . . . . . . . . . 54

2.1.2 Characterization measures . . . . . . . . . . . . . . . . 55

2.1.2.1 Centrality measures . . . . . . . . . . . . . . . . . . . . . . 55

2.1.2.2 A small visual example of centrality measures . . . . . . . . . . . . . . . 58

2.1.2.3 Generalized random walk accessibility . . . . . . . . . . . . . . 59

2.1.2.4 Global measures . . . . . . . . . . . . . . . . . . . . . . . . . . 61

2.1.3 Network optimization: Degree-degree correlation tuning . . . . . . . 62

2.1.4 Network models . . . . . . . . . . . . . . . . . . 63

2.1.4.1 Erdös - Rényi model . . . . . . . . . . . . . . . . . . 63

2.1.4.2 Barabási - Albert model . . . . . . . . . . . . . . . . . 64

2.1.4.3 Spatial models . . . . . . . . . . . . . . . . . 65

2.1.4.4 Uncorrelated Configuration Model (UCM) . . . . . . . . . . . . . 66

2.1.4.5 Random regular networks . . . . . . . . . . . . . . 67

$2.2 \quad$ Spectral characterization of networks . . . . . . . . . . 67

2.2.1 Adjacency matrix . . . . . . . . . . . . . . . . . . . . . . 68

2.2.2 Laplacian matrix . . . . . . . . . . . . . . . . . . . . . 69

2.2.3 Probability transition matrix . . . . . . . . . . . . . . . 70

2.2.4 Non-backtracking matrix . . . . . . . . . . . . . . . . 71

$2.3 \quad$ Multilayer network theory . . . . . . . . . . . . . . 72

2.3.1 Matrix representation . . . . . . . . . . . . . . 74

2.3.1.1 Definitions . . . . . . . . . . . . . . . . . . 74

2.3.1.2 The supra-adjacency matrix . . . . . . . . . . . . . . . . 75

2.3.1.3 Quotient graphs ................... . . 76

2.3.1.4 Aggregate Network and Network of Layers of a Multiplex Network . . . . . 76

2.3.1.5 Spectral relations between supra and cross-grained representations . . . . . 77

2.3.2 Tensorial representation . . . . . . . . . . . . 77 
2.3.2.1 Definitions . . . . . . . . . . . . . . . . . . 77

2.3.2.2 Projections . . . . . . . . . . . . . . . . . . . 78

2.3.2.3 Eigentensor problem . . . . . . . . . . . . . . . . 79

2.3.2.4 Interlacing properties . . . . . . . . . . . . . . . 79

$2.4 \quad$ Summary . . . . . . . . . . . . . . . . . . 81

3 FUNDAMENTALS OF SPREADING PROCESSES IN NETWORKS 83

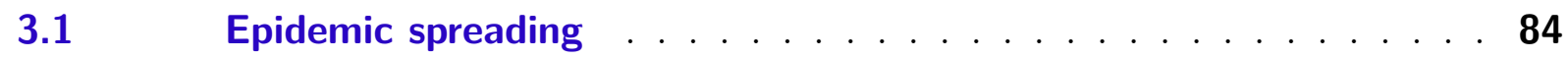

$3.2 \quad$ Rumor spreading . . . . . . . . . . . . . . . . 86

3.3 Comments on other spreading models . . . . . . . . . . . . . 88

3.4 Exact SIS Markov chain . . . . . . . . . . . . . . . . . 90

$3.5 \quad$ Mean field approaches . . . . . . . . . . . . . . . . 95

3.5.1 Mean Field (MF) . . . . . . . . . . . . . . . . . . . . . . . . . 95

3.5.2 Heterogeneous Mean Field (HMF) . . . . . . . . . . . . . 98

3.5.3 Quenched Mean Field (QMF) . . . . . . . . . . . . . . . . 101

3.5.3.1 Single-layer networks . . . . . . . . . . . . . . . . . . 101

3.5.3.2 Multilayer networks: SIS . . . . . . . . . . . . . . . . . . . 104

3.5.3.3 Multilayer networks: SIR . . . . . . . . . . . . 106

3.5.4 Pair Quenched Mean Field (PQMF) . . . . . . . . . . . . . 106

3.5.4.1 Analytical aspects . . . . . . . . . . . . . . . . 107

3.5.4.2 Numerical aspects . . . . . . . . . . . . . . . . . . . 109

3.5.4.3 Comments on the PQMF critical point predictions . . . . . . . . . . . . 110

3.5.4.4 Comparison with recurrent-state message-passing approach . . . . . . . . . 111

3.5.5 Synchronous cellular automaton . . . . . . . . . . . . . 113

3.5.5.1 Single-layer networks . . . . . . . . . . . . . . . . 113

3.5.5.2 Multilayer-layer networks . . . . . . . . . . . . . . . . 115

3.5.6 Asynchronous cellular automaton . . . . . . . . . . . 116

3.5.7 Comments on the generalization for multilayer networks . . . . . . 117

3.6 Comparison of continuous-time and cellular automaton models . . 119

3.7 Monte Carlo simulations . . . . . . . . . . . . . . . . . . . . 121

3.7.1 Continuous-time simulations: Poisson processes . . . . . . . . . . 121

3.7.2 The standard algorithm . . . . . . . . . . . . . . . . . 121

3.7.3 A different implementation . . . . . . . . . . . . . . 122

3.7.4 Quasi-stationary algorithm (QS) . . . . . . . . . . . . 125

3.7.4.1 Definition . . . . . . . . . . . . . . . . 125

3.7.4.2 Example: a numerical evaluation . . . . . . . . . . . . . . 127

3.7.5 Discrete-time simulations: Cellular automaton . . . . . . . . . . 129

3.7.6 A systematic accuracy evaluation . . . . . . . . . . . . . . . . . 131

3.7.6.1 Single-layer analyses . . . . . . . . . . . . . . . . . . . . 132

3.7.6.2 Multilayer analyses . . . . . . . . . . . . . . . 133 


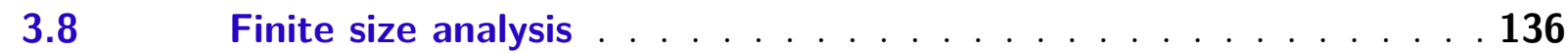

3.9 A brief comment on the nature of the critical point . . . . . . 141

$3.10 \quad$ Summary . . . . . . . . . . . . . . . . . . . . . . 142

4 NEW PERSPECTIVES ON MODELING SINGLE LAYER NET-

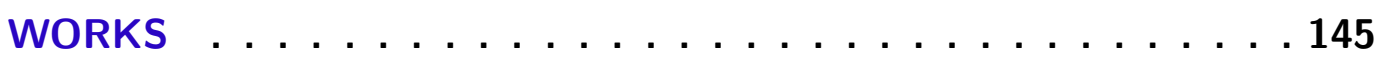

4.1 General Markov chain approach for disease and rumor spreading . 146

4.1.1 The general model . . . . . . . . . . . . . . . . . . . . . 147

4.1.1.1 First order approximation . . . . . . . . . . . . . . . . . . . . . . 149

4.1.1.2 The mean-field approximation . . . . . . . . . . . . . . . . . . 152

4.1.1.3 Homogeneously mixed population . . . . . . . . . . . . . . . . . 154

4.1.2 Analytical Analysis . . . . . . . . . . . . . . . . . . 155

4.1.2.1 Early time behavior . . . . . . . . . . . . . . . . . . . . . . . . 155

4.1.2.2 Steady state analysis . . . . . . . . . . . . . . . . . . . . 160

4.1.3 Special cases . . . . . . . . . . . . . . . . . . 160

4.1.3.1 Disease spreading . . . . . . . . . . . . . . . . . . . . . . . . . 161

4.1.3.2 Rumor spreading . . . . . . . . . . . . . . . . . . . . . . . . . . 161

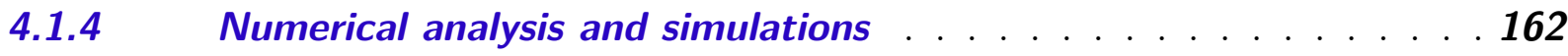

4.1.4.1 Phase diagrams . . . . . . . . . . . . . . . . . . . 162

4.1.4.2 Monte Carlo simulations . . . . . . . . . . . . . . . . . . . 165

4.1.5 Applications to real social systems . . . . . . . . . . . . . . 168

4.2 Role of centrality to identify influential spreaders . . . . . . . 173

4.2.1 Methods . . . . . . . . . . . . . . . . . . . . . . . 174

4.2.1.1 Monte Carlo simulations . . . . . . . . . . . . . . . . . . . . . 174

4.2.1.2 Database . . . . . . . . . . . . . . . . . . . . . . . . . . 175

4.2.2 Parameter analysis . . . . . . . . . . . . . . . 176

4.2.3 Spatial networks . . . . . . . . . . . . . . . . 178

4.2.4 Road networks . . . . . . . . . . . . . . . . . 180

4.2.5 Non-spatial networks . . . . . . . . . . . . . . . . . . . 183

$4.3 \quad$ Summary . . . . . . . . . . . . . . . . . . . . 186

5 ADVANCES IN MULTILAYER NETWORKS . . . . . . . . 189

5.1 Disease localization in multilayer networks . . . . . . . . . 190

5.1.1 Epidemic spreading on multilayer networks . . . . . . . . . . 191

5.1.2 Localization and spreading of diseases . . . . . . . . . . . . 192

5.1.3 2-Layer multiplex systems . . . . . . . . . . . . . . 193

5.1.3.1 Numerical solution . . . . . . . . . . . . . . . . . . . . . . . . . . 193

5.1.3.2 Spectral analysis . . . . . . . . . . . . . . . . . . . 196

5.1.3.3 Multiple susceptibility peaks . . . . . . . . . . . . . . . . . . 198

5.1.3.4 Second susceptibility peak analysis: Erdös-Rényi layers . . . . . . . . . . 199 
5.1.3.5 Finite size analysis: Erdös-Rényi layers . . . . . . . . . . . . . . . . . 202

5.1.4 3-Layer interconnected systems: the barrier effect . . . . . . . . . 206

5.1.4.1 Spectral analysis . . . . . . . . . . . . . . . . 206

5.1.4.2 Multiple susceptibility peaks . . . . . . . . . . . . . . . 208

5.2 On degree-degree correlations in multilayer networks . . . . . . . 209

5.2.1 Assortativity in multilayer networks . . . . . . . . . . . . . . . 210

5.2.2 Application to real data . . . . . . . . . . . . . . . . . 213

5.2.3 Epidemic spreading in correlated multilayer networks . . . . . . . . 217

$5.3 \quad$ Summary . . . . . . . . . . . . . . . . 218

6 NEW SPECTRAL ASPECTS OF MULTILAYER NETWORKS . . 221

6.1 Eigenvalue crossings and localization of eigenvectors in multilayer networks . . . . . . . . . . . . . . . . . . . . . 222

$6.1 .1 \quad$ 2-Layer case . . . . . . . . . . . . . . . . 222

6.1.1.1 Eigenvalue crossing . . . . . . . . . . . . . . . 223

6.1.1.2 Identical layers . . . . . . . . . . . . . . . . . . . 223

6.1.1.3 Similar layers . . . . . . . . . . . . . . . . . . . . . . . . 224

6.1.2 Spectrum on the 3-Layer case . . . . . . . . . . . . 225

6.2 Universal properties of multilayer networks . . . . . . . . . . 227

6.2.1 Model definition and problem statement . . . . . . . . . . . . . . . 228

6.2.2 Scaling analysis of Multilayer Networks . . . . . . . . . . . . . . . . 231

6.2.3 Scaling analysis of Multiplex Networks . . . . . . . . . . . . . . 233

6.2.3.1 Weighted Multiplex . . . . . . . . . . . . . . . . 233

6.2.3.2 Unweighted Multiplex . . . . . . . . . . . . . . . . 235

6.2.4 Application to real-world networks . . . . . . . . . . . . 236

$6.3 \quad$ Exploring the block structure of multilayer networks . . . . . . . 238

6.3.1 Problem definition . . . . . . . . . . . . . . . . . . 239

6.3.1.1 Quadratic eigenvalue problem . . . . . . . . . . . . . . 239

6.3.1.2 Interesting QEP properties . . . . . . . . . . . . . . . 240

6.3.1.3 2-Layer multiplex networks . . . . . . . . . . . . . . . . 241

6.3.2 Spectral analysis . . . . . . . . . . . . . . . . . 241

6.3.3 Supra-Laplacian matrix . . . . . . . . . . . . . . . . . . . 242

6.3.3.1 Structural transitions . . . . . . . . . . . . . . . . . . . 243

6.3.3.2 Bounds . . . . . . . . . . . . . . . . . . . . 244

6.3.3.3 Spectral properties as a function of the coupling $p \ldots . . \ldots 246$

6.3.4 Supra-adjacency matrix . . . . . . . . . . . . . . . . . . . . 248

6.3.4.1 Bounds . . . . . . . . . . . . . . . . . . . 248

6.3.4.2 Spectral properties as a function of the coupling $p \ldots \ldots$. . . . . . 250

$6.4 \quad$ Summary . . . . . . . . . . . . . . . 252 


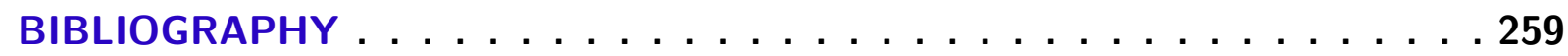

APPENDIX A GENERALIZED RANDOM WALK ACCESSIBILITY:

ADDITIONAL COMMENTS ........... . . 273

A.1 Accessibility in star graphs . . . . . . . . . . . . 273

A.1.1 First analytical approach . . . . . . . . . . . . . . . 273

A.1.2 Eigendecomposition analysis . . . . . . . . . . . . . . 274

A.2 Accessibility in ring graphs . . . . . . . . . . . . . 276

A.3 Accessibility in complete graphs . . . . . . . . . . 277

A.3.1 Eigendecomposition analysis . . . . . . . . . . . . 278

APPENDIX B INTERLACING PROPERTIES: PROOF OF EQUATION $2.50 \ldots \ldots 281$

APPENDIX C FUNDAMENTALS OF SPREADING PROCESSES: COM-

PLEMENTARY COMMENTS . . . . . . . . . 283

C.1 Formulation . . . . . . . . . . . . . . . 283

APPENDIX D LOCALIZATION IN INTERCONNECTED NETWORKS287

D.1 Localization on interconnected networks . . . . . . . . . . 287

D.2 Multiple susceptibility peaks: additional results . . . . . . . . . 289

APPENDIX E QUADRATIC EIGENVALUE PROBLEM: SPECTRAL

PROPERTIES . . . . . . . . . . . . . 291

E.1 Bounds . . . . . . . . . . . . . . . . 291

E.2 Comments on symmetric problems: HQEP . . . . . . . . . . . 292

E.3 Limits for sparse intrer-layer coupling: singular $\mathbf{D}=\mathbf{M}_{12}=\mathbf{M}_{21} \ldots 293$

ANNEX A ACCURACY OF NUMERICAL APPROXIMATIONS AND MONTE CARLO SIMULATIONS . . . . . . . . . . . 295 

CHAPTER

1

1

INTRODUCTION

Historically, the classical modeling approach assumes homogeneously mixed populations, which implies that the probability of two individuals in the population being connected is the same. Such formalism proved to be unrealistic, as most real systems present heterogeneously mixed populations, in which contacts among individuals frequently follow non-trivial patterns, such as power-law degree distributions (NEWMAN; PARK, 2003; BOCCALETTI et al., 2006; COSTA et al., 2007), degree-degree correlations (NEWMAN, 2002), cycles (WATTS, 1999), among many other features (BOCCALETTI et al., 2006; COSTA et al., 2007). This limitation is overcome by adopting the complex network framework. Regarding this formalism, a population is represented as a network (also referred to monoplex or single/mono-layer networks using the modern terminology), where individuals are portrayed as nodes and their relationships are given by edges connecting them. This theory is highly interdisciplinary and connects many different fields, from the elegant mathematical graph theory of Erdös and Rényi on random graphs to the concepts of phase transitions in thermodynamics. Modeling complex systems as networks (BOCCALETTI et al., 2006; COSTA et al., 2007; BARRAT; BARTHLEMY; VESPIGNANI, 2008; NEWMAN, 2010; COSTA et al., 2011) has proved to be a successful approach to characterize the structure and function of many real-world systems, from the biological domain to social and technological applications (COSTA et al., 2011). However, on single-layer networks only one type of connection is accounted for. This modeling is also limited because most natural and artificial systems, such as the brain, our society or modern transportation networks (KIVELÄ et al., 2014; BOCCALETTI et al., 2014) are made up of different constituents and/or various types of interaction. Indeed, their structure is organized into layers. For instance, in social networks, individuals can be connected according to different social ties, such as friendship or family relationship (e.g. (VERBRUGGE, 1979)). In transportation networks, routes of a single airline can be represented as a network, whose vertices (destinations) can be mapped into networks of different companies (CARDILLO 
et al., 2013). Gene co-expression networks consist of layers, each one representing a different signaling pathway or expression channel (LI et al., 2011). Therefore, mapping out these and similar systems such as monoplex networks can miss relevant information, which is not captured if the single layers were analyzed separately - neither when all the layers collapse together in an aggregated graph. Additionally, in most of these interconnected systems, the information travels not only among vertices of the same layer but also between pairs of layers. In order to overcome the aforementioned limitations and to allow a proper representation of the previously presented examples, multilayer networks have been introduced in recent years. In this formalism, systems are represented as a collection of layers, which are made of nodes and edges. In this context, edges can be of two types, intra or inter-layer, accounting for connections inside or between two different layers. Note that the original definition of networks is also considered by the multilayer, generalizing the previous one, but also including more complex and richer structures (KIVELÄ et al., 2014; BOCCALETTI et al., 2014).

As a consequence of the structural aspects mentioned above, dynamical processes were also studied on top of single and multilayer-structured populations. For instance, rumor and epidemic spreading, synchronization of coupled oscillators, diffusion processes, among many others. Interestingly, the introduction of heterogeneous structures has changed our understanding of their dynamical behavior. Take the case of disease spreading as an example. First, in homogeneous populations, disease always presents a phase transition from a disease-free state to an epidemic state. However, as observed by Pastor-Satorras and Vespignani in 2001 (PASTOR-SATORRAS; VESPIGNANI, 2001b; PASTOR-SATORRAS; VESPIGNANI, 2001a) and using the HMF (heterogeneous mean field), a disease outbreak takes place when the spreading rate, $\lambda$, is larger than the epidemic threshold (PASTORSATORRAS; VESPIGNANI, 2001b; PASTOR-SATORRAS; VESPIGNANI, 2001a), i.e., if $\lambda>\lambda_{c}^{H M F}=\mathbb{E}(k) / \mathbb{E}\left(k^{2}\right)$, where $\mathbb{E}\left(k^{m}\right)$ is the $m$-th moment of the degree distribution. More specifically, in the HMF theory a network is coarse-grained by the degree of its nodes, i.e all the nodes with a given degree are considered to be statistically equivalent, neglecting correlations. Therefore, considering scale-free networks (those for which the degree distribution follows a power law $P(k) \sim k^{-\zeta}$ with $\left.\zeta \leq 3\right)$ are particularly prone to spreading diseases, since $\lambda_{c} \rightarrow 0$ when $N \rightarrow \infty$. Contrasting with the HMF, in 2003 Wang proposed the QMF (quenched mean field) approach (WANG et al., 2003), in which the individual node probability is evaluated, including the full network structure, by means of the adjacency matrix $\mathbf{A}$. This approach predicts the critical point as $\lambda_{c}^{Q M F}=\frac{1}{\lambda_{1}(\mathbf{A})}$, where $\lambda_{1}(\mathbf{A})$ is the largest eigenvalue of $\mathbf{A}$. Complementing this analysis with the results of Chung et. al. (CHUNG; LU; VU, 2003) on the spectral properties of $\mathbf{A}$, hence $\lambda_{c}^{Q M F}={\sqrt{k_{\max }}}^{-1}$ if $\zeta>\frac{5}{2}$ and $\lambda_{c}^{Q M F} \approx \mathbb{E}(k) / \mathbb{E}\left(k^{2}\right)$ if $2<\zeta<\frac{5}{2}$. Intriguingly, both the HMF and the QMF agree in their predictions for $2<\zeta<\frac{5}{2}$, however the first predicts a finite threshold for $\zeta>3$, while the second predicts that the threshold vanishes when $k_{\max }$ 
diverges, regardless of the exponent $\zeta$. Interestingly, numerical results suggested that the QMF is qualitatively correct on power-law networks, therefore representing an improvement of the HMF approach (FERREIRA; CASTELLANO; PASTOR-SATORRAS, 2012). In order to explain the contradiction between both theories, in 2012 Goltsev et.al. (GOLTSEV et al., 2012) proposed a spectral theory approach explaining the observed discrepancies among the critical point predictions as a localization phenomena. Accordingly, if $\lambda_{1}(\mathbf{A})$ corresponds to a localized eigenstate, the disease remains in a finite set of nodes, e.g. a subgraph containing a huge hub. Conversely, if $\lambda_{1}(\mathbf{A})$ corresponds to a delocalized eigenstate, the disease infects a finite fraction of nodes.

Complementary to the aforementioned results for single-layer networks, also the theory of multilayer networks have been developed and used to study dynamical processes. Among the most important advances, there is the mathematical formulation following the tensorial (De Domenico et al., 2013) and matricial (BOCCALETTI et al., 2014; KIVELÄ et al., 2014) formalisms and the finding of a structural transition on the Laplacian algebraic connectivity by Radicchi and Arenas in 2013 (RADICCHI; ARENAS, 2013). From the dynamical point of view, also in 2013, Gómez et. al. formalized the diffusion process on multiplex networks (GOMEZ et al., 2013). Interestingly, they observed the super-diffusion, meaning that the time scale of the multiplex is smaller than that of each layer considered independently. Complementary, regarding epidemic spreading, in the same year Cozzo et. al. studied disease spreading processes on top of multiplex networks (COZZO et al., 2013), extracting important results from the perturbation theory. Among their main results, they observed a shift to the left in the phase diagram of the non-dominant layer, as a consequence of the activity of the dominant one. More precisely, the non-dominant layer experiences an unexpected earlier outbreak, where the number of infected individuals on such a layer is larger than zero even before its expected critical point. This phenomena appears as a consequence of the disease spreading through the inter-layer edges. Besides, they also proposed an approximation for the leading eigenvalue of the adjacency matrix, which helped them to explain their results. Indeed, the previously mentioned results emphasize the different scales of multiplex systems, which can be one of three phases: (i) the decoupled phase, when the layers behave independently, (ii) the multiplex phase, when the system acts as a whole and (iii) the network of layers phase, when the inter-layer connections dominate the processes.

On the other hand, shifting from the historical perspective to the practical and social viewpoints, the importance of such dynamical processes can be emphasized recalling the Influenza A pandemic, and specially, Dr Harvey Fineberg's presentation on May 2011 at the World Health Assembly: "Conclusion 3: The world is ill-prepared to respond to a severe influenza pandemic or to any similarly global, sustained and threatening public health emergency. Beyond the implementation of core public health capacities called for in the IHR (International Health Regulations), global preparedness can be advanced 
through research, strengthened health-care delivery systems, economic development in lowand middle-income countries and improved health status" (WHO, 2017b). Moreover, recently, the World Health Organization reported a new outbreak of Ebola in May 2017, two years after the first big outbreak (WHO, 2017a). Summarizing, epidemic and information spreading are important processes from a phenomenological point of view, and are directly applicable to social problems and are especially interesting both in mathematics and physics, as many of the results could be extended to other different dynamical processes on top of networks. From this perspective, we developed our work and wrote this thesis, aiming to contribute, both socially and technically, to the fields of mathematical epidemiology and information spreading.

\subsection{Objectives}

The main aim of this thesis is to have a better understanding of epidemic-like processes and the improvement of their modeling techniques. In order to pursue this aim, we divided our efforts into three connected goals, which were also separated into chapters in this thesis: (i) analysis and modeling of spreading processes on single-layer networks, (ii) analysis of epidemic spreading on multilayer analysis and (iii) spectral analysis of multilayer networks. More specifically and further dividing these goals, we organized them as follows:

1. Fundamentals of spreading processes in networks: To begin with, it is important to formally define our models establishing a coherent mathematical framework, which will be used throughout the thesis. Importantly, during our research, we found a of couple difficulties in the literature, especially regarding the comparison between analytical and numerical results with Monte Carlo simulations. In order to fill this gap, we formally defined the local rules, which define our dynamical processes and evaluated the exact approach and some mean field approximations. Next, a comparison between different approximations was also performed. To summarize, in this item, we performed a brief literature review, classifying different types of simulations and relating them to their specific mean-field approximation. Furthermore, we must emphasize that this analysis is focused on the framework and its applications and we do not aim to perform an extensive review of previous results regarding spreading processes since this was recently done in (PASTOR-SATORRAS et al., 2015). The development of this goal is presented in Chapter 3.

2. Modeling of spreading processes in single-layer networks: The analysis performed on the previous item revealed some gaps in the literature. Thus, we explored some new aspects of modeling spreading processes in networks. More specifically, we focused on generalizing models in order to represent various processes under the 
same formalism. In this goal, we value the applications of our methods. Thus, we valued a mathematical framework which would allow us to guarantee a low computational cost with the flexibility to model as many real processes as possible. In this case, we followed the discrete time Markov chain approach. Due to the mathematical complexity of the chosen Markov chain (many possible transitions, which is a consequence of the general approach chosen), we developed our theory under the single-layer formalism. However, it is worth mentioning that considering multilayer networks on such formalism is natural and was left as possible future work. The development of this goal is presented on Chapter 4, Section 4.1.

3. Analysis of spreading processes in single-layer networks: Here, we were interested in the role of centrality and its relationship with the initial conditions for absorbing spreading process, which also improve our understanding of the relationship between structure and dynamics in networks. More specifically, we focused on the SIR epidemic model and Maki - Thompson rumor model. This relationship was evaluated in terms of the correlations between dynamical properties and structural properties (centrality measures) aiming to find influential spreaders. This work was developed considering a single-layer framework because it was lacking in the literature, but also due to the stage of the multilayer literature at the time. More specifically, there are a set of small details regarding the multilayer theory that should be accounted for: (i) there were just a few centrality measures that properly considered the multilayer structure, (ii) the dynamical understanding of spreading processes on multilayer networks was not well developed, (iii) there is a parameter defining the coupling strength between layers, which would restrict some conclusions and increase the computational cost and (iv) the real networks available usually have different numbers of layer, which are also an additional challenge. Considering this, the single-layer analysis was considered to be the most suitable and meaningful. The development of this goal is presented in Chapter 4, Section 4.2.

4. Analysis of epidemic spreading in multilayer analysis: Besides analyzing single-layer systems, we were also interested in multilayer networks. Due to the current stage of our field, it was necessary, to begin with the basics, redefining the epidemic processes on multilayer structures and extending previous literature results for this new type of networks. As a natural consequence, new phenomena was also found. In this goal, we focused on the mathematical and experimental characterization of the new phenomena. Moreover, we also performed numerical experiments regarding the effects of degree-degree correlations on the epidemic spreading. Although this is a simpler task, the mathematical framework to measure correlations on multilayer networks was lacking in the literature. Thus, in order to fill this gap, we proposed an extended measure, which is able to measure correlations at different 
levels (the whole multiplex, a layer, the network of layers, among other possibilities). The development of this goal is presented in Chapter 4.

5. Spectral analysis of multilayer networks: A constant element throughout this thesis is the key role of spectral properties of the networks under study. For instance, while analyzing the critical behavior of most of the evaluated methods, the control parameter depends on the leading eigenvalue, as well as the leading eigenvector, which provides us with information about localization properties of our network. This connection between structure and dynamics motivated us to further study these properties but also focusing on multilayer networks, where some of these properties appeared as a natural consequence of their study. Furthermore, since multilayer networks are still in their infancy, as pointed out in (KIVELÄ et al., 2014), many important properties are still to be discovered. Therefore, to begin with, we focused on the spectral properties that have arisen naturally from the previous item, complementing them. Next, we followed a random matrix approach seeking universal properties. Finally, we explored the block structure of these systems willing to find more rigorous results in such systems. The development of this goal is presented in Chapter 6.

\subsection{Thesis organization}

This thesis is organized as follows: in Chapter 2 we define our networks (single and multilayer), present some characterization metrics, focusing on the concepts used in this thesis. Next, in Chapter 3 we formally define the spreading processes under study. We describe these processes beginning with the local rules that define the individual dynamics, regardless of the structure, then extend them to different structures. From the point of view of network dynamics, we start with the exact formulation, which is impractical for most problems, followed by a discussion of the mean field approximations. Additionally, in Chapter 3, we also discuss the numerical and simulation methods in order to properly describe and analyze the various aspects of this class of processes. This chapter is directly linked to goal 1 in the previous section. These two chapters establish the main background aspects, allowing us to proceed with our results. Thus, in Chapter 4 we present our first results, focusing on the modeling and analysis of spreading processes in single-layer networks, fulfilling goals 2 and 3 previously discussed. Afterwards, we focus on multilayer networks. Firstly, in Chapter 5, we present our results on layer-wise localization and the barrier effect of the SIS epidemic spreading, followed by the effects of degree-degree correlations on a similar process. This chapter covers goal 4 in the previous section. Finally, in Chapter 6, we explore the spectral properties of multilayer networks. This property emerged naturally from the analysis of made in chapter 5. In fact, sine the beginning of this thesis, the importance of such properties has become evident. Thus, in Chapter 6 we 
extended the analysis of the previous chapter. Furthermore, we also evaluate universal scaling on such systems, following a random matrix formalism and, finally, we propose a new formalism on the spectral analysis which takes advantage of the block structure of the matrices that represent multilayer networks. Note that this is related to goal 5, previously presented. To finalize, we present our overall conclusions in Chapter 7 , where we also show some possible future research lines.

\subsection{Contributions: list of publications}

In this section we provide a list of publications collected during the Ph.D., giving a short and systematic context in terms of the previous exposed goals.

- Multilayer Networks: Metrics and Spectral Properties (chapter in the book Interconnected Networks, Springer International Publishing) (COZZO et al., 2016), discussed in Chapter 2, Section 2.3.1. It is important to emphasize that most of the content of this chapter belongs to Emanuele Cozzo's thesis.

- A general Markov chain approach for disease and rumor spreading in complex networks (Journal of Complex Networks) (ARRUDA et al., 2017), shown in Objective 2 and discussed in Chapter 4, Section 4.1.

- Role of centrality for the identification of influential spreaders in complex networks (Physical Review E) (ARRUDA et al., 2014), shown in Objective 3 and discussed in Chapter 4, Section 4.2.

- Disease Localization in Multilayer Networks (Physical Review X) (ARRUDA et al., 2017), shown in Objective 4 and discussed in Chapter 5, Section 5.1, where we discuss the dynamical aspects of epidemic spreading on multilayer networks and also in Chapter 6, Section 6.1, where we focus on the spectral properties studied as a consequence of the dynamics.

- On degree-degree correlations in multilayer networks (Physica D: Nonlinear Phenomena) (ARRUDA et al., 2016), shown in Objective 4 and discussed in Chapter 5, Section 5.2.

- Scaling properties of multilayer random networks (Physical Review E) (MÉNDEZBERMÚDEZ et al., 2017), shown in Objective 5 and discussed in Chapter 6, Section 6.2 .

- A process of rumour scotching on finite populations (Royal Society Open Science) (ARRUDA et al., 2015). This paper was not included in the thesis because it proposes a new rumor model and we focused most of our analysis on the complete graph. Thus, we decided to leave it as a reference. 
- Diluted banded random matrices: Scaling behavior of eigenfunction and spectral properties (Journal of Physics A: Mathematical and Theoretical) (MENDEZBERMUDEZ et al., 2017). This paper was not included in the thesis as it does not deal with complex networks. However, it is interesting to emphasize that it was a necessary experiment before the paper Scaling properties of multilayer random networks (MÉNDEZ-BERMÚDEZ et al., 2017), which is part of the thesis. In this paper, we evaluated the diluted block banded model, which presents common features with the used models and was helpful in our analysis. 
CHAPTER

2

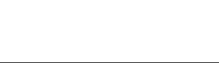

\section{STRUCTURAL ASPECTS OF COMPLEX \\ NETWORKS}

Throughout this thesis, we study two types of networks: single and multilayer networks. This chapter addresses the structural characterization of these systems. Real networks consist of thousands or even millions of interacting parts. Consequently, a proper definition of the mathematical objects and statistical characterization are fundamental to proceed with our analysis. Along these lines, in this chapter, we formally define these networks in terms of its adjacency matrix. In general, there are many ways to represent them. Here we focus on the matricial representation because it allows us to naturally link the structure with its spectral properties, which are especially interesting when analyzing dynamical processes. Additionally, we also present some key concepts of structural characterization of networks, regarding two levels: the nodal characterization, i.e centrality measures, and global measures. Moreover, we also briefly present network models, which are fundamental to analyzing dynamical processes in networks, as they provide a null model. Furthermore, a special section is aimed at analyzing spectral properties of these systems, as they play a key role in dynamical processes, connecting the structure and dynamics. In summary, this chapter allows the reader to become acquainted with the main fundamental points in the structural characterization of single and multilayer networks, paving the way for the next chapters.

\subsection{Classical network theory}

In this section, we provide a formal description of a network, defining it in terms of a graph, in Section 2.1.1. Afterwards, we present some basic network characterization measurements in Section 2.1.2, where we also discuss the local and global network characterization concepts. Next, in the last subsection, Section 2.1.4, we present some of the 
Figure 1 - The undirected network $G=([N],[L])$, where $[N]=\{0,1,2,3,4,5\}$ and $[L]=$ $\left\{l_{0 \rightarrow 1}, l_{0 \rightarrow 2}, l_{0 \rightarrow 3}, l_{0 \rightarrow 4}, l_{0 \rightarrow 5}, l_{5 \rightarrow 1}, l_{4 \rightarrow 5}, \ldots\right\}$, where the reciprocal edges were omitted. Its graphical representation is shown in (a), while its adjacency matrix is shown in (b).

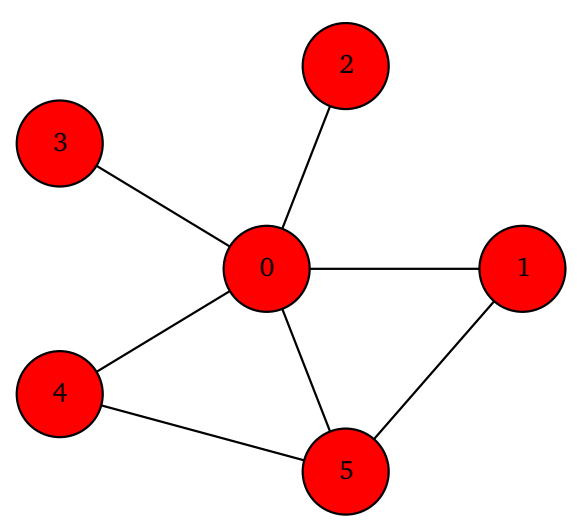

(a)

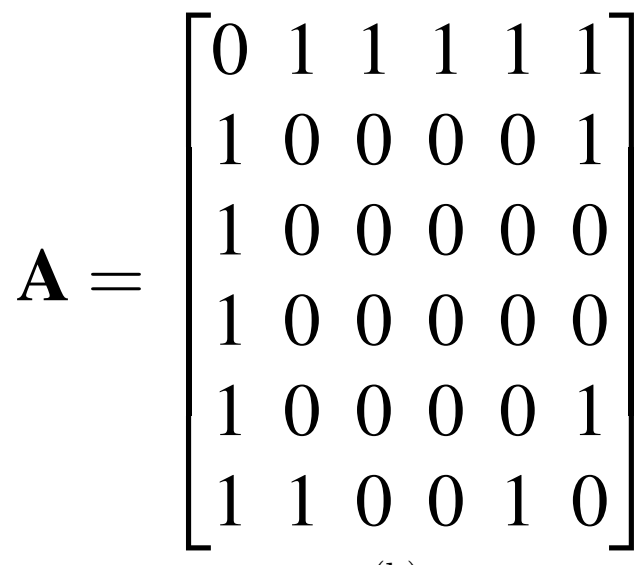

(b)

Source: Developed by the author.

main network models, which play a key role in the development of this thesis.

\subsubsection{Network representation}

A network is formally represented as a graph (BOCCALETTI et al., 2006). Consequently, a graph consists of two sets, $G=([N],[L])$ : (i) the set of nodes $[N]:=\{1,2, \ldots, N\}$ and the set of edges are ordered pairs of $[N], l_{i \rightarrow j}:=(i, j)$, i.e. $[L]:=\left\{l_{i \rightarrow j}, \forall i, j\right.$ are connected $\}$ and $|L|=M$. Observe that in undirected graphs, the pairs $l_{i \rightarrow j}$ are simply unordered implying that both $l_{i \rightarrow j} \in[L]$ and $l_{j \rightarrow i} \in[L]$. Often a graph is represented by its adjacency matrix, $\mathbf{A}$, where $\mathbf{A}_{i j}=1$ if there is an edge from node $i$ to node $j$, i.e. $l_{i \rightarrow j} \in[L]$, and $\mathbf{A}_{i j}=0$ otherwise. One might also associate a weight to each connection, making a socalled weighted network. This network is represented by $\mathbf{W}$, where the elements, $\mathbf{W}_{i j}$, of this matrix represent the weight between nodes $i$ and $j$. We remark that in the undirected case, $\mathbf{A}$ is symmetric, while in the directed case, this is usually false. It is worth mentioning that other representations are also possible, such as edgelists, adjacency lists, among other matrices. However, here we focus on the adjacency matrix due to its mathematical elegance and potential. Figure 1 exemplifies an undirected network, showing its adjacency matrix and its graphical representation.

Throughout this thesis, we restrict ourselves to some specific types of networks. We focus on finite undirected, unweighted, connected networks. In other words, we consider a finite number of nodes, where the all the edges present unitary weights and are undirected (present in both directions). Moreover, there is a path from any node $i$ to any other node $j$ on the network. In case we extend our results to other types of networks, we make it 
explicit, otherwise, those are our standard constraints.

\subsubsection{Characterization measures}

Proper characterization is an important task in the field of complex networks, allowing us to study their structure, but also supporting studies on the analysis/evaluation of the relationship between structure, dynamics and function of networks. This task can be made using different measurements. In a general statement, we might define the characterization task as the mapping of a system into a vector of measurements. Furthermore, citing (COSTA et al., 2007), Generic mappings can be used in order to obtain the characterization of the network in terms of a suitable set of measurements. In case the mapping is invertible, we have a complete representation of the original structure. Interestingly, as the authors also pointed out (COSTA et al., 2007), note, however, that the characterization and classification of networks do not necessarily require invertible measurements.

In order to summarize information, which concerns a specific feature found in the system, many characterization measures were already proposed in the literature (BOCCALETTI et al., 2006; COSTA et al., 2007). They can be divided following many criteria. However, here we divide them into centrality measurements, features that characterize nodes of a network, and global measures, which characterize the whole network. The first category, which is discussed in Section 2.1.2.1, enables us to compare different nodes within the same network. In other words, it quantifies the importance of a given node with respect to the other nodes. On the other hand, global measurements, which are discussed in Section 2.1.2.4 enable us to compare different networks and/or summarize an information of interest.

\subsubsection{Centrality measures}

As mentioned before, in principle some metrics can be considered to define the centrality of a node. Here, we provide the basic definitions of those used in the rest of the thesis. For more, please refer to (BOCCALETTI et al., 2006; COSTA et al., 2007). The most basic definition of centrality is the node degree, $k_{i}$, formally given in terms of its adjacency matrix as

$$
k_{i}=\sum_{j} \mathbf{A}_{i j}
$$

In this case, the most central node has the largest number of connections. In directed networks, the in-degree of the node is defined by $k_{i}^{i n}=\sum_{i=1}^{N} A_{i j}$ and the out-degree, $k_{i}^{\text {out }}=$ $\sum_{j=1}^{N} A_{i j}$. Note that the degree is given by $k_{i}=k_{i}^{i n}+k_{i}^{\text {out }}$. Regarding weighted networks, this concept is generalized in the so-called strength of the node $s_{i}=\sum_{j=1}^{N} W_{i j}$. Observe that the strength can be also generalized for directed networks. 
Alternatively, the centrality of a node can be defined in terms of the degree of its second neighbors, as strongly connected vertices can surround a central node. In this case, the average degree of the nearest neighbors of $i$ is defined as

$$
r_{i}=\frac{1}{k_{i}} \sum_{j \in \partial i} k_{j},
$$

where $\partial i$ is the set of nodes connected to $i$. Interestingly, it can be observed that the average neighborhood degree is related to epidemic spreading in networks (GLEESON et al., 2012; BARRAT; BARTHLEMY; VESPIGNANI, 2008).

Another property of interest is the shortest paths in the network. From this concept, we can also define centrality based on the distance between pairs of nodes. From this perspective, the more central the node is, the lower its total distance to all other nodes. The measurement presented here that follows this concept is the closeness centrality defined as (NEWMAN, 2010)

$$
C_{i}=\frac{N}{\sum_{j=1, j \neq i}^{N} d_{i j}},
$$

where $d_{i j}$ is the length of the shortest distance between nodes $i$ and $j$. Alternatively, the effective load of a node (also defined in terms of shortest paths) can be considered as a centrality measure. This measurement is called betweenness centrality and it quantifies the load as the number of times a node acts as a bridge along the shortest path between two other nodes (GIRVAN; NEWMAN, 2002). Thus, for a node $i$,

$$
B_{i}=\sum_{(a, b)} \frac{\sigma(a, i, b)}{\sigma(a, b)}
$$

where $\sigma(a, i, b)$ is the number of the shortest paths connecting nodes $a$ and $b$ that pass through node $i$ and $\sigma(a, b)$ is the total number of the shortest paths between $a$ and $b$. The sum is over all pairs $(a, b)$ of distinct nodes. In this case, a central node should be crossed by many paths and shows the higher values of $B_{i}$.

Moreover, considering another local property, an important quantity of interest is the number of triangles in a given structure. Thus, we call the clustering coefficient the measure that quantifies the occurrence of triangles in the networks. It is defined as (BOCCALETTI et al., 2006)

$$
c c(i)=\frac{3 N_{\triangle}(i)}{N_{3}(i)}
$$

where $N_{\triangle}(i)$ is the number of triangles involving the node $i$ and $N_{3}(i)$ is the number of triples centered around $i$. As argued in (NEWMAN, 2010), $c c(i)$ can be understood as a centrality measure in the sense that if two nodes are connected only via node $i$, this node can control the information flow. Thus, the clustering coefficient can be thought of as a local version of the betweenness centrality. Note that $c c(i)$ takes smaller values for more 
Figure 2 - An example of clustering coefficient. Three network configurations that result in different values of the clustering coefficient.

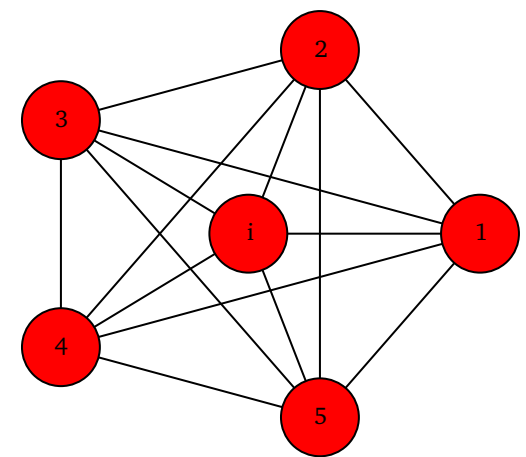

(a) $c c(i)=1$

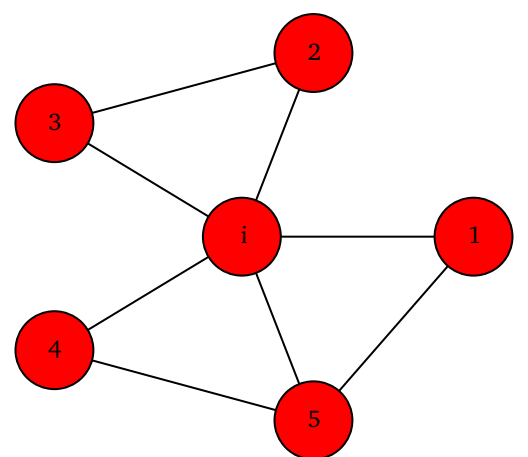

(b) $c c(i)=0.5$

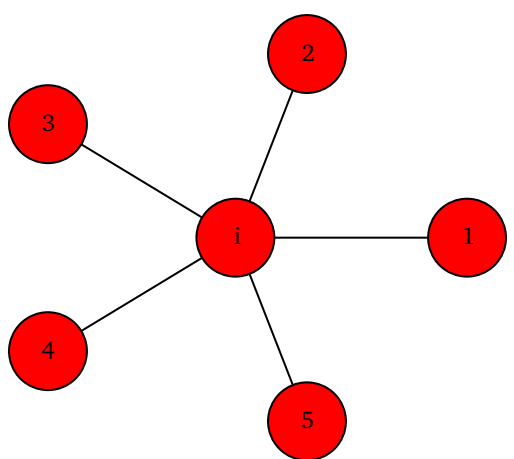

(c) $c c(i)=0$

Source: Developed by the author.

central nodes, contrary to the other centrality measures. Figure 2 illustrates the calculus of the clustering coefficient.

The $k$-shell decomposition, also called $k$-core, partitions a network into substructures, assigning an integer index to each node $i, k_{c}(i)$. Thus, $k_{c}(i)=k$ if $i$ belongs to $k$-core, but does not belong to $(k+1)$-core (SEIDMAN, 1983). Nodes with low values of $k_{c}$ are located at the periphery of the network. The most central nodes should have the highest values of coreness, whereas high-degree nodes located in the network periphery should display small values of coreness (KITSAK et al., 2010). Therefore, only hubs at the main core of networks present the highest values of $k_{c}$. Importantly, this measure showed to be correlated with epidemic spreading, more specifically with the SIR models (KITSAK et al., 2010) (for more on the SIR model, see Chapter 3). In fact, this relation is the object of our study in Chapter 4.

Next, considering the eigenstructure of the adjacency matrix we can define the socalled eigenvector centrality. This measurement considers that the centrality of each node is the sum of the centrality values of the nodes that it is connected to. Thus, the eigenvector centrality is defined as the components of the leading eigenvector, the eigenvector associated to the largest eigenvalue, of the adjacency matrix A. Formally,

$$
x_{i}=\lambda_{1}^{-1} \sum_{j} \mathbf{A}_{i j} x_{j}
$$

alternatively, in the matrix form we have the eigenvalue problem, $\mathbf{A} \mathbf{x}=\lambda_{1} \mathbf{x}$, where $\mathbf{x}$ is the right leading eigenvector (NEWMAN, 2010) and $\lambda_{1}$ is the largest eigenvalue. Observe that, contrasting with the previous measurements, which considers only local information, the eigenvector centrality considers the complete network structure. Besides, this measurement is closely connected to the spreading process in networks, as these processes relate to the structure by means of the adjacency matrix. In Chapter 3 we discuss this 
Figure 3 - Comparison of three different centrality measures: degree in (a), betweenness centrality in (b) and Page Rank in (c). The size of the nodes are proportional to the centrality of the node. The network used is the São Carlos road map, extracted using the Python library OSMNX (BOEING, 2017) (Version: 0.5.1 https://github.com/gboeing/osmnx).

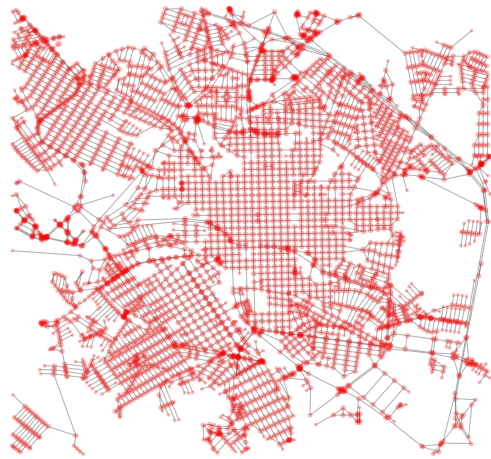

(a) Degree

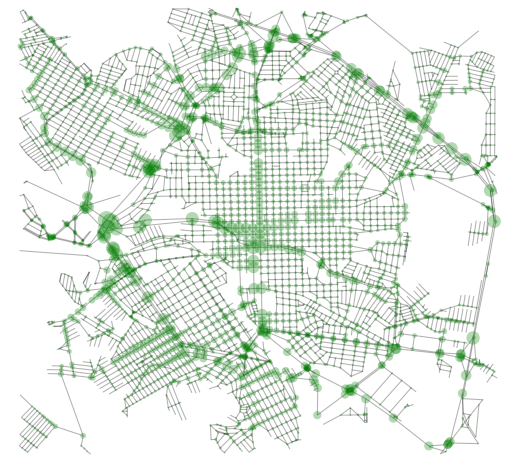

(b) Betweenness

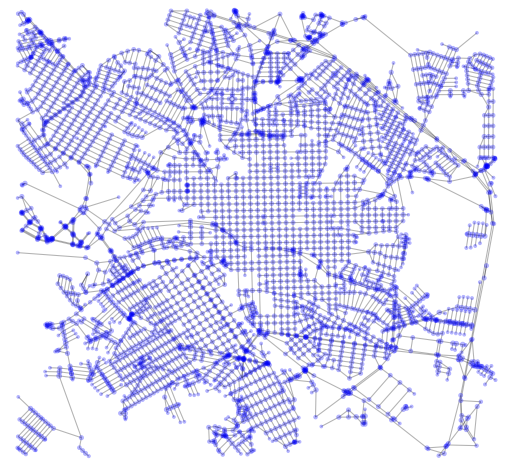

(c) Page Rank

Source: Developed by the author.

relationship, especially its role near the critical point for epidemic spreading.

Furthermore, the number of visits that a given node receives when an agent travels through the network without a preferential route can also be taken into account to quantify the node centrality. In this case, a possible measure is the Google PageRank (BRIN; PAGE, 1998). Formally, PageRank is calculated as

$$
\pi^{T}=\pi^{T} \mathbf{G}
$$

where $\mathbf{G}$ is the Google matrix, i.e.,

$$
\mathbf{G}=\kappa\left(\mathbf{P}+\frac{a e^{T}}{N}\right)+\frac{(1-\kappa)}{N} u u^{T},
$$

and $a$ is the binary vector called dangling node vector $\left(a_{i}\right.$ is equal to one if $i$ is a dangling node and 0 otherwise), $u$ is the all one vector of length $N$ and $\mathbf{P}_{i j}=\frac{\mathbf{A}_{i j}}{k_{j}}$ is the transition probability matrix of the respective network (for more on this matrix see Section 2.2.3). The original version of the algorithm considers $\kappa=0.85$ (BRIN; PAGE, 1998). The PageRank of a node $i, \pi_{i}$, is given by the $i$-th entry of the dominant eigenvector $\pi$ of $\mathbf{G}$, given that $\sum_{i} \pi_{i}=1$. $\pi_{i}$ can be understood as the probability of arriving at node $i$ after numerous steps following a random walk navigation through the network.

\subsubsection{A small visual example of centrality measures}

In order to illustrate the differences between centrality measures, in Figure 3 we show a comparison by means of visualization. The network used here is the São Carlos roadmap. Regarding the centralities, we chose three metrics, degree, betweenness centrality and Page Rank. Note that the first is based on a local property, the second on shortest 
paths and the last one on navigation properties. As commented previously, there is no single measurement that summarizes the importance of a single node. Thus, each metric captures a different property and quantifies it.

São Carlos is a city in São Paulo state, Brazil and its estimated population in 2017 was 246.088 inhabitants $^{1}$. Observe that the city center presents a regular pattern, where most of the nodes have four connections, while in the periphery we have different patterns even with long roads connecting distant parts of the city. This information is well captured by the betweenness centrality, while neglected by local measures such as the degree centrality. Thus, due to the nature of the analyzed network, we observe that the most suitable measure here is the betweenness centrality, which captures the main avenue of São Carlos (and its crossings) as the most important roads. Furthermore, we also notice a strong correlation between degree and the Page Rank centrality in this specific analysis.

\subsubsection{Generalized random walk accessibility}

In this section, we present the concept of random walks applied as the centrality measurement. More specifically, we use the accessibility (TRAVENCOLO; F. Costa, 2008; TRAVENCOLO; VIANA; COSTA, 2009), which is related to the diversity of access of individual nodes through random walks (TRAVENCOLO; F. Costa, 2008). This measure has been considered to identify the border of complex networks (TRAVENCOLO; VIANA; COSTA, 2009). Let $\mathbf{P}^{(h)}(i, j)$ be the probability of reaching node $j$ by performing random walks of length $h$ departing from $i^{2}$. The accessibility of node $i$ for a given distance $h$, by the exponential of the Shannon entropy (TRAVENCOLO; F. Costa, 2008), i.e.,

$$
\alpha_{h}(i)=\exp \left(-\sum_{j} P^{(h)}(i, j) \log P^{(h)}(i, j)\right)
$$

where $1 \leq \alpha_{h}(i) \leq N$. The maximum value corresponds to the case in which all nodes are reached with the same probability $1 / N$. Note that this metric was defined in a multilevel fashion, depending on parameter $h$ that defines the scale of the dynamics (TRAVENCOLO; F. Costa, 2008; TRAVENCOLO; VIANA; COSTA, 2009). In addition, although we are constrained to random walks, virtually any other type of dynamics yielding transition probabilities between adjacent nodes can be considered in the accessibility, which makes this measurement adaptable to the dynamics of each problem being studied.

In order to generalize the accessibility, here we introduce a new version of this metric, which is based on the matrix exponential operation (BHATIA, 1997). This matrix enables the calculus of the probability of transition considering walks of all lengths between any pair of nodes. Thus, if $\mathbf{P}$ is the transition matrix, the exponential of $\mathbf{P}$ is defined as

1 Data From IBGE/2017.

2 Note that $\mathbf{P}^{(h)}$ is the $h$-th power of the transition matrix $\mathbf{P}_{i j}=\frac{\mathbf{A}_{i j}}{k_{j}}$. 
(ARRUDA et al., 2014)

$$
\mathbf{W}=\sum_{k=0}^{\infty} \frac{1}{k !} \mathbf{P}^{k}=e^{\mathbf{P}} .
$$

Matrix $\mathbf{W}$ is based on a modified random walk, which penalizes longer paths. To construct this stochastic process we consider an usual random walk $\left(X_{n}\right)_{n \geq 0}$, where $X_{n}$ represents the node visited by the agent at time $n$. We take a collection of independent and identically distributed uniform random variables in the interval $(0,1)$, i.e. $\left\{U_{1}, U_{2}, \ldots\right\}$, which represents a kind of "fitness" associated to each step of the walk. Moreover, we assume independence between the collection of uniform random variables and the random walk. This modified random walk, which we call accessibility random walk (ARW), considers walks through the network such that all associated fitnesses along a trajectory are in ascending order. We say that node $j$ is visited by the ARW, at time $n$, if $X_{n}=j$ and $U_{1}<U_{2}<U_{3}<\cdots<U_{n}$. We denote by $\left(\tilde{X}_{n}\right)_{n \geq 0}$ the new process and note that $\left\{\tilde{X}_{n}=j\right\}$ implies $\left\{X_{n}=j\right\}$, but the opposite is not necessarily true. A quantity of interest is the number of visits that a given node $j$ receives when an agent travels through the network according to the ARW. This quantity can be written as $\sum_{n=1}^{\infty} I_{\left\{\tilde{X}_{n}=j\right\}}$, where $I_{A}$ is the indicator function of event $A$. We are interested in the mean of this value, by assuming that the agent starts from node $i$, i.e. $\sum_{n=1}^{\infty} \mathbb{E}\left(I_{\left\{\tilde{X}_{n}=j\right\}} \mid \tilde{X}_{0}=i\right)$. In order to compute this value we observe that the term of the sum is the probability $\mathbb{P}\left(\tilde{X}_{n}=j \mid \tilde{X}_{0}=i\right)$ which, by our definition, is equal to $\mathbb{P}\left(\left\{X_{n}=j\right\} \cap\left\{U_{1}<U_{2}<U_{3}<\cdots<U_{n}\right\} \mid X_{0}=i\right)$. This probability is exactly $(1 / n !) \mathbf{P}^{(n)}(i, j)$, where $\mathbf{P}^{(n)}(i, j)$ is the probability of the transition from $i$ to $j$ through walks of length $n$. Therefore, matrix $\mathbf{W}$ considered in Eq. (2.10) is a matrix of mean values associated to the ARW. Element $\mathbf{W}(i, j)$ provides the mean number of visits that node $j$ receives when the agent starts at node $i$ and follows the ARW.

Therefore, the probability of transition between any pair of nodes through ARW is given by

$$
\mathbf{P}=\frac{\mathbf{W}}{e}
$$

Note that matrix $\mathbf{W}$ weighs all walks by the inverse of the factorial of lengths. Therefore, this definition penalizes longer walks, i.e., the shortest walks receive more weight than the longest ones. We define the generalized expression for the accessibility as (ARRUDA et al., 2014)

$$
\alpha(i)=\exp \left(-\sum_{j} \mathbf{P}(i, j) \log \mathbf{P}(i, j)\right),
$$

which we call generalized random walk accessibility. Figure 4 illustrates this measure on the Zachary Karate-club network (ZACHARY, 1977).

We note that the exponential matrix is also considered in the definition of the communicability (ESTRADA; HATANO, 2008; ESTRADA; HATANO; BENZI, 2012). The difference is that the accessibility is based on the concept of diversity (HILL, 1973; 
Figure 4 - Illustration of the concept of accessibility (values calculated from Eq.2.12) in the Zachary Karate-club network (ZACHARY, 1977). Node size is also proportional to their accessibility values. Nodes at the center of the network present the highest accessibility.
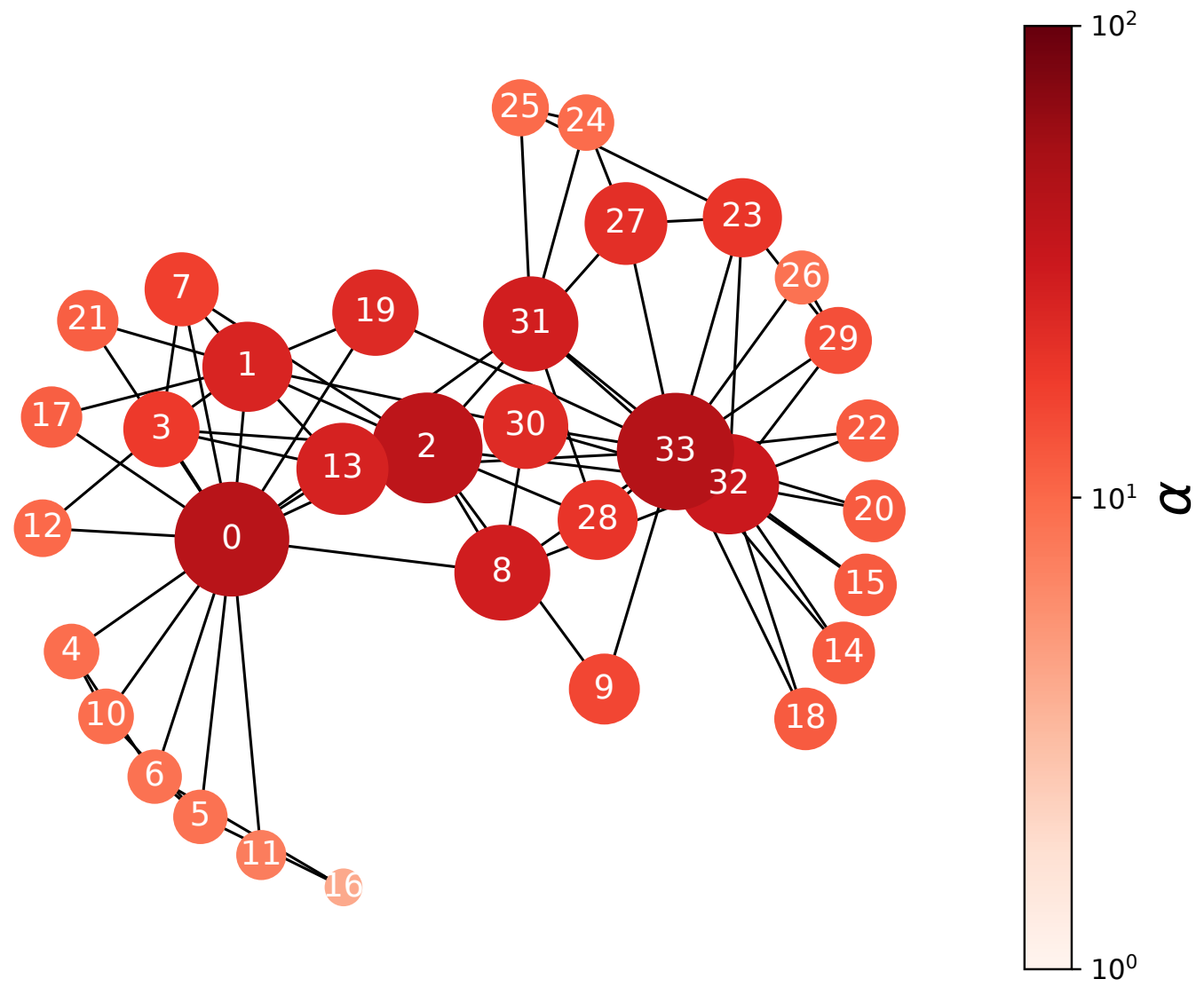

Source: Developed by the author.

JOST, 2006) whereas communicability is associated with the communication between any pair of nodes (ESTRADA; HATANO; BENZI, 2012). Moreover, the former is related to the probability transition matrix, whereas the latter to the adjacency matrix. Thus, there is no trivial relationship between these two metrics in irregular graphs.

In Appendix A we provide an in-depth analysis of this measurement in star graphs, which can be used as an approximation to highly heterogeneous systems, rings and complete graphs.

\subsubsection{Global measures}

Contrasting with the measurements previously presented, here we show some metrics that may be used to globally quantify a specific feature of our network. First of all, it can be observed that, in principle, any summary statistics ${ }^{3}$ might be applied to local

3 Summary statistics are used to summarize a set of observations. As examples, we can cite the average value or any statistical moment, the variation, the standard deviation, or any central moment. 
features. Formally, the $m$-th moment of any distribution is defined as

$$
\mathbb{E}\left(X^{m}\right)=\sum_{i} x_{i}^{m} \mathbb{P}\left(x_{i}\right),
$$

where $X$ is a random variable (with a countable set - discrete case), whose outcomes are $x_{i}$ and its occurrence is given by $\mathbb{P}\left(x_{i}\right)$. As an example, the average degree quantifies the average number of connections in a node found in a given network. Complementary, we might also be interested in the deviation from the average value. In order to quantify it, we may use the variance, defined as

$$
\mathbb{V}(X)=\mathbb{E}\left(X^{2}\right)-\mathbb{E}(X)^{2}
$$

where $\mathbb{E}\left(X^{2}\right)$ is the second moment and $\mathbb{E}(X)^{2}$ is the squared value of the first moment. Observe that the variance is measured in squared units. In order to express it in the same unit of the original data we might consider the standard deviation, which is simply given as $\sigma(X)=\sqrt{\mathbb{V}(X)}$.

Next, regarding correlations among nodes, the degree correlation can be calculated considering the Pearson correlation coefficient on the extremes of each edge (NEWMAN, 2002). This correlation is calculated by

$$
\rho^{P}=\frac{(1 / M) \sum_{j>i} k_{i} k_{j} A_{i j}-\left[(1 / M) \sum_{j>i}(1 / 2)\left(k_{i}+k_{j}\right) A_{i j}\right]^{2}}{(1 / M) \sum_{j>i}\left(k_{i}^{2}+k_{j}^{2}\right) A_{i j}-\left[(1 / M) \sum_{j>i}(1 / 2)\left(k_{i}+k_{j}\right) A_{i j}\right]^{2}},
$$

where $M$ is the number of edges and the superscript $P$ in $\rho^{P}$ stands for the Pearson correlation coefficient. Alternatively, the Spearman correlation coefficient (LITVAK; HOFSTAD, 2013) can also be used. This topic is briefly discussed in Chapter 5. If $\rho^{P}>0$, then the network is assortative, indicating that nodes with similar degrees tend to connect among themselves. On the other hand, if $\rho^{P}<0$, then the network is called disassortative, which indicates that nodes with higher degree tends to connect with low degree nodes. Case $\rho^{P}=0$ suggests that there is no degree correlation. Note that the Pearson correlation coefficient measures only the linear correlation.

\subsubsection{Network optimization: Degree-degree correlation tuning}

Under some circumstances, it is interesting to tune/optimize a specific network measurement, which can be a structural or even dynamical feature of the system. For instance, in Chapter 5 we are interested in evaluating different levels of assortative/disassortative structures and their impact on epidemic spreading. Thus, we describe an optimization method in terms of the assortative coefficient. However, we remark that it can be used for any global property of interest.

Thus, in order to control the level of degree-degree correlations in random networks, we consider a simulated annealing algorithm (KIRKPATRICK, 1984). This algorithm is 
based on two functions, i.e., (i) the perturbation function, which changes the system configuration, and (ii) the energy function, which is minimized. In our case, the perturbation function is a rewiring procedure that preserves the degree distribution of the network but changes the large-scale degree-degree correlations. The energy function is defined as $E_{t}=c\left(\rho_{t}+1\right)$, where $\rho_{t}$ is the network assortativity at time $t$ and $c$ is a constant related to the level of degree-degree correlation, i.e., $c=-1$ if the goal is to obtain an assortative network or $c=1$ if the goal is a disassortative network.

Given an initial network configuration, an initial temperature, $T$ and a cooling factor $\alpha$, the algorithm can be described by the following steps: (i) the energy function is initialized as $E_{0}$; (ii) while the number of iterations is less than a threshold or the optimal solution is not found (or good solution, given a tolerance) the following steps are performed: (iii) a rewiring preserving the degree distribution is executed, according to our perturbation function; (iv) the new energy function, $E_{t+1}$, is calculated; (v) if $E_{t}-E_{t+1}<0$ or $\exp \left(\frac{-\left(E_{t}-E_{t+1}\right)}{T}\right)<U(0,1)$, where $U(0,1)$ is a random number sampled from a uniform distribution in $[0,1]$, then the new solution is accepted; (vi) the temperature is updated, $T=\alpha T$; and (vii) increment the iteration counter. Observe that a worse state than the current one can be accepted with a probability $\exp \left(\frac{-\left(E_{t}-E_{t+1}\right)}{T}\right)$. This mechanism allows the system to avoid local minima. Following this procedure, we can generate random networks with a defined level of degree-degree correlation.

\subsubsection{Network models}

Network models are stochastic methods to generate networks with different structures (BOCCALETTI et al., 2006; COSTA et al., 2007; NEWMAN, 2010; BARRAT; BARTHLEMY; VESPIGNANI, 2008). The main goal of the theoretical approaches is to reproduce some features observed in real systems. One of the first network models was proposed by Erdös and Rényi (ER) (ERDÖS; RÉNYI, 1959). According to this model, nodes are connected according to a uniform probability, without any preference. Despite its mathematical elegance, it cannot describe properties of real networks, as discussed in (NEWMAN; PARK, 2003; NEWMAN, 2010). Thus, many other models have been proposed in the literature to overcome this limitation of the ER model. In this section, we briefly describe some important models that can be used to either mathematically evaluate a given model or describe a real network structure.

\subsubsection{Erdös - Rényi model}

In 1959, the mathematicians Paul Erdös and Alfred Renyi proposed a random model (ERDÖS; RÉNYI, 1959), where the probability of connection between two different nodes is the same. It is also called the ER model. This model was studied in-depth in (BOLLOBÁS, 2001). Besides its mathematical elegance, the structures built by this model 
Figure 5 - In (a) we present the degree distribution of an Erdös and Rényi network with $N=10^{5}$ and $\mathbb{E}(k)=20$, while in the inset, a small ER network is shown for visualization purposes. In (b) we present the degree distribution of a Barabási - Albert network with $N=5 \times 10^{5}$ and $\mathbb{E}(k)=10$, where the estimated $\zeta \approx 3.0$. In the inset, a small BA network is shown for visualization purposes. This figure and its approximation of $\zeta$ were generated using the code provided in http://tuvalu.santafe.edu/ aaronc/powerlaws/ (Accessed on 4th September, 2017).

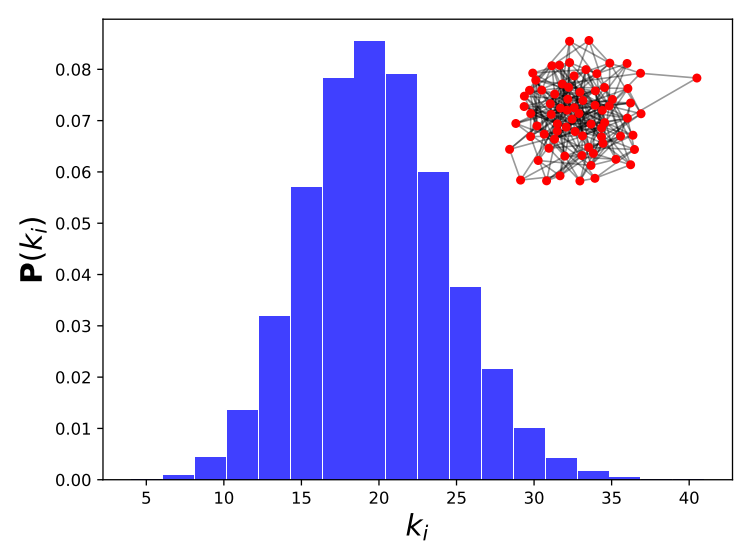

(a) ER

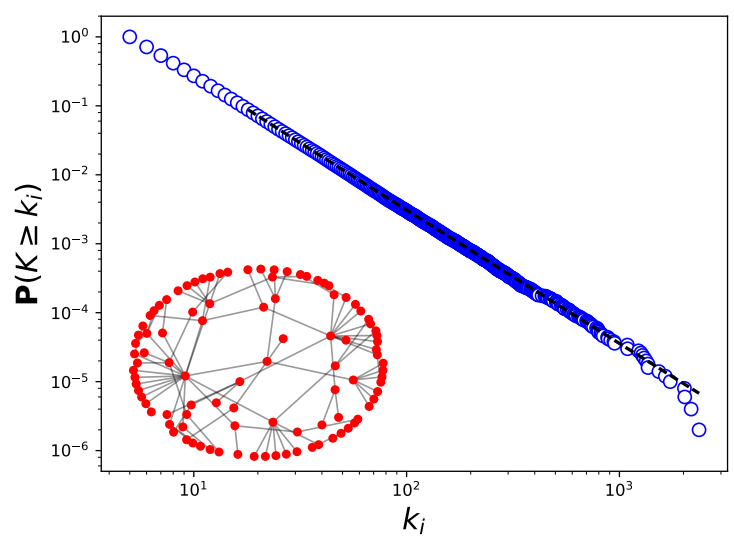

(b) BA

Source: Developed by the author.

do not represent real networks, as empirically verified by Newman in (NEWMAN; PARK, 2003).

Systematically, the algorithm that generates this model is given as: starting with a set of $N$ disconnected nodes, at each pair of nodes an edge is added with probability $p$ - and not added with a complementary probability $(1-p)$. Thus, each edge on the graph has the same probability and the graph is completely homogeneous. Obviously, the average number of connections for each node is $k=p(N-1)$. Note that its degree distribution is given by a binomial distribution since this is a Bernoulli process. Thus, for a large value of $N$, i.e. on the thermodynamic limit, and the average degree is kept constant, the degree distribution tends to a Poisson distribution, as a consequence of the law of rare events (BOLLOBÁS, 2001). Figure 5 (a) shows an example of ER degree distribution and a small visualization.

\subsubsection{Barabási - Albert model}

Barabási and Albert proposed a model which considers growth and preferential attachment rules to generate scale-free networks (BARABÁSI; ALBERT, 1999). It is also known as the BA model. In this case, a network is generated starting with a set of $m_{0}^{*}$ connected nodes. After that, a new node with $m^{*}$ edges is included in the network. The probability of the new node $i$ to connect with an existing node $j$ in the network is 
Figure 6 - In (a) we present a network visualization of the Waxman model (homogeneous), while in (b) the spatial scale free model proposed by Barthélemy (heterogeneous). Each networks have $N=200$. Observe that (a) does not present hubs and long-range connections are rare, while in (b) those features are more often observed.

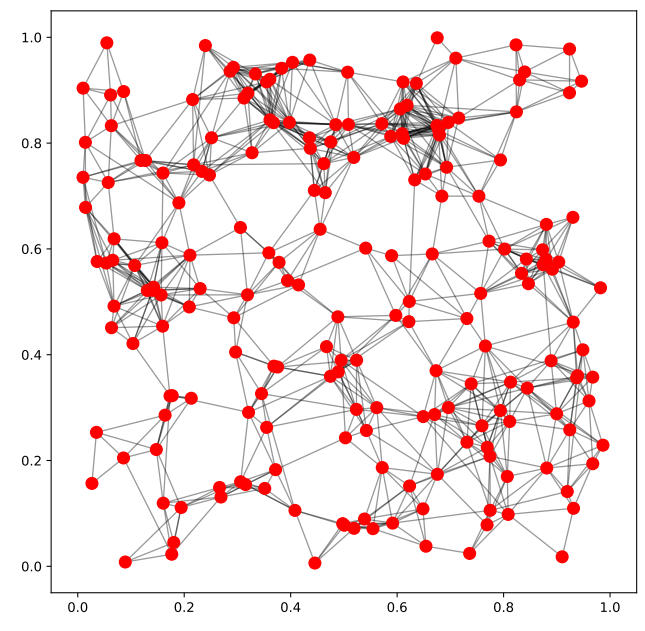

(a) Waxman model

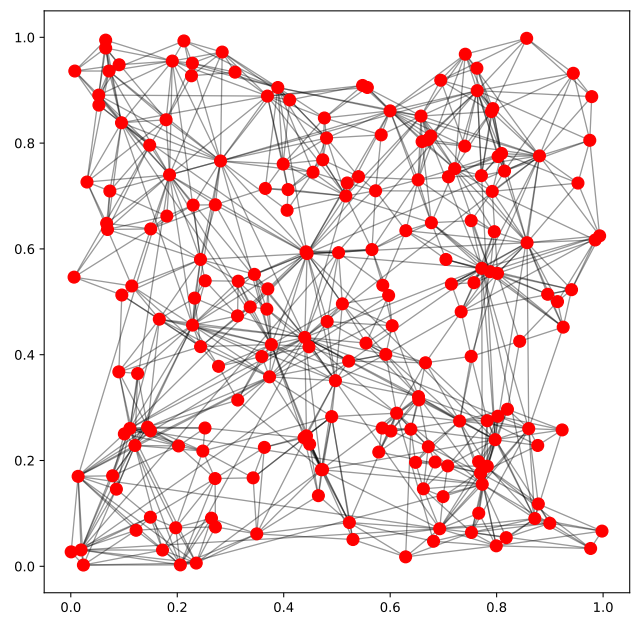

(b) Spatial scale free model

Source: Developed by the author.

proportional to the number of connections of $j$, formally,

$$
\mathbb{P}(i, j)=\frac{k_{j}}{\sum_{u} k_{u}}
$$

The most connected vertices have a greater probability of receiving new connections. Thus, networks generated by this model present a power-law degree distribution, $\mathbb{P}(k)=k^{-\zeta}$, where $\zeta=3$ in the thermodynamic limit $(N \rightarrow \infty)$ (BARABÁSI; ALBERT, 1999). For the sake of an example, we show Figure 5 (b), where we present the degree distribution of a BA network and a small visualization. Moreover, compared to Figure 5 (a) we can contrast homogeneous, ER, and heterogeneous, BA, degree distributions.

\subsubsection{Spatial models}

Firstly, regarding a homogeneous model, the model proposed by Waxman (WAXMAN, 1988) considers that nodes are uniformly distributed into a square of a unitary area. Each pair of nodes is connected according to a probability, that depends on their distances, given as:

$$
\mathbb{P}(i, j)=\eta_{s} \exp \left(-\eta_{s} d_{i j}\right)
$$

where $\eta_{s}$ is a parameter that controls the average degree and $d_{i j}$ is the Euclidean distance between nodes $i$ and $j$. This model generates networks with an exponential degree distri- 
bution, which means that the probability of a node having a degree different than $\mathbb{E}(k)$ decays exponentially.

Secondly, considering the model introduced by Barthélemy (BARTHÉLEMY, 2003; BARTHÉLEMY, 2011), it produces scale-free networks embedded in space. This model is called SSF, which stands for spatial scale-free. Considering a regular $d$ dimensional lattice with length $L$, the algorithm has three main steps. Initially, $n_{0}$ initial active nodes are selected at random. Next, an inactive node $i$ is randomly selected, and connected to an active node $j$ with probability

$$
\mathbb{P}(i, j) \propto \frac{k_{j}+1}{\exp \left(d_{i j} / r_{c}\right)},
$$

where $k_{j}$ is the number of connections of node $j, r_{c}$ is a finite scale parameter and $d_{i j}$ is the Euclidean distance between nodes $i$ and $j$. Finally, the node $i$ becomes active and the second and third steps are repeated until all nodes are active. For each node, the second and third steps are repeated $m^{*}$ times in order to set the average connectivity as $\mathbb{E}(k)=2 m^{*}$ (BARTHÉLEMY, 2003; BARTHÉLEMY, 2011). The parameter $r_{c}$ controls the clustering coefficient (WATTS, 1999) and assortativity (NEWMAN, 2002) of the network. Here we considered $r_{c}=0.05, L=1$ and $d=2$. These values are similar to those used in the original paper (BARTHÉLEMY, 2003).

Figure 6 shows an example of visualization of both models discussed in this subsection. In (a) the Waxman model, which produces a homogeneous network, while in (b) the SSF model, producing is a scale-free network that weighs two different mechanisms: (i) the spatial distribution of the nodes and (ii) the preferential attachment.

\subsubsection{Uncorrelated Configuration Model (UCM)}

Empirical evidence from many areas shows that real networks present a heterogeneous pattern of connections (BOCCALETTI et al., 2006; COSTA et al., 2007; NEWMAN, 2010; BARRAT; BARTHLEMY; VESPIGNANI, 2008). Many networks present a heavytailed degree distribution often approximated by a power-law of the form $\mathbb{P}(k) \sim k^{-\zeta}$. Thus, it is important to have an algorithm to generate a network with such a feature but random regarding other network properties. In fact, the so-called configuration model can generate a network with any given degree distribution (more specifically, with a given degree sequence). It is implemented as follows: (i) for each node we assign a degree $k_{i}$ based on the degree distribution $\mathbb{P}(k)$; (ii) the graph is constructed by randomly connecting pairs of nodes until every node is connected. Observe that there is no correlation associated with this process since the edges are created without taking into account the degree of the nodes that are connected.

Regarding uncorrelated scale-free networks, i.e. $\mathbb{P}(k) \sim k^{-\zeta}$, there is an upper bound for the maximum degree of the network of $k_{\max }=\frac{1}{\sqrt{N}}$ (imposing a structural cut- 
off, $k_{\text {max }} \sim N^{\frac{1}{2}}$ ), as shown in (CATANZARO; BOGUÑ́́; PASTOR-SATORRAS, 2005). This upper bound applies to networks without self-loops and multiple edges. Besides, allowing a free value of $k_{\max }=N$ would also introduce correlations in this kind of networks. In this case, for the natural cut-off, where $k_{\max } \sim N^{\frac{1}{(\zeta-1)}}$ (BARRAT; BARTHLEMY; VESPIGNANI, 2008), it is impossible to construct uncorrelated power-law distributions with $2<\zeta \leq 3$ without allowing multiple edges and self-loops (CATANZARO; BOGUÑ́́; PASTOR-SATORRAS, 2005).

The configuration model can be adapted to produce random networks with a distribution of triangles, allowing us to control the clustering coefficient (NEWMAN, 2009). This model can be used, for example, to quantify the impact of the triangles over a certain dynamical process. It is especially useful in social dynamics since this is a typical feature of this class of networks (BOCCALETTI et al., 2006; COSTA et al., 2007).

\subsubsection{Random regular networks}

Random regular networks $(\mathrm{RRN})$ are a class of homogeneous graphs where every node has the same degree, $k_{i}=k$, but their connections are made randomly. Formally we have that $\mathbb{P}\left(k_{i}=k\right)=1, \forall i \in[N]$. This model does not represent real systems, but it is helpful when analyzing and evaluating dynamical processes in networks. For instance, note that its homogeneity also implies a simplification of the mathematical modeling. Moreover, due to correlations, it is an interesting structural model in the accuracy evaluation of dynamical models, as seen in (Mata, Angélica S.; Ferreira, Silvio C., 2013), where the authors have used this network to evaluate their prediction to the critical point on epidemic spreading. Furthermore, in Chapter 3, we use this model in the accuracy evaluation of mean field approaches.

\subsection{Spectral characterization of networks}

This section describes the spectral properties of networks. Here we focus on singlelayer networks, but the generalization to multilayer networks is natural and straightforward, due to is matricial representation. Firstly, we must establish our constraints. Here we focus on undirected networks. In case we relax these constraints we will specify on the context. Note that directed networks do not have symmetric associated matrices. Historically, Augustin-Louis Cauchy proved the spectral theorem for self-adjoint matrices (or Hermitian matrices), i.e., that every real, symmetric matrix is diagonalizable. Furthermore, the spectral decomposition, eigenvalue decomposition, or eigendecomposition is the decomposition of a matrix on the underlying vector space on which the operator acts. Mathematically such decomposition of $\mathbf{M}$ is given as

$$
\mathbf{M}=\mathbf{V D}^{*} \mathbf{V}^{-1}
$$


Figure 7 - Spectrum of the adjacency matrix of an Erdös and Rényi network with $n=3000$ and $\mathbb{E}(k)=10$. The red line represents the spectral distribution given by the Wigner's semicircle law.

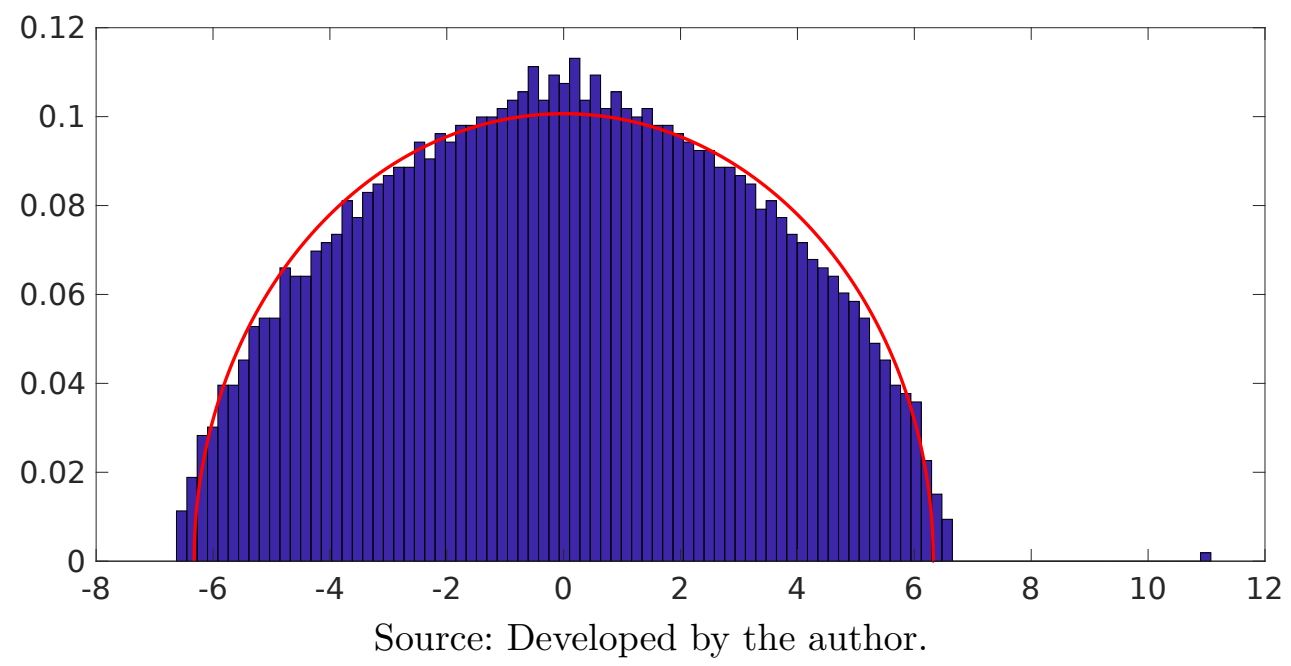

where $\mathbf{V}$ is a $N \times N$ matrix, whose $i$-th column is the eigenvector $\mathbf{V}_{i}$ and $\mathbf{D}^{*}$ is a diagonal matrix whose elements are the associated eigenvalues, $\mathbf{D}_{i i}^{*}=\lambda_{i}$.

The first natural structure is the adjacency matrix since it completely describes a network. Moreover, this matrix and its spectra also appear in the modeling of spreading processes, as will be shown in Chapter 3. A brief description of its spectral properties is shown in Section 2.2.1. The next matrix studied is the Laplacian matrix, Section 2.2.2, which also appears in the dynamical processes. However, its use is more frequent in synchronization processes (ARENAS et al., 2008) and community organization of networks (FORTUNATO, 2010). In Section 2.2.3, we discuss the probability transition matrix, which is closely connected to random walks and even epidemic processes in networks (GÓMEZ et al., 2010; ARRUDA et al., 2017). Finally, in Section 2.2.4 we discuss the nonbacktracking matrix which brings some interesting structural properties, such as communities (KRZAKALA et al., 2013) and their relation to spreading processes (SHRESTHA; SCARPINO; MOORE, 2015) (see also the discussion in Chapter 3, Section 3.5.4).

\subsubsection{Adjacency matrix}

The adjacency matrix completely describes a graph or network. As previously mentioned, it is a binary matrix, whose elements $i$ and $j$ are one if there is an edge between these nodes or mathematically,

$$
\mathbf{A}_{i j}= \begin{cases}1 & \text { if there is an edge connecting nodes } i \text { and } j \\ 0 & \text { otherwise. }\end{cases}
$$

As commented in (KRZAKALA et al., 2013), for sufficient dense Erdös and Rényi 
networks, the Wigner's semicirle law (WIGNER, 1958) can be applied. Formally, it states that the bulk of eigenvalues is distributed as

$$
\mathbb{P}(\lambda)=\frac{\sqrt{4 c-\lambda^{2}}}{2 \pi c}
$$

thus, the bulk of the spectrum lies in $[-2 \sqrt{c}, 2 \sqrt{c}]$ and $c$ is the average degree $c=\mathbb{E}(k)$, where we used the same notation as the original papers. Figure 7 shows an example of the spectral distribution of an Erdös and Rényi network with $n=3000$ and $\mathbb{E}(k)=10$ and its prediction from Wigner's semicircle law.

Next, restricting ourselves to uncorrelated power-law degree distributions, $P(k) \sim$ $k^{-\zeta}$, we can use previous results in the spectrum of graphs (CHUNG; LU; VU, 2003) and obtain the following expression for the lading eigenvalue

$$
\lambda_{\text {max }} \simeq \begin{cases}\sqrt{k_{\max }} & \zeta>\frac{5}{2} \\ \frac{\mathbb{E}\left(k^{2}\right)}{\mathbb{E}(k)} & 2<\zeta<\frac{5}{2}\end{cases}
$$

where $k_{\max }$ is the maximum degree of the network. Note that, in the thermodynamic limit, the maximum degree is a growing function of the network size.

Furthermore, regarding the leading eigenvalue, which is connected to many dynamical processes in networks (for more, see Chapter 3). We can guarantee, using the PerronFrobenius theorem, that any adjacency matrix has a unique largest real eigenvalue and that the corresponding eigenvector can be chosen to have strictly positive components.

\subsubsection{Laplacian matrix}

In order to introduce the Laplacian matrix, we show it in terms of the diffusion process, as it is more natural in this way. Assume that the flux of a given quantity is proportional to a control parameter times the differences between nodes, we have the following master equation

$$
\frac{d \psi_{i}}{d t}=C \sum_{j} \mathbf{A}_{i j}\left(\psi_{j}-\psi_{i}\right),
$$

where $\psi_{i}$ is the quantity under study, $C$ is the control parameter, which can be understood also as a coupling parameter. Thus, this equation can be rewritten in its matricial form, hence

$$
\frac{d \psi}{d t}+C \mathbf{L} \psi=0
$$

where $\psi$ is a vector. Interestingly, this differential equation as a similar form as the gas diffusion equation, except the Laplacian operator $\nabla^{2}$ has been replaced by the Laplacian matrix L. For this reason, we call it the Laplacian graph (NEWMAN, 2010). Thus, the Laplacian matrix can be formally defined, in its matricial formulation, as

$$
\mathbf{L}=\mathbf{D}-\mathbf{A},
$$


Figure 8 - Spectrum of the Laplacian matrix of an Erdös and Rényi network with $n=3000$ and $\mathbb{E}(k)=10$.

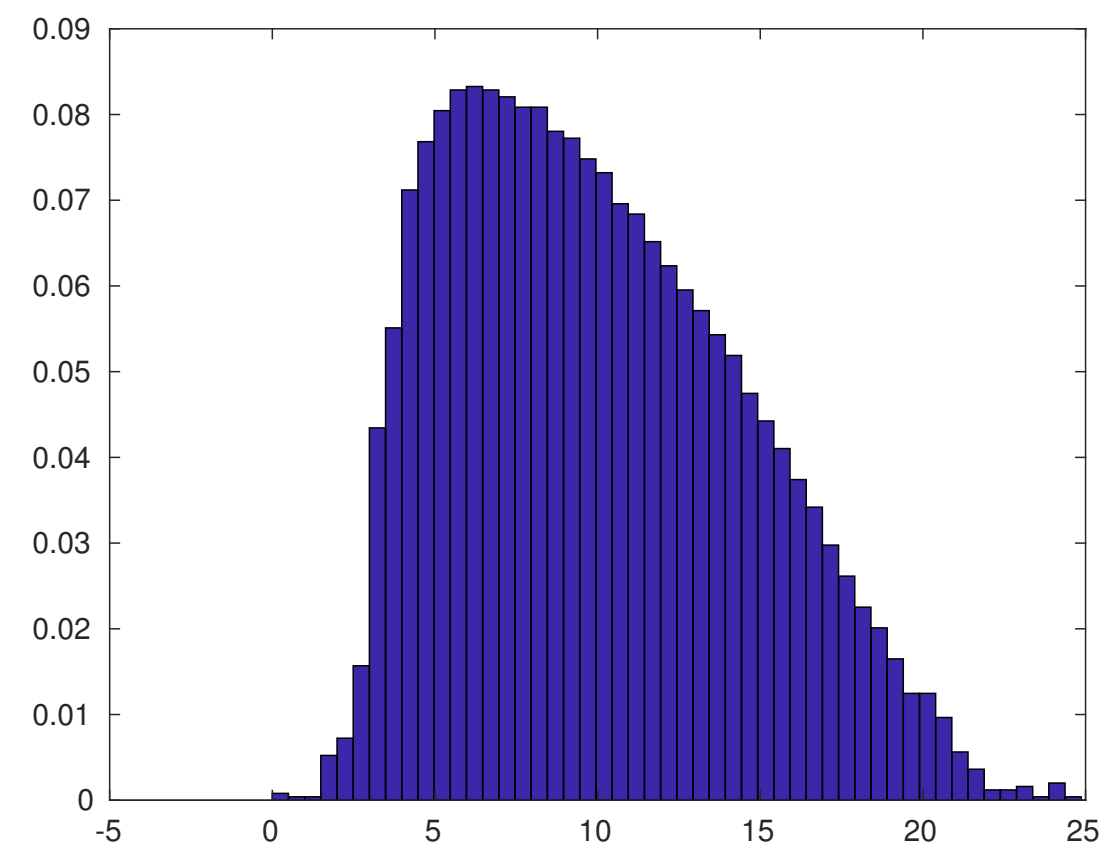

Source: Developed by the author.

where $\mathbf{D}$ is a diagonal matrix comprising the degree of each node, $\mathbf{D}_{i i}=k_{i}$ and $\mathbf{A}$ is the adjacency matrix. Observe that the sum of the rows of this matrix is zero for all of its rows. This also implies that zero is an eigenvalue. Furthermore, the Laplacian matrix is positive semi-definite and its smallest eigenvalue is zero. Therefore, our main interest lies in the second smallest eigenvalue, which is also called algebraic connectivity, and its correspondent eigenvector, called the Fiedler vector. These two objects are directly connected to the community structure (SHI; MALIK, 2000; FORTUNATO, 2010) and dynamical processes (NEWMAN, 2010). Moreover, for the sake of an example, in Figure 8 we show the spectral distribution of the Laplacian matrix. Note that all the eigenvalues are positive and zero is also an eigenvalue.

\subsubsection{Probability transition matrix}

The probability transition matrix was already presented in Section 2.1.2.3. It was defined as $\mathbf{P}_{i j}=\frac{\mathbf{A}_{i j}}{k_{j}}$. However, we can also define it in its matricial form as

$$
\mathbf{P}=\mathbf{D}^{-1} \mathbf{A}
$$

where $\mathbf{D}$ is a diagonal matrix comprising the degree of each node, $\mathbf{D}_{i i}=k_{i}$. Note that it is closely related with random walks (MEILA; SHI, 2001; TRAVENCOLO; F. Costa, 2008; 
Figure 9 - Spectrum of the Non-backtracking matrix of an Erdös and Rényi network with $n=$ 3000 and $\mathbb{E}(k)=10$.

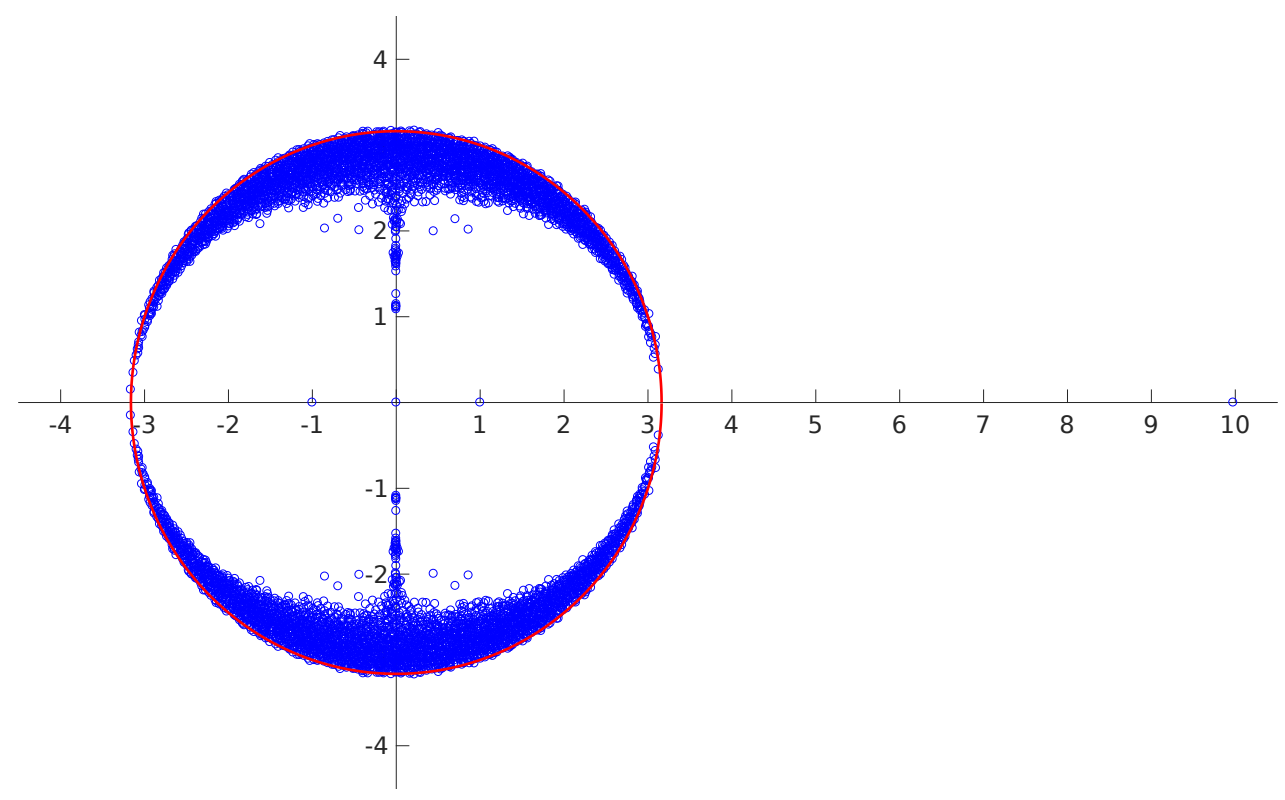

Source: Developed by the author.

TRAVENCOLO; VIANA; COSTA, 2009; ARRUDA et al., 2014) and spreading processes in graphs (GÓMEZ et al., 2010; ARRUDA et al., 2017).

In (MEILA; SHI, 2001), the authors show that the spectral properties of $\mathbf{P}$ are related to the problem of clustering proposed in (SHI; MALIK, 2000). In (MEILA; SHI, 2001) the authors propose to cut a graph into pieces using the following relation with the Laplacian matrix,

$$
\mathbf{L} v=\mu \mathbf{D} v .
$$

This model proved to be efficient in clustering tasks. In (MEILA; SHI, 2001) it was shown that $\mu=(1-\lambda)$, where $\lambda$ are solutions of

$$
\mathbf{P} v=\lambda v
$$

Besides, observe that the eigenvector is the same. This result can be easily obtained by multiplying Equation 2.27 on the left by $\mathbf{D}^{-1}$. The interest in this relationship lies in the fact that the probability matrix shows a strong relation with the group structure in the network and also the Laplacian matrix.

\subsubsection{Non-backtracking matrix}

Finally, in this section, we present the non-backtracking matrix, which is a $M \times M$ matrix defined in directed edges of the graph. Formally it is given as (KRZAKALA et al., 
2013)

$$
\mathbf{B}_{(i \rightarrow j, k \rightarrow l)}= \begin{cases}1 & \text { if } j=k \text { and } i \neq l \\ 0 & \text { otherwise. }\end{cases}
$$

As described in (KRZAKALA et al., 2013), the spectrum of $\mathbf{B}$ is less sensitive to high degree nodes. Furthermore, trees dangling off or disconnected from the graph simply contribute to zero eigenvalues. Similarly, it can be observed that unicyclic components yield to 0,1 and -1 eigenvalues. Besides these particularities, B's bulk is confined to a disk in the complex plane, whose radius is $\sqrt{c}$ (KRZAKALA et al., 2013). Additionally, similarly to the adjacency matrix, using the Perron-Frobenius theorem we can guarantee that the non-backtracking matrix has a unique largest real eigenvalue and that the corresponding eigenvector can be chosen to have strictly positive components. In order to exemplify these concepts, in Figure 9 we show the spectrum of the non-backtracking matrix for an Erdös and Rényi network with $n=3000$ and $\mathbb{E}(k)=10$.

As a result of the previously mentioned properties, B's spectrum can be reduced (the important part) to the spectrum of (HASHIMOTO, 1989; BASS, 1992; ANGEL; FRIEDMAN; HOORY, 2007; KRZAKALA et al., 2013)

$$
\mathbf{B}^{\prime}=\left(\begin{array}{cc}
0 & \mathbf{D}-\mathbf{I} \\
-\mathbf{I} & \mathbf{A}
\end{array}\right)
$$

which is a $2 N \times 2 N$ matrix. This observation drastically reduces the computational complexity. Note that in dense networks $M \in O\left(N^{2}\right)$. Moreover, we can re-write the eigenvalue problem in terms of the block matrices as

$$
\left(\begin{array}{cc}
0 & \mathbf{D}-\mathbf{I} \\
-\mathbf{I} & \mathbf{A}
\end{array}\right)\left(\begin{array}{l}
v_{1} \\
v_{2}
\end{array}\right)=\lambda\left(\begin{array}{c}
v_{1} \\
v_{2},
\end{array}\right)
$$

yielding to the following quadratic eigenvalue problem,

$$
\left(\lambda^{2} \mathbf{I}-\lambda \mathbf{A}+\mathbf{D}-\mathbf{I}\right) v_{2}=0 .
$$

Thus, the characteristic polynomial is given as

$$
\operatorname{det}\left[\lambda^{2} \mathbf{I}-\lambda \mathbf{A}+(\mathbf{D}-\mathbf{I})\right]=0,
$$

whose solutions are the eigenvalues of $\mathbf{B}^{\prime}$ and, obviously, also $\mathbf{B}$. It accounts for the $2 N$ of $\mathbf{B}$ 's eigenvalues, while the other $2(M-N)$ are \pm 1 . This equation is well-known in the theory of graph zeta functions (HASHIMOTO, 1989; BASS, 1992; ANGEL; FRIEDMAN; HOORY, 2007; KRZAKALA et al., 2013).

\subsection{Multilayer network theory}

Multilayer networks are very general objects. They are constituted by interacting layers, representing different means of contact. Consequently, layers are comprise nodes 
Figure 10 - Schematic Illustration of the 3 multilayer networks cases considered as examples. Left panels represent the original networks which give rise to three distinct configurations for the networks of layers. See the text for more details.

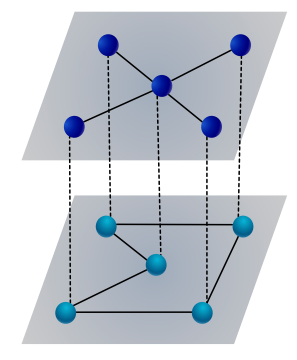

A

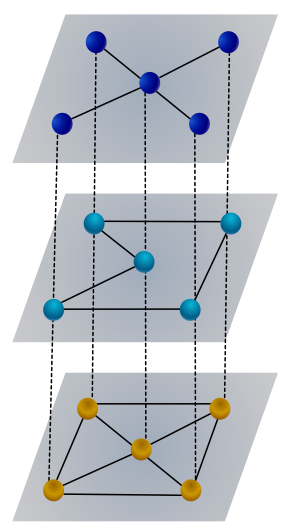

B

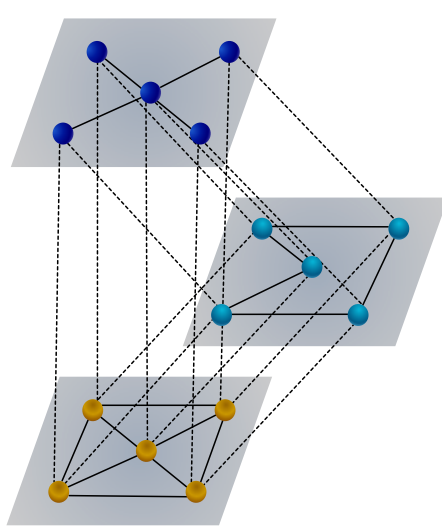

C

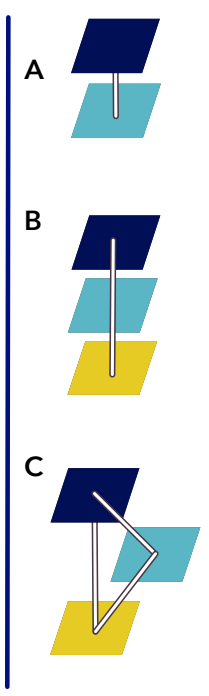

Source: Developed by the author.

and edges, modeling the interactions on the same mean. Indeed, multilayer networks do not impose any restrictions on inter and intra-layer connections. Interestingly, by introducing symmetries, we might also obtain meaningful and simplified objects, which describe real systems. It is arguable that these symmetries could reduce the generality of our objects. However, as an argument, we might observe that the foundations of physics lie in symmetries. Besides its mathematical simplification, the introduced symmetries naturally appear on the real systems justifying their uses. For instance, here we focus on two specific cases, the multiplex and the interconnected networks. In the multiplex case, the network of layers is a complete graph, i.e. each node has its counterpart in all layers (fully diagonal coupling). It is worth mentioning that one might also consider a multiplex with a different number of nodes in different layers (sparse diagonal coupling), however we focus on the first case. In the interconnected case, we can consider a different network of layers topologies and also sparsity on the inter-layer edges. However, we focus on the one-to-one case, where if there is a connection between two layers, all nodes on one layer have a counterpart on the other layer. On the other hand, if there is no connection between layers, none of the nodes are directly connected by inter-layer edges. Furthermore, as shown in (KIVELÄ et al., 2014) "interconnected networks are sets of networks in which some nodes from the various networks are adjacent to each other, but the edges that connect different networks need not indicate dependency relations. If the connections in interdependent networks and similar structures are limited in a certain way, then there is a relationship between them and multiplex networks". or the sake of clarity, we show a schematic illustration in Figure 10, where (a) and (c) represent 2 or 3-layer multiplex networks, while (b) represents 
an interconnected network. For more definitions, we refer the reader to (KIVELÄ et al., 2014).

In this section, we focus on the basic concepts of multilayer representations. Firstly, in Section 2.3.1, we focus on multiplex networks, defining them in terms of their supraadjacency matrix. Note that the generalization to the multilayer case is natural, however, the symmetries introduced in the multiplex case allow us to extract some spectral properties of this system. Next, in Section 2.3.2, we describe the tensorial notation, showing its projections and generalizing some concepts of the previous section to this formalism. It is important to emphasize that both formalisms are equally valid and choosing one over the other might depend on the application or even being a matter of taste. It is worth mentioning that the matrix notation provides a more straightforward notation for sparse coupling matrices as it allows a different number of nodes in each layer. This case on a tensor notation implies in many zeros in the tensor, which can be easily treated. On the other hand, the main advantage of the tensorial notation is the compactness of the equations and its projections, which appear naturally and also have physical meanings.

\subsubsection{Matrix representation}

In this section, we focus on the matrix representation of multiplex networks, which are probably the most natural extension of the single-layer theory to the multilayer formalism. In the following section, we formally define our mathematical objects, followed by the concepts of quotient graphs and coarse-grained versions of the original multiplex. Finally, we introduce the eigenvalue interlacing between the original multiplex network and its coarse-grained versions, which are the main results of this section. These concepts were formerly defined and shown in (COZZO et al., 2016).

\subsubsection{Definitions}

A multiplex network is a quadruple $\mathscr{M}=(\mathfrak{L}, \mathfrak{n}, \mathfrak{P}, \mathfrak{M}) . \mathfrak{L}=\{1, \ldots, m\}$ is an index set that we call the layer set. Here we have assumed $\mathfrak{L} \subset \mathscr{N}$ for practical reasons and without loss of generality. We indicate the general element of $\mathfrak{L}$ with Greek lower case letters. Moreover, $\mathfrak{n}$ is a set of nodes and $\mathfrak{P}=(\mathfrak{n}, \mathfrak{L}, \mathfrak{N}), \mathfrak{N} \subseteq \mathfrak{n} \times \mathfrak{L}$ is a binary relation. Finally, the statement $(n, \alpha) \in \mathfrak{N}$ is read node $n$ participates in layer $\alpha$. We call the ordered pair $(n, \alpha) \in \mathfrak{N}$ a node-layer pair, and we say that the node-layer pair $(n, \alpha)$ is the representative of node $n$ in layer $\alpha$.

On the other hand, $\mathfrak{M}=\left\{G_{\alpha}\right\}_{\alpha \in \mathfrak{L}}$ is a set of graphs, that we call layer-graphs, indexed by means of $\mathfrak{L}$. The node set of a layer-graph $G_{\beta} \in \mathfrak{M}$ is a sub-set $\mathfrak{n}_{\beta} \subset \mathfrak{N}$ such that $\mathfrak{n}_{\beta}=\{(n, \alpha) \in \mathfrak{P} \mid \alpha=\beta\}$, so the nodes of $G_{\beta}$ are node-layer pairs; therefore we say that node-layer pairs represent nodes in layers. The edge set of a graph $G_{\alpha} \in \mathfrak{M}$ is 
$\mathfrak{E}_{\beta} \subseteq \mathfrak{n}_{\beta} \times \mathfrak{n}_{\beta}$. Additionally, the binary relation $\mathfrak{P}$ can be identified with its graph $G_{\mathfrak{P}} . G_{\mathfrak{P}}$ has nodes set given by $\mathfrak{n} \cup \mathfrak{L}$, and edge set $\mathfrak{E}_{\mathfrak{P}}=\mathfrak{N}$, and we call it the participation graph.

Consider the graph $G_{\mathfrak{C}}$ on $\mathfrak{N}$ in which there is an edge between two node-layer pairs $(n, \alpha)$ and $(m, \beta)$ only if $n=m$; that is, only if the two edges in the graph $G_{\mathfrak{P}}$ are incident on the same node $n \in \mathfrak{n}$, which means that the two node-layer pairs represent the same node in different layers. We call $G_{\mathfrak{C}}$ the coupling graph. It is easy to notice that the coupling graph is composed by $n=|\mathfrak{n}|$ disconnected components that are clicks or isolated nodes. Each clique is formed by all the representatives of a node in the layers, we call the components of $G_{\mathfrak{C}}$ supra-nodes.

Let's now also consider graph $G_{\mathfrak{l}}$ on the same node set $\mathfrak{N}$, where there is an edge between two node-layer pairs $(n, \alpha),(m, \beta)$ only if $\alpha=\beta$; that is, only if the two edges in graph $G_{\mathfrak{P}}$ are incident on the same node $\alpha \in \mathfrak{L}$. We call $G_{\mathfrak{l}}$ the layer graph. It can be observed that graph is formed by $m=|\mathfrak{L}|$ disconnected components that are clicks.

Finally, we can define the supra-graph $G_{\mathscr{M}}$ as the union of the layer-graphs with the coupling graph: $G_{\mathscr{C}} \cup \mathfrak{M}$. $G_{\mathscr{M}}$ has node set $\mathfrak{N}$ and edge set $\bigcup_{\alpha} \mathfrak{E}_{\alpha} \cup \mathfrak{E}_{\mathfrak{C}} . G_{\mathscr{M}}$ is a synthetic representation of the Multiplex Network $\mathscr{M}$. It results that each layer-graph $G_{\alpha}$ is a sub-graph of $G_{\mathscr{M}}$ induced by $\mathfrak{n}_{\alpha}$. Furthermore, when all nodes participate in all layer-graphs, the Multiplex Network is said to be fully aligned (KIVELÄ et al., 2014) and the coupling graph is made of $n$ complete graphs of $m$ nodes.

It is useful to come back to our system of social agents as a paradigmatic multiplex network to make sense of the previous definitions. The layer set is the list of OSNs, for example, $\mathfrak{L}=\{$ Facebook,Twitter,Google +$\}$. Since for practical purposes we want a set of indexes that are natural numbers, we may say that: Facebook is 1, Twitter is 2, and Google + is 3. The set of nodes is the set of social actors, for example, $n=\{$ Marc, Alice, BiFi,Nick, Rose $\}$. The binary relations represent the participation of each of these agents in some OSNs, thus we have that a statement of the type Alice has a Facebook account is represented by the pair (Alice, 1 ), that is a node-layer pair. Each set of relationships in each OSN is represented by a graph, for example, the link [(Alice, 1$),($ Nick,1)] means that Alice and Nick are friends on Facebook. If Alice has a Facebook account and a Twitter account, but not a Google+ account, in the coupling graph we will have the connected component [(Alice, 1$),($ Alice, 2$)]$ that is the supra-node related to Alice. If only the BiFi, Nick, and Rose have Google+ accounts, in the layer graph we will have the connected component [(Bifi,3), (Nick,3), (Rose,3)].

\subsubsection{The supra-adjacency matrix}

The supra-adjacency matrix is the adjacency matrix of the supra-graph $G_{\mathscr{M}}$. Just as $G_{\mathscr{M}}, \mathbf{A}$ is a synthetic representation of the whole multiplex $\mathscr{M}$. By definition, it can be obtained from the intra-layer adjacency matrices and the coupling matrix in the following 
way:

$$
\mathbf{A}=\bigoplus_{\alpha} \mathbf{A}^{\alpha}+\mathscr{C}
$$

where the same consideration as in $\mathscr{C}$ applies for the indices. We also define $\overline{\mathbf{A}}=\bigoplus \mathbf{A}^{\alpha}$, and we call it the intra-layer adjacency matrix. Furthermore, one might weight the inter and intra-layer interactions differently. Thus, Equation 2.34 can be re-written as

$$
\mathbf{A}=\bigoplus_{m=1}^{M} \mathbf{A}^{(m)}+p \mathbf{C}
$$

where $p$ is a parameter that defines the strength of the inter-layer edges and $\mathbf{C}$ is the interlayer coupling matrix, whose elements represent the relations between nodes in different layers, thus implicitly containing the information of a network of layers (SÁNCHEZGARCÍA; COZZO; MORENO, 2014).

\subsubsection{Quotient graphs}

We next briefly introduce the notion of network quotient associated to a partition of the node set. Suppose that $V_{1}, \ldots, V_{m}$ is a partition of the node set of a network $G$ with adjacency matrix $A$, and write $n_{i}=\left|V_{i}\right|$. The quotient network $Q$ of $G$ is a coarse-grained representation of the network with respect to the partition. It has one node per cluster $V_{i}$ and an edge from $V_{i}$ to $V_{j}$ weighted by an average connectivity from $V_{i}$ to $V_{j}$

$$
b_{i j}=\frac{1}{\sigma} \sum_{\substack{k \in V_{i} \\ l \in V_{j}}} a_{k l} .
$$

Different choices are possible for the normalization parameter $\sigma: \sigma_{i}=n_{i}, \sigma_{j}=n_{j}$ or $\sigma_{i j}=\sqrt{n_{i} n_{j}}$. Depending on the choice for $\sigma$ we call the resulting quotient respectively: left, right or symmetric quotient. We can express the left quotient $Q_{l}(A)$ in matrix form. Consider the $n \times m$ characteristic matrix of the partition $S=s_{i j}$, with $s_{i j}=1$ if $i \in V_{j}$ and zero otherwise. Then

$$
Q_{l}(A)=\Lambda^{-1} S^{T} A S
$$

where $\Lambda=\operatorname{diag}\left\{n_{1}, \ldots, n_{m}\right\}$.

\subsubsection{Aggregate Network and Network of Layers of a Multiplex Network}

In the context of Multiplex Networks, two quotient graphs arise naturally (SÁNCHEZGARCÍA; COZZO; MORENO, 2014; COZZO et al., 2016) by considering coupled nodelayer pairs and layers. Supra-nodes partition the supra-graph, and the supra-nodes characteristic matrix $S_{n}$ is the associated characteristic matrix. Then, we define the aggregate network of the multiplex network as the quotient associated to that partition:

$$
\tilde{\mathbf{A}}=\Lambda^{-1} \mathscr{S}_{n}^{T} \mathscr{\mathscr { A }} \mathscr{S}_{n}
$$


where $\Lambda=\operatorname{diag}\left\{\kappa_{1}, \ldots, \kappa_{n}\right\}$ is the multiplexity degree matrix. Since, the Laplacian of the quotient is equal to the quotient of the Laplacian, the Laplacian of the aggregate network is given by:

$$
\tilde{\mathbf{L}}=\Lambda^{-1} \mathscr{S}_{n}^{T} \overline{\mathscr{L}}_{n}
$$

In the same way, layers partition the supra-graph, thus the network of layers is defined by

$$
\tilde{\mathbf{A}}_{\mathfrak{l}}=\Lambda^{-1} \mathscr{S}_{\mathfrak{l}}^{T} \overline{\mathscr{A}} \mathscr{S}_{\mathfrak{l}}
$$

and its Laplacian is given by

$$
\tilde{L}_{\mathfrak{l}}=\Lambda^{-1} S_{\mathfrak{l}}^{T} \bar{L} S_{\mathfrak{l}}
$$

\subsubsection{Spectral relations between supra and cross-grained representations}

The fundamental spectral result related to a quotient network is that adjacency eigenvalues of a quotient network interlace the adjacency eigenvalues of the parent network. That is, if $\mu_{i}, \ldots, \mu_{m}$ are the adjacency eigenvalues of the quotient network, and $\lambda_{i}, \ldots, \lambda_{n}$ are the adjacency eigenvalues of the parent network, the result is that

$$
\lambda_{i} \leq \mu_{i} \leq \lambda_{i+n-m}
$$

The same result applies for Laplacian eigenvalues. We can derive directly from that result a list of bounds for the supra-adjacency and the supra-Laplacian in terms of the aggregate network and of the network of layers (SÁNCHEZ-GARCÍA; COZZO; MORENO, 2014; COZZO et al., 2016). Besides, in the case of node aligned multiplex networks, we have that the eigenvalues of the Laplacian of the network of layers are a subset of the spectrum of the supra-Laplacian. This result is of special relevance in studying the structural properties of a multiplex network since it states that the adjacency (Laplacian) eigenvalues of the crossgrained representation of a multiplex interlace the adjacency (Laplacian) eigenvalues of the parent. In the case of a node-aligned multiplex, the Laplacian eigenvalues of the network of layers are a sub-set of the Laplacian eigenvalues of the parent Multiplex network.

\subsubsection{Tensorial representation}

\subsubsection{Definitions}

Here we use the representation formerly presented in (De Domenico et al., 2013). We also adopt the Einstein summation convention, in order to have more compact equations: if two indices are repeated, where one is a superscript and the other a subscript, then this operation implies a summation. Moreover, the result is a tensor whose rank lowers by

2. For instance, $A_{\beta}^{\alpha} A_{\alpha}^{\gamma}=\sum_{\alpha} A_{\beta}^{\alpha} A_{\alpha}^{\gamma}$. In our notation, we use Greek letters to indicate the components of a tensor. In addition, we use a tilde $(\tilde{*})$ to denote the components related 
to the layers, with dimension $m$, while the components without a tilde have dimension $n$ and are related to the nodes.

A multilayer network is represented as the fourth-order adjacency tensor $M \in$ $\mathbb{R}^{n \times n \times m \times m}$, which can represent various relations between nodes (De Domenico et al., 2013). Formally we have

$$
M_{\beta \tilde{\gamma}}^{\alpha \tilde{\delta}}=\sum_{\tilde{h}, \tilde{k}=1}^{m} C_{\beta}^{\alpha}(\tilde{h} \tilde{k}) E_{\tilde{\gamma}}^{\tilde{\delta}}(\tilde{h} \tilde{k})=\sum_{\tilde{h}, \tilde{k}=1}^{m} \sum_{i, j=1}^{n} w_{i j}(\tilde{h} \tilde{k}) \mathscr{E}_{\beta \tilde{\gamma}}^{\alpha \tilde{\delta}}(i j \tilde{h} \tilde{k})
$$

where $E_{\tilde{\delta}}^{\tilde{\gamma}}(\tilde{h} \tilde{k}) \in \mathbb{R}^{m \times m}$ and $\mathscr{E}_{\beta \tilde{\gamma}}^{\alpha \tilde{\delta}}(i j \tilde{h} \tilde{k}) \in \mathbb{R}^{n \times n \times m \times m}$ indicate the tensor in its respective canonical basis.

In addition to the tensor $M \in \mathbb{R}^{n \times n \times m \times m}$, we are usually interested in the weighted tensor, where the inter and intra layer edges have different weights. This tensor is denoted as

$$
\mathscr{R}_{\beta \tilde{\delta}}^{\alpha \tilde{\gamma}}(\lambda, \eta)=M_{\beta \tilde{\sigma}}^{\alpha \tilde{\eta}} E_{\tilde{\eta}}^{\tilde{\sigma}}(\tilde{\gamma} \tilde{\delta}) \delta_{\tilde{\delta}}^{\tilde{\gamma}}+\frac{\eta}{\lambda} M_{\beta \tilde{\sigma}}^{\alpha \tilde{\eta}} E_{\tilde{\eta}}^{\tilde{\sigma}}(\tilde{\gamma} \tilde{\delta})\left(U_{\tilde{\delta}}^{\tilde{\gamma}}-\delta_{\tilde{\delta}}^{\tilde{\gamma}}\right)
$$

and it is called supra contact tensor, whose name comes from its definition on epidemic spreading (ARRUDA et al., 2017). Note that the intra-layer edges are weighted by $\eta$, while the inter-layer edges are weighted by $\lambda$. Furthermore, since a scalar does not change the spectral properties of our tensor, we divide it by $\lambda$, remaining with just one parameter, the so-called coupling parameter, $\frac{\eta}{\lambda}$.

\subsubsection{Projections}

One of the advantages of the tensorial representation is the projections, which allow us to have very compact equations. In the context of multilayer networks, these projections often present a physical meaning, allowing us to characterise different levels of the system. First of all, observe that we can extract one layer by projecting the tensor $M_{\beta \tilde{\gamma}}^{\alpha \tilde{\delta}}$ to the canonical tensor $E_{\tilde{\delta}}^{\tilde{\gamma}}(\tilde{r} \tilde{r})$. Formally, from (De Domenico et al., 2013) we have

$$
M_{\beta \tilde{\gamma}}^{\alpha \tilde{\delta}} E_{\tilde{\delta}}^{\tilde{\gamma}}(\tilde{r} \tilde{r})=C_{\beta}^{\alpha}(\tilde{r} \tilde{r})=A_{\beta}^{\alpha}(\tilde{r})
$$

where $\tilde{r} \in\{1,2, \ldots, m\}$ is the selected layer and $A_{\beta}^{\alpha}(\tilde{r})$ is the adjacency matrix (rank-2 tensor). Moreover, aiming at having more compact and clear equations, we define the allone tensors $u_{\alpha} \in \mathbb{R}^{n}$ and $U^{\beta \tilde{\delta}} \in \mathbb{R}^{n \times m}$. Here, we restrict our analysis to multilayer networks with a diagonal coupling (KIVELÄ et al., 2014). In other words, each node can have at most one counterpart on the other layers. In addition, for the sake of simplicity,, we focus on unweighted and undirected connected networks, in which there is a path from each node to all other nodes.

Besides the adjacency tensor presented above, the network of layers (SÁNCHEZGARCÍA; COZZO; MORENO, 2014) also characterizes the topology of the system. In 
this reduced network representation, each node represents one layer and the edges between them codify the number of edges connecting those two layers. Formally we have,

$$
\Psi_{\tilde{\delta}}^{\tilde{\gamma}}=M_{\beta \tilde{\delta}}^{\alpha \tilde{\gamma}} U_{\alpha}^{\beta}
$$

where $\Psi_{\tilde{\delta}}^{\tilde{\gamma}} \in \mathbb{R}^{m \times m}$. Note that this a network presents self-loops, which are weighted by the number of edges on the layer. Additionally, since we assume that the layers have the same number of nodes, the edges of the network of layers have weights equal to the number of nodes $n$.

Another important reduction of the multilayer network is the so-called projection (De Domenico et al., 2013). This network aggregates all the information into one layer, including self-loops that stand for the number of layers in which a node appears. Mathematically, we have

$$
P_{\beta}^{\alpha}=M_{\beta \tilde{\gamma}}^{\alpha \tilde{\delta}} U_{\tilde{\delta}}^{\tilde{\gamma}}
$$

where $P_{\beta}^{\alpha} \in \mathbb{R}^{n \times n}$. Complementary, a version of the projection without self-edges is called the overlay network and is given as the contraction over the layers (De Domenico et al., 2013), i.e.,

$$
O_{\beta}^{\alpha}=M_{\beta \tilde{\gamma}}^{\alpha \tilde{\gamma}}
$$

Observe that the overlay network does not consider the contribution of the interlayer connections, whereas the projection does. As we will see later, comparisons between the assortativity of these two different representations of the system reveal the key role of such inter-links (for more details, please refer to 5.2).

\subsubsection{Eigentensor problem}

Here we describe the eigenvalue problem considering the tensorial representation. This eigenvalue problem can be generalized to the case of a rank-4 tensor leading to

$$
\mathscr{R}_{\beta \tilde{\delta}}^{\alpha \tilde{\gamma}} f_{\alpha \tilde{\gamma}}(\Lambda)=\Lambda f_{\beta \tilde{\delta}}(\Lambda)
$$

where $\Lambda$ is an eigenvalue and $f_{\beta \tilde{\delta}}(\Lambda)$ is the corresponding eigentensor. In addition, we are assuming that the eigentensors form an orthonormal basis. Importantly, the supra-contact matrix, $R$, in (COZZO et al., 2013) can be understood as a flattened version of the tensor

$\mathscr{R}_{\beta \tilde{\delta}}^{\alpha \tilde{\gamma}}(\lambda, \eta)$. Consequently, all the results for $R$ also apply to the tensor $\mathscr{R}$. As argued in (De Domenico et al., 2013), this supra-adjacency matrix corresponds to unique unfolding of the fourth-order tensor $M$ yielding square matrices. Following this unique mapping, we have the correspondence of the eigensystems.

\subsubsection{Interlacing properties}

Invoking the unique mapping presented in the previous sub-section and considering the results of (SÁNCHEZ-GARCÍA; COZZO; MORENO, 2014; COZZO et al., 2016), we 
Table 1 - Structure and spectra of the normalized network of layers $\Phi_{\tilde{\delta}}^{\tilde{\gamma}}(\lambda, \eta)$. The eigenvalues assume that the average degree of each layer, $\mathbb{E}\left(k^{l}\right)$, is the same, i.e. $\mathbb{E}\left(k^{l}\right)=\mathbb{E}(k), \forall l$.

\begin{tabular}{|c|c|c|c|c|}
\hline Network & \multicolumn{3}{|c|}{$\Phi_{\tilde{\delta}}^{\tilde{\gamma}}(\lambda, \eta)$} & Eigenvalues \\
\hline Line with 2 nodes & {$\left[\begin{array}{r}\mathbb{E}(k \\
\frac{1}{7}\end{array}\right.$} & $\begin{array}{l}=1) \\
\mathbb{E}(k\end{array}$ & $=2)]$ & $\begin{array}{l}\mathbb{E}(k)-\frac{\eta}{\lambda} \\
\mathbb{E}(k)+\frac{\eta}{\lambda}\end{array}$ \\
\hline Line with 3 nodes & {$\left[\begin{array}{c}\mathbb{E}\left(k^{l=1}\right) \\
\frac{\eta}{\lambda} \\
0\end{array}\right.$} & $\mathbb{E}\left(\begin{array}{c}\frac{\eta}{\lambda} \\
\frac{\eta}{\lambda}\end{array}\right.$ & $\mathbb{E}\left(\begin{array}{c}0 \\
\frac{\eta}{\lambda} \\
\left.k^{l=3}\right)\end{array}\right.$ & $\begin{array}{c}\mathbb{E}(k) \\
\mathbb{E}(k)-\sqrt{2} \frac{\eta}{\lambda} \\
\mathbb{E}(k)+\sqrt{2} \frac{\eta}{\lambda}\end{array}$ \\
\hline Multiplex & {$\left[\begin{array}{c}\mathbb{E}\left(k^{l=1}\right) \\
\frac{\eta}{\lambda} \\
\frac{\eta}{\lambda}\end{array}\right.$} & $\mathbb{E}\left(\begin{array}{c}\frac{\eta}{\lambda} \\
k^{l=2} \\
\frac{\eta}{\lambda}\end{array}\right)$ & $\mathbb{E}\left(k^{\frac{\eta}{\lambda}}\right)$ & $\begin{array}{l}\mathbb{E}(k)-\frac{\eta}{\lambda} \\
\mathbb{E}(k)-\frac{\eta}{\lambda} \\
\mathbb{E}(k)+2 \frac{\eta}{\lambda}\end{array}$ \\
\hline
\end{tabular}

can use the interlacing properties to relate the spectra of the multilayer network with the spectra of the network of layers. First of all, we define the normalized network of layers (for more see Section 2.3.2.2) in terms of the supra contact tensor as

$$
\Phi_{\tilde{\delta}}^{\tilde{\gamma}}(\lambda, \eta)=\frac{1}{n} \mathscr{R}_{\beta \tilde{\delta}}^{\alpha \tilde{\delta}}(\lambda, \eta) U_{\alpha}^{\beta}
$$

where we are implicitly assuming a multilayer network in which the layers have the same number of nodes and a dependency on the coupling parameters (the demonstration that this tensor is an unfolding of the matrix in (SÁNCHEZ-GARCÍA; COZZO; MORENO, 2014) is shown in Appendix B in order to have a more fluid text). Additionally, let's denote by $\mu_{1} \geq \mu_{2} \geq \ldots \geq \mu_{m}$ the ordered eigenvalues of $\Phi_{\tilde{\delta}}^{\tilde{\gamma}}(\lambda, \eta)$. Following (SÁNCHEZGARCÍA; COZZO; MORENO, 2014), the interlacing properties imply

$$
\Lambda_{n m-m+j} \leq \mu_{j} \leq \Lambda_{j}
$$

for $j=m, \ldots, 1$. As examples, Table 1 shows the spectrum of three simple networks of layers that can be computed analytically: a line with two and three nodes and a triangle. Figure 10 shows a schematic illustration of these 3 multilayer networks.

Furthermore, using similar arguments we can also obtain results for the normalized projection, formally given as

$$
\mathbf{P}_{\beta}^{\alpha}=\frac{1}{m} \mathscr{R}_{\beta \tilde{\delta}}^{\alpha \tilde{\delta}}(\lambda, \eta) U_{\tilde{\gamma}}^{\tilde{\delta}}
$$

whose ordered eigenvalues, denoted by $v_{1} \geq v_{2} \geq \ldots \geq v_{m}$, also interlace with the supra contact tensor satisfying

$$
\Lambda_{n m-n+j} \leq v_{j} \leq \Lambda_{j},
$$

for $j=n, \ldots, 1$. Finally, the adjacency tensor of an extracted layer also interlaces, yielding

$$
\Lambda_{n m-n+j} \leq \Lambda_{j}^{l} \leq \Lambda_{j},
$$


for $j=n, \ldots, 1$. These results show that the eigenvalue of the multilayer adjacency tensor is always larger than or equal to all the eigenvalues of the individual isolated layers as well as the network of layers.

\subsection{Summary}

In this chapter we formally defined the concept of networks (single and multilayer) focusing on the matricial representation, which is essential for the development of dynamical processes, the topic of the next chapter.

The measure and networks model described here are considered in the analysis of influential spreaders in networks and in the development of the new models of disease and rumor spreading, both discussed in Chapter 4. The centrality measures are useful to predict the capacity of propagation of each node, whereas the other measures are considered to describe the large-scale network organization. Indeed, the network models considered here generate networks with different properties, which are quantified by network measures. These models are fundamental to study the relationship between the structure and dynamics of networks as they can control the network organization while keeping the number of edges and nodes constant.

Finally, in the multilayer theory, we also defined our main objects, presenting two different representations, matricial and tensorial. It can be observed that both approaches are equivalent and have its advantages and disadvantages, as previously discussed. Besides the representation, we also presented the concept of eigenvalue interlacing, which allows us to extract useful information about our systems. The developed theory is essential for understanding Chapters 5 and 6, where we study epidemic spreading and its relation with the spectral properties of multilayer networks. 

CHAPTER

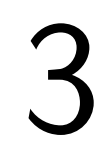

\section{FUNDAMENTALS OF SPREADING PROCESSES IN NETWORKS}

Epidemic-like spreading processes are paradigmatic, as they can describe not only the temporal unfolding and evolution of diseases but also of ideas, information and rumors in fields as diverse as biological, information and social sciences (PASTOR-SATORRAS et al., 2015). Due to their fundamental nature and simplicity, two particular models have received special attention by the scientific community, the susceptible-infected-susceptible (SIS) and the susceptible-infected-recovered (SIR). In both models, an infected individual spreads the disease to its neighbors at a given (spreading) rate and infected individuals recover at some other rate. The difference between both scenarios lies in the fact that in the SIS case, once recovered, infected individuals can catch the disease again, and, therefore, they go back to the susceptible state. On the contrary, in the SIR model, recovered individuals are supposed to acquire permanent immunity and do not play an active role in the spreading process anymore. There are many other variations of these two models, including more realistic and intricate compartmental models (PASTOR-SATORRAS et al., 2015). However, these two schemes are sufficient to capture the main phenomenology of disease dynamics - and many other contagion-like processes - including the onset of epidemics, while remaining simple. Moreover, with the advent of modern communication technologies and transportation means and the increasing availability of data (often in real time or with a highly detailed time resolution), previous theoretical-only models are fed with data (GLEESON et al., 2016), making data-driven simulations an effective tool for decision-making and designing efficient viral algorithms in the case of information dissemination.

In this chapter, we address a key subject of this thesis: the basic concepts on dynamical spreading processes in networks. We will follow a constructive way, defining the local rules, which naturally appear from analyzing biological systems. Next, we follow 
the mathematical formalisms, properly defining the Markov chain and obtaining the exact equations, but also the mean field approximations. Furthermore, here we also made a especial effort to properly classify and distinguish among the various approaches found in the literature. We classified the spreading processes into two classes according to the time distinction: (i) continuous-time and (ii) cellular automata approach. Moreover, the cellular automata approaches can be further classified into two classes according to their update scheme, which can be: (a) synchronous or (ii) asynchronous. Besides analytical aspects, we also discuss the correct association between a given mathematical framework and its simulation algorithm, also providing a systematic accuracy analysis.

\subsection{Epidemic spreading}

Firstly, we must define the set of local rules that characterize our dynamical processes. After that we formally define our mathematical model constructing a Markov chain that follow each set of rules in its appropriate state space. Denoting by $X$ individuals that are susceptible and then can be infected, by $Y$ individuals that are infected and can spread the disease to its neighbors by means of contact and, finally, by $Z$ recovered or removed individuals, which do not take part on the dynamical process ${ }^{1}$. Here we define our local rules using the following notation, $Y+X \stackrel{\lambda}{\rightarrow} Y+Y$, where two individuals in states $X$ and $Y$ perform a contact with a rate or probability $\lambda$, turning them into the state $Y$ and $Y$. We must emphasize that our notion of time is still not yet defined, therefore while defining our set of local rules we do not distinguish between rates and probabilities. This distinction is discussed throughout this chapter, especially in the mean field approximations, considering the continuous time approaches with the cellular automata formalism, in Sections 3.5 and 3.6. The first local rule presented here comprises the Susceptible-Infected (SI) model. It is modeled by (ANDERSSON; BRITTON, 2000; BARRAT; BARTHLEMY; VESPIGNANI, 2008; PASTOR-SATORRAS et al., 2015)

$$
Y+X \stackrel{\lambda}{\rightarrow} Y+Y
$$

where the disease is spread by the contact of an infected and a susceptible individual. Note that this process presents a single absorbing state. For a non-zero initial condition, this rule will always drive the system to the all infected state, where every connected individual of the population is infected. Although many diseases can be modeled using this rule, such as AIDS, most of the diseases present a curing mechanism. One way to model them is considering the Susceptible-Infected-Susceptible (SIS) model, which is given by the following set of local rules (ANDERSSON; BRITTON, 2000; BARRAT; BARTHLEMY;

1 As a curiosity, as stated in (NEWMAN, 2010): from the mathematical point of view, we are not concerned about whether the individuals in the $Z$ state are recovered or dead. For this reason, some people say that it stands for removed, rather than recovered. 
VESPIGNANI, 2008; PASTOR-SATORRAS et al., 2015)

$$
\begin{aligned}
Y+X & \stackrel{\lambda}{\rightarrow} Y+Y, \\
Y & \stackrel{\delta}{\rightarrow} X,
\end{aligned}
$$

where the spreading mechanism is the same as the SI model. However, we include a curing mechanism. Observe that, now regarding a finite population, the only absorbing state is the inactive state, where every individual in the population is healthy, $X$. As discussed in section 3.4, this model also presents a meta-state, in which the disease can sustain for a long time. Furthermore, in the thermodynamic limit, infinite system size, the system stays trapped in this state. The SIS dynamics capture some of the most important features of real diseases while being simple enough. In fact, it allows us to study the critical behavior of many more intricate dynamics. As examples of diseases modeled by this model, we may cite the flu. Although the SIS model represents a huge branch of diseases, there are many diseases where the individual acquires immunity after being cured, such as in the Susceptible-Infected-Recovered (SIR) model. In this case, the local rule that accounts for the recovering must be changed (ANDERSSON; BRITTON, 2000; BARRAT; BARTHLEMY; VESPIGNANI, 2008; PASTOR-SATORRAS et al., 2015). Formally, we have the SIR set of local rules defined as

$$
\begin{gathered}
Y+X \stackrel{\lambda}{\rightarrow} Y+Y, \\
Y \stackrel{\delta}{\rightarrow} Z,
\end{gathered}
$$

where the last rule was changed, removing the cured individual from the dynamics. Contrasting with the SIS, here we have infinitely many absorbing states on the thermodynamic limit. Among the examples, we may cite chickenpox and mumps. This model is also of great interest, as it contrasts with the SIS in terms of its absorbing states, allowing us to model different diseases. However, at the same time, it is still mathematically simple, allowing us to capture the main features of more intricate diseases. The final disease presented here is the Susceptible-Infected-Recovered-Susceptible (SIRS) model, defined by the following set of local rules (ANDERSSON; BRITTON, 2000; BARRAT; BARTHLEMY; VESPIGNANI, 2008; PASTOR-SATORRAS et al., 2015)

$$
\begin{aligned}
Y+X & \stackrel{\lambda}{\rightarrow} Y+Y, \\
Y & \stackrel{\delta}{\rightarrow} Z, \\
Z & \stackrel{\gamma}{\rightarrow} X,
\end{aligned}
$$

where we have two spontaneous transitions, one from $Y$ to $Z$ and from $Z$ to $X$. Note that this chain also presents just one absorbing state for finite populations, similarly to the SIS dynamics. It is not as well explored as the previous models due to its higher complexity. 
Next, in order to formally describe the evolution of this phenomenon, we construct a Markov chain $\left(\xi_{t}\right)_{t \geq 0}$ with state space ${ }^{2} \mathscr{S}=\{(1,0,0),(0,1,0),(0,0,1)\}^{[N]}$, where $[N]$ is the set of nodes and it can be continuous or discrete, as discussed next. More precisely, we define $\xi_{t}:=\left\{\left(X_{i}(t), Y_{i}(t), Z_{i}(t)\right): i \in[N]\right\}$, where $X_{i}(t), Y_{i}(t)$ and $Z_{i}(t)$ are Bernoulli random variables indicating whether the node $i \in[N]$ is susceptible, infected, or recovered at time $t$, respectively. Therefore, $(1,0,0),(0,1,0)$ and $(0,0,1)$ represent the states $X, Y$ and $Z$, respectively. Each point $\xi \in \mathscr{S}$ is called configuration. To construct the Markov chain, we consider random objects defined in the same suitable probability space $(\Omega, \mathscr{F}, \mathbb{P})$, where $\Omega$ is the sample space, $\mathscr{F}$ is a $\sigma$-algebra of subsets of $\Omega$, i.e., the set of configurations, and $\mathbb{P}$ is a probability measure function.

Furthermore, observe that, regarding the epidemic spreading processes in continuous time, it is modeled as a collection of independent Poisson processes with rates $\lambda$ and $\delta$. Thus, one might rescale time, obtaining the effective spreading rate $\tau=\frac{\lambda}{\delta}$, which is a suitable control parameter for our system, simplifying our analysis. However, in the discrete time case, $\lambda$ and $\delta$ are probabilities and this rescale is not always possible, as can be seen in the analysis of the cellular automata case, Section 3.5.5.

\subsection{Rumor spreading}

Along the same lines as the previous section, here we firstly present the set of local rules, then we perform a formal description of our model. The two most common rumor spreading models are Daley Kendall (DK) and Maki Thompson (MT). Denoting by $Y$ the ignorants, individuals that are not aware of a rumor/information, by $X$ the spreaders, which know and are willing to spread it and, finally, $Z$ the stiflers, individuals that are aware of the rumor/information, but are unwilling to spread it (they lost interest in it). Chronologically, beginning with the DK model (DALEY; KENDALL, 1964; DALEY; KENDALL, 1965), their rules are defined as

$$
\begin{array}{lll}
Y+X & \stackrel{\lambda}{\rightarrow} Y+Y, \\
Y+Y & \stackrel{\alpha}{\rightarrow} Z+Z, \\
Y+Z & \stackrel{\alpha}{\rightarrow} Z+Z,
\end{array}
$$

where both, the spreading and annihilation mechanisms are based on the contact, which contrasts with the epidemic spreading models previously described. Besides, observe that such mechanism is undirected, since on the contact of two spreaders both turn into stiflers. In fact this is the difference between the DK model and the Maki - Thompson (MT) models (MAKI; THOMPSON, 1973). In the latter, the contacts are assumed to be directed,

2 Note that for the SIS dynamics we only need one random variable, however, here we present the most general case. 
yielding to

$$
\begin{array}{lll}
Y+X & \stackrel{\lambda}{\rightarrow} Y+Y, \\
Y+Y & \stackrel{\alpha}{\rightarrow} Z+Y, \\
Y+Z & \stackrel{\alpha}{\rightarrow} Z+Z .
\end{array}
$$

Interestingly, in (LEBENSZTAYN; MACHADO; RODRÍGUEZ, 2011) the authors show that in the limit both models present similar behavior in terms of the final fraction of ignorants, but with different variances for finite sizes. Moreover, in (LEBENSZTAYN; MACHADO; RODRÍGUEZ, 2011) they re-parametrized the traditional rumor models of Daley and Kendal (DALEY; KENDALL, 1964; DALEY; KENDALL, 1965) and of Maki Thompson (MAKI; THOMPSON, 1973) and considered a new class of individuals, called uninterested, studying the final state and variance of the final fraction of the stiflers on finite populations.

Furthermore, González-Bailón et al. in (González-Bailón Sandra et al., 2011) analyzed the dynamics of protest recruitment using Twitter data from the mobilizations in Spain during 2011. They showed the existence of influential spreaders, similarly to what was found in the SIR epidemic spreading model (KITSAK et al., 2010). On the other hand, in (BORGE-HOLTHOEFER; MORENO, 2012) the authors found evidence of an absence of influential spreaders in complex networks. These findings motivated Borge-Holthoefer et al. to propose two different models presenting this feature (BORGE-HOLTHOEFER et al., 2012). The first considers an activity parameter, while the second aims to model the apathy, in which a node can receive the information, but is not interested in spreading it. Thus, an individual can be a stifler without passing through the spreader state. This model considers that after a contact, an ignorant turns into a spreader with a rate/probability $\lambda \eta$, while it can turn into a stifler directly with rate/probability $\lambda(1-\eta)$. Formally, their model's set of local rules are given as

$$
\begin{array}{ccc}
Y+X & \stackrel{\lambda \eta}{\longrightarrow} & Y+Y, \\
Y+X & \stackrel{\lambda(1-\eta)}{\longrightarrow} & Y+Z, \\
Y+Y & \stackrel{\alpha}{\rightarrow} & Z+Y, \\
Y+Z & \stackrel{\alpha}{\rightarrow} & Z+Z .
\end{array}
$$

In a similar manner as done for the epidemic spreading in the previous section, we can also describe the evolution of this phenomena constructing a Markov chain $\left(\xi_{t}\right)_{t \geq 0}$ with the same state space $\mathscr{S}=\{(1,0,0),(0,1,0),(0,0,1)\}^{[N]}$ as previously defined. Furthermore, to construct the Markov chain, we consider random objects defined in the same suitable probability space $(\Omega, \mathscr{F}, \mathbb{P})$, where $\Omega$ is the sample space, $\mathscr{F}$ is a $\sigma$-algebra of subsets of $\Omega$, i.e., the set of configurations, and $\mathbb{P}$ is a probability measure function. Regarding time, we 
should mention that most of the approaches in the literature consider continuous time approaches (DALEY; KENDALL, 1964; DALEY; KENDALL, 1965; MAKI; THOMPSON, 1973; LEBENSZTAYN; MACHADO; RODRÍGUEZ, 2011; BORGE-HOLTHOEFER et al., 2012). In other words, it implies that they are modeled with a collection of independent Poisson processes with rates $\lambda$ and $\alpha$. Moreover, we discuss a cellular automata approach for rumor spreading in Chapter 4, where we also propose a general framework on rumor/information and epidemic spreading.

\subsection{Comments on other spreading models}

So far we have discussed the key aspects of epidemic and rumor spreading in general contexts. Here we present/summarize some other literature results, focusing on the texts that motivated this thesis, especially, our model presented in Chapter 4, where we intend to propose a general framework generalizing rumor/information and epidemic spreading. For the sake of generality and given that we shall aim to develop a general model for spreading processes - no matter if it is a disease or a piece of information-, we consider throughout this thesis the following states: (i) susceptible or ignorant, which we denote by state $X$, (ii) infected or spreader, which is represented by state $Y$; and (iii) recovered or stifler, corresponding to state $Z$. The reader is also referred to Table 2 , where we have summarized the main rumor and epidemic models studied so far. The transitions between states in each model and the thresholds, when available, are also shown for completeness.

Regarding the epidemic models, we have already discussed the main aspects of their local rules. Additionally, in this chapter, we also show some of the most important mean-field approximations. In this case, we focus mainly on the SIS process, but the generalization to other dynamics is natural. Besides that and considering the models designed specifically for rumor or information spreading, it is important to present a few more references and comments.

Besides two main models of rumor spreading, DK and MT, we must also comment on some extensions. First of all, they have have been adapted to allow for heterogeneous contact patterns in (MORENO; NEKOVEE; PACHECO, 2004), which is also the first paper to report such a dynamical process in complex networks. Next, the generalization to a spontaneous loss of interest, as a complementary mechanism to the contact, was done in (NEKOVEE et al., 2007). As mentioned in the previous section, motivated by the verification of real influential spreaders in information spreading through Twitter (GonzálezBailón Sandra et al., 2011) and its absence in MT models (BORGE-HOLTHOEFER; MORENO, 2012), in (BORGE-HOLTHOEFER et al., 2012) the authors propose two models that overcome this deficiency. The first analyzes an activity parameter, considering that some individuals (or users in that context) are more active than others (BORGE- 
Table 2 - A brief literature review: A summary of previous epidemic and rumor spreading models. The states are (i) susceptible or ignorant $(X)$, (ii) infected or spreader $(Y)$ and (iii) recovered or stifler $(Z)$.

\begin{tabular}{|c|c|c|c|c|c|}
\hline & Model & Interactions & Threshold & Networks & Comments \\
\hline \multirow{4}{*}{ 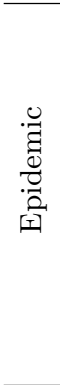 } & $\mathrm{SI}^{1}$ & $Y+X \stackrel{\lambda}{\rightarrow} Y+Y$ & - & Yes & Only two fixed points. \\
\hline & SIR & $\begin{array}{c}Y+X \stackrel{\lambda}{\rightarrow} Y+Y \\
Y \stackrel{\delta}{\rightarrow} Z\end{array}$ & $\lambda>{\frac{\delta}{\Lambda_{\max }}}^{2}$ & Yes & $\begin{array}{c}\text { Absorbing state, } Z ; \\
\text { Presence of influential spreaders. }\end{array}$ \\
\hline & SIS & $\begin{aligned} Y+X & \stackrel{\lambda}{\rightarrow} Y+Y \\
Y & \stackrel{\delta}{\rightarrow} X\end{aligned}$ & $\lambda>{\frac{\delta}{\Lambda_{\max }}}^{3}$ & Yes & $\begin{array}{l}\text { Presents an active steady state; } \\
\text { Discrete and continuous time. }\end{array}$ \\
\hline & SIRS & $\begin{aligned} Y+X & \stackrel{\lambda}{\rightarrow} Y+Y \\
& Y \stackrel{\delta}{\rightarrow} Z \\
& Z \stackrel{\gamma}{\rightarrow} X\end{aligned}$ & $\lambda>\delta \frac{\mathbb{E}(k)}{\mathbb{E}\left(k^{2}\right)}$ & Yes & $\begin{array}{l}\text { Presents an active steady state; } \\
\text { Short-term immunity. }\end{array}$ \\
\hline \multirow{5}{*}{ 节 } & Daley - Kendall & $\begin{array}{l}Y+X \stackrel{\lambda}{\rightarrow} Y+Y \\
Y+Y \stackrel{\alpha}{\rightarrow} Z+Z \\
Y+Z \stackrel{\alpha}{\rightarrow} Z+Z \\
\end{array}$ & $\frac{\lambda}{\alpha}>0$ & Yes & $\begin{array}{l}\text { Absorbing state, } R \\
\text { Undirected contact; }\end{array}$ \\
\hline & Maki - Thompson & $\begin{array}{l}Y+X \stackrel{\lambda}{\rightarrow} Y+Y \\
Y+Y \stackrel{\alpha}{\rightarrow} Z+Y \\
Y+Z \stackrel{\alpha}{\rightarrow} Z+Z\end{array}$ & $\frac{\lambda}{\alpha}>0$ & Yes & $\begin{array}{l}\text { Absorbing state, } Z ; \\
\text { Directed contact. }\end{array}$ \\
\hline & Nekovee et al. & $\begin{array}{c}Y+X \stackrel{\lambda}{\rightarrow} Y+Y \\
Y+Y \stackrel{\alpha}{\rightarrow} Z+Y \\
Y+Z \stackrel{\alpha}{\rightarrow} Z+Z \\
Y \stackrel{\delta}{\rightarrow} Z \\
\end{array}$ & $\frac{\lambda}{\delta} \geq \frac{\mathbb{E}(k)}{\mathbb{E}\left(k^{2}\right)}$ & Yes & $\begin{array}{c}\text { Absorbing state, } Z ; \\
\text { Presents a } \\
\text { "lost of interest" mechanism. }\end{array}$ \\
\hline & Borge et al. ${ }^{4}$ & $\begin{array}{c}Y+X \stackrel{\lambda \eta}{\longrightarrow} Y+Y \\
Y+X \stackrel{\lambda(1-\eta)}{\longrightarrow} Z+Y \\
Y+Y \stackrel{\alpha}{\rightarrow} Z+Z \\
Y+Z \stackrel{\alpha}{\rightarrow} Z+Z\end{array}$ & - & Yes & $\begin{array}{l}\text { Absorbing state, } Z \text {; } \\
\text { Presents an } \\
\text { apathy mechanism; } \\
\text { Models activity. }\end{array}$ \\
\hline & Kawachi et al. ${ }^{5}$ & $\begin{array}{c}Y+X \stackrel{\alpha_{x y} \theta_{x y}}{\longrightarrow} Y+Y \\
Y+X \stackrel{\alpha_{x y}\left(1-\theta_{x y}\right)}{\longrightarrow} Y+Z \\
Z+X \stackrel{\alpha_{x z} \theta_{x z}}{\longrightarrow} Z+Y \\
Z+X \stackrel{\alpha_{x z}\left(1-\theta_{x z}\right)}{\rightarrow} Z+Z \\
Y+Y \stackrel{\beta}{\rightarrow} Y+Z \\
Z+Y \stackrel{\stackrel{\gamma}{\rightarrow}}{ } Z+Z \\
Z+Z \stackrel{\lambda p}{\longrightarrow} Z+X \\
Y \stackrel{\eta_{y}}{\longrightarrow} X \\
Z \stackrel{\eta_{z}}{\rightarrow} X\end{array}$ & - & No & Presents an active steady state. \\
\hline
\end{tabular}

1 This models is similar to the Eden model (EDEN, 1961).

${ }^{2}$ This expression is obtained in details in (NEWMAN, 2010), assuming uncorrelated networks. In (BARRAT; BARTHLEMY; VESPIGNANI, 2008), the authors show another compartmental based approach, which yields $\frac{\lambda}{\delta}>\frac{\mathbb{E}(k)}{\mathbb{E}\left(k^{2}\right)-\mathbb{E}(k)}$. The first follows a quenched mean field (QMF) approach, where the process takes place in a fixed network, while the second expression is obtained considering the degree-based mean field (DMF) approach, where we assume that every node with the same degree is statistically equivalent.

3 This expression is obtained in details in (NEWMAN, 2010), assuming uncorrelated networks. In (BARRAT; BARTHLEMY; VESPIGNANI, 2008) the authors show that $\frac{\lambda}{\delta}>\frac{\mathbb{E}(k)}{\mathbb{E}\left(k^{2}\right)}$, that is completely analogous to the expression in the table, since $\Lambda_{\max }=\frac{\mathbb{E}\left(k^{2}\right)}{\mathbb{E}(k)}$ for a random network generated by the configuration model (CHUNG; LU; VU, 2003) considering scale-free networks in which $P(k) \sim k^{-\zeta}$ and $2<\zeta<\frac{2}{5}$. Observe that these expressions are valid in finite networks and the threshold tends to zero in the thermodynamic limit, since $\mathbb{E}\left(k^{2}\right) \rightarrow \infty$.

${ }^{4}$ Considering the Model II in (BORGE-HOLTHOEFER et al., 2012), which takes into account the apathy of the individuals.

${ }^{5}$ The authors considered even more interactions, but did not evaluate most of the possibilities numerically. In addition, the transitions follow the notation used in (KAWACHI et al., 2008) and are rates, not necessarily probabilities, as the authors follow a continuous time approach. 
HOLTHOEFER et al., 2012). This assumption seems to be reasonable for real-world modeling. On the other hand, the second aims to model the apathy, in which a node can receive the information, but is not interested in spreading it. This approach was discussed in the previous section.

Finally, another rumor model, which motivated our work, accounts for even more interactions was proposed in (KAWACHI et al., 2008). Although this model is quite general it does not consider a networked pattern of connections, which is present in many real data, for instance Twitter. Besides, they also considered a continuous time approach, which contrasts with our discrete time cellular automata approach in Chapter 4.

\subsection{Exact SIS Markov chain}

This exact SIS Markov chain models the process as it was formulated in Section 3.1, following the assumptions made in that section. Additionally, here we also assume a Markovian process given as a collection of independent Poisson processes. In other words, to each directed edge emanating from an infected individual, $i$, we associate a Poisson process with parameter $\lambda, N_{i \rightarrow j}^{\lambda}$, modeling the spread of the disease. Additionally, in order to model the cure of infected individuals, we associate a Poisson process with parameter $\delta, N_{i}^{\delta}$. Naturally, we also consider that our population interacts in a network given by the the adjacency matrix A. For simplicity, throughout the thesis we consider an undirected and connected ${ }^{3}$ network. Based on this simple set of assumptions, the time evolution of our process is described as

$$
\frac{d \mathbb{E}\left(Y_{i}\right)}{d t}=\mathbb{E}\left(-\delta Y_{i}+\lambda \sum_{k=1}^{N} \mathbf{A}_{k i} X_{i} Y_{k}\right)
$$

where $\mathbb{E}\left(Y_{i}\right)$ is the probability that node $i$ is infected. Complementary, $\mathbb{E}\left(X_{i}\right)=1-\mathbb{E}\left(Y_{i}\right)$ is the probability that node $i$ is susceptible. Besides, we might also define the effective spreading rate $\tau=\frac{\lambda}{\delta}$, where we can rescale time in terms of $\delta$ enabling us to have just one control parameter, $\tau$. In fact, Equation 3.1 is indeed exact, but it relies on the solution of $\mathbb{E}\left(Y_{i} Y_{j}\right)$, which can be obtained through the following formal definition (Van Mieghem, 2014)

$$
\frac{d \mathbb{E}\left(Y_{i} Y_{j}\right)}{d t}=\mathbb{E}\left(Y_{j} \frac{d Y_{i}}{d t}+Y_{i} \frac{d Y_{j}}{d t}\right)
$$

Note that applying 3.2 we will have a dependency on the product of three random variables. Such processes can be repeated until the product of $N$ random variables. Note that terms such as $E\left[Y_{i} Y_{i}\right]$ also appear naturally and they follow that $E\left[Y_{i} Y_{i}\right]=E\left[Y_{i}\right]$, as we are dealing with Bernoulli random variables. The same also applies to $E\left[X_{i} Y_{j}\right]=0$. Furthermore, as

3 A connected network is a network where there is a path from each node $i$ to each node $j$ in the network. In other words, it comprises just one component. 
can be easily seen, in the product of two random variables we have $\left(\begin{array}{c}N \\ 2\end{array}\right)$, while for three we have $\left(\begin{array}{c}N \\ 3\end{array}\right)$ and so forth, yielding to $\sum_{k=1}^{N}\left(\begin{array}{l}N \\ k\end{array}\right)=2^{N}-1$ (Van Mieghem, 2014). Consequently, the exact solution can be obtained solving the set of $2^{N}$ equations, which is prohibitive for practical purposes ${ }^{4}$. Thus, the main importance of such formulation falls on the physical insights it provides and their approximations, as discussed in Section 3.5.

Aiming to extract useful information from our model we should first define an order parameter ${ }^{5}$, which, in our case, must be a macro variable that captures the behavior of the whole system or a variable of interest. Here the order parameter is assumed to be the fraction of infected individual, formally defined as

$$
\rho=\frac{1}{N} \sum_{i}^{N} \mathbb{E}\left(Y_{i}\right)
$$

Next, we might formally define our notion of phase transition and epidemic threshold or, more generally, critical point. In our context of a process with an absorbing state, we consider two different phases: (i) the inactive phase, also called the healthy phase and the (ii) active phase or endemic. Considering the thermodynamic limit, where $N \rightarrow \infty$, a given an order parameter, which here the natural choice is the average fraction of infected individual, $\rho$, and a control parameter, here we consider $\tau$. In the inactive phase, the order parameter goes to zero, i.e. for $\tau<\tau_{c}, \rho=0$, while after the critical point the order parameter is larger than zero, i.e. $\tau>\tau_{c}, \rho>0$. It worth emphasizing that the phase transition will only appear in the thermodynamic limit. In this case, we have an infinite number of micro-states (for the same order parameter) and an infinite number of infected individuals allowing the dynamics to be trapped in that state.

Then, observing that $X_{i} \geq 0$ in Equation 3.1 we observe that (Van Mieghem, 2016)

$$
\frac{d w}{d t} \leq(\tau \mathbf{A}-\mathbf{I}) \mathbb{E}(w(t))
$$

where $w=\left[y_{1}, y_{2}, \ldots, y_{N}\right]$ is a binary vector containing the state of each node and whose solution is given by

$$
\mathbb{E}(w(t)) \leq \exp (\tau \mathbf{A}-\mathbf{I}) t \mathbb{E}(w(0)),
$$

where

$$
\exp ((\tau \mathbf{A}-\mathbf{I}) t)=\sum_{j}^{m}\left\{\sum_{k}^{m_{j}} Y_{m k} t^{k-1}\right\} e^{\Lambda_{m} t},
$$

where $Y_{m k}$ are linearly independent constant matrices and $\Lambda_{m}$ are eigenvalues of the adjacency matrix. Then, as reported in (Van Mieghem, 2016), before the critical point, the

4 For instance, observe that a network with $N=30$, we have $2^{N}=1073741824$ equations!

5 As a curiosity, following the Ehrenfest classification, the order parameter is defined as the first derivative of the free energy with respect to the external field. If it is continuous across the transition it is called a second order phase transition, which is the case of our classical SIS models. On the other hand, if it is discontinuous it is called a first order transition. 
Figure 11 - In (a) the graph representation of the exact Markov chain, where each node is a state, defined by its binary and integer representation. In (b) the numerical solution of the exact model for a small Erdös and Rényi network with $N=10, \mathbb{E}(k)=5, \delta=1$ and $\lambda=2$. Time is measured in $\frac{1}{\delta}$ units.

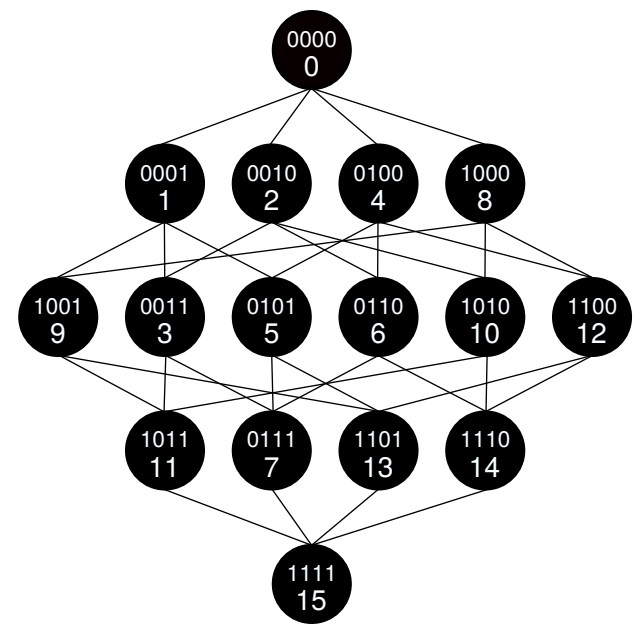

(a) Exact Markov chain

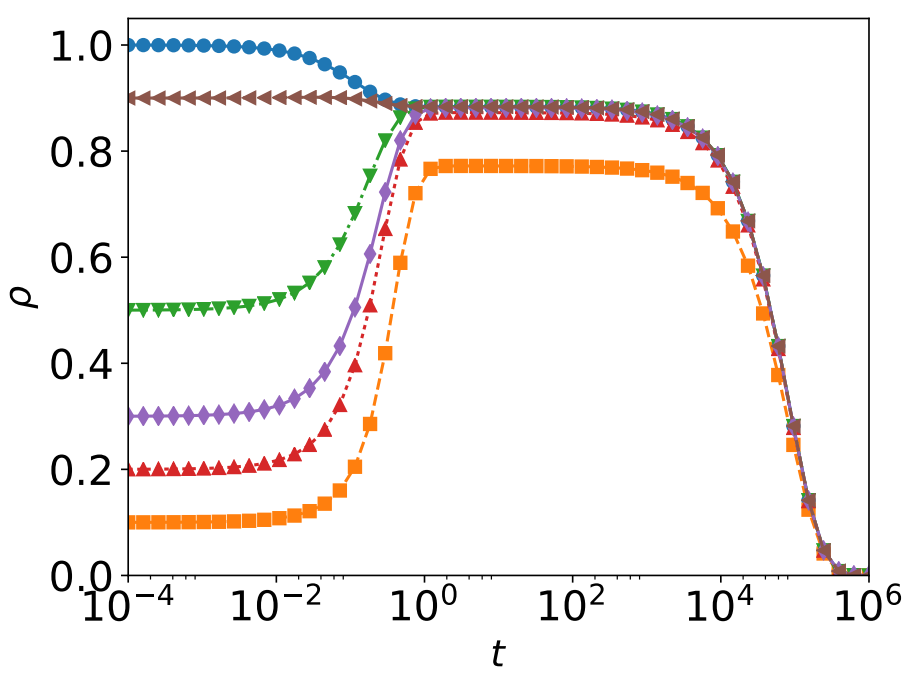

(b) Numerical solution

Source: Developed by the author.

disease must die exponentially fast when $\tau<\frac{1}{\Lambda_{1}}$. Note that this is the first time on the thesis where we relate the spectral properties of our graph with dynamical properties of our system. This will be common in the next part of our text.

Besides these conclusions, an interesting connection between the spectral properties of the Laplacian matrix (see Section 2.2) and the epidemic spreading might be explored from the order parameter definition, Equation 3.1 and after some algebra (Van Mieghem, 2016), hence

$$
\frac{d \rho(t)}{d t}=\frac{1}{N} \mathbb{E}\left(w^{T}(t)(\mathbf{L}-\tau \mathbf{I}) w(t)\right),
$$

where $w=\left[y_{1}, y_{2}, \ldots, y_{N}\right]$ is a binary vector containing the state of each node. In (Van Mieghem, 2016) the author proposed an approximate formula to describe the time evolution of the SIS process using just a single non-linear equation. Besides this proposition, by means of Equation 3.7, this clarifies the connection of the Laplacian matrix with this process, which is most often analyzed through the adjacency matrix. It is also true that this connection is not as natural/trivial as the adjacency matrix, but it must be studied and motivated us to further study the Laplacian graph, as depicted in Chapter 6.

As well as its mathematical elegance, there are other forms of expressing the exact processes. Next we use the infinitesimal generator approach to derive a set of equations which describes our dynamics. First of all, denoting the micro-state of our Markov chain as 
the binary vector $S_{i}=\left[y_{N}, y_{N-1}, \ldots, y_{1}\right]^{6}$, we can represent it as the integer $i=\sum_{k=1}^{N} y_{k}(i) 2^{k-1}$ (MIEGHEM; OMIC; KOOIJ, 2009; Van Mieghem, 2014). Figure 11 (a) shows a small example of this chain using its graph representation. Note that in state $i=0$, all bits of our micro-state vector are zero, implying that none of the individuals are infected. On the other hand, the micro-state $i=2^{N}-1$, implies the all one vector, which means that all the individuals hold the disease. In other words, we map our intuitive binary vector that contains the Bernoulli random variables previously defined, holding the micro-state of every node of our population, into an integer number. This procedure allows us to properly write the probability transitions between each micro-state. Since our process presents $2^{N}$ micro-states, the infinitesimal generator, $Q$, can be written as

$$
Q_{i j}= \begin{cases}\delta & \left\{\begin{array}{l}
j=i-2^{m-1} ; m=1,2, \ldots, N \\
\text { and } y_{m}(i)=1 \\
\lambda \sum_{k=1}^{N} \mathbf{A}_{m k} y_{k}(i)
\end{array}\right. \\
-\sum_{k=1}^{2^{N}} Q_{k j} & \begin{array}{l}
j=2^{m-1} ; m=1,2, \ldots, N \\
\text { and } y_{m}(i)=1
\end{array} \\
0 & \text { otherwise }\end{cases}
$$

whose solution is given as

$$
s^{T}(t)=s^{T}(0) \exp (Q t)
$$

where $s_{i}(t)=\mathbb{P}\left(Y_{1}(t)=y_{1}, Y_{2}(t)=y_{2}, \ldots, Y_{n}(t)=y_{n}\right)$ is the joint probability that defines the micro-state $i$. From the joint probability, we are able to obtain the nodal probability of $y_{j}=\mathbb{P}\left(Y_{j}=1\right)=\mathbb{E}\left(Y_{j}\right)$ as the sum over all the states of all nodes except the node $j$, which can be written as

$$
y_{j}(y)=\mathbb{P}\left(Y_{j}(t)=1\right)=\mathbb{E}\left(Y_{j}(t)\right)=\sum_{i=0}^{2^{N}-1} s_{i}(t) S_{i}(j)
$$

where $S_{i}(j)$ if the binary state of node $j$ in state $i$ (integer representation), where $i=$ $\sum_{k=1}^{N} y_{k}(i) 2^{k-1}$. Thus, we only sum the states where the node $j$ is infected. The order parameter can also be represented as

$$
\rho(t)=y(t)=\frac{1}{N} s^{T}(0) e^{Q t} \mathbf{M} u
$$

where $u$ is the all one vector and $\mathbf{M}$ is a $2^{N} \times N$ matrix with the bit-reversed binary state

6 The reversed order is important! 
representation. Formally,

$$
\mathbf{M}=\left[\begin{array}{ccccc}
0 & 0 & 0 & \cdots & 0 \\
1 & 0 & 0 & \cdots & 0 \\
0 & 1 & 0 & \cdots & 0 \\
1 & 1 & 0 & \cdots & 0 \\
0 & 0 & 1 & \cdots & 0 \\
\vdots & \vdots & \vdots & \vdots & \vdots \\
1 & 1 & 1 & \cdots & 1
\end{array}\right] .
$$

Furthermore, in Figure 11 (b) we have an example of numerical solution Equation 3.11. In this example we used a small Erdös and Rényi network with $N=10, \mathbb{E}(k)=5, \delta=1$ and $\lambda=2$.

Probably the most important results extracted from the exact Markov chain are concerned with its absorbing state. The first observation is that the SIS dynamics presents only one absorbing state, $i=0$, or in its binary representation $S_{0}=[0,0, \ldots, 0]$. Thus, in a finite population when time goes to infinity, it will always reach the absorbing. In (Van Mieghem, 2014) the author argues that, for a rate $\tau=\frac{\lambda}{\delta}$ above the epidemic threshold, the number of infected nodes over time presents two regions: (i) for small times the process tends exponentially fast to the meta-stable ${ }^{7}$ state (see Figure 11) and (ii) after that it also decays exponentially in $t$, but with a very small rate, towards the absorbing state. On the other hand, below the epidemic threshold, the number of infected nodes decay exponentially to the absorbing state, as shown above.

Besides, observe that Equation 3.2 allows us to perform a $n$-th order approximation by deriving the $n$-th random variables product equation and approximating the $(n+1)$-th products. Physically, what we are doing with this procedure is assuming that the $n$-th products are independent. These kinds of approximations are very common in the literature and are explored in Section 3.5, especially, the first order which is the so-called quenched mean field (QMF), while the second-order approximation is the pair quenched mean field (PQMF). In Section 3.7.6 we also perform a comparison of those two approximations and Monte Carlo simulations in order to validate and quantify the precision of both approximations.

7 In a metastable state, there are a set of possible states (micro-configurations) where the system remains trapped and there is a stochastic variation over time. In addition, note that there are many different configurations for which the fraction of infected nodes is the same. More formally, there is a set of states which have a finite probability larger than zero, configuring a meta-state. 


\subsection{Mean field approaches}

In this section, we explore the continuous and discrete time mean-field approximation in order to overcome the complexity of the exact approach, $O\left(2^{N}\right)$, which is prohibitive for large systems. Regarding the continuous time formulations, we follow a path of increasing complexity. In Section 3.5.1, we present the classical homogeneous mean-field approximation, which does not account for a network structured population. Next, in Section 3.5.2 we extend this model taking into account a heterogeneous population, however neglecting the actual network and considering an ensemble of networks, which are described by their degree distribution. Contrasting with the previous methods, in Section 3.5.3, we discuss a model which accounts for a fixed network structure, describing the probability of each individual on the population. This model proved to be very efficient in many situations, as we will discuss later, however it neglects structural correlations. Again, to overcome such a limitation we follow the pair-approximation, whose description is presented in Section 3.5.4. Complementary to the continuous time approach, considering a discrete time framework, we follow a cellular automaton process in order to describe the epidemic/rumor spreading, which is presented in Section 3.5.5.

In the following sections, we mainly explore the SIS model, focusing on the framework and the key concepts of each of the previously mentioned approximations. This description allows us to extend these concepts to other dynamical processes, which we will not show in detail. For instance in Section 3.7.6, we apply them and compare some of these approaches with Monte Carlo simulations, systematic evaluating its accuracy. Additionally, in Appendix $\mathrm{C}$ we present a complete table with all the derived equations for all the mean field approaches.

\subsubsection{Mean Field (MF)}

First of all, considering a homogeneous population, the probability of contact between any pair of nodes is approximately the same. This is true in the complete graph and can be a reasonable approximation for Erdös - Rényi networks. In a complete graph, each vertex has $(N-1)$ connections. Thus, each individual can contact any other with the same probability. Regarding regular graphs, which is also a homogeneous case, the probability of contact depends on the mean degree of the graph, $\mathbb{E}(k)$, which is also the number of contacts each individual has. The dynamical system that describes the SIS epidemic spreading model in such homogeneous population is described by the equation (ANDERSSON; BRITTON, 2000; BARRAT; BARTHLEMY; VESPIGNANI, 2008; PASTOR-SATORRAS et al., 2015)

$$
\frac{d \mathbb{E}(Y)}{d t}=\lambda \mathbb{E}(k) \mathbb{E}(Y)(1-\mathbb{E}(Y))-\delta \mathbb{E}(Y)
$$


where $\mathbb{E}(Y)$ is the fraction of the population that is infected and $\mathbb{E}(k)$ is the average number of connections in a regular graph. Observe that the fraction of susceptible individuals is given by $\mathbb{E}(X)=(1-\mathbb{E}(Y))$. In the steady-state and neglecting second order terms, i.e. $\mathbb{E}(Y)^{2}$, which is a linear stability analysis around the only absorbing state of the exact SIS model, $\mathbb{E}(Y)=0$, we obtain (BARRAT; BARTHLEMY; VESPIGNANI, 2008)

$$
R_{0}=\mathbb{E}(k) \frac{\lambda}{\delta}>1
$$

where $R_{0}$ is also called the basic reproduction number. This relationship shows a phase transition, where $R_{0}$ is the critical point, also called epidemic threshold. For $R_{0}<1$, the disease tends to die out and for $R_{0}>1$ it can sustain in the population, i.e. an endemic state. Note that it is also intuitive since we expect that the disease persists if there are more individuals being infected than cured. At this point, we must observe that the nonzero stable solution represents a meta-stable state on the exact dynamics. In such a case, the steady state is not an absorbing state in the Markov sense, as there is a set of possible states (micro-configurations) where the system remains trapped and there is a stochastic variation over time. In addition, note that there are many different configurations for which the fraction of infected nodes is the same. More formally, there is a set of states above the threshold, which has a finite probability larger than zero, configuring a metastate. The only absorbing state of this set of equations is thus the disease-free state since when it is reached the (micro and macro) dynamics stops. This meta-state will also be captured by the other mean field approaches and the same discussion applies for any of the other approximations described here. Moreover, this discussion is clearer and even more interesting in the quenched and pair-quenched mean field, where the heterogeneity is directly accounted for in the model and the individual probability is evaluated.

Although the SIS model represents and/or captures the behavior of a huge branch of diseases and captures interesting physical properties, there are many real diseases where the individual acquires immunity after being cured. Aiming to model such diseases we use the SIR model. Here, in order to exemplify the case where the Markov chain has an infinite number of absorbing states in the thermodynamic limit we, present the mean field approximation for the SIR model. In this case, another equation must be included to model hence (ANDERSSON; BRITTON, 2000; BARRAT; BARTHLEMY; VESPIGNANI, 2008; PASTOR-SATORRAS et al., 2015),

$$
\begin{cases}\frac{d \mathbb{E}(X)}{d t}= & -\lambda \mathbb{E}(k) \mathbb{E}(Y)(1-\mathbb{E}(Y)-\mathbb{E}(Z)) \\ \frac{d \mathbb{E}(Y)}{d t}= & \lambda \mathbb{E}(k) \mathbb{E}(X)(1-\mathbb{E}(Y)-\mathbb{E}(Z))-\delta \mathbb{E}(Y) \\ \frac{d \mathbb{E}(Z)}{d t}= & \delta \mathbb{E}(Y)\end{cases}
$$

Note $\mathbb{E}(X)+\mathbb{E}(Y)+\mathbb{E}(Z)=1$ and the sum of the derivatives is zero, which indicates that the population size is fixed. Moreover, observe that there is an infinite number of 
absorbing states, each one with a non-zero fraction of removed individuals. Intuitively, once an individual becomes recovered it no longer takes place in the dynamics. Formally, for any configuration where $\mathbb{E}(Y)=0$ we have a stable solution. Interestingly, observe that $\frac{d \mathbb{E}(Y)}{d t}$ is the same for the SIR and SIS epidemic models, allowing us to translate some properties of the SIS model to the SIR context. This can be done at the critical point, where the reproduction number says that, for an infinite population, the fraction of recovered individuals is greater than zero and the number of recovered individuals is also infinite. In other words, since the population is infinity and the fraction of recovered individuals is not null, it implies that we have an infinite number of revered individuals.

Finally in epidemic spreading, it is interesting to contrast the dependence of both models in the initial conditions. In the SIS case, we have the absorbing state and a metastable, which, for $R_{0}$ above the critical point, will be achieved, regardless of the initial conditions. On the other hand, in the SIR case, we have infinitely many absorbing states and a strong dependence on the initial conditions, since it limits the steady state.

Furthermore, considering a homogeneous population in the Maki - Thompson model, the set of equations that describes the dynamics is given as

$$
\begin{cases}\frac{d \mathbb{E}(X)}{d t}= & -\lambda \mathbb{E}(Y) \mathbb{E}(X) \\ \frac{d \mathbb{E}(Y)}{d t}= & \lambda \mathbb{E}(Y) \mathbb{E}(X)-\alpha(\mathbb{E}(Y) \mathbb{E}(Y)+\mathbb{E}(Y) \mathbb{E}(Z)) \\ \frac{d \mathbb{E}(Z)}{d t}= & \alpha(\mathbb{E}(Y) \mathbb{E}(Y)+\mathbb{E}(Y) \mathbb{E}(Z)),\end{cases}
$$

where variables $\mathbb{E}(X), \mathbb{E}(Y)$ and $\mathbb{E}(Z)$ can be interpreted as fractions of the population (or the probability of uniformly sampling an ignorant, spreader or stifler individual, respectively). Considering a homogeneous population, the independence of the random variables, $\mathbb{E}(Y X)=\mathbb{E}(Y) \mathbb{E}(X)$ (implicitly taken in the previous equation), is not a strong assumption, since the correlations tend to vanish in the thermodynamic limit, i.e. $N \rightarrow \infty$. This approach was initially proposed by (MAKI; THOMPSON, 1973) and largely explored in the literature, e.g. (LEBENSZTAYN; MACHADO; RODRÍGUEZ, 2011). Firstly, note that, as the SIR dynamics, the MT also have infinitely many absorbing states and a strong dependence on the initial conditions, since it limits the steady state. Next, we exemplify two fundamental results regarding the convergence of this process in the limit of an infinity population. Formally,

$$
\sqrt{N}\left(\frac{X_{\infty}}{N}-x_{\infty}\right) \Rightarrow \mathscr{N}\left(0, \sigma^{2}\right) \text { as } N \rightarrow \infty
$$

where $\Rightarrow$ denotes convergence in distribution and $\mathscr{N}\left(0, \sigma^{2}\right)$ is the Gaussian distribution with mean zero and variance

$$
\sigma^{2}=\frac{x_{\infty}\left(1-x_{\infty}\right)}{1-2 x_{\infty}}=0.27273575
$$


and

$$
x_{\infty}=\frac{-W_{0}(-2 \exp (-2))}{2}=0.20318787,
$$

where $W_{0}$ is the principal branch of the Lambert function. For a small validation experiment, see Section 3.7 and Figure 13, where we compare these theoretical predictions with Monte Carlo simulations in a finite graph.

\subsubsection{Heterogeneous Mean Field (HMF)}

The heterogeneous mean field (HMF) or Degree-based mean field (DBMF) was the first theoretical framework used to analyze the epidemic spreading processes in complex networks (PASTOR-SATORRAS; VESPIGNANI, 2001b; BOGUÑÁ; PASTOR-SATORRAS; VESPIGNANI, 2003; BARRAT; BARTHLEMY; VESPIGNANI, 2008; PASTOR-SATORRAS et al., 2015). This approach divides the population into groups according to the vertex degree, assuming that individuals with the same degree are statistically equivalent. Thus, this approximation describes the dynamics based on the network's degree distribution. Similarly to 3.13 (SIS), the HMF dynamical equation for the fraction of infected individuals with a given degree $k, \mathbb{E}\left(Y_{k}\right)$, is formally expressed by

$$
\frac{d \mathbb{E}\left(Y_{k}(t)\right)}{d t}=-\mathbb{E}\left(Y_{k}(t)\right)+\tau k\left(1-\mathbb{E}\left(Y_{k}(t)\right)\right) \sum_{k^{\prime}} \mathbb{P}\left(k^{\prime} \mid k\right) \mathbb{E}\left(Y_{k^{\prime}}(t)\right),
$$

where $\tau$ is the effective spreading rate and the contacts are accounted for based on the conditional probability $\mathbb{P}\left(k^{\prime} \mid k\right)$, which is the probability that a node with degree $k^{\prime}$ has a neighbor with degree $k$. Note that the fraction of susceptible individuals for a given degree $k$ is $\mathbb{E}\left(X_{k}\right)=\left(1-\mathbb{E}\left(Y_{k}\right)\right)$, as these quantities are complementary. This system of equations cannot be solved in a closed form for a general degree distribution (PASTOR-SATORRAS et al., 2015) due to the difficulty in estimating $\mathbb{P}\left(k^{\prime} \mid k\right)$ in correlated networks. Interestingly, observe that in homogeneous populations, the set of Equations 3.20, reduces to a single equation given by 3.13. A quantity of interest in this system is the critical point, which can be obtained by means of linear stability analyses (BOGUÑ́́; PASTOR-SATORRAS, 2002), similarly to the previous section. Considering only the first order terms of $\mathbb{E}\left(Y_{k}(t)\right)$ in Equation 3.20, we have

$$
\frac{d \mathbb{E}\left(Y_{k}(t)\right)}{d t} \simeq \sum_{k^{\prime}} J_{k k^{\prime}} \mathbb{E}\left(Y_{k^{\prime}}(t)\right)
$$

where $J_{k k^{\prime}}=-\delta_{k k^{\prime}}+\tau k \mathbb{P}\left(k^{\prime} \mid k\right)$ is the Jacobian matrix and $\delta_{k k^{\prime}}$ is the Kronecker delta function. The endemic state will emerge when $\tau \Lambda_{M}>1$, where $\Lambda_{M}$ is the largest eigenvalue of the connectivity matrix, defined as (BOGUÑÁ; PASTOR-SATORRAS, 2002)

$$
C_{k k^{\prime}}=k \mathbb{P}\left(k^{\prime} \mid k\right)
$$


which have real eigenvalues and its largest eigenvalue is real and positive as a consequence of the Perron - Frobenius theorem. Therefore, the endemic state takes place when

$$
\tau>\tau_{c}^{H M F}=\frac{1}{\Lambda_{1}}
$$

where $\Lambda_{1}$ is the largest eigenvalue of $C_{k k^{\prime}}$.

Regarding uncorrelated networks, we can derive closed expressions for the epidemic threshold. From this assumption, hence, the conditional probability does not depend on degree $k$. Formally, in uncorrelated networks we have $\mathbb{P}\left(k^{\prime} \mid k\right)=\frac{k^{\prime} \mathbb{P}\left(k^{\prime}\right)}{\mathbb{E}(k)}$ (BARRAT; BARTHLEMY; VESPIGNANI, 2008), which implies

$$
\frac{d \mathbb{E}\left(Y_{k}(t)\right)}{d t}=-\mathbb{E}\left(Y_{k}(t)\right)+\tau k\left[1-\mathbb{E}\left(Y_{k}(t)\right)\right] \Theta,
$$

where $\Theta$ is the probability of finding an infected node following a randomly chosen edge, which is formally expressed by

$$
\Theta=\sum_{k^{\prime}} \frac{k^{\prime} \mathbb{P}\left(k^{\prime}\right)}{\mathbb{E}(k)} \mathbb{E}\left(Y_{k^{\prime}}(t)\right) .
$$

From Equation 3.24 and imposing the steady state condition, $\frac{d \mathbb{E}\left(Y_{k}(t)\right)}{d t}=0$, we obtain

$$
\mathbb{E}\left(Y_{k}(t)\right)=\frac{\tau k \Theta(\tau)}{1+\tau k \Theta(\tau)} .
$$

Note the dependency of $\Theta$ on the effective spreading rate $\tau$. In addition, observe that the higher the node degree, the higher the probability of being infected. This observation is also a motivation to consider inhomogeneous degree distributions, as it clarifies the divergence between this approach and the homogeneous case. Combining Equations 3.25 and 3.26 , we obtain

$$
\Theta(\tau)=\frac{1}{\mathbb{E}(k)} \sum_{k} k \mathbb{P}(k) \frac{\tau k \Theta(\tau)}{1+\tau k \Theta(\tau)},
$$

which is a self-consistent equation admitting a non-zero solution when the threshold is satisfied (PASTOR-SATORRAS; VESPIGNANI, 2001b; BARRAT; BARTHLEMY; VESPIGNANI, 2008). The critical point can be obtained using a geometrical argument, as shown in (BARRAT; BARTHLEMY; VESPIGNANI, 2008) or considering an early time analysis (BARRAT; BARTHLEMY; VESPIGNANI, 2008). Mathematically it is given as

$$
\tau_{c}>\tau_{c}^{H M F}=\frac{\mathbb{E}(k)}{\mathbb{E}\left(k^{2}\right)} .
$$

Furthermore, observe that the threshold in Equation 3.28 can also be obtained from matrix $C_{k k^{\prime}}$, in Equation 3.22, and noticing that $C_{k k^{\prime}}=\frac{k k^{\prime} \mathbb{P}\left(k^{\prime}\right)}{\mathbb{E}(k)}$ has a unique eigenvalue $\Lambda_{1}=\frac{\mathbb{E}(k)}{\mathbb{E}\left(k^{2}\right)}$ (BOGUÑÁ; PASTOR-SATORRAS, 2002; BARRAT; BARTHLEMY; VESPIGNANI, 2008).

The results in 3.28 imply a null epidemic threshold in networks with $\mathbb{E}\left(k^{2}\right) \rightarrow \infty$ in the limit of the infinite size, which are the cases for power-law degree distributions with 
exponent $2<\zeta \leq 3$. This observation is crucial to understand the epidemic spreading in real networks. On one hand, scale-free networks can be interpreted as the ideal medium for disease spreading, as the epidemic threshold goes asymptotically to zero (as a function of the network size). On the other hand, the network heterogeneity allows us to develop a more efficient immunization procedures since we can better identify the most influential spreaders. These results are presented in more details in (BARRAT; BARTHLEMY; VESPIGNANI, 2008).

Regarding the definition of a critical point, it is important to highlight that the concept of phase transition only applies in the infinite size limit (the thermodynamic limit). However, in the literature of complex network dynamics, especially for epidemic spreading, it is usual to use the terms critical point and phase transition on finite systems, as we find a behavioral change in that point. More importantly, as shown, for scale-free networks this point vanishes in the thermodynamic limit. However, we still call it a phase transition.

Considering the prevalence, recovered by $\rho_{\infty}=\sum_{k} \mathbb{P}(k) \mathbb{E}\left(X_{k}\right)$, we can analyze its critical behavior in the vicinity of the epidemic threshold by solving Equation 3.27 (PASTORSATORRAS; VESPIGNANI, 2001a). After some manipulations, we obtain the critical behavior as $\rho_{\infty} \sim\left(\tau-\tau_{c}\right)^{v}$, where

$$
v^{H M F}=\left\{\begin{array}{lll}
\frac{1}{3-\zeta} & \text { for } & \zeta<3 \\
\frac{1}{\zeta-3} & \text { for } & 3<\zeta \leq 4 . \\
1 & \text { for } & \zeta \geq 4
\end{array}\right.
$$

On one hand, the epidemic threshold vanishes for $2<\zeta \leq 3$. On the other hand, it presents a slow growth of the prevalence near the critical point. The critical exponent, $v$, is larger than one in this case, which implies in a very slow growth near the critical point. This observation makes the spreading process on the top of these networks less threatening, as observed in (PASTOR-SATORRAS; VESPIGNANI, 2001b; PASTOR-SATORRAS et al., 2015).

Furthermore, regarding the SIR, it is important to mention that we should observe that the disease reaches an individual through one of its edges (BARRAT; BARTHLEMY; VESPIGNANI, 2008). Thus, we must exclude one of its neighbors when calculating $\Theta$. Thus, we should re-write Equation 3.25 as

$$
\Theta=\sum_{k^{\prime}} \frac{k^{\prime}-1}{k^{\prime}} \mathbb{P}\left(k^{\prime} \mid k\right) \mathbb{E}\left(Y_{k^{\prime}}(t)\right),
$$

which implies on $\Theta=\sum_{k^{\prime}} \frac{k^{\prime}-1}{\mathbb{E}(k)} \mathbb{P}\left(k^{\prime}\right) \mathbb{E}\left(Y_{k^{\prime}}(t)\right)$ for uncorrelated networks. In terms of the critical point, it implies that $\tau_{c}^{H M F}=\frac{\mathbb{E}(k)}{\mathbb{E}\left(k^{2}\right)-\mathbb{E}(k)}$ for the SIR (BARRAT; BARTHLEMY; VESPIGNANI, 2008). Besides, a similar argument must also be used in the MT dynamics and the HMF approach. 


\subsubsection{Quenched Mean Field (QMF)}

Quenched mean field (QMF), also called the Individual-based mean field (IBMF) or N-Intertwined mean field approximation (NIMFA) (MIEGHEM; OMIC; KOOIJ, 2009), is a theoretical approach based on nodal dynamics for a fixed network structure. This approach describes the time evolution of each node based on the state of the node and its neighbors. This method considers a set of $N$ equations to describe the time evolution of the probabilities. Implicitly it makes the assumption of independence between the state of the nodes. In addition, it is important to observe that QMF is always an upper bound to the exact equation (MIEGHEM; OMIC; KOOIJ, 2009) when considering a connected graph. This affirmation can be easily proven by observing that the conditional probabilities of node $j$ being infected given that another node $i$ is already infected are equal or larger than the probability of the node $j$. This occurs because the probability of the node $i$ cannot negatively influence the probability of the node $j$ (MIEGHEM; OMIC; KOOIJ, 2009; CATOR; Van Mieghem, 2014) ${ }^{8}$. Additionally, it is worth mentioning that in (FERREIRA; CASTELLANO; PASTOR-SATORRAS, 2012), based on extensive numerical analyses, the authors state that the QMF is an improvement over HMF as it provides a better estimate for the epidemic threshold.

Besides the dynamical aspects, briefly described above, this framework also enables us to take into account a single fixed network, allowing us to extend the spreading process to more complex structures, such as multilayer networks. It is noteworthy that dealing with multilayer networks using the HMF formalism would destroy the multilayer structure, turning it into a community structured network with heterogeneous spreading rates (inter and intra-layer rates). Thus, quenched formalisms are more adequate when modeling dynamical processes in multilayers. In this section, we will explore the basics of QMF in single-layers in Section 3.5.3.1 and extend it to multilayer networks in Section 3.5.3.2.

\subsubsection{Single-layer networks}

First of all, beginning with single-layer networks, similarly to the homogeneous case, Equation 3.13, and the HMF, Equation 3.20, the dynamical equations that describe such process are

$$
\frac{d \mathbb{E}\left(Y_{i}(t)\right)}{d t}=\lambda \sum_{j=0}^{N} \mathbf{A}_{i j} \mathbb{E}\left(Y_{j}(t)\right)-\mathbb{E}\left(Y_{i}(t)\right)\left(\lambda \sum_{j=0}^{N} \mathbf{A}_{i j} \mathbb{E}\left(Y_{j}(t)\right)+\delta\right)
$$

8 Formally, from (MIEGHEM; OMIC; KOOIJ, 2009), denoting $Y_{i}(t)$ the state of the node $i$ and time $t, \mathbb{P}\left(Y_{j}(t)=1 \mid Y_{i}(t)=1\right) \geq \mathbb{P}\left(Y_{j}(t)=1\right)$, while independence can be formally expressed as $\mathbb{P}\left(Y_{j}(t)=1, Y_{i}(t)=1\right)=\mathbb{P}\left(Y_{j}(t)=1\right) \mathbb{P}\left(Y_{i}(t)=1\right)$. Observing both equations it is clear that the QMF is an upper bound. As a reference, Cator and Van Mieghem proved rigorously that the states of any two nodes in the SIS model are non-negatively correlated for all finite graphs in (CATOR; Van Mieghem, 2014). 
or in matrix terms

$$
\frac{d V(t)}{d t}=\left(\lambda \operatorname{diag}\left(1-\mathbb{E}\left(Y_{i}(t)\right)\right) \mathbf{A}-\delta \mathbf{I}\right) V(t),
$$

where $V(t)=\left[\mathbb{E}\left(Y_{1}(t)\right), \mathbb{E}\left(Y_{2}(t)\right), \ldots, \mathbb{E}\left(Y_{n}(t)\right)\right]$, i.e. $V(t)$ is a vector consisting of $y_{i}(t)=$ $\mathbb{E}\left(Y_{i}(t)\right)$. It is noteworthy that considering the complete graph, we have a set of $N$ identical equations, in which all are equal to Equation 3.13. Furthermore, it is also interesting to note the relationship between QMF the HMF. The former takes into account a fixed network, considering a specified adjacency matrix. The latter considers an additional approximation, i.e., the annealed network, which assumes that every node with degree $k$ is statistically equivalent. From Equation 3.31, we can recover the HMF approach considering the annealed adjacency matrix (PASTOR-SATORRAS et al., 2015)

$$
\overline{\mathbf{A}}_{i j}=\frac{k_{j} \mathbb{P}\left(k_{i} \mid k_{j}\right)}{N \mathbb{P}\left(k_{i}\right)} .
$$

Moreover, the uncorrelated matrix is given as $\overline{\mathbf{A}}_{i j}=\frac{k_{i} k_{j}}{N \mathbb{E}(k)}$. Additionally to these matrices, we have to consider the degree-based average $y_{k}=\sum_{i \in k} \frac{y_{i}}{N \mathbb{P}(k)}$.

Assuming the existence of the steady state, we are able to calculate the steadystate probabilities for each node. We impose the steady state $\left.\frac{d y_{j}(t)}{d t}\right|_{t \rightarrow \infty}=0$ and denote by $y_{j \infty}$ its steady state solution. In addition, considering networks without self-loops $\left(A_{i i}=0\right)$. From 3.31,

$$
y_{i \infty}=\frac{\lambda \sum_{j=0}^{N} \mathbf{A}_{i j} y_{j \infty}}{\lambda \sum_{j=0}^{N} \mathbf{A}_{i j} y_{j \infty}+\delta}=1-\frac{1}{1+\tau \sum_{j=0}^{N} \mathbf{A}_{i j} y_{j \infty}} .
$$

The trivial solution implies that every node is healthy. However, if $\delta=0$, then every node is infected. In fact, this situation represents the SI epidemic process, when there is no cure. In addition, being more precise, $y_{i \infty}=1-O\left(\tau^{-1}\right)$ for large values of $\tau$.

Furthermore, interpreting Equation 3.34 as a recursive relationship we are able to obtain a useful bound (MIEGHEM; OMIC; KOOIJ, 2009). Formally given as

$$
y_{i \infty}=1-\frac{1}{1+\tau \sum_{j=0}^{N} \mathbf{A}_{i j} y_{j \infty}}=1-\frac{1}{1+\tau k_{i}-\tau \sum_{j=0}^{N} \mathbf{A}_{i j}\left(1-y_{j \infty}\right)} \leq 1-\frac{1}{1+\tau k_{i}} .
$$

This equation can be continuously iterated. Thus, for any effective spreading rate $\tau=\frac{\lambda}{\delta}>0$ we have the following bound for the probability of a node being infected

$$
0 \leq y_{i \infty} \leq 1-\frac{1}{1+\tau k_{i}}
$$

Moreover, this bound can be refined considering for $\tau \geq \frac{1}{k_{\min }}$ as shown in (MIEGHEM; OMIC; KOOIJ, 2009), hence

$$
1-\frac{1}{1+\frac{k_{i}}{k_{\min }}\left(\tau k_{\min }-1\right)} \leq y_{i \infty} \leq 1-\frac{1}{1+\tau k_{i}} .
$$


Finally, in order to evaluate the critical behavior of our system, we can also consider the steady state of Equation 3.32, reducing it to

$$
\mathbf{A} V_{\infty}-\operatorname{diag}\left(y_{i \infty}(t)\right)\left(\mathbf{A} V_{\infty}+\tau^{-1} u\right) V_{\infty}=0
$$

where $u$ is an all-one vector. Next, after some algebra (MIEGHEM; OMIC; KOOIJ, 2009), we have

$$
\frac{1}{\tau} V_{\infty}+\frac{1}{\tau} \sum_{k=2}^{\infty} V_{\infty}^{k}=\mathbf{A} V_{\infty}
$$

From such an equation and considering the vicinity of the epidemic threshold, $\tau=\tau_{c}+\varepsilon$, for an arbitrary small constant $\varepsilon>0$ we have $V_{\infty}=\varepsilon v$, where $v$ is the eigenvector belonging to the largest eigenvalue of the adjacency matrix A. Considering Equation 3.39 divided by $\varepsilon$ we have

$$
\mathbf{A} v=\frac{1}{\tau} v+\frac{\varepsilon}{\tau} v^{2}+O\left(\varepsilon^{2}\right)
$$

Regarding the epidemic threshold, we consider a sufficiently small $\varepsilon>0$, where only the first order terms remains, which reduces to

$$
\mathbf{A} v=\frac{1}{\tau} v
$$

which implies that $v$ is an eigenvector of $\mathbf{A}$, belonging to the eigenvalue $\frac{1}{\tau}$. The Perron - Frobenius theorem states that the components of the eigenvector associated with the largest eigenvalue, $\Lambda_{1}$, are all positive. Moreover, there is only one eigenvector of $\mathbf{A}$ with nonnegative elements. Hence, $\tau_{c}=\Lambda_{1}^{-1}$ and for $\tau<\tau_{c}$ the only possible solution is $V_{\infty}=0$. On the other hand, for $\tau>\tau_{c}$ there is a non-zero solution.

An important class of networks have power-law degree distribution, $P(k) \sim k^{-\zeta}$. For this class we can use previous results on the spectrum of graphs (CHUNG; LU; VU, 2003) and derive the following expression for the epidemic threshold as

$$
\tau_{c}^{Q M F} \simeq \begin{cases}\frac{1}{\sqrt{k_{\max }}} & \zeta>\frac{5}{2} \\ \frac{\mathbb{E}(k)}{\mathbb{E}\left(k^{2}\right)} & 2<\zeta<\frac{5}{2}\end{cases}
$$

where $k_{\max }$ is the maximum degree of the network. Note that, at the thermodynamic limit, there is a vanishing threshold for every network in which the maximum degree is a growing function of the network size, which is often the case for all random, non-regular networks, as pointed out in (PASTOR-SATORRAS et al., 2015).

Furthermore, this formalism also allows us to obtain an expression for the prevalence near the critical point (similarly to Section 3.5.2). In (Van Mieghem, P., 2012) the author shows the steady state fraction of infected nodes, $y_{\infty}=\rho=\frac{1}{N} \sum_{i} y_{i \infty}(\tau)$, depends on $\Lambda_{1}$, which is the lading eigenvalue and its associated eigenvector $v_{1}$, obeying

$$
\rho=\frac{1}{N} \frac{u^{T} v_{1}}{\Lambda_{1} \sum_{j=1}^{N}\left(v_{1}\right)_{j}^{3}}\left(\tau_{c}^{-1}-\tau^{-1}\right)+O\left(\tau_{c}^{-1}-\tau^{-1}\right)^{2},
$$


considering sufficiently small values of $\tau$ approaching from above. In addition, we can derive some bounds for the term $\sum_{j=1}^{N}\left(v_{1}\right)_{j}^{3}$. Using the Hölder inequality and considering a connected graph, after some algebra we have (Van Mieghem, P., 2012),

$$
\frac{1}{\sqrt{N}} \leq \sum_{j=1}^{N}\left(v_{1}\right)_{j}^{3} \leq \max _{1 \leq j \leq N}\left(v_{1}\right)_{j}
$$

Note that $\left(v_{1}\right)_{j}=\frac{1}{\sqrt{N}}$ in the regular graph (Van Mieghem, P., 2012), which implies that this bound is the best possible since both sides are reached in this case. Expression 3.42 allows us to study the critical exponent given its leading eigenvalue and its respective eigenvector.

Finally, an important phenomena can be seen in QMF, the localization of diseases, as pointed out in (GOLTSEV et al., 2012). Using the inverse participation ratio, we can infer if the disease is localized in a few set of nodes, a localized state, or if the disease is active in the network as a whole. Formally, if the inverse participation ratio ${ }^{9}$ of the leading eigenvector of the adjacency matrix (a normalized eigenvector), $\operatorname{IPR}\left(f\left(\Lambda_{1}\right)\right) \sim O(1)$, then the disease is localized in a few nodes, hubs, and the epidemic grows slowly as a function of $\lambda$. On the other hand, if $\operatorname{IPR}\left(f\left(\Lambda_{1}\right)\right) \sim O\left(\frac{1}{\sqrt{N}}\right)$, the disease is present in the whole networks, configuring a true active state (GOLTSEV et al., 2012; WANG et al., 2017). This concept will be further explored in the context of multilayer networks in Chapters 5 and 6.

\subsubsection{Multilayer networks: SIS}

Following the QMF approach, given a fixed network, we model the spreading process using the tensorial notation defined in Section 2.3.2, the equations describing the systems dynamics read as (ARRUDA et al., 2017)

$$
\frac{d \mathbb{E}\left(Y_{\beta \tilde{\delta}}(t)\right)}{d t}=-\mathbb{E}\left(Y_{\beta \tilde{\delta}}(t)\right)+\left(1-\mathbb{E}\left(Y_{\beta \tilde{\delta}}(t)\right)\right) \tau \mathscr{R}_{\beta \tilde{\delta}}^{\alpha \tilde{\gamma}}(\lambda, \eta) \mathbb{E}\left(Y_{\alpha \tilde{\gamma}}(t)\right)
$$

where the supra contact tensor is defined as

$$
\mathscr{R}_{\beta \tilde{\delta}}^{\alpha \tilde{\gamma}}(\lambda, \eta)=M_{\beta \tilde{\sigma}}^{\alpha \tilde{\eta}} E_{\tilde{\eta}}^{\tilde{\sigma}}(\tilde{\gamma} \tilde{\delta}) \delta_{\tilde{\delta}}^{\tilde{\gamma}}+\frac{\eta}{\lambda} M_{\beta \tilde{\sigma}}^{\alpha \tilde{\eta}} E_{\tilde{\eta}}^{\tilde{\sigma}}(\tilde{\gamma} \tilde{\delta})\left(U_{\tilde{\delta}}^{\tilde{\gamma}}-\delta_{\tilde{\delta}}^{\tilde{\gamma}}\right),
$$

which encodes the contacts. It has a similar role to the matrix $R$ in (COZZO et al., 2013) (see Section 3.5.5.2 for more details). Notice that we have implicitly assumed that the random variables $Y_{\beta \tilde{\delta}}$ are independent. Naturally, the order parameter, also called macrostate variable, is defined as the average of the individual probabilities, formally given by

$$
\rho=\frac{1}{n m} \mathbb{E}\left(Y_{\beta \tilde{\delta}}\right) U^{\beta \tilde{\delta}} .
$$

9 The inverse participation ratio is defined as $\operatorname{IPR}\left(f\left(\Lambda_{i}\right)\right)=\sum_{i}^{N} f\left(\Lambda_{i}\right)^{4}$, where $f\left(\Lambda_{i}\right)$ is the normalized eigenvector associated with the eigenvalue $\Lambda_{i}$. 
Furthermore, regarding the epidemic threshold, analogously to the results for single-layer systems we have a critical point given as

$$
\left(\frac{\delta}{\lambda}\right)_{c}=\Lambda_{1}
$$

where $\Lambda_{1}$ is the largest eigenvalue of $\mathscr{R}$. The complete derivation is similar to the singlelayer, presented in the previous subsection. Observe that the eigen-structure of the tensor $\mathscr{R}$ is the same as the matrix $R$ in (COZZO et al., 2013), since it can be understood as a flattened version of the tensor $\mathscr{R}_{\beta \tilde{\delta}}^{\alpha \tilde{\gamma}}(\lambda, \eta)$. As argued in (De Domenico et al., 2013), the supra-adjacency matrix corresponds to a unique unfolding of the fourth-order tensor $\mathscr{R}$ yielding square matrices. Moreover, if $\eta M_{v \tilde{\delta}}^{\xi \tilde{\gamma}} E_{\xi}^{v}(\beta \beta) \ll \lambda M_{\beta \tilde{\gamma}}^{\alpha \tilde{\xi}} E_{\tilde{\xi}}^{\tilde{\gamma}}(\tilde{\delta} \tilde{\delta})$, the critical point is dominated by the individual layer behavior and the epidemic threshold is approximated to that of an SIS model in single-layers, when considering the union of $m$ disjoint networks. Consequently, the epidemic threshold is determined by the largest eigenvalue, considering all layers. The same conclusion was reached in (COZZO et al., 2013) using the perturbation theory in the supra-contact matrix.

Finally, extending the bounds we found for single-layer networks, the nodal probability on the steady state can be bounded by (ARRUDA et al., 2017)

$$
1-\frac{1}{1+\frac{d_{\beta \tilde{\delta}}}{d^{\min }}\left[\left(\frac{\lambda}{\delta}\right) d^{\min }-1\right]} \leq \mathbb{E}\left(Y_{\beta \tilde{\delta}}^{\infty}\right) \leq 1-\frac{1}{\left(\frac{\lambda}{\delta}\right) d_{\beta \tilde{\delta}}+1}
$$

where $\mathbb{E}\left(Y_{\beta \tilde{\delta}}^{\infty}\right)$ denotes the probability that node $\beta$ in layer $\tilde{\delta}$ is in the steady state regime, $d_{\beta \tilde{\delta}}=\mathscr{R}_{\beta \tilde{\delta}}^{\alpha \tilde{\gamma}}(\lambda, \eta) U_{\alpha \tilde{\gamma}}$ and $d^{\text {min }}=\operatorname{Min}\left\{d_{\beta \tilde{\delta}}\right\}$. The derivation and analysis of these bounds are similar to the ones presented in the previous subsection. Observe that the higher $d^{\text {min }}$, the closer the lower and upper bounds. In the extreme case $\left(\frac{\lambda}{\delta}\right) \rightarrow \infty$ the bounds approach each other and all nodes tend to be infected. Phenomenologically, the latter parameter configuration models the limiting case of a SI-like scenario, where $\delta=0$. In such a dynamical process, all individuals are infected in the steady state.

The model presented in this subsection, considering SIS spreading processes in multilayer networks using a continuous time approach was initially proposed by Arruda et.al. (ARRUDA et al., 2017) and will be explored in depth throughout the thesis. From the dynamical point of view the phenomena of layer-wise localization and the barrier effect were observed, which is explored in Chapter 5. From the spectral viewpoint a transition on the spectral distribution, from the union of the individual spectral distribution to the distribution of the network of layers was observed. Moreover, localization phenomena can also be explained by means of eigenvector localization along the same lines as (GOLTSEV et al., 2012), also mentioned in the previous section. Those results are presented in Chapter 6 . 


\subsubsection{Multilayer networks: SIR}

For the sake of completeness, besides the SIS epidemic model, we can also consider the SIR model. Contrasting with the SIS, which have just one absorbing state (inactive), the SIR has many absorbing states. In fact, considering an infinite population we have an infinite number of absorbing states. We introduce the recovered and susceptible states, denoted here by $x_{\beta \tilde{\delta}}$ and $z_{\beta \tilde{\delta}}$, respectively. Then, using a similar notation as in the latter section and associating Poisson processes to nodes and edges, we have the dynamical set of equations

$$
\left\{\begin{array}{l}
\frac{d \mathbb{E}\left(Y_{\beta \tilde{\delta}}\right)}{d t}=-\delta \mathbb{E}\left(Y_{\beta \tilde{\delta}}\right)+\mathbb{E}\left(Z_{\beta \tilde{\delta}}\right) \lambda \mathscr{R}_{\beta \tilde{\delta}}^{\alpha \tilde{\gamma}}(\lambda, \eta) \mathbb{E}\left(Y_{\alpha \tilde{\gamma}}\right) \\
\frac{d \mathbb{E}\left(X_{\beta \tilde{\delta}}\right)}{d t}=\delta \mathbb{E}\left(Y_{\beta \tilde{\delta}}\right) \\
\frac{d \mathbb{E}\left(Z_{\beta \tilde{\delta}}\right)}{d t}=-\mathbb{E}\left(Z_{\beta \tilde{\delta}}\right) \lambda \mathscr{R}_{\beta \tilde{\delta}}^{\alpha \tilde{\gamma}}(\lambda, \eta) \mathbb{E}\left(Y_{\alpha \tilde{\gamma}}\right)
\end{array}\right.
$$

Note that, similarly to the previous cases, the Poisson processes on the nodes model the recovering, whereas on the edges, model the spreading. Additionally, observe that the critical point obtained for the SIS case also applies here. Note, however, that the physical meaning of such a transition changes. After the critical point, the number of recovered individuals scales with the number of nodes in the network. In other words, the fraction of recovered individuals is larger than zero. On the other hand, before the critical point, this fraction goes to zero in the thermodynamic limit. Interestingly, the number of recovered individuals will be at least the number of individuals initially infected due to the nature of such dynamics.

\subsubsection{Pair Quenched Mean Field (PQMF)}

As shown in Section 3.4, we can obtain a $n$-th order approximation, iterating Equation 3.2. Here we extend the quenched mean field to the pair approximation. In other words we evaluate the probabilities $\mathbb{E}\left(Y_{i}(t) Y_{j}(t)\right)$ for each edge on the network. The approximation in this case considers that the pairs $\mathbb{E}\left(Y_{i}(t) Y_{j}(t)\right)$ are independent, contrary to the node independence assumption on the QMF. This approximation enables us to account for structural correlations, improving the accuracy of the QMF framework. This framework was previously studied in (CATOR; Van Mieghem, 2012; Mata, Angélica S.; Ferreira, Silvio C., 2013). Regarding our notation, here the time dependence of our random variables is often suppressed in order to have shorter equations.

In the following section, we discuss the PQMF formulation from the analytical viewpoint, in Section 3.5.4.1, where we develop a new set of equations, relating this approximation with the non-backtracking (for more details, see Section 2.2). Besides its simplicity, to the best of our knowledge, this was not previously done in the literature. 
Additionally, we show the relationship between the presented formalism with the message passing approach for recurrent state, proposed in (SHRESTHA; SCARPINO; MOORE, 2015). Next, in Section 3.5.4.2, we explore this model numerically, emphasizing its approximations. Finally, we also compare this model with the recurrent message passage approach, discussing their similarities and differences in Section 3.5.4.4.

Here we will only consider single-layer networks to avoid unnecessary complexity, but the model can be easily extended to multilayer networks in two different manners: (i) considering a supra-adjacency matrix instead of the adjacency matrix, but considering that the inter-layer elements are weighted by $\frac{\eta}{\lambda}$ or (ii) re-writing the following equations on the tensorial form, but considering the tensor $\mathscr{R}_{\beta \tilde{\delta}}^{\alpha \tilde{\gamma}}(\lambda, \eta)$ instead of $\mathbf{A}$. Both approaches are equivalent and were previously explored in this thesis.

\subsubsection{Analytical aspects}

Starting with a fixed network, for each node, we express its probabilities over time following Equation 3.1 and describe the evolution of $\mathbb{E}\left(X_{i} Y_{j}\right)$ using the relation 3.2, hence for each edge we have

$$
\begin{gathered}
\frac{d \mathbb{E}\left(X_{i} Y_{j}\right)}{d t}=\mathbb{E}\left(-(\delta+\lambda) X_{i} Y_{j}+\delta Y_{i} Y_{j}+\lambda \sum_{j \leftarrow k} \mathbf{B}_{(i \leftarrow j, j \leftarrow k)} X_{i} X_{j} Y_{k}-\lambda \sum_{i \leftarrow k} \mathbf{H}_{(i \leftarrow j, i \leftarrow k)} X_{i} Y_{j} Y_{k}\right), \\
\frac{d \mathbb{E}\left(Y_{i} Y_{j}\right)}{d t}=\mathbb{E}\left(-2 \delta Y_{i} Y_{j}+\lambda X_{i} Y_{j}+\lambda \sum_{j \leftarrow k} \mathbf{B}_{(i \leftarrow j, j \leftarrow k)} Y_{i} X_{j} Y_{k}+\lambda \sum_{i \leftarrow k} \mathbf{H}_{(i \leftarrow j, i \leftarrow k)} X_{i} Y_{j} Y_{k}\right)
\end{gathered}
$$

where $\mathbf{B}_{(i \leftarrow j, j \leftarrow k)}$ is the non back-tracking matrix (for more, see Section 2.2.4) and $\mathbf{H}_{(i \leftarrow j, i \leftarrow k)}$ is a new matrix defined as

$$
\mathbf{H}_{(i \leftarrow j, k \leftarrow l)}= \begin{cases}1 & \text { if } i=k \text { and } j \neq l \\ 0 & \text { otherwise. }\end{cases}
$$

Note that we denote the indices of the matrix by arrows, e.g. $i \leftarrow j$, in order to easily understand the physical meaning of these terms. Interestingly, in the literature, in (CATOR; Van Mieghem, 2012) all possible pairs were accounted for in the modeling, generating a system of $N^{2} \times N^{2}$. On the other hand, in (Mata, Angélica S.; Ferreira, Silvio C., 2013), the authors describe the system only for the edges, reducing the complexity to $(M+N) \times(M+N)$, where $M$ is the number of edges. Note that although for dense networks $M \in O\left(N^{2}\right)$, in sparse networks, this consideration drastically reduces the computational cost of numerical evaluations of those equations. Besides, both models are the same, they differ in terms of their approximation concerning the product of three terms, i.e. $\mathbb{E}\left(Y_{i} Y_{j} Y_{k}\right)$, as we will discuss later. 
Next, using some basic probability and expectation properties and the relation $X_{i}+Y_{i}=1$, we have the following relation,

$$
\mathbb{E}\left(X_{i} Y_{j}\right)=\mathbb{E}\left(Y_{j}\right)-\mathbb{E}\left(Y_{i} Y_{j}\right)
$$

Then, deriving Equation 3.53 and substituting Equations 3.50 and 3.51, hence

$$
\frac{d \mathbb{E}\left(Y_{j}\right)}{d t}=\mathbb{E}\left(-\delta Y_{j}+\lambda \sum_{j \leftarrow k} \mathbf{B}_{(i \leftarrow j, j \leftarrow k)} X_{j} Y_{k}\right),
$$

where $\mathbb{E}\left(Y_{j}\right)$ are the components of a vector with $M$ elements, each one associated with an edge. Physically, it is the probability of an edge that an edge from $j$ to $i$ has a susceptible source $j$. This equation is not intuitive to analyze, but it is convenient for a linear stability analysis near the absorbing state, $Y_{i}=0, \forall i \in \mathscr{V}$. The fixed point is $\mathbb{E}\left(Y_{i}\right)=\mathbb{E}\left(Y_{i} Y_{j}\right)=$ $\mathbb{E}\left(Y_{i} X_{j}\right)=0$. In order to simplify 3.54 , we use the relation 3.53 implying that

$$
\frac{d \mathbb{E}\left(Y_{j}\right)}{d t}=\mathbb{E}\left(-\delta Y_{j}+\lambda\left(\sum_{j \leftarrow k} \mathbf{B}_{(i \leftarrow j, j \leftarrow k)} Y_{k}-\sum_{j \leftarrow k} \mathbf{B}_{(i \leftarrow j, j \leftarrow k)} Y_{j} Y_{k}\right)\right) .
$$

Finally, assuming that $\mathbb{E}\left(Y_{j}\right) \approx \mathbb{E}\left(X_{i} Y_{j}\right) \approx \varepsilon$, which also imply $\mathbb{E}\left(Y_{i} Y_{j}\right) \approx 0$, due to the relation 3.53. Physically, we can imagine a scenario where just a small fraction of individuals in our population is infected. Thus, it is expected that, randomly sampling an edge, it is very much more likely to sample an edge connecting a spreader and susceptible $\left(\mathbb{E}\left(Y_{i} X_{j}\right)\right)$, than two spreaders $\left(\mathbb{E}\left(Y_{i} Y_{j}\right)\right)^{10}$. Considering this, proceeding with the linear stability analysis we have

$$
\frac{d \varepsilon}{d t}=\left(\mathbf{B}_{(i \leftarrow j, j \leftarrow k)}-\frac{\delta}{\lambda} \mathbf{I}\right) \varepsilon
$$

where $\varepsilon$, is a vector. Next, observe that the expression 3.55 presents repeated elements, weighting the individual states differently. In order to correct this expression and show that it also captures the critical behavior we can write the order parameter as

$$
\frac{d \rho^{*}}{d t} \approx d^{T}\left(\mathbf{B}_{(i \leftarrow j, j \leftarrow k)}-\frac{\delta}{\lambda} \mathbf{I}\right) \varepsilon
$$

where $\rho^{*}=\sum_{i}^{N} d_{i} \varepsilon_{i}$ is the approximated order parameter near the critical point, $\varepsilon$ is a vector with expectation $\mathbb{E}\left(Y_{i}\right) \approx \varepsilon_{i}$, with the appropriate dimensions and $d^{T}=\left[\left(k_{l}^{*}\right)^{-1}\right]$, $\forall l=1,2, \ldots, M$ is a vector composed by $k_{l}^{*}$, which is the degree of the node $j$, from which the edge $l$ points to. In other words, for the directed edge $l$, which connects $i$ to $j$, thus $k_{l}^{*}=k_{j}$. Note that this vector presents repeated components, which weighs the infection probability, correcting the contributions in Equation 3.55. Next, in order to determine ${ }_{10}$ Complementary, observe that $\mathbb{E}\left(X_{i} X_{j}\right)=\mathbb{E}\left(X_{j}\right)-\mathbb{E}\left(X_{i} Y_{j}\right)$. Then assuming $\mathbb{E}\left(X_{i} X_{j}\right) \approx 1$ and $\mathbb{E}\left(X_{j}\right) \approx 1-\varepsilon$ (by construction, as it is the fraction of infected individuals in the population and we are performing our analysis around the absorbing state), hence $\mathbb{E}\left(X_{i} Y_{j}\right) \approx \varepsilon$ 
the critical point, one might observe that $d^{T}$ is composed by positive elements. Thus, the smallest value of $\left(\frac{\delta}{\lambda}\right)$ from which the product $\left(\mathbf{B}_{(i \leftarrow j, j \leftarrow k)}-\frac{\delta}{\lambda} \mathbf{I}\right) \varepsilon$ is positive is the leading eigenvalue of $\mathbf{B}$. Considering this, near the critical point we can re-write Equation 3.57 as

$$
\frac{d \rho^{*}}{d t} \approx\left(\Lambda_{1}-\frac{\delta}{\lambda}\right) d^{T} \varepsilon=\left(\Lambda_{1}-\frac{\delta}{\lambda}\right) \rho^{*},
$$

where $\varepsilon$ is approximated by the leading eigenvector of $\mathbf{B}$ near the critical point, as also shown in Equation 3.56. Therefore, this equation implies that the obtained critical point for the PQMF is given as

$$
\tau_{c}^{P Q M F}=\frac{1}{\Lambda_{1}}
$$

where $\Lambda_{1}$ is the leading eigenvalue of the non-backtracking matrix, B. Interestingly, as argued in (SHRESTHA; SCARPINO; MOORE, 2015), $\Lambda_{1}(\mathbf{B}) \leq \Lambda_{1}(\mathbf{A})$ for single-layer networks, implying that the obtained critical point is indeed an improvement in comparison to the QMF. Besides, Equation 3.58 explicitly shows the critical point. Observe that the Perron-Frobenius theorem assures that the leading eigenvector consists of positive elements. Furthermore, Equation 3.58 emphasizes the contributions of the eigenvector to the critical behavior. Interestingly, the product $d^{T} \varepsilon$ geometrically as $\cos (\theta)=\frac{d^{T} \varepsilon}{\|d\|\|\| \varepsilon \|}$, where $\theta$ is the angle between $d$ and $\varepsilon$.

\subsubsection{Numerical aspects}

So far we have dealt with the analytical aspects of the PQMF equations. In addition to, it is also enlightening to numerically evaluate our models. By doing so, here we discuss our model from the numerical point of view. First of all, we must discuss how to properly approximate the expectation of the product of three random variables. In (CATOR; Van Mieghem, 2012) the approximation used was

$$
\mathbb{E}\left(Y_{i} Y_{j} Y_{k}\right)=\mathbb{E}\left(Y_{i} Y_{j}\right) \mathbb{E}\left(Y_{k}\right)
$$

which is not the standard, as commented in (Mata, Angélica S.; Ferreira, Silvio C., 2013), where the authors use the standard approximation, given as

$$
\mathbb{E}\left(Y_{i} Y_{j} Y_{k}\right)=\frac{\mathbb{E}\left(Y_{i} Y_{j}\right) \mathbb{E}\left(Y_{j} Y_{k}\right)}{\mathbb{E}\left(Y_{j}\right)} .
$$

Throughout this thesis, we will use the standard approximation schema, since it is the most appropriate and accurate. Curiously, in (CATOR; Van Mieghem, 2012), the authors discuss their approximation observing their limitations in small networks. Interestingly, the approximation shown in Equation 3.61 is also an upper bound for $\mathbb{E}\left(Y_{i} Y_{j} Y_{k}\right)$. Note that, from the Bayes rule we have

$$
\begin{aligned}
\mathbb{E}\left(Y_{i} Y_{j} Y_{k}\right) & =\mathbb{P}\left(Y_{i}=1, Y_{j}=1, Y_{k}=1\right)=\mathbb{P}\left(Y_{i}=1 \mid Y_{j}=1, Y_{k}=1\right) \mathbb{P}\left(Y_{j}=1, Y_{k}=1\right)= \\
& =\mathbb{P}\left(Y_{i}=1 \mid Y_{j}=1, Y_{k}=1\right) \mathbb{P}\left(Y_{j}=1 \mid Y_{k}=1\right) \mathbb{P}\left(Y_{k}=1\right) .
\end{aligned}
$$


While from Equation 3.61 we have

$$
\begin{aligned}
\frac{\mathbb{E}\left(Y_{i} Y_{j}\right) \mathbb{E}\left(Y_{j} Y_{k}\right)}{\mathbb{E}\left(Y_{j}\right)} & =\frac{\mathbb{P}\left(Y_{i}=1 \mid Y_{j}=1\right) \mathbb{P}\left(Y_{k}=1 \mid Y_{j}=1\right)\left(\mathbb{P}\left(Y_{j}=1\right)\right)^{2}}{\mathbb{P}\left(Y_{j}=1\right)}= \\
& =\mathbb{P}\left(Y_{i}=1 \mid Y_{j}=1\right) \mathbb{P}\left(Y_{k}=1 \mid Y_{j}=1\right) \mathbb{P}\left(Y_{j}=1\right) .
\end{aligned}
$$

Then, comparing Equations 3.62 and 3.63 we can show that

$$
\mathbb{E}\left(Y_{i} Y_{j} Y_{k}\right) \geq \frac{\mathbb{E}\left(Y_{i} Y_{j}\right) \mathbb{E}\left(Y_{j} Y_{k}\right)}{\mathbb{E}\left(Y_{j}\right)},
$$

due to $\mathbb{P}\left(Y_{i}=1 \mid Y_{j}=1, Y_{k}=1\right) \geq \mathbb{P}\left(Y_{i}=1 \mid Y_{j}=1\right)$ since the contribution of $Y_{k}=1$ is always positive. This is naturally expected since another infected individual will only increase the conditional probabilities. In this manner, we prove that that, similarly to the QMF, the PQMF is also an upper bound for the probability (for the average value). Furthermore, it is an improvement over the QMF bounds.

Thus, Equation 3.1 is kept the same, but the expectation operator is constrained to the random variables, as we are dealing with static networks. Mathematically,

$$
\frac{d \mathbb{E}\left(Y_{i}\right)}{d t}=-\delta \mathbb{E}\left(Y_{i}\right)+\lambda \sum_{k=1}^{N} \mathbf{A}_{k i} \mathbb{E}\left(X_{i} Y_{k}\right)
$$

where the terms $\mathbb{E}\left(X_{i} Y_{k}\right)$ are calculated using the standard approximation. After some algebra we have,

$\frac{d \mathbb{E}\left(X_{i} Y_{j}\right)}{d t} \approx \delta \mathbb{E}\left(Y_{j}\right)-\lambda \mathbb{E}\left(X_{i} Y_{j}\right)+\lambda \frac{1-\mathbb{E}\left(Y_{i}\right)-\mathbb{E}\left(X_{i} Y_{j}\right)}{1-\mathbb{E}\left(Y_{j}\right)} \sum_{j \leftarrow k} \mathbf{B}_{(i \leftarrow j, j \leftarrow k)} \mathbb{E}\left(X_{j} Y_{k}\right)-\lambda \frac{\mathbb{E}\left(X_{i} Y_{j}\right)}{1-\mathbb{E}\left(Y_{i}\right)} \sum_{i \leftarrow k} \mathbf{H}_{(i \leftarrow j, i \leftarrow k)} \mathbb{E}\left(X_{i} Y_{k}\right)$,

which can be solved using a numerical approach, such as Runge-Kutta. Intuitively, note that the probability

$$
\mathbb{P}\left(Y_{i}=1 \mid Y_{j}=1\right)=\frac{\mathbb{P}\left(Y_{i}=1 \cap Y_{j}=1\right)}{\mathbb{P}\left(Y_{j}=1\right)}=\frac{\mathbb{E}\left(Y_{i} Y_{j}\right)}{\mathbb{E}\left(Y_{j}\right)},
$$

which is the same that appears multiplying the summations. Thus, the first summation is the positive contribution of the neighbors of $j$, given that $i$ and $j$ are both susceptible. On the other hand, for the second summation it contributes negatively because it accounts for the probability that both ends of the edge have infected individuals.

\subsubsection{Comments on the PQMF critical point predictions}

It is noteworthy that the critical point obtained in (Mata, Angélica S.; Ferreira, Silvio C., 2013) is not a closed form equation and was only obtained for some regular structures. In (Mata, Angélica S.; Ferreira, Silvio C., 2013), the authors show, for the general case, that the critical point can be obtained numerically finding the null eigenvalues of

$$
L_{i j}^{*}=-\left(1+\frac{\lambda^{2} k_{i}}{2 \lambda+2}\right) \delta_{i j}+\frac{\lambda(2+\lambda)}{2 \lambda+2} \mathbf{A}_{i j}
$$


This equation can be easily obtained performing a quasi-static approximation in the infinite time limit in Equation 3.50 and substituting it on Equation 3.1. In order to obtain a good approximation of the critical point we employed a binary search on $\lambda$, seeking $\lambda_{\max }\left(L^{*}\right)=0$ with a tolerance parameter of tol $=10^{-6}$. In most of the networks evaluated our method converged in less than 20 iterations. Those estimations are denoted here as $\tau_{c}^{s P Q M F}$, where sPQMF stands for standard PQMF. In Section 3.8 we evaluate the accuracy of this expression and compare it with the other mean field approximations discussed here.

Although our approximation, Equation 3.59, agrees with Equation 3.59 on the predictions for the the random regular case, there is no general map between Equations 3.59 and 3.68. It is important to emphasize that the approximations performed to obtain the critical point in Equations 3.59 and 3.68 are different. In fact, they diverge on many cases, as numerically shown in Section 3.8. The formulation of Section 3.5.4.1 is interesting because it connects the epidemic spreading processes with the non-backtracking matrix. This relationship was previously observed in (RADICCHI; CASTELLANO, 2016), where the authors proposed a centrality measure based on non-backtracking matrix as a tool for the identification of influential spreaders and also in (SHRESTHA; SCARPINO; MOORE, 2015), where the authors proposed a recurrent-state message-passing approach for the SIS epidemic process. On the other hand, regarding the critical point estimation presented in Equation 3.59, it is not a bound for the critical point. In other words, it can be above or bellow the real critical point, contrasting with Equation 3.68, which is an improved upper bound (in comparison with the QMF predictions).

\subsubsection{Comparison with recurrent-state message-passing approach}

In order to set the scene, (KARRER; NEWMAN, 2010) proposed a message passing formulation to tackle the SIR epidemic spreading process. In such formalism, the diseases are interpreted as messages in the network. Interestingly, this formalism is exact on trees. However, it does not translate directly to SIS epidemic spreading processes due to the recurrence of node states. For instance, in the SIR, once a node is recovered it passes from $Y \rightarrow Z$ and it will remain in that state. On the other hand, in the SIS, a note is always changing from $Y \rightarrow X$ and $X \rightarrow Y$. In this context, aiming to overcome these limitations, in (SHRESTHA; SCARPINO; MOORE, 2015) the authors proposed a recurrent-state message-passing approach. Recently, in (WANG et al., 2017), the authors wrote a short literature review, summarizing some of the previous results and pointing to (Wang Wei et al., 2016), where there is a classification of these methods into three categories:(i) Mean-Field like; (ii) Quenched Mean field and (iii) dynamical message passing. The above-mentioned references show the importance of message-passing approaches and in the rest of this section we will compare this approach with the pair approximation in order to see their similarities and differences. 
In (SHRESTHA; SCARPINO; MOORE, 2015), the authors studied the SIRS, SEIS and the SIS models. Here we only discuss the SIS case, but all the conclusions can also be extended to those models. Reproducing the equations proposed in (SHRESTHA; SCARPINO; MOORE, 2015) and using their notation (exception made to the rates, where we use our standard notation in order to avoid confusion), we have the following set of dynamical equations

$$
\begin{gathered}
\frac{d I_{i}}{d t}=-\delta I_{i}+\lambda S_{i} \sum_{j \in \partial i} I_{j \leftarrow i}, \\
\frac{d I_{i \leftarrow j}}{d t}=-\delta I_{i \leftarrow j}+\lambda S_{j} \sum_{k \in \partial j \backslash i} I_{k \leftarrow j}
\end{gathered}
$$

where the contacts are directed, $I_{i}(t)$ is the probability that node $i$ is infected on time $t$, complementary, $S_{i}(t)$ is the probability that it is susceptible and $I_{j \leftarrow i}$ is the probability that $j$ is infected by its neighbors at time $t$ in absence of node $i$ (WANG et al., 2017). From this framework, using linear stability analysis, in (SHRESTHA; SCARPINO; MOORE, 2015), the authors derived the critical point as

$$
\tau_{c}^{r M P}=\frac{1}{\Lambda_{1}}
$$

where $\Lambda_{1}$ is the leading eigenvalue of the non-backtracking matrix. Note that this result agrees perfectly with our PQMF approach.

From the comparison between Equations 3.1 and 3.69 and application of Bayes theorem, we observe the following relations

$$
\mathbb{E}\left(X_{i} Y_{k}\right)=\mathbb{P}\left(Y_{k}=1 \mid X_{i}=1\right) \mathbb{P}\left(X_{i}=1\right)=S_{i} I_{j \leftarrow i}
$$

where we included the term $S_{i}$ inside the summation on 3.69. Note that $\mathbb{P}\left(X_{i}=1\right)=S_{i}$, which suggests that $I_{j \leftarrow i}$ can be interpreted as the conditional probability $\mathbb{P}\left(Y_{k}=1 \mid X_{i}=1\right)$ in the Markov chain context. In order for both approaches to be the same we, must have that $\frac{d S_{i} I_{i \leftarrow j}}{d t}$ is also the same as 3.66. Expanding it we have,

$$
\frac{d S_{i} I_{i \leftarrow j}}{d t}=S_{i} \frac{d I_{i \leftarrow j}}{d t}+\frac{d S_{i}}{d t} I_{i \leftarrow j},
$$

which is not exact the same as 3.69. For instance, observe that in (SHRESTHA; SCARPINO; MOORE, 2015), by construction, the authors avoid the contribution of the interaction on edge $i \leftarrow j$ on $I_{i \leftarrow j}$, while it appears naturally on the summations of $\mathbb{E}\left(X_{i} Y_{k}\right)$. In (SHRESTHA; SCARPINO; MOORE, 2015), they showed that the recurrent-state messagepassing performs well in many simulations, which suggests to us that neglecting this term does not drastically affect the overall result. Additionally, they also showed that this method performed better, compared with Monte Carlo simulations, in their experiments. However, in their experiments just small networks were used, the Zachary's karate club, with $N=34$ and an Erdös and Rényi network with $N=100$ and average degree $\mathbb{E}(k)=3$. 
Thus, we believe that further investigations in this approach are needed. On the other hand, in Section 3.7.6 we extensively and systematically tested the QMF and the PQMF formalisms, comparing their results with Monte Carlo simulations.

\subsubsection{Synchronous cellular automaton}

Besides continuous time approaches, we can also model spreading processes as a discrete time Markov chains (DTMC). There is more than one possible epidemic/rumor spreading model that consider discrete time. Here, in this section, we will discuss cellular automaton. Briefly, synchronous cellular automaton (CA) treating time as discrete and nodal states are updated synchronously. In other words, the state of every individual in the population is updated together in a synchronous manner. In contrast, an asynchronous cellular automaton is able to update individuals independently, so that the new state of a cell affects the calculation of states in neighboring cells. Our main interest resides in the synchronous case, which will be presented for single and multilayer networks in Sections 3.5.5.1 and 3.5.5.2, respectively. Furthermore, we briefly discuss the practical aspects of the asynchronous case in Section 3.5.6, as it was widely used in the literature as a simulation method.

Importantly, in continuous time approaches $\lambda$ and $\delta$ are rates and, in this context, the probabilities are obtained after the multiplication by the time interval, i.e. $\lambda d t$ and $\delta d t$. Here, in discrete time formalisms, they assume a different role and are interpreted directly as probabilities. Thus, throughout this section and whenever we use CA formalisms they are assumed to be probabilities without further comments.

\subsubsection{Single-layer networks}

The cellular automaton (CA) approach was initially proposed in (GÓMEZ et al., 2010), where the authors consider a discrete time approach for the SIS dynamics. This method presents some similarities with the formalism presented in Section 3.5.3.1, as both consider a fixed network. However, there are some fundamental differences, such as reinfection. In other words, an individual can be cured and reinfected in the same time step. Another important point of this formalism is the synchronous behavior. This means that at every time step, each infected node intends to spread the disease, which contrasts to the continuous time, where the events occur based on exponential time. In fact, this is the most important difference between their Markov chains. Finally, the cellular automaton approach also allows us to introduce a parameter that controls the activity of the individuals. This parameter drives the dynamics from a contact process $(\mathrm{CP})^{11}$, where just one contact is performed (one contact per individual, but all the infected individuals try to spread the disease), to a reactive process (RP), where every contact is performed at

11 This is not a standard CP as it considers the reinfection. 
the same time (each infected individual tries to spread the disease to all of their neighbors). Following the SIS, we have the following discrete-time system equations

$$
y_{i}(t+1)=\left(1-y_{i}(t)\right)\left(1-s_{i}(t)\right)+y_{i}(t)(1-\delta)+y_{i}(t) \delta\left(1-s_{i}(t)\right),
$$

where $y_{i}(t)$ is the probability of node $i$ is infected at time $t$, while $s_{i}(t)$ is the probability of the node $i$ not being infected at time $t$, formally given by

$$
s_{i}(t)=\prod_{j=1}^{N}\left(1-\lambda \mathbf{R}_{j i} y_{j}(t)\right) .
$$

The term $(1-y(t))$ is the probability of the node being susceptible at time $t$ and $\left(1-s_{i}(t)\right)$ indicates the probability of this node becoming infected by at least one of its neighbors. Analyzing our model term by term, we have that $y(t)(1-\delta)$ which takes into account the recovering of an infected node. Finally, $y(t) \delta\left(1-s_{i}(t)\right)$ indicates the probability of a node being cured and also reinfected in the same time interval. Note that one might prefer a model without reinfections. This can easily be done by just deleting the term $y_{i}(t) \boldsymbol{\delta}\left(1-s_{i}(t)\right)$ in Equation 3.74. The authors argue that this formulation generalizes the previous approaches. Furthermore, the activity can be modeled in terms of random walkers leaving the node $i$ at each time step, hence,

$$
\mathbf{R}_{i j}=1-\left(1-\frac{\mathbf{W}_{i j}}{\sum_{j} \mathbf{W}_{i j}}\right)^{\kappa},
$$

where $\kappa$ represents the number of trials to spread the disease. Note the extreme cases are $\kappa=1$, which implies $\mathbf{R}_{i j}=\mathbf{P}_{i j}$, the probability transition matrix (see Section 2.2), which represents the contact process $(\mathrm{CP})$. On the other hand, $\lim _{\kappa \rightarrow \infty} \mathbf{R}_{i j}=\mathbf{A}_{i j}$, represents the reactive process $(\mathrm{RP})$.

The steady state in a discrete time system implies $y_{i}(t+1)=y(t)$. Considering this condition in Equation 3.74 we have

$$
y_{i}=\left(1-s_{i}\right)-(1-\delta) y_{i} s_{i}
$$

where the time dependency is suppressed. Moreover, the prevalence can be obtained in a similar manner to the QMF considering the average value as

$$
\rho=\frac{1}{N} \sum_{i=1}^{N} y_{i} .
$$

The existence of the critical point $\tau_{c}$ is assumed and considering that the parameters $\lambda, \delta$ and $\kappa$ are the same for every node. Regarding the epidemic threshold, it can be obtained by means of linear stability analyses neglecting second-order terms. Equation 3.75 is given as

$$
s_{i}(t) \approx 1-\lambda \sum_{j=1}^{N} \mathbf{R}_{j i} y_{j}(t) .
$$


Inserting $s_{i}(t)$ in 3.77 and considering an arbitrary small value of $0<p_{i} \ll 1$, we have the system

$$
\left(\mathbf{R}-\tau^{-1} \mathbf{I}\right) p=0
$$

where $p$ is a vector. This system has non-trivial solutions only if $\tau^{-1}$ is an eigenvalue of $\mathbf{R}$. Hence, the lowest value of $\tau$ is

$$
\tau_{c}^{C A}=\frac{1}{\Lambda_{1}}
$$

where $\Lambda_{1}$ is the largest eigenvalue of $\mathbf{R}$. Note that the RP case coincides with the QMF predictions (see Section 3.5.3.1). Moreover, in (GÓMEZ et al., 2010) the authors also evaluated this formalism over the HMF approximations in order to derive the epidemic threshold. They showed that the influence of the reinfection terms for the $\mathrm{CP}$ and RP cases is given by non-linear terms of spreading and recovering rates.

In this section, we presented the SIS modeled as a cellular automaton in a very specific scenario, where reinfections are allowed. In order to allow rumors and more intricate disease dynamics, such as SIR and SIRS, we will revisit this approach generalizing it. These results are the discussion of Chapter 4, where we study in depth the formal and applied aspects of cellular automata in single-layer networks.

\subsubsection{Multilayer-layer networks}

Once again, here we opt for the tensorial notation on the multilayer network modeling, firstly presented in its matricial form (COZZO et al., 2013), next extended to multilayers in (ARRUDA et al., 2016). However it is important to emphasize that this choice is merely a matter of taste. Using the same contact tensor, $\mathscr{R}_{\alpha \tilde{\gamma}}^{\beta \tilde{\delta}}$, defined on Section 3.5.3.2. Observe that we focus on the RP case in this section, but the $\mathrm{CP}$ can be easily obtained, simply changing the contact tensor. Denoting the probability of node $\beta$, in layer $\tilde{\delta}$, becoming infected at time $t$ as $y_{\beta}(t)$, the discrete time evolution equation for this probability is described as

$$
\begin{aligned}
y_{\beta \tilde{\delta}}(t+1)= & \left(1-y_{\beta \tilde{\delta}}(t)\right)\left(1-q_{\beta \tilde{\delta}}(t)\right)+(1-\delta) y_{\beta \tilde{\delta}}(t) \\
+ & \delta\left(1-q_{\beta \tilde{\delta}}(t)\right) y_{\beta \tilde{\delta}}(t)
\end{aligned}
$$

where the probability that a node will not be infected by any of its neighbors at time $t$ is given as

$$
q_{\beta \tilde{\delta}}(t)=\prod_{\alpha} \prod_{\tilde{\gamma}}\left(1-\lambda \mathscr{R}_{\alpha \tilde{\gamma}}^{\beta \tilde{\delta}}(\lambda, \gamma) y_{\alpha \tilde{\gamma}}\right)
$$

Observe that in Equation 3.82, the indices $\beta \tilde{\delta}$ are not dummy and there is no summation on it. A more formal notation would be obtained substituting $y_{\beta \tilde{\delta}}$ by $y_{\eta \tilde{\sigma}} E^{\eta \tilde{\sigma}}(\beta \tilde{\delta})$. The implicit summation has only one term different from zero, which is $y_{\beta} \tilde{\delta}$. 
Finally, the macro-state variable is given as the average of the individual probabilities, similar to Equation 3.46, we have

$$
\rho=\frac{1}{n m} \mathbb{E}\left(Y_{\beta \tilde{\delta}}\right) U^{\beta \tilde{\delta}},
$$

where $U^{\beta \tilde{\delta}}$ is the all one tensor.

At this point, it is interesting to compare the spreading of a disease on the aggregate network, with the full multiplex structure. This can easily be done, by changing the the contact tensor, $\mathscr{R}_{\alpha \tilde{\gamma}}^{\beta \tilde{\delta}}$, by the tensor $P_{\beta}^{\alpha}$, defined in Equation $2.47^{12}$. Contrasting with the full multilayer model, observe that in the process in the aggregated network each individual chooses a layer with uniform probability, then spreads the disease to all neighbors in that layer. On the other hand, in the model where we account for the full multilayer, Equation 3.82, each node can infect its neighbors in any layer. Furthermore, the critical point also changes, yielding to

$$
\left(\tau_{c}^{C A}\right)_{P}=\frac{1}{\Lambda_{1}}
$$

where $\Lambda_{1}$ is the largest eigenvalue of the aggregated adjacency matrix. Interestingly, applying the interlacing results (see Section 2.3.2.4) we have

$$
\left(\tau_{c}^{C A}\right)_{P} \geq \tau_{c}^{C A}
$$

This result implies that the spreading process in the multilayer structure is more efficient, or in the worst case as efficient as the process in the aggregate network (SÁNCHEZGARCÍA; COZZO; MORENO, 2014). Note that we do not conclude anything about the whole phase diagram. We just use the term efficient in terms of the critical point. This comparison was formerly presented in (SÁNCHEZ-GARCÍA; COZZO; MORENO, 2014; COZZO et al., 2016). The examples shown here exemplify the importance of considering the multilayer structure and the role of the aggregated network. Observe that a similar conclusion can be obtained using the network of layers and its interlacing properties.

Additionally, observe that the tensor $P_{\beta}^{\alpha}$ of the aggregated network have rank 2 and might be interpreted as a weighted matrix and the framework presented in this section reduces to the single-layer case, presented in the previous section.

\subsubsection{Asynchronous cellular automaton}

As mentioned before, we will briefly describe and discuss some concepts of this approach, but without going into too much detail. The main aim of this section is to recognize

12 Note that this example concerns the RP case. The CP case can be obtained after some manipulations on the tensor $P_{\beta}^{\alpha}$. 
and characterize this schema as an asynchronous cellular automaton. It is important because such approach was widely used in the literature, especially in the first studies on epidemic (PASTOR-SATORRAS; VESPIGNANI, 2001b; PASTOR-SATORRAS; VESPIGNANI, 2001a; MORENO; GÓMEZ; PACHECO, 2003) and rumor spreading (MORENO; NEKOVEE; PACHECO, 2004; BORGE-HOLTHOEFER; MORENO, 2012). Recently, more sophisticated simulation methods were proposed, as we will discuss and compare in Section 3.7. It is noteworthy that the simulations of the cellular automata described in Sections 3.5.5.1 and 3.5.5.2 follow a synchronous pattern of updating and their proper simulation methods will be discussed in Section 3.7.5.

First of all, beginning with the SIS model, the simulation can be easily described as two steps. For each infected node, which can be selected sequentially or in a random order it: (i) spread the disease to all its susceptible neighbors with probability $\lambda$ and after that (ii) it recovers to a susceptible with probability $\delta$. It is noteworthy that the SIR models only differ in the second step, where, with a probability $\delta$, the individual turns into recovered.

Besides epidemic spreading, this framework also applies to rumor spreading. However, it presents a couple of subtleties. Regarding the Maki - Thompson model, for each spreader, which can be selected sequentially or in a random order, it contacts its neighbors and, if: (i) its neighbor is ignorant then the rumor is spread with probability $\lambda$, but, if (ii) the neighbor is another spreader or a stifler it must also become a stifler with a probability $\alpha$. If we stop contacting the neighbors at the same time, it becomes a stifler and we call this a truncated process (TP). However, if we contact just one of the neighbors, we call this a contact process (CP) (BORGE-HOLTHOEFER; MORENO, 2012). In addition to truncated and contact processes, the only change we need to perform in order to implement the Daley Kendall model concerns the contact of two spreaders. In the MT model, just the individual makes the contact change its state, while in the DK model both must change its state.

We must emphasize that this simulation method captures many interesting features, for instance, it was used to study the analysis of influential spreaders in epidemic and rumor spreading on (BORGE-HOLTHOEFER; MORENO, 2012; ARRUDA et al., 2014). However, it also presents a downside, it does not predict the time correctly and is mainly applicable for steady state analysis. As mentioned before, more sophisticated methods were proposed, also capturing other features. Those methods are presented on Section 3.7.

\subsubsection{Comments on the generalization for multilayer networks}

In most of this chapter, we focused on single-layer networks. This was done because it is more intuitive to pose the problems following this formulation and then extend the 
established formalism to multilayer networks. In addition, such a generalization is natural and does not demand much effort.

First of all, it is important to observe that the use of MF and HMF approaches in the analysis of dynamical processes in multilayer networks is debatable. To begin with, let us use the HMF as an example. Observe that the HMF considers that a collection of nodes is statistically equivalent and interacts (with a given rate) with other groups in the same way. Thus, it is impossible to distinguish between a multilayer network of a single-layer network with a community structure. In other words, the equations would be the same if we have a rate for the spreading inside and outside the communities. The same argument also applies to the MF. Going further, note that in the MF the coarse graining would account for even less information. Obviously, these approaches might be useful in certain circumstances and are also mathematically correct, but we focus our efforts on the QMF and PQMF, which preserve the multilayer structure.

First of all, considering the QMF and the SIS or SIR, one can obtain a multilayer model just by changing the adjacency matrix by

$$
\mathbf{A}_{i j}^{\text {Supra }}(\lambda, \eta)=\left\{\begin{array}{ll}
1 & \text { if } i \text { is connected to } j \text { by an intra-layer edge } \\
\frac{\eta}{\lambda} & \text { if } i \text { is connected to } j \text { by an inter-layer edge } \\
0 & \text { otherwise }
\end{array},\right.
$$

where $\lambda$ and $\eta$ are the intra and inter-layer spreading rates. Regarding the MT process, one might use matrix 3.87 in the spreading (in the terms that appear on the multiplications by $\lambda$ ) and another matrix, but similarly defined, in the stifling terms (on the terms that appear in the multiplications by $\alpha$ ). Mathematically, $\mathbf{A}_{i j}^{\text {Supra }}(\alpha, v)$, where $v$ is the interlayer stifling rate. Here, in the experiments of Section 3.7.6 we considered $\eta=v$.

Next, regarding the PQMF approach, we follow a similar path, redefining the non backtracking matrix as

$$
\mathbf{B}_{(i \rightarrow j, k \rightarrow l)}^{\text {Supra }}(\lambda, \eta)= \begin{cases}1 & \text { if } j=k, i \neq l \text { and } k \rightarrow l \text { is an inter-layer edge } \\ \frac{\eta}{\lambda} & \text { if } j=k, i \neq l \text { and } k \rightarrow l \text { is an intra-layer edge } \\ 0 & \text { otherwise, }\end{cases}
$$

where $\lambda$ and $\eta$ are the intra and inter-layer spreading rates. Besides the non backtracking matrix, we also must consider

$$
\mathbf{H}_{(i \leftarrow j, k \leftarrow l)}^{\text {Supra }}=\left\{\begin{array}{ll}
1 & \text { if } i=k \text { and } j \neq l \text { and } k \rightarrow l \text { is an inter-layer edge } \\
\frac{\eta}{\lambda} & \text { if } i=k \text { and } j \neq l \text { and } k \rightarrow l \text { is an intra-layer edge } \\
0 & \text { otherwise. }
\end{array} .\right.
$$

Note that a similar comment also applies for the MT dynamics, where one might use different matrices for spreader and stifling processes. 


\subsection{Comparison of continuous-time and cellular automa- ton models}

So far we have been using a constructive and intuitive way to show the models and analyze them. However, at this point, a couple of important points arise and a more formal approach might be useful. In this section, we tackle the comparison of continuous-time and synchronous cellular automaton models. One might interpret that both formalisms model the same process (in terms of simulations), differing just by a proper translation of the rates into probabilities. However this statement is not always true. Observe that in the continuous time processes only one event takes place at a time, in fact, the probability goes to zero quadratically as the time interval goes to zero, $O\left(\Delta t^{2}\right)$. On the other hand, the opposite behavior is expected on the cellular automaton case, where every node performs its contacts during the time interval $\Delta t$.

First of all, denote the infinitesimal generator of the continuous Markov chain given by the QMF as (MIEGHEM; OMIC; KOOIJ, 2009)

$$
Q_{i}(t)=P_{i}(t)-\mathbf{I}=\left[\begin{array}{cc}
-\mathbb{E}\left(q_{1}^{i}(t)\right) & \mathbb{E}\left(q_{1}^{i}(t)\right) \\
\delta & -\delta
\end{array}\right],
$$

where

$$
\mathbb{E}\left(q_{1}^{i}(t)\right)=\lambda \sum_{i} \mathbf{A}_{i j} \mathbb{E}\left(Y_{j}\right)
$$

Next, a possible discretization of the SIS process is the sampled time Markov chain (Van Mieghem, 2014) of our QMF formalism, Equation 3.31. The obtained equation is given as

$$
\mathbb{E}\left(Y_{i}(t+\Delta t)\right)-\mathbb{E}\left(Y_{i}(t)\right)=-\delta \Delta t \mathbb{E}\left(Y_{i}\right)+\lambda \Delta t \sum_{k=1}^{N} \mathbf{A}_{k i} \mathbb{E}\left(X_{i}(t)\right) \mathbb{E}\left(Y_{k}(t)\right),
$$

where $\Delta t$ is the time interval and $\delta \Delta t$ is the recovering probability, while $\lambda \Delta t$ is the spreading probability. Note that it coincides with the Euler method for numerical solutions of differential equations. In (Van Mieghem, 2014)it was shown that for a proper value of $\Delta t<\frac{1}{\max _{i} q_{i}}$, where $q_{i}=\sum_{j=1, j \neq i}^{N} Q_{i} i j=-Q_{i} i i \geq 0$, the sampled time Markov chain is exact (not approximated) to the QMF model (Van Mieghem, 2014). Following this rule, the sampling rate will always be greater than the fastest possible transition rate, $\max _{i} q_{i}$.

The transition probabilities of the sampled time Markov chain can be written as

$$
P_{i}(t)=\left[\begin{array}{cc}
1-\mathbb{E}\left(q_{1}^{i}(t)\right) \Delta t & \mathbb{E}\left(q_{1}^{i}(t)\right) \Delta t \\
\delta \Delta t & 1-\delta \Delta t
\end{array}\right]
$$

where $\mathbb{E}\left(q_{1}^{i}(t)\right)$ is given by Equation 3.91. On the other hand, the transition probability matrix of the cellular automaton can be written as

$$
P_{i}^{C A}(t)=\left[\begin{array}{cc}
s_{i}(t) & \left(1-s_{i}\right) \\
\delta s_{i}(t) & (1-\delta)+\delta\left(1-s_{i}(t)\right)
\end{array}\right]
$$


where $s_{i}(t)$ is the probability of the node $i$ not being infected at time $t$, defined in 3.75 . Thus, comparing Equations 3.93 and 3.94 it suggests that both Markov chains are different, representing different processes. Despite its differences, both were designed to represent the SIS process and follow the same set of local rules. In order to further explore their similarities it is enlightening to explore an approximate version of the RP case without reinfections ${ }^{13}$. Here it is important to consider only the first order terms in $s_{i}$, denoted as $\tilde{s}_{i}(t) \approx 1-\lambda \sum_{i} \mathbf{R}_{i j} y_{j}$. Observe that this approximation was on the linear stability analysis in order to find the critical point of our model in 3.5.5.1. Following this approximation, the probability transition can be written as:

$$
P_{i}^{C A^{\prime}}(t)=\left[\begin{array}{cc}
\tilde{s}_{i}(t) & \left(1-\tilde{s}_{i}(t)\right) \\
\delta & (1-\delta)
\end{array}\right]=\left[\begin{array}{cc}
1-\lambda \sum_{i} \mathbf{A}_{i j} y_{j} & \lambda \sum_{i} \mathbf{A}_{i j} y_{j} \\
\delta & (1-\delta)
\end{array}\right]
$$

which is the same probability transition matrix as 3.93. This approximated model explains why the QMF and the $\mathrm{CA}(\mathrm{RP})$ models have the same critical point.

Besides the comparison between continuous time approaches and cellular automata, observe that MF, QMF and PQMF are all course grained versions of the same Markov chain, allowing us to understand them as approximations of the same process. In addition, it is also noteworthy that in (FENNELL; MELNIK; GLEESON, 2016) the authors examined numerical simulation, showing how synchronous cellular automata can bias discrete time formalisms if compared to the continuous time formalisms.

In summary, following the infinitesimal generator approach of the QMF formalism we were able to write the sampled time Markov chain and its probability transition matrix. Comparing this matrix and the respective matrix for the CA framework, we have shown evidence that both methods are not the same process. Despite its differences, we were also able to show that a first order approximation of the CA without reinfections presents the same probability transition matrix, which explains why, even being different processes, both models predict the same critical point. Moreover, similar conclusions must be taken for other models, such as the SIR. As a final comment, it should be mentioned that we are not inferring if one model is better than the other, we are just showing where they are similar and where they differ. Choosing the best model for a given real application is a different task and in one case the researcher must choose one over the other depending on their needs and restrictions. The main contribution of this result is helping researchers to properly understand which model is concerned with which process and what the adequate simulation algorithms are, which will be the discussion of our next section.

$\overline{13}$ The CA without reinfections is described by $y_{i}(t+1)=\left(1-y_{i}(t)\right)\left(1-s_{i}(t)\right)+y_{i}(t)(1-\delta)$. 


\subsection{Monte Carlo simulations}

In this section, we will leave aside the mathematical aspects of epidemic and rumor modeling and focus on the computational perspectives, aiming to model the processes described in Section 3.1. We will focus our descriptions on the multilayer case, since the single-layer approaches can be understood as a special case of the multilayer case, a multilayer with just one layer. Besides, the performance of the algorithms will not be our main concern.

Firstly we will describe the continuous time simulations in Section 3.7.1, followed by the discrete time approach, focusing on the synchronous cellular automaton case, in Section 3.7.5. Next, we will discuss the formal and practical aspects of the quasi-stationary algorithm (QS) as a simulation method on the study of absorbing state Markov chains. To round up, we will make an extensive comparison, in terms of accuracy, between numerical solutions of QMF and PQMF equations and Monte Carlo simulations. These experiments cover SIS, SIR and MT processes on top of single and multilayer structures and are presented in Section 3.7.6.

\subsubsection{Continuous-time simulations: Poisson processes}

First of all, we must restate our assumptions in the continuous time formulations, but here extending to multilayer networks. Therefore, SIS dynamics are modeled associating a Poisson process to each of the elementary dynamical transitions: intra and interlayer spreading and the recovery from the infected state. The first two processes are associated with the edges of the graph and are characterized by the parameters $\lambda$ and $\eta$, respectively. The latter transition is modeled in the node, also via a Poisson process with parameter $\delta$. In the following we present two statistically exact Gillespie-like algorithms (GILLESPIE, 1977).

\subsubsection{The standard algorithm}

The method used in (FERREIRA; CASTELLANO; PASTOR-SATORRAS, 2012; MATA; FERREIRA, 2015) is adapted here to the case of multilayer networks. For more references on this algorithm we also refer to (SANDER; COSTA; FERREIRA, 2016a; COTA; FERREIRA, 2017). At each time step, the time is incremented by $\Delta t=\frac{1}{\left(\delta N_{i}+\lambda N_{k}+\eta N_{m}\right)}$, where $N_{i}$ is the number of infected nodes, and $N_{k}$ and $N_{m}$ are the number of intra-layer and inter-layer edges emanating from them, respectively. With probability $\frac{\delta N_{i}}{\left(\delta N_{i}+\lambda N_{k}+\eta N_{m}\right)}$, one randomly chosen infected individual becomes susceptible. On the other hand, with probability $\frac{\lambda N_{k}}{\left(\delta N_{i}+\lambda N_{k}+\eta N_{m}\right)}$, one infected individual, chosen with a probability proportional to its intra-layer degree, spreads the disease to an edge chosen uniformly random. Finally, with probability $\frac{\eta N_{m}}{\left(\delta N_{i}+\lambda N_{k}+\eta N_{m}\right)}$ one infected individual, chosen with a probability propor- 
tional to its inter-layer degree, propagates the disease to an edge chosen uniformly. If an edge between two infected individuals is selected during the spreading, nothing happens, only time is incremented. The process is iterated following this set of rules, simulating the continuous process described by the SIS scenario.

It is noteworthy that a generalization from this framework to other dynamical processes is trivial. Beginning with the SIR, the procedure is basically the same, however, instead of curing the node and classifying it as susceptible again, we must label it as recovered. Once there are no more spreaders the dynamics stop. On the other hand, in order to simulate the MT process, we must adapt our framework. This process is modeled associating a Poisson process to each of the elementary dynamical transitions: intra and interlayer spreading and stifling. The first two processes are associated with the edges of the graph and are characterized by the parameters $\lambda$ and $\eta$, respectively. The latter two transitions are also modeled on the edge via a Poisson process with parameters $\alpha$ and $v$. Contrasting with the SIS and SIR dynamics, the annihilation mechanisms are based on the contact between individuals, requiring us to also associate these processes on the edges. We also remark that the DK dynamics would also present a similar modeling, however when the annihilation processes take place, both ends of an edge must change, as the process is undirected, contrasting with the MT approach. Here in this thesis we only follow the MT models.

From the computational viewpoint, the MT dynamics is modeled as follows. Firstly, given an initial condition, at each time step, the time is incremented by $\Delta t=\frac{1}{\left((\lambda+\alpha) N_{k}+(\eta+v) N_{m}\right)}$, where $N_{k}$ and $N_{m}$ are the number of intra-layer and inter-layer edges emanating from spreaders, respectively. With probability $\frac{\lambda N_{k}}{\left((\lambda+\alpha) N_{k}+(\eta+v) N_{m}\right)}$ a spreader, chosen with a probability proportional to its intra-layer degree, spreads the rumor/information to an edge chosen uniformly randomly. On the other hand, with probability $\frac{\alpha N_{k}}{\left((\lambda+\alpha) N_{k}+(\eta+v) N_{m}\right)}$ a spreader, chosen with a probability proportional to its intra-layer degree, can be stifled by an edge chosen uniformly randomly if the contacted neighbor is another spreader or a stifler. Similar actions are taken regarding inter-layer edges, however with probabilities $\frac{\eta N_{m}}{\left((\lambda+\alpha) N_{k}+(\eta+v) N_{m}\right)}$, for spreading and probability $\frac{v N_{m}}{\left((\lambda+\alpha) N_{k}+(\eta+v) N_{m}\right)}$ for stifling processes. The next time is incremented by $\Delta t$ and this set of rules is applied until we reach the absorbing state. In other words, until there are no spreaders on the population.

\subsubsection{A different implementation}

Alternatively, instead of choosing uniform probabilities in order to decide which Poisson process we should execute, we can also simulate by a sampling exponential time for each process. These times refer to the next event (note that the times must be accumulative), thus choosing the smallest among all times. Considering the SIS as an example, a spreader has random exponential times associated with its edges, whose parameter is 
Figure 12 - Multiplex network composed by two Erdös and Rényi layers, $m=2$, and $n=10^{4}$ nodes on each layer, where the first with $\mathbb{E}(k)=16$, while the second with, $\mathbb{E}(k)=12$. The effective spreading rate $\tau=0.15$ and $\eta=0.01$. The continuous lines represents the first (QMF) and second (PQMF) order approximations on the whole population, the order parameter $\rho$, while the dot-dashed and dashed lines represents the average of infected individuals on the first and second layers, respectively. Each colored line represents one of the 50 independent Monte Carlo simulations.

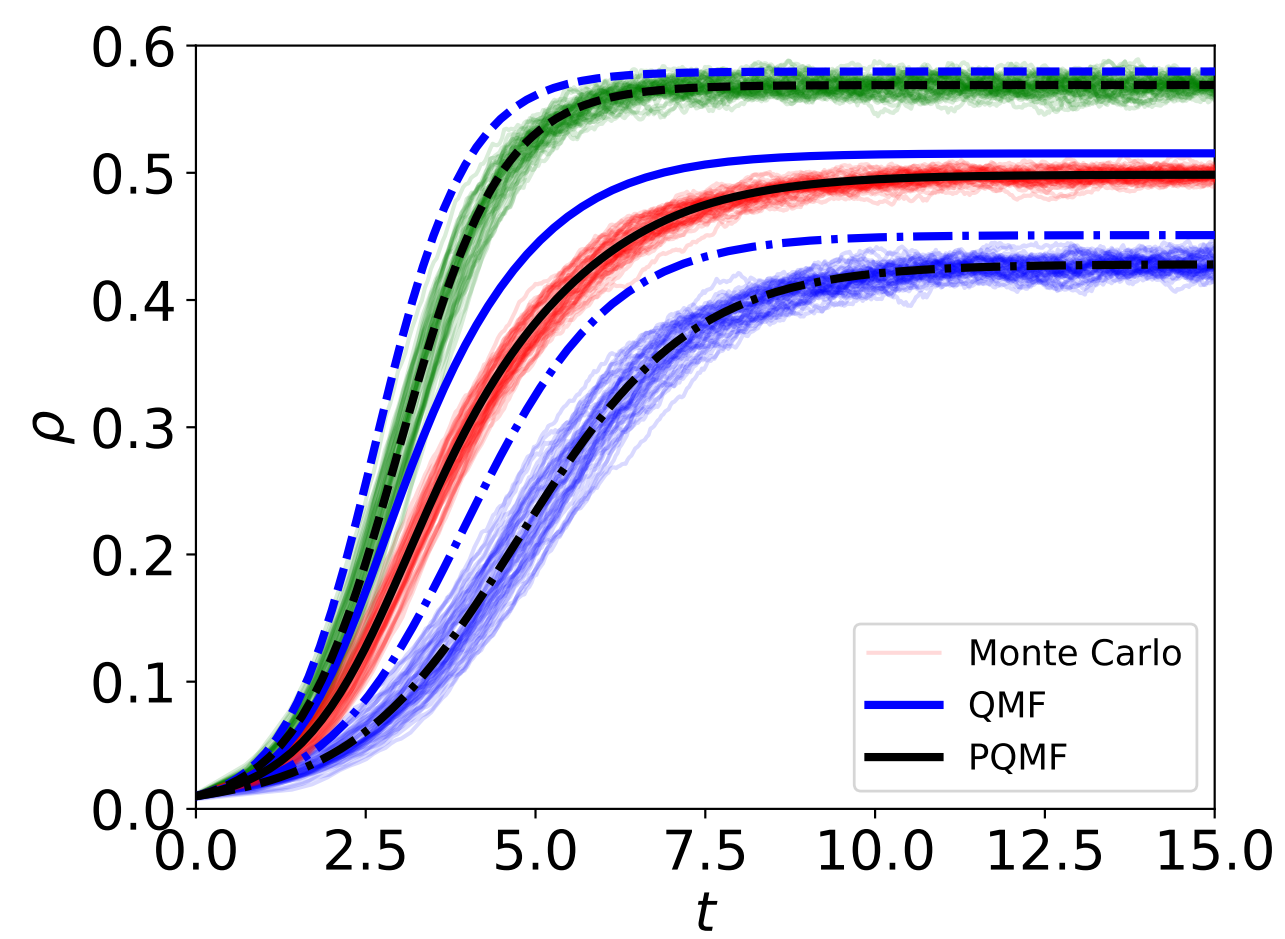

Source: Developed by the author.

$\lambda, T+\Delta t$ (where $T$ is the actual time of the simulation and $\Delta t$ is the random exponential time), and an exponential random number, with parameter $\delta$, modeling the cure, similarly $T+\Delta t$. The same is reproduced for all infected individuals. Supposing that the next event regards a spreading, as the edge is considered to be directed, it is guaranteed it emanates from a spreader, then its respective neighbor becomes infected at that time. If it was already infected nothing happens. Next, the time is updated, $T^{\prime}=T+\Delta t$. Moreover, after spreading the disease, a new time is associated, allowing the individual to spread another time on that same edge, $T^{\prime}+\Delta t^{\prime}$ (where $\Delta t^{\prime}$ is the new exponentially sampled random number, the time for the next event). On the other hand, if the next event is associated with a curing process, the node makes its state susceptible and all its Poisson processes are deleted and, obviously, time must be incremented, $T^{\prime}=T+\Delta t$. Note that choosing a collection of exponentially distributed values, as described in the previous section, leads to the same outcome. Thus, the main advantage of this approach is to capture the time behavior of the process slight more precisely being more intuitive, as we implement each Poisson process independently. Additionally, it makes the algorithm easier to 
Figure 13 - Distribution of 500 simulations over a complete graph with $N=2 \times 10^{3}$ nodes in order to validate the simulation. Comparing with the results shown in Section 3.5.1 (Equations 3.18 and 3.19) we obtained an error of 0.00014 on the average and 0.0166 on the variance of the simulations. Additionally, we performed the Kolmogorov Smirnov test obtaining $D=0.0408$, suggesting that our data is normally distributed.

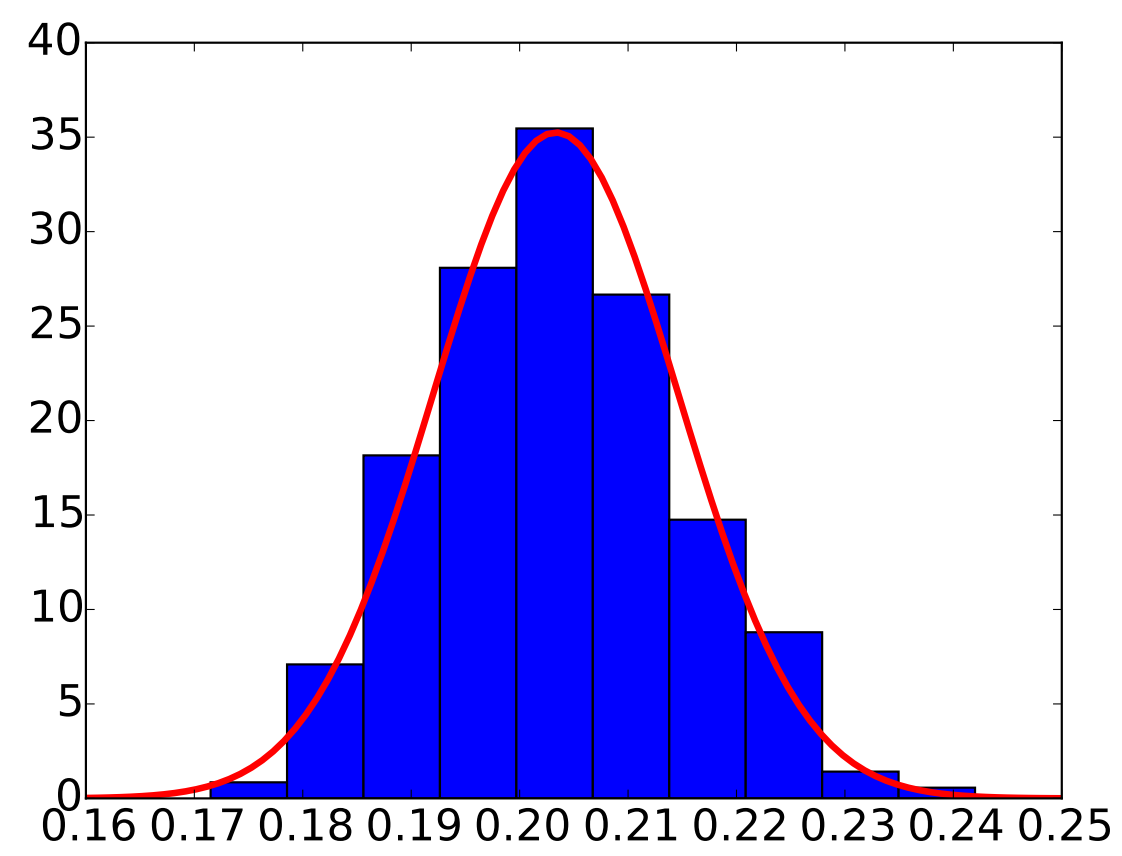

Source: Developed by the author.

study non-Markovian and heterogeneous processes.

As an example, in Figure 12, we show an example of this algorithm compared to the first, QMF, and second-order approximations, PQMF over time. In this example we considered a multiplex network composed by two Erdös and Rényi layers, $m=2$, and $n=5 \times 10^{3}$ nodes on each layer, where the first with $\mathbb{E}(k)=16$, while the second with, $\mathbb{E}(k)=12$. In this figure, we simulated the SIS with an effective spreading rate of $\tau=0.15$.

A similar approach also applies to the other dynamical processes, e.g. SIR, MT. We must only consider the rates associated with the processes and the rules that must be applied. For instance, in Figure 13, we show the simulations over a complete graph with $N=2 \times 10^{3}$ nodes in order to validate our simulations. Here we compare the results obtained over 500 simulations and Equations 3.18 and 3.19 for the theoretical expected fraction of ignorants and its variance. The errors obtained were 0.00014 on the average and 0.0166 on the variance of the simulations. As the last comparison, we performed a Kolmogorov - Smirnov test obtaining $D=0.0408$, suggesting that our data is also normally distributed, as expected by the predictions shown in Section 3.5.1. 
As a comment on computational cost, we must emphasize that this algorithm is much more costly than the standard one, and it will only be used in this chapter, while in Chapter 5 together with the QS method (see next section) we use the standard algorithm.

\subsubsection{Quasi-stationary algorithm (QS)}

In this section, we first formally define the Quasi-stationary algorithm (QS) in the next section, followed by some numerical examples of stars and random regular networks, which allow us to discuss important features related to the critical behavior of epidemic spreading in networks.

\subsubsection{Definition}

In this section, we explore the quasi-stationary algorithm (QS) in two steps. To begin with, we discuss some of its basic aspects, showing the main mechanism behind this algorithm. This analysis follows the approach shown in (DICKMAN; VIDIGAL, 2002). Next, from the computational point of view, we show how it is implemented and how we can use it to precisely determine the critical point on the SIS dynamics (FERREIRA; CASTELLANO; PASTOR-SATORRAS, 2012; MATA; FERREIRA, 2015). Near the critical point, fluctuations are often high, driving the system to the absorbing state on finite system sizes. This complication made the determination of such a point not a trivial task. In order to avoid it we might use the quasi-stationary distribution, where the system is restricted to active states, but still represents the original process.

Firstly, consider a general stochastic process with an absorbing state, denoted by $\left(Y_{t}^{*}\right)_{t \geq 0}$, where $Y_{t}^{*}=0$ is the absorbing state. The probability that the process does not fall into the absorbing sate, also called survival probability, is given as (DICKMAN; VIDIGAL, 2002)

$$
P(t)=\sum_{n^{*} \geq 1} p_{n^{*}}(t)
$$

where $P(t)$ is the survival probabilities, while $p_{n^{*}}(t)$ is the probability of having $n^{*}$ infected individuals (or occupied sites in a more general context) in the population. Thus, we can define the QS probability, $\bar{p}_{n^{*}}$ from the following condition

$$
p_{n^{*}}(t)=P(t) \bar{p}_{n^{*}} \quad n^{*} \geq 1,
$$

where $\bar{p}_{n^{*}}$ is time-independent, since we are taking the limit of infinity time. Note that, by construction, $\bar{p}_{0}=0$. The normalization

$$
\sum_{n^{*} \geq 1} \bar{p}_{n^{*}}=1
$$

also apply. 
Our process was defined in a single variable denoting the number of infected individuals, $n^{*}$. Thus, denoting the transition rates as $W_{m^{*}, n^{*}}$, where the dynamics transit from $Y^{*}=n^{*}$ to $Y^{*}=m^{*}$, for the epidemic spreading we have

$$
\left\{\begin{array}{l}
W_{n^{*}-1, n^{*}}=n \\
W_{n^{*}+1, n n^{*}}=\tau \sum_{i=1}^{N} \sum_{k=1}^{N} \mathbf{A}_{k i} X_{i} Y_{k},
\end{array}\right.
$$

where $\sum_{k=1}^{N} Y_{k}=n^{*}$. Note that one might want to describe the SIS stochastic process in its micro-state and re-define the rates $W_{S_{i}, S_{j}}$, where $S_{i}$ is a micro-state. However here we are interested in the macro-state chain. More specifically, it resembles the infinitesimal generator presented in 3.8. Therefore, we have the following master equation

$$
\frac{d p_{n^{*}}}{d t}=W_{n^{*}, n^{*}+1} p_{n^{*}+1}+W_{n^{*}, n^{*}-1} p_{n^{*}-1}-W_{n^{*}-1, n^{*}} p_{n}-W_{n^{*}+1, n^{*}} p_{n^{*}},
$$

which describes $p_{n^{*}}$. After deriving Equation 3.96 and applying the relation observed in the master Equation 3.100 it yields to $\frac{d P}{d t}=-p_{1}(t)$, which under the QS hypothesis can be expressed as

$$
\frac{1}{P} \frac{d P}{d t}=-p_{1}(t)
$$

which leads to (DICKMAN; VIDIGAL, 2002)

$$
\tau=\frac{1}{\bar{p}_{1}},
$$

allowing us to precisely obtain the critical point by means of the QS distribution. Moreover, observe that the order parameter may also be obtained through the the QS distribution as $\rho^{Q S}=\sum_{n \geq 1} \bar{p}_{n^{*}}$. Interestingly, in the thermodynamic limit $\rho^{Q S}$ converges to $\rho$. Before the critical point the distribution $\bar{p}_{n}$ is concentrated on a finite number of individuals, which means that just one, or a finite fraction, of individuals is infected. Thus, in the thermodynamic limit the order parameter goes to zero, $\rho^{Q S}=\frac{m^{*}}{N} \rightarrow 0=\rho$, where $m^{*}$ is a finite number.

Next, from a computational point of view, the quasi-stationary method (FERREIRA; CASTELLANO; PASTOR-SATORRAS, 2012; MATA; FERREIRA, 2015) restricts the dynamics to non-absorbing states and can be implemented as follows. Every time the process tries to visit an absorbing state, it is substituted by an active configuration previously visited and is stored in a list with $M$ configurations, constantly updated. With a probability $p_{r}$ a random configuration in such a list is replaced by the actual configuration. In order to extract meaningful statistics from the quasi-stationary distribution, denoted by $\bar{p}_{n^{*}}$, where $n^{*}$ is the number of infected individuals, the system must be in the stationary state and a large number of samples must be extracted. Thus, we let the simulations run during a relaxation time $t_{r}$ and extract the distribution $\bar{p}_{n^{*}}$ during a sampling time $t_{a}$. The threshold can be estimated using the modified susceptibility (FERREIRA; 
Figure 14 - Finite size analysis for a random regular graph with $\mathbb{E}(k)=10$ from $N=10^{3}$ to $N=$ $3 \times 10^{4}$ considering the fraction of infected individuals (left panel) and susceptibility (right panel) as a function of the spreading rate $\lambda$ and recovering rate $\delta=1$. The dashed lines represent the QMF predictions for the critical point.
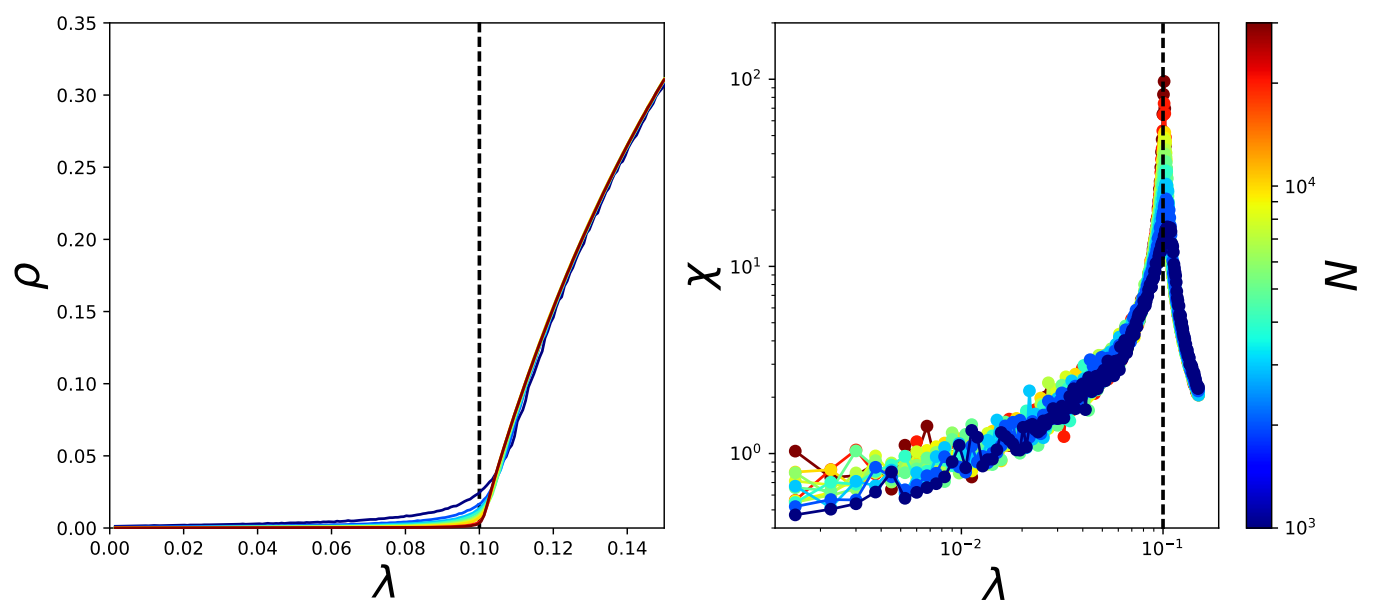

Source: Developed by the author.

CASTELLANO; PASTOR-SATORRAS, 2012), given by

$$
\chi=\frac{\mathbb{E}\left(\left(n^{*}\right)^{2}\right)-\mathbb{E}\left(n^{*}\right)^{2}}{\mathbb{E}\left(n^{*}\right)}=n m\left(\frac{\mathbb{E}\left(\left(\rho^{Q S}\right)^{2}\right)-\mathbb{E}\left(\rho^{Q S}\right)^{2}}{\mathbb{E}\left(\rho^{Q S}\right)}\right)
$$

where $\rho^{Q S}$ is the quasi-stationary distribution $\bar{p}_{n^{*}}$. As argued in (FERREIRA; CASTELLANO; PASTOR-SATORRAS, 2012; MATA; FERREIRA, 2015) the susceptibility presents a peak at the phase transition in finite systems. This measure is the coefficient of variation of the temporal distribution of states over time in the steady state. Note that the magnitude of the susceptibility $\chi$ is not of primary interest to us, but rather the position of its maximum value with respect to $\lambda / \delta$, as it will coincide with the critical threshold for sufficiently large systems.

\subsubsection{Example: a numerical evaluation}

Complementing the previous section, here we show a practical application of the QS algorithm in two paradigmatic examples: (i) the random regular network (for more, see Section 2.1.4.5) and (ii) Star. The first is a completely homogeneous network, where every node has the same connectivity, $\mathbb{E}\left(k_{i}\right)=k$, for $k_{i}=1,2, \ldots, N$, while the star is a heterogeneous case, where the central node has $N$ neighbors, while the leafs have only one connection. Despite its simplicity, this model was previously used in (ARRUDA et al., 2014) as an approximation for hubs in scale-free networks. Additionally, both models are extreme cases and are of special interest in modeling. For instance, in (Mata, Angélica S.; 
Figure 15 - Finite size analysis for a star from $N=10^{3}$ to $N=10^{5}$ considering the fraction of infected individuals (left panel) and susceptibility (right panel) as a function of the spreading rate $\lambda$ and recovering rate $\delta=1$. The dashed lines represent the PQMF predictions for the critical point.
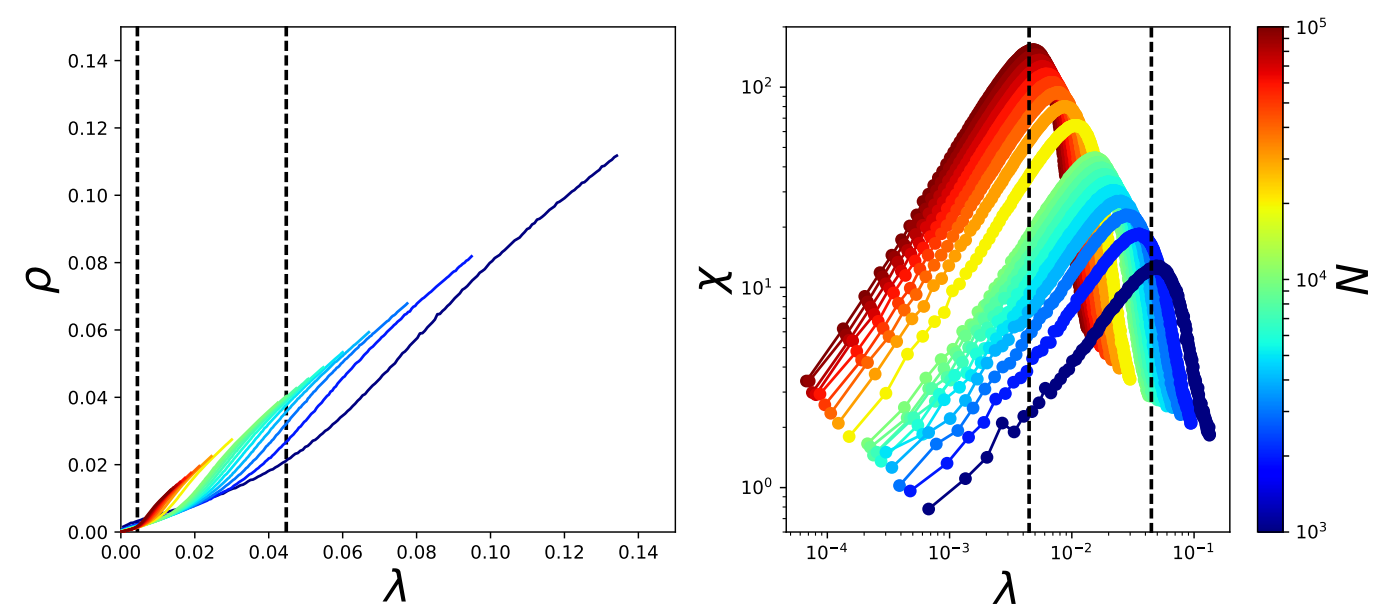

Source: Developed by the author.

Ferreira, Silvio C., 2013) the authors used these two networks to discuss the accuracy of the PQMF predictions of the critical point.

Figure 14 shows the fractions of infected individuals and their respective susceptibility as a function of the spreading rate and size for RRNs. As expected by the mean field approaches, the critical point remains fixed as we change the system size. Additionally, we also observe that the peak of susceptibility also scales with the system size, suggesting that it diverges on the thermodynamic limit. Consequently, these results suggest a second order phase transition. It is noteworthy that similar results are also expected for Erdös Rényi networks and complete graphs. Complementary, we also show the predictions of the QMF for the critical point, which seems to be reasonable in this experiment. Note that in reference (Mata, Angélica S.; Ferreira, Silvio C., 2013), the authors compared the QMF and PQMF predictions in RRNs, showing that the PQMF are more accurate than the QMF in this class of networks. This is indeed true and verified in our experiments (results not shown). However they conducted their experiments in sparse networks, $\mathbb{E}(k)=6$ on (Mata, Angélica S.; Ferreira, Silvio C., 2013), while we used a denser network, $\mathbb{E}(k)=10$. Finally, it should be mentioned that the denser the networks, the more precise QMF is. For the sake of discussion, observe that $\left(\tau_{R R N}^{Q M F}\right)_{c} \approx \frac{1}{k}$, while $\left(\tau_{R R N}^{P Q M F}\right)_{c} \approx \frac{1}{k-1}$.

Complementary, Figure 15 shows the fractions of infected individuals and their respective susceptibility as a function of the spreading rate and size for the stars. Contrasting with the results for the homogeneous case, here the critical point tends to zero as the system size goes to infinity. As previously discussed, it cannot be considered a 
Figure 16 - Example of discrete Monte Carlo simulations. Phase diagrams for epidemic spreading considering the $\mathrm{CA}(\mathrm{RP})$ formalism. In (a) the SIS model, considering two cases, with or without reinfections at the same time step. In (b) the SIR model. The continuous lines are the analytical solutions, while the symbols are an average of $10^{2}$ the Monte Carlo simulation, $\eta=0.5$ fixed in all simulations. The multiplex considered is composed by two scale-free networks, $P(k) \sim k^{\zeta}$, the first with $\zeta \approx 2.8$, while the second with $\zeta \approx 3.0$. The variance is the size of the symbols.

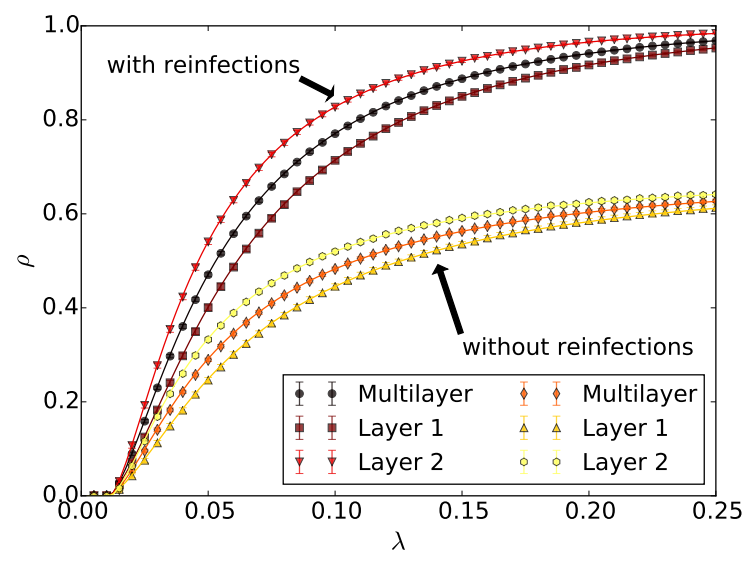

(a) Susceptible-Infected-Susceptible (SIS)

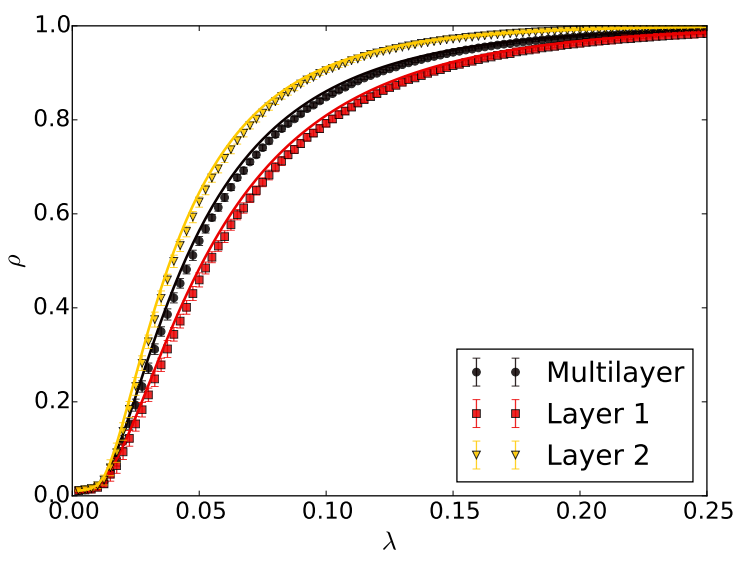

(b) Susceptible-Infected-Recovered (SIR)

Source: Developed by the author.

phase transition in the classical sense, as phase transitions are defined in the thermodynamic limit. However, as also previously mentioned, in the literature of complex networks we still use the term phase transition and critical point for the observed phenomena on scale-free networks. Regarding the critical point predictions, in the star the QMF approach does not present an accurate prediction, confirming the results of (Mata, Angélica S.; Ferreira, Silvio C., 2013). In Figure 15, the dashed lines present the PQMF prediction, $\left(\tau_{\text {Star }}^{P Q M F}\right)_{c} \approx \sqrt{\frac{2}{N-1}}$. For the sake of comparison, the QMF prediction is given as $\left(\tau_{\text {Star }}^{Q M F}\right)_{c} \approx \frac{1}{\sqrt{N-1}}$. Both formulas were extracted from (Mata, Angélica S.; Ferreira, Silvio C., 2013). It is interesting to observe that both formulas present a different behavior as a function of $N$.

Finally, is worth mentioning that more in-depth and proper evaluation of the accuracy of the critical point predictions in power-law networks were performed referring to (FERREIRA; CASTELLANO; PASTOR-SATORRAS, 2012), where the authors compare numerical and theoretical results for the HMF and QMF approaches.

\subsubsection{Discrete-time simulations: Cellular automaton}

In addition to the continuous time algorithms, we must also discuss the particularities of the cellular automaton formalism. As shown in Section 3.6, the most traditional 
approaches in the epidemic spreading modeling, the continuous time and the cellular automaton, represent different Markov chains and therefore different processes. Although both follow the same set of local rules, their formal process differs. Thus, we must also provide a Monte Carlo simulation that corresponds to the cellular automaton formalism. Here we focus on the synchronous case.

Firstly, regarding the SIS dynamics. Denoting by $Y_{i}(t)$ the state of the node $i$ at time $t$, where it is $Y_{i}(t)=1$ if node $i$ is infected and 0 otherwise. Thus, starting from an initial condition $S_{i}(0)$ for all $i$, the algorithm is described in four steps, et each time step, $t$ : (i) the state of every node should be copied, $Y_{i}(t+1)=Y(t)$, next (ii) every infected individual at time $t,\left\{i \mid Y_{i}(t)=1\right\}$, will be cured with probability $\delta, Y_{i}(t+1)=0$, then (iii) every infected individual at time $t,\left\{i \mid Y_{i}(t)=1\right\}$, will contact all its neighbors, denoted by $j$ (considering the neighbor status at time $t+1$, which allows the reinfection ${ }^{14}$ ), in the RP or to a single random neighbor if in the CP case, spreading the disease to each susceptible neighbor contacted with probability $\lambda$ and finally, then $Y_{j}(t+1)=1$ (iv) time is incremented $t^{\prime}=t+1$. The main difference between the SIS to the SIR process is that in the former case the infected individuals will become susceptible again, while in the latter they will be recovered and removed from the dynamics. These processes must be iterated for a fixed number of steps, $T_{\max }$, or if there are no more infected individuals (an absorbing state).

Figure 16 shows three examples of phase diagrams for our algorithm, SIS with and without reinfection and SIR. Moreover, we show a small comparison of these approaches and the mathematical formalism presented in Section 3.5.5. Further experiments concerning the accuracy of these models are presented in Section 3.7.6 and Chapter 4.

Regarding the comparison between the synchronous and asynchronous cases, there is no proof that it would or would not converge to the same results in specific cases. In fact, many there are a couple of examples where both show different outcomes, as shown in (CORNFORTH; GREEN; NEWTH, 2005). However, we must observe that the asynchronous case must be similar for the SIR case, as the removed nodes do not play a role in the dynamics. We remark that the time in both approaches is different, but both present the same final result in terms of the final fraction of infected individuals. Note also that the asynchronous processes are often described as firstly spreading, then curing (see the description in Section 3.5.6), while here we opted for the opposite. This is justified since the update is always synchronous and, in principle, the order would not change the outcome. Note that $Y_{i}(t+1)$ never affects $Y_{i}(t)$. However curing first allows us to easily

$\overline{14}$ Note that we not are changing the state of node in the same time window. Thus, we are allowing reinfections. If we do not allow reinfection we should consider only the nodes that still are infected at $t+1$. In other words, we would use only the states at $t+1$, following a sequential algorithm. It is also interesting to mention that, without reinfection, just in the state vector is necessary and the actions can be made in-place. 
Figure 17 - Estimated errors in the bars and their standard error in the deviations. From left to right, we present the error of the SIS, SIR and MT processes with respect to the QMF and PQMF mean filed approaches in four different structures, uncorrelated, assortative, disassortative and a clustered network. The networks have $N=10^{4}$, see text for more details on their structural properties.
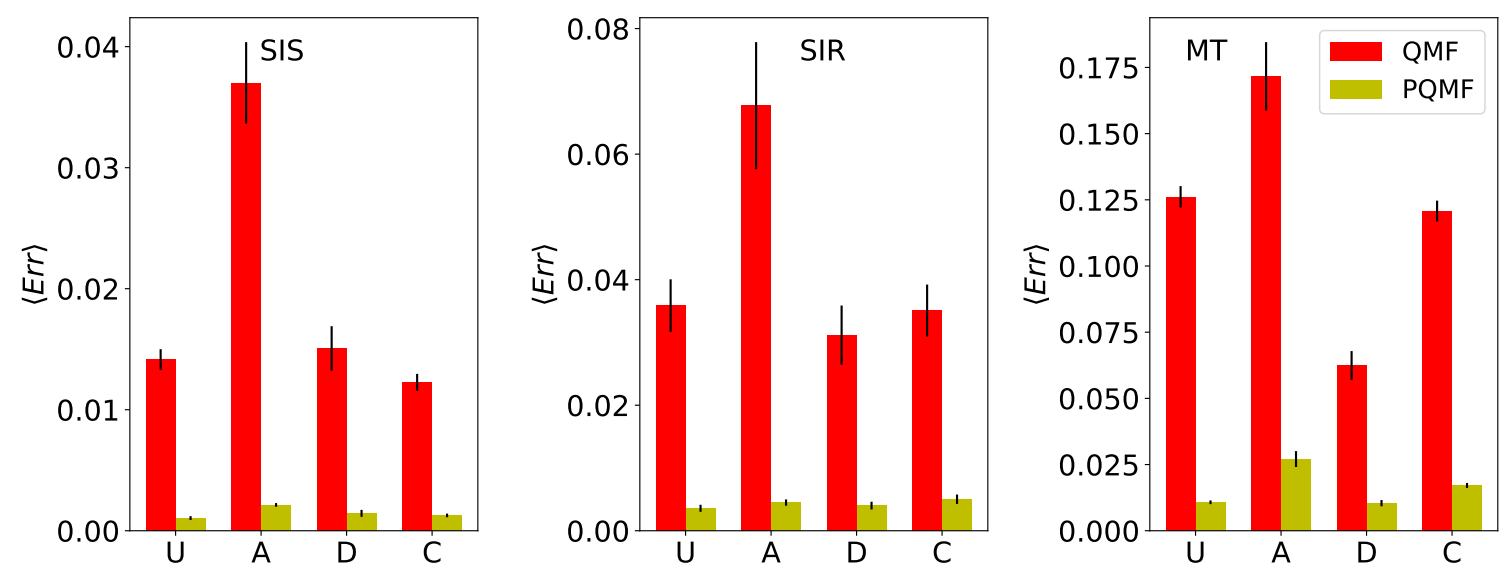

Source: Developed by the author.

model the reinfections with no further steps in our algorithm.

Some modifications were made to adapt this scheme to other contexts, as discussed in Chapter 4. Among the generalizations, we take into account the MT rumor spreading and more intricate disease dynamics, such as SIRS.

\subsubsection{A systematic accuracy evaluation}

Similarly to (GLEESON et al., 2012), where the author evaluates the accuracy of many mean field models, here we also perform an accuracy evaluation. However, we focus on specific spreading dynamics models. More specifically, we focus on the QMF and PQMF approaches, since it was already shown that the QMF is qualitatively better than the HMF (FERREIRA; CASTELLANO; PASTOR-SATORRAS, 2012), providing a better estimative of the critical point. Along with this section, we perform an extensive analysis regarding three models: SIS, SIR and MT. Moreover, we evaluate different structures, including single and multilayer networks, with and without correlations and triangles. Firstly, in Section 3.7.6.1, we study the accuracy of these models in single layers. Secondly, we use the previously analyzed networks to build 2-Layer multiplex networks. Then, in Section 3.7.6.2, based on those structures we evaluate their accuracy while changing the coupling parameter. 


\subsubsection{Single-layer analyses}

In order to compare the accuracy of different mean field approximations, we extract a phase diagram, $(\rho, \lambda)$ (with $\delta=1$, hence $\tau=\lambda$ ), from the mean field approach and compare it with Monte Carlo simulations. The error is quantified/estimated using the average of the absolute error among the Monte Carlo runs,

$$
\operatorname{Err}_{i}=\frac{\sum_{i}\left|\rho_{i}^{M C}-\rho^{M F}\right|}{n_{\exp }},
$$

where $n_{\exp }$ is the number simulations performed, $\rho_{i}^{M C}$ is the result of one Monte Carlo simulation, while $\rho^{M F}$ is its prediction following a mean field approach and its standard error $^{15}$ is calculated as

$$
S E=\frac{s}{\sqrt{n_{\text {points }}}}
$$

where $n_{\text {points }}$ is the number of points in the phase diagram $(\rho, \lambda)$ and $s$ is the sample standard deviation of $\operatorname{Err}_{i}$. In these experiments we performed 50 independent Monte Carlo runs for each $\lambda$ from $\lambda=0.01$ to $\lambda=0.49$ in steps of $\lambda=0.01$. The simulations and numerical experiments were executed until $t_{\max }=10^{2}$. Additionally, aiming to reduce the noise on the curve of the SIS, we consider a temporal average on the meta-state. Formally,

$$
\rho^{M C}=\frac{1}{t_{s}} \int_{t_{s}}^{t_{\max }} \rho(t) \mathrm{d} t=\frac{1}{t_{s}} \sum_{t \geq t_{s}}^{t \leq t_{\max }} \rho(t) \Delta t,
$$

where $t_{s}$ is the sampling time, which was assumed to be $t_{s}=50$ and on the discrete equation $\rho(t) \Delta t$ is interpreted as the macro-state times the time it was presented in the meta-state. In words, $\rho^{M C}$ is the temporal average of the states visited in the meta-state, observing that they are properly weighted by the time the system spent on that state. This can be done because we know that there is a stochastic fluctuation over the average value, which is the value we are aiming to capture by the mean field methods. Note that it does not apply to the SIR and MT dynamics due to the nature of their absorbing states.

From the structural point of view, in this subsection we focused on four scale-free networks: (i) scale free uncorrelated network with $P(k) \sim k^{-2.7}, N=10^{4}$ and $\mathbb{E}(k) \approx 7.85$, assortativity and clustering coefficient close to zero, denoted as (U), (ii) correlated scale free network with $P(k) \sim k^{-2.7}, N=10^{4}$ and $\mathbb{E}(k) \approx 7.85$, disassortative with $\rho^{P}=-0.39$, and clustering coefficient close to zero, denoted by (D), (iii) correlated scale free network with $P(k) \sim k^{-2.7}, N=10^{4}$ and $\mathbb{E}(k) \approx 7.85$, assortative with $\rho^{P}=0.30$, and clustering

$\overline{15}$ Note that we are estimating the error based on independent samples of it. The formula of $S E$ is derived from our knowledge about the variance of a sum of independent random variables. Now, suppose that $X_{1}, X_{2}, \ldots, X_{n_{p}}$ are $n_{p}$ independent observations from a population that has a mean $\mu$ and standard deviation $\sigma$, then the variance of the total $T=\left(X_{1}+X_{2}+\cdots+X_{n_{p}}\right)$ is $n \sigma^{2}$. Hence, the variance of $T / n_{p}$ is $\frac{1}{n_{p}{ }^{2}} n_{p} \sigma^{2}=\frac{\sigma^{2}}{n_{p}}$, yielding to the standard deviation of $T / n_{p}$ must be $\sigma / \sqrt{n_{p}}$. 
coefficient close to zero, denoted by (A) and (iv) scale free network with clustering coefficient (HOLME; KIM, 2002), $N=10^{4}$ and $\mathbb{E}(k) \approx 8$, assortativity coefficient $\rho^{P}=-0.07$ and clustering coefficient $\mathbb{E}(c c)=0.215$, denoted by $(\mathrm{C})$.

In Figure 17, we summarize our experiments in terms of the errors. The observed errors are consistently small for the second-order approximation, PQMF, as expected. It is arguable that the first order approximation, QMF, is a good approximation in most cases. However, in the MT dynamics, it predicts the processes dynamics poorly, presenting a huge error. Regarding the structural correlations, we also observed assortative structures seem to pose a bigger challenge for the approximations, even when compared with disassortative and networks with a high clustering coefficient.

Since we observed and quantified that the PQMF is evidently more accurate than the QMF it is important to comment on both approaches' computational cost. Considering a graph with $N$ nodes and $M$ edges, the QMF can be solved using $O(N)$ equations, while the PQMF $O(N+M)^{16}$. If this graph is dense, it also implies that $E \in O\left(N^{2}\right)$. Note, however, in dense graphs the PQMF presents a huge computational cost. However, luckily, in dense networks, the QMF is expected to perform better, since the dynamical correlations tend to vanish in a complete graph on the thermodynamic limit. In other words, for a large and dense enough graph the dynamical correlations are expected to be smaller and the approximations $\mathbb{E}(X Y) \approx \mathbb{E}(X) \mathbb{E}(Y)$ become better and better as we approach the thermodynamic limit (MIEGHEM; OMIC; KOOIJ, 2009).

\subsubsection{Multilayer analyses}

Extending the results of the previous section to the multilayer framework, we are able to quantify the accuracy of mean field approaches in this structure. In addition to the networks previously evaluated, we also considered the RRN (for more, see Section 2.1.4), since it presents a well defined critical point. Besides, since every node in such a network has the same degree, this network may also present some dynamical correlations too, being a good proxy for our experiments. Thus, we tested our methods in four different multiplex networks: (i) ARR (Assortative+RRN - randomly coupled), (ii) AAA (Assortative+Assortative - Assortativelly coupled), (iii) ACA (Assortative+Clustered Assortativelly coupled) and (iv) ACR (Assortative+Clustered - randomly coupled).

Figure 18 shows the results for the comparison of Monte Carlo simulations and QMF and PQMF mean field approaches in terms of the errors. Additionally, each phase diagram may be found in Annex A. Again, the errors observed are consistently small for the second-order approximation, $\mathrm{PQMF}$, which was expected. We might also observe that this observation suggests that one of the main causes of this error is the dynamical

16 Note that the number of equation of SIS are $N(\mathrm{QMF})$ and $N+M(\mathrm{PQMF})$ for the SIS and $2 N(\mathrm{QMF})$ and $2 N+2 M(\mathrm{PQMF})$ for SIR and MT. 
Figure 18 - Estimated errors in the bars and their standard errors in the deviations. From top to bottom, we present the error of the SIS, SIR and MT processes with respect to the QMF and PQMF mean filed approaches in four different structures: ARR (Assortative+RRN - randomly coupled), AAA (Assortative+Assortative - Assortativelly coupled), ACA (Assortative+Clustered - Assortativelly coupled) and ACR (Assortative+Clustered - randomly coupled). From left to right, we change the coupling parameter $\eta=10^{-3}, \eta=10^{-2}, \eta=10^{-1}$. The networks have $n=10^{4}$ and $m=2$, see text for more details on their structural properties.
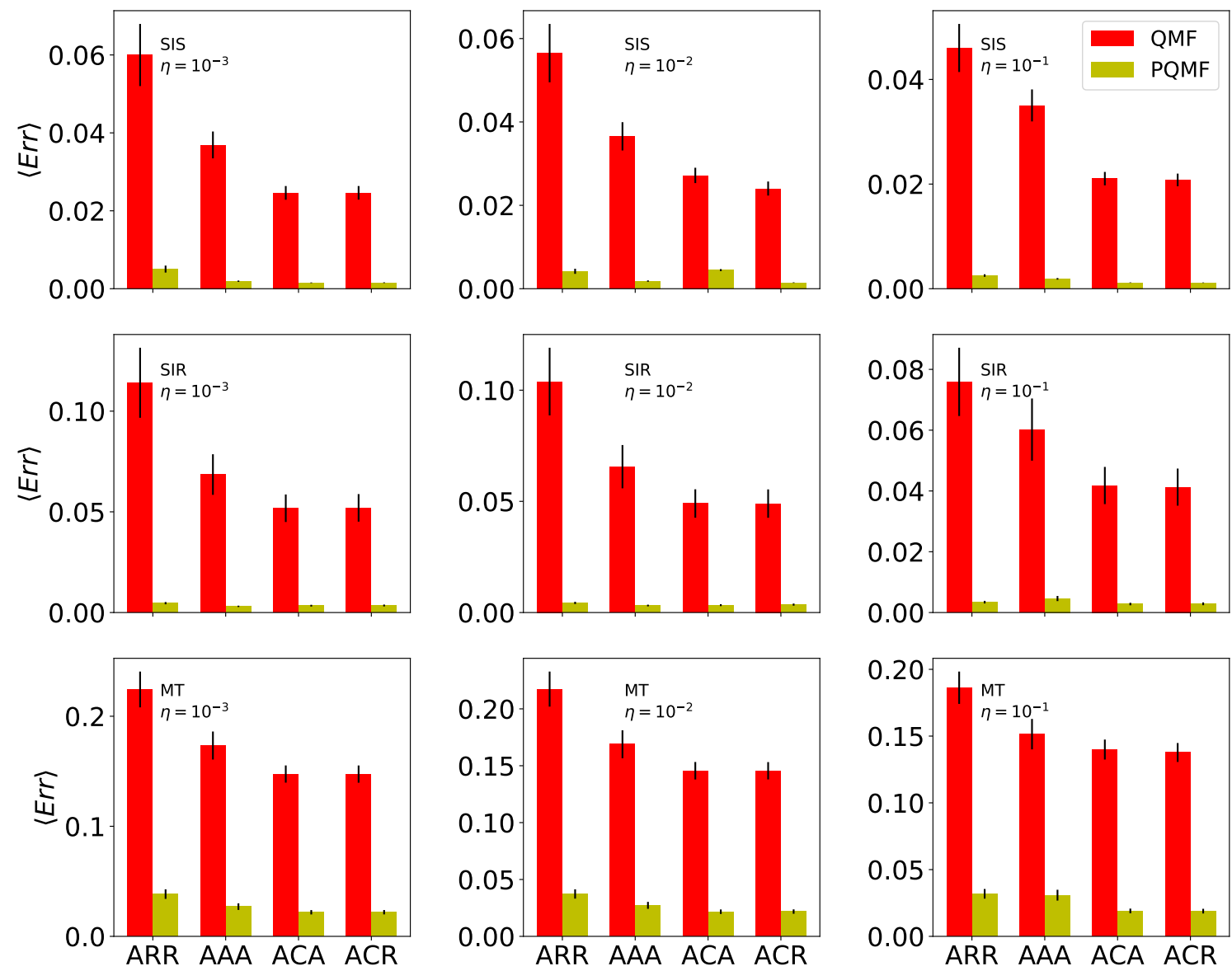

Source: Developed by the author.

correlations neglected by the first order approximation. Also similarly to the single layer case, here it is arguable that the QMF can be considered a good approximation in most cases. Obviously, it always depends on the application, but in general, the QMF can qualitatively predict the behavior of our dynamics. Moreover, it has the interesting advantage of being an upper bound for the average value. However, in the MT dynamics, it predicts the processes dynamics poorly, presenting a huge error. In this case, the most appropriate choice is the PQMF. Interestingly, we do not observe a huge difference while changing the coupling parameter $\eta$.

Regarding the structural correlations, we also observed that random regular and 
Figure 19 - Estimated errors in the bars and their standard errors in the deviations. From above to bottom, we present the error of the SIS with reinfections, SIS and SIR processes with respect to the CA approach in four different structures: ARR (Assortative+RRN - randomly coupled), AAA (Assortative+Assortative - Assortativelly coupled), ACA (Assortative+Clustered - Assortativelly coupled) and ACR (Assortative+Clustered - randomly coupled). From left to right, we change the coupling parameter $\eta=10^{-3}, \eta=10^{-2}, \eta=10^{-1}$. The networks have $n=10^{4}$ and $m=2$, see text for more details on their structural properties.
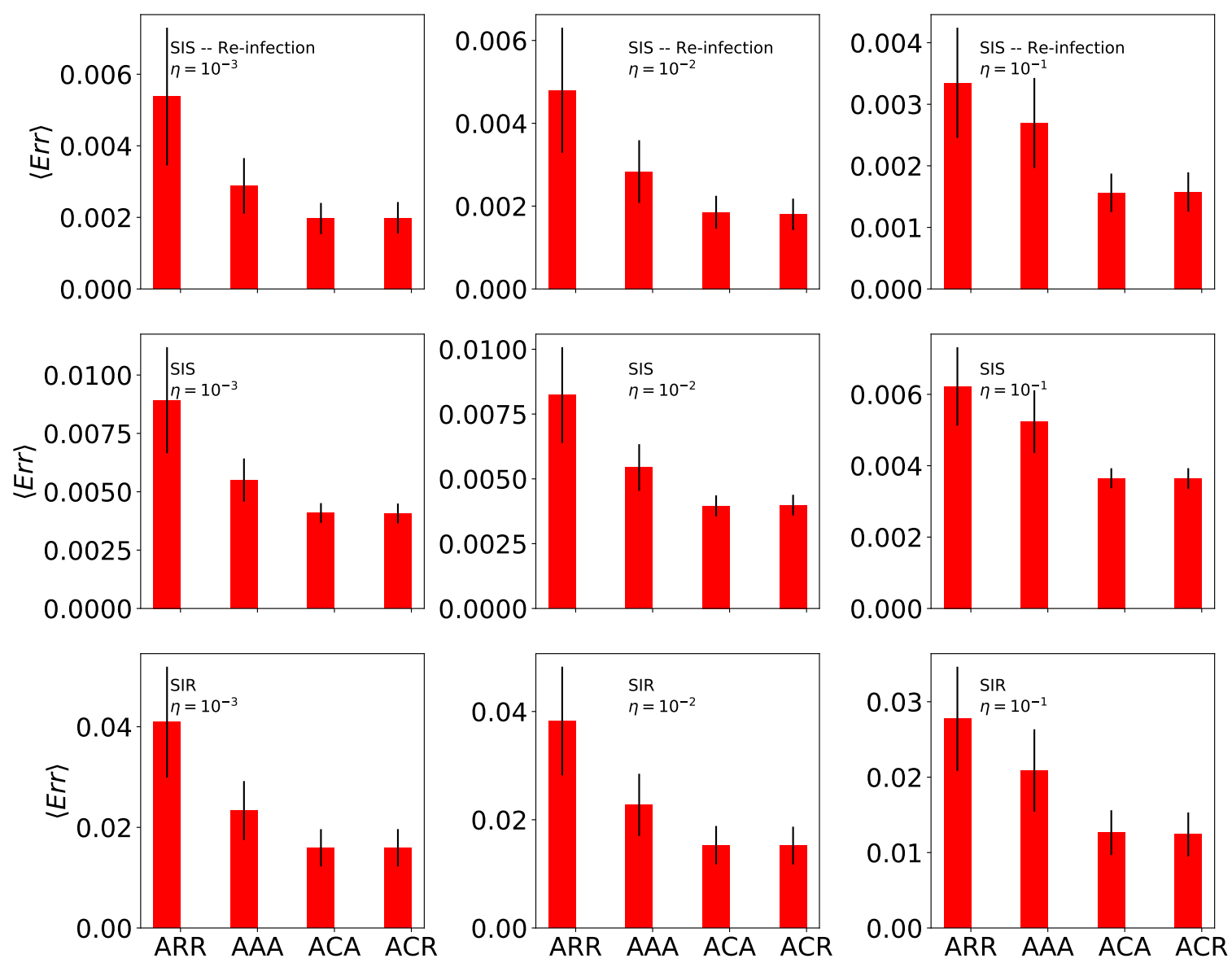

Source: Developed by the author.

assortative structures seem to pose the biggest challenge for the approximations. It is interesting to mention that regular networks (lattices) are still a big challenge in the predictions by means of mean field approaches due to their long shortest paths. Intuitively, the state of nodes in lattices present plenty of dynamical correlations, especially in sparse cases. Actually, it also tends to decrease in denser cases, as previously argued.

Contrasting with the previous experiments, now we are interested in the $\mathrm{CA}(\mathrm{RP})$ methods and, obviously, its own simulation scheme, as described in Section 3.7. Doing so, in Figure 19 we show the results for the comparison of Monte Carlo simulations and $\mathrm{CA}(\mathrm{RP})$ mean field approaches in terms of the errors. CA approaches presented a class 
Figure 20 - Finite size scaling for a power-law network with $\mathbb{P}(k) \sim k^{-2.25}$. In (a) the susceptibility as a function of the spreading rate $\lambda$ with $\delta=1$, while in (b) the scaling of the the susceptibility peak and its respective fraction of infected individuals, both obtained using the QS algorithm.

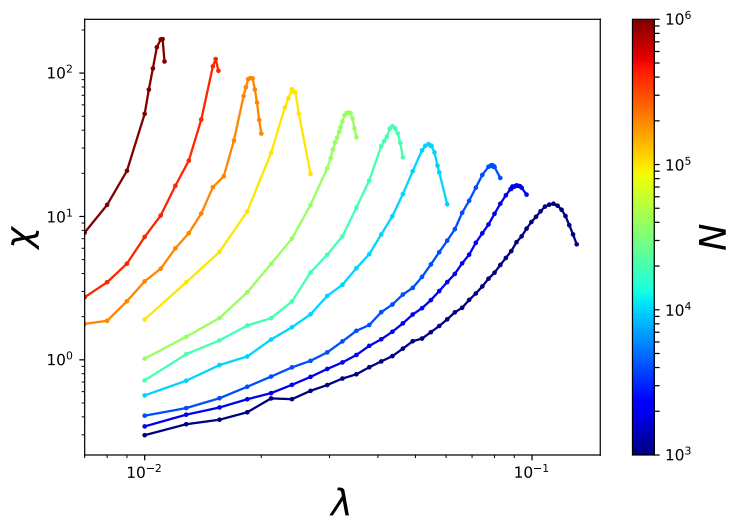

(a) Susceptibility

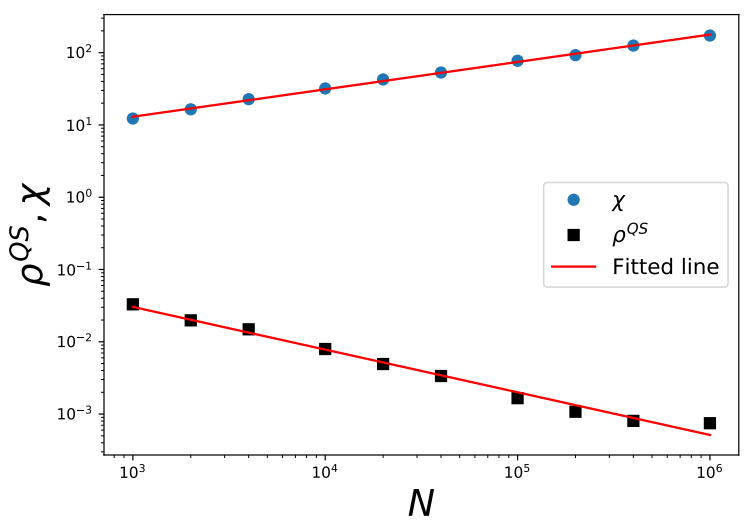

(b) Numerical Fitting

Source: Developed by the author.

of really precise models. The observed errors are remarkably small. In this case, the SIR models presented the largest error among all the CA approaches studied. Note that the CA follows a first order approximation and the error is comparable to the second order models on continuous time approaches. Here we just focused on the RP case, where the error is really small. However, is worth mentioning that the CP cases presented a poor result and it was not presented here since we present a generalized model in Chapter 4 and this error is evaluated in this specific context.

It is important to remark that in this section we are not inferring if one approach is better than the other. We are just systematically comparing them with Monte Carlo simulations, which are in turn also a computational model too. Modeling is a complex task and the nature of the problem and its restrictions (computational restrictions for instance) may help us to choose the proper model, which can be continuous, discrete, CA-like and so on.

\subsection{Finite size analysis}

In this section, we present an example of finite size analysis with the SIS dynamics on top of uncorrelated power-law single layer network. In order to estimate the susceptibility and the order parameter, we use the QS algorithm. On the simulations in this section we used the QS list with $M=25$ elements and $t_{r}$ from $10^{5}$ to $10^{4}$, while $t_{a}$ from $10^{7}$ to $10^{5}$ depending on the network size. An important consequence of the critical behavior of a dynamical system is its scaling as the system size grows. As discussed in (FERREIRA; 
Figure 21 - Finite size scaling for a power-law network with $\mathbb{P}(k) \sim k^{-2.75}$. In (a) the susceptibility as a function of the spreading rate $\lambda$ with $\delta=1$, while in (b) the scaling of the the susceptibility peak and its respective fraction of infected individuals, both obtained using the QS algorithm.

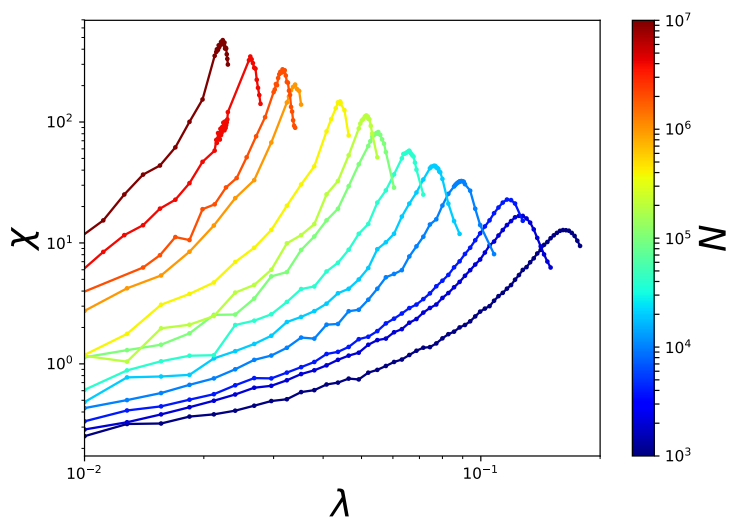

(a) Susceptibility

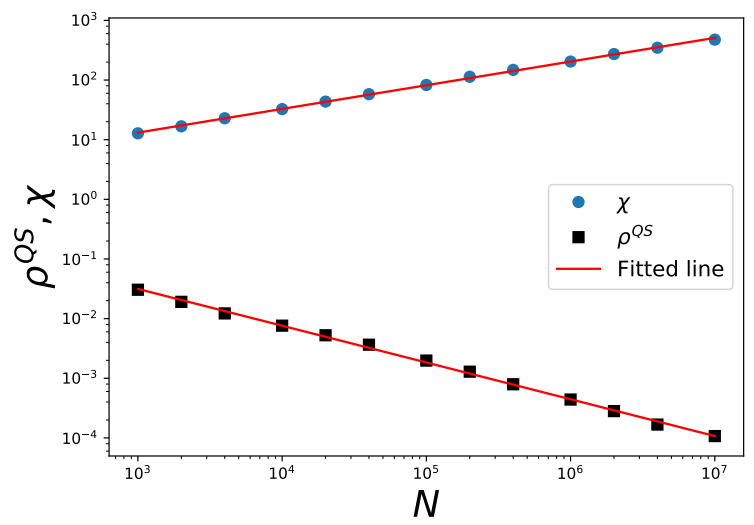

(b) Numerical Fitting

Source: Developed by the author.

Figure 22 - Finite size scaling for a power-law network with $\mathbb{P}(k) \sim k^{-3.5}$. In (a) the susceptibility as a function of the spreading rate $\lambda$ with $\delta=1$, while in (b) the scaling of the the susceptibility peak and its respective fraction of infected individuals, both obtained using the QS algorithm.

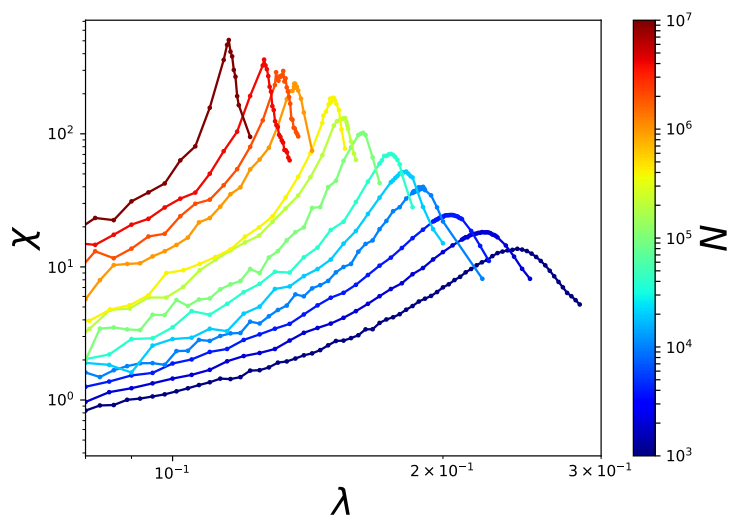

(a) Susceptibility

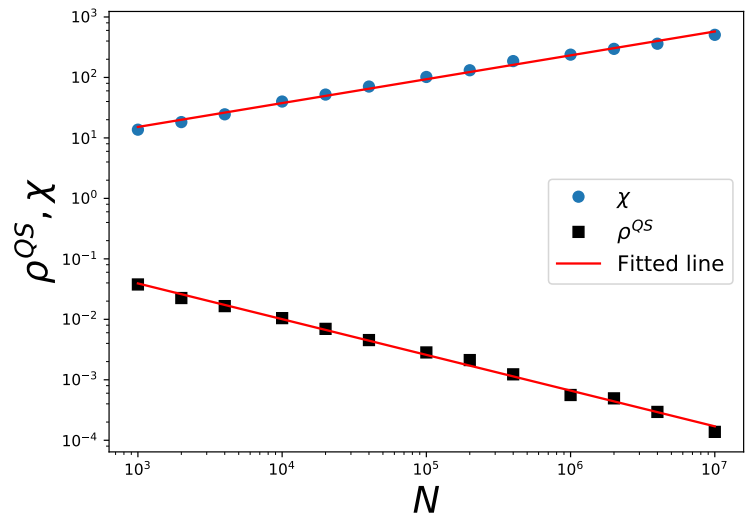

(b) Numerical Fitting

Source: Developed by the author.

CASTELLANO; PASTOR-SATORRAS, 2012), considering a system with finite critical point in the thermodynamic limit, it is expected that $\tau_{p}(N)-\tau_{c}(\infty) \sim N \frac{-1}{\bar{n} u}$, where $\tau_{p}(N)$ is the spreading ratio, which is obtained by the susceptibility peak and $\tau_{c}(\infty)$, which is the same point on the thermodynamic limit. Note that $\tau_{p}(N)$ is rounded by finite size effects. 
In this manner, at the critical point, we have that

$$
\rho^{Q S} \sim N^{\frac{\beta^{\prime}}{\bar{v}}}, \quad \chi \sim N^{\frac{\beta^{\prime}+\gamma^{\prime}}{\bar{v}}} .
$$

From the previously established equations, we can estimate the exponents $\frac{\beta^{\prime}}{\bar{v}}$ and $\frac{\beta^{\prime}+\gamma^{\prime}}{\bar{v}}$, simply estimating the susceptibility peaks for different system sizes and fitting a line in the log-log plots of the desired quantity versus $N$.

The finite size scaling experiments were performed in the same manner as (FERREIRA; CASTELLANO; PASTOR-SATORRAS, 2012), where the authors compare the theoretical critical point predictions with their respective numerical simulations. Similarly to (FERREIRA; CASTELLANO; PASTOR-SATORRAS, 2012), here we also study three different power-law degree distributions, $\mathbb{P}(k) \sim k^{-\zeta}$, for $\zeta=2.25, \zeta=2.75$ and $\zeta=3.5$. Note that those exponents were chosen due to the adjacency matrix spectral properties and its consequences on the critical point predictions, as shown in sections 2.2.1 and 3.5.3. In this manner, observe that for $2<\zeta<\frac{5}{2}$ the QMF and HMF predictions coincide, i.e. $\tau_{c}^{Q M F}=\tau_{c}^{H M F}=\frac{\mathbb{E}(k)}{\mathbb{E}\left(k^{2}\right)}$, while for $\frac{5}{2}<\zeta<3$ the QMF predictions scale with $\tau_{c}^{Q M F}=\frac{1}{\sqrt{k_{\max }}}$ (which implies that $\tau_{c}^{Q M F} \sim N^{-\frac{1}{4}}$ for the uncorrelated case, for more, see Section 2.1.4.4) and $\tau_{c}^{H M F}=\frac{\mathbb{E}(k)}{\mathbb{E}\left(k^{2}\right)}$. In this manner we are able to compare both predictions, reproducing the results obtained in (FERREIRA; CASTELLANO; PASTOR-SATORRAS, 2012). Finally, on the case $\zeta>3$ the second moment of the degree distribution is finite, yielding to a finite critical point prediction on the HMF theory. However, it contrasts with the QMF theory, which still predicts a vanishing critical point. Regarding the case $\zeta>3$, we might emphasize that multiple susceptibility peaks were initially observed in (FERREIRA; CASTELLANO; PASTOR-SATORRAS, 2012) and studied in details in (MATA; FERREIRA, 2015), where the authors relate such phenomena with localization properties of those networks. In (MATA; FERREIRA, 2015), the authors associated the role of outliers on the degree distribution with the multiple susceptibility peaks. Aside from that, they also proposed an hard upper cutoff on the degree distribution as $k_{\max }=k_{\min } N^{\frac{0.75}{\zeta-1}}$, which suppress the emergence of outliers (MATA; FERREIRA, 2015), also called rigid cutoff. Here we follow such model on our scaling analysis in order to avoid the complications of $\zeta>3$, but we refer the reader to (MATA; FERREIRA, 2015) for a deeper discussion on this type of structure.

Our experiments on power-law single layer networks are presented as follows:

- Power-law $2<\zeta<\frac{5}{2}$ : First of all, we consider uncorrelated networks with degree distribution of the form $\mathbb{P}(k) \sim k^{-2.25}$. Figure 20 shows on (a) the susceptibility curves, while on (b) the scaling as a function of the system size $N$. In this manner, by means of a simple linear fitting using the least squared method we obtained the 
following exponents

$$
\frac{\beta^{\prime}}{\bar{v}} \approx-0.619, \quad \frac{\beta^{\prime}+\gamma^{\prime}}{\bar{v}} \approx 0.378
$$

It is noteworthy that in (FERREIRA; CASTELLANO; PASTOR-SATORRAS, 2012) the authors estimated $\frac{\beta^{\prime}}{\bar{v}} \approx-0.65$ and $\frac{\beta^{\prime}+\gamma^{\prime}}{\bar{v}} \approx 0.37$, which are close to the obtained values, validating our analyses. Besides, in (FERREIRA; CASTELLANO; PASTORSATORRAS, 2012) the authors evaluated systems up to $N=10^{7}$, which may be one of the reasons of the small differences found.

- Power-law $\frac{5}{2}<\zeta<3$ : The next experiment regards uncorrelated networks with degree distribution of the form $\mathbb{P}(k) \sim k^{-2.75}$. Figure 21 shows on (a) the susceptibility curves, while on (b) the scaling as a function of the system size $N$. In this manner, we obtained the following exponents

$$
\frac{\beta^{\prime}}{\bar{v}} \approx-0.617, \quad \frac{\beta^{\prime}+\gamma^{\prime}}{\bar{v}} \approx 0.397 .
$$

Note that in (SANDER; COSTA; FERREIRA, 2016b) the authors estimated the exponent associated with $\rho^{Q S}$ for $\zeta=2.7$ and obtained $\frac{\beta^{\prime}}{\bar{v}} \approx 0.612$, which again is very close to our experiments, validating our analyses.

- Power-law $\zeta>3$ : Finally, we consider uncorrelated networks with degree distribution of the form $\mathbb{P}(k) \sim k^{-3.5}$, however adopting a hard cutoff as $k_{\max }=k_{\min } N^{\frac{0.75}{\zeta-1}}$. Figure 22 shows on (a) the susceptibility curves, while on (b) the scaling as a function of the system size $N$. In this manner, we obtained the following exponents

$$
\frac{\beta^{\prime}}{\bar{v}} \approx-0.591, \quad \frac{\beta^{\prime}+\gamma^{\prime}}{\bar{v}} \approx 0.395 .
$$

It is important to emphasize that systems with $\zeta>3$ present a very rich dynamical behavior and here we showed a really simple case, where the outliers of the degree distribution play a smaller role than the uncorrelated case, i.e. $k_{\max }=N^{\frac{1}{2}}$. As previously mentioned, such case was deeply studied in (MATA; FERREIRA, 2015).

Next, since we estimated the critical points for networks in those three different regimes, we might also compare them with the mean field predictions, similarly to (FERREIRA; CASTELLANO; PASTOR-SATORRAS, 2012; Mata, Angélica S.; Ferreira, Silvio C., 2013), but, aside from the QMF and HMF already considered in (FERREIRA; CASTELLANO; PASTOR-SATORRAS, 2012), we also evaluate the PQMF predictions, in a similar manner as (Mata, Angélica S.; Ferreira, Silvio C., 2013). Those results are shown in Figure 23, where we considered three different uncorrelated power-law networks with: $\zeta=2.25\left(k_{\max }=N^{\frac{1}{2}}\right)$ in $(\mathrm{a}), \zeta=2.75\left(k_{\max }=N^{\frac{1}{2}}\right)$ in (b) and $\zeta=3.5$ (rigid cutoff $k_{\max }=k_{\min } N^{\frac{0.75}{\zeta-1}}$, similarly to (MATA; FERREIRA, 2015)) in (c) and their susceptibility 
Figure 23 - Evaluation of the HMF, QMF and PQMF mean field predictions for the critical point considering different system sizes. The critical points were estimated using the QS algorithm on top of three different uncorrelated power-law networks with: $\zeta=2.25$ in (a), $\zeta=2.75$ in (b) and $\zeta=3.5$ (rigid cutoff) in (c).

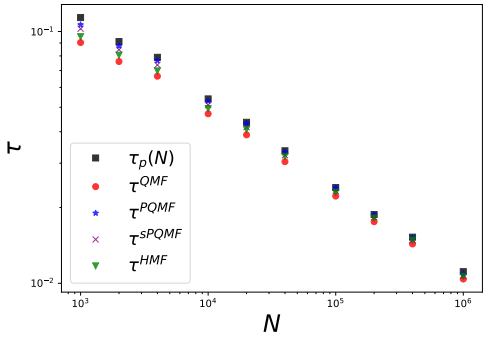

(a) $\zeta=2.25$

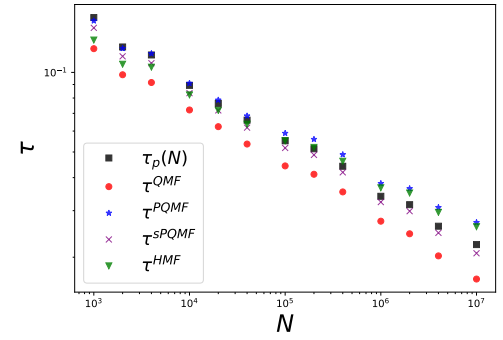

(b) $\zeta=2.75$

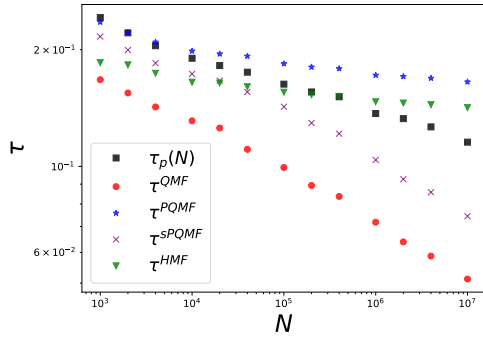

(c) $\zeta=3.5$ (rigid cutoff)

Source: Developed by the author.

plots are shown on Figures 20, 21 and 22 respectively. First of all, considering $\zeta=2.25$ our results agree with similar experiments performed in (FERREIRA; CASTELLANO; PASTOR-SATORRAS, 2012; Mata, Angélica S.; Ferreira, Silvio C., 2013) and all the predictions are apparently exact in the thermodynamic limit. Besides, note that the QMF and HMF present the same scale since both coincide. This was already pointed in (FERREIRA; CASTELLANO; PASTOR-SATORRAS, 2012) for the QMF and HMF, but here we extend such observation for the PQMF approach. Additionally, the most accurate predictions are the inverse of the leading eigenvalue of the non-backtracking matrix, followed by the standard PQMF, HMF, and QMF. Next, considering $\zeta=2.75$, a similar result was presented in (Mata, Angélica S.; Ferreira, Silvio C., 2013), where the authors validated sPQMF predictions. In this case, the QMF and the sPQMF predictions seem to scale correctly and the SPQMF present a very good approximation for the critical point in agreement with (Mata, Angélica S.; Ferreira, Silvio C., 2013). Interestingly, the nonbacktracking predictions seem to scale similarly to the HMF. Although it performs better in some cases, both seams to lead to wrong predictions on the large system limit. Finally, the last analyzed case is $\zeta=3.5$ with a rigid cutoff $k_{\max }=k_{\min } N^{\frac{0.75}{\zeta-1}}$, similarly to the analysis performed in (MATA; FERREIRA, 2015), where the authors were suppressing the effects of outliers on the degree distribution. Our results are similar to the ones obtained in (MATA; FERREIRA, 2015), where the authors observed that the critical point are also close to the second peak for uncorrelated models (with $k_{\max }=N^{\frac{1}{2}}$ ) and also close to the HMF predictions. These results are not shown here since it is out of the scope of this section. In this case, we observed that none of the predictions follows the correct trend, but the HMF and the non-backtracking approaches present a better result.

In summary, in this section, we evaluated different types of power-law networks aiming to exemplify the finite size analysis and how it can be useful in the analysis of 
phase transitions. Additionally, we also validated/verified previous results on the literature (FERREIRA; CASTELLANO; PASTOR-SATORRAS, 2012; Mata, Angélica S.; Ferreira, Silvio C., 2013; MATA; FERREIRA, 2015), where those experiments were previously performed. Finally, regarding the matricial analysis of the PQMF using the non-backtracking matrix (see Section 3.5.4) it is important to emphasize that despite its critical point predictions, an important feature of the analysis performed on Section 3.5.4 is the connection between the epidemic spreading process and the non-backtracking matrix, which was already observed in (RADICCHI; CASTELLANO, 2016).

\subsection{A brief comment on the nature of the critical point}

To round up this chapter we present a short discussion on the nature of the critical point and its relationship with state localization, hub activation and, reactivation. In this manner, we summarize some of the comments already presented in this chapter but focusing on the physical/phenomenological point of view. This section is mainly centered in the reference (BOGUÑÁ; CASTELLANO; PASTOR-SATORRAS, 2013), where the authors develop a very good discussion on the nature of the critical point.

In (GOLTSEV et al., 2012; BOGUÑÁ; CASTELLANO; PASTOR-SATORRAS, 2013) the authors analyzed the effects of eigenvector localization on the SIS steady state. They observed that on power-law networks with $\zeta<\frac{5}{2}$ the leading eigenvector is delocalized, implying that for $\tau>\tau_{c}^{Q M F}$ the disease is spread over the whole network. In other words, it implies that there is a finite density of infected individuals. On the other hand, for $\zeta>\frac{5}{2}$ this eigenvector is localized, implying that the system is active in a few set of nodes, comprising the hubs and their neighbors. This would lead to a number of infected nodes that scales sublinearly with the system size, thus it does not constitute a true active state (GOLTSEV et al., 2012; BOGUÑÁ; CASTELLANO; PASTOR-SATORRAS, 2013).

Next, following a similar view, in (LEE; SHIM; NOH, 2013), the authors (partially) considered dynamical correlation effects, restricting themselves to hubs and their neighbors. In this manner, the activity of a node is characterized by its lifetime, which is a function of the spreading rate and its degree, i.e. $\hat{\tau}(k, \lambda)$. When hubs are directly connected, the lifetime is sufficiently large to sustain the disease. In this scenario, above the critical point, $\tau_{c}^{Q M F}$, there is an endemic state configured by hubs mutual reinfection. On the other hand, if the hubs are not directly connected this reinfection mechanism is not possible. Therefore, in (LEE; SHIM; NOH, 2013), the authors suggest that above $\tau_{c}^{Q M F}$ we have a Griffiths phase (VOJTA, 2006). In this phase, the density of infected nodes decays slowly than exponentially, while the true phase transition is located at a higher value of $\tau$. Importantly, such analyses points to a $\tau_{c}>0$ in power-law networks with $\zeta>3$ 
and a vanishing critical point only for $\zeta<3$.

Furthermore, in (BOGUÑÁ; CASTELLANO; PASTOR-SATORRAS, 2013), the authors presented numerical and analytical evidence of a vanishing critical point for any small world network with degree distribution decaying slower than exponentially, which includes power-law networks with any $\zeta$. This result contrasts with the interpretations given in (GOLTSEV et al., 2012; LEE; SHIM; NOH, 2013), where a finite threshold is predicted for $\zeta>3$. Similarly to (LEE; SHIM; NOH, 2013), in (BOGUÑ́́; CASTELLANO; PASTOR-SATORRAS, 2013), the authors also consider the dynamical correlations, but they do not restrict themselves to hubs immediate neighbors. In fact, as pointed in (CHATTERJEE; DURRETT, 2009; BOGUÑÁ; CASTELLANO; PASTOR-SATORRAS, 2013) the direct connection is not a necessary condition to the hub reinfection mechanism. Moreover, the authors also presented arguments suggesting that perturbations can propagate up to distances of order $\ln (N)$. In summary, the main result of (BOGUÑÁ; CASTELLANO; PASTOR-SATORRAS, 2013) relies on a null epidemic threshold in the thermodynamic limit in all random small world network with degree distribution decaying slower than exponentially.

Finally, in (FERREIRA; SANDER; PASTOR-SATORRAS, 2016), the authors extend the ideas presented in (BOGUÑÁ; CASTELLANO; PASTOR-SATORRAS, 2013) proposing a criterion to evaluate the nature of the critical point. Their analysis is based on the comparison between hub refection and recovering time scales. Formally, if the lifespan is larger than the infection time the epidemic process is triggered by means of hub activation (hubs are reinfecting each other), leading to a vanishing threshold in the thermodynamic limit. Conversely, considering a scenario where the recovery time scale is smaller than the infection time scale the process is triggered by a collective process. In this manner, it yields to a standard phase transition. Additionally, in (FERREIRA; SANDER; PASTOR-SATORRAS, 2016), the authors also argue that in the process triggered by hub activation and reactivation the QMF theories are qualitative correct because it accounts for the whole structure. On the other hand, when the transition is triggered by a collective process, the HMF should be qualitatively correct since it works on an annealed version of the network.

\subsection{Summary}

In this chapter, we established the basic concepts of spreading processes in complex networks, focusing on the framework, allowing the reader to extend those ideas to more complex and intricate processes. Here we also made a especial effort to properly classify and distinguish among the various approaches found in the literature. We classified the spreading processes into two classes according to the time distinction: (i) continuous- 
time and (ii) cellular automata approach. Moreover, the CA approaches can be classified further in two classes according to their update scheme, which can be: (a) synchronous or (ii) asynchronous. Note that the cellular automata follow a discrete-time, however, it is interesting to observe that the sampled time Markov chain is also described in terms of discrete time, but is exact to the QMF if the time interval is small enough. This discussion was performed in Section 3.6. In this section, we also discussed some differences between these approaches.

Regarding the analytical aspects, for each process in this chapter, we started defining the local rules, then formally defining their correspondent Markov chain. Regarding the presented mathematical approaches, we used the exact SIS model to discuss some key points, such as finite size effects and the critical behavior. Obviously, the exact Markov chain is not practical due to its computational cost. In order to overcome this difficulty, we followed the mean field approaches. In increasing order of complexity, we followed the simplest MF, the HMF, QMF and also the PQMF, which we also related with nonbacktracking matrix and the message passing approach. It is noteworthy that, to the best of our knowledge, this was not found in the previous literature. For a complete table of all the derived equations for all the mean field approaches and dynamical processes discussed here, please see Appendix C.

On the other hand, considering the simulations, we also discussed the proper simulation algorithm for each case (continuous-time or cellular automata), contextualizing with previous literature results. In this part, we focused on the correct association between a given mathematical framework and its simulation algorithm. Going further, we also performed an accuracy analysis between the mean field approaches QMF and PQMF in relation to the Monte Carlo simulations previously discussed. To round up, we also discussed the QS algorithm, which is a powerful tool in the analysis of stochastic processes with a single absorbing state, which is precisely the case of SIS epidemics.

We also remark that the main aim of this chapter was not to perform an extensive literature review, which was recently done in (PASTOR-SATORRAS et al., 2015). The main objective here was to present those formalisms, clarifying the main ideas behind them and allowing the reader to deal with different specific situations.

Finally, we emphasize that this chapter is essential in terms of understanding of our work, as it defines the dynamical processes we are dealing with in different aspects: analytically, numerically and simulations. More specifically, in Chapter 4, Section 4.1 we use the synchronous cellular automata to model and unify rumor and epidemic spreading. Next, in Chapter 4, Section 4.2 we follow a the asynchronous cellular automata on the study of influential spreaders in complex networks. Finally, in Chapter 5 we analyze epidemic spreading processes on top of multilayer networks, in Section 5.1, characterizing the phenomena of layer localization, while in Section 5.2, we analyze the effects of assortative 
on spreading, as an application of our proposed generalization of the assortative measure for multilayer networks. 
CHAPTER

4

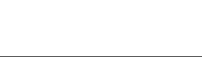

\section{NEW PERSPECTIVES ON MODELING SINGLE LAYER NETWORKS}

Spreading phenomena are ubiquitous in nature (HETHCOTE, 2000; COSTA et al., 2011; PASTOR-SATORRAS et al., 2015). Rumors and viruses spread from person to person, worms contaminate computers worldwide and innovations are diffused from place to place. The advent of new technology and modern means of transportation has led to radical changes in classical transmission channels, making in many cases, natural and manmade systems more prone to contagion processes. On the other hand, new tools have been developed to study such phenomena, for instance, by explicitly dealing with the topology and dynamics of so-called complex networks, which are nothing else but the backbone on top of which information and diseases propagate (BARRAT; BARTHLEMY; VESPIGNANI, 2008; NEWMAN, 2010).

Epidemic and rumor spreading processes are similar in many aspects. Indeed, the creation mechanism is the same as in classical models: with a given (spreading) rate/probability, a disease (rumor) is transmitted to any of the neighbors of an infected (spreader) individual. On the contrary, the annihilation mechanisms are completely different by the very nature of the processes being studied. In disease contagion, spreaders die out because they recover from the infection with a given probability. This is regardless of their neighbors' dynamical states and of any interaction. Rumor-like processes are fundamentally different: in traditional models, rumors decay as a result of the interactions between spreaders and other individuals that already know the rumor - no matter whether they are actually propagating it (spreaders) or have already stopped (stiflers). Despite the differences between these two contagion processes, they have been studied using the same methodological approaches, albeit independently (PASTOR-SATORRAS et al., 2015). Surprisingly, the phenomenological similarities between them have not been fully exploited and there is not a general framework that helps us study both processes under the same formulation. 
Admittedly, rumor-like dynamics on complex topologies have been studied only at the mean-field level, and approaches such as the discrete-time Markov chains are not available yet. Here, we fill the existing gap and propose a general information-spreading model that captures the dynamical behavior of epidemic and rumor spreading processes. This analysis is presented in Section 4.1.

Furthermore, focusing on processes with infinitely many absorbing states ${ }^{1}$, e.g. SIR, MT, among others, the identification of the most influential spreaders in networks is important to control and understand the spreading capabilities of the system, as well as to ensure efficient information diffusion such as in rumor-like dynamics. Recent works have suggested that identifying influential spreaders is not independent of the dynamics being studied (KITSAK et al., 2010; BORGE-HOLTHOEFER; MORENO, 2012). For instance, the key disease spreaders might not necessarily be so when it comes to analyzing social contagion or rumor propagation. Additionally, it has been shown that different metrics (degree, coreness, etc.) might identify different influential nodes even for the same dynamical processes with a diverse degree of accuracy. In Section 4.2, we investigate how nine centrality measures correlate with the disease and rumor spreading capabilities of the nodes in different synthetic and real-world (both spatial and non-spatial) networks.

\subsection{General Markov chain approach for disease and rumor spreading}

Here, we fill the existing gap and propose a general information-spreading model that captures the dynamical behavior of epidemic and rumor spreading processes. Our model includes, as particular cases, not only the traditional models of rumor and epidemic spreading, but also other mechanisms such as apathy (BORGE-HOLTHOEFER et al., 2012), forgetting (KAWACHI et al., 2008), loss of interest (NEKOVEE et al., 2007; KAWACHI et al., 2008) and a new mechanism proposed here that characterizes cases in which the interest in the rumor can be recovered once lost. We thoroughly study, both analytically and numerically, the dynamics of the model in random synthetic graphs, as well as in real social networks. Moreover, we analyze several plausible scenarios and obtain the corresponding critical spreading probabilities for the contact and reactive limiting cases, in addition, to analyzing the early time dynamics of the spreading process. We also perform extensive numerical simulations and show that the discrete Markov chain approach developed here is highly accurate, both at the micro and the macroscopic levels. Finally, we discuss potential applications of this framework in the context of social contagion and the design of new viral algorithms for efficient information dissemination.

1 Infinitely many absorbing states on the thermodynamic limit. 
Figure 24 - A simplified diagram of all possible transitions between the three different dynamical states in our model. $X, Y$ and $Z$ stand for ignorant or susceptible, spreader and stifler or recovered, respectively. Most of the transitions involve interactions between two individuals, however, spontaneous ones are also allowed (represented by dashed lines).

A

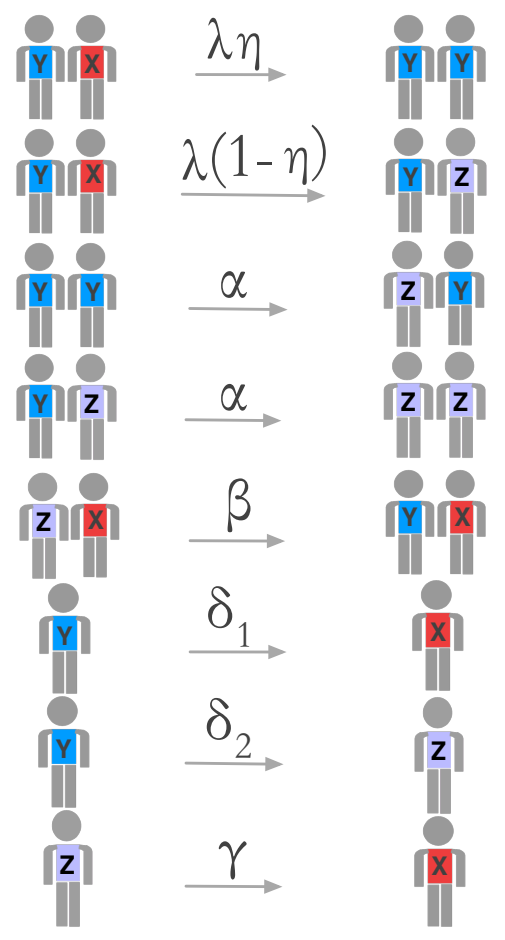

B
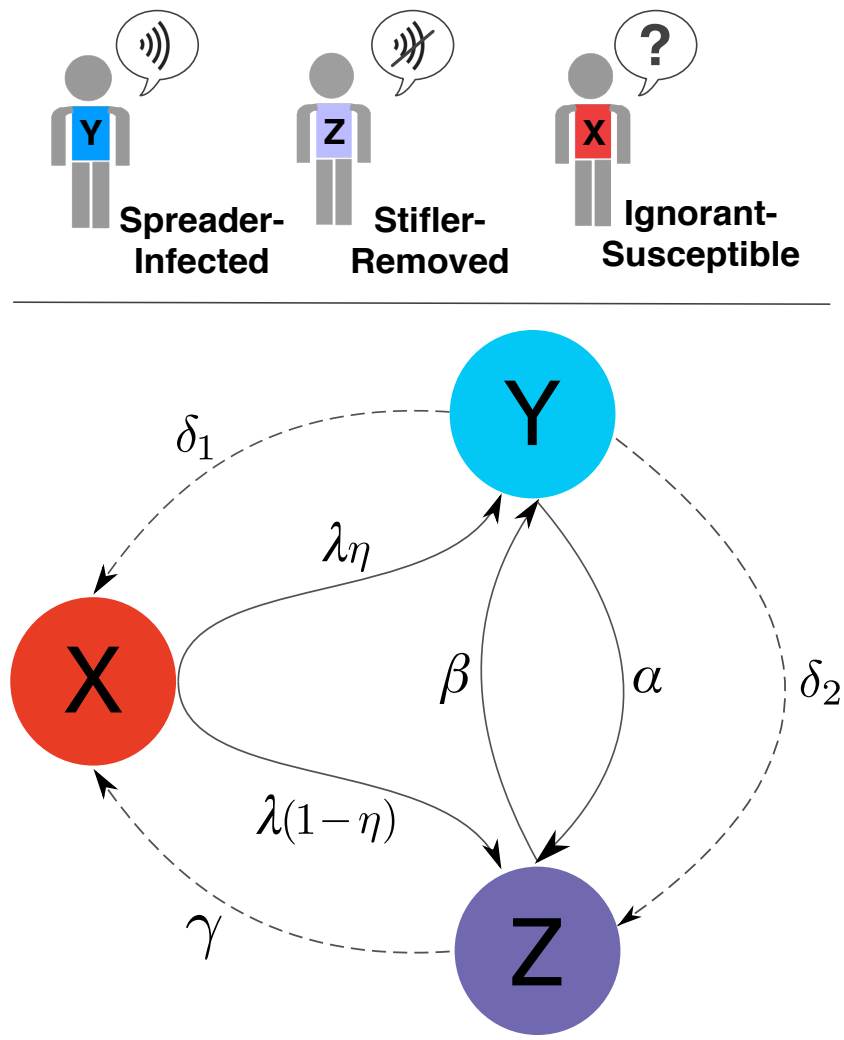

Source: Developed by the author.

\subsubsection{The general model}

Here we introduce a general model that captures all the features of the models described in Section 3.3. We consider a spreading dynamics in a population of $N$ individuals whose contact pattern is given by a network. The structure of the interaction graph is encoded by a network with adjacency matrix $\mathbf{A}$, as previously defined in Section 2.1.1. We consider undirected networks, i.e., $A_{i j}=A_{j i}$, for all $i, j$. The spreading process described by the model could refer to the transmission of a rumor, a disease or information that could be disseminated (also, a malware over a communication network, innovations, etc). In order to be clearer and precise, we will henceforth use terminology that mostly refers to information transmission, but we stress that depending on the transitions that one allows to take place, the dynamics could represent other spreading processes. Therefore, let's assume that a "piece of information" is being transmitted. An individual holding this piece of information and willing to spread it is called a spreader, whose state is represented by 
$Y$. On the other hand, a stifler, whose state is denoted by $Z$, is a subject who knows the information and does not spread it. An individual who is not aware of the information is called ignorant, and its state is denoted as $X$. Thus, notation $X, Y$ and $Z$, refers to subjects (or system elements such as devices) that have not participated in the spreading process, which are active, and that have taken part in the dynamics but are not active any longer, respectively. Hence, when we deal with an infectious disease, $X, Y$ and $Z$ are, respectively, the susceptible, infective and removed states.

The general model described here includes the transitions represented in Figure 24. Specifically, at each time step, the spreading dynamics proceeds as follows for any given node $i$ :

(i) An individual in state $Y$ changes to state $X$ with probability $\delta_{1}$. This, for instance, refers to the case in which in a rumor process, a spreader forgets the rumor (meaning the piece of news becomes old and therefore $Y$ is ignorant again). It also represents transitions of the type infected $\rightarrow$ susceptible in an SIS model.

(ii) An individual in state $Y$ changes to state $Z$ with probability $\delta_{2}$. For rumor processes, it represents the case in which a spreader spontaneously (that is, not as a result of interactions with other $Y$ or $Z$ individuals) loses interest in further propagating the rumor and becomes a stifler. It also accounts for transitions of the type infected $\rightarrow$ recovered in an SIR model.

(iii) If transitions (i) and (ii) do not happen, which occurs with probability $\left(1-\delta_{1}-\delta_{2}\right)$, then spreader $i$ interacts with its neighbors. The outcome of such interactions are:

(iii.1) If the individual contacted (the one at the other end of the edge) is in state $X$, then the latter turns into $Y$ with probability $\lambda \eta$. This transition is the classical susceptible $\rightarrow$ infected one in disease models for $\eta=1$. Traditional rumor models also include the very same transition (ignorant $\rightarrow$ spreader, also for $\eta=1$ ), but here, in order to be as general as possible, we also consider that an ignorant $X$ can directly go to stifler class $Z$ with probability $(1-\eta)$, thus the probability that a transition $X \rightarrow Z$ occurs is $\lambda(1-\eta)$. This mimics information dissemination in systems such as Twitter, in which reading the tweet does not imply that the user spreads it -as a matter of fact, the most common situation is that in which the user does not retweet the piece of news (BORGE-HOLTHOEFER et al., 2012).

(iii.2) On the contrary, if the neighbor of the spreader $i$ is in state $Y$ or $Z$, then $i$ turns into $Z$ with probability $\alpha$. Note that this spreader $\rightarrow$ stifler transition is only found in rumor models and it is not present in disease contagion. 
(iv) Finally, at variance with traditional rumor models (MAKI; THOMPSON, 1973; DALEY; KENDALL, 1964), we also ascribe an active role to stiflers $Z$. We assume that individuals in state $Z$ can go back to state $X$ with probability $\gamma$. This represents scenarios in which stiflers might spontaneously "forget" the rumor, thus becoming ignorants again. We also note that this transition can be identified with a recovered $\rightarrow$ susceptible one in SIRS disease models, in which it is assumed that after some time, individuals might lose their acquired immunity and become susceptible to catch the same disease again.

(v) If (iv) does not happen, which occurs with probability $(1-\gamma)$, then node $i$ in state $Z$ interacts with its neighbors. If the contact is with a subject in state $X$ (ignorant), the stifler (state $Z$ ) might recover interest in the rumor propagation and with probability $\beta$ turns into $Y$. This transition mimics cases in which an individual who knows the rumor but is not transmitting it, learns that the rumor is still active and new, and therefore starts spreading it again.

In summary, our model presents the following transitions:

$$
\begin{array}{rcc}
Y+X & \stackrel{\lambda \eta}{\rightarrow} & Y+Y, \\
Y+X & \stackrel{\lambda(1-\eta)}{\rightarrow} & Y+Z, \\
Y+Y & \stackrel{\alpha}{\rightarrow} & Z+Y, \\
Y+Z & \stackrel{\alpha}{\rightarrow} & Z+Z, \\
Z+X & \stackrel{\beta}{\rightarrow} & Y+X, \\
Y & \stackrel{\delta_{1}}{\rightarrow} & X, \\
Y & \stackrel{\delta_{2}}{\rightarrow} & Z, \\
Z & \stackrel{\gamma}{\rightarrow} & X,
\end{array}
$$

and our next goal will be to describe a process with such transitions through a suitable Markov chain approach.

\subsubsection{First order approximation}

In order to describe the evolution of this phenomenon in a given network made up by a set of nodes $[N]:=\{1,2, \ldots, N\}$, and adjacency matrix $\mathbf{A}$, we constructed a

discrete time Markov chain $\left(\xi_{t}\right)_{t \geq 0}$ with state space $\mathscr{S}=\{(1,0,0),(0,1,0),(0,0,1)\}^{[N]}$. More precisely, we define $\xi_{t}:=\left\{\left(X_{i}(t), Y_{i}(t), Z_{i}(t)\right): i \in[N]\right\}$, where $X_{i}(t), Y_{i}(t)$ and $Z_{i}(t)$ are Bernoulli random variables indicating whether the node $i \in[N]$ is an ignorant (susceptible), a spreader (infected) or a stifler (recovered) at time $t$, respectively. Therefore, $(1,0,0),(0,1,0)$ and $(0,0,1)$ represent the states $X, Y$ and $Z$, respectively. Each point 
$\xi \in \mathscr{S}$ is called configuration. To construct the Markov chain, we consider random objects defined in the same suitable probability space $(\Omega, \mathscr{F}, \mathbb{P})$, where $\Omega$ is the sample space, $\mathscr{F}$ is a $\sigma$-algebra of subsets of $\Omega$, i.e., the set of configurations, and $\mathbb{P}$ is a probability measure function. For every $i, j \in[N]$ we take the following independent collections, each of independent and identically distributed (i.i.d.) random variables

$\begin{array}{lcc}\text { Collection } & \text { Associated to } & \text { Influence a choice in } \\ \left\{U_{i}(t)\right\}_{t \geq 0} & Y & \text { state } Y \\ \left\{I_{i}^{\gamma}(t)\right\}_{t \geq 0} & Z & \text { state } Z \\ \left\{I_{i j}^{\lambda}(t)\right\}_{t \geq 0} & Y+X & \text { state } Y \\ \left\{I_{i}^{\alpha}(t)\right\}_{t \geq 0} & Y+Y \text { or } Y+Z & \text { state } Y \\ \left\{I_{i}^{\beta}(t)\right\}_{t \geq 0} & Z+X & \text { state } Z \\ \left\{I_{i}^{\eta}(t)\right\}_{t \geq 0} & Y+X & \text { state } X\end{array}$

where $U_{i}(1)$ is a random variable uniformly distributed in $(0,1)$, and $I_{i}^{\gamma}(1), I_{i j}^{\lambda}(1), I_{i}^{\alpha}(1), I_{i}^{\beta}(1)$ and $I_{i}^{\eta}(1)$ are Bernoulli random variables with parameter $\gamma, \lambda, \alpha, \beta$ and $\eta$, respectively. In addition, for each node $i$, we consider a sequence of i.i.d. random objets uniformly distributed on the neighbors of $i$, i.e., a sequence $U_{i}^{n b}(1), U_{i}^{n b}(2), \ldots$ such that $P\left(U_{i}^{n b}(1)=\right.$ $j)=1 / k_{i}$, for all $j \in[N]$ satisfying $A_{i j}=1$, where $k_{i}=\sum_{j \in[N]} A_{i j}$ is the number of neighbors of node $i$.

The main idea is to define a stochastic process that evolves according to the realization of the random variables defined above. For example, let's think of a rumor process. If at a fixed time $t$ node $i$ is in state $Y$, then it forgets the rumor if $U_{i}(t)<\delta_{1}$ (transition (i) above), it loses interest in the propagation whenever $\delta_{1} \leq U_{i}(t)<\delta_{1}+\delta_{2}$ (transition (ii) above), or contacts its neighbors when $U_{i}(t) \geq \delta_{1}+\delta_{2}$ (i.e., transition (iii) above). In the last situation, if node $i$ contacts node $j$, and $j$ is in state $X$, then the rumor is propagated from $i$ to $j$ if $I_{i j}^{\lambda}(t)=1$. On the other hand, if the contacted node $j$ is a spreader, then $i$ turns into a stifler, i.e., $I_{i}^{\alpha}(t)=1$. We can proceed in a similar way to represent the remaining transitions and interactions of the process. Given the above description, it is not difficult to see that the transitions of this stochastic process can be written, for each 
$i \in[N]$ and $t \geq 0$, as:

$$
\left\{\begin{aligned}
X_{i}(t+1)= & X_{i}(t) A_{i}(t)+Y_{i}(t) 1_{\left\{U_{i}^{t}<\delta_{1}\right\}}+Z_{i}(t) I_{i}^{\gamma}(t) \\
Y_{i}(t+1)= & X_{i}(t)\left(1-A_{i}(t)\right) I_{i}^{\eta}(t)+ \\
& Y_{i}(t) 1_{\left\{U_{i}^{t} \geq \delta_{1}+\delta_{2}\right\}} B_{i}(t)+ \\
& Z_{i}(t)\left(1-I_{i}^{\gamma}(t)\right)\left(1-C_{i}(t)\right), \\
Z_{i}(t+1)= & X_{i}(t)\left(1-A_{i}(t)\right)\left(1-I_{i}^{\eta}(t)\right)+ \\
& Y_{i}(t)\left(1_{\left\{U_{i}^{t} \geq \delta_{1}+\delta_{2}\right\}}\left(1-B_{i}(t)\right)+1_{\left\{\delta_{1} \leq U_{i}^{t}<\delta_{1}+\delta_{2}\right\}}\right)+ \\
& Z_{i}(t)\left(1-I_{i}^{\gamma}(t)\right) C_{i}(t),
\end{aligned}\right.
$$

where $1_{\{A\}}$ is the indicator function, which is equal to 1 if the condition $A$ is satisfied and 0 otherwise and $A_{i}(t), B_{i}(t)$ and $C_{i}(t)$ are Bernoulli random variables indicating that a node $i$, given the influence of its neighbors, will not be informed, will not become a stifler, or will not recover the interest in rumor propagation, from time $t$ to time $t+1$, respectively. Observe that these variables depend on the contacts between node $i$ and its neighbors.

Here we study two limiting cases, namely, the contact process (CP), in which each node performs only one contact per unit time; and the fully reactive process $(\mathrm{RP})$, in which each vertex contacts all its neighbors at each time step. The contact based variables for the $\mathrm{CP}$ are given by

$$
\left\{\begin{array}{l}
A_{i}(t)=\prod_{j=1}^{N}\left[1-I_{j i}^{\lambda}(t) 1_{\left\{U_{j}^{n b}(t)=i\right\}} 1_{\left\{U_{j}^{t} \geq \delta_{1}+\delta_{2}\right\}} Y_{j}(t)\right], \\
B_{i}(t)=1-\sum_{j=1}^{N}\left[I_{i}^{\alpha}(t) 1_{\left\{U_{i}^{n b}(t)=j\right\}}\left(Y_{j}(t)+Z_{j}(t)\right)\right], \\
\left.C_{i}(t)=1-\sum_{j=1}^{N}\left[I_{i}^{\beta}(t) 1_{\left\{U_{i}^{n b}(t)=j\right\}} X_{j}(t)\right)\right] .
\end{array}\right.
$$

On the other hand, the contact based variables for the RP are

$$
\left\{\begin{array}{l}
A_{i}(t)=\prod_{j=1}^{N}\left[1-I_{i}^{\lambda}(t) A_{j i} 1_{\left\{U_{j}^{t} \geq \delta_{1}+\delta_{2}\right\}} Y_{j}(t)\right], \\
B_{i}(t)=\prod_{j=1}^{N}\left[1-I_{i}^{\alpha}(t) A_{i j}\left(Y_{j}(t)+Z_{j}(t)\right)\right], \\
\left.C_{i}(t)=\prod_{j=1}^{N}\left[1-I_{i}^{\beta}(t) A_{i j} X_{j}(t)\right)\right] .
\end{array}\right.
$$

Therefore, the contact based variables of the node $i$, i.e., $A_{i}(t), B_{i}(t)$ and $C_{i}(t)$ in Equations (4.2), (4.3) and (4.4) are dependent on the parameters $\lambda, \alpha$ and $\beta$, the state of its neighbors and the process, i.e., whether it is a $\mathrm{CP}$ or a RP. The variables $B_{i}(t)$ and $C_{i}(t)$ describe the feedback from the contacts to the node. Note that in rumor dynamics, interactions can also change the state of the spreading node, returning an immediate feedback, 
in contrast to epidemic spreading models (PASTOR-SATORRAS et al., 2015) where the state of the spreader changes at a rate that is independent of the interaction network. These relations modeled by $B_{i}(t)$ and $C_{i}(t)$ are absent in the recent work by Stanoev et al. (STANOEV; DANIEL; LJUPCO, 2014), since their formalism does not allow for instantaneous feedback over node $i$. We also highlight that the CP and RP mimic different situations in, for instance, social networks. The CP models one-to-one communication, when the rumors spread in friendship networks, email networks or networks in which each individual interacts with only one contact at each time step. On the other hand, the $\mathrm{RP}$ is best suited for one-to-many information dissemination, as in Twitter-like networks, since when a user posts a tweet, all its followers receive this information.

Finally, we also point out that Equation (4.2) ensures the existence of a function $f$ such that

$$
\xi_{t+1}=f\left(\xi_{t}, \mathscr{I}_{t}\right)
$$

where

$$
\mathscr{I}_{t}:=\bigcup_{i=1}^{N}\left\{U_{i}(t), I_{i}^{\gamma}(t), I_{i j}^{\lambda}(t), I_{i}^{\alpha}(t), I_{i}^{\beta}(t), I_{i}^{\eta}(t), U_{i}^{n b}(t)\right\}
$$

is the collection of all the (independent) random variables at time $t$. This in turns implies that $\left(\xi_{t}\right)_{t \geq 0}$ is a discrete-time Markov chain.

\subsubsection{The mean-field approximation}

Although the above Markovian description of the model is exact, its analysis is rather complex. This is because, while the Markov chain $\left(\xi_{t}\right)_{t \geq 0}$ is defined from the realizations of independent random variables through (4.2), the state of each node as well as of its neighbors have dependencies, whose complexity might change according to the network. We solve this difficulty by considering a mean-field approximation for the Markovian description. We denote the probabilities that a node $i$ is in state $X, Y$ and $Z$ at time $t$ by $p_{i}^{X}(t), p_{i}^{Y}(t)$ and $p_{i}^{Z}(t)$, respectively, and note that

$$
\begin{aligned}
& p_{i}^{X}(t):=\mathbb{P}\left(X_{i}(t)=1\right)=\mathbb{E}\left(X_{i}(t)\right), \\
& p_{i}^{Y}(t):=\mathbb{P}\left(Y_{i}(t)=1\right)=\mathbb{E}\left(Y_{i}(t)\right), \\
& p_{i}^{Z}(t):=\mathbb{P}\left(Z_{i}(t)=1\right)=\mathbb{E}\left(Z_{i}(t)\right),
\end{aligned}
$$

where $\mathbb{E}$ denotes expected values. Therefore, the mean-field approximation is obtained by considering expected values in Equations (4.2), (4.3) and (4.4). We also assume that there are no dynamical correlations at first order. In other words, we assume that the expected values of variable pairs factorize. The resulting system of equations obtained for a node 
$i \in[N]$, for all $t \geq 0$, is given by

$$
\begin{cases}p_{i}^{X}(t+1)= & p_{i}^{X}(t) a_{i}(t)+p_{i}^{Y}(t) \delta_{1}+p_{i}^{Z}(t) \gamma \\ p_{i}^{Y}(t+1)= & p_{i}^{X}(t)\left(1-a_{i}(t)\right) \eta+ \\ & p_{i}^{Y}(t)\left(1-\delta_{1}-\delta_{2}\right) b_{i}(t)+ \\ & p_{i}^{Z}(t)(1-\gamma)\left(1-c_{i}(t)\right), \\ p_{i}^{Z}(t+1)= & p_{i}^{X}(t)\left(1-a_{i}(t)\right)(1-\eta)+ \\ & p_{i}^{Y}(t)\left(1-\delta_{1}-\delta_{2}\right)\left(1-b_{i}(t)\right)+p_{i}^{Y}(t) \delta_{2} \\ & p_{i}^{Z}(t)(1-\gamma) c_{i}(t),\end{cases}
$$

where $a_{i}(t), b_{i}(t)$ and $c_{i}(t)$ are the probabilities that a node $i$, given a contact with its neighbors, remains at state $X$ (e.g., it is not informed), does not change to state $Z$ from state $Y$ (that is, will not become a stifler), or remains at state $Z$ (that is, will not recover the interest in rumor propagation) in the time interval from $t$ to $t+1$, respectively (see also Figure24). These probabilities depend on the number of contacts per unit time. Thus, the contact based probabilities for the $\mathrm{CP}$ are given by

$$
\left\{\begin{array}{l}
a_{i}(t)=\prod_{j=1}^{N}\left[1-\lambda P_{i j}\left(1-\delta_{1}-\delta_{2}\right) p_{j}^{Y}(t)\right], \\
b_{i}(t)=1-\sum_{j=1}^{N}\left[\alpha P_{i j}\left(p_{j}^{Y}(t)+p_{j}^{Z}(t)\right)\right], \\
\left.c_{i}(t)=1-\sum_{j=1}^{N}\left[\beta P_{i j} p_{j}^{X}(t)\right)\right]
\end{array}\right.
$$

where $P_{i j}:=A_{i j} / k_{i}$. On the other hand, the contact based probabilities for the RP are

$$
\left\{\begin{array}{l}
a_{i}(t)=\prod_{j=1}^{N}\left[1-\lambda A_{j i}\left(1-\delta_{1}-\delta_{2}\right) p_{j}^{Y}(t)\right] \\
b_{i}(t)=\prod_{j=1}^{N}\left[1-\alpha A_{i j}\left(p_{j}^{Y}(t)+p_{j}^{Z}(t)\right)\right] \\
\left.c_{i}(t)=\prod_{j=1}^{N}\left[1-\beta A_{i j} p_{j}^{X}(t)\right)\right] .
\end{array}\right.
$$

The system of Equations (4.6) describes the micro-state evolution of the system, i.e., the evolution of the probabilities of each node to be in a given state. The macro-state variables can be defined as the average of the individual probabilities, namely,

$$
\rho^{\ell}=\frac{1}{N} \sum_{i=1}^{N} p_{i}^{\ell}(\infty)
$$

where $p_{i}^{\ell}(\infty)$ is the probability of node $i$ to be in state $\ell$, with $\ell \in\{X, Y, Z\}$, as $t \rightarrow \infty$. Note that the generalization for weighted and directed networks is straightforward. In weighted networks, it is necessary to consider the weight matrix whose rows must add up to one. 
Figure 25 - Phase diagram of the steady state $(t \rightarrow \infty)$ considering the complete infinity graph. The dynamical parameters have been set to $\delta_{1}=\delta_{2}=\gamma=0.1, \lambda=1, \beta=0.5$ and $\eta=1$.

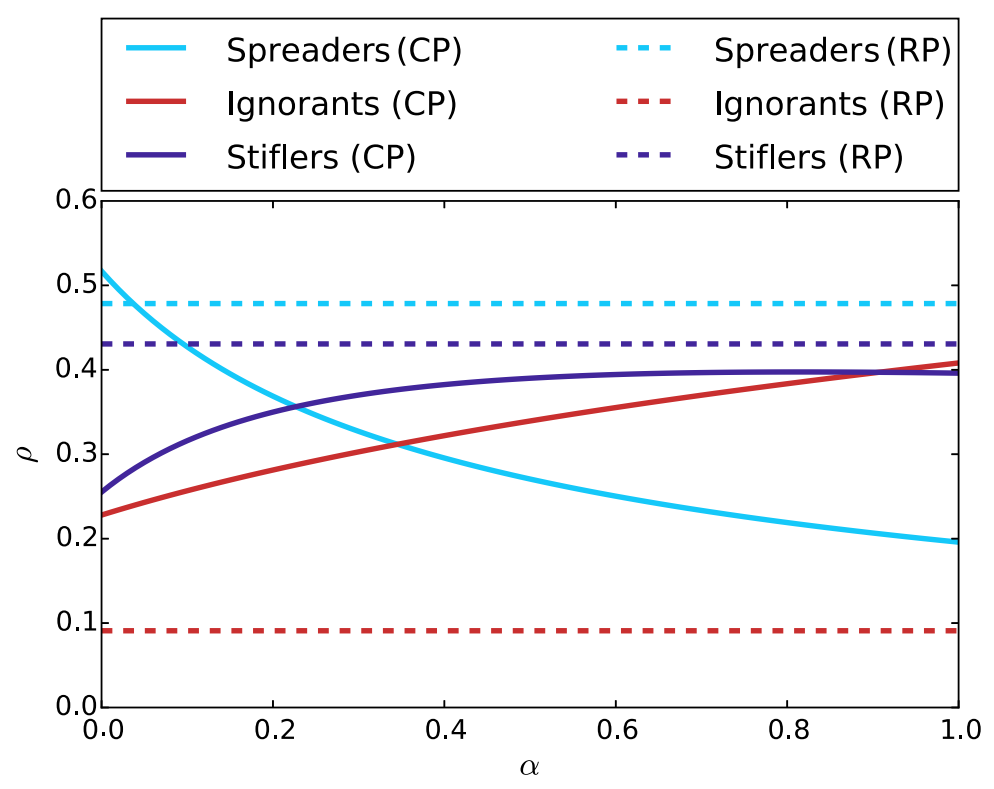

Source: Developed by the author.

In directed networks, the degree must be substituted by the out-degree, $k_{\text {out }}$. For the $\mathrm{CP}$ case, the directed network must be an ergodic Markov chain. Another possibility of generalization is the consideration of heterogeneous parameters, which is treated naturally in the set of Equations (4.6), (4.7) and (4.8). In the following analysis, we assume that the parameters have the same values for every node. In addition, we consider all networks as undirected and unweighted.

Furthermore, it is important to emphasize the differences between our model and the continuous formulation and consequently its embedded Markov chain, which is also a discrete time approach. In the continuous formulation, the probability of two events taking place at the same time vanishes as $\Delta t \rightarrow 0$. This implies that just one contact or one spontaneous process happens at a time. Along the same lines, the embedded Markov chain also captures this dynamics, however, the time in this approach is discrete. Finally, we also remark that in our approach every node performs its contacts in a time step -no matter whether a CP or an RP. Note that our formulation is similar to a cellular automaton as every node changes its probabilities based on their neighbor probabilities.

\subsubsection{Homogeneously mixed population}

For the sake of comparison and completeness, we next study our model on a homogeneously mixed population, i.e., we evaluate our model on a complete graph when the number of nodes goes to infinite. For a complete graph with $N$ nodes, and for all 
$i \in[N]$, we have that $A_{i j}=1$, for all $j \in[N] \backslash\{i\}$, which implies $P_{i j}=\frac{1}{N-1}$. We obtain for the CP, from the set of Equations (4.7), and by taking the thermodynamic limit $N \rightarrow \infty$, that the probability that a node does not perform a transition to another state after a pairwise interaction at time $t$ is given by

$$
\left\{\begin{array}{l}
a(t)=e^{\left[-\lambda\left(1-\delta_{1}-\delta_{2}\right) p^{Y}(t)\right]} \\
b(t)=1-\alpha\left[p^{Y}(t)+p^{Z}(t)\right] \\
c(t)=1-\beta p^{X}(t)
\end{array}\right.
$$

Note that we omit the index $i$ in our notation, as the probabilities are the same for every node. We observe that the probability $a(t)$ decays exponentially fast as a function of $p^{Y}(t)$, with a parameter $\tau^{-1}=\lambda\left(1-\delta_{1}-\delta_{2}\right)$. After including the probabilities (4.10) into Equation (4.6), we obtain the steady state behavior of this simplified model shown in Figure 25. As can be seen, the expected values for the densities of ignorant, spreaders and stiflers depend on $\alpha$, which is the tuning parameter in this scenario.

Interestingly enough, the behavior of the RP scenario is radically different. For the RP, after taking the limit $N \rightarrow \infty$ in Equation (4.8), we obtain $a(t)=0, b(t)=0$ and $c(t)=0$, as those probabilities are based on the product of infinite terms with absolute values less than unity. As a consequence, the system of Equations (4.6) for the RP becomes in the limit $N \rightarrow \infty$

$$
\left\{\begin{array}{l}
p^{X}(t+1)=p^{Y}(t) \delta_{1}+p^{Z}(t) \gamma \\
p^{Y}(t+1)=p^{X}(t) \eta+p^{Z}(t)(1-\gamma) \\
p^{Z}(t+1)=p^{X}(t)(1-\eta)+p^{Y}(t)\left(1-\delta_{1}\right)
\end{array}\right.
$$

The evolution of the fraction of spreaders, ignorants and stiflers are also shown in Figure 25. Note that these quantities are constant in $\alpha$ in sharp contrast to the results for the CP.

\subsubsection{Analytical Analysis}

In this section, we evaluate our model in terms of its early time behavior and perform the steady-state analysis. We derivate some closed expressions for the thresholds of the CP and RP cases in terms of the spectra of the probability transition and the adjacency matrices, respectively.

\subsubsection{Early time behavior}

Consider the first steps of the process. For a small time $\tilde{t}$ we may assume that $p_{i}^{Y}(t) \approx \varepsilon_{i}^{Y}$, and $p_{i}^{Z}(t) \approx \varepsilon_{i}^{Z}$, for any $t \leq \tilde{t}$, where $\varepsilon_{i}^{Y}$ and $\varepsilon_{i}^{Z}$ are constants such that $0 \leq \varepsilon_{i}^{Y} \ll$ 1 , and $0 \leq \varepsilon_{i}^{Z} \ll 1$. This in turns implies that $p_{i}^{X}(t)=1-p_{i}^{Y}(t)-p_{i}^{Z}(t) \approx 1-\varepsilon_{i}^{Y}-\varepsilon_{i}^{Z} \approx 1$. 
This approximation takes into account that the information spreading starts from at most a few spreaders. Neglecting second-order terms for the RP in Equation (4.8) we obtain, for $t \leq \tilde{t}$

$$
\left\{\begin{array}{l}
a_{i}(t) \approx 1-\sum_{j=1}^{N}\left[\lambda A_{j i}\left(1-\delta_{1}-\delta_{2}\right) p_{j}^{Y}(t)\right] \\
b_{i}(t) \approx 1-\sum_{j=1}^{N}\left[\alpha A_{i j}\left(p_{j}^{Y}(t)+p_{j}^{Z}(t)\right)\right] \\
\left.c_{i}(t) \approx 1-\sum_{j=1}^{N}\left(\beta A_{i j} p_{j}^{X}(t)\right)\right) .
\end{array}\right.
$$

Notice that

$$
c_{i}(t) \approx 1-\beta k_{i}
$$

since $p_{i}^{X}(t) \approx 1$, for $i=1,2, \ldots, N$. Substituting Equations (4.12) and (4.13) in the system of Equations (4.6), we obtain, for the equations of $p_{i}^{X}$ and $p_{i}^{Y}$,

$$
\left\{\begin{array}{l}
-\lambda\left(1-\delta_{1}-\delta_{2}\right) \sum_{j=1}^{N} A_{i j} \varepsilon_{j}^{Y}+\delta_{1} \varepsilon_{i}^{Y}+\gamma \varepsilon_{i}^{Z} \approx 0 \\
\eta \lambda\left(1-\delta_{1}-\delta_{2}\right) \sum_{j=1}^{N} A_{i j} \varepsilon_{j}^{Y}+\left(1-\delta_{1}-\delta_{2}\right) \varepsilon_{i}^{Y}+\beta k_{i}(1-\gamma) \varepsilon_{i}^{Z} \approx \varepsilon_{i}^{Y}
\end{array}\right.
$$

where $\varepsilon_{j}^{Y}+\varepsilon_{j}^{Z} \approx 0, \forall j=1,2, \ldots, N$. Isolating $\varepsilon_{i}^{Z}$ in the second equation, we get

$$
\varepsilon_{i}^{Z}=\frac{\left(\delta_{1}+\delta_{2}\right) \varepsilon_{i}^{Y}-\eta \lambda\left(1-\delta_{1}-\delta_{2}\right) \sum_{j=1}^{N} A_{i j} \varepsilon_{j}^{Y}}{\beta k_{i}(1-\gamma)},
$$

which describes $\varepsilon_{i}^{Z}$ as a combination of $\varepsilon_{j}^{Y}, \forall j=1,2, \ldots N$. Substituting Equation (4.15) in the first Equation of (4.14), we have

$$
\left(-1-\frac{\eta \gamma}{\beta k_{i}(1-\gamma)}\right) \lambda\left(1-\delta_{1}-\delta_{2}\right) \sum_{j=1}^{N} A_{i j} \varepsilon_{j}^{Y}+\delta_{1} \varepsilon_{i}^{Y}+\gamma \frac{\varepsilon_{i}^{Y}\left(\delta_{1}+\delta_{2}\right)}{\beta k_{i}(1-\gamma)} \approx 0
$$

whose factorization is

$$
\sum_{j=1}^{N}\left[A_{i j}-\delta_{i j}\left(\frac{\gamma\left(\delta_{1}+\delta_{2}\right)+\delta_{1}(1-\gamma) \beta k_{i}}{\lambda\left(1-\delta_{1}-\delta_{2}\right)\left((1-\gamma) \beta k_{i}+\gamma \eta\right)}\right)\right] \varepsilon_{i}^{Y} \approx 0 .
$$

Notice that Equation (4.17) does not decouple the structure and the dynamics of the system, as it is not possible to isolate the terms depending on $k_{i}$. However, it is possible to evaluate the threshold for $\beta(1-\gamma) \approx 0$. In this case, the system has nontrivial solutions when $\left(\frac{\delta_{1}+\delta_{2}}{\eta \lambda\left(1-\delta_{1}-\delta_{2}\right)}\right)$ is an eigenvalue of $A$. Thus, the critical value, which depends on the largest eigenvalue of the adjacency matrix $\mathbf{A}$, is given by

$$
\Lambda_{\max }^{A} \approx\left(\frac{\delta_{1}+\delta_{2}}{\eta \lambda\left(1-\delta_{1}-\delta_{2}\right)}\right)_{c}
$$

where $(\cdot)_{c}$ denotes the critical point. Considering the critical point as a function of $\lambda$, i.e., assuming that the other dynamical parameters are fixed, the threshold vanishes in the thermodynamic limit for scale-free networks with a divergent second moment, similarly to epidemic spreading (PASTOR-SATORRAS; VESPIGNANI, 2001a). 
Figure 26 - Numerical evaluation of equation (4.6) considering the RP for $\beta=0, \delta_{1}=\gamma=0.25$, $\delta_{2}=0.2$ and varying the spreading probability $\lambda$. The network considered is a scalefree network with $\zeta \approx 2.7, N=10^{4}$ and $\mathbb{E}(k) \approx 10$. The dashed line indicates the critical point (Equation $((4.18)))$ for $\eta=1$, whereas the continuous line corresponds to the critical value for the case in which $\eta=0.5$.

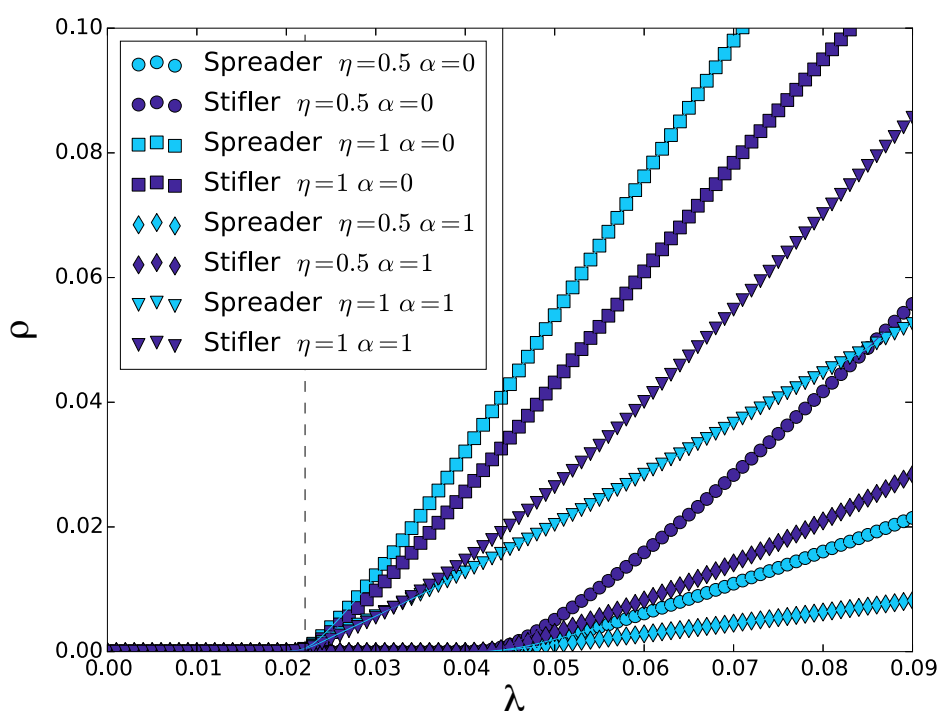

Source: Developed by the author.

The numerical evaluation of Equation (4.18) is shown in Figure 26. As it can be seen, there is a good agreement between the theoretical predictions (Equation (4.18)) and the numerical solution of the system (4.6). The calculation was performed for a scale-free network with $\zeta \approx 2.7, N=10^{4}$ and $\mathbb{E}(k) \approx 10$. As initial conditions, we considered that each node is set as a spreader with uniform probability $Y_{i}(0)=0.01, \forall i \in\{1,2, \ldots N\}$. The values of $\lambda$ in the figure are close to zero, as $\lambda$ depends on the inverse of the leading eigenvalue. We can also see that the limiting cases for $\alpha$ do not change the critical point. Furthermore, the density of nodes that holds the rumor, i.e., of spreaders and stiflers, is higher for $\alpha=0$ and $\eta=1$, while the lowest density is obtained for $\alpha=1$ and $\eta=0.5$. This observation reinforces the role of stifling rates, similarly to what happens in classical rumor models.

The approximation of the critical point in the RP is restricted by $\beta(1-\gamma) \approx 0$, as there is an explicit dependence on the degree $k_{i}$. However, for the CP the critical point can be evaluated without any constraint. The contact based probability in Equation (4.7) can be obtained using the same set of equations as in the RP (Equation (4.12)). Notice that only the first equation, i.e., that for $a_{i}(t)$, is an approximation, whereas the equations for $b_{i}(t)$ and $c_{i}(t)$ are exact. Following the same approach as for the RP, the expression for the critical point for the $\mathrm{CP}$ is similar to that in Equation (4.17) - the only two changes are (i) the use of the matrix $\mathbf{P}$ instead of $\mathbf{A}$ and (ii) $\sum_{j} P_{i j}=1, \forall j=1,2, \ldots N$. Another 
Figure 27 - Numerical evaluation of Equation (4.6) considering the CP with parameters $\delta_{1}=$ $\gamma=0.15, \delta_{2}=0.1$ and varying the spreading probability $\lambda$. The network considered is a scale-free network with $\zeta \approx 2.7, N=10^{4}$ and $\mathbb{E}(k) \approx 100$. In panel (a) $\eta=0.5$ and $\alpha=1$, whereas in (b) we have set $\eta=1$ and also explored the limiting cases of $\alpha=0$ and $\alpha=1$. The vertical lines correspond to the critical point Equation(4.18) evaluated for the parameters used here.
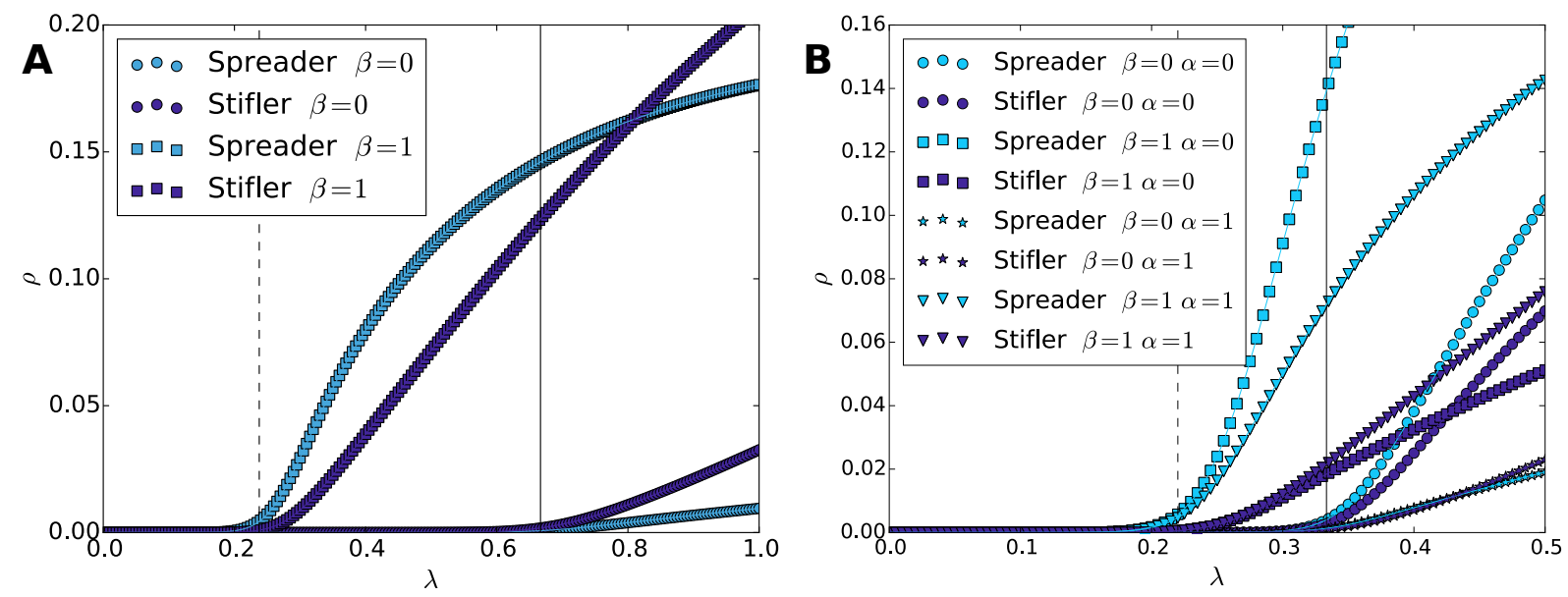

Source: Developed by the author.

important result is that the leading eigenvalue of the transition probability matrix is always equal to unity (MIEGHEM, 2011). Thus, the critical point for the CP is given by

$$
\Lambda_{\max }^{P}=1 \approx\left(\frac{\gamma\left(\delta_{1}+\delta_{2}\right)+\delta_{1}(1-\gamma) \beta}{\lambda\left(1-\delta_{1}-\delta_{2}\right)((1-\gamma) \beta+\gamma \eta)}\right)_{c} .
$$

Note that this expression does not depend on the network structure, as the leading eigenvalue $\Lambda_{\max }^{P}$ is the same for every connected network.

The numerical evaluation of the expression (4.19) is shown in Figure 27 considering a scale-free network with $\zeta \approx 2.7, N=10^{4}$ and $\mathbb{E}(k) \approx 100$. In (a) we consider $\eta=0.5$ and $\alpha=1$, while in (b) we assume $\eta=1$. Similarly to the RP case, here we also observe a very good agreement between the theoretical results and the numerical evaluation of the system (4.6) for all parameter values explored. Thus, the conclusions are similar to the $\mathrm{RP}$ scenario, the only difference being the average degree of the network, a dependency that we shall analyze in more detail later on.

The parameters of the model (with the exception of $\alpha$ that does not affect first order terms) can be used as control parameters of the system's dynamics. In particular, it is interesting to analyze the effect of $\beta$, which has been introduced here to account for the possibility of recovering the interest in the rumor. Isolating $\beta$ in Equation (4.19), we obtain

$$
\beta_{c} \approx \frac{\gamma\left(\delta_{1}+\delta_{2}-\eta \lambda\left(1-\delta_{1}-\delta_{2}\right)\right)}{\lambda\left(1-\delta_{1}-\delta_{2}\right)-\delta_{1}(1-\gamma)}
$$

which defines the minimum value of $\beta$ that allows for the spreading of the rumor to a 
Figure 28 - Numerical evaluation of Equation (4.6) considering the CP and, without loss of generality, fixing the parameters $\delta_{1}=\gamma=\delta_{2}=0.2, \lambda=1$ and varying the parameter $\beta$. The network considered is a scale-free network with $\zeta \approx 2.7, N=10^{4}$ and $\mathbb{E}(k) \approx$ 100.

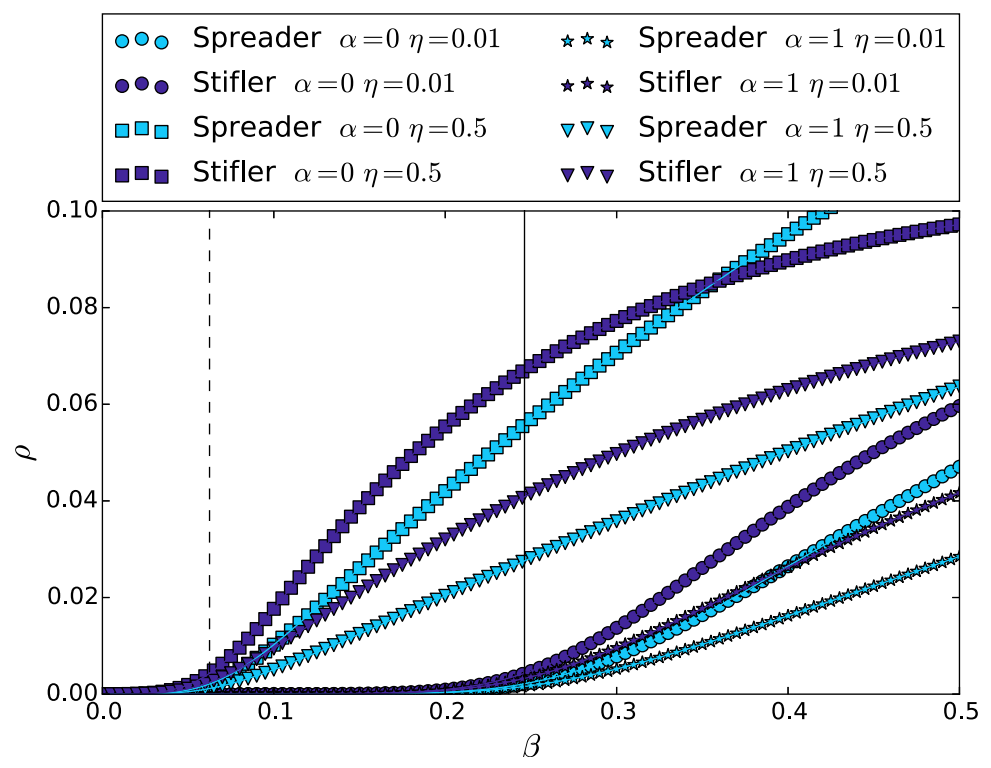

Source: Developed by the author.

macroscopic fraction of the population for a given spreading rate $\lambda$-as is the case in most applications. Figure 28 shows the evaluation of equation (4.6) for the CP near the critical point considering a scale-free network with $\zeta \approx 2.7, N=10^{4}$ and $\mathbb{E}(k) \approx 100$, and, without loss of generality, fixing the parameters $\delta_{1}=\gamma=\delta_{2}=0.2, \lambda=1$ and varying the parameter $\beta$. Similarly to the results shown in Figures 26 and 27, our approximations agree with the simulated values.

As for the other important parameter, $\alpha$, that defines at which rate spreaders decay into stiflers after $Y-Y$ or $Y-Z$ interactions, we stress that any approximation neglecting second-order terms does not involve parameter $\alpha$, as it controls the stifling rate in the expression for $b_{i}(t)$. In other words, $b_{i}$ is a conditional probability, and it is multiplied by the probability of an individual being a spreader. Thus, $\alpha$ does not affect the thresholds for the RP, since it always appears in second-order terms (see Fig 26). The same occurs for the CP, as shown in Figure 27. Note that while the macroscopic behavior of the system (the expected final densities of the different classes) is different for each parameter analyzed, the threshold is always the same. An equivalent analysis was done in (NEKOVEE et al., 2007), where the authors proposed a model with a rate similar to $\delta_{2}$ (in our model) and showed the independence of the threshold in parameter $\alpha$. 


\subsubsection{Steady state analysis}

In the previous section, we analyzed the early time evolution of the system aiming at finding its critical point. In this section, for the sake of completeness, we perform a similar analysis for the steady state solution. An analogous approach was used in (GÓMEZ et al., 2010), but for the specific case of an SIS model. At the steady state, we can assume, for $t$ large enough, $p_{i}^{I}(t) \approx \pi_{i}^{I}, p_{i}^{Y}(t) \approx \pi_{i}^{Y}$ and $p_{i}^{R}(t) \approx \pi_{i}^{R}$, for $i=1,2, \ldots, N$. Neglecting second-order terms, inserting (4.12) into the system (4.6) and after some algebra, the steady-state solution is

$$
\left\{\begin{array}{l}
\pi_{i}^{I}=\frac{\left(\delta_{1}+\delta_{2} \gamma+\delta_{1}(-1+\gamma) c_{i}\right)}{\left(\delta_{2}\left(1+\gamma-a_{i}\right)+\delta_{1}\left(2+\eta\left(-1+a_{i}\right)-a_{i}+(-1+\gamma) c_{i}\right)-\left(-1+a_{i}\right)\left(1-c_{i}+\gamma\left(-1+\eta+c_{i}\right)\right)\right)} \\
\pi_{i}^{Y}=\frac{\left(-1+a_{i}\right)\left(1-c_{i}+\gamma\left(-1+\eta+c_{i}\right)\right)}{\left(\delta_{2}\left(-1-\gamma+a_{i}\right)+\delta_{1}\left(-2+\eta+a_{i}-\eta a_{i}+c_{i}-\gamma c_{i}\right)+\left(-1+a_{i}\right)\left(1-c_{i}+\gamma\left(-1+\eta+c_{i}\right)\right)\right)} \\
\pi_{i}^{R}=\frac{-\left(\left(\left(\delta_{1}+\delta_{2}-\delta_{1} \eta\right)\left(-1+a_{i}\right)\right)\right.}{\left.\left(\delta_{2}\left(1+\gamma-a_{i}\right)+\delta_{1}\left(2+\eta\left(-1+a_{i}\right)-a_{i}+(-1+\gamma) c_{i}\right)-\left(-1+a_{i}\right)\left(1-c_{i}+\gamma\left(-1+\eta+c_{i}\right)\right)\right)\right)} .
\end{array}\right.
$$

where the time dependence no longer appears.

Considering that $Y_{i}=\varepsilon_{i}$, where $0 \leq \varepsilon_{i} \ll 1$, and after substitution in the second equation of the system (4.21), we obtain

$$
\varepsilon_{i}\left(\delta_{2} \gamma+\delta_{1}\left(c_{i}-\gamma c_{i}-1\right)\right)=\left(a_{i}-1\right)\left(1-c_{i}+\gamma\left(\eta+c_{i}-1\right)\right),
$$

Taking into account the approximation for $a_{i}(t)$ in Equation (4.12), we have

$$
\varepsilon_{i}\left(\delta_{2} \gamma+\delta_{1}\left(k_{i} \beta(\gamma-1)-\gamma\right)\right)=-\lambda\left(1-\delta_{1}-\delta_{2}\right)\left((1-\gamma) k_{i} \beta+\gamma \eta\right) \sum_{j=1}^{N} A_{i j} \varepsilon_{j}
$$

which is the same Equation as (4.17). This result is for the RP process. For the CP case, we only need to change the adjacency matrix $\mathbf{A}$ by the transition probability matrix $\mathbf{P}$. It is also worth highlighting that for an arbitrary choice of model parameters, in general, and at variance with classical rumor models, there is no absorbing state corresponding to the absence of spreaders. Regarding the parameter configurations where we have infinitely many absorbing states in the thermodynamic limit, we mention the classical SIR and Maki - Thompson models. Besides, observe that the SI model also has an absorbing state, which is the configuration in which every node is infected.

\subsubsection{Special cases}

The model proposed includes new transitions, formulates rumor dynamics in terms of discrete Markov chains and generalizes several previous spreading models (see Section 3.3). It is instructive to show how to obtain some of the main epidemic and rumor models from our approach, which we will do next. 


\subsubsection{Disease spreading}

We can obtain a particular case of the SIS model by setting $\eta=1, \delta_{2}=\gamma=0$, $\beta=0$ and $\alpha=0$ in our model (see Figure 24). This model is not exactly an SIS model, as we assume that each node cannot spread the disease and become susceptible at the same time step. This modification implies that the parameters used in the traditional models change. However, in both cases the SIS dynamics can be studied in terms of the final fraction of spreaders. From Equation (4.18), the epidemic threshold is given by

$$
\Lambda_{\max } \approx\left(\frac{\delta_{1}}{\lambda\left(1-\delta_{1}\right)}\right)_{c} .
$$

Comparing with the results in Table 2, the recovery probability in this SIS model is thus given by $\delta=\frac{\delta_{1}}{\left(1-\delta_{1}\right)}$, while the spreading probability is $\lambda$.

In (GÓMEZ et al., 2010), the authors used a discrete time Markov chain approach to model the SIS dynamics considering a reinfection term and a parameter that allows us to explore a family of contact-based scenarios, including the $\mathrm{CP}$ and the fully $\mathrm{RP}$ as limiting cases. Concerning the reinfection, our model does not include such a feature, however, we note that reinfection within the same time step is rarely taken into account in SIS like models. On the other hand, the second ingredient can be easily incorporated into our framework by exchanging matrix $\mathbf{A}$ by the following matrix

$$
R_{i j}=1-\left(1-\frac{A_{i j}}{k_{i}}\right)^{\kappa_{i}}
$$

where $\kappa_{i}$ is the activity parameter. A similar case was exposed in Section 3.5.5.1 and Equation 3.76. Observe that the limiting cases are obtained setting $\kappa_{i}=1, \forall i=1,2, \ldots, N$ for the CP, and $\kappa_{i} \rightarrow \infty, \forall i=1,2, \ldots, N$ for the fully RP.

The SIR model is obtained from our model by setting $\eta=1, \delta_{1}=\gamma=0, \beta=0$

and $\alpha=0$. In this case, the recovery rate is $\delta=\frac{\delta_{2}}{\left(1-\delta_{2}\right)}$. Additionally, our framework also includes the SIRS scenario as advanced before. By considering the model introduced in (CHEN; SUN, 2014) (see also Table 2) the SIRS scheme is recovered by setting $\eta=1$, $\delta_{1}=0, \beta=0$ and $\alpha=0$. The system can then be written as a function of $\lambda, \delta_{2}$ and $\gamma$. From Equation (4.18), this reduction implies that the threshold is given by

$$
\Lambda_{\max } \approx\left(\frac{\delta_{2}}{\lambda\left(1-\delta_{2}\right)}\right)_{c}
$$

which depends only on $\boldsymbol{\delta}_{2}$ and $\boldsymbol{\lambda}$.

\subsubsection{Rumor spreading}

Besides the Daley - Kendall model, all rumor models in Table 2 can be obtained from our general model. However, it is worth mentioning that the reason why the DK 
model is not covered by our model relies on the fact that it considers edge sampling and undirected contacts (see Section 3.3). The difference between our approach and the previous models is that our approach does not allow an individual to perform two transitions (or two attempts) at the same time step.

The MK model can be obtained by considering $\eta=1, \beta=0, \delta_{1}=\delta_{2}=\gamma=0$ in Equation (4.6), letting the system be a function of $\alpha$ and $\lambda$. This model does not have a critical threshold. On the other hand, it is possible to obtain the threshold for the variant introduced by Nekovee et al. (NEKOVEE et al., 2007), which considers a spontaneous process to model the loss of interest in the propagation of the rumor. The probability of turning a spreader into a stifler is called $\bar{\delta}$ in the original paper. This parameter is similar to $\delta_{2}$ here, which leads us to a threshold equivalent to that in (NEKOVEE et al., 2007)

$$
\Lambda_{\max } \approx\left(\frac{\delta_{2}}{\lambda\left(1-\delta_{2}\right)}\right)_{c}
$$

Finally, the model by Borge-Holthoefer et al. (BORGE-HOLTHOEFER et al., 2012) considers a feature that is very common in human communication through online social networks, namely, apathy, in which an ignorant might turn into a stifler after being informed - this is often the case observed in Twitter for instance, where the fact that a tweet appears on a user's timeline does not directly imply that the user spreads it further. This feature has been used to identify influential spreaders in rumor-like dynamics (González-Bailón Sandra et al., 2011; BORGE-HOLTHOEFER et al., 2012; De Domenico et al., 2013; ARRUDA et al., 2014). The model by Borge-Holthoefer et al. (BORGEHOLTHOEFER et al., 2012) is recovered from our approach by setting $\beta=0, \delta_{1}=\delta_{2}=$ $\gamma=0$ in Equation (4.6). Note that the system's dynamics depends on $\eta, \alpha$ and $\lambda$ in this scenario.

\subsubsection{Numerical analysis and simulations}

Once we have got some analytical insights, we next compare results from extensive Monte Carlo (MC) simulations with the numerical solution of the system of Equations (4.6) that describes the dynamics of the model, showing that they agree at the micro (e.g., at the individual level) and macro (e.g., at the system level) scales. We obtain the respective phase diagrams for the limiting cases of $\mathrm{CP}$ and $\mathrm{RP}$ for several combinations of the model's parameters, as well as for different topologies of the underlying network of contacts.

\subsubsection{Phase diagrams}

First, we present the $(\lambda, \beta)$ phase diagrams for the CP and the RP cases. They are obtained by solving the dynamical set of Equations (4.6) and varying these parameters 
Figure $29-(\lambda, \beta)$ phase diagrams at the stationary state for the CP case on a scale-free network with degree distribution $P(k) \sim k^{-\zeta}$ and $\zeta \approx 2.7$. The network is made up of $N=10^{4}$ nodes and has an average degree $\mathbb{E}(k) \approx 100$. The rest of the dynamical parameters have been set to $\eta=0.01, \delta_{1}=\delta_{2}=\gamma=0.1$ and $\alpha=1.0$. The intensity of the color (as given by the legend) represents the final fraction of ignorants (panel (a)), spreaders (panel (b)) and stiflers (panel (c)). The continuos lines are the analytical values for the critical point.

a)

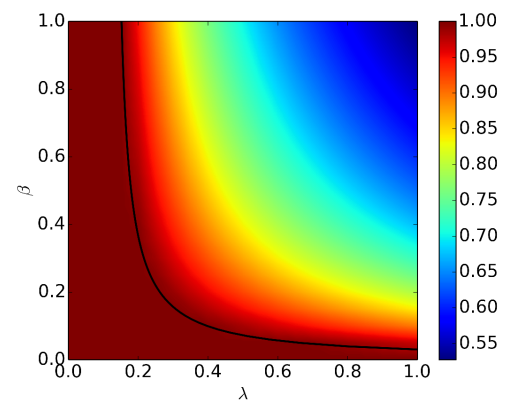

b)

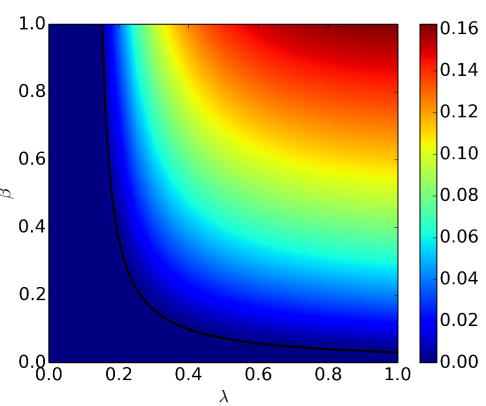

c)

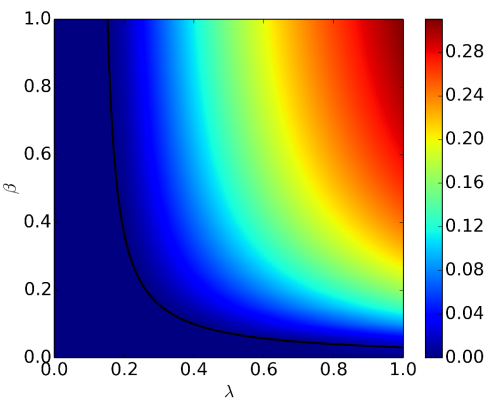

Source: Developed by the author.

Figure $30-(\lambda, \beta)$ phase diagrams as in Figure 29 but for the RP. The underlying network of contacts has the same features except for the average degree that is $\mathbb{E}(k) \approx 10$. The rest of the parameters are $\eta=0.01, \delta_{1}=\delta_{2}=\gamma=0.25$ and $\alpha=1.0$. The intensity of the color (as given by the legend) represents the final fraction of ignorants (panel (a)), spreaders (panel (b)) and stiflers (panel (c)).

a)

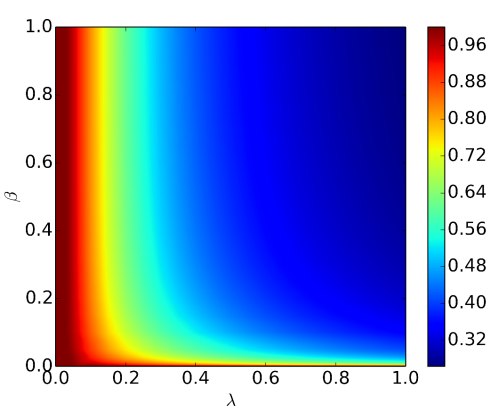

b)

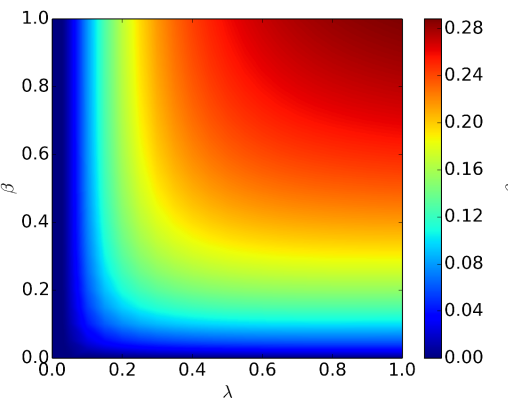

c)

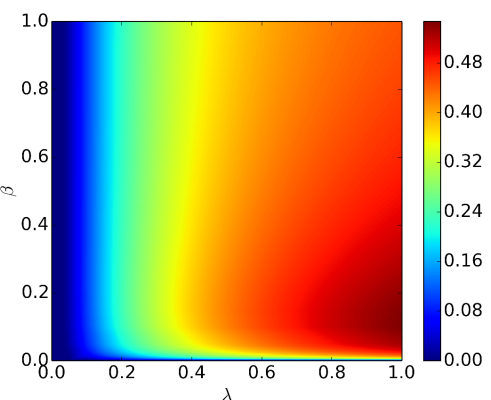

Source: Developed by the author.

from 0 to 1 in intervals of $\Delta \lambda=\Delta \beta=5 \times 10^{-2}$. In addition, when $\beta<0.05(\lambda<0.05)$, i.e., near the critical region, we set $\Delta \lambda=\Delta \beta=5 \times 10^{-3}$.

Figures 29 and 30 show the $(\boldsymbol{\lambda}, \boldsymbol{\beta})$ phase diagrams obtained from the numerical evaluation of the system of Equations (4.6) for the CP and the RP, respectively. In the two figures, we represent (color-coded) in the two figures the fraction of ignorants, spreaders and stiflers in panels (a), (b) and (c), respectively. The underlying network is the same in both cases (scale-free graph with $\zeta \approx 2.7, N=10^{4}$ ), except for the average degree, which is $\mathbb{E}(k) \approx 100$ in Figure 29 and $\mathbb{E}(k) \approx 10$ in Figure 30 . The rest of the parameters 
Figure 31 - Phase diagram at the steady state for (a) a CP considering a scale-free network with $\zeta \approx 2.7, N=10^{4}$ and $\mathbb{E}(k) \approx 100$, and (b) a RP simulated on top of a scale-free network with $\zeta \approx 2.7, N=10^{4}$ and $\mathbb{E}(k) \approx 10$. The simulations were carried out using the following dynamical parameters: $\delta_{1}=\delta_{2}=\gamma=0.1, \lambda=1, \beta=0.5$ and $\eta=1$. The continuous lines are the theoretical predictions, whereas the symbols are the results of MC simulations. The standard deviation has approximately the size of the symbols.
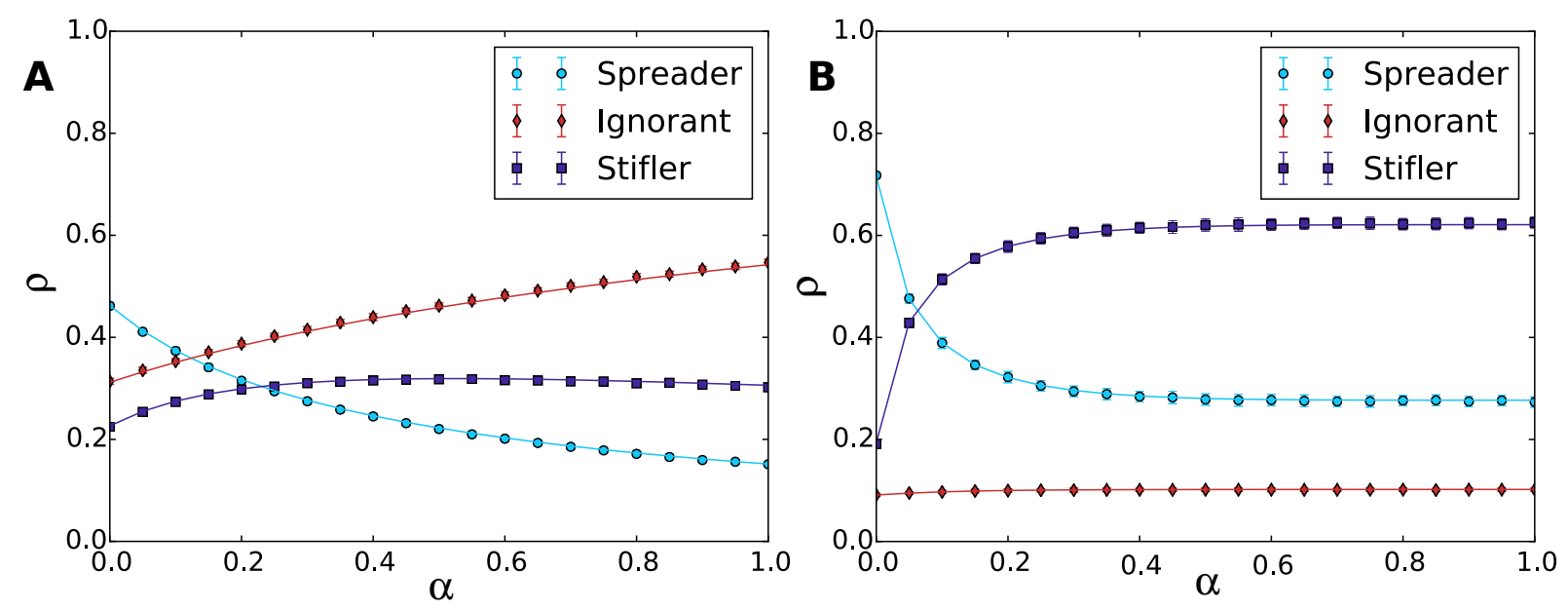

Source: Developed by the author.

have been set to $\eta=0.01, \delta_{1}=\delta_{2}=\gamma=0.1$ and $\alpha=1.0$ for the $\mathrm{CP}$ and to $\eta=0.01$, $\delta_{1}=\delta_{2}=\gamma=0.25$ and $\alpha=1.0$ for the RP. As discussed in the previous section, an analytical expression for the critical point in the CP scenario can be obtained (Equation (4.19)), which is indicated by the continuous black line. This is not possible for the RP.

The comparison of the results shown in Figures 29 and 30 indicates that there are important differences in the system's behavior for the CP and RP schemes, notably with respect to the final fraction of stiflers, which is in most practical scenarios the quantity that we would like to be as high as possible - as that would mean that the piece of information reached a large fraction of the population and therefore that it was spread efficiently. The $\mathrm{CP}$ exhibits a critical point that does not depend on the network structure, and although it cannot be evaluated for the RP in a closed form, the numerical solutions show that this scenario is more complex with some interesting dependencies on the network structure and the dynamical parameters of the model. As a matter of fact, as it can be seen in Figure $30 \mathrm{c}$, there is a nonlinear effect in $\beta$ for fixed values of $\lambda$. Admittedly, for large values of $\lambda \geq 0.7$, when $\beta$ starts to increase from zero, the final density of stiflers also grows. However, at some point (roughly around $\beta \approx 0.1$ ), this density reaches its maximum value and starts decreasing beyond that value of $\beta$. 
Figure 32 - Probability of finding a node $i$ in any of the dynamical states of the model $(X, Y$ or $Z$ ) obtained by solving analytically the system of Equations (4.6) as compared with Monte Carlo simulations. The probabilities are calculated by averaging $10^{3}$ simulations. Panel (a) corresponds to the $\mathrm{CP}$, whereas the RP is represented in panel (b).

a)

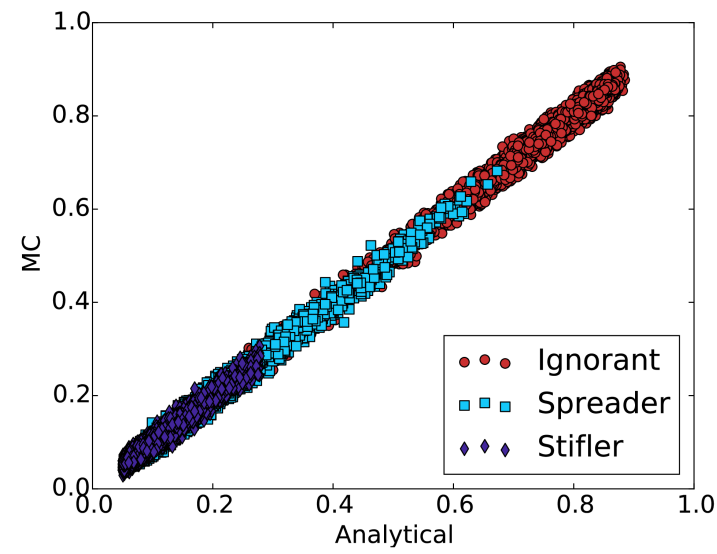

b)

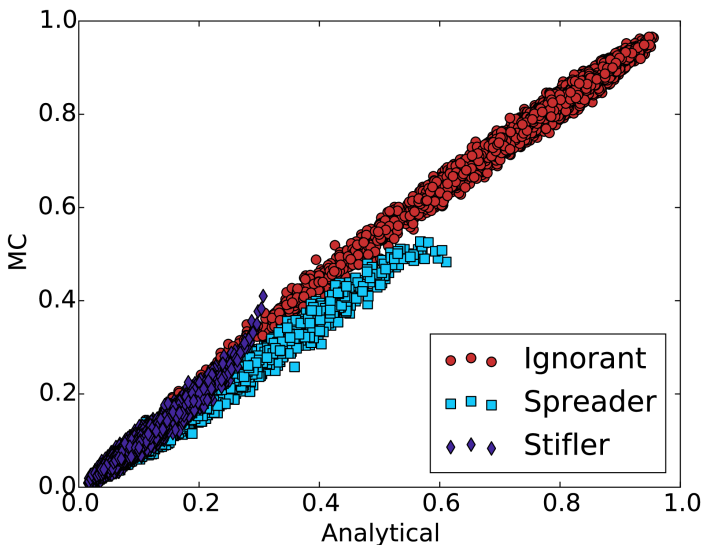

Source: Developed by the author.

\subsubsection{Monte Carlo simulations}

In order to check whether our analytical and numerical solutions are accurate at describing both individual states and the macroscopic behavior of the system, we performed large-scale Monte Carlo simulations. All results reported henceforth are averages taken over at least $100 \mathrm{MC}$ simulations with an initial fraction of spreaders and ignorants equal to $\rho^{Y}(0)=0.01$ and $\rho^{X}(0)=0.99$, respectively.

In Figure 31, we present results for the dependency of the densities of $X, Y$ and $Z$ as a function of $\alpha$ with all the other model parameters fixed. The $(\rho, \alpha)$ plots correspond to the CP (panel (a)) and the RP (panel (b)) scenarios. As can be seen, the continuous lines, which are obtained by numerically evaluating Equation (4.6) perfectly match results from MC simulations in both limiting cases. In order to check whether this agreement is also verified at the individual scale, we have represented in a scatter plot, see Figure 32 , the probability of finding an individual in the ignorant, spreader or stifler class at the stationary state. Despite finite size effects and stochastic fluctuations, the analytical results capture the micro dynamical states in the large $t$ limit, since there is a strong correlation between the probabilities obtained from MC and the solutions of the system Equation (4.6). These results thus convincingly show that one can explore the model in terms of either the individual probabilities or the macroscopic expected values by solving the equations describing the system's dynamics, without the need to rely on extensive and costly MC simulations. This is, from a practical point of view, an added value of the proposed framework, as we shall discuss later on.

Spreading processes have been shown to be greatly affected by the topological 
Figure 33 - Phase diagram of the system's dynamics at the steady state as a function of $\alpha$. In all panels, the rest of the parameters were fixed to $\delta_{1}=\delta_{2}=\gamma=0.1, \lambda=1, \beta=0.5$ and $\eta=1$ and the continuous lines are the theoretical predictions, while the symbols correspond to the MC simulations. Panels (a) and (b) show results obtained varying the exponent $\zeta$ of the power law degree distribution, $P(k) \sim k^{-\zeta}$, of the underlying networks of contacts of size is $N=10^{4}$ and $\mathbb{E}(k) \approx 100(\mathrm{CP}$, panel (a)) and $\mathbb{E}(k) \approx 10$ $(\mathrm{RP}$, panel (b)). We considered the following exponents $\zeta: 2.3(\bullet), 2.5(\times), 2.7(\square)$, $3.3(\nabla)$ and $3.5(\diamond)$. The effects of the network size are shown in Panel $(\mathrm{c})$ for scalefree networks $\left(P(k) \sim k^{-\zeta}\right.$ with $\left.\zeta \approx 2.7\right)$ with $\mathbb{E}(k) \approx 100$ (main plot) and $\mathbb{E}(k) \approx 10$ (inset) and the following sizes: $N=5 \times 10^{2}(\bullet), N=10^{3}(\square), N=5 \times 10^{3}(\diamond)$ and $N=10^{4}(\nabla)$. Finally, in panel $(\mathrm{d})$, we represent results obtained for the $\mathrm{RP}$ and different average degrees: $\mathbb{E}(k) \approx 10(\bullet), \mathbb{E}(k) \approx 20(\times), \mathbb{E}(k) \approx 35(\square), \mathbb{E}(k) \approx 45$ $(\nabla)$ and $\mathbb{E}(k) \approx 60(\diamond)$. The rest of the network's parameters are the same as in panel (c).

a)

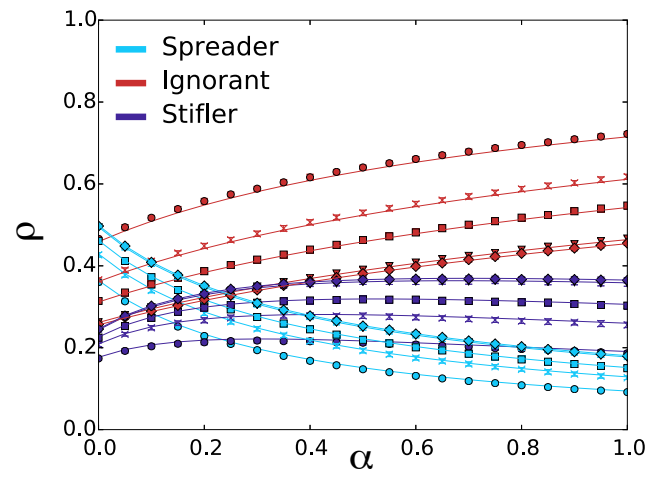

c)

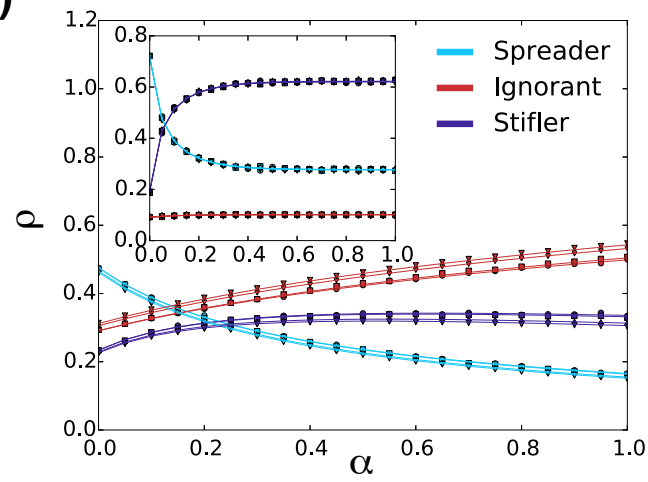

b)

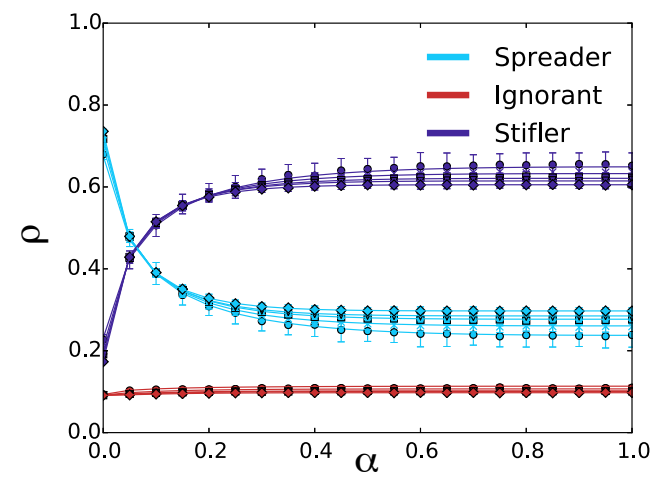

d)

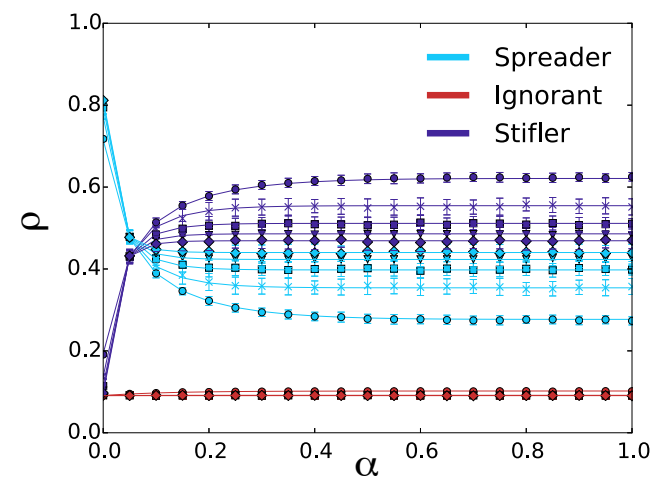

Source: Developed by the author.

features of the networks on top of which they take place. It is therefore of further interest to investigate network effects on the dynamical evolution of the spreading phenomenon. To this end, we have explored the impact of three characteristics of the contact networks, namely, the exponent of the degree distribution $P(k)$ for scale-free graphs - for which $P(k) \sim k^{-\zeta}$, the size of these networks and finally how dense they are by tuning the average degree of the network's nodes. Figure 33 shows the results of these analyses. First, we note that in all cases, the previous agreement between the numerical solution of 
the system's equations and MC simulations still holds. Other aspects worth highlighting include the fact that the value of $\zeta$ influences the outcomes of the spreading process mainly for the $\mathrm{CP}$, unless $\zeta$ is larger than 3. This is because, for larger exponents, the networks are effectively equivalent to homogeneous graphs, and therefore there are no hubs anymore, leading to fairly similar results regardless of the specific value of $\zeta$. Another interesting effect of the network structure, and in particular of $\zeta$, is the one observed in Figure 33b. Admittedly, the final density of ignorants is independent from this network parameter. In other words, variations of the exponent mainly affect the number of spreaders and stiflers, with the number of ignorants remaining roughly constant.

As for finite size effects, our results, see Figure 33c, show that they do not appear to play a major role in the rumor spreading dynamics. This is not the case with respect to the last parameter analyzed, the average degree of the network. As it can be seen in Figure 33d, the steady-state fractions of spreaders and stiflers strongly depends on the density of connections, while the fraction of ignorants is (as it happened with respect to $\zeta$ ) almost the same for different average degrees. In summary, it seems that the most important dependencies with respect to the networks' topological properties are given by their heterogeneity (characterized by $\zeta$ ) and the density of connections (e.g., $\mathbb{E}(k)$ ). Both features mainly affect the ratio between spreaders and stiflers at the stationary state but do not significantly affect the final number of ignorants. Altogether, and from a practical point of view, it can be concluded that, with the exception of the heterogeneity of the degree distribution, the size and average degree of the networks have a somewhat minimal impact on the propagation of the rumor, at least in what concerns its main outcome: the number of individuals that learned the rumor - both spreaders and stiflers represent states in which individuals are aware of the news, that is, they have been reached by the contagion process (but also see below).

Finally, we also show Figure 34 where the numerical solution of the system (4.6) and Monte Carlo simulations are in good agreement not only in the large $t$ limit but also when we explore the system's behavior at intermediate times, i.e., in transient states. The figure shows the evolution in time of the fraction of ignorants, spreaders and stiflers for both the $\mathrm{CP}$ and RP limiting cases when the substrate network is a scale-free graph with $\zeta \approx 2.7, N=10^{4}$ and $\mathbb{E}(k) \approx 100$. As we will see in the next section, the fact that the temporal dynamics can be captured accurately by numerically solving the set of Equations (4.6) could be used to study real spreading dynamics for which highly resolved temporal data is available.

We round off this section by pointing out some limitations of the mean field approximation. Gleeson et al. (GLEESON et al., 2012) discussed that the accuracy of the mean field theory is higher when three main assumptions are satisfied, i.e., (i) vanishing local clustering, (ii) non-modular network organization and (iii) absence of dynamical 
Figure 34 - Time evolution of the average probabilities for the (a) CP and (b) RP. The continuous lines are the theoretical predictions, while the symbols are obtained by Monte Carlo simulations.

a)

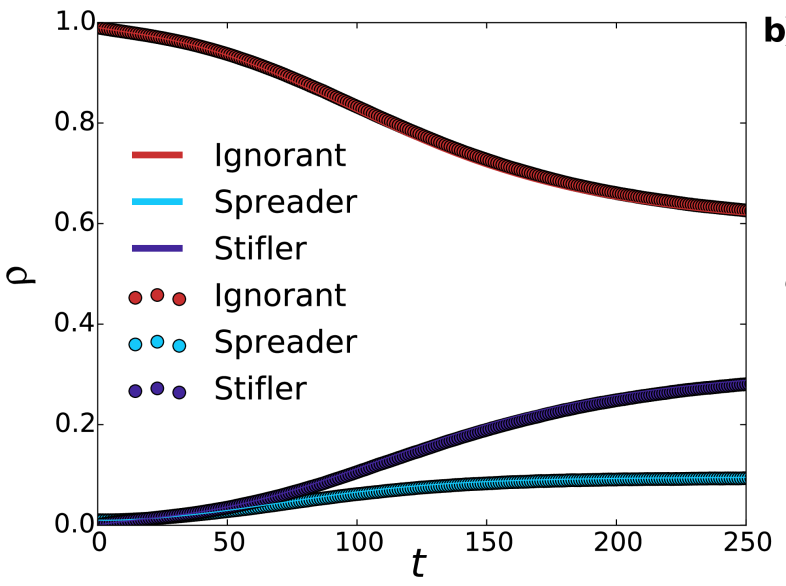

b)

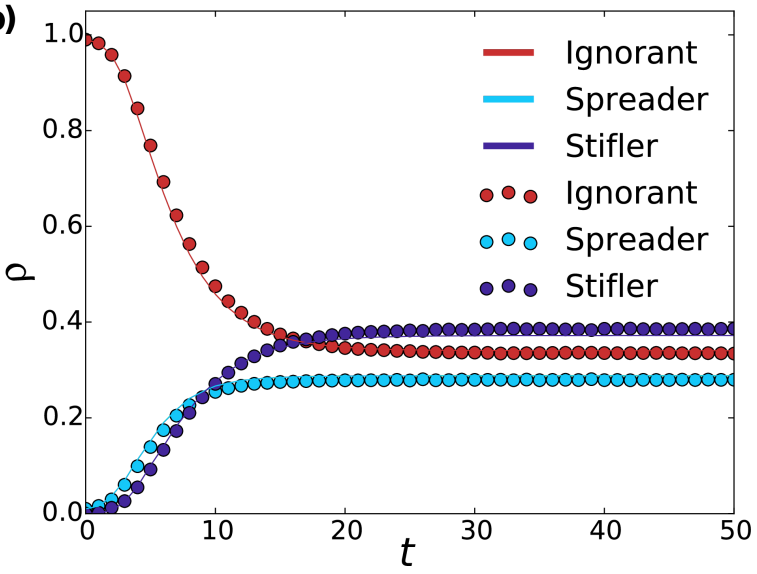

Source: Developed by the author.

correlations. Conditions (i) and (ii) depends on the structure of the network. They are satisfied in our analysis, because we consider the configuration model, which satisfies the properties (i) and (ii). We will next analyze how our model performs in real online networks. However, the dynamical correlations might represent a major source of error, as we assume that the state of each node is independent of the state of the rest of vertices. Nevertheless, a node is more likely to be informed from one of its neighbors than from other vertices. In (GLEESON et al., 2012), the authors exemplified this effect by considering an SIS dynamics, suggesting that the error is reduced with the increase of the mean first neighbor degree. To quantify the error between our numerical experiments and MC simulations, we set the dynamical parameters to $\delta_{1}=\delta_{2}=\gamma=0.1, \lambda=1$ and vary $\alpha$. The differences between the theoretical predictions and MC simulations are then quantified by the absolute error. We found (results not shown) that, for the RP, the error vanishes as soon as $\mathbb{E}(k) \geq 10$, whereas, for the case of a $\mathrm{CP}$, the error is close to zero only for $\mathbb{E}(k) \geq 100$, as shown in Figure 35 .

\subsubsection{Applications to real social systems}

Up to now, we have explored the model in computer-generated networks. While these networks share some of the topological features that have been found in real-world systems, they do not account for all of them, especially when it comes to clustering properties or several kinds of correlations. It is therefore important to run our model on top of real systems and check whether the results reported so far also hold for more realistic topologies. In doing so, we will also show how the model could be used to obtain a better understanding of the mechanisms driving real spreading phenomena. In what follows, we inspect whether the model gives accurate results at the microscopic scale for 
Figure 35 - Phase diagram at the steady state for the CP considering a scale-free network with $\zeta \approx 2.7, N=10^{4}$ and the dynamical parameters $\delta_{1}=\delta_{2}=\gamma=0.1$ and $\lambda=1$. The continuous lines are obtained by the least squares method.

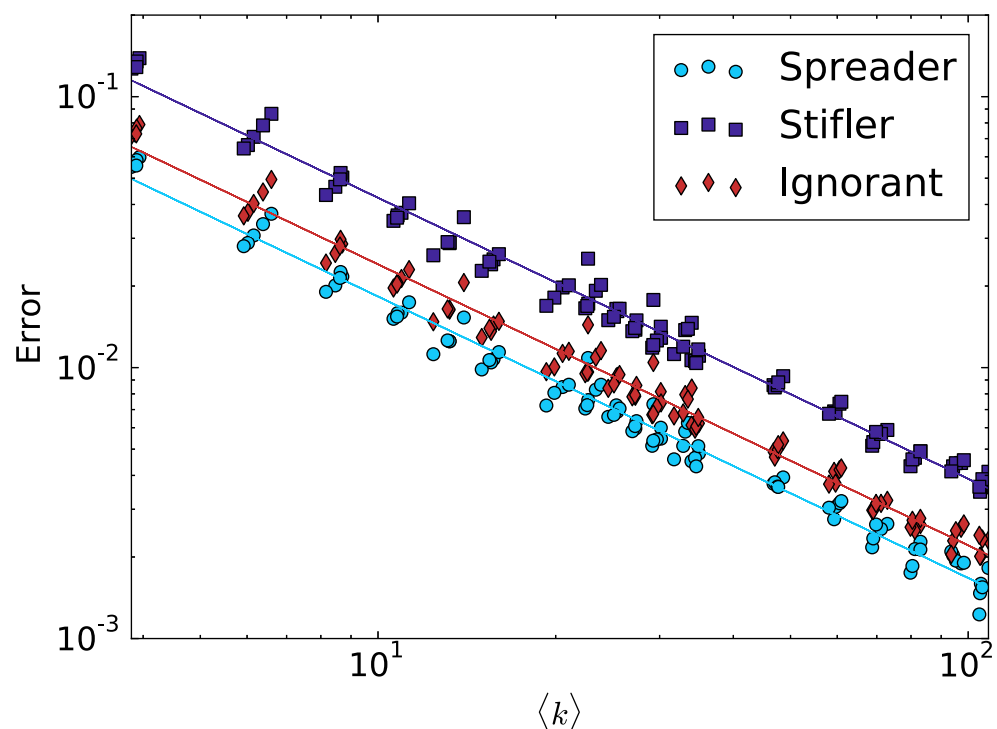

Source: Developed by the author.

both the CP and the RP scenarios, as we have done before for the synthetic networks. Finally, we also reproduce the temporal dynamics of a real spreading process that took place over online social networks (Twitter in our analysis) when the confirmation of the existence of the Higgs boson was announced.

To verify that our model performs well also in real networks, we use two social networks. The first is an email network, in which messages are mostly spread as contact processes (that is, one-to-one communication at a time), and the second network considered is the contact patterns of Twitter. The latter is an online social system where the information dynamics is mainly of the form one-to-all, that is, a user posts a message that reaches to out all the user's followers at the same time, thus corresponding to the $\mathrm{RP}$ limit in our formulation. We also consider $\eta=0.01$ to simulate the apathy as in (BORGE-HOLTHOEFER et al., 2012).

The email contact network was created from emails exchanged between users within the Universitat Rovira i Virgili (GUIMERA et al., 2003). The network is composed by $N=1133$ nodes and $\mathbb{E}(k)=9.6$; connections are directed and unweighted. However, here we consider an undirected version of this network. The Twitter network was extracted from the mobilizations in Spain during 2011 (BORGE-HOLTHOEFER et al., 2011; González-Bailón Sandra et al., 2011; BORGE-HOLTHOEFER; RIVERO; MORENO, 2012). Here we consider a simplified version of this network, composed by $N=85712$ nodes and $\mathbb{E}(k)=109.9$, with undirected and unweighted contacts. Both net- 
Figure 36 - Application of the proposed discrete time Markov chain formulation to the analysis of spreading processes that take place on top of social networks. Panels (a) and (b) show the probability of node $i$ belonging to state $X, Y$ or $Z$ obtained by solving numerically the system of Equations (4.6)) and by Monte Carlo simulations. Panel (a) corresponds to a RP that takes place over the Twitter network, where as panel (b) has been obtained simulating a $\mathrm{CP}$ on the email network. The model parameters: $\lambda=1.0, \eta=0.01, \delta_{1}=\delta_{2}=\gamma=0.01, \beta=1.0$ and $\alpha=1.0$ for the RP; and $\lambda=1.0$, $\eta=0.01, \delta_{1}=\delta_{2}=\gamma=0.1, \beta=1.0$ and $\alpha=1.0$. Results for MC simulations are averages over $10^{3}$ different simulations. Panel (c) depicts the time evolution of the Higgs boson rumor spreading, taking into account tweets of the dataset shown in (De Domenico et al., 2013). The dashed lines represent the real data, while the continuous lines are the numerical solutions of our model. The vertical lines mark the three time windows used, with the following parameters: (i) $0 \leq t \leq 77, \lambda=0.00025$, $\alpha=0.0002, \delta_{2}=0.0001$ and $\eta=0.85$; (ii) $77<t \leq 106, \lambda=0.021, \alpha=0.00075$, $\delta_{2}=0.0015$ and $\eta=0.17$; (iii) $t>106, \lambda=0.065, \alpha=0.002, \delta_{2}=0.002$ and $\eta=0.01$.

a)

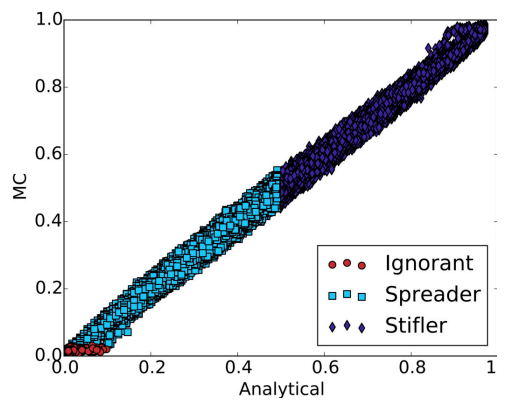

b)

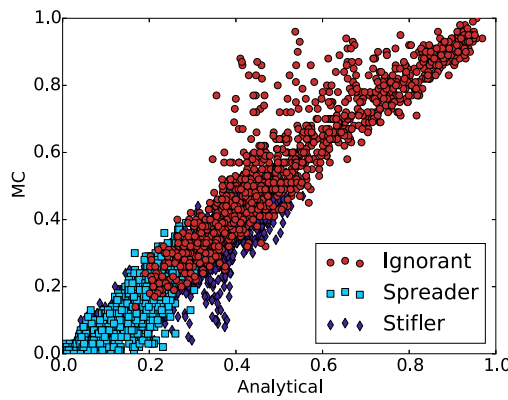

c)

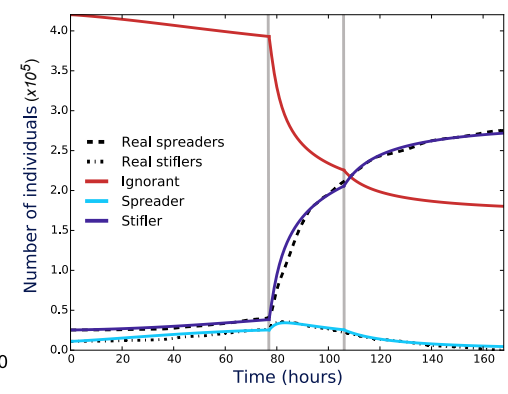

Source: Developed by the author.

works have an average clustering coefficient $\mathbb{E}(c c) \approx 0.22$. In addition, while the email network is slightly assortative, $r=0.08$, the Twitter network is disassortative $r=-0.14$.

We compare the results obtained via MC simulations of the model with the numerical solution of Equation (4.6) in Figure 36a and b using Twitter and email networks as underlying graphs, respectively. The results show that despite the new topological features of the real networks, the agreement is still very good. Panel (a) corresponds to an $\mathrm{RP}$ and the probabilities of finding a node in each of the three possible states at the large time limit match fairly well. This indicates that the new topological ingredients that were not present in the synthetic networks play a minor role when it comes to evaluating the accuracy of the discrete Markov chain formulation - or at the very least for this particular network. Panel (b) on the contrary shows the results obtained when simulating a $\mathrm{CP}$ on top of the email network. Again, the numerical solutions of the set of equations describing the dynamics of the system agree well with MC simulations, albeit having larger deviations and more dispersion along the diagonal line. This could be due to the fact that $\mathbb{E}(k)=9.6$ for the email network, and as discussed and shown earlier (see Figure 35 ), for a $\mathrm{CP}$ errors are vanishingly small only beyond $\mathbb{E}(k) \approx 100$. Other new topological 
characteristics or even some dynamical correlations (GLEESON et al., 2012) might have an impact as well. Additionally, note that another possible source could be the relatively small size $(N=1133)$ of the email network. Admittedly, the Twitter network also has triangles, modular organization and degree-degree correlations (BORGE-HOLTHOEFER et al., 2012; BORGE-HOLTHOEFER et al., 2011), but it is an order of magnitude larger.

We also apply the formalism to simulate the time evolution of a real information dissemination process. Specifically, we have modeled the temporal dynamics of the rumor spreading on Twitter during and after the announcement of the discovery of a new particle with the features of the elusive Higgs boson on 4th July, 2012. This dataset was formerly analyzed in (De Domenico et al., 2013). Here, we consider the giant component of an undirected network of the friend/follow network, which is composed by $N=456626$ individuals, with $\mathbb{E}(k)=54.79$, an average clustering coefficient $\mathbb{E}(c c)=0.189$ and assortativity $r=-0.098$. This database describes the timestamps (in seconds) of mentions, replies and retweets. In our analysis, we consider these three timestamps as events. However, if two events occur at the same time and are generated by the same user, we consider them as just one event. For instance, if a user retweets a mention, we would have two events at the same time, one for the mention and the other for the retweet. In such a case, we consider it as just one tweet.

Moreover, how to assign the state a node belongs to (i.e., spreader or stifler) is not trivial, because we cannot distinguish between: (i) a spreader turns into a stifler, then recovers the interest in the rumor and becomes a spreader again or (ii) a spreader that remains as a spreader during the observation time. Here, we assume that an individual is an ignorant if he/she did not tweet about the Higgs boson. An ignorant becomes a spreader when he/she first tweets about this topic and remains as such up to its last tweet, when he/she becomes a stifler. The only special case considered is the users that tweeted just once. In such a case, we consider that these individuals are spreaders during just one-time step, i.e., 1 unit time, and they become stiflers the next time-stamp. Additionally, the initial conditions are given by the activities before the observation time window, which means starting with a certain fraction of spreaders and also stiflers, since some users that tweeted about the Higgs boson do not tweet during the observation time window. Note that the modeling assumes that a user is aware of the rumor only if he/she tweets about it. Finally, it is important to define $\Delta t$. For our analysis, we have assumed that each discrete time window represents 1 hour of the real data. This choice implies that we are not able to distinguish events that occur at a faster rate. However, setting $\Delta t=1 h$ drastically reduces the computational cost. It should be mentioned that our aim here is to verify whether our model is capable of describing the behavior observed in the real data, not to perform a detailed forensic analysis of the actual rumor spreading process.

In order to fit simulations of the model with the data, we assume that the forget- 
ting mechanisms can be neglected, as the total observation time is reasonably small (1 week), implying that $\delta_{1}=\gamma=0$. Moreover, we assume $\beta=0$, as we consider that there is no transition from a stifler to a spreader, i.e., the possibility of recovering the interest in the rumor is neglected - this is in part also due to the constraint that we cannot distinguish such transitions in real data. Additionally, observe that $\delta_{2}$ is the probability that a spreader loses the interest about the rumor spontaneously, while $\alpha$ represents the probability of turning into stiflers after contacting spreaders or stiflers. Note that $\eta, \gamma$ and $\alpha$ might produce similar effects, depending on the defined time-steps.

Figure 36c compares the time evolution of the spreading dynamics as extracted from the real data with results obtained from our model using three different time windows. The latter is needed as it is known that in viral processes such as the one we are analyzing, there are different phases of the dynamics: an early stage in which the number of messages exchanged increases slowly (subcritical regime) followed by an explosive period (critical and supercritical regimes) that signals the moment beyond which the piece of news goes viral and finally a phase in which the active spreaders die out and stop propagating the rumor any farther (BORGE-HOLTHOEFER et al., 2012). For the first time window, we thus assign initial probabilities of being in state $X, Y$ or $Z$ as given by the real data, but considering them to be the same for every node. For the following time windows, these probabilities come from the output of the previous simulation window. In the first time window, $0 \leq t \leq 77$, we consider $\lambda=0.00025, \alpha=0.0002, \delta_{2}=0.0001$ and $\eta=0.85$. In the second, $77<t \leq 106, \lambda=0.021, \alpha=0.00075, \delta_{2}=0.0015$ and $\eta=0.17$. Finally, for $t>106, \lambda=0.065, \alpha=0.002, \delta_{2}=0.002$ and $\eta=0.01$.

The results show that the model is indeed able to accurately reproduce the temporal evolution of the real spreading dynamics. We, however, stress that we chose those sets of parameters by simple inspection, i.e., we did not apply any fitting algorithm. In order to obtain a better fit, one may use statistical inference tools or even a simulated annealing algorithm. As this is beyond the scope of this work, we will leave this line of research as a potential future work. The simulation performed is nevertheless worth carrying out. The fact that a model like the one discussed here could be adapted without the use of sophisticated fitting algorithms to describe the temporal dynamics of a real rumor spreading process is an important step towards having a better understanding of the mechanisms at work in this real contagion dynamics. Admittedly, without knowing the parameters, real-time projections of the temporal evolution of the rumor dynamics cannot be done. But once we know the parameters that give the best matching with the real data, we are in a position to know which mechanisms (that is, what transitions) are more important and which are not, thus gaining valuable phenomenological insights. In turn, given the universal features behind the spreading processes, this would allow us to perform other analyses, such as detecting who are potential candidates to be influential spreaders in other online social systems or for the propagation of other rumors in the very 
same network.

\subsection{Role of centrality to identify influential spreaders}

In the previous section, we dealt with the modeling aspects of epidemic and rumor spreading in complex networks. Here, in this section, we change our perspective, from modeling to focusing on the relation between the structure of the system and its emergent dynamical properties. Considering this, finding determinant structural factors is important, as a better knowledge would allow controlling the function of the system, which for the scope of this section, means determining which network properties are more closely related to information and virus diffusion. In particular, we will focus our attention on one topological feature: centrality. Since the most central nodes can diffuse their influence to the whole network faster than the rest of nodes, it is expected that such agents are the most influential spreaders. Recently, Kitsak et al. (KITSAK et al., 2010) found shreds of evidence that confirmed this hypothesis for the case of epidemic outbreaks. The authors verified that the most influential spreaders can be forecasted from the $k$-shell decomposition analysis. These agents are located within the core of the network and do not need to be the most connected. Silva et al. (SILVA; VIANA; COSTA, 2012) explored the correlations between the heterogeneous spread and central attributes of the vertices that were first seeded with a disease, finding that the degree and accessibility are measured mostly related to the efficient spread of the disease. On the other hand, Borge-Holthoefer and Moreno (BORGE-HOLTHOEFER; MORENO, 2012) showed that, for standard rumor models, it is not possible to identify the most influential spreaders using the same metrics.

Although many works have provided evidence on the presence of influential spreaders in epidemic spreading, the conclusions are not general. Indeed, there is no general consensus on the definition of network "centrality", because there are many measures able to quantify the centrality of a node, each one considering specific concepts (NEWMAN, 2010). For instance, betweenness and closeness centrality take into account only the shortest distance between pairs of nodes (BOCCALETTI et al., 2006; NEWMAN, 2010), ignoring alternative paths. At the same time, the $k$-core decomposition may eliminate important sets of vertices, which can be connected to the main core through nodes with a small number of links (SEIDMAN, 1983). Thus, to overcome such a lack of a universal definition of node centrality, it is necessary to look at additional measures. In this section, we study the problem of identifying influential spreaders using nine centrality measures in order to complement previous studies (KITSAK et al., 2010; BORGE-HOLTHOEFER; MORENO, 2012). Moreover, we use our new metric, the generalized accessibility, as a centrality measure that is based on random walks (for more on its definition, please see Section 2.1.2.3 and also Appendix A). We observe that in social and scale-free networks, 
the accessibility, average neighborhood degree and closeness centrality are the measures most related to rumor spreading. Other measures, such as the $k$-core and degree correlate well only with epidemic spreading in social networks, as found previously in (KITSAK et al., 2010; BORGE-HOLTHOEFER; MORENO, 2012).

Another important result is related to the kind of networks analyzed in this work. Despite the fact that many diffusion processes take place in spatially embedded networks (BARTHÉLEMY, 2011), previous studies have disregarded spatial networks (KITSAK et al., 2010; BORGE-HOLTHOEFER; MORENO, 2012; SILVA; VIANA; COSTA, 2012). These networks have several topological constraints that greatly influence the way that connections are established, and thus, one expects an impact in network centrality metrics and consequently in the spreading dynamics. Here, we intend to fill this gap by exploring the role of centrality measures in predicting the spreading capabilities of nodes of spatial networks. Specifically, we consider both real networks (road networks of four countries) and artificial spatial networks with exponential and power-law degree distributions and find that correlation between spreading capacity and centrality measures in spatial networks differ significantly from those observed in non-spatial networks.

\subsubsection{Methods}

In this section, we define our simulation algorithms and the considered dataset. We remark that other possible combinations are possible, but, since we performed an extensive evaluation we believe that our results are reasonable general.

\subsubsection{Monte Carlo simulations}

In this study, we focused on the asynchronous cellular automaton simulation algorithm, described in Section 3.5.6. As described in chapter 3 we have different possible algorithms. Here we opted for this approach as it allows us to compare with previous results in the literature. For instance, the works by Kitsak et al. (KITSAK et al., 2010) and Borge-Holthoefer and Moreno (BORGE-HOLTHOEFER; MORENO, 2012), which are important references in terms of identifying influential spreaders in epidemic and rumor spreading, respectively, use the asynchronous cellular automaton method. Thus, we follow the same algorithm, allowing us to compare our results with the literature results.

Furthermore, since we are interested in the relation between structure and dynamics, our analyses are performed in terms of the correlation between a centrality measure and a dynamical measurement. More specifically, the Spearman correlation coefficient between a centrality measure and the final fraction of infected individuals. 
Table 3 - Structural properties of the complex networks.

\begin{tabular}{|c|c|c|c|c|c|c|c|c|c|c|c|}
\hline & Network & $N$ & $\mathbb{E}(k)$ & $\mathbb{E}\left(c c_{i}\right)$ & $\mathbb{E}\left(B_{i}\right)$ & $\mathbb{E}\left(C_{i}\right)$ & $\mathbb{E}\left(r_{i}\right)$ & $\mathbb{E}(\pi)$ & $\mathbb{E}(\alpha)$ & $\mathbb{E}\left(x_{i}\right)$ & $\mathbb{E}\left(k_{c}\right)$ \\
\hline \multirow{6}{*}{ 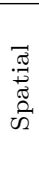 } & Japan & 2130 & 3.792 & 0.24 & $3.731 \times 10^{4}$ & 0.03 & 4.290 & $4.695 \times 10^{-4}$ & 6.95 & $2.892 \times 10^{-3}$ & 2.523 \\
\hline & England & 4460 & 3.415 & 0.14 & $8.163 \times 10^{4}$ & 0.03 & 3.557 & $2.242 \times 10^{-4}$ & 6.65 & $1.401 \times 10^{-3}$ & 2.062 \\
\hline & United States & 6443 & 3.098 & 0.09 & $1.605 \times 10^{5}$ & 0.02 & 3.302 & $1.552 \times 10^{-4}$ & 6.178 & $9.328 \times 10^{-4}$ & 2.038 \\
\hline & Germany & 3555 & 3.068 & 0.08 & $5.944 \times 10^{4}$ & 0.03 & 3.173 & $2.813 \times 10^{-4}$ & 6.243 & $2.668 \times 10^{-3}$ & 1.988 \\
\hline & Spatial SF & 5000 & 3.998 & 0.04 & $1.226 \times 10^{4}$ & 0.17 & 9.291 & $2.000 \times 10^{-4}$ & 9.793 & $6.001 \times 10^{-3}$ & 2.000 \\
\hline & Waxman & 4883 & 4.078 & 0.14 & $4.598 \times 10^{4}$ & 0.05 & 4.863 & $2.048 \times 10^{-4}$ & 8.071 & $1.433 \times 10^{-3}$ & 2.570 \\
\hline \multirow{5}{*}{ 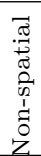 } & Advogato & 5054 & 15.58 & 0.25 & $5.748 \times 10^{3}$ & 0.31 & $9.962 \times 10^{1}$ & $1.979 \times 10^{-4}$ & 28.92 & $6.819 \times 10^{-3}$ & 8.137 \\
\hline & e-mail & 1133 & 9.622 & 0.22 & $1.475 \times 10^{3}$ & 0.28 & $1.790 \times 10^{1}$ & $8.826 \times 10^{-4}$ & 17.88 & $1.764 \times 10^{-2}$ & 5.349 \\
\hline & Political blogs & 1222 & 27.36 & 0.32 & $1.061 \times 10^{3}$ & 0.37 & $1.001 \times 10^{2}$ & $8.183 \times 10^{-4}$ & 33.08 & $1.681 \times 10^{-2}$ & 14.82 \\
\hline & Google+ & 23613 & 3.319 & 0.17 & $3.580 \times 10^{4}$ & 0.25 & $7.270 \times 10^{2}$ & $4.235 \times 10^{-5}$ & 15.13 & $2.301 \times 10^{-3}$ & 1.669 \\
\hline & $\mathrm{BA}$ & 10000 & 3.999 & $5.76 \times 10^{-3}$ & $2.005 \times 10^{4}$ & 0.20 & $1.706 \times 10^{1}$ & $1.000 \times 10^{-4}$ & 10.57 & $3.108 \times 10^{-3}$ & 2.000 \\
\hline
\end{tabular}

\subsubsection{Database}

We performed numerical simulations of epidemic and rumor spreading processes on top of real-world and artificial networks. Table 3 presents some network properties of the road maps and networks generated by the spatial models (for more on the artificially generated networks, please see Section 2.1.4).

First of all, the road networks used in the following experiments have been extracted from the maps available as a portable format $(p d f)$ on the United Nations website ${ }^{2}$. Initially, the maps have been pre-processed in order to eliminate irrelevant information and keep only the main roads. After that, the skeletonization procedure has extracted the so-called skeleton of the image (COSTA; Cesar Jr, 2000). The node identification was performed by applying an 8-connected hit-or-miss convolution filter (DOUGHERTY, 1992). Finally, a label propagation procedure was implemented by each node. When two pairs of labels $i$ and $j$ find each other, a connection is established between them. Here, we have considered the networks extracted from maps of Germany, Japan, England and the United States.

Next, the social networks considered here are: (i) the email contact network obtained from messages exchanged between users within the Universitat Rovira i Virgili (GUIMERA et al., 2003); (ii) the political blogs network, composed of hyperlinks between web blogs obtained over the period of two months preceding the U.S. Presidential Election of 2004 (ADAMIC; GLANCE, 2005) ; (iii) the advogato network, which is an online community dedicated to free software development launched in 1999 (MASSA; SALVETTI; TOMASONI, 2009; KONECT, 2014a) and (iv) the Google+ network, which consists of users connected according to their circles of friends (MCAULEY; LESKOVEC, 2012; KONECT, 2014b). Avogato, political blogs and Google+ networks are directed networks. Moreover, advogato is also a weighted network. However, here we consider only the unweighted and undirected versions of these networks. In addition, our analysis uses only the nodes in the giant component.

2 http://www.un.org 
Figure 37 - Correlation between spreading capacity and the degree, $k, \mathrm{k}$-core index, $k_{c}$, and accessibility, $\alpha$, covering all possible combinations of parameters $((\boldsymbol{\lambda}, \boldsymbol{\delta})$ for epidemic spreading (SIR model) and $(\lambda, \alpha)$ for rumor propagation (MT-TP model), for the road networks of US and Germany.

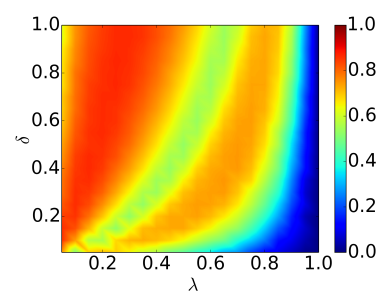

(a) US: SIR $(\alpha)$

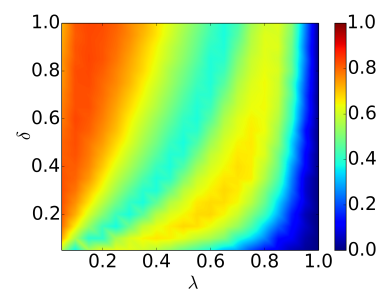

(e) US: SIR $(k)$

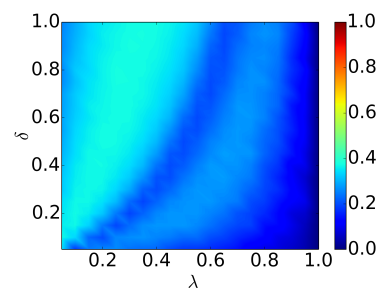

(i) US: SIR $\left(k_{c}\right)$

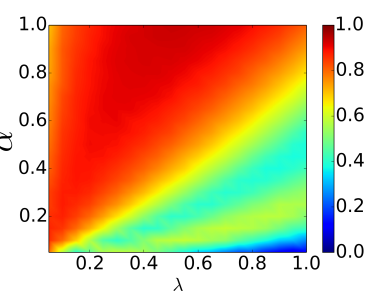

(b) US: MT-TP $(\alpha)$

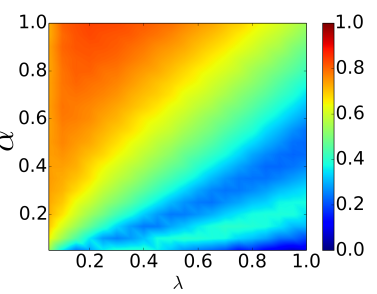

(f) US: MT-TP $(k)$

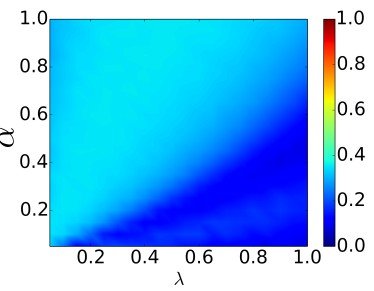

(j) US: MT-TP $\left(k_{c}\right)$

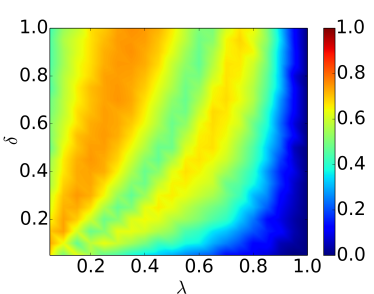

(c) Germany: $\operatorname{SIR}(\alpha)$

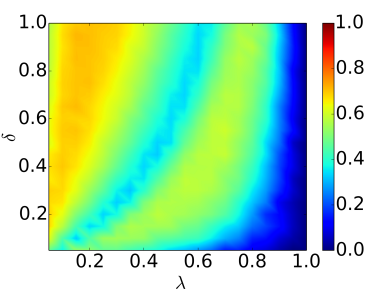

(g) Germany: SIR $(k)$

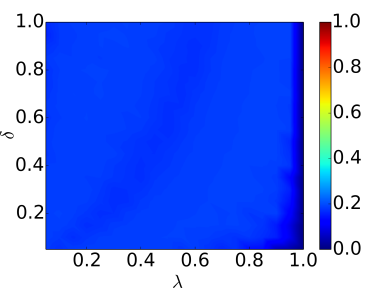

(k) Germany: SIR $\left(k_{c}\right)$

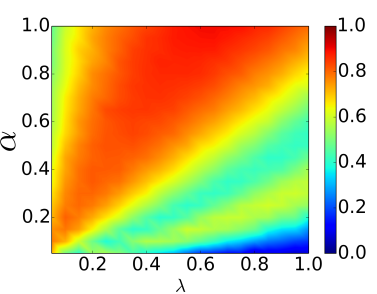

(d) Germany: MT-TP $(\alpha)$

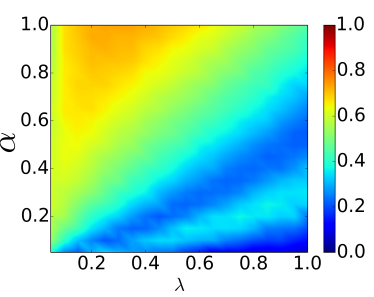

(h) Germany: MT-TP $(k)$

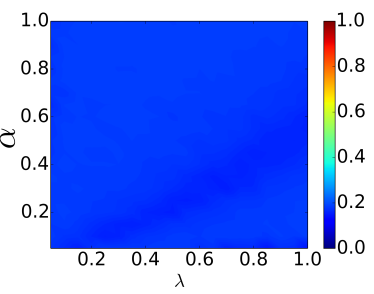

(l) Germany: MT-TP $\left(k_{c}\right)$

Source: Developed by the author.

\subsubsection{Parameter analysis}

The efficiency of a particular node in the spreading process depends not only on its topological characteristics but also on the parameters of the epidemic and rumor models. Here we calculate the above correlations between spreading capacity and the degree, $k$, $\mathrm{k}$-core index, $k_{c}$, and accessibility, $\alpha(i)$, covering all possible combinations of parameters $((\boldsymbol{\lambda}, \boldsymbol{\delta})$ for epidemic spreading and $(\boldsymbol{\lambda}, \boldsymbol{\alpha})$ for rumor propagation). Figure 37 shows the results for the road networks of US and Germany. The accessibility is the most correlated with the spreading capacity in all cases, whereas the k-core yields the smallest correlation for all sets of parameters. On the other hand, the degree is most correlated with the spreading capacity in the SIR model for small values of $\lambda$. In this case, the propagation reaches only the immediate neighbors of the infected node and thus, the nodes with the largest degrees are the most efficient as far as the spreading is concerned. In addition, note that the correlation decreases for $\lambda$ close to one because the epidemics will succeed to reach most of the nodes, regardless of the initial seeded node. For the rumor spreading, 
Figure 38 - Correlation between spreading capacity and the degree, $k$, k-core index, $k_{c}$, and accessibility, $\alpha$, covering all possible combinations of parameters $((\lambda, \delta)$ for epidemic spreading (SIR model) and $(\lambda, \alpha)$ for rumor propagation (MT-TP model), for the email contact network and political blogs (polblogs).

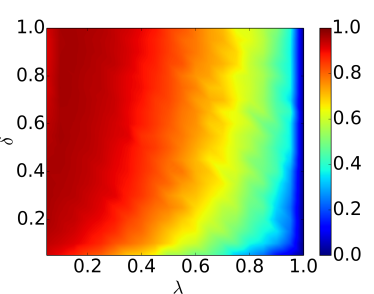

(a) Email: $\operatorname{SIR}(\alpha)$

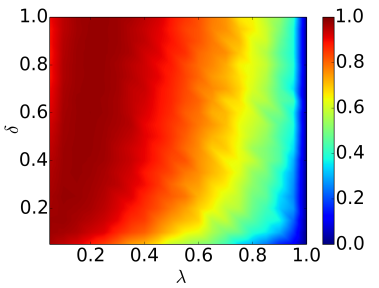

(e) Email: SIR (k)

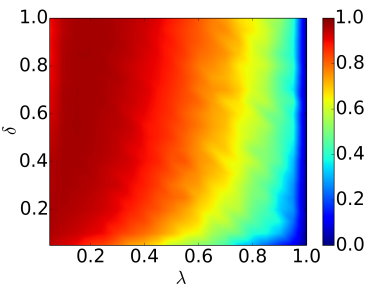

(i) Email: $\operatorname{SIR}\left(k_{c}\right)$

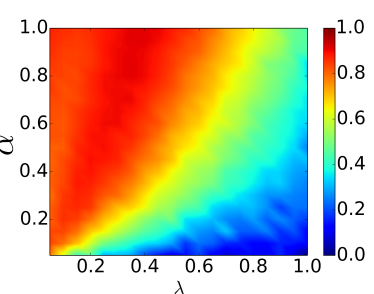

(b) Email: MT-TP $(\alpha)$

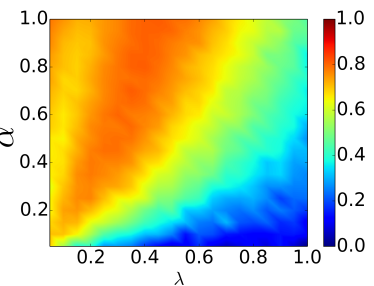

(f) Email: MT-TP $(k)$

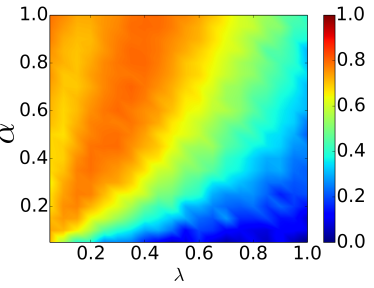

(j) Email: MT-TP $\left(k_{c}\right)$

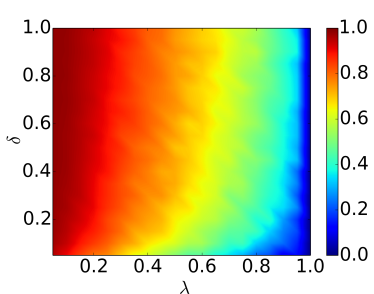

(c) polblogs: SIR $(\alpha)$

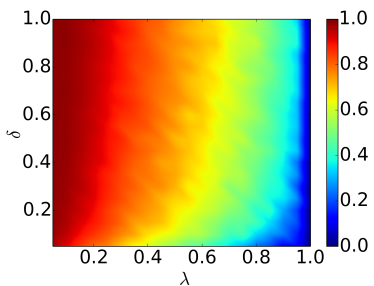

(g) polblogs: SIR $(k)$

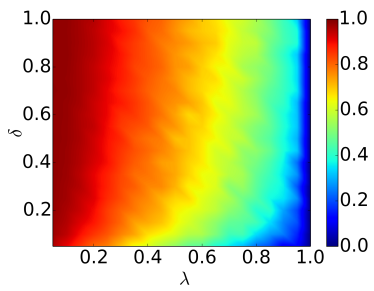

(k) polblogs: SIR $\left(k_{c}\right)$

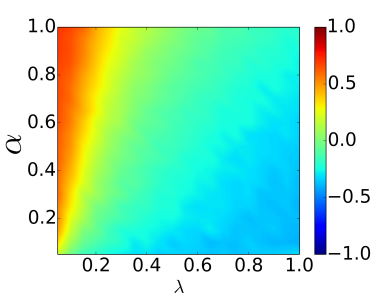

(d) polblogs: MT-TP $(\alpha)$

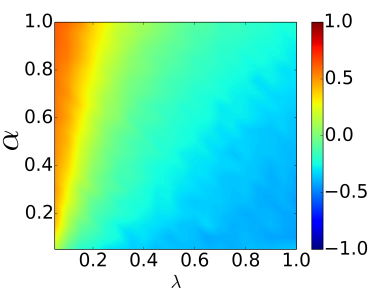

(h) polblogs: MT-TP $(k)$

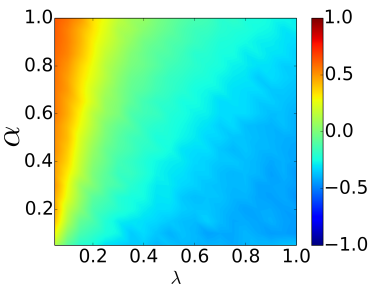

(1) polblogs: MT-TP $\left(k_{c}\right)$

Source: Developed by the author.

the correlation between the accessibility and the final fraction of stiflers is the highest for $\alpha>\lambda$. The same relationship is observed for the degree, but the correlations are smaller than those observed for the accessibility measure.

Figure 38 shows the results for the social networks of email and political blogs cases. The results indicate that the degree, the k-core and the accessibility yield similar correlations, which point out that the central nodes show the highest values of such measures simultaneously. Thus, these networks tend not to have peripheral hubs. The highest correlations occur for small values of $\lambda$, indicating that the infections or rumors do not propagate past the first neighbors of the origin and thus the nodes with the largest degree are the most efficient in spreading. These nodes also present the highest values of accessibility and k-core. For $\lambda$ close to one, the correlation is close to zero, because most of the nodes will become recovered or stifler independently of the initial spreader.

Having completed the previous analysis, we consider in the next sections the following parameter values: (i) $\lambda=0.8, \delta=1.0$ and (ii) $\lambda=0.3, \delta=1.0$ for the epidemic 
Figure 39 - The percentage of recovered individuals in the SIR epidemic spreading model $(\lambda=0.3, \delta=1.0)$ according to the local measures for the US road network: (a) degree; (b) clustering coefficient; (c) average degree of the nearest neighbors; (d) betweenness centrality; (e) PageRank; (f) k-core index; (g) eigenvector centrality and (h) closeness centrality.

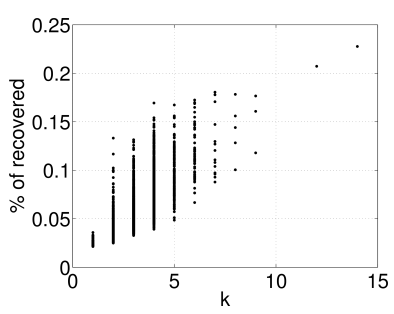

(a)

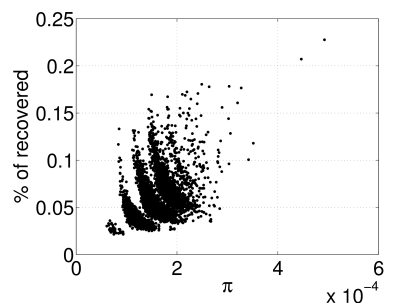

(e)

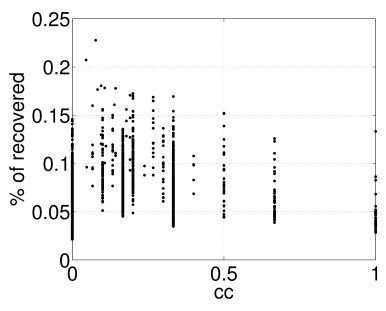

(b)

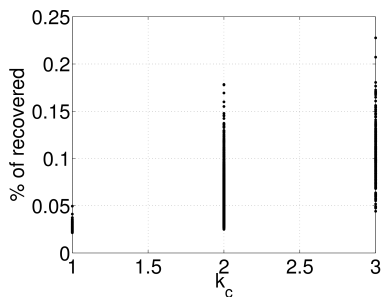

(f)

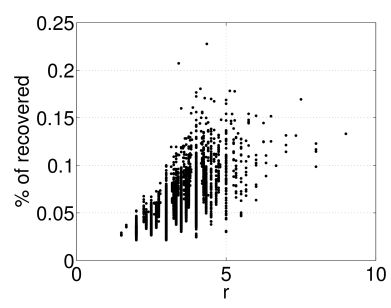

(c)

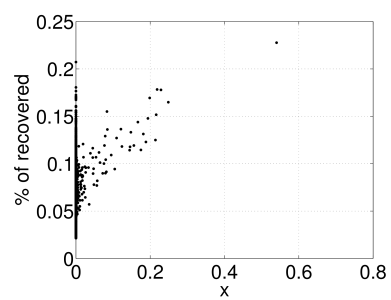

(g)

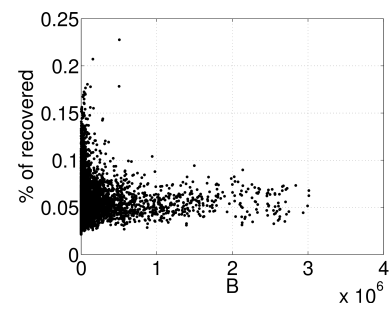

(d)

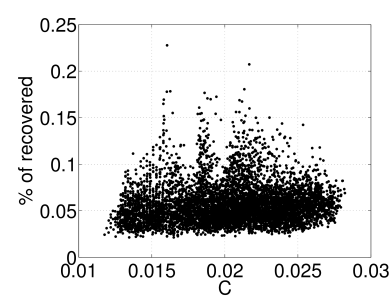

(h)

Source: Developed by the author.

spreading and (i) $\lambda=0.8, \alpha=1.0$, (ii) $\lambda=0.8, \alpha=0.3$, (iii) $\lambda=0.3, \alpha=1.0$ and (iv) $\lambda=0.3, \alpha=0.3$ for the rumor dynamics.. Note that previous investigations used $\alpha=1$ (KITSAK et al., 2010) and $\delta=1$ (BORGE-HOLTHOEFER; MORENO, 2012) for epidemic and rumor spreading, respectively. Here, we consider also the cases in which the spreading rate is higher than the stifling rate and one situation in which both have the same values. These values represent the cases in which the dynamics are dependent on the network structure, i.e., correlations are higher than zero, as we can see in Figs 37 and 38 .

\subsubsection{Spatial networks}

As outlined in Section 2.1.2.1, we have studied different centrality metrics: the degree $(k)$, clustering coefficient $(c c)$, betweenness centrality $(B)$, average neighborhood degree $(r)$, PageRank $(\pi)$, eigenvector centrality $(x)$, k-core index $\left(k_{c}\right)$, closeness centrality $(C)$ and accessibility $(\alpha)$. We have considered only the unweighted and undirected versions of these measures. Table 3 presents the average values obtained for the road maps and networks generated by the Waxman and scale-free spatial models. Spatial networks are sparse, have large characteristic path lengths and non-zero clustering coefficients. In addition, scale-free spatial networks have the smallest average geodesic distance due to the presence of hubs. 
Figure 40 - The percentage of stiflers on the MT (TP) rumor model $(\lambda=0.3, \alpha=1.0)$ according to the local measures for the United States network: (a) degree; (b) clustering coefficient; (c) average degree of the nearest neighbors; (d) betweenness centrality; (e) PageRank; (f) k-core index; (g) eigenvector centrality and (h) closeness centrality.

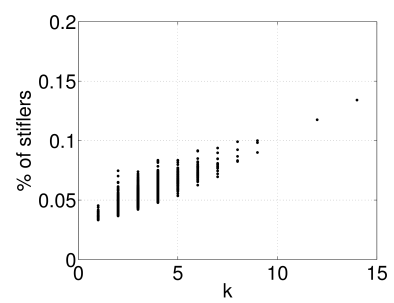

(a)

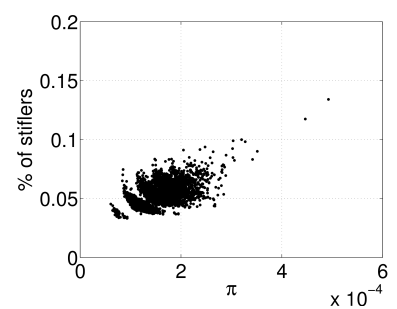

(e)

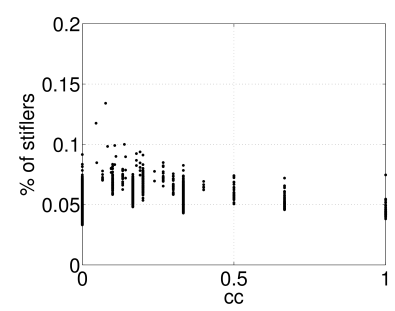

(b)

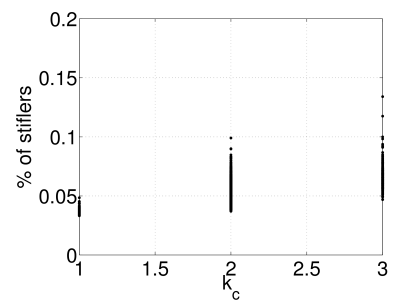

(f)

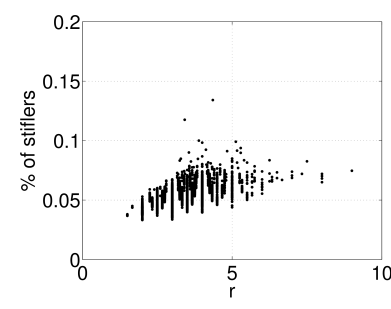

(c)

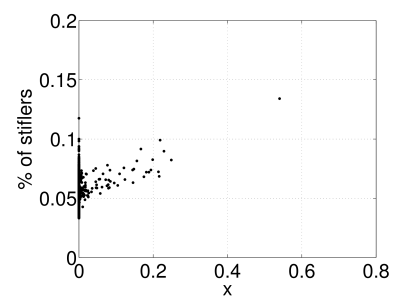

$(\mathrm{g})$

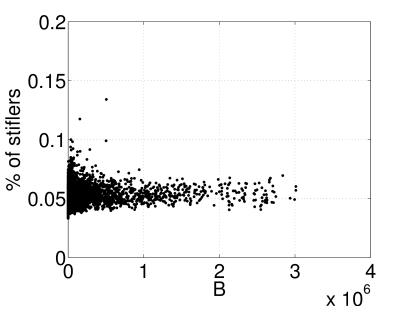

(d)

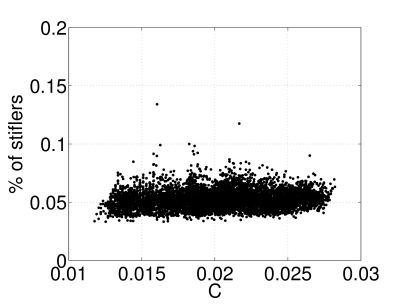

(h)

Source: Developed by the author.

Figure 41 - The percentage of recovered individuals on the SIR epidemic spreading model $(\boldsymbol{\lambda}=$ $0.3, \delta=1.0)$ according to the accessibility measure for the road networks of (a) Japan, (b) England, (c) United States and (d) Germany.

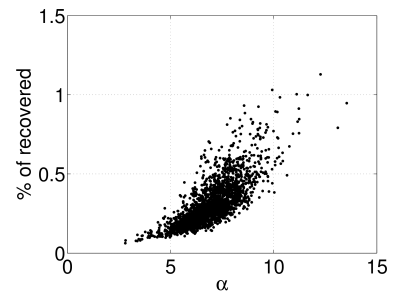

(a)

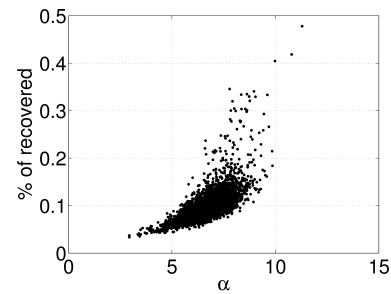

(b)

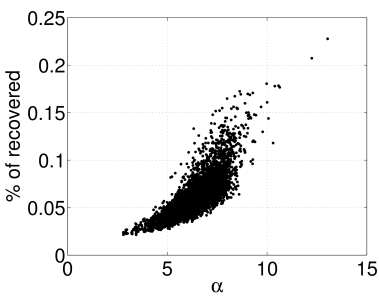

(c)

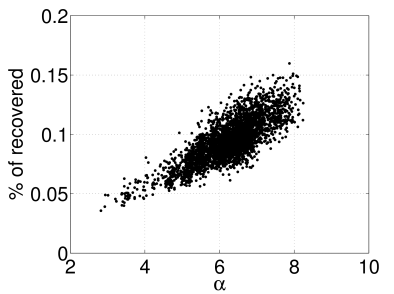

(d)

Source: Developed by the author.

We have conducted numerical simulations of the SIR (epidemic) and MT (rumor) models to inspect correlations between nodes' centrality (as given by the different metrics above) and the final dynamical outcome of the system, the latter being measured by the density of removed and stiflers after the dynamics have come to an end, respectively. These correlations have been determined by the Spearman rank correlation coefficient, which is defined as the Pearson correlation coefficient between the ranked variables (WOLFE; HOLLANDER, 1973). The reason for our choice is that the Spearman coefficient quantifies monotonic relationships, whereas the Pearson correlation measures linear relationships. As shown below, these correlations can be monotonic, but not necessarily linear. 
Figure 42 - The percentage of stiflers on the MT (TP) rumor model $(\lambda=0.3, \alpha=1.0)$ according to the accessibility measure for the road networks of (a) Japan, (b) England, (c) United States and (d) Germany.

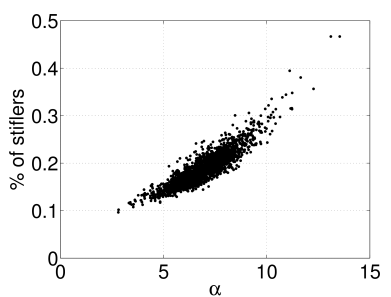

(a)

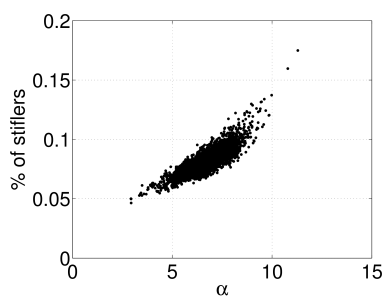

(b)

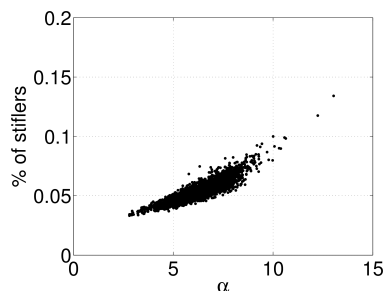

(c)

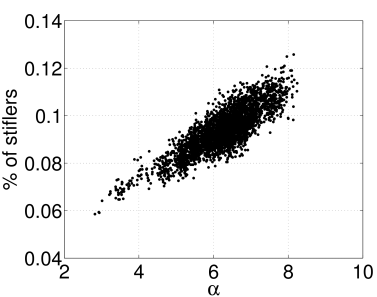

(d)

Source: Developed by the author.

Figures 39 and 40 show the scatter plots for the epidemic and rumor dynamics in the US road network, respectively. The strongest correlation corresponds to the degree centrality, while for other metrics, correlations are weak and positive, though not zero. On the contrary, the clustering coefficient leads to a negative correlation because the more central a node is, the smaller its clustering coefficient is. On the other hand, Figures 41 and 42 show that the correlations between the generalized random walk accessibility and the potential of rumor and epidemic spreading processes are almost linear and positive for all road networks analyzed.

Furthermore, Table 4 shows that for both spreading processes, the highest correlations between a centrality measure and the impact of the disease or rumor correspond to the case of the generalized accessibility centrality, whose values are often higher than 0.7. Interestingly, the $k$-core centrality yields small correlation values, contrary to what was observed in (KITSAK et al., 2010), which considered networks not embedded in space. However, this result agrees with (BORGE-HOLTHOEFER; MORENO, 2012), in the case of rumor dynamics. The node degree is highly correlated with the final fraction of recovered nodes, but less if we look at the results corresponding to the final fraction of stiflers, mainly for the case of an MT model simulated using a contact process setting, again as found in (BORGE-HOLTHOEFER; MORENO, 2012). Moreover, PageRank, closeness and betweenness centrality metrics do not show significant correlations with disease and rumor spreading capabilities - except when the parameter $\alpha$ in rumor models is small, in which case the closeness gives high correlation. It is also worth noticing that the eigenvector centrality shows high correlation only for the spatial scale-free network model.

\subsubsection{Road networks}

Focusing on real networks, Figure 43 shows results obtained for the generalized random walk accessibility of each node for the road networks of Japan, England, United 
Table 4 - Correlation between centrality measures and the final fraction of recovered individuals (SIR model) or the final fraction of stiflers (MT model for the contact (CP) or truncated (TP) cases). The measures are the degree $(k)$, clustering coefficient $(c c)$, betweenness centrality $(B)$, average neighborhood degree $(r)$, PageRank $(\pi)$, eigenvector centrality $(x)$, k-core index $\left(k_{c}\right)$, closeness centrality $(C)$ and accessibility $(\alpha)$. The highest correlations are in bold.

\begin{tabular}{|c|c|c|c|c|c|c|c|c|c|c|c|}
\hline Process & Rates & Network & $k$ & $c c$ & $B$ & $r$ & $\pi$ & $x$ & $k_{c}$ & $C$ & $\alpha$ \\
\hline \multirow{12}{*}{ SIR } & \multirow{6}{*}{$\lambda=0.8, \delta=1.0$} & Japan & 0.40 & 0.11 & 0.24 & 0.26 & 0.27 & 0.27 & 0.35 & 0.47 & 0.47 \\
\hline & & England & 0.55 & 0.10 & 0.26 & 0.38 & 0.30 & -0.04 & 0.21 & 0.27 & 0.58 \\
\hline & & USA & 0.60 & 0.25 & 0.19 & 0.53 & 0.28 & 0.49 & 0.26 & 0.41 & 0.73 \\
\hline & & Germany & 0.54 & 0.05 & 0.42 & 0.35 & 0.20 & 0.22 & 0.19 & 0.34 & 0.63 \\
\hline & & Spatial SF & 0.81 & 0.25 & 0.65 & -0.04 & 0.75 & 0.35 & - & 0.32 & 0.60 \\
\hline & & Waxman & 0.74 & 0.34 & 0.47 & 0.29 & 0.62 & 0.05 & 0.70 & 0.19 & 0.73 \\
\hline & \multirow{6}{*}{$\lambda=0.3, \delta=1.0$} & Japan & 0.65 & 0.30 & 0.31 & 0.65 & 0.37 & 0.36 & 0.65 & 0.41 & 0.79 \\
\hline & & England & 0.68 & 0.13 & 0.27 & 0.61 & 0.31 & -0.07 & 0.38 & 0.21 & 0.76 \\
\hline & & USA & 0.77 & 0.38 & 0.10 & 0.68 & 0.38 & 0.59 & 0.37 & 0.14 & 0.86 \\
\hline & & Germany & 0.69 & 0.08 & 0.46 & 0.42 & 0.31 & 0.22 & 0.19 & 0.25 & 0.74 \\
\hline & & Spatial SF & 0.70 & 0.39 & 0.70 & 0.46 & 0.49 & 0.72 & - & 0.66 & 0.91 \\
\hline & & Waxman & 0.68 & 0.30 & 0.42 & 0.58 & 0.45 & 0.04 & 0.72 & 0.30 & 0.81 \\
\hline \multirow{24}{*}{ MT-TP } & \multirow{6}{*}{$\lambda=0.8, \alpha=1.0$} & Japan & 0.50 & -0.08 & 0.43 & 0.48 & 0.29 & 0.33 & 0.45 & 0.42 & 0.81 \\
\hline & & England & 0.54 & -0.20 & 0.43 & 0.49 & 0.22 & -0.03 & 0.34 & 0.32 & 0.85 \\
\hline & & USA & 0.67 & 0.11 & 0.22 & 0.62 & 0.30 & 0.55 & 0.33 & 0.24 & 0.90 \\
\hline & & Germany & 0.57 & -0.24 & 0.62 & 0.41 & 0.20 & 0.24 & 0.18 & 0.33 & 0.88 \\
\hline & & Spatial SF & 0.63 & 0.29 & 0.71 & 0.55 & 0.40 & 0.75 & - & 0.70 & 0.94 \\
\hline & & Waxman & 0.56 & 0.06 & 0.52 & 0.46 & 0.37 & 0.04 & 0.58 & 0.45 & 0.76 \\
\hline & \multirow{6}{*}{$\lambda=0.8, \alpha=0.3$} & Japan & 0.17 & -0.02 & 0.22 & 0.23 & 0.04 & 0.26 & 0.21 & 0.66 & 0.35 \\
\hline & & England & 0.32 & -0.07 & 0.31 & 0.38 & 0.05 & 0.05 & 0.26 & 0.60 & 0.53 \\
\hline & & USA & 0.26 & 0.00 & 0.25 & 0.29 & 0.07 & 0.08 & 0.12 & 0.83 & 0.43 \\
\hline & & Germany & 0.29 & -0.12 & 0.46 & 0.28 & 0.01 & 0.45 & 0.17 & 0.67 & 0.52 \\
\hline & & Spatial SF & 0.40 & 0.16 & 0.43 & 0.28 & 0.27 & 0.41 & - & 0.37 & 0.53 \\
\hline & & Waxman & 0.61 & 0.13 & 0.51 & 0.38 & 0.47 & 0.06 & 0.62 & 0.31 & 0.74 \\
\hline & \multirow{6}{*}{$\lambda=0.3, \alpha=1.0$} & Japan & 0.77 & 0.22 & 0.43 & 0.61 & 0.54 & 0.25 & 0.59 & 0.28 & 0.88 \\
\hline & & England & 0.77 & 0.03 & 0.34 & 0.53 & 0.47 & -0.07 & 0.30 & 0.16 & 0.83 \\
\hline & & USA & 0.84 & 0.32 & 0.19 & 0.63 & 0.50 & 0.56 & 0.35 & 0.13 & 0.91 \\
\hline & & Germany & 0.73 & 0.01 & 0.45 & 0.40 & 0.39 & 0.17 & 0.19 & 0.20 & 0.79 \\
\hline & & Spatial SF & 0.34 & 0.32 & 0.53 & 0.71 & 0.12 & 0.89 & - & 0.84 & 0.77 \\
\hline & & Waxman & 0.84 & 0.30 & 0.56 & 0.59 & 0.64 & 0.06 & 0.77 & 0.25 & 0.94 \\
\hline & \multirow{6}{*}{$\lambda=0.3, \alpha=0.3$} & Japan & 0.37 & 0.00 & 0.32 & 0.50 & 0.13 & 0.35 & 0.42 & 0.49 & 0.68 \\
\hline & & England & 0.42 & -0.09 & 0.34 & 0.52 & 0.07 & 0.01 & 0.37 & 0.38 & 0.71 \\
\hline & & USA & 0.54 & 0.12 & 0.15 & 0.64 & 0.16 & 0.54 & 0.33 & 0.28 & 0.80 \\
\hline & & Germany & 0.42 & -0.20 & 0.54 & 0.41 & 0.06 & 0.29 & 0.18 & 0.39 & 0.73 \\
\hline & & Spatial SF & 0.42 & 0.31 & 0.53 & 0.62 & 0.19 & 0.71 & - & 0.65 & 0.84 \\
\hline & & Waxman & 0.44 & 0.08 & 0.41 & 0.46 & 0.25 & 0.09 & 0.51 & 0.55 & 0.64 \\
\hline \multirow{24}{*}{ MT-CP } & \multirow{6}{*}{$\lambda=0.8, \alpha=1.0$} & Japan & 0.42 & 0.05 & 0.30 & 0.57 & 0.16 & 0.32 & 0.48 & 0.42 & 0.73 \\
\hline & & England & 0.43 & -0.10 & 0.32 & 0.56 & 0.08 & -0.05 & 0.36 & 0.32 & 0.73 \\
\hline & & USA & 0.57 & 0.16 & 0.13 & 0.682 & 0.17 & 0.55 & 0.34 & 0.24 & 0.82 \\
\hline & & Germany & 0.45 & -0.18 & 0.52 & 0.44 & 0.07 & 0.26 & 0.18 & 0.35 & 0.75 \\
\hline & & Spatial SF & 0.26 & 0.27 & 0.42 & 0.69 & 0.02 & 0.75 & - & 0.71 & 0.73 \\
\hline & & Waxman & 0.50 & 0.13 & 0.39 & 0.57 & 0.27 & 0.04 & 0.59 & 0.38 & 0.72 \\
\hline & \multirow{6}{*}{$\lambda=0.8, \alpha=0.3$} & Japan & 0.17 & 0.01 & 0.18 & 0.28 & 0.01 & 0.27 & 0.26 & 0.67 & 0.36 \\
\hline & & England & 0.24 & 0.00 & 0.24 & 0.37 & -0.03 & 0.16 & 0.29 & 0.70 & 0.43 \\
\hline & & USA & 0.29 & 0.04 & 0.20 & 0.36 & 0.04 & 0.20 & 0.16 & 0.74 & 0.47 \\
\hline & & Germany & 0.20 & -0.07 & 0.41 & 0.24 & -0.05 & 0.51 & 0.16 & 0.81 & 0.40 \\
\hline & & Spatial SF & 0.28 & 0.22 & 0.36 & 0.46 & 0.10 & 0.48 & - & 0.44 & 0.61 \\
\hline & & Waxman & 0.54 & 0.16 & 0.44 & 0.55 & 0.34 & 0.10 & 0.64 & 0.44 & 0.76 \\
\hline & \multirow{6}{*}{$\lambda=0.3, \alpha=1.0$} & Japan & 0.60 & 0.18 & 0.36 & 0.66 & 0.34 & 0.28 & 0.56 & 0.31 & 0.82 \\
\hline & & England & 0.58 & -0.02 & 0.31 & 0.60 & 0.25 & -0.10 & 0.31 & 0.18 & 0.77 \\
\hline & & USA & 0.68 & 0.26 & 0.12 & 0.71 & 0.30 & 0.56 & 0.35 & 0.14 & 0.85 \\
\hline & & Germany & 0.55 & -0.10 & 0.45 & 0.47 & 0.20 & 0.17 & 0.18 & 0.21 & 0.74 \\
\hline & & Spatial SF & 0.23 & 0.27 & 0.42 & 0.71 & 0.01 & 0.81 & - & 0.77 & 0.70 \\
\hline & & Waxman & 0.68 & 0.24 & 0.45 & 0.67 & 0.45 & 0.06 & 0.72 & 0.26 & 0.87 \\
\hline & \multirow{6}{*}{$\lambda=0.3, \alpha=0.3$} & Japan & 0.37 & 0.03 & 0.27 & 0.54 & 0.12 & 0.33 & 0.45 & 0.46 & 0.68 \\
\hline & & England & 0.40 & -0.08 & 0.32 & 0.53 & 0.06 & -0.01 & 0.36 & 0.36 & 0.69 \\
\hline & & USA & 0.54 & 0.14 & 0.13 & 0.65 & 0.14 & 0.53 & 0.33 & 0.27 & 0.79 \\
\hline & & Germany & 0.40 & -0.20 & 0.52 & 0.43 & 0.03 & 0.27 & 0.18 & 0.39 & 0.72 \\
\hline & & Spatial SF & 0.27 & 0.27 & 0.43 & 0.67 & 0.03 & 0.71 & - & 0.66 & 0.75 \\
\hline & & Waxman & 0.44 & 0.10 & 0.38 & 0.51 & 0.23 & 0.05 & 0.53 & 0.46 & 0.65 \\
\hline
\end{tabular}

States and Germany. In Japan, the most influential spreaders are the cities of Nagoya, Osaka and Hiroshima. Tokyo is highly connected but does not have the same spreading capability of these cities, since it is a peripheral hub. London, Liverpool and Manchester have the highest values of accessibility in England, while in the US, the cities with the highest accessibility are New York, Houston, Dallas and Chicago - interestingly enough, 
Figure 43 - Network visualization of the real road networks of (a) Japan, (b) England, (c) United States and (d) Germany. The colors represent the values of the accessibility.

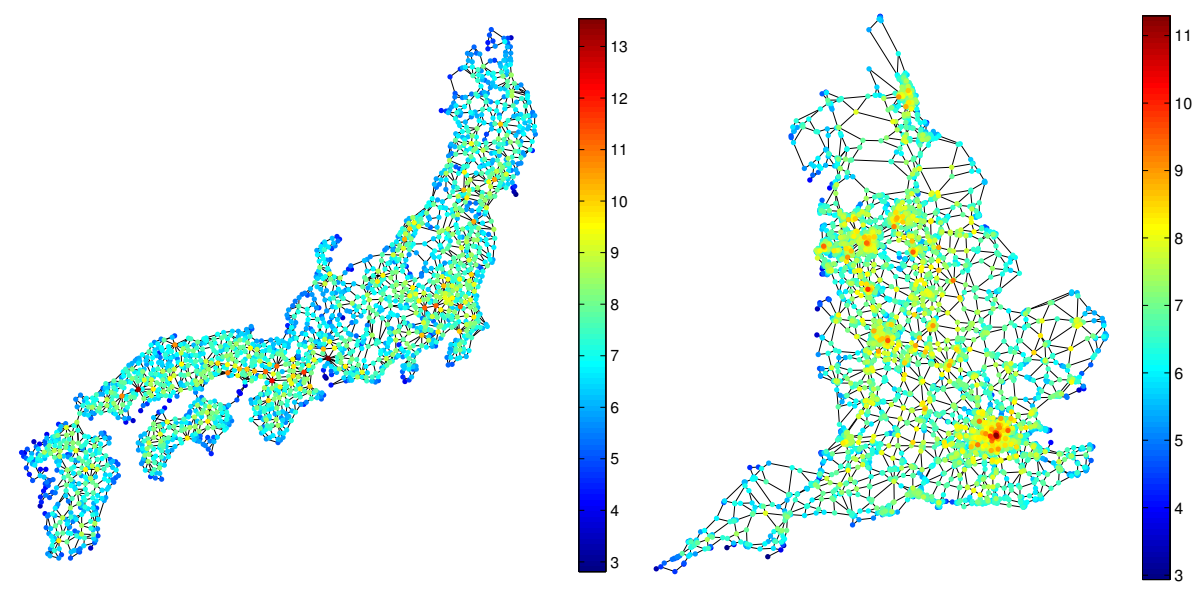

(a)

(b)

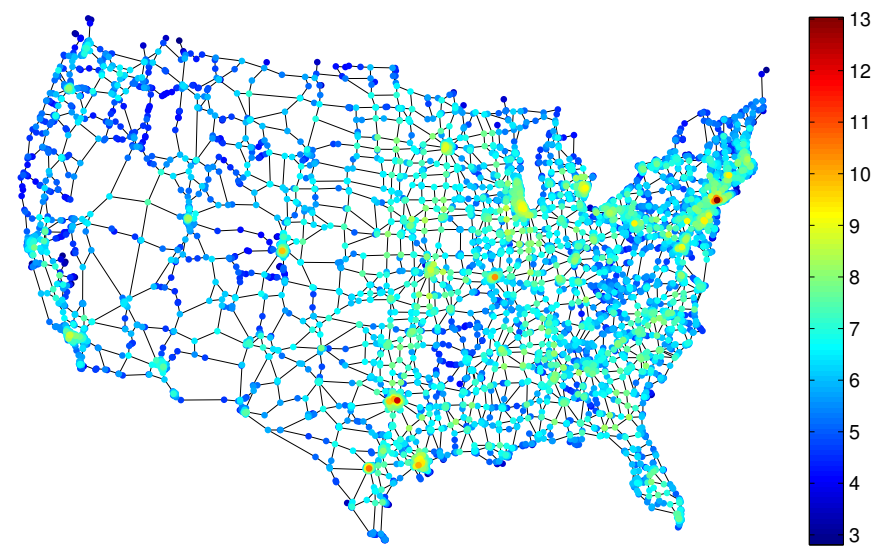

(c)

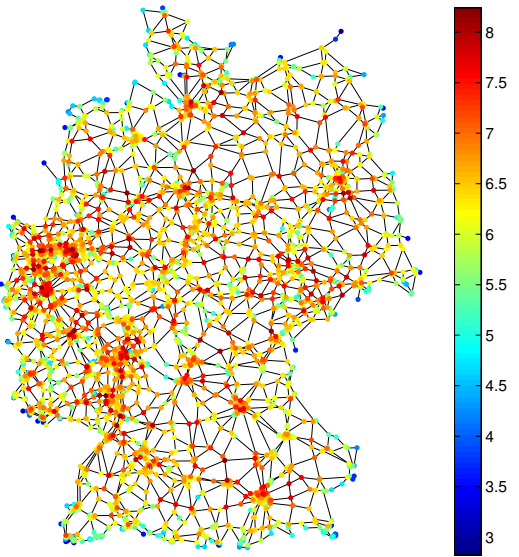

(d)

Source: Developed by the author.

these cities are also air transportation hubs. Finally, Berlin, München and Düsseldorf have the highest accessibility in Germany. Note that nodes at the border of the countries present the smallest values of accessibility. Therefore, this measure can be considered to identify the borders of networks, as previously pointed out for the original definition of accessibility in (TRAVENCOLO; VIANA; COSTA, 2009).

Figure 44 presents the probability distribution of the accessibility. For all cases, the distribution is asymmetric, presenting a long tail for higher values of accessibility, and centered at the same value. It is interesting to note that Germany and England have the smallest variation in the accessibility, whereas Japan has the highest one. This fact can be related to Japan's uneven terrain, which directly influences how the highways are distributed. 
Figure 44 - Probability distribution of the accessibility in the road networks of Japan, Germany, US and England.

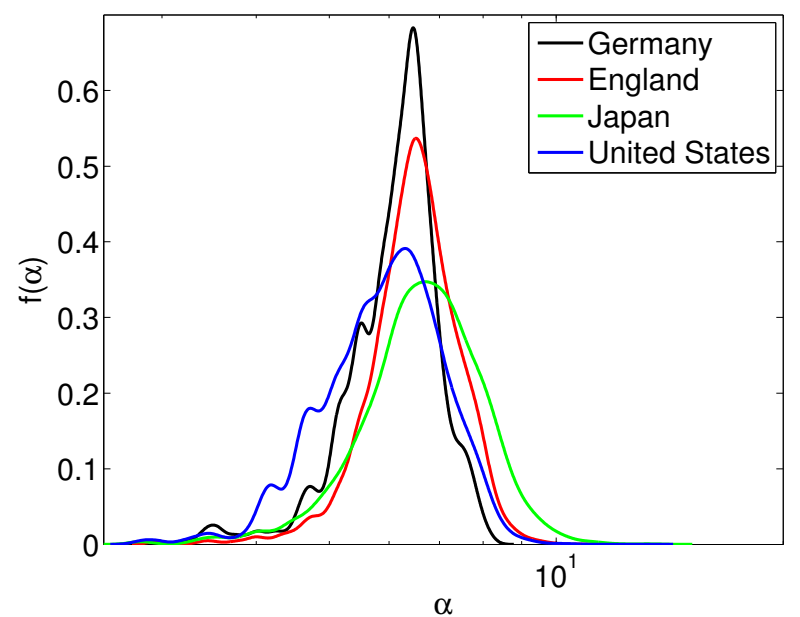

Source: Developed by the author.

Figure 45 - Probability distribution of the accessibility in the social networks of advogato, email, political blogs and Google+.

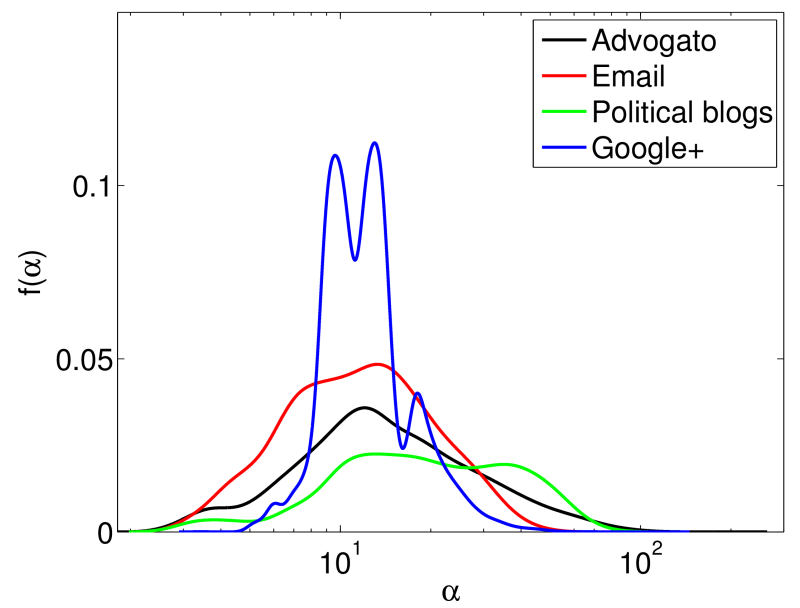

Source: Developed by the author.

\subsubsection{Non-spatial networks}

We have also studied what happens to non-spatial networks using the same set of measurements considered in Secs. 2.1.4.3 and 2.1.2.1. Table 3 presents the average values of these measures calculated in the social networks and in synthetic BA networks. Table 5 presents the Spearman correlation coefficient calculated between the centrality metrics and the final fraction of stiflers or recovered nodes in the epidemic and rumor processes, respectively. The results agree with the analysis of epidemic spreading presented in (KITSAK et al., 2010) and with the study of rumor diffusion in (BORGE-HOLTHOEFER; MORENO, 2012). In the case of the SIR model, the $k$-core and degree centralities are the most correlated with the final fraction of recovered nodes. Thus, the main hubs in the 
Table 5 - Correlation between centrality measures and the final fraction of stiflers (MT model for the contact $(\mathrm{CP})$ or truncated (TP) cases) or recovered individuals (SIR model). The measures are the degree $(k)$, clustering coefficient $(c c)$, betweenness centrality $(B$, average neighborhood degree $(r)$, PageRank $(\pi)$, eigenvector centrality $(x)$, k-core index $\left(k_{c}\right)$, closeness centrality $(C)$ and accessibility $(\alpha)$. The highest correlations are in bold.

\begin{tabular}{|c|c|c|c|c|c|c|c|c|c|c|c|}
\hline Process & Rates & Network & $k$ & $c c$ & $B$ & $r$ & $\pi$ & $x$ & $k_{c}$ & $C$ & $\alpha$ \\
\hline \multirow{10}{*}{ SIR } & \multirow{5}{*}{$\lambda=0.8 . \delta=1.0$} & advogato & 0.76 & 0.47 & 0.66 & 0.14 & 0.71 & 0.65 & 0.76 & 0.63 & 0.70 \\
\hline & & email & 0.67 & 0.41 & 0.57 & 0.09 & 0.64 & 0.58 & 0.67 & 0.57 & 0.63 \\
\hline & & polblogs & 0.57 & 0.29 & 0.51 & 0.04 & 0.55 & 0.55 & 0.57 & 0.55 & 0.57 \\
\hline & & Google+ & 0.81 & 0.67 & 0.61 & 0.17 & 0.64 & 0.50 & 0.81 & 0.48 & 0.63 \\
\hline & & BA & 0.19 & 0.13 & 0.39 & 0.48 & -0.02 & 0.45 & - & 0.49 & 0.60 \\
\hline & \multirow{5}{*}{$\lambda=0.3 . \delta=1.0$} & advogato & 0.97 & 0.40 & 0.85 & 0.19 & 0.92 & 0.88 & 0.97 & 0.84 & 0.92 \\
\hline & & email & 0.97 & 0.34 & 0.85 & 0.25 & 0.94 & 0.88 & 0.96 & 0.89 & 0.94 \\
\hline & & polblogs & 0.89 & 0.25 & 0.78 & -0.08 & 0.86 & 0.85 & 0.89 & 0.82 & 0.88 \\
\hline & & Google+ & 0.81 & 0.67 & 0.61 & 0.23 & 0.60 & 0.56 & 0.81 & 0.54 & 0.68 \\
\hline & & BA & 0.18 & 0.18 & 0.48 & 0.73 & -0.10 & 0.66 & - & 0.72 & 0.77 \\
\hline \multirow{20}{*}{ MT-TP } & \multirow{5}{*}{$\lambda=0.8 . \alpha=1.0$} & advogato & 0.23 & 0.17 & 0.26 & 0.55 & 0.17 & 0.38 & 0.22 & 0.43 & 0.35 \\
\hline & & email & 0.62 & 0.19 & 0.61 & 0.13 & 0.60 & 0.56 & 0.58 & 0.60 & 0.64 \\
\hline & & polblogs & -0.16 & -0.09 & -0.06 & 0.35 & -0.17 & -0.16 & -0.21 & -0.07 & -0.13 \\
\hline & & Google+ & 0.04 & 0.03 & 0.00 & 0.78 & -0.07 & 0.27 & 0.04 & 0.25 & 0.40 \\
\hline & & BA & 0.16 & 0.19 & 0.48 & 0.76 & -0.12 & 0.70 & - & 0.75 & 0.78 \\
\hline & \multirow{5}{*}{$\lambda=0.8 \cdot \alpha=0.3$} & advogato & 0.05 & 0.15 & 0.10 & 0.51 & 0.02 & 0.20 & 0.05 & 0.27 & 0.16 \\
\hline & & email & 0.29 & 0.22 & 0.30 & 0.06 & 0.28 & 0.24 & 0.26 & 0.28 & 0.30 \\
\hline & & polblogs & -0.37 & -0.01 & -0.26 & 0.48 & -0.37 & -0.34 & -0.39 & -0.22 & -0.33 \\
\hline & & Google+ & 0.00 & 0.03 & -0.05 & 0.69 & 0.01 & 0.11 & 0.004 & 0.09 & 0.27 \\
\hline & & BA & 0.14 & 0.19 & 0.47 & 0.77 & -0.11 & 0.79 & - & 0.82 & 0.72 \\
\hline & \multirow{5}{*}{$\lambda=0.3 . \alpha=1.0$} & advogato & 0.54 & 0.11 & 0.47 & 0.64 & 0.45 & 0.74 & 0.55 & 0.76 & 0.73 \\
\hline & & email & 0.77 & 0.03 & 0.71 & 0.59 & 0.70 & 0.89 & 0.76 & 0.91 & 0.89 \\
\hline & & polblogs & 0.19 & 0.05 & 0.19 & 0.41 & 0.17 & 0.19 & 0.16 & 0.31 & 0.26 \\
\hline & & Google+ & 0.20 & 0.17 & 0.14 & 0.84 & -0.12 & 0.63 & 0.20 & 0.61 & 0.65 \\
\hline & & BA & 0.35 & 0.11 & 0.46 & 0.34 & 0.20 & 0.47 & - & 0.48 & 0.51 \\
\hline & \multirow{5}{*}{$\lambda=0.3 \cdot \alpha=0.3$} & advogato & 0.36 & 0.19 & 0.31 & 0.57 & 0.29 & 0.53 & 0.37 & 0.57 & 0.52 \\
\hline & & email & 0.70 & 0.19 & 0.62 & 0.35 & 0.65 & 0.71 & 0.70 & 0.74 & 0.77 \\
\hline & & polblogs & -0.16 & 0.09 & -0.12 & 0.52 & -0.18 & -0.13 & -0.18 & -0.02 & -0.10 \\
\hline & & Google+ & 0.14 & 0.16 & 0.05 & 0.76 & -0.01 & 0.36 & 0.15 & 0.34 & 0.47 \\
\hline & & BA & 0.33 & 0.19 & 0.59 & 0.67 & 0.06 & 0.67 & - & 0.73 & 0.85 \\
\hline \multirow{20}{*}{ MT-CP } & \multirow{5}{*}{$\lambda=0.8 . \alpha=1.0$} & advogato & 0.47 & 0.14 & 0.35 & 0.52 & 0.36 & 0.63 & 0.50 & 0.62 & 0.65 \\
\hline & & email & 0.69 & 0.19 & 0.56 & 0.57 & 0.61 & 0.81 & 0.73 & 0.79 & 0.81 \\
\hline & & polblogs & 0.29 & 0.14 & 0.21 & 0.26 & 0.25 & 0.28 & 0.29 & 0.34 & 0.34 \\
\hline & & Google+ & 0.40 & 0.32 & 0.31 & 0.55 & -0.12 & 0.84 & 0.40 & 0.76 & 0.75 \\
\hline & & BA & 0.56 & 0.19 & 0.78 & 0.63 & 0.30 & 0.74 & - & 0.80 & 0.94 \\
\hline & \multirow{5}{*}{$\lambda=0.8 . \alpha=0.3$} & advogato & 0.29 & 0.14 & 0.21 & 0.35 & 0.21 & 0.38 & 0.31 & 0.39 & 0.41 \\
\hline & & email & 0.45 & 0.22 & 0.36 & 0.32 & 0.40 & 0.48 & 0.47 & 0.50 & 0.52 \\
\hline & & polblogs & 0.01 & 0.14 & -0.01 & 0.26 & -0.03 & 0.02 & 0.00 & 0.07 & 0.05 \\
\hline & & Google+ & 0.32 & 0.27 & 0.23 & 0.52 & -0.10 & 0.64 & 0.33 & 0.60 & 0.64 \\
\hline & & BA & 0.24 & 0.21 & 0.58 & 0.80 & -0.02 & 0.87 & - & 0.91 & 0.79 \\
\hline & \multirow{5}{*}{$\lambda=0.3 \cdot \alpha=1.0$} & advogato & 0.52 & 0.08 & 0.39 & 0.59 & 0.40 & 0.72 & 0.55 & 0.71 & 0.73 \\
\hline & & email & 0.64 & 0.05 & 0.55 & 0.68 & 0.55 & 0.83 & 0.67 & 0.82 & 0.80 \\
\hline & & polblogs & 0.51 & 0.14 & 0.39 & 0.25 & 0.46 & 0.52 & 0.51 & 0.56 & 0.57 \\
\hline & & Google+ & 0.37 & 0.28 & 0.32 & 0.55 & -0.15 & 0.89 & 0.38 & 0.84 & 0.75 \\
\hline & & BA & 0.82 & 0.10 & 0.67 & -0.01 & 0.74 & 0.31 & - & 0.34 & 0.52 \\
\hline & \multirow{5}{*}{$\lambda=0.3 \cdot \alpha=0.3$} & advogato & 0.46 & 0.15 & 0.34 & 0.50 & 0.35 & 0.60 & 0.48 & 0.60 & 0.63 \\
\hline & & email & 0.68 & 0.23 & 0.55 & 0.50 & 0.60 & 0.76 & 0.71 & 0.76 & 0.79 \\
\hline & & polblogs & 0.19 & 0.14 & 0.13 & 0.27 & 0.15 & 0.18 & 0.18 & 0.24 & 0.23 \\
\hline & & Google+ & 0.39 & 0.32 & 0.30 & 0.55 & -0.11 & 0.80 & 0.40 & 0.74 & 0.74 \\
\hline & & BA & 0.67 & 0.19 & 0.79 & 0.49 & 0.43 & 0.66 & - & 0.72 & 0.89 \\
\hline
\end{tabular}

social networks are located in the center of the network, because they have the highest coreness, suggesting that such networks tend not to present peripheral hubs. Moreover, correlations are stronger when the parameter $\lambda$ is decreased. On the contrary, the random walk accessibility yields the highest Spearman correlation for BA networks and for political blogs (for $\lambda=0.3$ ), although the correlation values are close to those obtained for the degree and $k$-core. All the remainder metrics exhibit smaller correlation coefficients than the $k$-core, $k$ and $\alpha$.

With respect to the rumor dynamics, the $\mathrm{CP}$ and $\mathrm{TP}$ cases present different results. In the first case, the eigenvector and accessibility centralities are strongly correlated with 
Figure 46 - Scatter plots of the accessibility measure and the final percentage of recovered individuals on the SIR epidemic spreading model $(\lambda=0.3, \delta=1.0)$ for the networks of (a) advogato, (b) political blogs, (c) e-mail, (d) Google+.

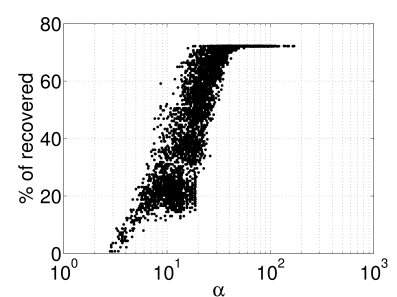

(a)

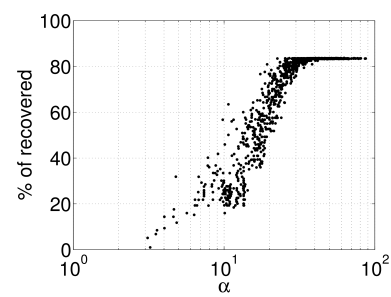

(b)

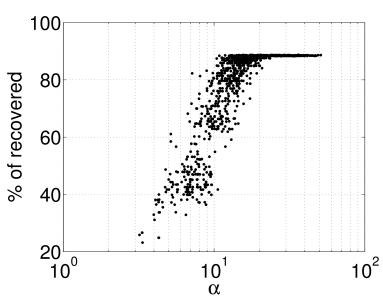

(c)

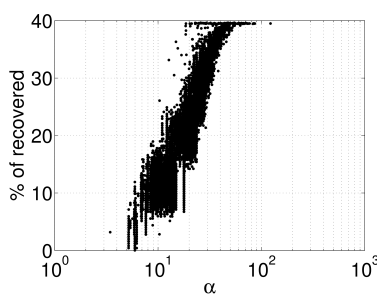

(d)

Source: Developed by the author.

Figure 47 - Scatter plots of the accessibility measure and the final percentage of stiflers on the MT (TP) rumor model $(\lambda=0.3, \alpha=1.0)$ for the networks of (a) advogato, (b) political blogs, (c) e-mail, (d) Google+.

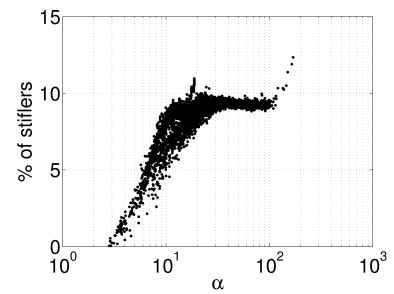

(a)

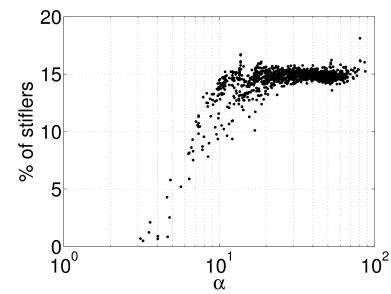

(b)

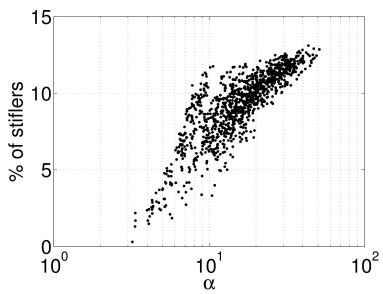

(c)

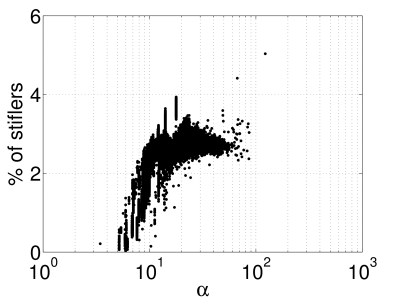

(d)

Source: Developed by the author.

the final fraction of stiflers, whereas, for the second case, closeness centrality and average neighborhood degree show the highest correlations. Considering the TP case with a stifling rate $\lambda=1$, if the spreading rate is high, the average neighborhood degree is more related to the dynamics. However, for lower spreading rates the distance from one node to the rest of the network is more critical. This property is shown in Table 5. Note that $r$ presents higher correlations for higher spreading rates, whereas the closeness centrality is more correlated when spreading rates are smaller. This analysis suggests that shortest paths become more important for information propagation proportionally to the inverse of the spreading rate. Furthermore, the $k$-core and degree centralities have not been found to exhibit strong correlations with the final fraction of stiflers, supporting the results in (BORGE-HOLTHOEFER; MORENO, 2012). Finally, we note that at variance with previous cases, for the rumor dynamics on non-spatial networks, there is no single metric that has yielded the highest correlations for all the networks analyzed. In particular, the accessibility centrality does not seem to be in this case as distinct as before, likely because, as seen in Figure 45, the distributions of accessibility in nonspatial networks are asymmetric, with different mean values and characterized by a long tail distribution. 
Furthermore, we verified that the generalized accessibility is more related to spreading processes in spatial networks than in non-spatial networks. Indeed, Table 5 shows that this metric is the structural property that exhibits the highest correlation in most of the cases when the underlying network is spatial. Figures 46 and 47 show that the relationship between the accessibility and centrality measures are almost linear in spatial networks, whereas, in non-spatial networks, such relationship is also almost linear, but only for values below a given threshold. Beyond that value, the fraction of stiflers and recovered nodes reaches a plateau, which is the maximum value of the dynamic measure in the networks. This plateau reduces the Spearman correlation between the accessibility and the fraction of stiflers since the relationship between these structural and dynamical measures is better defined for low values of accessibility. Therefore, due to the higher distances in spatial networks, the value of accessibility does not saturate (i.e., there is no plateau), resulting in higher correlations.

\subsection{Summary}

We devoted this chapter to study some aspects of single-layer networks epidemiclike processes, basically modeling and influential spreaders identification. In the first section, we focused our study on the theoretical and numerical analysis of the model, and showed that results obtained by numerically solving the system of equations describing the system's dynamics are in good agreement with extensive Monte Carlo simulations for three different scales: macro, micro and temporal. Regarding analytical results, we have obtained closed forms for the thresholds and the steady state densities of individuals in the different dynamical classes for the CP scenario and some special cases of the RP. Additionally, we thoroughly analyzed the influence of the model parameters, as well as several network properties in the spreading dynamics. Our findings indicate that using synthetic networks could help to have a first insight into what the effects are of the different mechanisms at work with a high degree of accuracy. Finally, we studied the propagation of rumors considering real networks on top of which both a contact process and a reactive process might take place. Importantly, we were able to reproduce the time evolution of a rumor propagation using Twitter's user activity during and after the announcement of the discovery of the Higgs boson.

Our formalism is general and covers many models in the literature. This opens up new opportunities to analyze real data. It is also worth stressing that in information spreading in real systems such as online social networks, rumor models play an important role. In this work, we have provided a framework that paves the way to developing new algorithms that could explore accurately and very fast different mechanisms and scenarios for viral information spreading. At variance with disease spreading, in which one is constrained to model a real outbreak when it comes to designing new ways to efficiently 
disseminate information, one is free to design the mechanisms that would optimize such a spreading process. In other words, the viral process can be designed without being constrained to fit a given past or ongoing outbreak. To this end, performing Monte Carlo simulations would be prohibitively costly given the size of the parameters' phase space. This practical hurdle might be surmounted by using the discrete Markov chain approach proposed here, as the computational cost of solving the set of equations is significantly low as compared to Monte Carlo simulations. This is of special relevance when we are dealing with online social systems, whose sizes range from a few hundreds of individuals to millions of users.

On the other hand, in the second section, we studied the relationship between the centrality of a node and the outcome of epidemic and rumor processes initiated in that node by means of extensive numerical simulations on top of several complex networks. We have considered nine network centrality metrics and two different kinds of networks: spatial and non-spatial ones. Networks generated by the Barabási-Albert, Waxman and scale-free spatial models were also considered. We proposed a generalization of the accessibility measure introduced in (TRAVENCOLO; F. Costa, 2008) (for more on its definition, please see Section 2.1.2.3 and also Appendix A), which allows the quantification of the potential of each node in accessing in a balanced and homogeneous manner other nodes. Such generalization takes into account walks of all lengths weighted by the inverse of the factorial of their lengths.

Furthermore, we verified that the generalized accessibility is more related to spreading processes in spatial networks than in non-spatial networks. Indeed, Table 5 shows that this metric is the structural property that exhibits the highest correlation in most of the cases when the underlying network is spatial. Figs. 46 and 47 show that the relationship between the accessibility and centrality measures are almost linear in spatial networks, whereas, in non-spatial networks, this relationship is also almost linear, but only for values below a given threshold. Beyond that value, the fraction of stiflers and recovered nodes reaches a plateau, which is the maximum value of the dynamic measure in the networks. This plateau reduces the Spearman correlation between the accessibility and the fraction of stiflers as the relationship between these structural and dynamical measures is better defined for low values of accessibility. Therefore, due to the higher distances in spatial networks, the value of accessibility does not saturate (i.e., there is no plateau), resulting in higher correlations. The previous conclusions can be understood by looking with more care to the meaning of the new metric discussed here. The definition of the accessibility in terms of random walks is strictly related to the spreading processes (PINTO; THIRAN; VETTERLI, 2012) and it is defined in terms of the diversity index of order one (HILL, 1973). Thus, the higher the number of neighbors that a node can access with similar probability, the higher the expected number of infected nodes. Therefore, the accessibility quantifies how many nodes can be effectively accessed during the spreading 
process.

The goals explored in this chapter motivated us to further study epidemic-like processes on more complex and realistic structures. Furthermore, it motivated us to focus on the structure - dynamics relation, especially on spectral properties of our adjacency matrix. Interestingly, in the first section, 4.1, we related the critical point of a complicated spreading dynamics with the adjacency matrix leading eigenvalue. Additionally, in our second result section, 4.2, we connected the concept of influential spreaders with centrality measures. In this process, we also evaluated the eigenvector centrality, which is deeply connected with the concepts of localization of eigenstates (for more, see Chapter 3 for its definition). Curiously, the correlations between the eigenvector centrality and the final fraction of individuals on the absorbing state are reasonable for various cases, especially regarding SIR on social networks (see Tables 4 and 5). 
CHAPTER

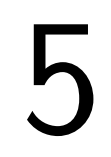

\section{ADVANCES IN MULTILAYER NETWORKS}

The vast majority of studies so far deal with single-layered networks, despite the fact that many real systems exhibit a large degree of interconnectivity and hence should be modeled as multilayer networks (KIVELÄ et al., 2014). These systems represent multimodal, multicategorical or temporal interactions, for instance social relations, the ecosystem formed by different online social networks or modern transportation systems (KIVELÄ et al., 2014). Cozzo et al.(COZZO et al., 2013) showed that disregarding the multilayer structure can lead to misleading conclusions, missing fundamental aspects of the critical dynamics of spreading-like processes. These findings reinforce the importance of a more detailed investigation of contagion processes on multilayer networks. In this chapter, we explore two aspects and their consequences on epidemic spreading in multilayer networks: (i) eigenstate localization and (ii) the degree-degree correlations.

To begin with, in Section 5.1, we develop a theoretical and computational framework for the analysis of disease spreading, generalizing the results of reference (MIEGHEM; OMIC; KOOIJ, 2009) to multilayer networks. An analysis of the continuous counterpart (presented in Section 3.5.3.2) to the model presented in (COZZO et al., 2013) (for more, see Section 3.5.5.2) is provided in terms of the tensorial notation introduced in (De Domenico et al., 2013) (for more see 2.3.2). Our methodology allows for several new results. First, we are able to write down in a compact form the equations describing the disease dynamics in a multilayer system. Secondly, we derive the corresponding epidemic thresholds for the SIS and SIR cases as well as establish bounds for the prevalence of the disease in the SIS scenario (results presented in Sections 3.5.3.2 and 3.5.3.3 and directly applied here). In this chapter, more precisely in Section 5.1, we identify previously unnoticed multiple susceptibility peaks and disease localization, which are traced back to the very topological nature of the system and described in terms of the eigenvalue spectra of the supra-contact tensor and the localization of eigenstates. In addition, we explore the notion of localization of eigenstates, formerly applied in epidemic spreading in (GOLTSEV et al., 2012), to 
inspect localization transitions, which were verified by multiple susceptibility peaks.

Next, in Section 5.2, we study degree-degree correlations in multilayer systems and propose a way to generalize previous assortativity metrics by considering the tensorial formulation (for more see 2.3.2). Our approach also covers a weighted version of assortativity (LEUNG; CHAU, 2007) and the case in which the assortativity is given by the Spearman correlation coefficient, generalizing the definition in (LITVAK; HOFSTAD, 2013). As an application, we study the influence of degree-degree correlations on epidemic spreading in multilayer networks. We verify that the impact of the disease depends on degree-degree correlations and also on the level of heterogeneity between the layers. It is important to observe that degree-degree correlations are a fundamental property of single-layer networks, impacting the spreading of diseases, synchronization phenomena and systems' resilience (BARRAT; BARTHLEMY; VESPIGNANI, 2008; NEWMAN, 2010). Additionally, it has been reported that different correlations arise in different kinds of networks: social networks are in general assortative, meaning that highly connected nodes tend to link with each other, whereas technological and biological systems have disassortative structures, in which high degree nodes are likely attached to low degree nodes (NEWMAN, 2002). However, for networks made up of more than one layer, only recently, Nicosia and Latora (NICOSIA; LATORA, 2015) considered the correlation between the degrees in two different layers. However, their methodology is only for node-aligned multiplex networks, which are special cases of multilayer networks (see (KIVELÄ et al., 2014)). In fact, multiplex networks are made up of $N$ nodes that can be in one or more interacting layers. The links in each layer represent a given mode of interaction between the set of nodes belonging to that layer, whereas links connecting different layers stand for the different modes of interaction between objects involved in (KIVELÄ et al., 2014).

\subsection{Disease localization in multilayer networks}

The concepts of localization were initially introduced in the context of disease spreading in (GOLTSEV et al., 2012), where the authors discuss the nature of the critical point for localized and delocalized system. They showed that the disease can be localized in a few set of nodes, a so-called localized state, or it can spread throughout the whole network, a delocalized state. Furthermore, they showed that those two cases can be distinguished using the inverse participation ratio. Formally, if the inverse participation ratio of the leading eigenvector of the adjacency matrix (a normalized eigenvector), $\operatorname{IPR}\left(f\left(\Lambda_{1}\right)\right) \sim O(1)$, then the disease is localized on a few nodes, hubs, and the epidemic grow slowly as a function of $\lambda$. On the other hand, if $\operatorname{IPR}\left(f\left(\Lambda_{1}\right)\right) \sim O\left(\frac{1}{\sqrt{N}}\right)$, the disease is present in the whole network, configuring a true active state (GOLTSEV et al., 2012; WANG et al., 2017). Interestingly, in multilayer networks, we also found a new phenomenon, where the disease is localized on a layer, which, in its turn, constitutes an 
infinite fraction of nodes. We call this phenomenon the layer-wise localization.

Firstly, we recall the main ideas on epidemic spreading on multilayer networks, in Section 5.1.1. Next, we discuss the mathematical characterization of localization of diseases in Section 5.1.2, followed by the analysis of 2-Layers multiplex systems in Section 5.1.3, where we discuss various new aspects of epidemic spreading processes in this network. Finally, on Section 5.1.4 we extend our analysis to 3-Layer systems, showing the barrier effect, where one intermediate layer might act as a barrier to the spreading.

\subsubsection{Epidemic spreading on multilayer networks}

The concepts and discussions about epidemic spreading in multilayer networks were already made in Chapter 3. However, here we reproduce some of its main aspects in order to make the text more intuitive and fluid.

First of all, following Chapter 3, Section 3.5.3.2, we model the continuous-time SIS disease dynamics associating a Poisson process to each of the elementary dynamical transitions: intra and inter layer spreading and the recovery from the infected state. The first two processes are associated to the edges of the graph and are characterized by the parameters $\lambda$ and $\eta$, respectively. The latter transition is modeled in the node, also via a Poisson process with parameter $\boldsymbol{\delta}$. Using the tensorial notation defined in Section 2.3.2, the equations describing the systems dynamics read as

$$
\frac{d \mathbb{E}\left(Y_{\beta \tilde{\delta}}(t)\right)}{d t}=-\mathbb{E}\left(Y_{\beta \tilde{\delta}}(t)\right)+\left(1-\mathbb{E}\left(Y_{\beta \tilde{\delta}}(t)\right)\right) \tau \mathscr{R}_{\beta \tilde{\delta}}^{\alpha \tilde{\gamma}}(\lambda, \eta) \mathbb{E}\left(Y_{\alpha \tilde{\gamma}}(t)\right)
$$

where $Y_{\beta \tilde{\delta}}(t)$ is a Bernoulli random variable that is $Y_{\beta \tilde{\delta}}(t)=1$ if the node $\beta$ on layer $\tilde{\delta}$ is infected in time $t^{1}$ and the supra contact tensor is defined as

$$
\mathscr{R}_{\beta \tilde{\delta}}^{\alpha \tilde{\gamma}}(\lambda, \eta)=M_{\beta \tilde{\sigma}}^{\alpha \tilde{\eta}} E_{\tilde{\eta}}^{\tilde{\sigma}}(\tilde{\gamma} \tilde{\delta}) \delta_{\tilde{\delta}}^{\tilde{\gamma}}+\frac{\eta}{\lambda} M_{\beta \tilde{\sigma}}^{\alpha \tilde{\eta}} E_{\tilde{\eta}}^{\tilde{\sigma}}(\tilde{\gamma} \tilde{\delta})\left(U_{\tilde{\delta}}^{\tilde{\gamma}}-\delta_{\tilde{\delta}}^{\tilde{\gamma}}\right),
$$

which encodes the contacts. Notice that we have implicitly assumed that the independence of the random variables $Y_{\beta \tilde{\delta}}$. Finally, the natural order parameter, is defined as the average of the individual probabilities, formally given by

$$
\rho=\frac{1}{n m} \mathbb{E}\left(Y_{\beta \tilde{\delta}}\right) U^{\beta \tilde{\delta}}
$$

Additionally, it is interesting to recall some important results from Section 3.5.3.2. As emphasized in Chapter 3, a key concept in dynamical processes is the critical point. On multilayer networks it is given as

$$
\left(\frac{\delta}{\lambda}\right)_{c}=\Lambda_{1}
$$

1 Note that, from this definition, $\mathbb{E}\left(Y_{\beta \tilde{\delta}}(t)\right)=\mathbb{P}\left(Y_{\beta \tilde{\delta}}(t)=1\right)$ is the probability the node $\beta$ on layer $\tilde{\delta}$ is infected in time $t$. 
where $\Lambda_{1}$ is the largest eigenvalue of $\mathscr{R}$. For a more precise derivation, we refer to Chapter 3, Section 3.5.3. Another interesting result is the nodal infection probability bounds, which are given as

$$
1-\frac{1}{1+\frac{d_{\beta \tilde{\delta}}}{d^{\min }}\left[\left(\frac{\lambda}{\delta}\right) d^{\mathrm{min}}-1\right]} \leq \mathbb{E}\left(Y_{\beta \tilde{\delta}}^{\infty}\right) \leq 1-\frac{1}{\left(\frac{\lambda}{\delta}\right) d_{\beta \tilde{\delta}}+1}
$$

where $\mathbb{E}\left(Y_{\beta \tilde{\delta}}^{\infty}\right)$ denotes the probability that node $\beta$ in layer $\tilde{\delta}$ is in the steady state regime, $d_{\beta \tilde{\delta}}=\mathscr{R}_{\beta \tilde{\delta}}^{\alpha \tilde{\gamma}}(\lambda, \eta) U_{\alpha \tilde{\gamma}}$ and $d^{\min }=\operatorname{Min}\left\{d_{\beta \tilde{\delta}}\right\}$.

Now, changing our perspective to the computational point of view, since we are following a continuous-time approach the appropriate simulation scheme is described in Section 3.7.2. Note that we followed the standard algorithm of the development of this work. Additionally, as we are interested in the critical behavior of our system, we also used the QS Method to extract statistically meaningful data from our dynamics. For more on this method, please see Section 3.7.4. Finally, we must recall that the critical point can be estimated using the modified susceptibility (FERREIRA; CASTELLANO; PASTOR-SATORRAS, 2012), which is given as

$$
\chi=\frac{\mathbb{E}\left(\left(n^{*}\right)^{2}\right)-\mathbb{E}\left(n^{*}\right)^{2}}{\mathbb{E}\left(n^{*}\right)}=n m\left(\frac{\mathbb{E}\left(\left(\rho^{Q S}\right)^{2}\right)-\mathbb{E}\left(\rho^{Q S}\right)^{2}}{\mathbb{E}\left(\rho^{Q S}\right)}\right),
$$

where $\rho^{Q S}$ is the quasi-stationary distribution $\bar{p}_{n^{*}}$, from which $n^{*}$ is the number of infected nodes on the quasi-stationary process (for more, see 3.7.4). As argued in (FERREIRA; CASTELLANO; PASTOR-SATORRAS, 2012; MATA; FERREIRA, 2015), the susceptibility presents a peak at the phase transition on finite systems.

\subsubsection{Localization and spreading of diseases}

To begin with, we investigate the behavior of the system near the phase transition and whether the phenomenon of disease localization shows up. These two issues were explored for monoplex networks in (Van Mieghem, P., 2012) and (GOLTSEV et al., 2012), respectively, but have not been addressed for the case of multilayer systems. The nodal probabilities can be written as a linear combination of the eigenbasis of $\mathscr{R}$ as

$$
X_{\beta \tilde{\delta}}=\sum_{\Lambda} c(\Lambda) f_{\beta \tilde{\delta}}(\Lambda)
$$

where $c(\Lambda)$ are the projections of $X_{\beta \tilde{\delta}}$ on the eigentensors $f$. Similarly to (GOLTSEV et al., 2012), substituting this expression in the middle term of Equation 3.34, we obtain

$$
c(\Lambda)=\sum_{\alpha \tilde{\gamma}} \frac{\lambda \sum_{\Lambda^{\prime}} c\left(\Lambda^{\prime}\right) \Lambda^{\prime} f_{\alpha \tilde{\gamma}}\left(\Lambda^{\prime}\right) f_{\alpha \tilde{\gamma}}(\Lambda)}{\lambda \sum_{\Lambda^{\prime}} c\left(\Lambda^{\prime}\right) \Lambda^{\prime} f_{\alpha \tilde{\gamma}}\left(\Lambda^{\prime}\right)+\delta} .
$$


Considering only the contributions of the first eigenvalue and eigentensor, for $\lambda \geq$ $\lambda_{c}$, the first order approximation of the macro state parameter is $\rho \approx \alpha_{1} \tau$, where $\tau=$ $\left(\frac{\lambda}{\delta} \Lambda_{1}-1\right)$, which yields

$$
\alpha_{1}=\frac{f_{\beta \tilde{\delta}}\left(\Lambda_{1}\right) U^{\beta \tilde{\delta}}}{n m\left(f_{\beta \tilde{\delta}}\left(\Lambda_{1}\right)\right)^{3} U^{\beta \tilde{\delta}}} .
$$

This expression is exact if there is a gap between the first two eigenvalues (Van Mieghem, P., 2012; GOLTSEV et al., 2012). Furthermore, considering two eigentensors we have $\rho \approx \alpha_{1} \tau+\alpha_{2} \tau^{2}$. Besides, following a similar approach as in (GOLTSEV et al., 2012) we can use the inverse participation ratio:

$$
\operatorname{IPR}(\Lambda) \equiv\left(f_{\beta \tilde{\delta}}(\Lambda)\right)^{4} U^{\beta \tilde{\delta}}
$$

In the limit of $n m \rightarrow \infty$, if the $\operatorname{IPR}(\Lambda)$ is of order $O(1)$ the eigentensor is localized and the components of $f_{\beta \tilde{\delta}}(\Lambda)$ are of order $O(1)$ only for a few nodes. On the other hand, if $\operatorname{IPR}(\Lambda) \rightarrow 0$ then this state is delocalized and the components of $f_{\beta \tilde{\delta}}(\Lambda) \sim O\left(\frac{1}{\sqrt{n m}}\right)$. Additionally, another possible scenario, completely different form the traditional single layer one, is possible if we consider a layer-wise localization, i.e localization on layers, instead of on a fraction of nodes. In this case, the $\operatorname{IPR}(\Lambda)$ will be of order $O(1 / n)$ in the localized phase, whereas it will be of order $O(1 / \mathrm{nm})$ in the delocalized phase. This is because, in the layer-wise localized phase the components of the eigentensor are of order $O(1 / \sqrt{n})$ for all the nodes in the dominant layer and of order zero for nodes in other layers. Observing that, it can be observed that the correct finite-size scaling to take in order to characterize such a transition is $m \rightarrow \infty$, i.e., the number of layers goes to infinity while the number of nodes per layer stays constant. In fact, in this limit, $\operatorname{IPR}(\Lambda)$ will vanish on one side of the transition point while remaining finite on the other side. Thus, we can observe localized states also in the case where there is no possibility for localization in each of the layers if isolated.

\subsubsection{2-Layer multiplex systems}

In this section, we numerically study 2-layer multiplex systems. First, we focus on the phase diagram of the spreading process as a function of the inter and intralayer spreading rates for both SIS and SIR scenarios. Next, we analyze the spectral properties of such systems, compared to results in Section 2.2. Finally, we perform Monte Carlo simulations that show the existence of multiple susceptibility peaks on multiplex networks. The latter results are analyzed in terms of the spectral properties of $\mathscr{R}(\lambda, \eta)$.

\subsubsection{Numerical solution}

Results shown in this section are the numerical solutions of the ODE systems 3.44 (SIS) and 3.49 (SIR) using a Runge-Kutta (4,5) algorithm (DORMAND; PRINCE, 1980). 
Figure 48 - Phase diagrams over a 2-Layer multiplex system, where each layer is a scale-free network with $n=10^{4}$ nodes, for a fixed value of $\delta=1$. (a) Density of spreaders as a function of the parameters $\eta$ and $\lambda$. (b) Density of recovered individuals as a function of the parameters $\eta$ and $\lambda$. Colors represent the fraction of spreaders and the white line is the threshold calculated using Equation 3.47.

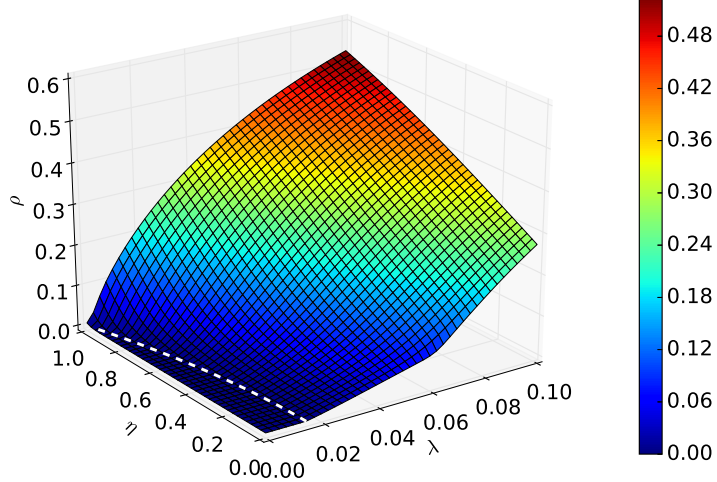

(a) SIS

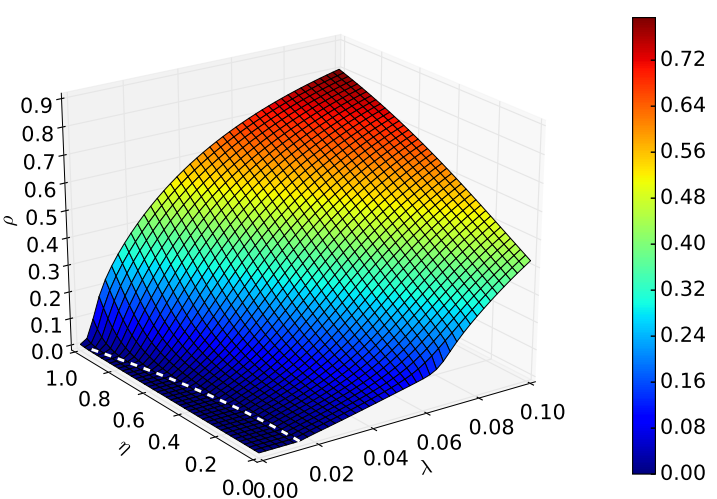

(b) SIR

Source: Developed by the author.

Figure 49 - Individual layer behavior over a 2-Layer multiplex system. Each layer has $n=10^{4}$ for a fixed value of $\delta=1$. The results considering both layers are shown in (a), while the dynamics in the individual layers are shown in (b) $\left(P(k) \sim k^{-2.5}\right)$ and (c) $\left(P(k) \sim k^{-4.5}\right)$. The arrows indicate the leading eigenvalues of the layers.

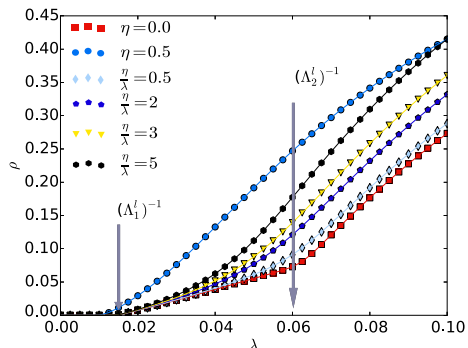

(a) Global

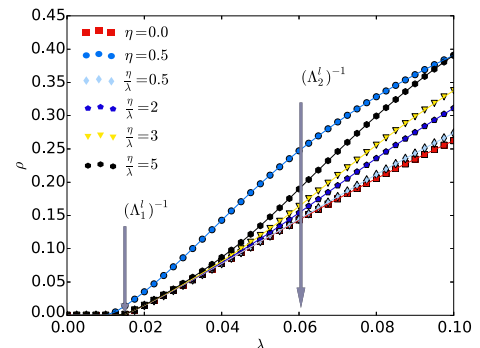

(b) First layer

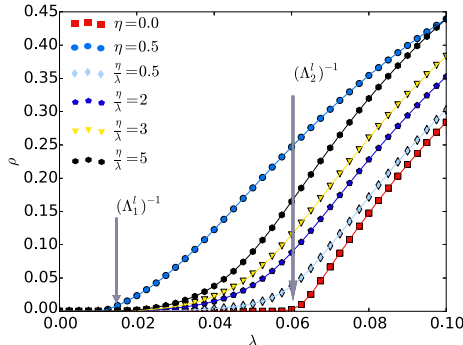

(c) Second layer

Source: Developed by the author.

We consider a 2 layer multiplex network $(m=2)$, where each layer has $n=10^{4}$ nodes. In order to build a multiplex network where the epidemic thresholds associated to the individual layers are well separated, we must guarantee that $\Lambda_{1}^{l} \gg \Lambda_{2}^{l}$. Therefore, we chose the degree distribution of the first layer to be $P(k) \sim k^{-2.5}$, whereas that of the second layer is $P(k) \sim k^{-4.5}$. Both layers are created using the uncorrelated configuration model (VIGER; LATAPY, 2005). Moreover, we consider a multilayer network in which every node has its counterpart on the other layer. This pairing of nodes of different layers is made randomly. Each result is the solution considering one single (and fixed) multiplex network. 
Figure 48 shows the phase diagram considering the average fraction of spreaders for the SIS dynamics (or recovered for the SIR dynamics) as the macro-state variable as a function of the spreading parameters $\lambda$ and $\eta$ for a given recovering rate $\delta=1$. The dashed white line denotes the epidemic threshold obtained from Equation 3.47. In (a) we show the SIS scenario, while (b) corresponds to the SIR model. In both cases, it is possible to observe two changes in the system's behavior. The first in the epidemic threshold, while the second near the epidemic threshold of the second layer. In addition, we note the agreement between the theoretical epidemic thresholds and the numerical results. Furthermore, the higher the $\eta$, the lower the epidemic threshold, which is a consequence of the eigentensor problem. Also note that $\rho$ increases for a fixed $\lambda$ as $\eta$ increases, even for $\lambda \sim 0$, which means that in such extreme cases, the disease spreads mainly on the interlayer edges.

Figure 49 shows the phase diagram for $\delta=1$ and different values of the parameter $\eta$ for the SIS dynamics. For $\eta=0$ we have no inter-layer spreading, while for $\eta=0.5$ we have a fixed spreading rate, regardless of the intra-layer rates. In addition, we also evaluated cases where the ratio $\frac{\eta}{\lambda}$ is constant. In Figure 49 (a) we have the global behavior of the system, which is an average of the individual behavior of the layers, represented in panels (b) and (c) since both layers have the same number of nodes. Furthermore, we also observe that the two individual networks show different behaviors near the epidemic threshold (Van Mieghem, P., 2012). The first layer (Figure 49 (b)) has a lower epidemic threshold than the second. However $\rho$ grows (as a function of $\lambda$ ) slower than in the second. This feature can be observed clearly in Figure 49 (b) and (c), where we show results for $\eta=0$, that is, when there is no spreading between the layers.

Considering the discrete system, Cozzo et al. (COZZO et al., 2013) verified the shifting on the non-dominated layer (the smallest amongst all individual eigenvalues) as the ratio $\frac{\eta}{\lambda}$ increases. Here we observe the same effect, as can be seen in Figure 49 (c). Additionally, we can also note another global change approximately beyond $\lambda>\left(\Lambda_{2}^{l}\right)^{-1}$. Our findings suggest the possibility of multiple phase transitions due to the multiplex structure of the network. It is noteworthy that in spite of the similarities between our continuous model and the discrete model (COZZO et al., 2013), both represent slightly different processes. In the continuous case, two events cannot happen at the same time. On the other hand, in the discrete model, every node contacts its neighbors in one discrete time step. Despite these differences, the results show that both the continuous and discrete formulations are phenomenologically similar. For more on this discussion, see Chapter 3 and Section 3.6. 
Figure 50 - Spectral properties of the tensor $\mathscr{R}(\lambda, \eta)$ as a function of the ratio $\frac{\eta}{\lambda}$ for a multiplex with two layers, the first with $\zeta \approx 2.2$, while the second $\zeta \approx 2.8$. Both have $\mathbb{E}(k) \approx 8$. In the left panel, we present the inverse participation ratio $(\operatorname{IPR}(\Lambda))$ of the two larger eigenvalues and the individual layer contributions, while in the right panel, we show the leading eigenvalues. Every curve is composed by $10^{3} \log$ spaced points, in order to have enough resolution.
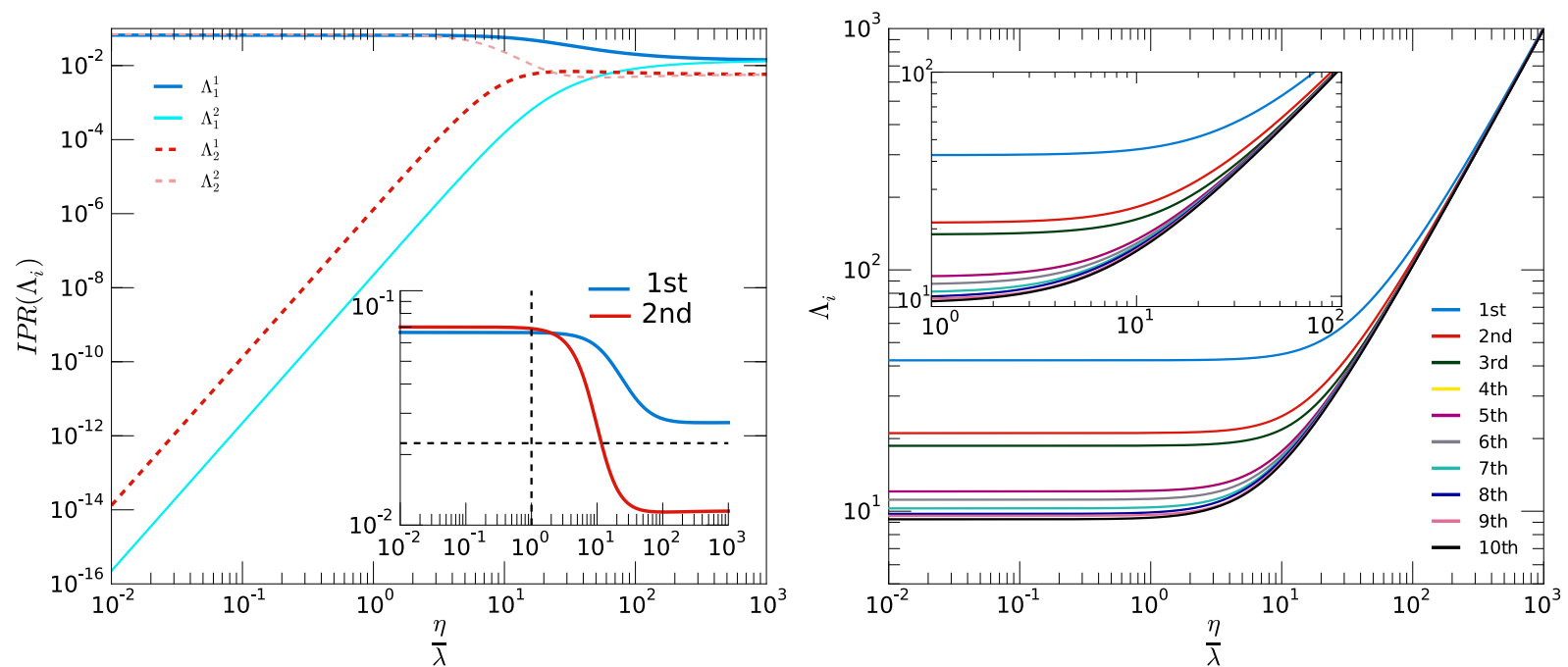

Source: Developed by the author.

\subsubsection{Spectral analysis}

Since the epidemic process is described through the supra adjacency tensor $\mathscr{R}(\lambda, \eta)$, its spectral properties give us some insights about the whole process, especially about the critical properties of the systems under analysis. In this section, we focus on the spectral analysis of this tensor as a function of the ratio $\frac{\eta}{\lambda}$ considering a 2-layer multiplex network with two different layers, i.e., there is a distance between the leading eigenvalues of each layer. Some important aspects of the spectral properties are discussed in the next chapter, more precisely in Section 6.1.1, where we present an analytical approach to the problem of eigenvalue crossings in Section 6.1.1.1. We focus on two special cases in increasing order of complexity: (i) the identical case, presented in Section 6.1.1.2, where both layers are exactly the same - i.e., there is a high correlation between the degree in each layer -; and (ii) the non-identical case, discussed in Section 6.1.1.3, where both layers have the same degree distribution, but different configurations.

Firstly, in this section, we focus on the case of two different layer structures, with spaced leading eigenvalues. Considering a multiplex network made up of two scale-free networks with $\zeta \approx 2.2$ and $\zeta \approx 2.8$. Both layers have $\mathbb{E}(k) \approx 8$ and $n=10^{3}$ nodes on each layer and the leading eigenvalues are $\Lambda_{1}^{1}=42.64$ for the first and $\Lambda_{1}^{2}=21.29$ for the second.

Figure 50 shows the spectral properties of the tensor $\mathscr{R}(\lambda, \eta)$ as a function of the 
Figure 51 - Diagram of the contribution of each layer to the $\operatorname{IPR}(\Lambda)$ for different values of the spreading ratio $\frac{\eta}{\lambda}$. The dashed line represents the case where both layers have the same contribution, i.e. a line with a slope one. In the inset we show the angle $\theta$ between the vector composed by the contributions of each layer to the $\operatorname{IPR}(\Lambda)$, $v=\left[\operatorname{IPR}\left(\Lambda_{1}^{1}\right), \operatorname{IPR}\left(\Lambda_{1}^{2}\right)\right]^{T}$, and the $x$-axis. The multiplex network used here is composed of two Erdös-Rényi networks, both with $n=5 \times 10^{4}$, the first layer $\mathbb{E}(k)=16$ $\left(\left(\Lambda_{1}^{1}\right)^{-1} \approx 0.0625\right)$, while the second $\mathbb{E}(k)=12\left(\left(\Lambda_{1}^{2}\right)^{-1} \approx 0.0833\right)$.

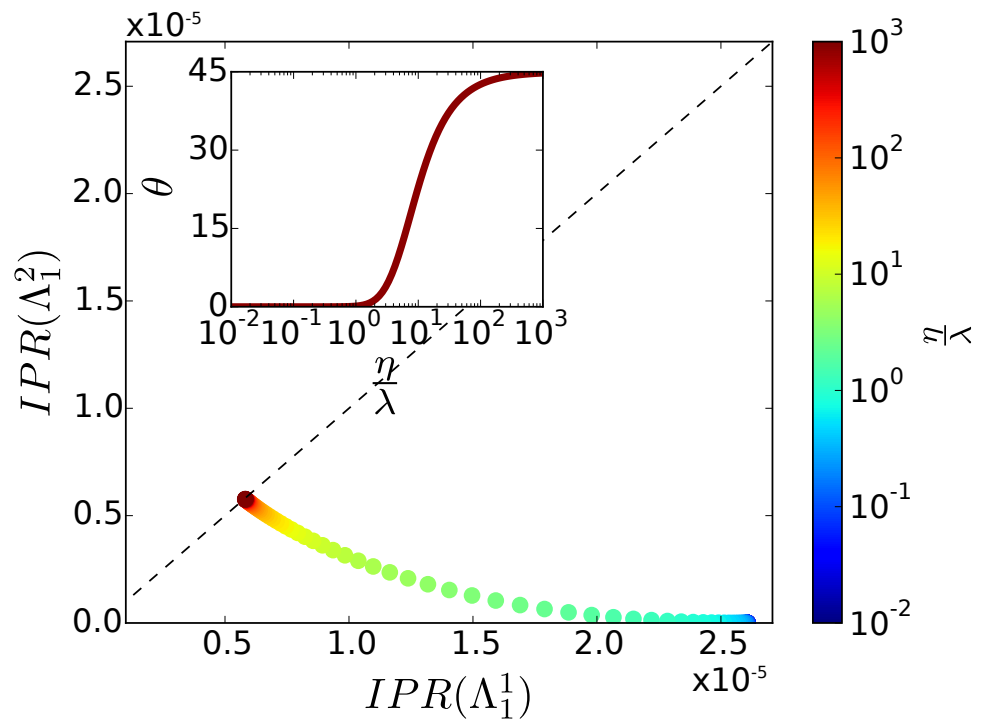

Source: Developed by the author.

ratio $\frac{\eta}{\lambda}$. In contrast to the identical layers (see Section 6.1.1.2) and the case of statistically equivalent layers (Section 6.1.1.3), Figures 63 and 64, where some eigenvalues increase while others decrease, here all the observed eigenvalues always increase. Moreover, we do not observe any crossing or near-crossing behavior. Regarding $\operatorname{IPR}(\Lambda)$, the same pattern as for the similar case is found: for small values of $\frac{\eta}{\lambda}$ and considering the first eigenvalue, the system appears to be localized in the first layer and delocalized in the second, while for $\operatorname{IPR}\left(\Lambda_{2}\right)$, it is the contrary. For larger values of $\frac{\eta}{\lambda}$, both layers contribute equally to the $\operatorname{IPR}(\Lambda)$. Furthermore, the main difference we observe for the current setup with respect to the two similar networks (see Figure 64, presented in Section 6.1.1), is that now no drastic change in the inverse participation ratio is found, as expected, since there is no near-crossing.

In Figure 50, we can also extract an important numerical result regarding the perturbation theory. We observed that in our case, considering a two spaced-individual layer eigenvalue problem, the leading eigenvalue can be approximated by the largest leading eigenvalue of the individual layers for $\frac{\eta}{\lambda} \lesssim 1$, this approximation becomes poorer as $\frac{\eta}{\lambda}$ increases, but it can be acceptable up to $\frac{\eta}{\lambda} \lesssim 10$, within a certain error. Apart from that, note that both eigenvalues tend to increase, while its difference tends to decrease. 
Secondly, analyzing the eigenfunction properties in homogeneous networks, Figure 51 shows the contribution of each layer to the $\operatorname{IPR}(\Lambda)$ considering different values $\frac{\eta}{\lambda}$. This experiment was calculated over a multiplex network composed by two Erdös-Rényi networks, both with $n=5 \times 10^{4}$, the first layer $\mathbb{E}(k)=16$, while the second $\mathbb{E}(k)=12$. Observe that for lower values of $\frac{\eta}{\lambda}$, the main contribution comes from one layer, configuring a layer-wise localized state and consequently placed on one the $x$-axis of Figure 51 . Then, increasing the ratio $\frac{\eta}{\lambda}$ we also increase the inverse participation ratio of the second layer, however decreasing the inverse participation ratio of the first layer, implying that the points tend to be on the diagonal line with slope one, where the contributions of both IPRs are the same. This observation is also confirmed by angle, $\theta$, between the vector composed by the IPR contributions, $v=\left[\operatorname{IPR}\left(\Lambda_{1}^{1}\right), \operatorname{IPR}\left(\Lambda_{1}^{2}\right)\right]^{T}$, and the x-axis, where we observe it changing from zero to 45 degrees.

\subsubsection{Multiple susceptibility peaks}

Mata and Ferreira showed that it is possible to have multiple susceptibility peaks in monoplex networks (MATA; FERREIRA, 2015). They studied the behavior of an SIS model in networks with $\zeta>3$. Here we show that this phenomena also appear, in a natural way, on multilayer networks. Motivated by the findings reported in the latter sections, especially by the presence of a second change in the slope of $\rho$ as observed in Figures 48 and 49, we have performed extensive Monte Carlo simulations using the QSmethod with the aim of determining as accurately as possible the points at which the transitions take place for a 2-layer multiplex network. Here we use the scale free multiplex built up in Section 5.1.3.2, as the leading eigenvalues of each layer are spaced. Note that our numerical simulations are performed in a fixed network, as we follow the quenched formalism.

Figure 52 shows that for low values of the ratio $\frac{\eta}{\lambda}$, both networks are weakly coupled and the system exhibits two well-defined susceptibility peaks (vertical dotted lines). However, as this ratio increases the peak signaling the presence of the second critical point decreases and eventually vanishes. In our simulations, we observed that up to $\frac{\eta}{\lambda} \approx 1$, the second peak, although less defined, is still present. Beyond the latter point, only one peak remains. As $\frac{\eta}{\lambda}$ further increases, the position of the critical point remains the same, and the peak is even more well defined. Interestingly enough, if the ratio $\frac{\eta}{\lambda}$ continues to increase - in our case beyond $\frac{\eta}{\lambda} \gtrsim 10$ - the critical point shifts to the left to values that are even smaller than the smallest critical point of the individual layers. It is worth highlighting that a similar qualitative behavior can be seen in the results shown in Figure 48 (a), where one can also observe a second change in the slope of $\rho$ near the leading eigenvalue of the second layer. This change also vanishes as the intra-layer spreading increases. 
As the tensor $\mathscr{R}(\lambda, \eta)$ plays a major role in the spreading process, our spectral results can help understand the observed critical dynamics. In epidemiological terms or in general for contagion processes - , the localization of the disease on a certain layer means that most of the spreading is expected to take place on the nodes of that layer. Moreover, in addition to the layer-wise localization, one can also have localization effects on specific nodes or groups of nodes, for instance.

In order to analytically explain this phenomenon, we evaluate $\operatorname{IPR}(\Lambda)$ for the two leading eigenvalues, as this measure indicates the localization of an eigenstate, see Section 5.1.3.2 (results shown in Figure 50). Comparing the susceptibility and $\operatorname{IPR}(\Lambda)$, we observe that $\operatorname{IPR}\left(\Lambda_{2}\right)$ starts decaying for $\frac{\eta}{\lambda} \approx 1$ and crosses the value $\frac{1}{\sqrt{n m}}$, at which the associated eigenvector delocalizes, for $\frac{\eta}{\lambda} \approx 10$, comparing well with the point at which the second peak in the susceptibility decays and finally disappears. Moreover, $\operatorname{IPR}\left(\Lambda_{1}\right)$ decays from $3 \lesssim \frac{\eta}{\lambda} \lesssim 10$, which coincides with the range where the remaining maximum in the susceptibility reaches higher values and is better defined. More interestingly, note that $\operatorname{IPR}\left(\Lambda_{1}\right)$ is mainly composed by the contributions of the first layer for a lower spreading ratio, suggesting that it is localized in this layer. Therefore, our results suggest that the $\operatorname{IPR}(\Lambda)$ is a proper measure to detect and predict the observed localization phenomena and potentially for $m$ localization transitions, as will be shown in Section 5.1.4.

Regarding the definition of a critical point, it is important to highlight, again, that the concept of phase transition only applies in the infinite size limit (the thermodynamic limit). However, in the literature of complex network dynamics, especially for epidemic spreading, it is usual to use the terms critical point and phase transition on finite systems, since we find a behavioral change at that point. More importantly, for scale-free networks, this point vanishes in the thermodynamic limit. Following the usual convention on the complex network literature, the first susceptibility peak observed in all the experiments can be classified as a critical point of a phase transition. In this point, the dynamics range from a disease-free state to an endemic state. On the other hand, the second susceptibility peak cannot receive this classification, as the process is already in an endemic state. Although it cannot be considered as a critical point, we have a transition from a layerwise localized state to a delocalized state. In other words, before the second susceptibility peak most of the events take place in only one layer (the one with largest individual eigenvalue), while after this point both layers are active and spread the disease.

\subsubsection{Second susceptibility peak analysis: Erdös-Rényi layers}

The second peak in the susceptibility curve suggests the existence of a second order phase transition. However, from its existence alone we cannot conclude this unequivocally, since although this point is related to the delocalization of the disease, the system is already in an endemic phase (upper critical regime in Physics jargon). Observe 
Figure 52 - Susceptibility, $\chi$, as a function of the spreading rate $\lambda$ for different ratios of inter and intra-layer spreading ratings, $\frac{\eta}{\lambda}$ for a fixed value of $\delta=1$ over a 2-Layer multiplex system, where each layer has $n=10^{3}$, the first with $\zeta \approx 2.2$, while the second $\zeta \approx 2.8$. Both have $\mathbb{E}(k) \approx 8$. The simulated values are $\frac{\eta}{\lambda}=0.1,0.2,0.3,0.4,0.5,0.6,0.7$, $0.8,0.9,1.0,1.1,1.2,1.3,1.4,1.5,1.6,2,3,4,5,6,7,8,9,10,20,30$.

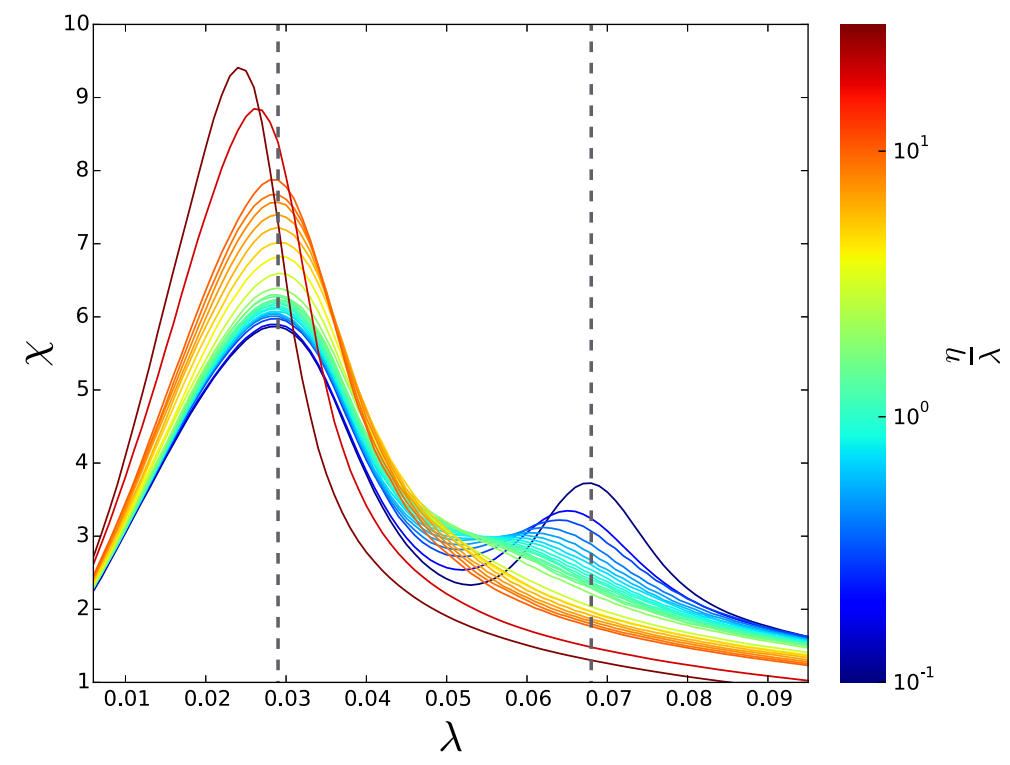

Source: Developed by the author.

that if $\eta \in O\left(\frac{1}{N}\right)$, in the thermodynamic limit we would have a phase transition. However, this configuration cannot be considered as a multilayer network, since both layers are (virtually) decoupled. Additionally, observe that we only analyzed layers without correlation. These features can also introduce different phenomenologies, some were briefly explored in (ARRUDA et al., 2016), however for discrete-time.

In order to better understand the second peak of susceptibility, we analyze a 2-Layer multiplex network composed by two Erdős-Rényi networks, in which we can precisely control the mean degree and consequently the epidemic threshold by fixing the number of edges. Furthermore, we must observe that for scale-free networks with a divergent second moment of its degree distribution, the epidemic threshold vanishes in the thermodynamic limit (PASTOR-SATORRAS et al., 2015). On the other hand, ErdösRényi networks always have a non-zero and finite critical point. Aside from that, as the nodes in this a network are statistically equivalent, the probabilities $X_{\beta \tilde{\delta}}$ are expected to be approximately the same. Henceforth, we assume that the first layer has a higher connectivity, that is, a lower epidemic threshold.

First of all, analyzing the layers individually for $\frac{\lambda}{\delta}>\left(\Lambda_{1}^{1}\right)^{-1} \geq \Lambda_{1}^{-1}$ the first layer is in its upper critical regime (endemic state), while the second layer still is in its sub-critical regime (disease-free state). Then, for a coupling parameter, $\eta>0$, the probability of a 
Figure 53 - Time evolution of the fraction of infected nodes in the second layer for $\delta=1$, different values of $\eta=10^{-4}, 10^{-3}, 10^{-2}, 10^{-1}$ and different values of spreading rate: (a) $\lambda=$ 0.078 , (b) $\lambda=0.083$, (c) $\lambda=0.085$ and (d) $\lambda=0.088$. The multiplex network used is composed of two Erdös-Rényi networks, both with $n=5 \times 10^{4}$, the first layer $\mathbb{E}(k)=16\left(\left(\Lambda_{1}^{1}\right)^{-1} \approx 0.0625\right)$, while the second $\mathbb{E}(k)=12\left(\left(\Lambda_{1}^{2}\right)^{-1} \approx 0.0833\right)$.
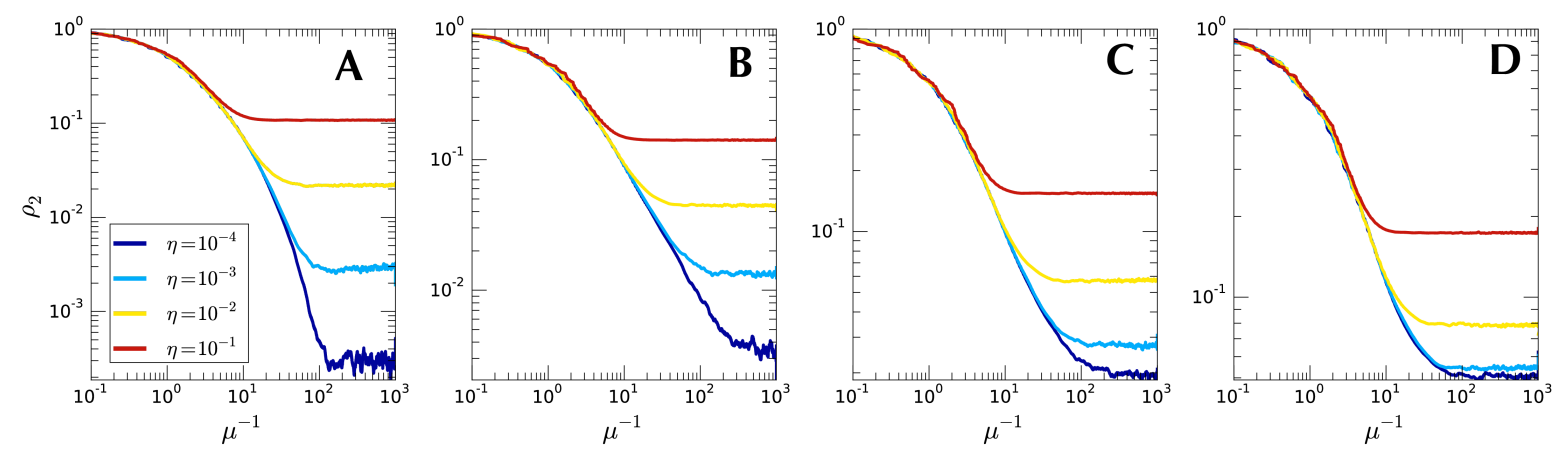

Source: Developed by the author.

node in the second layer being infected also increases. In fact, for Erdős-Rényi layers, it will be always larger than zero. Therefore, we can map this problem into a $\boldsymbol{\varepsilon}$-SIS model (Van Mieghem; CATOR, 2012), where each node has a probability of experiencing a spontaneous infection. Note that such a model does not present an absorbing state. In this mapping, we are interested in the behavior of the second layer and consider that the self-infection $\varepsilon$ is determined by the contribution of the first layer by means of the contacts between nodes in different layers, which are Poisson processes with parameter $\eta$. This would imply that we would not have a second order phase transition. However, we have a transition from a layer-wise localized system, in which only the first layer is active and able to sustain the disease for long times, to a delocalized system, where both layers are active.

In order to explore the time evolution of the system for a set of parameters near the second susceptibility peak, we run the continuous simulation 50 times and perform a moving average filter over a sampling of the original time series, resulting in $5 \times 10^{4}$ points. This approach gives us an average curve over time. Note that for continuous simulations the number of points can vary from one run to another. Both networks used have $n=5 \times 10^{4}$, the first $\mathbb{E}(k)=16\left(\left(\Lambda_{1}^{1}\right)^{-1} \approx 0.0625\right)$, while the second $\mathbb{E}(k)=12$ $\left(\left(\Lambda_{1}^{2}\right)^{-1} \approx 0.0833\right)$.

Figure 53 shows the time evolution of a disease spreading in the second layer for different values of $\lambda$ and $\eta$. The initial conditions for these experiments consider that the first layer has an initial probability of a node being infected equal to 0.01, while in the second every node is a spreader. Note that we chose this initial condition for visual purposes, as any initial condition would result in a similar steady-state regime. 
Thus, during the transient state we observe a decay of the fraction of infected individuals, then, at the meta-state that configures the steady state, we observe a stochastic variation centered on the average value. Besides, these fluctuations tend to increase near a "critical point". We observe that for $\left(\Lambda_{1}^{1}\right)^{-1}>\frac{\lambda}{\delta}>\left(\Lambda_{2}^{1}\right)^{-1}$ for $\eta=10^{-4}$ the incidence is very low, of order $O\left(\frac{1}{N}\right)$, however, larger than zero. As we increase the value of $\lambda$, we drive the system to its active state, being able to sustain the disease and spreading it by the intra-edges contacts. Besides, by increasing $\eta$ we are able to increase the incidence of the disease due to the intra-edge contacts. Near the critical point of the second layer, $\frac{\lambda}{\delta}=\left(\Lambda_{2}^{1}\right)^{-1}=0.833$, we can observe some features that are similar to a transition. From below, we observe that the lower the value of $\eta$, the longer it takes for the system to reach the steady state, similar to what it is expected in phase transitions. On the other hand, slightly above the critical point, the time to get into the steady state decreases and the curves for $\eta=10^{-4}$ and $\eta=10^{-3}$ get closer. This suggests that the effects of intra-layer spreadings are the main source of spreading. Finally, for $\frac{\lambda}{\delta}$ sufficiently large, we observe the same behavior for all values of $\eta$, i.e. all of them are in an active state.

In addition to the analysis shown in this section, we also inspected in detail the steady state for different system sizes, showing that neither the fluctuations diverge nor the final fraction of infected individuals goes to zero on the second layer. This analysis suggests that we do not have a second order phase transition but that the dynamics changes from a layer-wise localized to a delocalized phase. In this reach phenomenological scenario, the transition point is still of great importance for practical purposes, for instance when it comes to studying immunization policies. These complementary results are shown in the following section.

\subsubsection{Finite size analysis: Erdös-Rényi layers}

In this section, we analyze the behavior of a 2-layer multiplex network at the steady state considering different sizes. Such a multiplex was built considering two Erdős - Rényi networks with a fixed mean degree. As mentioned previously, we chose this type of network because their epidemic threshold does not vanish at the thermodynamic limit, which contrasts with the scale-free networks. Thus, we have a well-defined critical point that can be precisely tuned regardless of the network size. Following the usual convention on the complex network literature, the first susceptibility peak observed in our experiments can be classified as a critical point of a phase transition. At this point, the dynamics go from a disease-free state to an endemic state. However, the second susceptibility peak cannot be classified as a second order phase transition, since the disease is already in an endemic state. Although it cannot be considered as a critical point, before the second susceptibility peak most of the events take place on only one layer (the one with the largest individual eigenvalue), while after this point both layers are active and spreading the disease. 
Figure 54 - Final number of infected nodes in the second layer (with lowest individual eigenvalue) as a function of the size of the layers on the main panels, while in the insets we present the fraction of infected nodes on the left and the standard deviation in the steady state on the right. The parameters used in the simulations are shown on the tile of each panel. They are a combination of the parameters $\lambda=0.078,0.083,0.085,0.088$ and $\eta=10^{-4}, 10^{-3}, 10^{-2}, 10^{-1}$. Furthermore, the layer sizes are $n=2 \times 10^{3}, 3 \times 10^{3}, 4 \times 10^{3}, 5 \times 10^{3}, 6 \times 10^{3}, 7 \times 10^{3}, 8 \times 10^{3}, 9 \times 10^{3}, 10^{4}, 2 \times$ $10^{4}, 3 \times 10^{4}, 4 \times 10^{4}$ and $5 \times 10^{4}$ and $m=2$ in all cases.
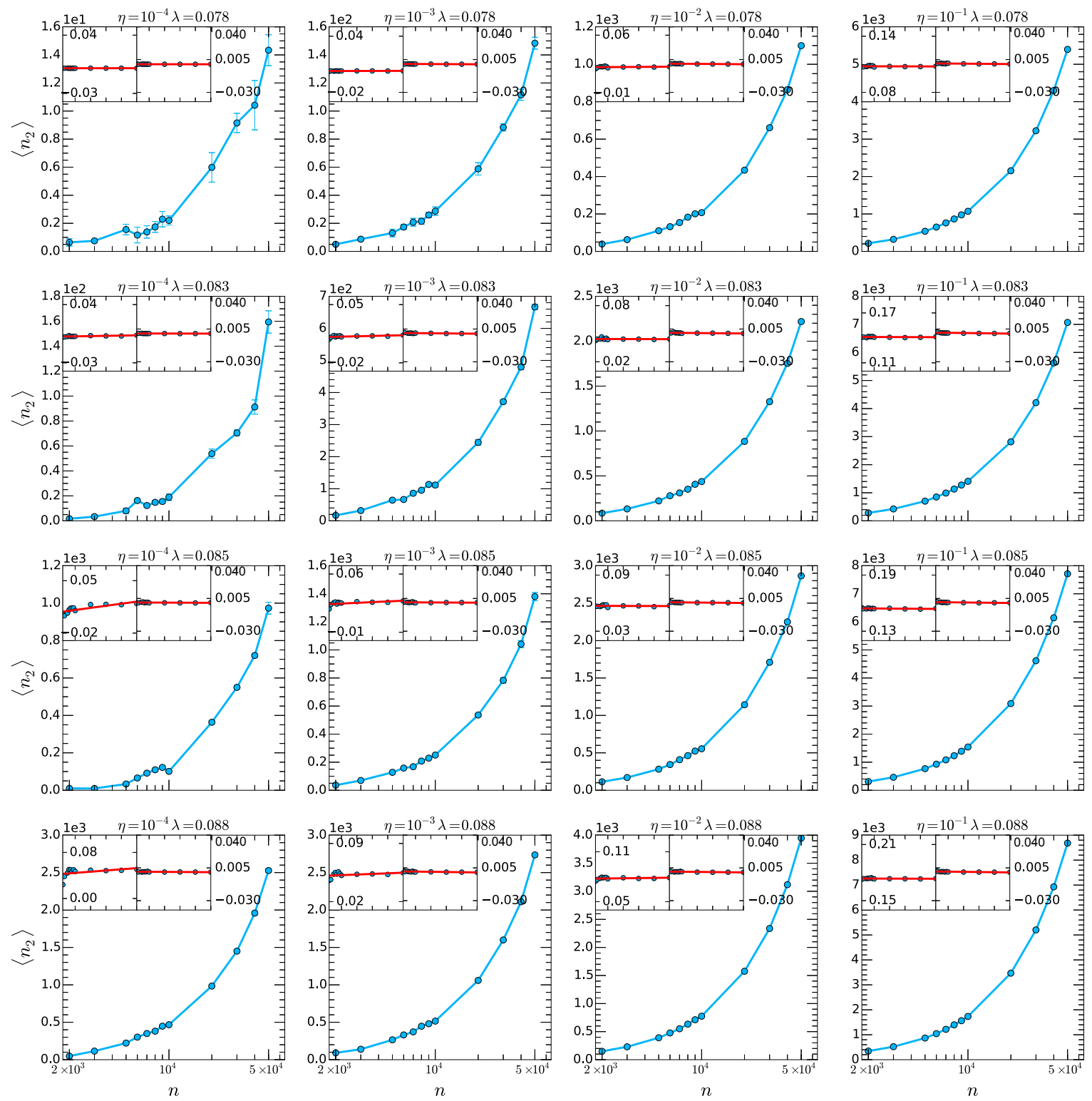

Source: Developed by the author.

Similarly to the experiments shown in Section 5.1.3.4, here we run the continuous simulation 50 times and perform a moving average filter over a sampling of the original time series, resulting in $5 \times 10^{4}$ points. The simulations are run up to $t=10^{3}$. Note that for continuous simulations the number of points can vary from one run to another. The 
Figure 55 - Final fraction of infected nodes on the layer with lowest individual eigenvalue as a function of the the size of the layers. The colors represent different values of $\eta$, while on we have $\lambda=0.078$ in (a), $\lambda=0.083$ in (b) $\lambda=0.085$ in (c) and $\lambda=0.088$ in (d). Furthermore, the layer sizes are $n=2 \times 10^{3}, 3 \times 10^{3}, 4 \times 10^{3}, 5 \times 10^{3}, 6 \times 10^{3}, 7 \times 10^{3}, 8 \times$ $10^{3}, 9 \times 10^{3}, 10^{4}, 2 \times 10^{4}, 3 \times 10^{4}, 4 \times 10^{4}$ and $5 \times 10^{4}$ and $m=2$ in all cases. Each curve is the result of a parameter $\eta$, from bottom to top $\eta=10^{-4}, 10^{-3}, 10^{-2}, 10^{-1}$.
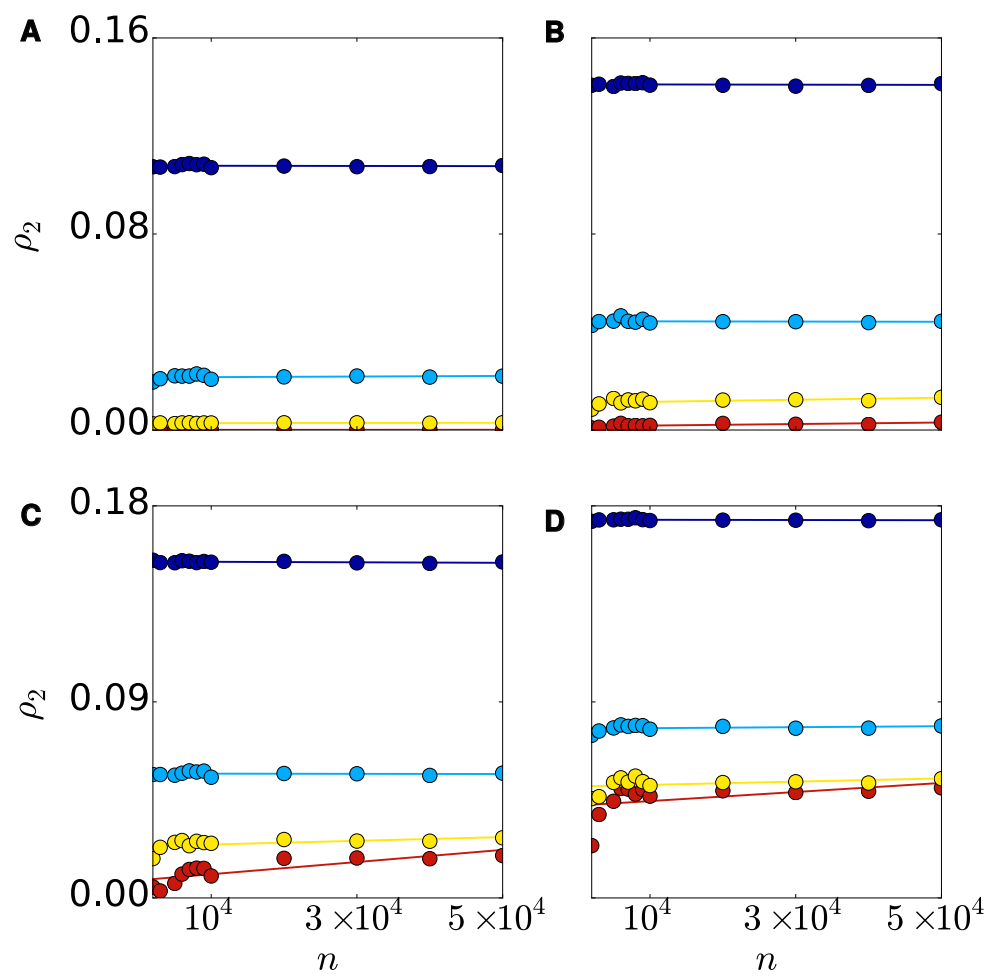

Source: Developed by the author.

steady state statistics are estimated for $t \geq 950$ or in other words, the last 50 time units. In contrast with the previous section, here we are interested in comparing results for different network sizes, $n=2 \times 10^{3}, 3 \times 10^{3}, 4 \times 10^{3}, 5 \times 10^{3}, 6 \times 10^{3}, 7 \times 10^{3}, 8 \times 10^{3}, 9 \times 10^{3}, 10^{4}, 2 \times$ $10^{4}, 3 \times 10^{4}, 4 \times 10^{4}$ and $5 \times 10^{4}$ and $m=2$ in all cases. Besides, we considered the mean degree as $\mathbb{E}(k)=16$ for the first layer and $\mathbb{E}(k)=12$ for the second. We expect that the second susceptibility peak appears near the epidemic threshold of the second layer individually, i.e. $\lambda \approx 0.083$.

Figure 54 presents the number of infected nodes in the steady-state on the layer with the lowest individual eigenvalue as a function of the size of the layers and a combination of the parameters $\lambda=0.078,0.083,0.085,0.088$ (near the individual critical point of the second layer) and $\eta=10^{-4}, 10^{-3}, 10^{-2}, 10^{-1}$. Besides, in the insets, we have the information about the average fraction (left inset of each panel) and its fluctuations, measured by the standard deviation (right inset on each panel). The straight lines in red were obtained by a least squares regression method. 
Figure 56 - Finite size analysis of the susceptibility. In the main panel, we have the susceptibility as a function of $\lambda$ for different sizes of 2 layer multiplex network, where the first layer have $\mathbb{E}(k)=16$ and the second $\mathbb{E}(k)=12$. In this experiment, we fixed the ratio $\frac{\eta}{\lambda}=0.01$. In the inset, we show the susceptibility of the two peaks as a function of the layer size, where the blue symbols refer to the first peaks, while the green symbols refer to the second peak. Besides, the red lines are a linear fitting of these points. The layer sizes evaluated are $n=3 \times 10^{3}, 4 \times 10^{3}, 5 \times 10^{3}, 6 \times 10^{3}, 7 \times 10^{3}, 8 \times 10^{3}, 9 \times$ $10^{3}, 10^{4}, 2 \times 10^{4}, 3 \times 10^{4}, 4 \times 10^{4}, 5 \times 10^{4}, 10^{5}$.

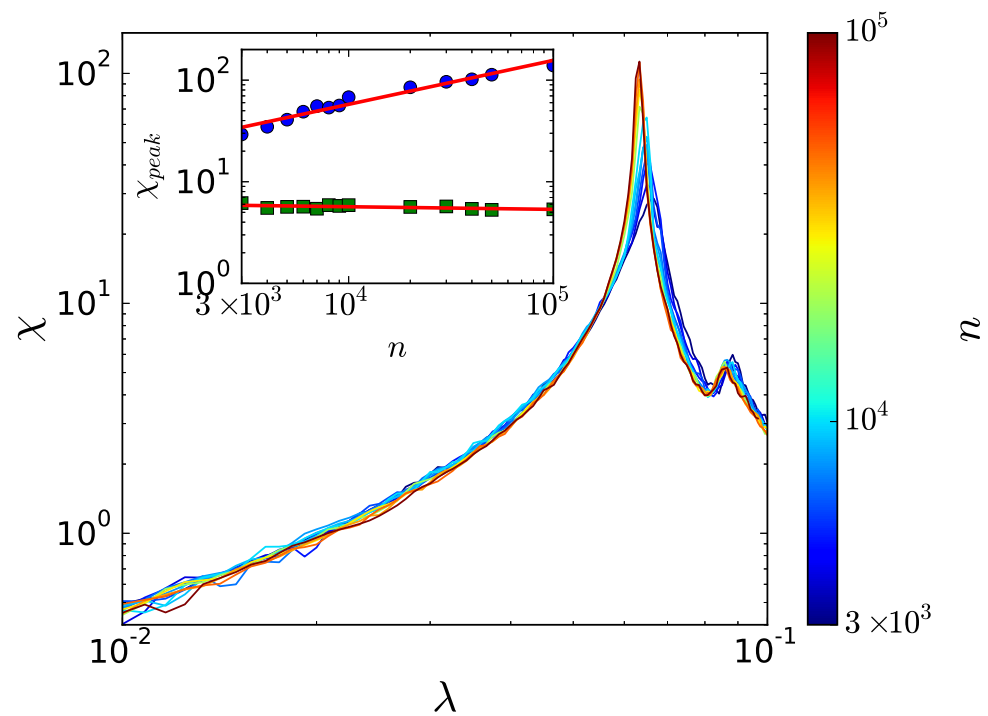

Source: Developed by the author.

We observe an approximately linear behavior of the number of infected nodes in the second layer as a function of the number of nodes in this layer (see the main panels of Figure 54). Consequently, the fraction $\rho_{2}$ also presents a linear trend (see the left inset on each panel of Figure 54). In fact, it presents a flat pattern, i.e approximately constant. Besides, the number of infected nodes is always larger than zero, since it is not a diseasefree state. Furthermore, we also observed that the fluctuations tend to be very low (see the right inset on each panel of Figure 54). Regarding the fluctuations, it is noteworthy that in a phase transition they tend to diverge, which does not happen in our analysis, thus also ruling out a second order phase transition as far as it is concerned. We also note that fluctuations are slightly higher for lower spreading rates, as can be seen by the error bars for $\lambda=0.078$, which is explained by the delocalization of our system.

Furthermore, in Figure 55 we present the comparison of steady state fractions. In each panel, we fix a value of $\lambda$ and compare different values of $\eta$. It emphasizes the influence of $\eta$ on the final fraction of infected nodes on the second layer. Note that for $\eta=10^{-4}$ and small networks the behavior exhibits a growing trend. This is due to the fact that for networks with $n<10^{4}$ the contribution of the first layer can be effectively 
neglected. In fact, observe that for $n \geq 10^{4}$, the fraction of infected nodes on the second layer follows a flat pattern (see Figure 55 (c) and (d)).

Finally, in Figure 56, we present a finite size analysis of the susceptibility for different sizes, ranging from $n=3 \times 10^{3}$ to $n=10^{5}$ and $m=2$ layers. Each curve was obtained by the QS algorithm, where we simulated 120 points from $\lambda=10^{-2}$ to $\lambda=10^{-1}$. In this experiment, we fixed the ratio $\frac{\eta}{\lambda}=0.01$. Additionally, we also used a moving average filter with two points for visualization purposes. In the inset, we show the scaling of the susceptibility of both peaks, where the first shows an increasing trend, while the second presents an approximately constant behavior. The increasing trend of the first peak is a consequence of the phase transition, suggesting a divergence of this parameter on the thermodynamic limit. On the other hand, the second peak is related to the localization phenomena and, contrasting with the first peak, this peak does not diverge, neither does it seem to vanish.

\subsubsection{3-Layer interconnected systems: the barrier effect}

Following the main ideas of the last sections, we explore the spreading dynamics in multilayer networks with more than two layers. Specifically, we have carried out numerical simulations for a 3-layer system. We generate multiplex networks using three scale-free networks, with $\zeta \approx 2.3, \zeta \approx 2.6$ and $\zeta \approx 2.9$, with $\mathbb{E}(k) \approx 8$ and $n=10^{3}$ nodes on each layer. Note that we consider three layers with spaced individual leading eigenvalues in order to investigate whether multiple susceptibility peaks are a generic phenomenon of multilayer systems. Note that we have two possible topologies for the network of layers: (i) a line graph and (ii) a triangle (which is a node-aligned multiplex). Consequently, the first can be arranged in three possible configurations by changing the central layer. That is, we have four possible systems. In this section, we focus on two configurations, the multiplex case and the line $(2.3+2.9+2.6)$. Both cases summarize the richness of dynamical processes on interconnected networks, presenting a new phenomenon, the barrier effect of an intermediate layer. We proceed by analyzing the spectral properties of this multilayer system in terms of the inverse participation ratio and the susceptibility. Regarding the other interconnected networks, we present these complementary results and analyses in the next chapter, more precisely in Section 6.1.2. Additionally, in Section 6.1.2 we show that increasing $\frac{\eta}{\lambda}$, also increases the role of the inter-layer edges relative to the intra-layer ones. Consequently, the structure of the network of layers imposes itself more strongly in the eigenvalues of the entire interconnected structure.

\subsubsection{Spectral analysis}

Figure 57 shows the $\operatorname{IPR}\left(\Lambda_{1}\right)$ of tensor $\mathscr{R}$. In the main panel, we present the individual contribution of each layer, while in the insets we have the total $\operatorname{IPR}\left(\Lambda_{1}\right)$. In 
Figure 57 - Spectral properties of the tensor $\mathscr{R}(\lambda, \eta)$ as a function of the ratio $\frac{\eta}{\lambda}$ for a multiplex with two layers with the same degree distribution (different random realizations of the configuration model) and connected to its counterpart on the other layer. In the left panel, we present the inverse participation ratio $(\operatorname{IPR}(\Lambda))$ of the two larger eigenvalues and the individual layer contributions, while in right panel, we show the leading eigenvalues. Every curve is composed by $10^{3} \log$ spaced points, in order to have enough resolution. In (a) we have the line $(2.3+2.9+2.6)$, while in $(b)$ the multiplex case.
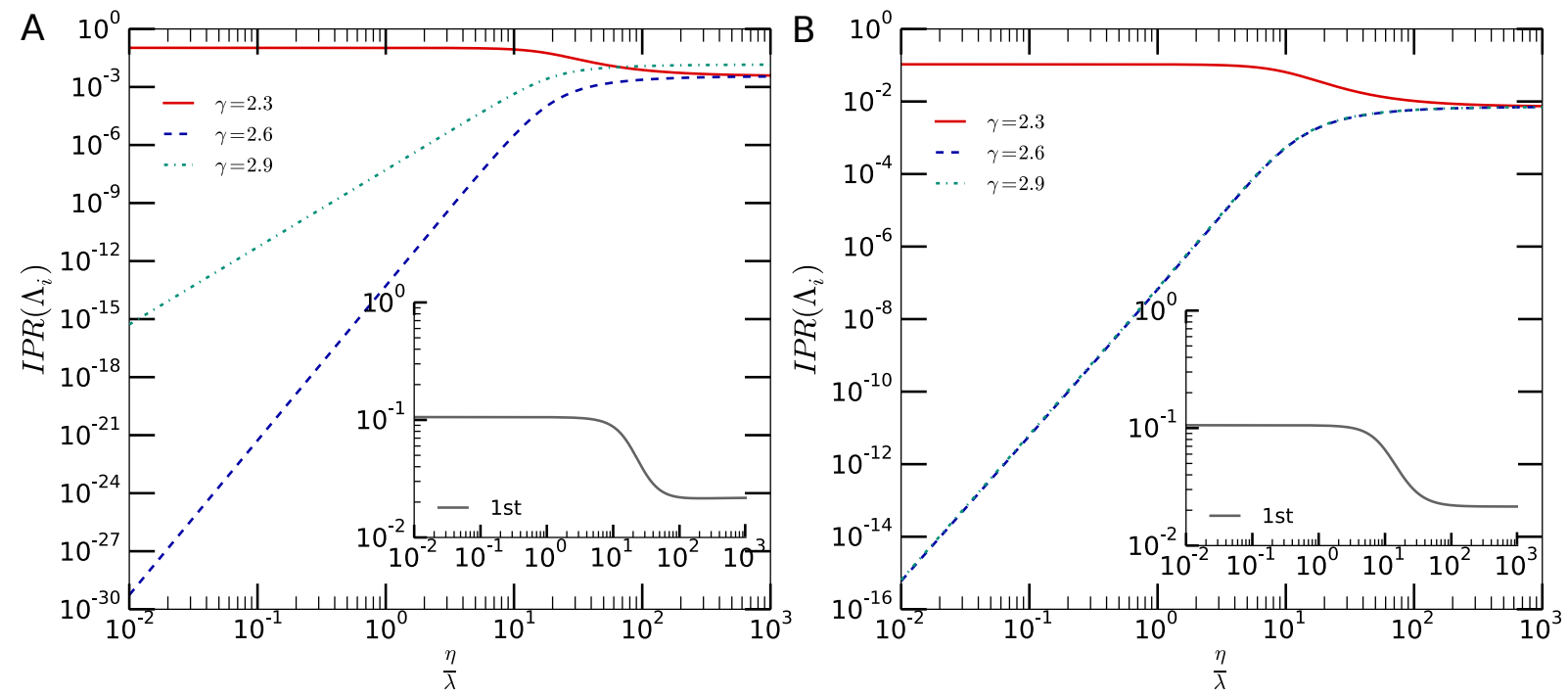

Source: Developed by the author.

the left panel, we have the line $(2.3+2.9+2.6)$, whereas in the right panel we have the multiplex network. In this section, we focus on the spectral comparison of two cases: (i) the lines $(2.3+2.6+2.9)$ and $(2.3+2.9+2.6)$ and (ii) the line $(2.6+2.3+2.6)$ and the multiplex network. Additionally, the reader is referred to Appendix D.1, specifically to Figure 82 for complementary results.

An interesting phenomenon can be observed comparing the different configurations of the network of layers. The largest eigenvalue of the whole system, $\Lambda_{1}$, has its associated eigenvector localized in the dominant layer, that is, in the layer generated using $\zeta=2.3$. Regarding the line configuration, depending on the position of that layer in the whole system - i.e., central or peripheral layer - the contribution of the non-dominant layers to the $\operatorname{IPR}\left(\Lambda_{1}\right)$ varies. In particular, when the dominant layer corresponds to an extreme node of the network of layers, the contribution of the other two layers will be ordered according to the distance to the dominant one. Consequently, when the dominant layer is in the center of the network of layers, the contributions of the non-dominant ones are comparable (see Figure 82 on Appendix D.1 for complementary results).

Furthermore, for the first eigenvalue, which is usually enough to analyze the localization as a first order approximation, we observe that the layer with the largest eigenvalue 
Figure 58 - Susceptibility $\chi$ as a function of $\lambda$ considering all three layer configurations and many different ratios $\frac{\eta}{\lambda}$, which is represented by the color of the lines. The recovering rate is $\delta=1$. The simulated values are $\frac{\eta}{\lambda}=0.05,0.06,0.07,0.08,0.09,0.1,0.2,0.3$, $0.4,0.5,0.6,0.7,0.8,0.9,1.0,2,3,4,5,6,7,8,9,10,20$. In (a) we have the line $(2.3+2.9+2.6)$, while in (b) the multiplex case.
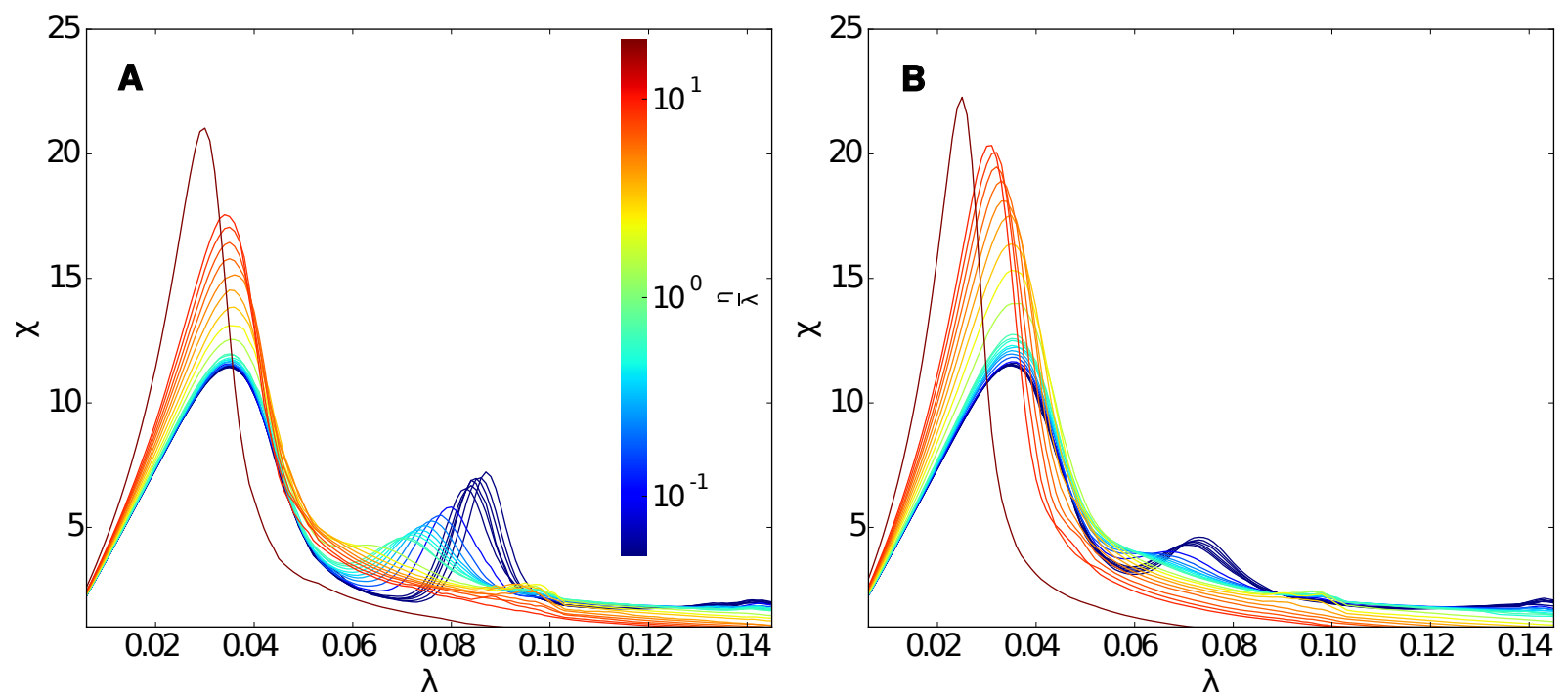

Source: Developed by the author.

dominates the dynamics. In addition, note the similarities between the multiplex and the line configuration $(2.6+2.3+2.6)$ (see also Figure 82, Appendix D.1), where the nondominant layers behave similarly. This is because, for small values of $\frac{\eta}{\lambda}$, the effect of the extra edge in the network of layers (closing the triangle) is of order $\eta^{2}$ and so is the similar behavior observed for the two configurations. As $\frac{\eta}{\lambda}$ grows, the symmetry in the node-aligned multiplex dominates the eigenvector structure and the contributions of all layers are comparable. As we show next, the different contributions of the layers to the total $\operatorname{IPR}\left(\Lambda_{1}\right)$ are at the root of the multiple susceptibility peaks observed.

\subsubsection{Multiple susceptibility peaks}

Figure 58 shows the susceptibility as a function of $\lambda$ for different ratios of $\frac{\eta}{\lambda}$. We observe three well-defined peaks on such curves when the ratio $\frac{\eta}{\lambda}$ is small. In addition, similar to the 2-layer case, these peaks tend to become less defined and vanish as the ratio $\frac{\eta}{\lambda}$ increases. The third peak is less defined than the others because the average number of infected nodes is larger in this case. Consequently, the susceptibility tends to be lower, as it measures the variance in relation to the average. This an observation suggests that it could be harder to observe peaks for non-dominating layers that have an individual critical point too far from the dominating layer.

Except for the line $(2.3+2.9+2.6)$, all figures are similar and present similar peaks, implying that the susceptibility peaks occur approximately at the same point (for 
a complementary analysis see Appendix D.2 and Figure 83). On the other hand, the line $(2.3+2.9+2.6)$ shows a slightly different behavior for the second peak, that is found for a larger value of $\lambda$ than for the other cases. This result suggests that when the layer with the largest eigenvalue is located at the center of the line, it can effectively act as a barrier to the disease. In addition, it can be observed that the extra inter-edges of the multiplex case do not lead to radical changes in the transition points. We remark that the susceptibility does not measure the fraction of spreaders in the steady state. Thus, despite the similarities of these curves, the phase diagrams for the incidence of the disease are different.

Coming back to what is observed for the network of layers described by the line $(2.3+2.9+2.6)$, an interesting phenomenon arises, namely, the formation of barriers to the epidemic spreading. Since the middle layer has the lowest individual eigenvalue among the layers, it creates a barrier effect "delaying" the second transition. Moreover, we observe that this transition also vanishes for higher values of the ratio $\frac{\eta}{\lambda}$, if compared to the other cases. This can be related to the inverse participation ratio of $\Lambda_{1}, \operatorname{IPR}\left(\Lambda_{1}\right)$, shown in Figure 57. Note that, for the line $(2.3+2.9+2.6)$, the contribution of the layer $\zeta=2.6$ is the lowest. As shown in Section 5.1.3.1 (and in (COZZO et al., 2013)), for a 2-layer multiplex, the non-dominant layer has its critical point shifted to a lower value of the spreading rate, which means that the outbreak takes place before it would have happened if that layer were isolated. However, here such shifting is compromised by the fact that the central layer is unable to sustain the epidemic process, acting effectively as a barrier for disease contagion. Apart from this new effect, the system behaves qualitatively similar to the 2-layer scenario.

Regarding the third peak, note that it is less defined than the others because the average number of infected nodes is larger in this case. Consequently, the susceptibility tends to be lower, as it measures the variance in relation to the average. The comparison of Figures 58 (b) and 83 shows that there is no difference in the position of the susceptibility peaks. As mentioned, the only observed difference is the barrier effect, shown in Figure 58 (a). We also remark the similarities between the line $(2.6+2.3+2.6)$ and the multiplex case, which emphasize the role of the central node. In that line configuration, the layer with $\zeta=2.3$ spreads its influence to both layers, which is similar to the multiplex case, however with fewer intra-edges.

\subsection{On degree-degree correlations in multilayer networks}

In this section, we study degree-degree correlations in multilayer systems and propose a way to generalize previous assortativity metrics by considering the tensorial formulation introduced in (De Domenico et al., 2013) (see also Section 2.3.2). Our approach 
also covers a weighted version of assortativity (LEUNG; CHAU, 2007) and the case in which the assortativity is given by the Spearman correlation coefficient, generalizing the definition in (LITVAK; HOFSTAD, 2013). Moreover, it is worth mentioning the generalization for weighted and directed networks (TELLER et al., 2014). The study of a real dataset corresponding to the airport transportation network shows a contrasting behavior between the analyses of each layer independently and altogether, which reinforces the need for such a generalization of the assortativity measure.

\subsubsection{Assortativity in multilayer networks}

Using the tensorial notation, presented in Section 2.3.2.1 and following Einstein's summation convention, the assortativity coefficient (originally proposed in (NEWMAN, 2002)) can be written as

$$
\rho\left(\mathscr{W}_{\beta}^{\alpha}\right)=\frac{\mathscr{M}^{-1} \mathscr{W}_{\beta}^{\alpha} Q^{\beta} Q_{\alpha}-\left[1 / 2 \mathscr{M}^{-1}\left(\mathscr{W}_{\beta}^{\alpha} Q_{\alpha} u^{\beta}+\mathscr{W}_{\beta}^{\alpha} Q^{\beta} u_{\alpha}\right)\right]^{2}}{\mathscr{M}^{-1}\left(\mathscr{W}_{\beta}^{\alpha}\left(Q_{\alpha}\right)^{2} u^{\beta}+\mathscr{W}_{\beta}^{\alpha}\left(Q^{\beta}\right)^{2} u_{\alpha}\right)-\left[1 / 2 \mathscr{M}^{-1}\left(\mathscr{W}_{\beta}^{\alpha} Q_{\alpha} u^{\beta}+\mathscr{W}_{\beta}^{\alpha} Q^{\beta} u_{\alpha}\right)\right]^{2}}
$$

where $u$ is the 1 -tensor, which is a tensor of rank 1 and has all elements equal to $1, \mathscr{W}_{\beta}^{\alpha}$ is a second order tensor that summarizes the information that is being extracted and $\mathscr{M}=\mathscr{W}_{\beta}^{\alpha} U_{\alpha}^{\beta}$ is a normalization constant.

Let us explain in more details all terms appearing in the expression of $\rho\left(\mathscr{W}_{\beta}^{\alpha}\right)$. First, we define

$$
Q^{\alpha}=\mathscr{W}_{\beta}^{\alpha} u^{\beta},
$$

which is a 1-contravariant tensor and

$$
Q_{\beta}=\mathscr{W}_{\beta}^{\alpha} u_{\alpha}
$$

which is a 1-covariant tensor. Moreover, the indices are related to the direction of the relationships between nodes. This choice ensures a more general expression, capturing degree correlations on non-symmetric tensors and, consequently, in directed and weighted networks.

Due to the multiplex nature of such systems, we obtain different types of correlations, which can be uncovered by operating the adjacency tensor. As operations on the nodes we can cite the single layer extraction (Equation 2.45), the projected network (Equation 2.47) and the overlay network (Equation 2.48). Complementary, if we extract the network of layers (Equation 2.46), the correlation between different layers can also be evaluated. For more, see Section 2.3.2.2 and reference (De Domenico et al., 2013). In other words, we define the tensor $\mathscr{W}_{\tilde{\delta}}^{\tilde{\gamma}}$ as one of the above-cited projections.

Regarding the overlay and the projected networks, extracting degree-degree correlations, nodes with similar degrees connected in the same or different layers contribute 
positively to the assortativity coefficient. On the other hand, the connections between hubs and low degree nodes in the same or different layers decrease the assortativity. Selfedges always increase the assortativity, which yields different values of assortativity for the overlay and the projected networks. This gives information on the nature of the coupling between different replicas of the same object among different layers.

Complementary, if we extract the network of layers (Equation 2.46, Section 2.3.2.2), the correlation between different layers can also be evaluated. We use $\mathscr{W}_{\tilde{\delta}}^{\tilde{\gamma}}=\Psi_{\tilde{\delta}}^{\tilde{\gamma}}$. It is important to stress that the components of this adjacency tensor are not binary, but weighted by the number of edges between each layer. Moreover, also in this case, the resulting tensor presents self-edges that encode the information about the density of connections inside a single layer. Finally, we can consider only interlayer relationships over two different layers. This information is extracted by projecting the adjacency tensor on the canonical base as

$$
\mathscr{W}_{\beta}^{\alpha}=C_{\beta}^{\alpha}(\tilde{r} \tilde{h})=M_{\beta \tilde{\gamma}}^{\alpha \tilde{\delta}} E_{\tilde{\delta}}^{\tilde{\gamma}}(\tilde{r} \tilde{h}) .
$$

Note that this is only applicable to multilayer networks and does not make sense in multiplex networks, since in the latter case the coupling is diagonal.

Moreover, in some applications, it is interesting to calculate a pair-wise correlation between a set of nodes, for instance, between couple of layers. Thus, we propose a new operation, that we call selection, which is a projection over a selected set of layers:

$$
\mathscr{W}_{\beta}^{\alpha}(\mathscr{L})=S_{\beta}^{\alpha}(\mathscr{L})=M_{\beta \tilde{\gamma}}^{\alpha \tilde{\delta}} \Omega_{\tilde{\delta}}^{\tilde{\gamma}}(\mathscr{L})
$$

where $\Omega_{\tilde{\delta}}^{\tilde{\gamma}}$ is a tensor used to select the set of layers we consider in the projection $(\mathscr{L})$. The components of the tensor are equal to unity when the layers $\tilde{\delta}$ and $\tilde{\gamma}$ are selected, and zero otherwise. Note that by selecting all layers together we recover the 1-tensor $U_{\tilde{\delta}}^{\tilde{\gamma}}$ and consequently Equation 2.47. Another special case is $\tilde{\delta}=\tilde{\gamma}$, which yields Equation 2.45, or the layer extraction. The tensor can also be generalized to weigh different layers. In this case, each element of $\Omega_{\tilde{\delta}}^{\tilde{\gamma}}$ contains the weight of the relationship between two layers $\tilde{\delta}$ and $\tilde{\gamma}$. This projection is similar to the covariance matrix in statistics, which generalizes the concept of variance. The covariance between two variables is quantified in each entry of the matrix and the main diagonal has the variance of each variable. Thus, we can define a matrix that generalizes the assortativity in a similar way the covariance matrix generalizes the concept of variance, i.e.

$$
\mathbf{S}_{\tilde{\delta}}^{\tilde{\gamma}}=\rho\left(S_{\beta}^{\alpha}(\mathscr{L}=\{\tilde{\gamma}, \tilde{\delta}\})\right)
$$

which belongs to a $\mathbb{R}^{L \times L}$ space. We call $\mathbf{S}$ the P-assortativity matrix.

Also in this case, a similar operation for the overlay network can be considered, yielding

$$
\mathscr{W}_{\beta}^{\alpha}(\mathscr{L})=Z_{\beta}^{\alpha}(\mathscr{L})=\sum_{\tilde{h} \in \mathscr{L}}^{L} C_{\beta}^{\alpha}(\tilde{h} \tilde{h})
$$


Figure 59 - Example of an airport transportation multilayer network. Each layer represents an airline, in which each node represents an airport and the edges are flights between two airports. This visualization was generated using MuxViz (De Domenico; PORTER; ARENAS, 2014).

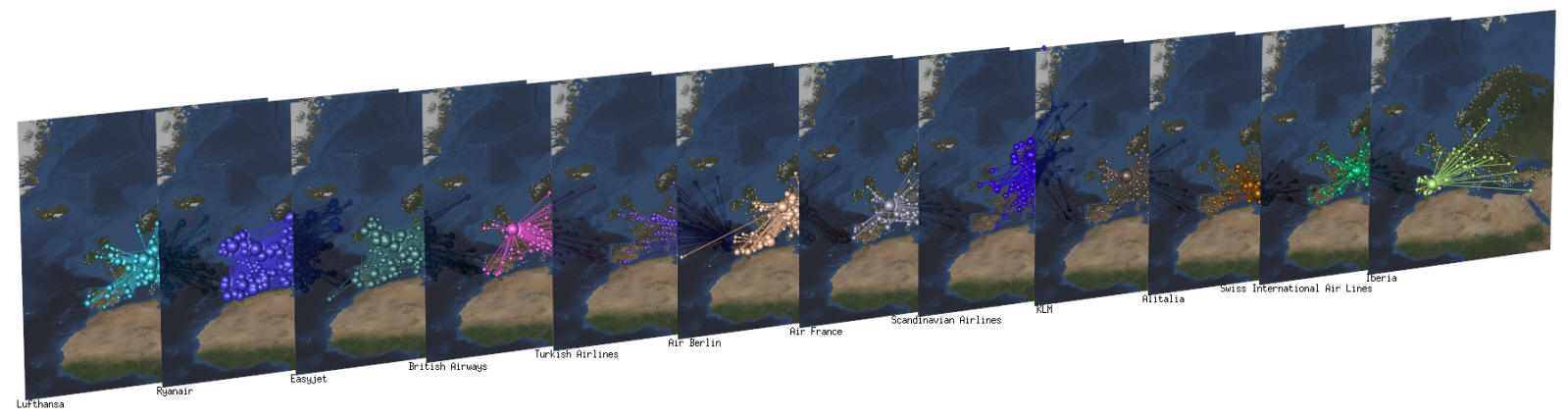

Source: Developed by the author.

which can also be generalized in a similar way to Equations 5.15 and 5.16, resulting in the matrix

$$
\mathbf{Z}_{\tilde{\delta}}^{\tilde{\gamma}}=\rho\left(Z_{\beta}^{\alpha}(\mathscr{L}=\{\tilde{\gamma}, \tilde{\delta}\})\right)
$$

We call $\mathbf{Z}$ the $O$-assortativity matrix. A similar interlayer correlation was also proposed in (NICOSIA; LATORA, 2015), where the authors suggested measuring the degree correlation between two different layers of the replica of the same object (or node). Furthermore, they proposed three different ways: the Pearson correlation coefficient, Spearman rank correlation and the Kendall's $\tau$ index. However, it is worth pointing out that such an approach does not consider the intra-layer relationship because it is only for node-aligned multiplex networks (KIVELÄ et al., 2014). Here, we generalize this measure in terms of tensorial notation.

Finally, the assortativity coefficient can also be defined in terms of the Spearman rank correlation (LITVAK; HOFSTAD, 2013), as the traditional definition of this coefficient based on the Pearson correlation (NEWMAN, 2002) can lead to incomplete results, as discussed in (LITVAK; HOFSTAD, 2013). The generalization of assortativity coefficient proposed here considers the Spearman rank correlation coefficient by changing Equations 5.12 and 5.13. Specifically, instead of considering the values of $Q^{\alpha}$ and $Q_{\beta}$, one substitutes them by their respective ranks ${ }^{2}$. This transformation is performed by using

$$
Q^{\alpha}=\operatorname{rank}\left(\mathscr{W}_{\beta}^{\alpha} u^{\beta}\right)
$$

and

$$
Q_{\beta}=\operatorname{rank}\left(\mathscr{W}_{\beta}^{\alpha} u_{\alpha}\right)
$$

$\overline{2}$ One should not confuse rank in this context with the tensorial rank. Here it is the position in the ordered set of values, whereas the rank of a tensor is the number of covariant and contravariant indices. 
Table 6 - Structural properties of the airport transportation multilayer networks.

\begin{tabular}{|l|c|c|c|c|c|}
\hline Network & $N$ & $\mathscr{M}$ & $\mathbb{E}\left(Q^{\alpha}\right)$ & $\rho^{P}\left(\mathscr{W}_{\beta}^{\alpha}\right)$ & $\rho^{S}\left(\mathscr{W}_{\beta}^{\alpha}\right)$ \\
\hline Network of Layers $\left(\Psi_{\beta}^{\alpha}\right)$ & 37 & 30398.0 & 821.568 & 0.377 & 0.286 \\
Overlay $\left(O_{\beta}^{\alpha}\right)$ & 450 & 7176.0 & 15.947 & -0.050 & -0.025 \\
Projected network $\left(P_{\beta}^{\alpha}\right)$ & 450 & 30398.0 & 67.551 & 0.795 & 0.560 \\
\hline
\end{tabular}

where $\operatorname{rank}\left(X_{i}\right)$ is the rank of the tensor $X_{i}$.

We henceforth denote by $\rho^{P}\left(\mathscr{W}_{\beta}^{\alpha}\right)$ and $\rho^{S}\left(\mathscr{W}_{\beta}^{\alpha}\right)$ the Pearson and Spearman correlation coefficients, respectively. Furthermore, we adopt $\left(\mathbf{S}^{P}\right)_{\tilde{\delta}}^{\tilde{\gamma}}$ and $\left(\mathbf{S}^{S}\right)_{\tilde{\delta}}^{\tilde{\gamma}}$ for the pair-wise correlation matrices using the Pearson and Spearman correlation coefficients, respectively. The same notation can be used for the matrices $\left(\mathbf{Z}^{P}\right)_{\tilde{\delta}}^{\tilde{\gamma}}$ and $\left(\mathbf{Z}^{S}\right)_{\tilde{\delta}}^{\tilde{\gamma}}$. Monoplex assortativity, i.e. assortativity in single-layer networks (NEWMAN, 2002), is recovered by considering the adjacency matrix, $\mathscr{W}_{\beta}^{\alpha}=A_{\beta}^{\alpha}$, and consequently $Q^{\alpha}$ and $Q_{\beta}$ are analogous to in-degree and out-degree, respectively. Note that $Q^{\alpha}=Q_{\beta}$ for undirected networks. Moreover, $\mathscr{M}$ is equal to twice the number of edges, recovering the equation introduced in (NEWMAN, 2002), which also captures correlations of weighted networks, as shown in (LEUNG; CHAU, 2007).

Each approach presented here gives a different descriptor of the multilayer structure. For instance, the projected and overlay networks gather the information of all layers into a single layer structure, aiming at describing the whole system using single descriptors. Besides those, we also provide a pair-wise descriptor which gives another type of information. Moreover, there is also the network of layers, that possesses information about yet another level of the system. Thus, our approach gives a set of metrics that capture information about the whole multilayer structure. However, it is worth mentioning that the interpretation and choices depend on the application.

\subsubsection{Application to real data}

We analyze the airport transportation network (COLIZZA et al., 2006), whose multilayer representation was studied in (CARDILLO et al., 2013). The network comprises 450 airports and 37 companies, which are mapped as nodes and layers, respectively. More specifically, in each layer, the edges represent the directed flights operated by a given company and nodes, airports. Figure 59 shows a representation of 12 layers of such a multilayer network. The inter-layer connections link the airports shared by pairs of different companies. This approach gives us a multilayer network that is not a node-aligned multiplex network, since the latter considers a diagonal coupling between all nodes in all layers. Note that the way proposed in (CARDILLO et al., 2013) to create the aggregated monoplex network is the union of all layers considering multiple edges as single 
Table 7 - Structural properties of each layer of the airport transportation multilayer networks.

\begin{tabular}{l|c|c|c|c|c}
\hline Company & $n$ & $\mathscr{M}$ & $\mathbb{E}\left(Q^{\alpha}\right)$ & $\rho^{P}\left(C_{\beta}^{\alpha}\right)$ & $\rho^{S}\left(C_{\beta}^{\alpha}\right)$ \\
\hline Lufthansa & 106 & 488.0 & 4.604 & -0.668 & -0.473 \\
Ryanair & 128 & 1202.0 & 9.391 & -0.321 & -0.348 \\
Easyjet & 99 & 614.0 & 6.202 & -0.428 & -0.470 \\
British Airways & 65 & 132.0 & 2.031 & -0.775 & -0.754 \\
Turkish Airlines & 86 & 236.0 & 2.744 & -0.697 & -0.567 \\
Air Berlin & 75 & 368.0 & 4.907 & -0.501 & -0.434 \\
Air France & 59 & 138.0 & 2.339 & -0.637 & -0.661 \\
Scandinavian Airlines & 66 & 220.0 & 3.333 & -0.681 & -0.521 \\
KLM & 63 & 124.0 & 1.968 & -1.000 & -1.000 \\
Alitalia & 51 & 186.0 & 3.647 & -0.572 & -0.538 \\
Swiss International Air Lines & 48 & 120.0 & 2.500 & -0.728 & -0.618 \\
Iberia & 35 & 70.0 & 2.000 & -0.900 & -0.838 \\
Norwegian Air Shuttle & 52 & 174.0 & 3.346 & -0.511 & -0.523 \\
Austrian Airlines & 67 & 144.0 & 2.149 & -0.823 & -0.744 \\
Flybe & 43 & 198.0 & 4.605 & -0.560 & -0.489 \\
Wizz Air & 45 & 184.0 & 4.089 & -0.350 & -0.381 \\
TAP Portugal & 42 & 106.0 & 2.524 & -0.779 & -0.610 \\
Brussels Airlines & 44 & 86.0 & 1.955 & -1.000 & -1.000 \\
Finnair & 42 & 84.0 & 2.000 & -0.915 & -0.858 \\
LOT Polish Airlines & 44 & 110.0 & 2.500 & -0.658 & -0.598 \\
Vueling Airlines & 36 & 126.0 & 3.500 & -0.438 & -0.456 \\
Air Nostrum & 48 & 138.0 & 2.875 & -0.571 & -0.569 \\
Air Lingus & 45 & 116.0 & 2.578 & -0.670 & -0.625 \\
Germanwings & 44 & 134.0 & 3.045 & -0.628 & -0.482 \\
Panagra Airways & 45 & 116.0 & 2.578 & -0.625 & -0.593 \\
Netjets & 94 & 360.0 & 3.830 & -0.106 & 0.107 \\
Transavia Holland & 40 & 114.0 & 2.850 & -0.585 & -0.535 \\
Niki & 36 & 74.0 & 2.056 & -0.838 & -0.784 \\
SunExpress & 38 & 134.0 & 3.526 & -0.797 & -0.542 \\
Aegean Airlines & 38 & 106.0 & 2.789 & -0.583 & -0.560 \\
Czech Airlines & 42 & 82.0 & 1.952 & -1.000 & -1.000 \\
European Air Transport & 53 & 146.0 & 2.755 & -0.416 & -0.423 \\
Malev Hungarian Airlines & 35 & 68.0 & 1.943 & -1.000 & -1.000 \\
Air Baltic & 45 & 90.0 & 2.000 & -0.844 & -0.812 \\
Wideroe & 45 & 180.0 & 4.000 & -0.293 & -0.311 \\
TNT Airways & 53 & 122.0 & 2.302 & -0.415 & -0.346 \\
Olympic Air & 37 & 86.0 & 2.324 & -0.754 & -0.662 \\
\hline
\end{tabular}

ones. This is in contrast to our approach, because we consider the projections and overlay networks as weighted networks, thus retaining the information of the number of different connections between the same pair of airports.

Previous studies (COLIZZA et al., 2006; CARDILLO et al., 2013) showed that the airport transportation network presents the rich-club effect, which refers to the tendency of highly central nodes to be connected among themselves. This is also captured by the assortativity as shown in Table 6 , where we verify that the projected network has positive assortativity coefficients, agreeing with previous analyses. However note that the 
Figure 60 - Pair-wise assortativity coefficient using Spearman rank correlation, $\rho^{S}\left(\mathbf{S}_{\beta}^{\alpha}\right)$. Observe that the main diagonal presents the same coefficient considering the layer extraction operation, $\rho^{S}\left(C_{\beta}^{\alpha}(\tilde{r} \tilde{r})\right)$

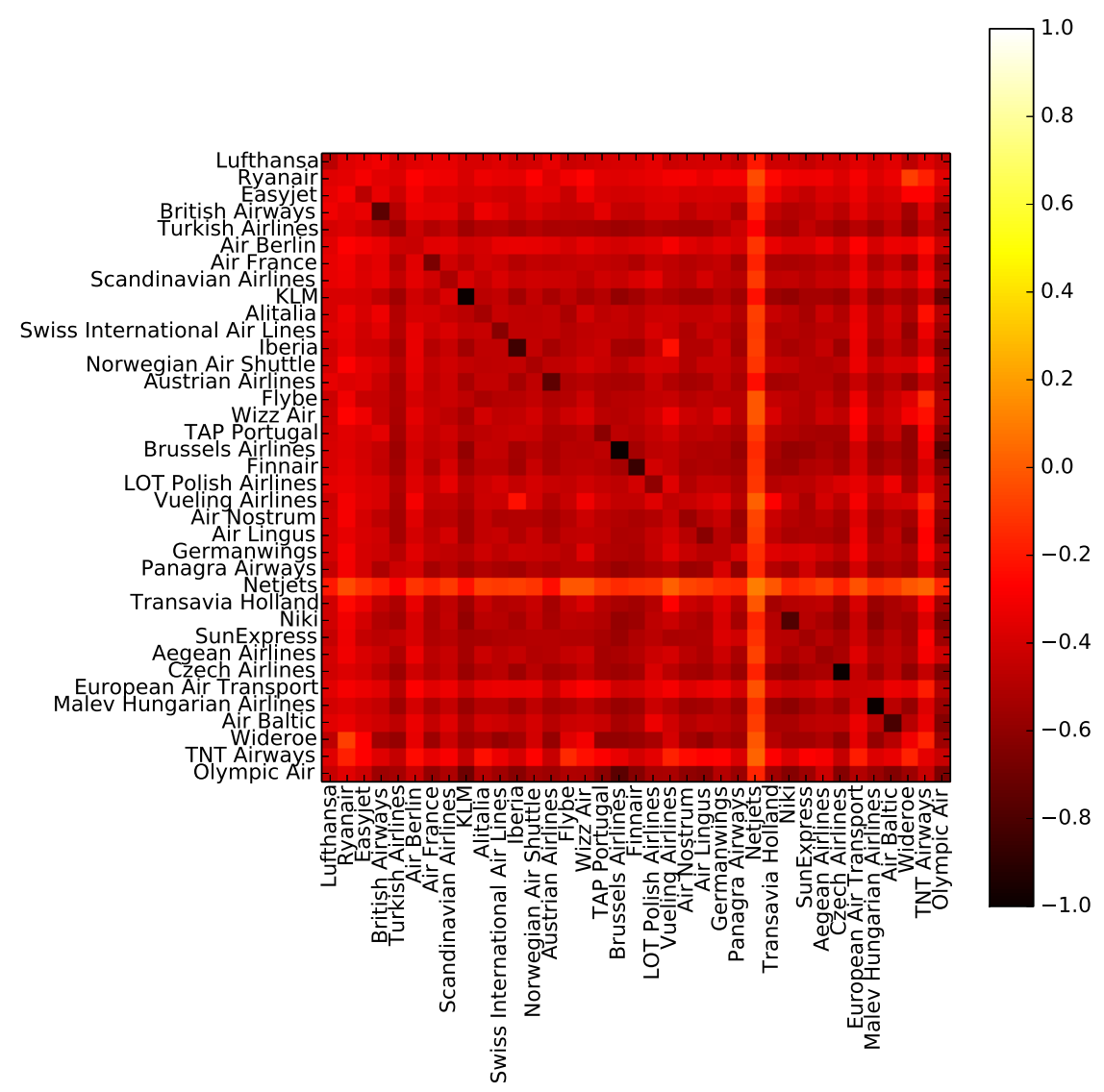

Source: Developed by the author.

projection has a positive value of the assortativity, whereas the overlay has a negative one. Thus, the assortativity of the projection indicates that many companies share hubs airport, not that hubs connect between them. This apparent contradiction results from the fact that the rich-club effect is masked out in the overlay setup by a large number of peripheral nodes connecting to hubs.

The analysis of each layer separately (see Table 7) shows a different result, where most of the layers are disassortative. The only exception is the Netjet layer, which presents a positive coefficient for the rank correlation. Usually, the companies focus their activities on one city or country, for example, Lufthansa in Germany or Air France in France, and have flights to other airports where their activity is lower. This leads to the disassortative behavior of each layer. Additionally, the disassortative correlations found in single layers is more significant than that of the overlay representation, which can be explained by noticing that hubs of a company are peripheral (or secondary) airports for other companies, but when the layers are collapsed they are also hubs in the overlay network and are 
Figure 61 - Pair-wise assortativity coefficient using Spearman rank correlation, $\rho^{S}\left(\mathbf{Z}_{\beta}^{\alpha}\right)$. Observe that the main diagonal presents the same coefficient considering the layer extraction operation, $\rho^{S}\left(C_{\beta}^{\alpha}(\tilde{r} \tilde{r})\right)$

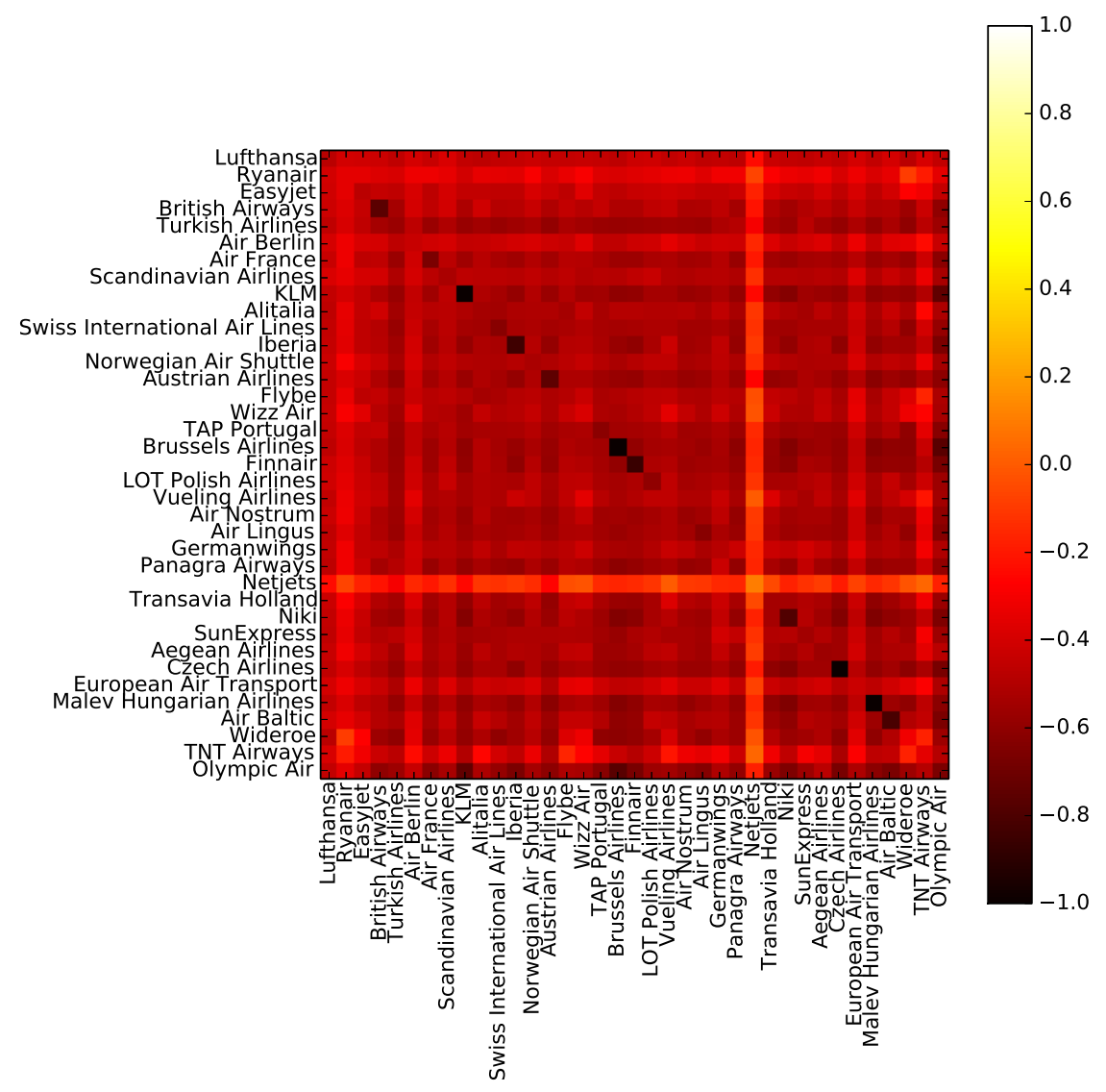

Source: Developed by the author.

connected.

Figures 60 and 61 shows the pair-wise correlation between layers. Interestingly, the latter is disassortative, in contrast to the results obtained for the projected network, but of the same sign as those computed for the overlay representation (see Table 6). Furthermore, our construction of the adjacency tensor leads to an assortative network of layers, suggesting that bigger companies tend to share similar airports. This analysis agrees with (CARDILLO et al., 2013), where the authors argued that the main airports are connected to each other via direct flights. In addition, considering the Pearson correlations, the $O$-assortativity matrix presents lower values if compared to the $P$-assortativity matrix due to the intra-layer contributions, as discussed before. 
Figure 62 - Phase diagram for epidemic spreading on different levels of assortativity. (a) First layer with $\zeta \approx 3$ and $\mathbb{E}(k) \approx 17$, and second with $\zeta \approx 2.8$ and $\mathbb{E}(k) \approx 12$. (b) First layer with $\zeta \approx 3$ and $\mathbb{E}(k) \approx 17$, and second with $\zeta \approx 4.5$ and $\mathbb{E}(k) \approx 13$. All networks are composed by $n=10^{4}$ nodes. We adopt $\frac{\eta}{\lambda}=2$ and $\delta=1$. The continuous lines are the analytical solution (Equation 3.82), while the symbols are obtained from Monte Carlo simulations, averaging over $10^{2}$ runs. The standard deviation is of the size of the symbols.

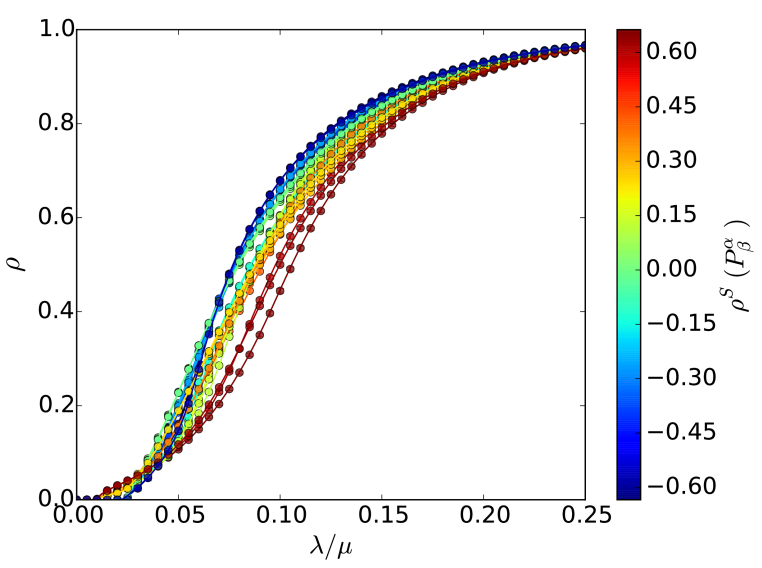

(a)

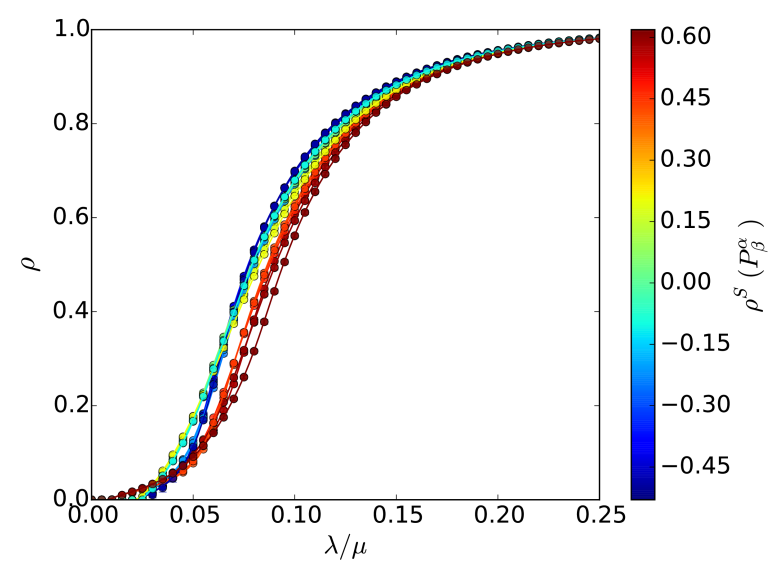

(b)

Source: Developed by the author.

\subsubsection{Epidemic spreading in correlated multilayer networks}

We investigate the effects of degree-degree correlations on epidemic spreading. To this end, we consider a classical SIS (Susceptible-Infected-Susceptible) model. Here, we adopt the discrete formulation presented in (COZZO et al., 2013), CA approach, considering a fully reactive process (RP), and also perform the appropriate Monte Carlo simulations of the epidemic process. We also assume the possibility of re-infection, that is, an infected individual can be cured and re-infected in the same time interval. Furthermore, it is convenient to consider the ratio between intra-layer and inter-layer spreading probabilities as a constant (COZZO et al., 2013), here we set $\frac{\eta}{\lambda}=2$. However, we find similar results considering other ratios. For more on its definitions, please see Chapter 3 , more specifically, Section 3.5.5.2, where we formally define our model.

In order to quantify the effect of degree-degree correlations on the spreading process, we generate two scale-free networks, with degree distribution $P(k) \approx k^{-\zeta}$, according to the configuration model (VIGER; LATAPY, 2005). The first layer has $\zeta \approx 3$ and $\mathbb{E}(k) \approx 17$, whereas the other one we evaluate in two different configurations: (i) $\zeta \approx 4.5$ and $\mathbb{E}(k) \approx 13$ and (ii) $\zeta \approx 2.8$ and $\mathbb{E}(k) \approx 12$. Both networks are composed by $n=10^{4}$ nodes.

Thus, using the simulated annealing algorithm described in Section 2.1.3, we tune the assortativity on the individual layers. We can have three different configurations for 
each layer, i.e., (i) one assortative, (ii) one disassortative and (iii) one non-assortative. These individual layers are connected, forming a multiplex network. In this case, we can have three different configurations: (i) assortative: densely connected nodes from one layer is connected to densely connected nodes in the other layer, (ii) disassortative: hubs in one layer are connected to low degree nodes in the other layer, and (iii) random: nodes in different layers are randomly connected. Thus, our data set is composed of 27 multiplex networks presenting different levels of assortativity. We also consider $\frac{\eta}{\lambda}=2$ and $\delta=1$. Similar results are found for different values of $\frac{\eta}{\lambda}$.

Figure 62 shows the simulations of the SIS dynamics on top of 2-Layer multiplex networks with different levels of assortativity. We can see a good agreement between the Monte Carlo simulation and the theoretical macro-state variable (see Equation 3.82), although we assume that there is no correlation between the state of each random variable. Each network has different values of the epidemic threshold and also exhibits different behaviors near the threshold. Indeed, the epidemic threshold for assortative networks is at a lower transmission probability. This happens because the disease has a faster initial growth rate and a shorter duration in assortative networks than in disassortative networks. Nevertheless, disassortative networks show higher values of $\rho$ for larger values of $\gamma / \lambda$. This result agrees with the analysis of single layer networks in (KISS; GREEN; KAO, 2008). Notice that the same behavior is observed for Figures 62(a) and 62(b), although in (a) the network is more heterogeneous than in (b). In fact, for more heterogeneous networks, the influence of degree-degree correlations is reduced.

\subsection{Summary}

In this chapter, we have studied disease spreading on top of multilayer networks. We considered the tensorial notation (for more, see Chapter 3 and reference (De Domenico et al., 2013)) and both the continuous-time and discrete-time formalisms in Sections 5.1 5.2 , respectively.

Firstly, we studied the localization properties of multilayer networks, in Section 5.1. In this case, our main results concern the emergence and vanishing of multiple susceptibility peaks as a function of the ratio between the inter-layer and intra-layer spreading rates and their relation to the spectral properties of the multilayer, which also revealed the phenomenon of disease localization, and in particular, its relation to the spectral properties of the supra contact tensor. Using the QS-Method and Monte Carlo simulations, we have been able to precisely determine the transition points. We remark that the first susceptibility peak is a phase transition, from a disease-free state to an endemic, but localized, state. On the other hand, the second peak is a transition from a localized to a delocalized state, which is not a second order phase transition. Additionally, we have proposed an 
analytical approach based on using the inverse participation ratio to characterize such transitions as a localization phenomenon, thus also connecting with (GOLTSEV et al., 2012).

A detailed exploration of the parameter space showed that as the ratio between the inter-layer and intra-layer spreading rates increases, the peaks of the susceptibility measured for the non-dominant layers tend to occur at lower values of $\lambda$ and vanish as $\frac{\eta}{\lambda}$ increases up to a point in which only one susceptibility peak is observed, which is a true phase transition. Interestingly enough, our results point out that such a transition can take place for even lower values of $\lambda$ than the inverse of the largest leading eigenvalue among all individual layers.

Furthermore, another important finding presented in Section 5.1 is the opposite phenomenon, namely, the barrier effect, which happens when the susceptibility peak takes place at a larger value of $\lambda$ than that expected as a consequence of the multiplex topology. Specifically, if the layers are arranged in such a way that the one with the smallest leading eigenvalue is at the center of the network of layers (for instance, as it happens for the line $(2.3+2.9+2.6)$ configuration), then the corresponding transition could be delayed due to the barrier effect.

Complementary, in Section 5.2, we focus on the effects of degree-degree correlations on the analysis of epidemic spreading. To do so, we have generalized the metric used to calculate assortativity of multilayer networks. Our approach consists of reducing the dimension of the adjacency tensor and applying the Pearson correlation coefficient on the extremes of each edge. We follow the tensorial approach, which helps us to have a compact, algorithmic and general formulation, covering various topological representations and possibilities, such as overlay and projected networks, and also pair-wise measurements. The calculation of the Spearman rank correlation is also possible from our formulation. Additionally, as an example, we studied the airport transportation network, where we verified that the individual analysis of the overlay or the projected networks can lead to misleading conclusions.

Finally, regarding the effects of degree-degree correlations on epidemic spreading. The results obtained from Monte Carlo simulations and theoretical analysis using a discrete-time Markov Chain formulation, $\mathrm{CA}(\mathrm{RP})$, in terms of tensors show that the level of assortativity and heterogeneity between layers influence the spreading process. More specifically, we verified that assortative networks show a smaller epidemic threshold and that the disease has a faster initial growth rate in these networks, but a shorter duration. On the contrary, the fraction of infected individuals is larger in disassortative networks. Finally, we have also shown that degree-degree correlations have a larger impact on the spreading dynamics when the coupled networks have similar levels of heterogeneity.

Interestingly, the findings of this chapter also emphasize the key role of spectral 
properties adjacency and contact tensor (or matrices). Note that the performed analysis raises questions on other layer configurations and how the spectra and its eigenvectors change as a function of the coupling $\frac{\eta}{\lambda}$ and also the system size. These ideas are the subject of our next chapter, where we complement the analysis we began here and study the spectral properties of multilayer networks in more details. 
CHAPTER

\section{6}

\section{NEW SPECTRAL ASPECTS OF MULTILAYER NETWORKS}

As motivated by the previous chapter, here we extend the spectral analysis performed in Section 5.1 for different layer configurations to similar and identical layers. This analysis motivates us to look more carefully at the problem of eigenvalue crossings and near crossings. Moreover, and as a natural consequence of the results of the previous chapter, we also evaluate the inverse participation ratio. Both analyses were performed as a function of the coupling parameter $\frac{\eta}{\lambda}$. Furthermore, we also observed a transition in the spectra of the supra-contact tensor, from the spectra resulting from the union of the individual layers to the spectra of the network of layers. This behavior implies that other dynamics and more complex structures can also be significantly affected by the interconnected nature of the system. These phenomena are explored in Section 6.1.

Additionally, shifting from the behavior as a function of the coupling parameter to an analysis regarding size, we study whether there are universal scaling properties in multilayer systems. In Section 6.2, we perform a scaling analysis of the eigenfunction localization properties of multilayer networks using random matrix theory (RMT) models and techniques. We explore multilayer networks whose networks of layers (COZZO et al., 2016) are of two types: (i) a line and (ii) a complete graph, node-aligned multiplex networks (for more, see Section 2.3). We demonstrate that the normalized localization length of the eigenfunctions of multilayer random networks exhibits a well-defined scaling function and also test the scaling law on real-world networks.

Finally, we change our perspective from the numerical experiments to the analytical approach. As shown in Section 6.3, the block nature of the multilayer matrices enable us to derive interesting results based on an interpretation of the traditional eigenvalue problem. More specifically, we reduce the dimensionality of our matrices but increasing the power of the characteristic polynomial, i.e a polynomial eigenvalue problem. This ap- 
proach may sound counterintuitive at first, but it allows us to relate the quadratic problem for a 2-Layer multiplex system with the spectra of the aggregated network, derive bounds for the spectra among many other interesting analytical insights. The main motivation for this approach can be found in (LANCASTER, 1966; GOHBERG; LANCASTER; RODMAN, 1982; TISSEUR; MEERBERGEN, 2001). Finally, we must also mention that this formalism is still in its development and was left as our last chapter because we believe it will allow us to derive new important results for the multilayer network theory in the future. In other words, it is also a new perspective for the structural analysis of multilayer networks.

\subsection{Eigenvalue crossings and localization of eigenvectors in multilayer networks}

On the one hand, the analysis performed in the previous chapter, more specifically in Section 5.1, produced a better understanding of the physical meaning of localization and mainly on layer-wise localization. On the other hand, it also opened up new paths for the investigation on the spectral properties of multilayer networks. More formally, it opened up questions on how the eigenfunctions are influenced by the structure in the multilayer in terms of the network of layers, but also in correlations between different layers.

Here, we explore the 2-Layer case in Section 6.1.1, analytically discussing eigenvalue crossing phenomena and numerically evaluating the identical and similar layers case. Note that the different layer case was previously discussed in Section 5.1.3.2 in the previous chapter. Additionally, the 3-Layer case is discussed in Section 6.1.2, complementing the results presented in Section 5.1.4, where we were mainly interested in the transition on the spectra of the supra-contact tensor, from the spectra resulting from the union of the individual layers to the spectra of the network of layers.

\subsubsection{2-Layer case}

In this section, we focus on the spectral analysis of the tensor $\mathscr{R}(\lambda, \eta)$ as a function of the ratio $\frac{\eta}{\lambda}$. First of all, we present an analytical approach to the problem of eigenvalue crossings in Section 6.1.1.1, then we focus our numerical analysis on two special cases in increasing order of complexity: (i) the identical case, where both layers are exactly the same. Thus, there is a high correlation between the degree in each layer, presented in Section 6.1.1.2; (ii) the non-identical case, where both layers present the same degree distribution, but different configurations on Section 6.1.1.3. The case in which both layers are structurally different and their individual leading eigenvalues are spaced was presented in the previous chapter. 


\subsubsection{Eigenvalue crossing}

Let us analyze the spectra of a simple setup: multiplex networks composed of $l$ identical layers. This class of networks provides insights about the spectral behavior as a function of $\left(\frac{\eta}{\lambda}\right)$. Although they are not very realistic a priori, there are situations in which this representation is helpful: for instance, in the context of disease contagion, one might think of a multi-strain disease in which each strain propagates in a different layer allowing co-infection of the host population.

The adjacency tensor can be written as

$$
\mathscr{R}_{\beta \tilde{\delta}}^{\alpha \tilde{\gamma}}(\lambda, \eta)=A_{\beta}^{\alpha} \delta_{\tilde{\delta}}^{\tilde{\gamma}}+\frac{\eta}{\lambda} \delta_{\beta}^{\alpha} K_{\tilde{\delta}}^{\tilde{\gamma}}
$$

where $A_{\beta}^{\alpha}$ is the 2-rank layer adjacency tensor, $K_{\tilde{\gamma}}^{\tilde{\delta}}$ is the adjacency tensor of the network of layers, which is a complete graph on the multiplex case, and $\delta_{\beta}^{\alpha}$ is the Kronecker delta. Observe that the sum of two Kronecker products, $\bar{A}=I_{m} \otimes A+\frac{\eta}{\lambda} K_{m} \otimes I_{n}$, where $I_{n}$ is the identity matrix of size $n$ and $K_{m}$ is the adjacency matrix of the complete graph with $m$ nodes is the unfolding of the adjacency tensor in this case. Thus, the eigenvalue problem can be written as

$$
\mathscr{R}_{\beta \tilde{\delta}}^{\alpha \tilde{\gamma}} f_{\alpha \tilde{\gamma}}=A_{\beta}^{\alpha} \delta_{\tilde{\delta}}^{\tilde{\gamma}} f_{\alpha \tilde{\gamma}}+\frac{\eta}{\lambda} \delta_{\beta}^{\alpha} K_{\tilde{\delta}}^{\tilde{\gamma}} f_{\alpha \tilde{\gamma}}
$$

where the sum of the eigenvalues of $A, \Lambda_{i}^{l}$, and $K, \mu_{i}$, are also eigenvalues of the adjacency tensor, hence $\mathscr{R}_{\beta \tilde{\delta}}^{\alpha \tilde{\gamma}} f_{\alpha \tilde{\gamma}}=\left(\Lambda_{i}^{l}+\frac{\eta}{\lambda} \mu_{j}\right) f_{\alpha \tilde{\gamma}}, i=1,2, \ldots n$ and $j=1,2, \ldots m$. Then,

$$
\left(\Lambda_{i}^{l}+\frac{\eta}{\lambda} \mu_{j}\right)=\left(\Lambda_{k}^{l}+\frac{\eta}{\lambda} \mu_{s}\right)
$$

The eigenvalues of the complete graph are $\mu_{1}=m-1$, and $\mu_{i}=-1, \forall i>1$, yielding to

$$
\frac{\eta}{\lambda}=\frac{\Lambda_{k}^{l}-\Lambda_{i}^{l}}{m}
$$

which imposes crossings on the eigenvalues of the adjacency tensor for identical layers, as $\left(\frac{\eta}{\lambda}\right)$ is a continuous parameter.

\subsubsection{Identical layers}

Considering a multiplex network made up of two layers with the same configuration. Each layer of the multiplex is a network composed by $n=1000, \mathbb{E}(k) \approx 6, \Lambda^{l}=14.34$, with degree distribution $P(k) \sim k^{-2.7}$. Aside from the intra-edge configuration, we also impose that inter-edges connect a node with its counterpart on the other layer, i.e., every node has the same intra-degree on all layers. This constraint imposes a high correlation between the degrees in each layer.

Figure 63 shows the spectral behavior of such a multiplex as a function of the parameter $\left(\frac{\eta}{\lambda}\right)$. In the right panel, we represent the inverse participation ratio of the first 
Figure 63 - Spectral properties of the tensor $\mathscr{R}(\lambda, \eta)$ as a function of the ratio $\frac{\eta}{\lambda}$ for a multiplex with two layers with the exact same degree distribution and connected to its counterpart on the other layer. In the right panel, we present the inverse participation ratio $(\operatorname{IPR}(\Lambda))$ of the three larger eigenvalues, while in the left panel, we show the leading eigenvalues. Every curve is composed by $10^{3} \mathrm{log}$ spaced points, in order to have enough resolution.
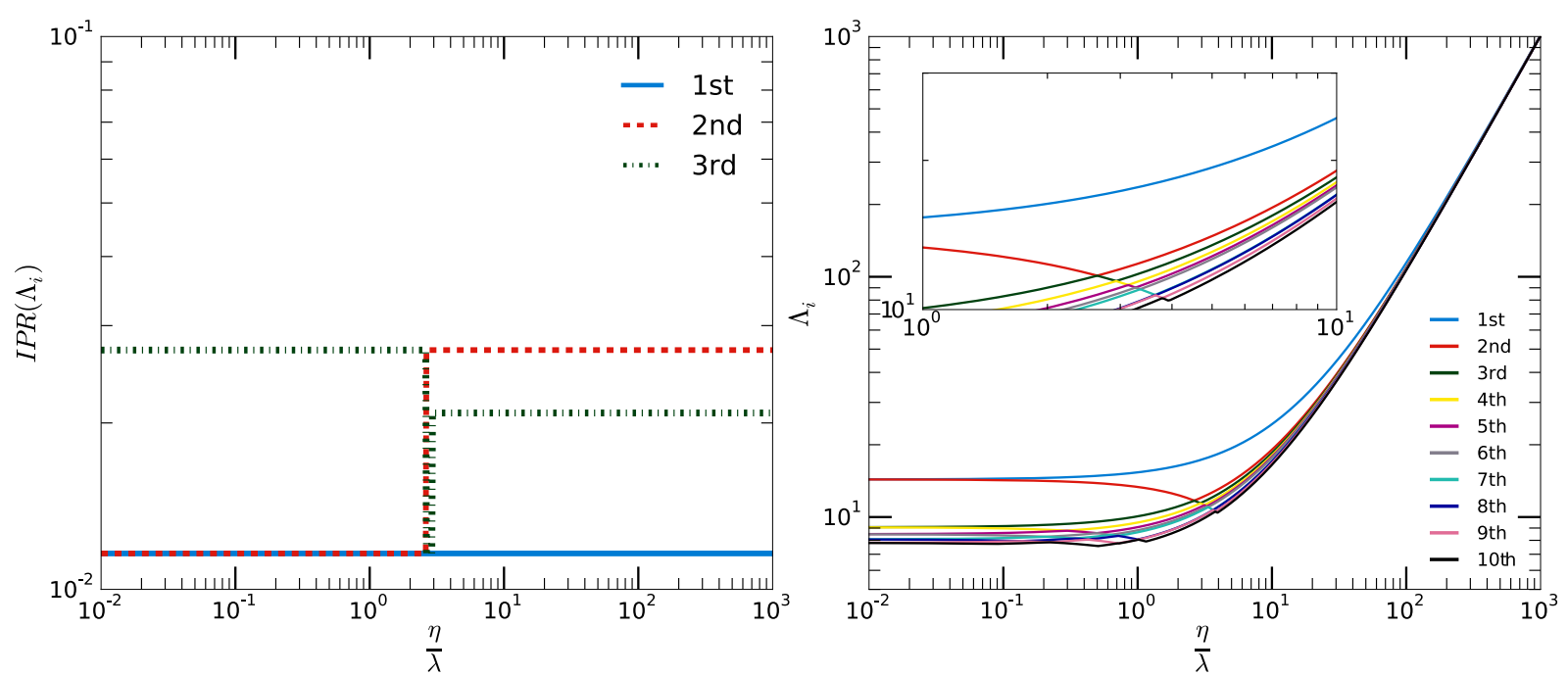

Source: Developed by the author.

three eigenvalues, while in the left panel, we plot the first ten eigenvalues. When the ratio $\frac{\eta}{\lambda}=0$ the eigenvalues have multiplicity two, as can be seen on the left side of the left panel (approximately, since the figure starts from $10^{-2}$ ). More importantly, those eigenvalues tend to behave differently: one increases, while the other tends to decrease. This behavior leads to the eigenvalue crossing (see Section 6.1.1.1). The inset of the left panel zooms out the region where the crossing takes place. Note that the eigenvalues cross at the same value for which the inverse participation ratio shows an abrupt change. Indeed, the jump in the $\operatorname{IPR}(\Lambda)$ has its roots in the interchange of the eigenvectors associated to each of the eigenvalues that are crossing. Moreover, we stress that the abrupt change observed for $\operatorname{IPR}(\Lambda)$ is always present in such scenarios, but it could be either from the lower to the higher values or vice versa depending on the structure of the layers.

\subsubsection{Similar layers}

In addition to the identical case, we have also considered a multiplex network composed of two layers with the same degree distribution (i.e. the same degree sequence), with $P(k) \sim k^{-2.7}$, but different random realizations of the configuration model. Furthermore, the inter-edges follow the same rule as before, connecting nodes with their counterparts on the other layer assuring that every node has the same intra-degree on all layers. Each layer of the multiplex network is composed by $n=1000$ and $\mathbb{E}(k) \approx 6$. As each layer is a different realization of the configuration model, both present a slightly different leading 
Figure 64 - Spectral properties of the tensor $\mathscr{R}(\lambda, \eta)$ as a function of the ratio $\frac{\eta}{\lambda}$ for a multiplex with two layers with the same degree distribution (different random realizations of the configuration model) and connected to its counterpart on the other layer. In the right panel, we present the inverse participation ratio $(\operatorname{IPR}(\Lambda))$ of the two larger eigenvalues and the individual layer contributions, while in the left panel, we show the leading eigenvalues. Every curve is composed by $10^{3} \log$ spaced points, in order to have enough resolution.
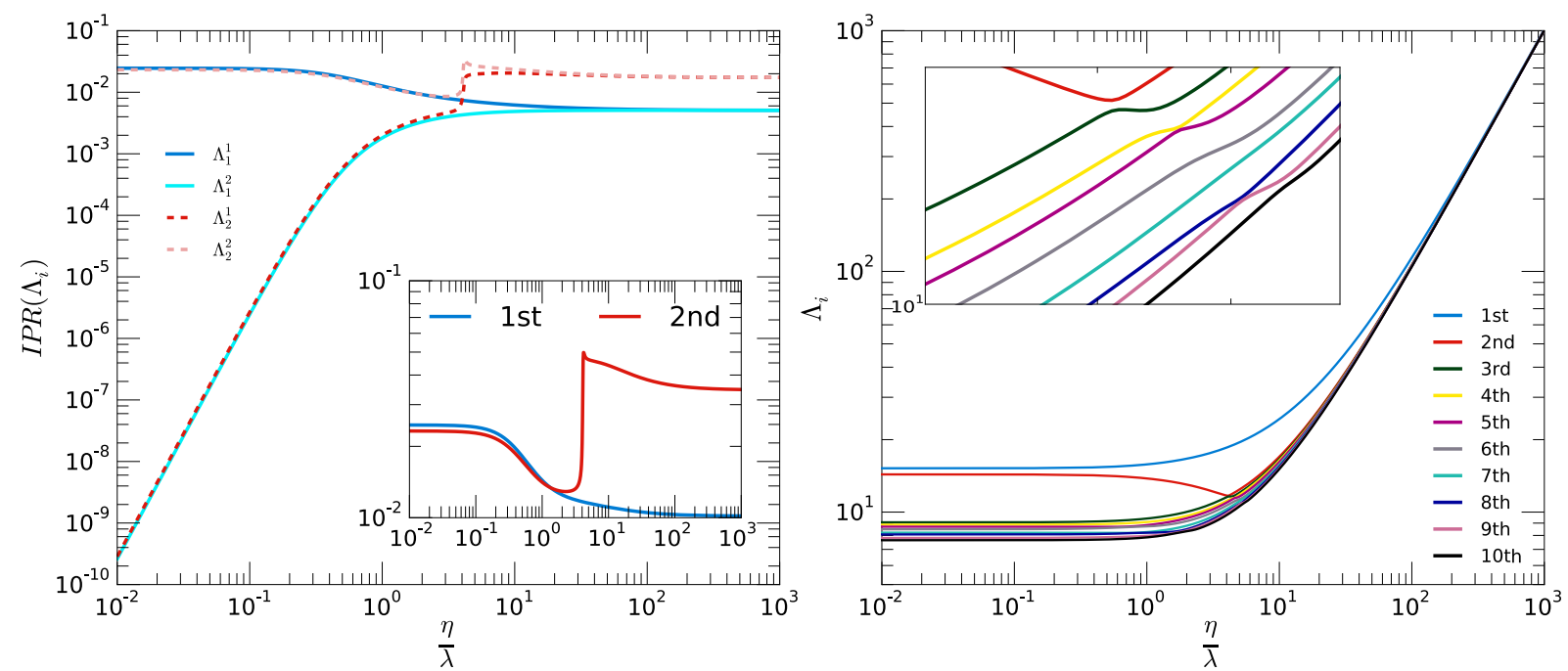

Source: Developed by the author.

eigenvalue, the first $\Lambda_{1}^{1}=15.21$ and the second $\Lambda_{1}^{2}=14.34$.

Figure 64 shows the spectral behavior of such a multiplex in terms of the largest eigenvalues, in the left panel, and the $\operatorname{IPR}(\Lambda)$, in the right panel. Here, in addition to the global inverse participation ratio, we also present the contribution of each layer to this measure. This analysis is meaningless in the identical case as the contribution is the same. As shown in the figure, we observe that for small values of $\frac{\eta}{\lambda}$, with regards to the first eigenvalue, the system is localized on the first layer and delocalized on the second. On the other hand, the picture changes when we focus on the second eigenvalue, as it is localized on the second layer, but delocalized on the first. For larger values of $\frac{\eta}{\lambda}$, both layers contribute equally to $\operatorname{IPR}(\Lambda)$. Analogously to the identical case, there is a change on $\operatorname{IPR}\left(\Lambda_{2}\right)$, which seems to be related to the changes on $\Lambda_{2}$, as one can see in the left panel and in the inset. Note that for this case, there is no crossing, i.e., the eigenvalues avoid the crossing - also referred to as near-crossing.

\subsubsection{Spectrum on the 3-Layer case}

In multilayer networks comprising three or more layers, the introduced complexity plays an important role. Thus, as the structure of the network of layers is no longer trivial, we shall find important differences regarding the spectra of such tensors for the different 
Figure 65 - Distribution of the eigenvalues. On the rows, from top to bottom, for the interconnected networks of Lines $2.3+2.6+2.9,2.3+2.9+2.6,2.6+2.3+2.9$ and the multiplex. On the columns, from left to right, we varied the ratios $\frac{\eta}{\lambda}=1,10,100$ and 1000 respectively. All histograms were built with 100 bins.
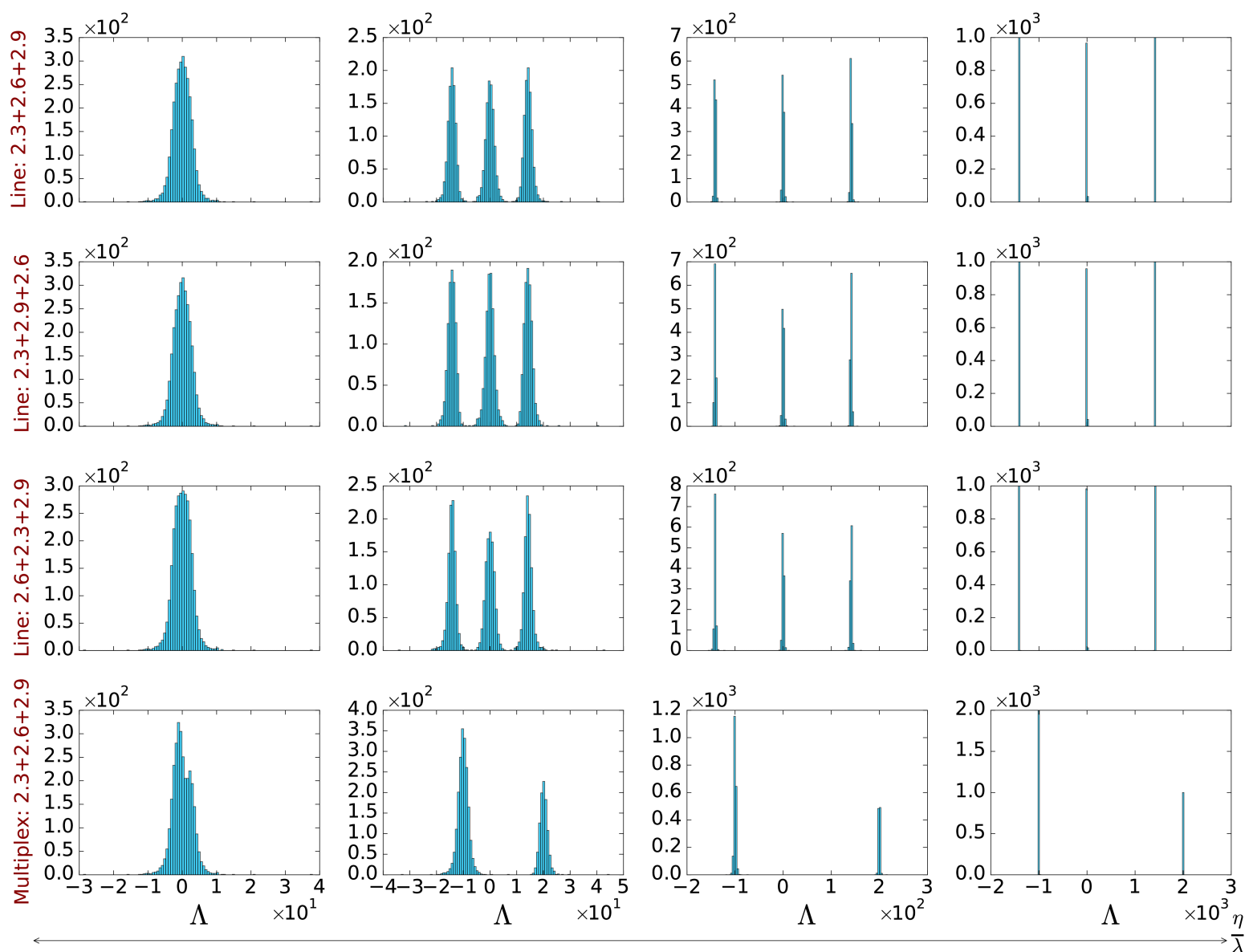

Source: Developed by the author.

topologies of the network of layers.

Figure 65 shows the spectrum of the four configurations of networks when varying the ratio $\frac{\eta}{\lambda}=1,10,100$ and 1000 . Observe that we do not show the ratio $\frac{\eta}{\lambda}=0$ as it is just the union of the individual layers' spectrum. For $\frac{\eta}{\lambda}=1$, the four configurations are very similar, especially the line graphs. In this case, the inter-layer edges are treated in the same way as the intra-layer ones. In other words, they are ignored and the network can be interpreted as a monoplex network. As the spreading ratio increases, the spectrum tends to be clustered near the values of the eigenvalues of the network of layers. This spectra was analytically calculated in Section 2.3.2.4 and shown in Table 1 in Chapter 2.

Regarding the triangle configuration, the clustering of the spectrum as $\frac{\eta}{\lambda}$ increases is even clearer. Triangles present the lowest eigenvalue with multiplicity two. In the extreme case of $\frac{\eta}{\lambda} \gg 1$, see Figure 65 , we have $2 / 3$ of the values near the left extreme value 
while $1 / 3$ is near the leading eigenvalue. On the other hand, for the line configurations, the frequencies of the eigenvalue distribution is related to the position of the central layer. However, in the limiting cases, these differences are reduced. This pattern is naturally related to the increase of the spreading ratio: When $\frac{\eta}{\lambda}$ increases, so does the role of the inter-layer edges relative to the intra-layer ones. Consequently, the structure of the network of layers imposes itself more strongly on the eigenvalues of the entire interconnected structure. This comes as a consequence of the interlacing theorems shown in Section 2.3.2.4.

Our findings can be related to the structural transition shown in (RADICCHI; ARENAS, 2013), where the authors evaluated the supra-Laplacian matrix as a function of the inter-layer weights. Their main result is an abrupt structural transition from a decoupled regime, where the layers seem to be independent, to a coupled regime where the layers behave as one single system. Here, we are interested in the supra-adjacency tensor, however, we found a similar phenomenological behavior and a structural change of the system as a function of the inter-layer weights, which in our case are determined by a dynamical process.

\subsection{Universal properties of multilayer networks}

As already largely discussed throughout this thesis, real systems are naturally structured in levels or interconnected substructures, which in turn consist of nodes organized in networks (KIVELÄ et al., 2014). These systems are nowadays referred to as multilayer networks (KIVELÄ et al., 2014; COZZO et al., 2016). The study of these systems is important as many critical properties of several phenomena are determined by their topology, and specifically by the spectral and eigenfunction properties of the adjacency and the Laplacian matrices of the networks. One particularly suitable approach to address the relation between the structure and the dynamics of a networked system is given by the Random Matrix Theory (RMT). RMT has numerous applications in many different fields, from condensed matter physics to financial markets (AKEMANN; BAIK; Di Francesco, 2015). In the case of complex networks, the use of RMT techniques might reveal universal properties ${ }^{1}$, which are always of interest, as they can reduce the set of parameters describing the system and provide relations that allow us to deduce its behavior from those few global parameters.

1 For instance, the nearest neighbor spacing distribution of the eigenvalues of the adjacency matrices of various model networks follow Gaussian Orthogonal Ensemble statistics (BANDYOPADHYAY; JALAN, 2007). In addition, the analysis of Erdös-Rényi networks shows that the level spacing distribution and the entropic eigenfunction localization length of the adjacency matrices are universal for fixed average degrees (J. A. Mendez-Bermudez; PERON, 2015). 
In this section, we study whether there are universal scaling properties in multilayer systems. We perform a scaling analysis of the eigenfunction localization properties of multilayer networks using RMT models and techniques. We explore multilayer networks whose networks of layers are of two types: (i) a line and (ii) a complete graph (node-aligned multiplex networks). In the first case, we study weighted layers coupled by weighted matrices, whereas in the latter case, weighted and binary layers coupled by identity matrices are considered. We demonstrate that the normalized localization length of the eigenfunctions of multilayer random networks exhibits a well-defined scaling function and also test the scaling law on real-world networks. Our results can be used to predict or design the localization features of the eigenfunctions of multilayer random networks and to better understand critical properties that depend on eigenfunction properties.

\subsubsection{Model definition and problem statement}

Here, in this section we follow the matricial notation (for more on its definition, see Section 2.3.1), $\mathbf{A}=\bigoplus_{m=1}^{M} \mathbf{A}^{(m)}+p \mathbf{C}$, where $\bigoplus$ represents the direct sum, $p$ is a parameter that defines the strength of the inter-layer edges and $\mathbf{C}$ is the interlayer coupling matrix, whose elements represent the relations between nodes in different layers, thus implicitly containing the information of a network of layers (SÁNCHEZ-GARCÍA; COZZO; MORENO, 2014). As a consequence, eigenvalue crossings, structural transitions (COZZO; MORENO, 2016), near crossings (ARRUDA et al., 2017) or localization problems (GOLTSEV et al., 2012; ARRUDA et al., 2017) (see also Section 5.1) are inherent to the network spectra, depending on $p$ for multilayer networks. Regarding dynamical processes, $p$ plays a fundamental role. For instance, in diffusion processes, it can drive the multilayer system to a super-diffusion regime (GOMEZ et al., 2013). Likewise, in contagion dynamics, in which $p$ is associated with the ratio of intra and interlayer spreading rates, there can be both localized and delocalized states (ARRUDA et al., 2017) depending on the value of $p$. Here, we restrict ourselves to $p=1$ : for $p \ll 1$ the layers can be considered as uncoupled, while for $p \gg 1$ the topology of the network of layers dominates the spectral properties (SÁNCHEZ-GARCÍA; COZZO; MORENO, 2014; COZZO; MORENO, 2016). Thus, $p=1$ represents a suitable intermediary case (multilayer phase).

We define two ensembles of multilayer random networks as adjacency matrices. As the first model we consider a network of layers on a line (see Figures 10(a) and (b) for examples of lines with two or three layers respectively) whose adjacency matrix $\mathbf{A}$ has the form

$$
\mathbf{A}=\left(\begin{array}{cccc}
\mathbf{A}^{(1)} & \mathbf{C}^{(1,2)} & \cdots & 0 \\
\mathbf{C}^{(2,1)} & A^{(2)} & & 0 \\
\vdots & & \ddots & \mathbf{C}^{(M-1, M)} \\
0 & 0 & \mathbf{C}^{(M, M-1)} & \mathbf{A}^{(M)}
\end{array}\right)
$$


where $\left(C^{\left(m, m^{\prime}\right)}\right)_{i, j}=\left(C^{\left(m, m^{\prime}\right)}\right)_{j, i}^{\mathrm{T}}$ are real rectangular matrices of size $N_{m} \times N_{m^{\prime}}$ and 0 represents null matrices. Furthermore, we consider a special class of matrices $A^{(m)}$ and $C^{\left(m, m^{\prime}\right)}$ which are characterized by the sparsities $\tilde{\alpha}_{A}$ and $\tilde{\alpha}_{C}$, respectively. In other words, since with a probability $\tilde{\alpha}_{*}$ their elements can be removed, these matrices represent ErdösRényi-type random networks. Notice that when the $N_{m}$ are all the same $N_{m}=$ constant $\equiv n$, which is the case we explore here. Also, the adjacency matrix $\mathbf{A}$ has the structure of a block-banded matrix of size $N=m \times n$. In addition, we consider this model as a model of weighted networks; i.e., the non-vanishing elements $\mathbf{A}_{i, j}$ are independent Gaussian variables with zero mean and variance $1+\delta_{i, j}$. We justify the addition of self-loops and random weights to edges by recognizing that in real-world networks the nodes and the interactions between them are in general non-equivalent. Moreover, with this prescription we retrieve well known random matrices (METHA, 2004) in the appropriate limits: a diagonal random matrix is obtained for $\tilde{\alpha}_{A}=\tilde{\alpha}_{C}=0$ (Poisson case), whereas the Gaussian Orthogonal Ensemble (GOE) is recovered when $\tilde{\alpha}_{A}=\tilde{\alpha}_{C}=1$ and $n=2$. For the sake of simplicity, and without loss of generality, in this work we consider the case where $\tilde{\alpha} \equiv \tilde{\alpha}_{A}=\tilde{\alpha}_{C}$. As an example, this network model can be applied to transportation networks, where the interlayer edges represent connections between two different means of transport. An obvious constraint is that no layer can be connected to more than two layers. In addition to the above configuration, we are also interested in the node-aligned multiplex case.

There is a well known RMT model known as the banded random matrix (BRM) model which was originally introduced to emulate quasi-one-dimensional disordered wires of length $N$ and width $b$ (with $b \ll N$ ). The BRM ensemble is defined as the set of $N \times N$ real symmetric matrices whose entries are independent Gaussian random variables with zero mean and variance $1+\delta_{i, j}$ if $|i-j|<b$ and zero otherwise. Therefore $b$ is the number of nonzero elements in the first matrix row which equals 1 for diagonal, 2 for tridiagonal, and $N$ for matrices of the GOE. There are several numerical and theoretical studies available for this model, see for example Refs. (G. Casati; IZRAILEV, 1990; EVANGELOU; ECONOMOU, 1990; FYODOROV; MIRLIN, 1991; G. Casati; MOLINARI, 1991; FYODOROV; MIRLIN, 1992; MIRLIN; FYODOROV, 1993; FYODOROV; MIRLIN, 1993; FYODOROV; MIRLIN, 1994; IZRAILEV, 1995; T. Kottos; RUFFO, 1996; G. Casati; MASPERO, 1997; SILVESTROV, 1997; T. Kottos; IZRAILEV, 1998; T. Kottos; POLITI, 1999; WANG, 2002). In particular, outstandingly, it has been found (G. Casati; IZRAILEV, 1990; EVANGELOU; ECONOMOU, 1990; FYODOROV; MIRLIN, 1991; FYODOROV; MIRLIN, 1992; MIRLIN; FYODOROV, 1993; FYODOROV; MIRLIN, 1993; FYODOROV; MIRLIN, 1994; IZRAILEV, 1995) that the eigenfunction properties of the BRM model, characterized by the scaled localization length $\beta$, are universal for the fixed ratio $x=b^{2} / N$. More specifically, it was numerically and theoretically shown 
that the scaling function

$$
\beta=\frac{\tilde{\gamma} x}{1+\tilde{\gamma} x}
$$

with $\tilde{\gamma} \sim 1$, holds for the BRM model. Admittedly, the ensemble of adjacency matrices of the multilayer network with layers on a line, see Equation (6.5), can be considered as a nonhomogeneous diluted version of the BRM model. Therefore, motivated by the similarity between these two matrix models, we propose the study of eigenfunction properties of the adjacency matrices of multilayer and multiplex random networks as a function of the parameter

$$
x=\frac{b_{\mathrm{eff}}^{2}}{N}
$$

where $b_{\text {eff }} \equiv b_{\text {eff }}(N, \tilde{\alpha})$ is the adjacency matrix effective bandwidth and $N=m \times n$.

A commonly accepted tool to characterize quantitatively the complexity of the eigenfunctions of random matrices (and of Hamiltonians corresponding to disordered and quantized chaotic systems) is the information or Shannon entropy $S$. This measure provides the number of principal components of an eigenfunction in a given basis. In fact, $S$ has been already used to characterize the eigenfunctions of the adjacency matrices of random network models; see some examples in Refs. (G. Zhu; LI, 2008; GONG; TONG, 2006; S. Jalan; LI, 2010; PASSERINI; SEVERINI, 2012; G. Menichetti; BIANCONI, 2014; J. A. Mendez-Bermudez; PERON, 2015). The Shannon entropy for the eigenfunction $\Psi^{l}$ is given as $S=-\sum_{n=1}^{N}\left(\Psi_{n}^{l}\right)^{2} \ln \left(\Psi_{n}^{l}\right)^{2}$ and can compute the scaled localization length as (IZRAILEV, 1990)

$$
\beta=\exp \left(\mathbb{E}(S)-S_{\mathrm{GOE}}\right)
$$

where $S_{\mathrm{GOE}} \approx \ln (N / 2.07)$, used as a reference, is the entropy of a random eigenfunction with Gaussian distributed amplitudes (i.e., an eigenfunction of the GOE). With this definition ${ }^{2} \beta$ can take values in the range $(0,1]$. Here, as well as in BRM model studies, we look for the scaling properties of the eigenfunctions of our random network models through $\beta^{3}$.

2 In the case of the multilayer network with layers on a line, when $\tilde{\alpha}=0$ (i.e., when all vertices in the network are isolated), since the eigenfunctions of the adjacency matrices of Equation (6.5) have only one non-vanishing component with magnitude equal to one, $\mathbb{E}(S)=0$ and $\beta \approx$ $2.07 / N$. On the other hand, when all nodes in this multilayer network are fully connected we recover the GOE and $\mathbb{E}(S)=S_{\mathrm{GOE}}$. Thus, the fully chaotic eigenfunctions extend over the $N$ available vertices in the network and $\beta=1$.

3 To this end, we use exact numerical diagonalization to obtain eigenfunctions $\Psi^{l}(l=1 \ldots N)$ of the adjacency matrices of large ensembles of multilayer random networks characterized by $m, n$, and $\tilde{\alpha}$. We perform the average $\mathbb{E}(S)$ taking half of the eigenfunctions, around the band center, of each adjacency matrix. 
Figure 66 - (a) Scaled localization length $\beta$ as a function of $x=b_{\text {eff }}^{2} / N$ for ensembles of multilayer networks characterized by the sparsity $\tilde{\alpha}$. The dashed line close to the data for $\tilde{\alpha}=0.8$ is Equation (6.6) with $\tilde{\gamma}=1.4$. Horizontal dot-dashed lines at $\beta \approx 0.12$ and 0.88 are shown as a reference, see the text. (b) Logarithm of $\beta /(1-\beta)$ as a function of $\ln (x)$. Upper inset: Power $\delta$, from the fittings of the data with Equation (6.10), as a function of $\tilde{\alpha}$. Lower inset: Enlargement in the range $\ln [\beta /(1-\beta)]=[-2,4]$ including data for $\tilde{\alpha}=0.6,0.8$, and 1. Lines are fittings of the data using Equation (6.10).
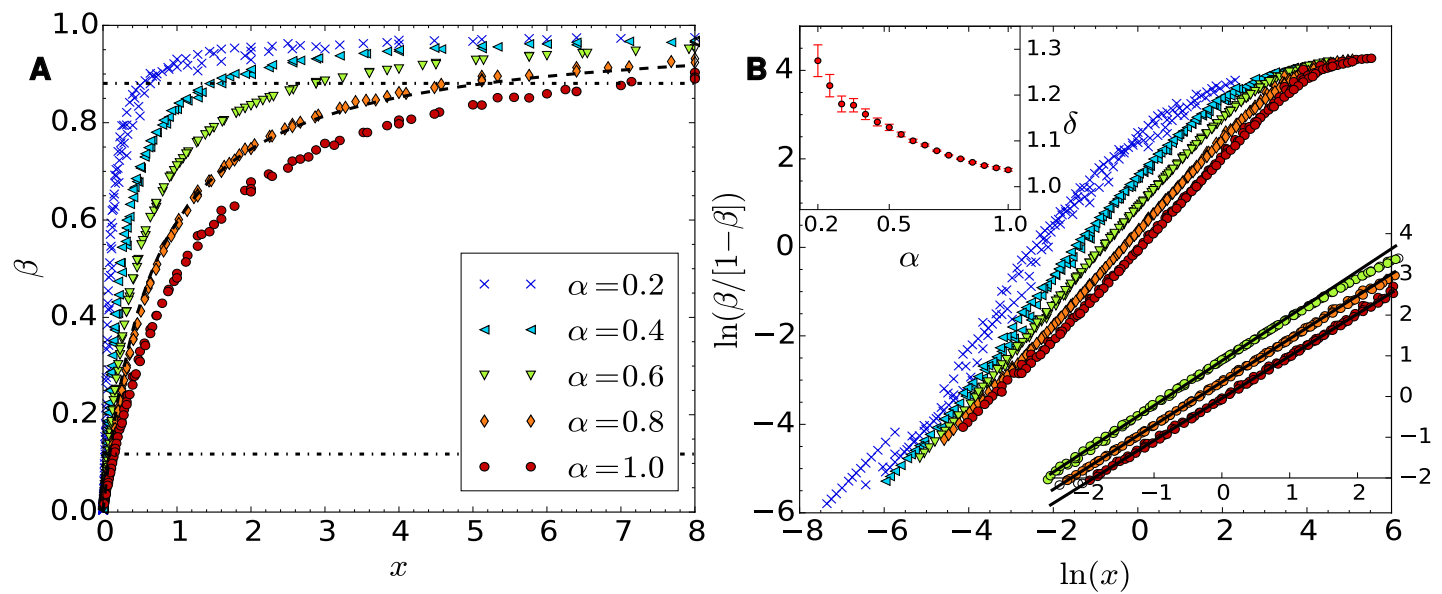

Source: Developed by the author.

\subsubsection{Scaling analysis of Multilayer Networks}

We now analyze in detail the multilayer network model with adjacency matrix given by Equation (6.5). In Figure 66(a), we present $\beta$ as a function of $x$, see Equation (6.7), for ensembles of networks characterized by the sparsity $\tilde{\alpha}$. We have defined $b_{\text {eff }}$ as the average number of non-vanishing elements per adjacency-matrix row,

$$
b_{\text {eff }}=2 n \tilde{\alpha} .
$$

We observe that the curves of $\beta$ vs. $x$ in Figure 66(a) have a functional form similar to that for the BRM model. To show this we are including Equation (6.6) (black dashed line) with $\tilde{\gamma}=1.4$ (the value of $\tilde{\gamma}$ reported in Ref. (G. Casati; IZRAILEV, 1990) for the BRM model) which is very close to our data for $\tilde{\alpha}=0.8$. In addition, in Figure 66(b) the logarithm of $\beta /(1-\beta)$ as a function of $\ln (x)$ is presented. The quantity $\beta /(1-\beta)$ was useful in the study of the scaling properties of the BRM model (G. Casati; IZRAILEV, 1990; FYODOROV; MIRLIN, 1992) because $\beta /(1-\beta)=\tilde{\gamma} x$, which is equivalent to scaling (6.6), implies that a plot of $\ln [\beta /(1-\beta)]$ vs. $\ln (x)$ is a straight line with unit slope. Even though, this statement is valid for the BRM model in a wide range of parameters (i.e., for $\ln [\beta /(1-\beta)]<2)$, it does not apply to our multilayer random network model; see Figure $66(\mathrm{~b})$. In fact, from this figure we observe that plots of $\ln [\beta /(1-\beta)]$ vs. $\ln (x)$ are straight lines (in a wide range of $x$ ) with a slope that depends on the sparsity $\tilde{\alpha}$. Therefore, we propose the scaling law

$$
\frac{\beta}{1-\beta}=\tilde{\gamma} x^{\delta}
$$


Figure $67-$ (a) $\beta$ as a function of $x^{*}$ [as defined in Equation (6.12)] for ensembles of multilayer networks with $\tilde{\alpha} \in[0.2,1]$ in steps of 0.05 . Inset: Data for $\tilde{\alpha} \in[0.5,1]$ in steps of 0.05 . Dashed lines are Equation (6.13). (b) Logarithm of $\beta /(1-\beta)$ as a function of $\ln \left(x^{*}\right)$ for $\tilde{\alpha} \in[0.2,1]$ in steps of 0.05. Inset: Enlargement in the range $\ln [\beta /(1-\beta)]=[-2,2]$ including curves for $\tilde{\alpha} \in[0.5,1]$ in steps of 0.05. Dashed lines are Equation (6.11).
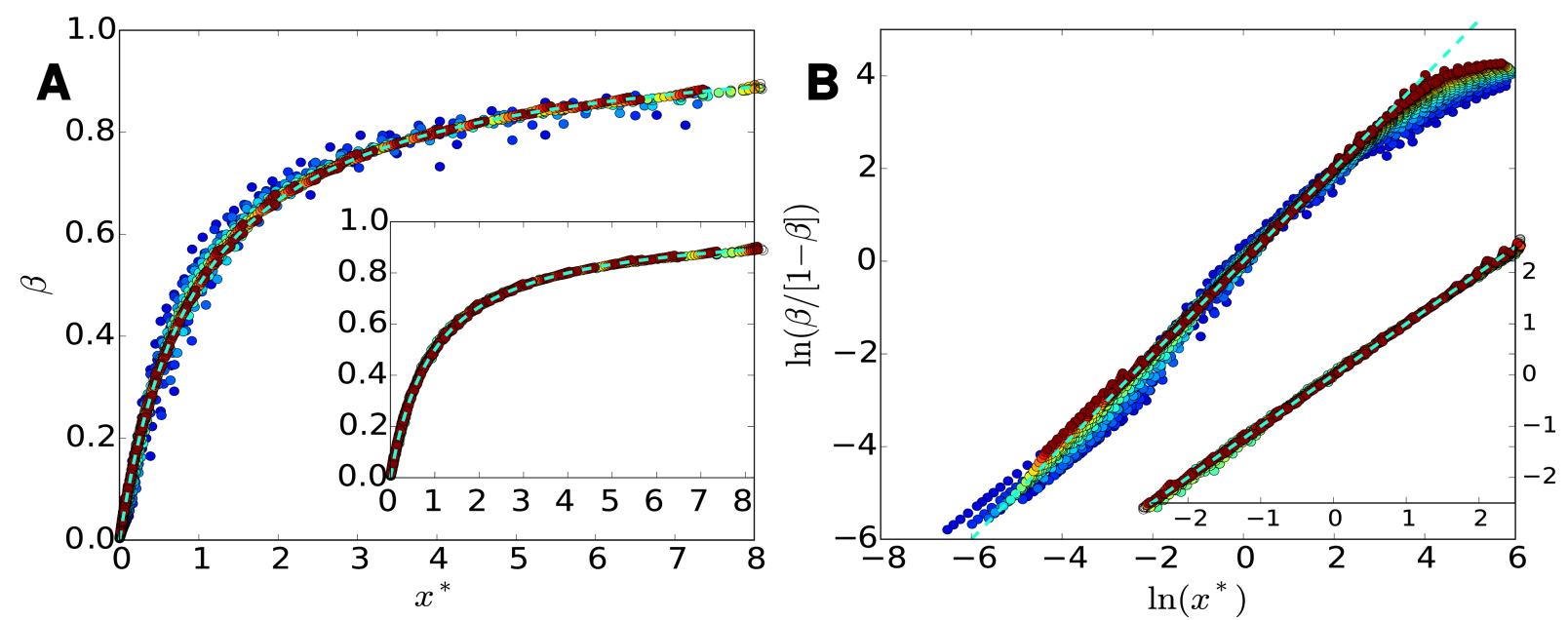

Source: Developed by the author.

where both $\tilde{\gamma}$ and $\boldsymbol{\delta}$ depend on $\tilde{\alpha}$. Indeed, Equation (6.10) describes well our data, mainly in the range $\ln [\beta /(1-\beta)]=[-2,2]$, as can be seen in the inset of Figure 66(b) where we show the numerical data for $\tilde{\alpha}=0.6,0.8$ and 1 and include fittings through Equation (6.10). We stress that the range $\ln [\beta /(1-\beta)]=[-2,2]$ corresponds to a reasonable large range of $\beta$ values, $\beta \approx[0.12,0.88]$, whose bounds are indicated with horizontal dotdashed lines in Figure 66(a). Finally, we notice that the power $\delta$, obtained from the fittings of the data using Equation (6.10), is very close to unity for all the sparsity values we consider here (see the upper inset of Figure 66(b)).

Therefore, from the analysis of the data in Figure 66, we are able to write down a universal scaling function for the scaled localization length $\beta$ of the eigenfunctions of our multilayer random network model as

$$
\frac{\beta}{1-\beta}=x^{*}
$$

where the scaling parameter $x^{*}=\tilde{\gamma} x^{\delta}$, as a function of the multilayer network parameters, is given by

$$
x^{*} \equiv \tilde{\gamma}\left(\frac{4 n \tilde{\alpha}^{2}}{m}\right)^{\delta} .
$$

To validate Equation (6.11) in Figure $67(\mathrm{~b})$ we present again the data for $\ln [\beta /(1-\beta)]$ shown in Figure 66(b) but now as a function of $\ln \left(x^{*}\right)$. It can be observed that curves for different values of $\tilde{\alpha}$ fall on top of Equation (6.11) for a wide range of the variable $x^{*}$. Moreover, the collapse of the numerical data is excellent in the range $\ln [\beta /(1-\beta)]=[-2,2]$ for $\tilde{\alpha} \geq 0.5$, as shown in the inset of Figure 67(b). Additionally, we rewrite Equation (6.11) 
into the equivalent, but explicit, scaling function for $\beta$ :

$$
\beta=\frac{x^{*}}{1+x^{*}}
$$

In Figure 67(a) we confirm the validity of Equation (6.13). We emphasize that the universal scaling given in Equation (6.13) extends outside the range $\beta \approx[0.12,0.88]$, for which Equation (6.10) was shown to be valid, see the main panel of Figure 67(a). Clearly, the collapse of the numerical data following Equation (6.13) is remarkably good for $\tilde{\alpha} \geq 0.5$, as shown in the inset of Figure 67(a).

Furthermore, we verify below that scaling (6.13) is also applicable to node-aligned multiplex networks - which are relevant for certain applications-, once $b_{\text {eff }}$ is properly defined.

\subsubsection{Scaling analysis of Multiplex Networks}

In the node-aligned multiplex case, whose network of layers is a complete graph, the coupling matrices are restricted to identity matrices and all layers have the same number of nodes, see Figure 1(b). The adjacency matrix of a node-aligned multiplex is given as

$$
\mathbf{A}=\left(\begin{array}{ccccc}
A^{(1)} & I & I & \cdots & I \\
I & A^{(2)} & I & & I \\
I & I & A^{(3)} & & I \\
\vdots & & & \ddots & I \\
I & I & I & I & A^{(M)}
\end{array}\right)
$$

Similarly to the multilayer model of Equation 6.5, this configuration is characterized by the sparsity $\tilde{\alpha}$ which we choose to be constant for all the $M$ matrices $A^{(m)}$ of size $n \times n$ composing the adjacency matrix $\mathbf{A}$ of size $L=m \times n$. Additionally, the configuration (6.14) is considered in two different setups: weighted and unweighted multiplex without selfloops. In the weighted case, the non-vanishing elements of the matrices $A^{(m)}$ are chosen as independent Gaussian variables with zero mean and variance $1+\delta_{i, j}$. A realistic example of this configuration is online social systems, where each layer represents a different online network (e.g., Facebook, Twitter and Google+, etc). In the unweighted case, the nonvanishing elements of $A^{(m)}$ are equal to unity. In (6.14), $I$ are identity matrices of size $n \times n$.

\subsubsection{Weighted Multiplex}

Now we consider weighted multiplex networks (i.e., where the non-vanishing elements of the adjacency matrices $A^{(m)}$ in (6.14) are chosen as independent Gaussian variables with zero mean and variance $\left.1+\delta_{i, j}\right)$. We follow the same methodology as in 
Figure 68 - (a) Scaled localization length $\beta$ as a function of $x=b_{\text {eff }}^{2} / N$ for ensembles of weighted multiplex networks characterized by the sparsity $\tilde{\alpha}$. The black dashed line corresponds to Equation 6.6 with $\tilde{\gamma}=1.4$. Horizontal black dot-dashed lines at $\beta \approx 0.12$ and 0.88 are shown as a reference, see the text. (b) Logarithm of $\beta /(1-\beta)$ as a function of $\ln (x)$. Upper inset: Power $\delta$, from the fittings of the data with Equation 6.8, as a function of $\tilde{\alpha}$. Lower inset: Enlargement in the range $\ln [\beta /(1-\beta)]=[-2,2]$ including data for $\tilde{\alpha}=0.6,0.8$, and 1. Lines are fittings of the data with Equation 6.8.
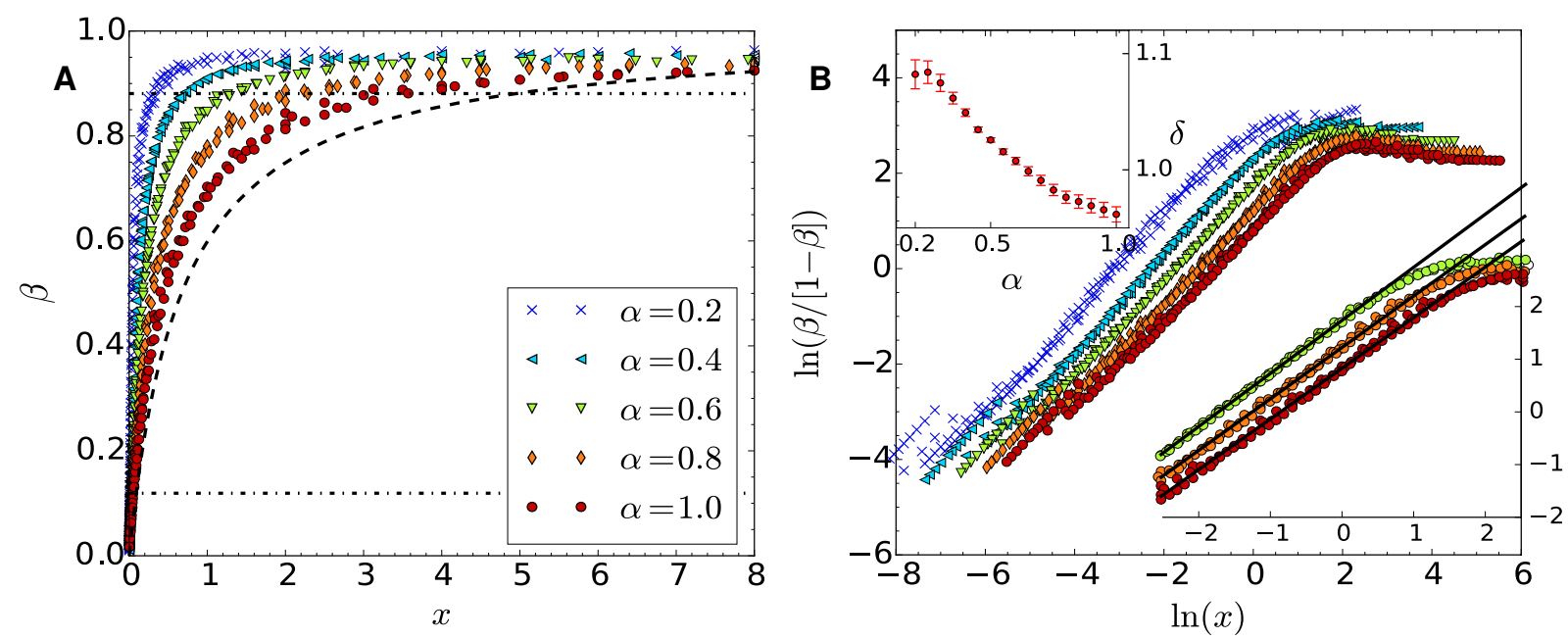

Source: Developed by the author.

the multilayer case. Thus, in Figure 68(a) we first present curves of $\beta$ vs. $x$; however, we redefine $b_{\text {eff }}$ as

$$
b_{\text {eff }}=n \tilde{\alpha},
$$

which is the average number of non-vanishing elements per row inside the adjacencymatrix band in the multiplex setup. From Figure 68(a) we observe that the curves of $\beta$ vs. $x$ have functional forms similar to those for the multilayer model (compare with Figure 66(a)), however, with larger values of $\beta$ for given values of $x$. As a reference, we also include Equation 6.6 (black-dashed line) with $\tilde{\gamma}=1.4$, corresponding to the BRM model, which is even below the data for $\tilde{\alpha}=1$. Moreover, in Figure 68(b) we show the logarithm of $\beta /(1-\beta)$ as a function of $\ln (x)$. As in the multilayer case, here we observe that plots of $\ln [\beta /(1-\beta)]$ vs. $\ln (x)$ are straight lines mainly in the range of $\ln [\beta /(1-\beta)]=[-2,2]$ with a slope that depends on the sparsity $\tilde{\alpha}$. We indicate the bounds of this range with horizontal dot-dashed lines in Figure 68(a). Therefore, the scaling law of Equation 6.8 is also valid here. Indeed, in the upper inset of Figure 68(b) we report the power $\boldsymbol{\delta}$ obtained from fittings of the data with Equation 6.8.

In order to validate the scaling hypothesis of Equation 6.8 for the node-aligned multiplex setup, in Figure 69(b) we present the data for $\ln [\beta /(1-\beta)]$ shown in Figure $68(\mathrm{~b})$, but now as a function of $\ln \left(x^{*}\right)$. We observe that curves for different values of $\tilde{\alpha}$ fall on top of Equation 6.9 for a wide range of the variable $x^{*}$. Moreover, the collapse of the numerical data on top of Equation 6.9 is excellent in the range of $\ln [\beta /(1-\beta)]=[-2,2]$ 
Figure $69-$ (a) $\beta$ as a function of $x^{*}$ [as defined in Equation 6.10] for ensembles of weighted multiplex networks with $\tilde{\alpha} \in[0.2,1]$ in steps of 0.05 . Inset: Data for $\tilde{\alpha} \in[0.5,1]$ in steps of 0.05. Dashed lines in main panel and inset are Equation 6.11. (b) Logarithm of $\beta /(1-\beta)$ as a function of $\ln \left(x^{*}\right)$ for $\tilde{\alpha} \in[0.2,1]$ in steps of 0.05 . Inset: Enlargement in the range $\ln [\beta /(1-\beta)]=[-2,2]$ including curves for $\tilde{\alpha} \in[0.5,1]$ in steps of 0.05 . Dashed lines in main panel and inset are Equation 6.9.
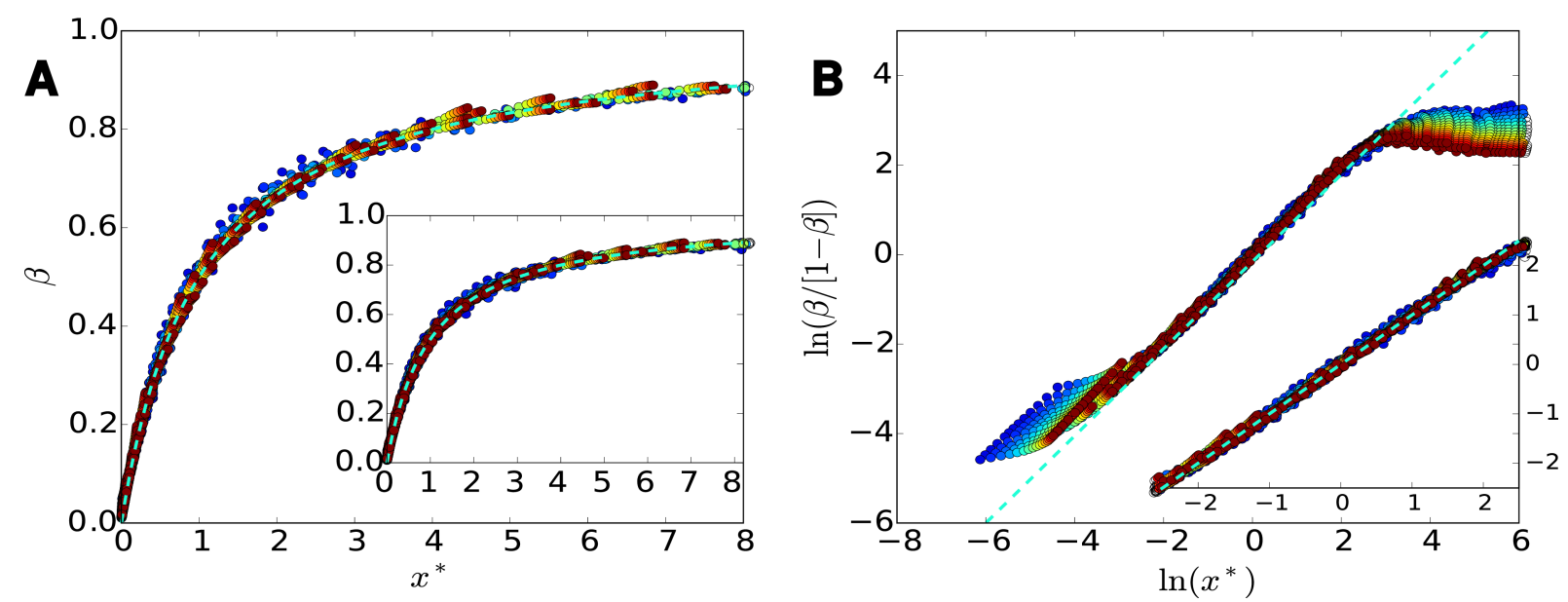

Source: Developed by the author.

for $\tilde{\alpha} \geq 0.5$, as shown in the inset of Figure 69(b). Finally, in Figure 69(a) we confirm the validity of Equation 6.11 which is as good here as for the multilayer case. We emphasize that the collapse of the numerical data on top of Equation 6.11 is remarkably good for $\tilde{\alpha} \geq 0.5$, as shown in the inset of Figure $69(\mathrm{a})$.

\subsubsection{Unweighted Multiplex}

The last analyzed scenario is the binary multiplex case. We recall that, in contrast to the two previous random network models, this model does not include weighted self-loops. Therefore the Poisson limit is not recovered when $\tilde{\alpha} \rightarrow 0$ and $\beta$ is not well defined there. Thus, we will compute $\beta$ for values of $x$ as smaller as the adjacency-matrix diagonalization produces meaningful results. Also, as for the weighted multiplex, we use here the effective bandwidth given in Equation (6.15).

The conducted experiments are similar to the previous ones. Then, in Figs. 70(a) and $70(\mathrm{~b})$ we present curves of $\beta$ vs. $x$ and $\ln [\beta /(1-\beta)]$ vs. $\ln (x)$, respectively. Here, due to the absence of self-loops we observe important differences with respect to the previous cases: In particular, the curves $\beta$ vs. $x$ present minima at given small values of $x$. This feature can be seen clearer in Figure $70(\mathrm{~b})$ since it is magnified there. Also, from Figure 70 (b) we can notice that the range where $\ln [\beta /(1-\beta)]$ is a linear function of $\ln (x)$ has been shifted upwards for all the values of $\tilde{\alpha}$ considered. Therefore, we perform fittings to the curves $\ln [\beta /(1-\beta)]$ vs. $\ln (x)$ with Equation 6.8 in the interval $\ln [\beta /(1-\beta)]=[0,3.75]$; the bounds of this interval are marked as dot-dashed lines in Figure 70(a). The corresponding 
Figure 70 - (a) Scaled localization length $\beta$ as a function of $x=b_{\text {eff }}^{2} / N$ for ensembles of unweighted multiplex networks characterized by the sparsity $\tilde{\alpha}$. The black dashed line corresponds to Equation 6.6 with $\tilde{\gamma}=1.4$. Horizontal black dot-dashed lines at $\beta \approx 0.5$ and 0.98 are shown as a reference, see the text. (b) Logarithm of $\beta /(1-\beta)$ as a function of $\ln (x)$. Lower inset: Enlargement in the range $\ln [\beta /(1-\beta)]=[-2,4]$ including data for $\tilde{\alpha}=0.6,0.7$, and 0.85 . Lines are fittings of the data with Equation 6.9 .
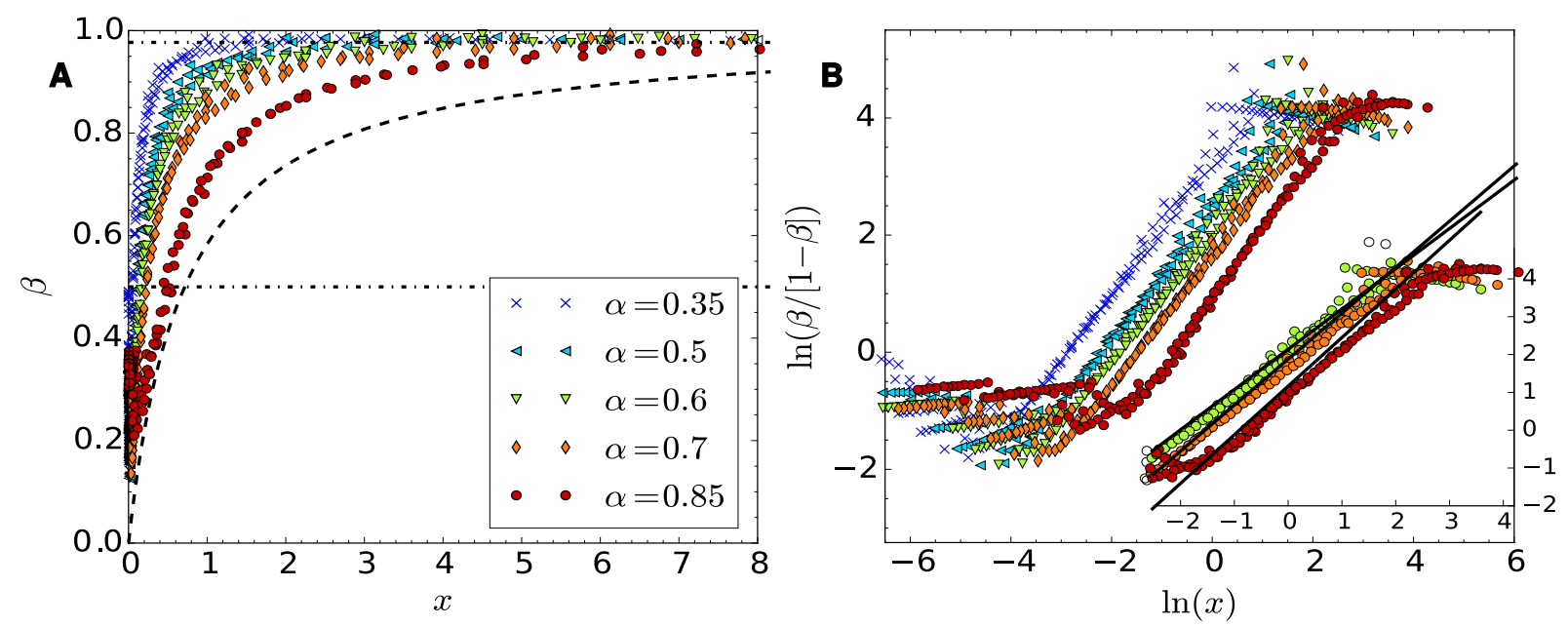

Source: Developed by the author.

values of delta are reported in Figure 72(a). Now, under the above conditions, we validate our scaling hypothesis by plotting $\beta$ vs. $x^{*}$ and $\ln [\beta /(1-\beta)]$ vs. $\ln \left(x^{*}\right)$, see Figs. 71(a) and 71(b), respectively. Remarkably, we observe a clear scaling behavior also in the unweighted multiplex case (despite the minima in the curves $\beta$ vs. $x^{*}$ for small $x^{*}$ ).

\subsubsection{Application to real-world networks}

The methodology was applied to 7 real networks, where 2 are biological networks and 5 from the social domain. The biological ones are constructed using the (BioGRID, thebiogrid.org) Biological General Repository for Interaction Datasets (C. Stark, B.-J. Breitkreutz, T. Reguly, L. Boucher, A. Breitkreutz, and M. Tyers, 2006; M. De Domenico; ARENAS, 2015) and consider different types of interactions: (i) direct interaction, (ii) association and (iii) physical association. On the other hand, regarding the social networks, each layer represents a different type of interaction: (i) on Pedgett Florentine families (J. F. Padgett, 1993), they are marriage alliances and business relationships; (ii) on Vickers chan 7th graders (VICKERS; CHAN, 1981), they are based on the answer to 3 questions, "Who do you get on with in the class?", "Who are your best friends in the class?" and "Who would you prefer to work with?"; (iii) on Lazega law firm (LAZEGA, 2001), they are cowork, friendship and advice; (iv) on Krackhardt high tech (KRACKHARDT, 1987) they are advice, friendship and "reports to" and (v) on Kapferer tailor shop (KAPFERER, 1972), they are "instrumental" (work- and assistance-related) and "societal" (friendships), 
Figure $71-$ (a) $\beta$ as a function of $x^{*}$ [as defined in Equation 6.10] for ensembles of unweighted multiplex networks with $\tilde{\alpha} \in[0.2,1]$ in steps of 0.05 . Inset: Data for $\tilde{\alpha} \in[0.5,1]$ in steps of 0.05. Dashed lines in main panel and inset are Equation 6.11. (b) Logarithm of $\beta /(1-\beta)$ as a function of $\ln \left(x^{*}\right)$ for $\tilde{\alpha} \in[0.2,1]$ in steps of 0.05 . Inset: Enlargement in the range $\ln [\beta /(1-\beta)]=[-1,4]$ including curves for $\tilde{\alpha} \in[0.5,1]$ in steps of 0.05 . Dashed lines in main panel and inset are Equation 6.9.
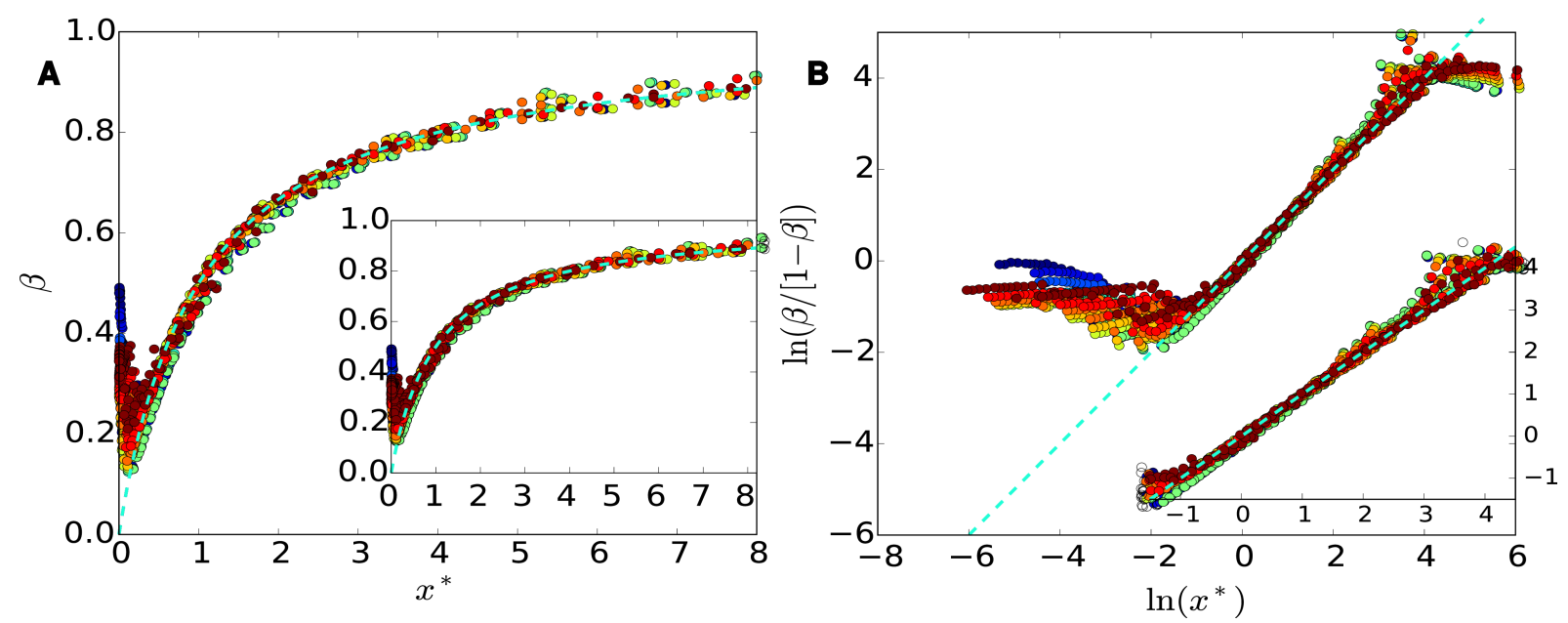

Source: Developed by the author.

recorded at two different times. For more information, please see Table 8.

Here, we tested the scaling law for $\beta$ on the above real-world multiplex networks. Here we give details regarding the calculation of $\tilde{\gamma}$ and $\delta$ used to compute $x^{*}=x^{*}(n, m, \bar{\alpha})$. First note that since the real networks we are analyzing are binary multiplex, we use the unweighted multiplex model of subsection 6.2.3.2 as the reference model. Also, recall that we have already obtained $\tilde{\gamma}$ and $\delta$ for several values of $\tilde{\alpha}$, indeed we used those values of $\tilde{\gamma}$ and $\delta$ to produce the scaled curves of Figure 71 . However, in order to provide $\tilde{\gamma}$ and $\delta$ corresponding to the specific values of $\overline{\tilde{\alpha}}$ of the real-world networks, see Table I, we proceed as follows. First we consider the region of $\tilde{\alpha}<0.2$, not explored in subsection 6.2.3.2. Again we extract $\tilde{\gamma}$ and $\delta$ from the fittings of the curves $\ln [\beta /(1-\beta)]$ vs. $\ln (x)$ with Equation6.8. But in contrast to the region $\tilde{\alpha} \geq 0.2$, where we performed the fittings in the fixed interval $\ln [\beta /(1-\beta)]=[0,3.75]$, now we have to adjust the fitting interval because the linear behavior of the curves $\ln [\beta /(1-\beta)]$ vs. $\ln (x)$ diminishes by decreasing $\tilde{\alpha}$. Thus, in Figure 72 we report the values of $\tilde{\gamma}$ and $\delta$ obtained for $\tilde{\alpha}<0.2$ (in steps of 0.01 ) and for $\tilde{\alpha} \geq 0.2$ (in steps of 0.05 ). Then, we perform a cubic spline interpolation (see continuous lines in Figure 72); therefore given a specific value of $\overline{\tilde{\alpha}}$ we retrieve interpolated values of $\tilde{\gamma}$ and $\delta$ that we use to compute $x^{*}=x^{*}(n, m, \overline{\tilde{\alpha}})$ with Equation6.10.

Finally, we test the scaling law for $\beta$ for a number of real-world multiplex networks, see Table 8. We proceed as follows. First, since these networks are highly nonhomogenous, we compute an average sparsity $\bar{\alpha}$. Then, with Equation (6.12) we obtain the corresponding $x^{*}=x^{*}(n, m, \bar{\alpha})$, as previously discussed. Therefore, we can already use 
Figure $72-(\mathrm{a}) \delta$ and (b) $\tilde{\gamma}$ as a function of $\tilde{\alpha}$ for ensembles of unweighted multiplex networks (symbols). Continuous lines are cubic spline interpolations of the data.
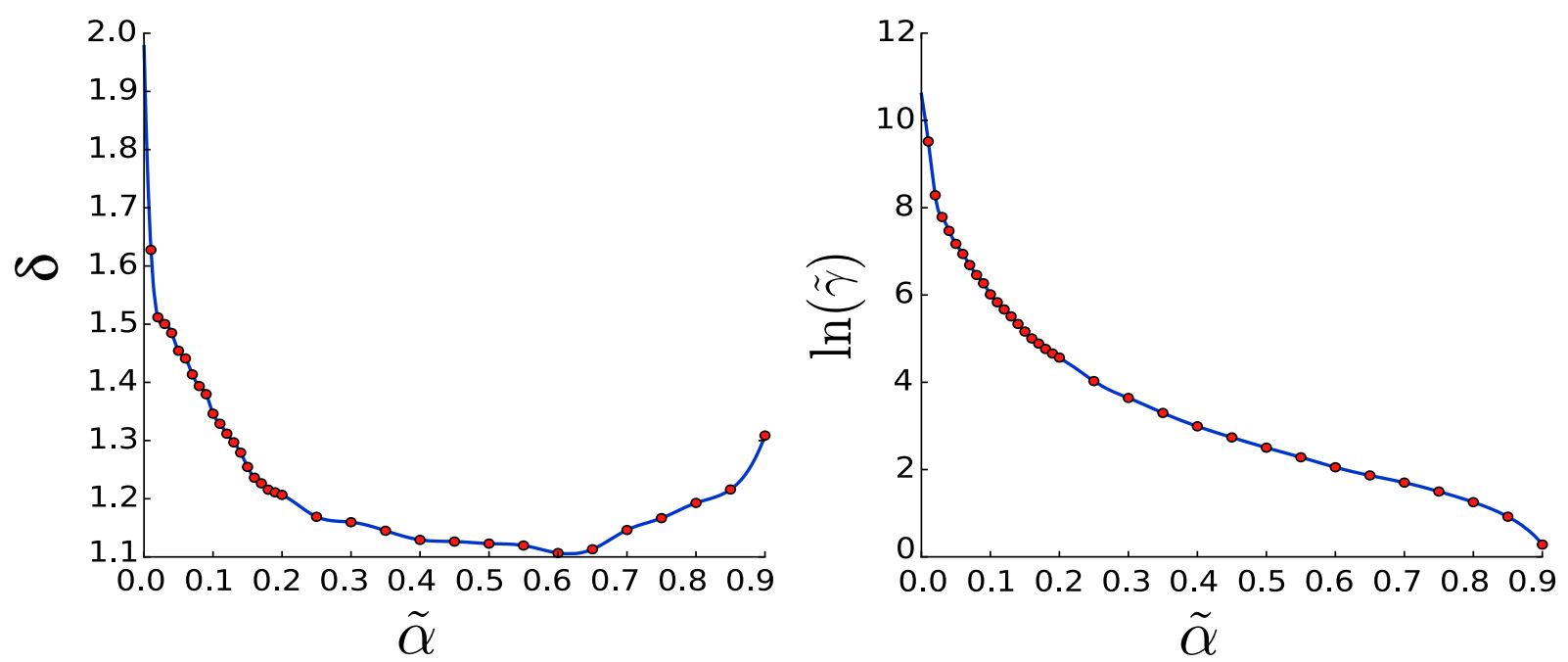

Source: Developed by the author.

Table 8 - Parameter values of the networks reported in Figure 73.

\begin{tabular}{l|c|c|c|l}
\hline Network name & $n$ & $m$ & $\bar{\alpha}$ & $x^{*}$ \\
\hline Kapferer Tailor Shop $(\circ)$ & 39 & 4 & 0.1862 & 29.417 \\
Oryctolagus genetic $(\square)$ & 144 & 3 & 0.0044 & 0.1056 \\
HepatitusC genetic $(\diamond)$ & 105 & 3 & 0.0076 & 0.5292 \\
Krackhardt High Tech $(\triangle)$ & 21 & 3 & 0.3873 & 22.596 \\
Padgett Florentine Families $(\triangleleft)$ & 16 & 2 & 0.1458 & 19.957 \\
Vickers Chan 7th Graders $(\triangleright)$ & 29 & 3 & 0.4252 & 32.707 \\
Lazega Law Firm $(\nabla)$ & 71 & 3 & 0.2231 & 92.037 \\
\hline
\end{tabular}

Equation (6.13) to predict the eigenfunction localization length of these networks that we will compare to the values of $\beta$ computed directly from them. In Figure 73 we report all data we obtained. Remarkably, although these real networks are binary multiplexed (basically different to the weighted multilayer networks used to derive the scaling of $\beta$ ) and highly non-homogenous, we observe a reasonably good general correspondence between the actual values of $\beta$ (symbols) and the corresponding prediction (dashed line).

\subsection{Exploring the block structure of multilayer networks}

From the previous studies, the importance of the block structure of multiplex networks becomes clear. In fact, the symmetries introduced by the multilayer formalism may allow us to mathematically analyze the spectral properties of our system. Additionally, as observed in the introduction of our analysis of Universal properties of multilayer networks in Section 6.2, a multilayer network can be understood as a function of the coupling parameter $p$. Here we also explore this dependency, analyzing how the spectral properties 
Figure $73-\ln [\beta /(1-\beta)]$ as a function of $\ln \left(x^{*}\right)$ for several real-world multiplex networks, see symbol code in Table 8. Dashed line is Equation (6.11).

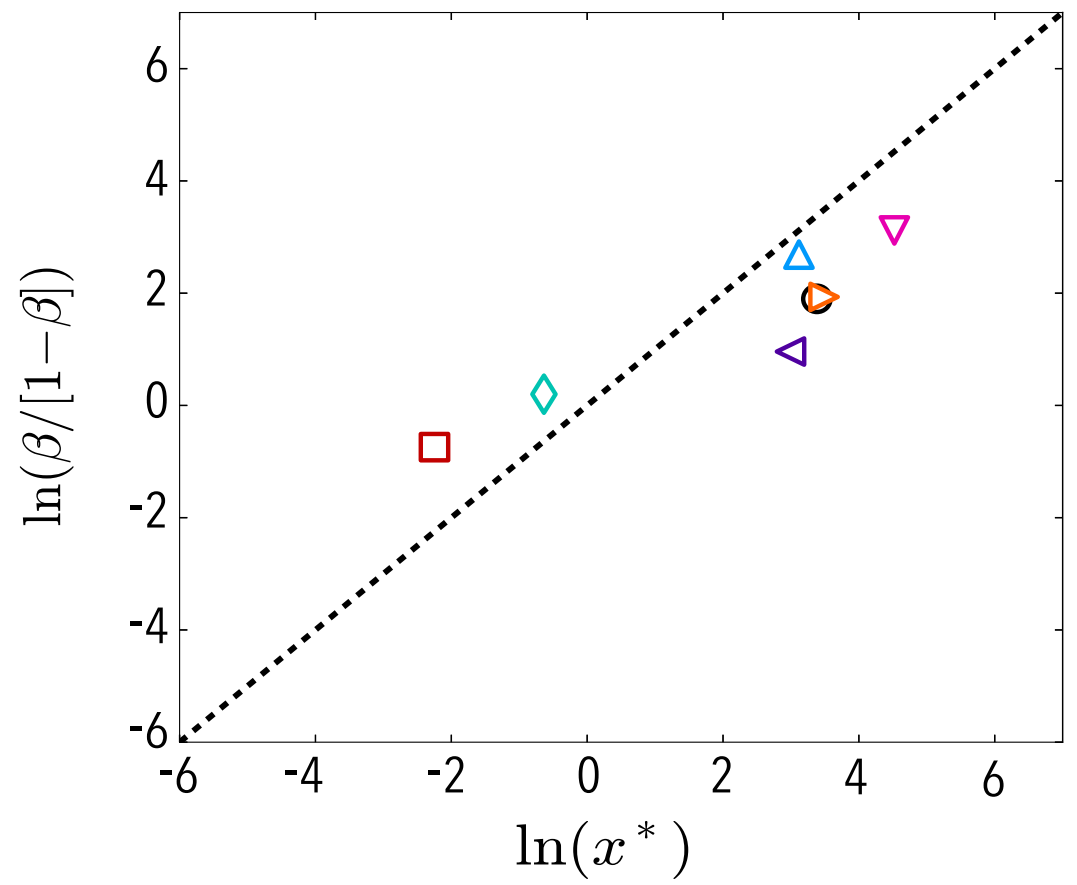

Source: Developed by the author.

of our networks change as we change the coupling parameters. The obtained results may, potentially, be used for designing and controlling of networks. However, the main goal of this section is mathematical. Here we focus our attention on 2-Layer multiplex systems composed of undirected edges and the same number of nodes on each layer. In the following sections, we will slightly relax these assumptions, but this constraint will be useful for our first analysis on the problem. Firstly, in Section 6.3.1.1, we define the general problem considering any matrix $M$. Next, in Section 6.3.1.2, we show some important definitions and identities. In the following part, in Section 6.3.1.3, we constraint ourselves to the multiplex case. Finally, we also apply the developed formalism to the case of supra adjacency and Laplacian matrices (for more on its definitions and spectral properties, please see Chapter 2 and Section 2.2). The results of this section are complemented by the appendix E, where more derivations are made, such as bounds for the spectra (SectionE.1), comments on symmetric problems (Section E.2) and a discussion on inter-layer sparse coupling matrices (Section E.3).

\subsubsection{Problem definition}

\subsubsection{Quadratic eigenvalue problem}

In addition to the eigenvalue problem, one might also think of a higher order matricial problem (order in the sense of a polynomial eigenvalue problem). Along these lines, the 
quadratic eigenvalue problem (QEP) (LANCASTER, 1966; GOHBERG; LANCASTER; RODMAN, 1982; TISSEUR; MEERBERGEN, 2001) is defined as

$$
\mathbf{Q}(\lambda)=\mathbf{A} \lambda^{2}+\mathbf{B} \lambda+\mathbf{C}
$$

which is a matrix polynomial of degree 2 , called $\lambda$-matrix, whose solutions are given by

$$
\operatorname{det}(\mathbf{Q}(\lambda))=0
$$

We emphasize that the quadratic eigenvalue problem is a special case of polynomial eigenvalue problems (LANCASTER, 1966), for an arbitrary matrix polynomial order in $\lambda$.

In addition to the eigenvalues, we have the right and left eigenvectors defined as

$$
\begin{array}{r}
\mathbf{Q}(\lambda) x=0, \\
y^{T} \mathbf{Q}(\lambda)=0,
\end{array}
$$

without loss of generality we assume in the following that the eigenvectors are unitary. Note, however, that if the right eigenvector $x$ is normalized, then the eigenvector of the standard eigenvalue problem, $\left[\begin{array}{ll}y & x^{T}\end{array}\right]^{T}$, is not normalized. Next, we assume that $x$ is unitary to simplify the equations.

\subsubsection{Interesting QEP properties}

From (LANCASTER, 1966) we can extract a couple of interesting definitions and relations regarding Quadratic eigenvalue problems. The main goal of this section is to present the main mathematical objects related to QEPs, helping us to relate them with the usual eigenvalue problem, in which we have much more intuition.

Definition 6.3.1. Jordan triple (LANCASTER, 1966): Denoting by $\mathbf{J}$ the Jordan matrix, and defining $(\mathbf{X}, \mathbf{J}, \mathbf{Y})$ the Jordan triple of $\mathbf{Q}(\boldsymbol{\lambda})$, where $\mathbf{X}$ and $\mathbf{Y}$ are the right and left eigenvectors. We also have that $\left(\mathbf{Y}^{*}, \mathbf{J}^{*}, \mathbf{Y}^{*}\right)$ the Jordan triple of $\mathbf{Q}(\boldsymbol{\lambda})^{*}$. Note that the matrix $\mathbf{J}$ is diagonal if all the eigenvalues are simple.

Observe that $\mathbf{X} \in \mathbb{R}^{N \times 2 N}$, where $\mathbf{X}=\left[x_{1}, \ldots, x_{2 n}\right]$ is composed by the right eigenvectors, while $\mathbf{Y} \in \mathbb{R}^{2 N \times N}$, where $\mathbf{Y}=\left[y_{1}, \ldots, y_{2 n}\right]^{T}$ is composed by its left eigenvectors. Finally, $\mathbf{J} \in \mathbb{R}^{2 N \times 2 N}$.

Definition 6.3.2. The left set of eigenvectors Y (LANCASTER, 1966): The left eigenvectors can be defined in terms of the right eigenvectors and the matrix $\boldsymbol{J}$ as

$$
\mathbf{Y}=\left[\begin{array}{c}
\mathbf{X} \\
\mathbf{X J}
\end{array}\right]^{-1}\left[\begin{array}{l}
\mathbf{0} \\
\mathbf{I}
\end{array}\right]
$$


Definition 6.3.3. Useful identities (LANCASTER, 1966):

$$
\begin{gathered}
\mathbf{X Y}=\mathbf{0}, \\
\mathbf{X J Y}=\mathbf{I} \\
\mathbf{X J}^{2}+\mathbf{B X J}+\mathbf{C X}=\mathbf{0} \\
\mathbf{J}^{2} \mathbf{Y}+\mathbf{J Y B}+\mathbf{Y C}=\mathbf{0}
\end{gathered}
$$

\subsubsection{2-Layer multiplex networks}

A general form of any matrix (adjacency, Laplacian or probability transition) of a multilayer network composed by two layers is given by a block matrix. Thus, the standard eigenvalue problem can be expressed as

$$
\left[\begin{array}{ll}
\mathbf{M}_{11} & \mathbf{M}_{12} \\
\mathbf{M}_{21} & \mathbf{M}_{22}
\end{array}\right]\left[\begin{array}{l}
v_{1} \\
v_{2}
\end{array}\right]=\lambda\left[\begin{array}{l}
v_{1} \\
v_{2}
\end{array}\right]
$$

where $\mathbf{M}_{12}=\mathbf{M}_{21}^{T}$ if $\mathbf{M}$ is symmetric, which can be the case in many situations as we assume undirected edges. Interpreting it as a system of equations and isolating $v_{1}$ in the first row, the dependence of the components of the eigenvector is expressed as

$$
v_{1}=-\mathbf{M}_{21}^{-1}\left(\mathbf{M}_{22}-\lambda \mathbf{I}\right) v_{2}
$$

Finally, inserting it into the second row, we have

$$
\left[\left(\mathbf{M}_{11}-\lambda \mathbf{I}\right) \mathbf{M}_{21}^{-1}\left(\mathbf{M}_{22}-\lambda \mathbf{I}\right)-\mathbf{M}_{12}\right] v_{2}=0
$$

which is a QEP, whose coefficient matrices are

$$
\begin{aligned}
& \mathbf{A}=\mathbf{M}_{12}^{-1}, \\
& \mathbf{B}=-\left(\mathbf{M}_{11} \mathbf{M}_{12}^{-1}+\mathbf{M}_{12}^{-1} \mathbf{M}_{22}\right) \\
& \mathbf{C}=\mathbf{M}_{11} \mathbf{M}_{12}^{-1} \mathbf{M}_{22}-\mathbf{M}_{12}
\end{aligned}
$$

In our context, exchanging $\mathbf{M}_{11}$ and $\mathbf{M}_{22}$ does not change the system, neither the solutions. However, if the polynomial considering the first layer is $\mathbf{Q}(\lambda)$, then, for the second one (relabling the layer indices) is $\mathbf{Q}(\lambda)^{T}$. In this way we found a relation between the right and left eigenvectors and these two possible configurations of our system. Formally, such an observation implies $x=v_{2}$ and $y=v_{1}$. Although in this chapter we focus on static networks where only the coupling matrices are a function of a coupling parameter or function, all the presented formalism can be extended to more general problems.

\subsubsection{Spectral analysis}

Up to this point we have defined our main mathematical tools, making as less constraints as possible. Now we restrict ourselves to diagonal coupling matrices and assume 
linear function of the parameter $p, \mathbf{M}_{12}=p \mathbf{D}$, where $\mathbf{D}$ is a diagonal invertible matrix (such constraint will be relaxed later). Then, defining the scalar equation that describes each eigenvalue as the product of $\mathbf{Q}(\lambda)$, by its left and right eigenvectors we have

$$
y^{T} \mathbf{Q}(\lambda) x=a\left(y^{T}, x\right) \lambda^{2}+b\left(y^{T}, x\right) \lambda+c\left(y^{T}, x\right)=0,
$$

where $a\left(y^{T}, x\right)=y^{T} \mathbf{A} x, b\left(y^{T}, x\right)=y^{T} \mathbf{B} x$ and $c\left(y^{T}, x\right)=y^{T} \mathbf{C} x$. Since this is a scalar quadratic equation, its solution is given by

$$
\lambda^{ \pm}(x)=\frac{-b\left(y^{T}, x\right) \pm \sqrt{\Delta\left(y^{T}, x\right)}}{2 a\left(y^{T}, x\right)},
$$

where $\Delta\left(y^{T}, x\right)=b\left(y^{T}, x\right)^{2}-4 a\left(y^{T}, x\right) c\left(y^{T}, x\right)$. Additionally, differentiating Equation 6.31 by $p$ we obtain information on how the eigenvalues change as $p$ changes. Formally we have

$$
\frac{\partial y^{T} \mathbf{Q}(\lambda) x}{\partial p}=y^{T} \frac{\partial \mathbf{Q}(\lambda)}{\partial p} x+y^{T} \mathbf{Q}(\lambda) \frac{d x}{d p}+\frac{d y}{d p} \mathbf{Q}(\lambda) x=0
$$

where

$$
\frac{\partial \mathbf{Q}(\lambda)}{\partial p}=2 \lambda \mathbf{D}^{-1} \frac{d \lambda}{d p}+\frac{d \lambda}{d p} \mathbf{B}+\lambda \frac{\partial \mathbf{B}}{\partial p}+\frac{\partial \mathbf{C}}{\partial p} .
$$

Note that the eigenvalues and eigenvectors are also a function of $p$. Moreover, observe for non-crossing points the relations $\frac{d y^{T}}{d p} \mathbf{Q}(\lambda) x=0$ and $y^{T} \mathbf{Q}(\lambda) \frac{d x}{d p}=0$ holds, since the derivatives are bounded for non-crossing points. However, on the crossings we have two eigenvectors associated to the same eigenvalue, which imply two solutions of the derivatives. Then, isolating the derivative of $\lambda$ we have

$$
\frac{d \lambda}{d p}=\frac{y^{T}\left(-\lambda \frac{\partial \mathbf{B}}{\partial p}-\frac{\partial \mathbf{C}}{\partial p}\right) x}{y^{T}\left(2 \lambda \mathbf{D}^{-1}+\mathbf{B}\right) x} .
$$

As an application, this relation can be used to drive a system from different regimes. For instance, considering the adjacency matrix, one can use this equation in order to chose an edge or set of edges to be removed (or weighted) in order to reduce the leading eigenvalue and consequently the critical point of spreading processes, such as epidemic spreading. The same also applies to the Lapplacian. Obviously matrix under study depends on the process. Moreover, another application would be the design of a numerical method to follow the right eigenvalues as a function of $p$.

\subsubsection{Supra-Laplacian matrix}

Our main formalism is now developed and we will show some applications on the supra-Laplacian matrix. This matrix is related to many dynamical processes, for instance diffusion and synchronization of coupled oscillators. Firstly, in Section 6.3.3.1, we discuss the so-called structural transition (RADICCHI; ARENAS, 2013), which we were able 
Figure 74 - Reproduction of a similar experiment as presented in (RADICCHI; ARENAS, 2013) for the algebraic connectivity and the Fiedler vector, here divided as $v=\left[v_{a}, v_{b}\right]$. Additionally, we also show our prediction of the structural transition.

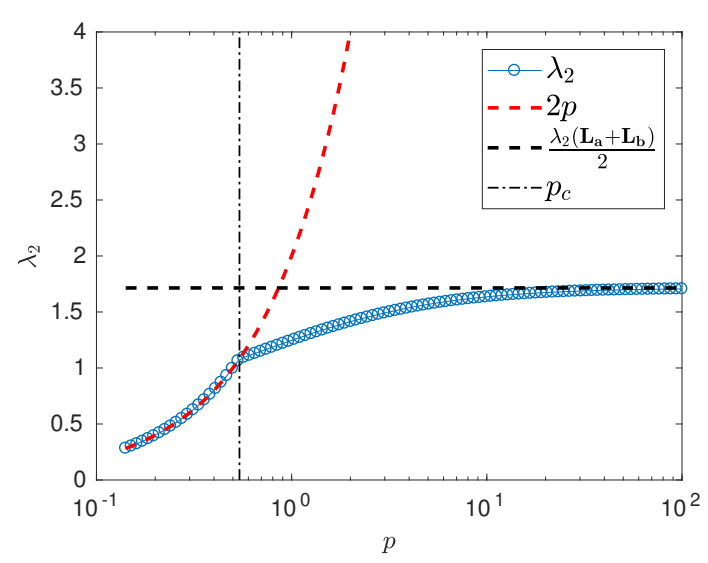

(a) Algebraic connectivity.

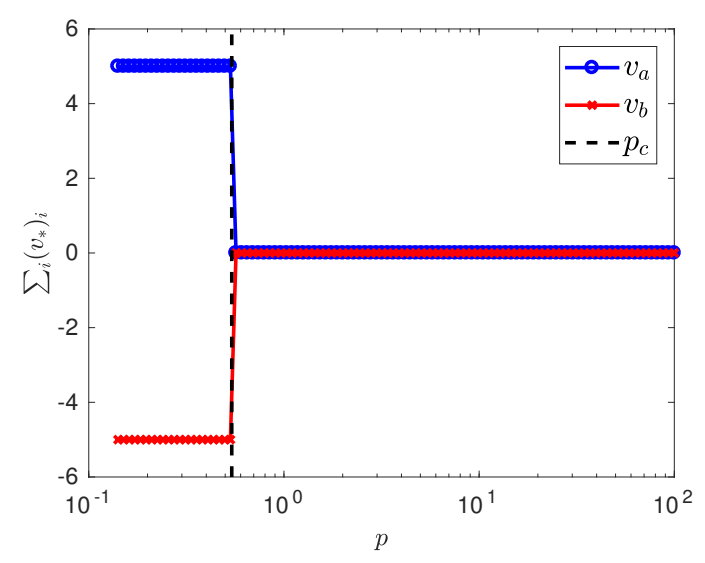

(b) Fiedler vector analysis.

Source: Developed by the author.

to calculate, however avoiding the mathematical difficulties found in (Darabi Sahneh; SCOGLIO; Van Mieghem, 2015). Next in Section 6.3.3.2 we extract some bounds for the supra-Laplacian matrix. Then, on Section 6.3.3.3, we evaluate its behavior as a function of the coupling parameter $p$.

To begin with, the most general Laplacian matrix consider a coupling matrix $M_{12}=-p \mathbf{D}$, where $\mathbf{D}$ is a diagonal matrix. The QEP of such matrix is defined by $\mathbf{A}=\mathbf{D}^{-1}$, $\mathbf{B}=-\left(\mathbf{L D}^{-1}+\mathbf{D}^{-1} \mathbf{L}_{\mathbf{b}}+2 p \mathbf{I}\right)$ and $\mathbf{C}=\mathbf{L D}^{-1} \mathbf{L}_{\mathbf{b}}+p\left(\mathbf{L}+\mathbf{L}_{\mathbf{b}}\right)$. The analysis of such QEP is not trivial, since the matrices are not symmetric, however the comparison with the diagonal coupling can give us some insights on the behavior of more general cases.

\subsubsection{Structural transitions}

Here, our main interest lies on the second smallest eigenvalue, which is also called algebraic connectivity, and its correspondent eigenvector, called Fiedler vector. In (RADICCHI; ARENAS, 2013) the authors found a structural transition on the algebraic connectivity on the supra-Laplacian matrix. Figure 74, reproduce their findings. However, in (Darabi Sahneh; SCOGLIO; Van Mieghem, 2015) the authors obtained an exact formula for the critical value of $p=p_{c}$, where such transition takes place. Here, we show an alternative form, where we can easily derive the structural transitions (RADICCHI; ARENAS, 2013) points using our formalism.

Firstly, we discuss the structural transition presented in (RADICCHI; ARENAS, 2013). Here, our main interest lies in the second smallest eigenvalue, which is also called algebraic connectivity, and its correspondent eigenvector, called Fiedler vector. More specif- 
ically, in (RADICCHI; ARENAS, 2013), the authors found a structural transition on the algebraic connectivity on the supra-Laplacian matrix. Figure 74, reproduces their findings. It is noteworthy that this transition points were also calculated in (Darabi Sahneh; SCOGLIO; Van Mieghem, 2015) using two different methods: eigenvalue sensitivity analysis and Shur's complement approach. Both derivations are quite complicated, contrasting with our approach, where the solutions is given using simple arguments. Note, however, that our approach presents a different expression if compared to the method presented in (Darabi Sahneh; SCOGLIO; Van Mieghem, 2015), but both expressions have the same final result. We did not prove the equivalence mathematically, but just verified their equivalence numerically.

Next, it is well known that $\lambda=2 p$ is an eigenvalue of the supra-Laplacian and the crossing points are a consequence of this eigenvalue crossing the bounded part of the supra-Laplacian spectra, producing the so-called structural transitions. In this manner, from our definition of QEP, we have that

$$
\operatorname{det}(\mathbf{Q}(2 p))=\operatorname{det}\left(\mathbf{L}+\mathbf{L}_{\mathbf{b}}\right) \operatorname{det}\left(\mathbf{L}_{\mathbf{b}} \mathbf{L}\left(\mathbf{L}+\mathbf{L}_{\mathbf{b}}\right)^{\dagger}-p \mathbf{I}\right)
$$

which have two possible solutions: (i) $\operatorname{det}\left(\mathbf{L}+\mathbf{L}_{\mathbf{b}}\right)=0$, which is always true, since the sum of two Laplacian matrices is also the Laplacian of the aggregated network and also have determinant equals to zero and (ii) the solution of $\operatorname{det}\left(\mathbf{L}_{\mathbf{b}} \mathbf{L}\left(\mathbf{L}+\mathbf{L}_{\mathbf{b}}\right)^{\dagger}-p \mathbf{I}\right)$, which are the crossing points or eigenvalues of multiplicity larger than one. Since it is also an eigenvalue problem in terms of $p$, so we have $p^{*}=\lambda_{i}\left(\mathbf{L}_{\mathbf{b}} \mathbf{L}\left(\mathbf{L}+\mathbf{L}_{\mathbf{b}}\right)^{\dagger}\right)$. There are $N$ possible values of $p$ that solve 6.36, each one representing one crossing. Observe that it only cross the $N$ lowest eigenvalues. The first crossing is trivial, at $p=0$, the second is the so-called structural transition, which impact on dynamical processes. This expression is different from the previous one presented in the literature, however both present the same solutions, as we numerically verified.

\subsubsection{Bounds}

In this section, we aim to find bounds for the spectra of the supra-Laplacian matrix. For a general idea of the concepts used here, we refer to Appendix E. Thus, the QEP of the supra Laplacian can be bounded considering the individual bounds of $\mathbf{B}$, which is an semi-positive definite Hermitian matrix, leading to

$$
2 p \leq-b\left(x^{T}, x\right) \leq 2 p+\lambda_{\max }\left(\mathbf{L}+\mathbf{L}_{\mathbf{b}}\right)
$$

Besides, the discriminant function is also bounded by

$$
\begin{gathered}
\min \left\{x^{T}\left(\left(\mathbf{L}-\mathbf{L}_{\mathbf{b}}\right)^{2}-2 p\left(\mathbf{L}+\mathbf{L}_{\mathbf{b}}\right)+4 p^{2} \mathbf{I}\right) x\right\} \leq \Delta\left(x^{T}, x\right) \\
\Delta\left(x^{T}, x\right) \leq \max \left\{x\left(\left(\mathbf{L}-\mathbf{L}_{\mathbf{b}}\right)^{2}\right)+4 p^{2} \mathbf{I}\right\}
\end{gathered}
$$


Figure 75 - Evaluation of the eigenvalues $\lambda(\mathbf{L})$ as a function of the coupling parameter $p$ of a multiplex network composed by two Erdös Renyi layers with $n=10^{3}$ nodes and the first layer have average degree $\mathbb{E}(k)=12$, while the second with $\mathbb{E}(k)=16$. The continuous lines are the upper bounds, while the dashed lines the lower bounds.

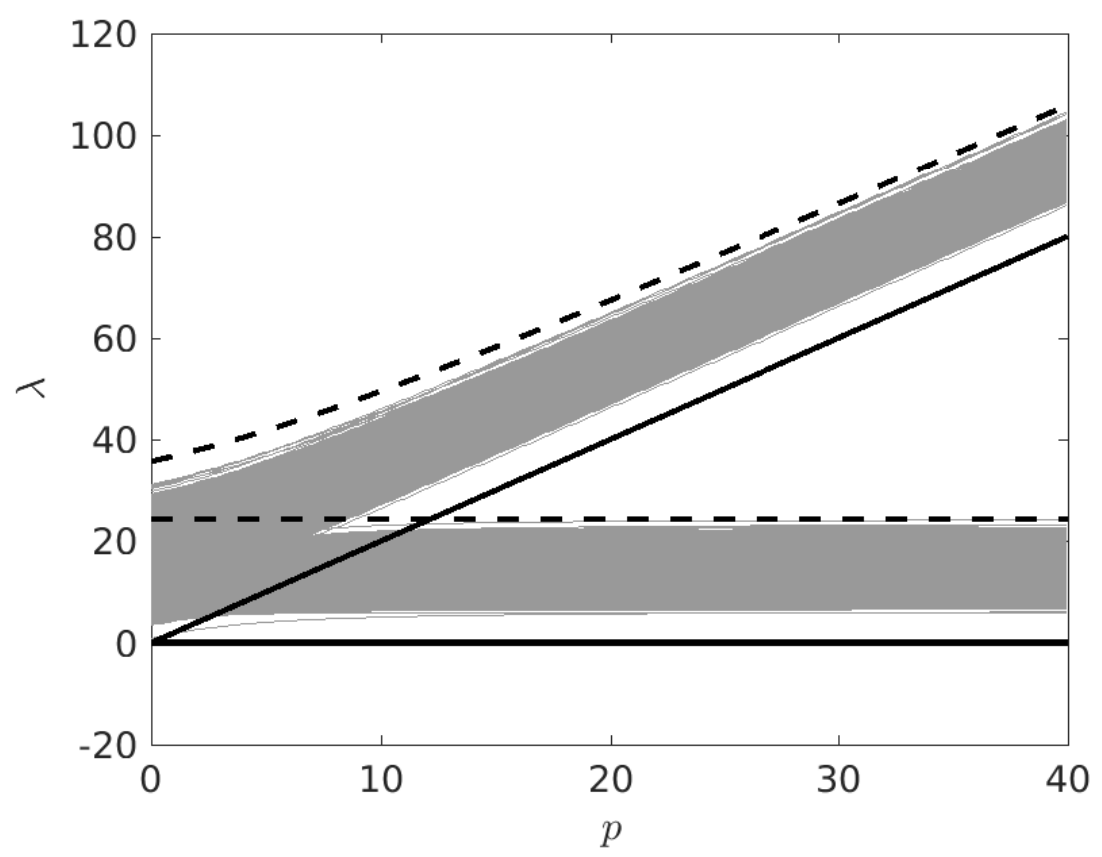

Source: Developed by the author.

where the upper bound can be defined as a function of the spectral properties of $\left(\mathbf{L}-\mathbf{L}_{\mathbf{b}}\right)^{2}$. On the other hand, regarding the lower bound, it can be improved by realizing that that the matrix $\Delta=\left(\mathbf{L}-\mathbf{L}_{\mathbf{b}}\right)^{2}-2 p\left(\mathbf{L}+\mathbf{L}_{\mathbf{b}}\right)+4 p^{2} \mathbf{I}$, defined on Appendix E.1, is semipositive definite for undirected networks, $\Delta \succeq 0$. In this manner, $\left(\mathbf{L}-\mathbf{L}_{\mathbf{b}}\right)^{2}-2 p\left(\mathbf{L}+\mathbf{L}_{\mathbf{b}}\right)+$ $4 p^{2} \mathbf{I} \succeq 0$, hence $\left(\mathbf{L}-\mathbf{L}_{\mathbf{b}}\right)^{2}+4 p^{2} \mathbf{I} \succeq 2 p\left(\mathbf{L}+\mathbf{L}_{\mathbf{b}}\right)$, implying that $\lambda_{i}\left(\left(\mathbf{L}-\mathbf{L}_{\mathbf{b}}\right)^{2}+4 p^{2} \mathbf{I}\right) \geq$ $\lambda_{i}\left(2 p\left(\mathbf{L}+\mathbf{L}_{\mathbf{b}}\right)\right)^{4}$. From those properties, we can establish the lower bound as $4 p^{2}$. Formally,

$$
4 p^{2} \leq \Delta\left(x^{T}, x\right) \leq \lambda_{\max }\left(\left(\mathbf{L}-\mathbf{L}_{\mathbf{b}}\right)^{2}\right)+4 p^{2}
$$

The previous bounds imply that in the asymptotic analysis formalism we have $\Delta\left(x^{T}, x\right) \in$ $\Theta\left(p^{2}\right)$. Moreover, observe that lower and upper bound converge to each other as the layers are similar. On the extreme case of identical layers we have $\Delta\left(x^{T}, x\right)=$ Finally, combining the formerly obtained bounds we have,

$$
0 \leq \lambda^{-}\left(x^{T}, x\right) \leq \frac{1}{2} \lambda_{\max }\left(\mathbf{L}+\mathbf{L}_{\mathbf{b}}\right)
$$

$\overline{4}$ In addition, lets recall that if $\mathbf{M}_{1}-\mathbf{M}_{2} \succeq 0$. For semi-positive matrices $\mathbf{M}_{1}$ and $\mathbf{M}_{2}$, if $\mathbf{M}_{1} \succeq \mathbf{M}_{2}$, then $\lambda_{i}\left(\mathbf{M}_{1}\right) \geq \lambda_{i}\left(\mathbf{M}_{2}\right)$, where the eigenvalues are in descending order. 
and

$$
2 p \leq \lambda^{+}\left(x^{T}, x\right) \leq p+\frac{\lambda_{\max }\left(\mathbf{L}+\mathbf{L}_{\mathbf{b}}\right)}{2}+\frac{\sqrt{\lambda_{\max }\left(\left(\mathbf{L}-\mathbf{L}_{\mathbf{b}}\right)^{2}\right)+4 p^{2}}}{2} .
$$

Interestingly, this bounds con be analyzed in terms of its asymptotic behavior (approximation), where for a sufficiently large value of $p$ they can be approximated to

$$
\begin{gathered}
0 \leq \lambda^{-}\left(x^{T}, x\right) \leq \frac{\lambda_{\max }\left(\mathbf{L}+\mathbf{L}_{\mathbf{b}}\right)}{2} \\
2 p \leq \lambda^{+}\left(x^{T}, x\right) \leq 2 p+\frac{\lambda_{\max }\left(\mathbf{L}+\mathbf{L}_{\mathbf{b}}\right)}{2} .
\end{gathered}
$$

Observe that from the asymptotic point of view we have $\lambda^{-}(x) \in \Theta(1)$ and $\lambda^{+}(x) \in \Theta(p)$. Additionally, we can establish a condition when such bounds can be adopted.

As an example, in Figure 75 we present the evaluation of the eigenvalues as a function of the coupling parameter $p$ of a multiplex network composed by two Erdös Renyi layers with $n=10^{3}$ nodes and the first layer have average degree $\mathbb{E}(k)=12$, while the second with $\mathbb{E}(k)=16$.

\subsubsection{Spectral properties as a function of the coupling $p$}

One of the main interests in the spectral properties of multiplex networks is its behavior as a function of the coupling parameter $p$. Thus, let us consider the simplest case, where $\mathbf{D}=\mathbf{I}$. In this case, we have a monic polynomial matrix, where $\mathbf{B}$ is the aggregated network, which is semi-positive definite. Besides, $\mathbf{C}$ is a matrix that contains the product of both layers and accounts for similarities between them. Thus, from Equation 6.35 can be expressed as

$$
\frac{d \lambda_{i}}{d p}=\frac{\left(2 \lambda_{i} y^{T} x-\hat{b}\left(y^{T}, x\right)\right)}{\left(2\left(\lambda_{i}-p\right) y^{T} x-\hat{b}\left(y^{T}, x\right)\right)}
$$

where $\hat{b}\left(y^{T}, x\right)=y^{T}\left(\mathbf{L}+\mathbf{L}_{\mathbf{b}}\right) x$ and $y^{T} x=\cos (\boldsymbol{\theta})$ is the cosine of the angle between left and right eigenvectors of our QEP. Observe that part of the spectra have $\frac{d \lambda_{i}}{d p} \rightarrow 0$, while the other part have $\frac{d \lambda}{d p} \rightarrow 2$ as $p$ increases, which can be proved as follows. Firstly, suppose that $\lambda_{i}$ is constant as a function of $p$, then $\frac{d \lambda_{i}}{d p} \rightarrow 0$ because the denominator grows as a function of $p$ and the numerator is bounded as supposed. Secondly, suppose that $\lambda_{i}$ grows with $p^{r}$, where $r<1$. In this case $\frac{d \lambda_{i}}{d p} \rightarrow 0$, by the same arguments as before, since the linear function of the denominator dominates it. However, if $r=1$ we have $\frac{d \lambda_{i}}{d p} \rightarrow 2$, since both grow linearly. Finally, with $p^{r}$, where $r>1$, both the numerator and denominator are dominated by $p^{r}$, which imply that for large $p$ the derivative $\frac{d \lambda_{i}}{d p} \rightarrow 1$, which is also a contradiction, since it was supposed to be a linear function of $p$. Thus, we conclude 
Figure 76 - Evaluation of the eigenvalues $\lambda(\mathbf{L})$ as a function of the coupling parameter $p$ of a multiplex network composed by two Erdös Renyi layers with $n=10^{3}$ nodes and the first layer have average degree $\mathbb{E}(k)=12$, while the second with $\mathbb{E}(k)=16$. The coupling matrix is $\mathbf{D}=\frac{n}{\sum_{i}^{n} i} \operatorname{diag}(1,2, \ldots, n)$. The continuous lines is the adapted upper bound, while the dashed line is the adapted lower bound.

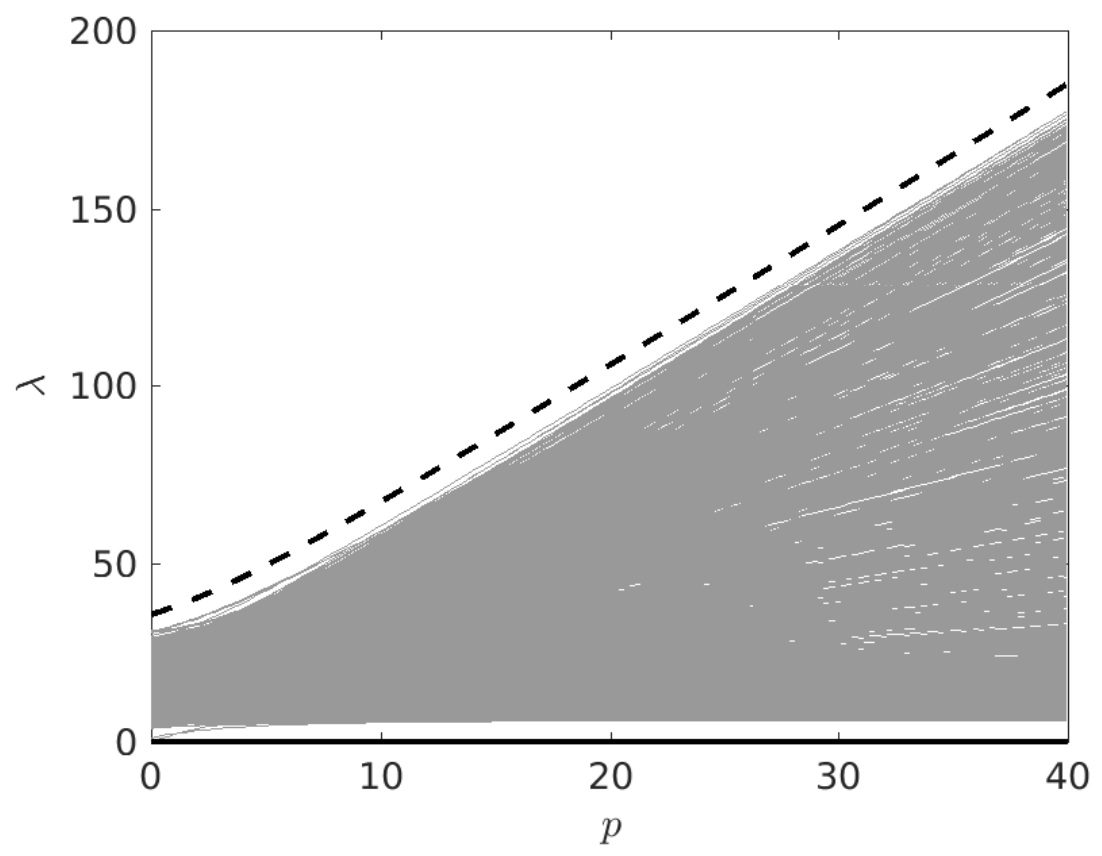

Source: Developed by the author.

that the derivatives of $\lambda_{i}$, for large values of $p$, cannot grow faster than linearly and their growth will be one of two values, 0 or 2 . Those results are in agreement with the previously obtained bounds. Additionally, as an example, in Figure 75 we can also observe such behavior.

Although for the simplified case we have two possible solutions at large $p$, observe that the above arguments fail on the case of general coupling matrix. From Equation 6.35 and the definition of the Laplacian QEP we conclude that only the denominator of 6.35 changes for a different choice of $\mathbf{D}$, as the terms which have dependencies on $\mathbf{D}$ vanishes on the partial derivatives at the numerator. Such denominator follow the general form $y^{T}\left(2 \lambda_{i} \mathbf{D}^{-1}-2 p \mathbf{I}-\mathbf{L D}^{-1}-\mathbf{D}^{-1} \mathbf{L}_{\mathbf{b}}\right) x$. Thus, different coupling weights can change the spectral behavior for different for large $p$. For instance, if $\mathbf{D}=\mathbf{I}$ the spectral distribution for large $p$ is bimodal, as pointed by arguments above, however, if $\mathbf{D}=\frac{n}{\sum_{i}^{n} i} \operatorname{diag}(1,2, \ldots, n)^{5}$, this behavior changes completely and the eigenvalues change with different rates, suggesting that iit can present a continuous bulk. This argument is valid for for infinity size

$5 \operatorname{diag}(\mathbf{D})$ have identically spaced numbers and unitary average, due to the term $\frac{n}{\sum_{i}^{n} i}$, allowing the comparison with any other figure on this paper. 
networks, since for finite size networks and a large value of $p$, gaps between eigenvalues may appear due to different rates of growth (as a function of $p$ ). This example is shown on Figure 76. Furthermore, we also found an empirical function that seems to bound the spectra as a function of $p$ in this experiment. The lower bound is trivial, as it is a semipositive definite matrix. The upper bound can be obtained correcting $\tilde{p}=\max \{\operatorname{diag}(\mathbf{D})\} p$, hence

$$
0 \leq \lambda_{i} \leq \tilde{p}+\frac{\lambda_{\max }\left(\mathbf{L}+\mathbf{L}_{\mathbf{b}}\right)+\sqrt{\lambda_{\max }\left(\left(\mathbf{L}-\mathbf{L}_{\mathbf{b}}\right)^{2}\right)+4 \tilde{p}^{2}}}{2} .
$$

From Figure 76 we observe that this bound is not as close to the largest eigenvalue as the homogeneous case, but it is interesting to observe this function and its relationship with Equation 6.44, as it suggest this correction.

In addition to a non-homogeneous coupling matrix, the last case studied is the sparse coupling. The analytical part of this study was presented on Appendix E.3. As predicted, each uncoupled node imply in a pair of eigenvalues that does not depend on $p$. Due to the nature of the Laplacian matrix, where just one eigenvalue varies with $p$, while the other remains bounded, the set of bounded eigenvalues increases by one. For example, if we have $\tilde{n}$ uncoupled nodes, the bounded part have $n+\tilde{n}$ eigenvalues, while the "unbounded" part have $n-\tilde{n}$ eigenvalues. Note that the upper bound for the bounded part is not $\frac{1}{2} \lambda_{\max }\left(\mathbf{L}+\mathbf{L}_{\mathbf{b}}\right)$ anymore. However the general upper bound for $\mathbf{D}=\mathbf{I}$ seems to be also an upper bound for the sparse problem, as we numerically verified. The figures for those experiments were not shown, since they are visually similar to Figure 75.

\subsubsection{Supra-adjacency matrix}

In addition to the supra-Laplacian case, in this Section we evaluate the supraadjacency matrix. Following a similar analysis, in Section 6.3.4.1 we establish bounds for the supra-adjacency spectra. Next, in Section 6.3.4.2, we evaluate its behavior as a function of the coupling parameter $p$. Thus, the general QEP for the supra-adjacency matrix is defined as $\mathbf{A}=\mathbf{D}^{-1}, \mathbf{B}=-\left(\mathbf{A}_{a} \mathbf{D}^{-1}+\mathbf{D}^{-1} \mathbf{A}_{b}\right)$ and $\mathbf{C}=\mathbf{A}_{a} \mathbf{D}^{-1} \mathbf{A}_{b}-p^{2} \mathbf{D}$.

\subsubsection{Bounds}

Similarly to the previous case, in this section, we aim to find bounds for the spectra of the supra-adjacency matrix. For a general idea of the concepts used here, we refer to Appendix E. For the sake of simplicity, concerns the case in which $\mathbf{D}=\mathbf{I}$, where we can find these bounds. Beginning with $\mathbf{B}$, we can bound it based on its eigenvalues as

$$
\lambda_{\min }\left(\mathbf{A}_{\mathbf{a}}+\mathbf{A}_{\mathbf{b}}\right) \leq-b(x) \leq \lambda_{\max }\left(\mathbf{A}_{\mathbf{a}}+\mathbf{A}_{\mathbf{b}}\right)
$$


Figure 77 - Evaluation of the eigenvalues $\lambda_{i}(\mathbf{A})$ as a function of the coupling parameter $p$ of a multiplex network composed by two Erdös Renyi layers with $n=10^{3}$ nodes and the first layer have average degree $\mathbb{E}(k)=12$, while the second with $\mathbb{E}(k)=16$. The continuous lines are the upper bounds, while the dashed lines the lower bounds.

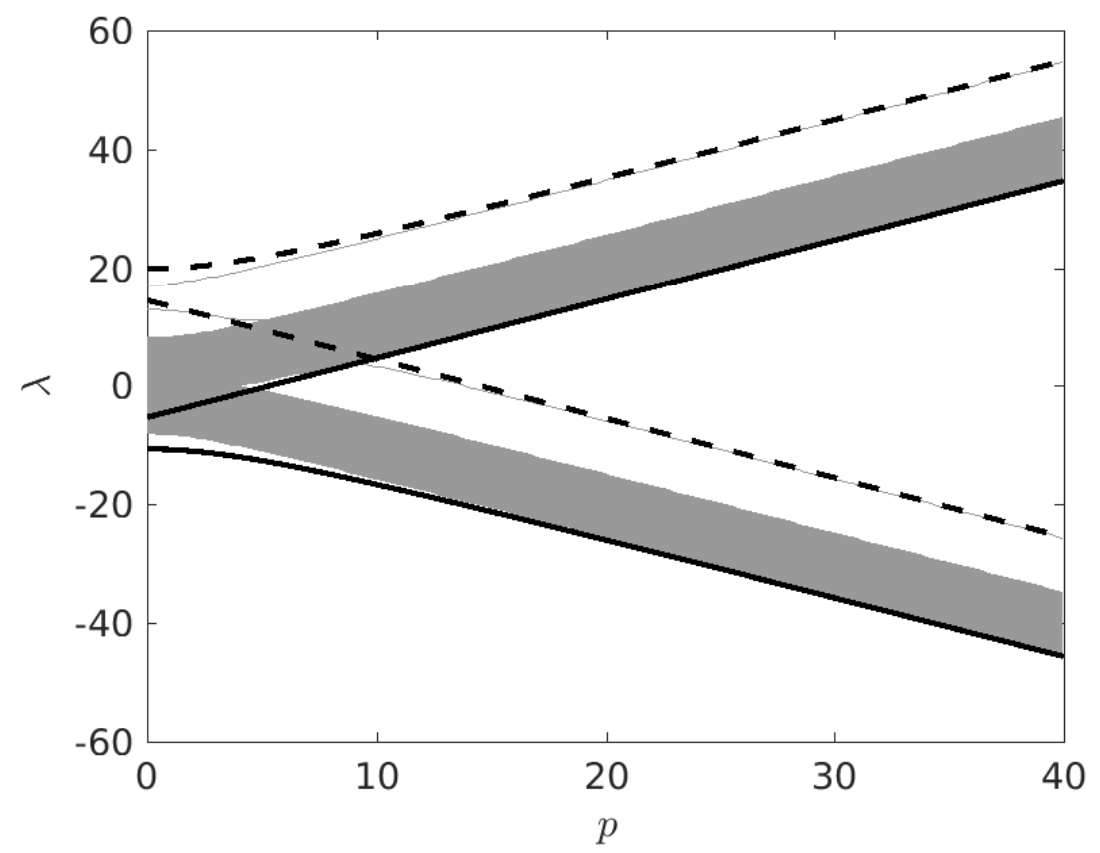

Source: Developed by the author.

Similarly, for the discriminant we have

$$
\begin{gathered}
\lambda_{\min }\left(\left(\mathbf{A}_{\mathbf{a}}-\mathbf{A}_{\mathbf{b}}\right)^{2}\right) \leq \Delta\left(x^{T}, x\right)^{2} \\
\Delta\left(x^{T}, x\right) \leq \lambda_{\max }\left(\left(\mathbf{A}_{\mathbf{a}}-\mathbf{A}_{\mathbf{b}}\right)^{2}\right)
\end{gathered}
$$

Combining those bounds we can bound both solutions by

$$
\begin{aligned}
& \frac{1}{2}\left(\lambda_{\min }\left(\mathbf{A}_{\mathbf{a}}+\mathbf{A}_{\mathbf{b}}\right)-\sqrt{\lambda_{\max }\left(\left(\mathbf{A}_{\mathbf{a}}-\mathbf{A}_{\mathbf{b}}\right)^{2}\right)+4 p^{2}}\right) \leq \lambda^{-} \\
& \quad \leq \frac{1}{2}\left(\lambda_{\max }\left(\mathbf{A}_{\mathbf{a}}+\mathbf{A}_{\mathbf{b}}\right)-\sqrt{\lambda_{\max }\left(\left(\mathbf{A}_{\mathbf{a}}-\mathbf{A}_{\mathbf{b}}\right)^{2}\right)+4 p^{2}}\right) .
\end{aligned}
$$

and

$$
\begin{aligned}
& \frac{1}{2}\left(\lambda_{\min }\left(\mathbf{A}_{\mathbf{a}}+\mathbf{A}_{\mathbf{b}}\right)+\sqrt{\lambda_{\max }\left(\left(\mathbf{A}_{\mathbf{a}}-\mathbf{A}_{\mathbf{b}}\right)^{2}\right)+4 p^{2}}\right) \leq \lambda^{+} \\
& \quad \leq \frac{1}{2}\left(\lambda_{\max }\left(\mathbf{A}_{\mathbf{a}}+\mathbf{A}_{\mathbf{b}}\right)+\sqrt{\lambda_{\max }\left(\left(\mathbf{A}_{\mathbf{a}}-\mathbf{A}_{\mathbf{b}}\right)^{2}\right)+4 p^{2}}\right) .
\end{aligned}
$$

Figure 77 shows an example of this bounds and its behavior as a function of $p$. In addition, these bounds asymptotically converges (approximation) to

$$
p \pm \frac{\lambda_{\min }\left(\mathbf{A}_{\mathbf{a}}+\mathbf{A}_{\mathbf{b}}\right)}{2} \leq \lambda^{ \pm}(x) \leq p \pm \frac{\lambda_{\max }\left(\mathbf{A}_{\mathbf{a}}+\mathbf{A}_{\mathbf{b}}\right)}{2} .
$$


Figure 78 - Evaluation of the eigenvalues $\lambda_{i}(\mathbf{L})$ as a function of the coupling parameter $p$ of a multiplex network composed by two Erdös Renyi layers with $n=10^{3}$ nodes and the first layer have average degree $\mathbb{E}(k)=12$, while the second with $\mathbb{E}(k)=16$. The coupling matrix is $\mathbf{D}=\frac{n}{\sum_{i}^{n} i} \operatorname{diag}(1,2, \ldots, n)$. The continuous lines is the adapted upper bound, while the dashed line is the adapted lower bound.

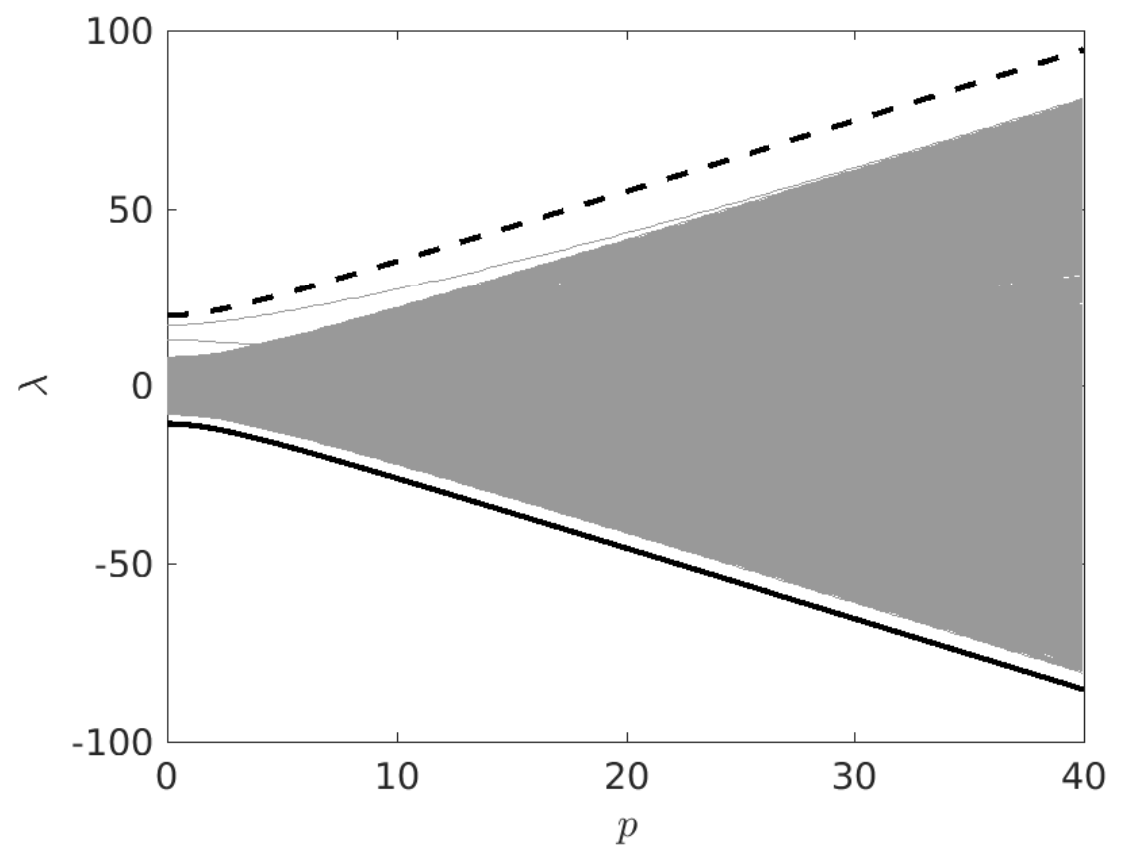

Source: Developed by the author.

In other words, the spectral density of the adjacency matrix is bimodal and part of the eigenvalues grow linearly with $p$, while the other part decrease at the same rate.

\subsubsection{Spectral properties as a function of the coupling $p$}

Interestingly, note that on previous chapters the coupling parameter $p$ was physically interpreted as the spreading ratio, $\frac{\eta}{\lambda}=p$, playing a major role in dynamical processes in multilayer networks. Thus, understanding its behavior as a function of $p$ can have important practical implications on the understanding of epidemics on this systems. Here we focus on its first derivative, which is given as

$$
\frac{d \lambda_{i}}{d p}=\frac{2 p y^{T} \mathbf{D} x}{\left(2 \lambda_{i} y^{T} \mathbf{D}^{-1} x+b\left(y^{T}, x\right)\right)}
$$

where $x$ and $y^{T}$ are the right and left eigenvectors associated with $\lambda_{i}$.

Firstly, focusing on $\mathbf{D}=\mathbf{I}$ and using similar results as applied to the Laplacian case we can suppose that $\lambda_{i}$ is a constant function of $p$ or a function of $p^{r}$ with $r<1$, however it would give us $\frac{d \lambda_{i}}{d p} \sim p$, which is a contradiction. Next we can suppose that it is a linear function of $p$, which implies $\frac{d \lambda_{i}}{d p} \rightarrow \pm 1$, depending on the sign of the linear 
Figure 79 - Evaluation of the eigenvalues $\lambda_{i}(\mathbf{A})$ as a function of the coupling parameter $p$ of a multiplex network composed by two Erdös Renyi layers with $n=10^{3}$ nodes and the first layer have average degree $\mathbb{E}(k)=12$, while the second with $\mathbb{E}(k)=16$. The coupling matrix is sparse. The continuous lines is the adapted upper bound, the dashed line is the adapted lower bound and the dotted line was obtained experimentally at the largest value of $p$ and extended for reference.

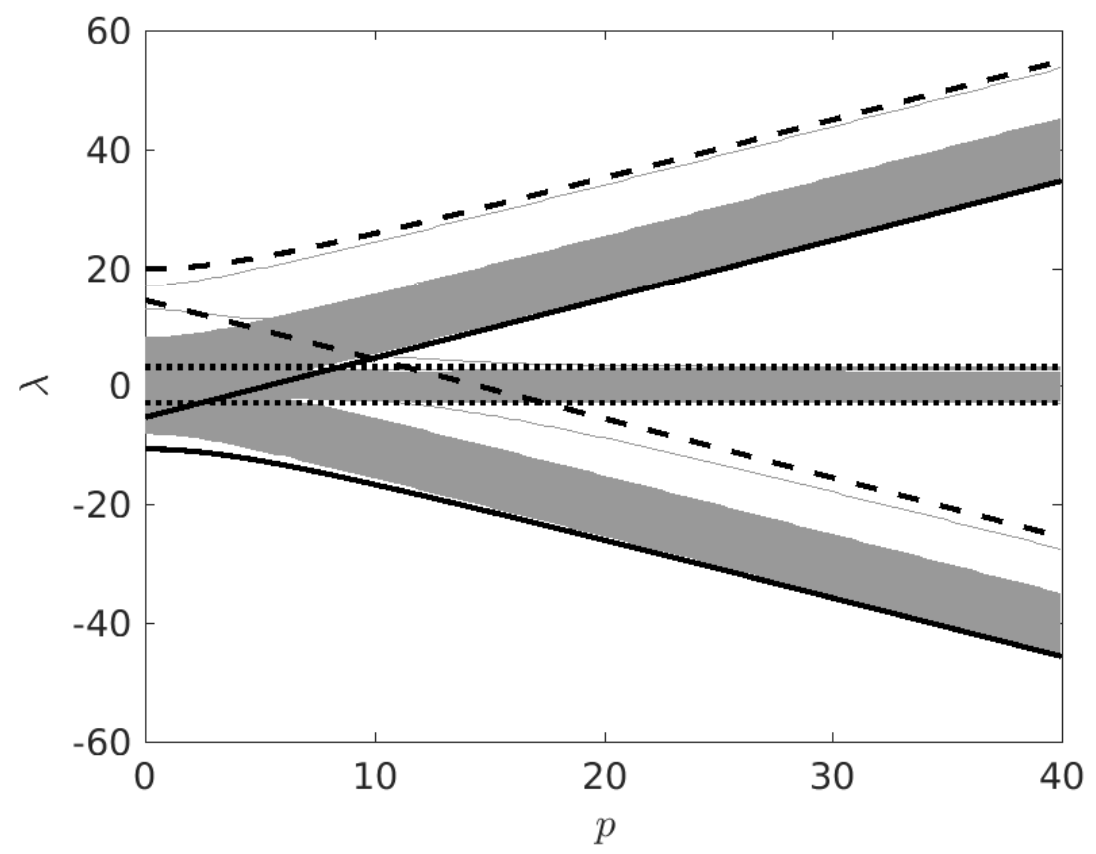

Source: Developed by the author.

coefficient. Finally, supposing that it is a function of $p^{r}$ with $r>1$ we obtain that $\frac{d \lambda_{i}}{d p} \rightarrow 0$, since the denominator grows faster than the numerator, which again is a contradiction. Therefore, based on such an analysis we infer that the first derivative of $\lambda_{i}$ can assume only $\frac{d \lambda_{i}}{d p} \rightarrow \pm 1$

Secondly, for the general case observe that both, the numerator and the denominator of 6.50 vary as a function of $\mathbf{D}$. Additionally, it weights the product of the components of the eigenvectors, which allow the derivatives to assume more values, even a "continuous bulk" instead of the bimodal distribution of the diagonal homogeneous case, similarity to the case discussed for the supra-Laplacian matrix, where we also use the coupling matrix $\mathbf{D}=\frac{n}{\sum_{i}^{n} i} \operatorname{diag}(1,2, \ldots, n)$ for dialectical purposes. Additionally, we show the spectral evolution as a function of $p$ for the non-homogeneous case in Figure 78. Similarly to the Laplacian case, also show evidence that the bounds can be corrected using $\tilde{p}=\max \{\operatorname{diag}(\mathbf{D})\} p$, hence

$$
\begin{aligned}
& \frac{1}{2}\left(\lambda_{\min }\left(\mathbf{A}_{\mathbf{a}}+\mathbf{A}_{\mathbf{b}}\right)-\sqrt{\lambda_{\max }\left(\left(\mathbf{A}_{\mathbf{a}}-\mathbf{A}_{\mathbf{b}}\right)^{2}\right)+4 \tilde{p}^{2}}\right) \leq \lambda_{i} \\
& \lambda \leq \frac{1}{2}\left(\lambda_{\max }\left(\mathbf{A}_{\mathbf{a}}+\mathbf{A}_{\mathbf{b}}\right)+\sqrt{\lambda_{\max }\left(\left(\mathbf{A}_{\mathbf{a}}-\mathbf{A}_{\mathbf{b}}\right)^{2}\right)+4 \tilde{p}^{2}}\right) .
\end{aligned}
$$


Here we can also obtain a similar conclusion as for the supra-Laplacian case. From Figure 78 we observe that the corrected bounds are not as close to the homogeneous case, but it is interesting to observe such function and its relation with Equation 6.50, since it suggest such correction.

Finally, we evaluate the sparse coupling case, whose analytical study was presented in Appendix E.3. As predicted, each uncoupled node imply in a pair of eigenvalues that does not depend on $p$. In this manner, if we have $\tilde{n}$ uncoupled nodes, the central part of the spectra will have $2 \tilde{n}$ eigenvalues that does not change as a function of $p$. Next, $n-\tilde{n}$ grow with linearly with $p$, while the other $n-\tilde{n}$ eigenvalues with $-p$. This is illustrated in Figure 79.

\subsection{Summary}

In this chapter, we numerically evaluated the spectral properties of multilayer networks as a function of two features, firstly the coupling strength ${ }^{6}$ in Section 6.1 and size, on Section 6.2. The first is a natural extension of our results presented in the previous chapter, while the second concerns a random matrix theory approach, seeking for universal properties. Finally, in Section 6.3, we explore the block structure of multilayer networks, reinterpreting the standard eigenvalue problem, whose dimension is $N \times N$, as a polynomial eigenvalue problem, which has a lower dimension, i.e. $n \times n$. Consequently, the problem is also of a higher order, $m$. We introduced this theory using 2-Layer multiplex networks and applying it to supra-adjacency and Laplacian matrices.

Regarding our first results, we must remark that we have observed a transition on the spectra of the supra-contact tensor, from the spectra resulting from the union of the individual layers to the spectra of the network of layers. This behavior implies that other dynamics and more complex structures can also be significantly affected by the interconnected nature of the system. In addition, we have also characterized analytically the phenomenon of eigenvalue crossing on the supra-contact tensor for the case of two identical layers. It is worth noticing that any dynamical process that is described by the same matrix will be affected by this effect.

Furthermore, considering our study on random matrices and universal properties, we demonstrated that the normalized localization length $\beta$ of the eigenfunctions of multilayer random networks scales as $x^{*} /\left(1+x^{*}\right)$. Here, $x^{*}=\gamma\left(b_{\text {eff }}^{2} / L\right)^{\delta}$; where $b_{\text {eff }}$ is the effective bandwidth of the network's adjacency matrix, $L$ is the adjacency matrix size, and $\delta \sim 1$. We showed that such a scaling law is robust covering weighted multilayer and both weighted and unweighted node-aligned multiplex networks. Moreover, the scal-

6 On spreading dynamics the physical meaning of this parameter is the ratio between intra and interlayer spreading rates 
ing law was validated in real-world networks. Our results might shed additional light on the critical properties and structural organization of multilayer systems. Interestingly enough, our findings might be used to either predict or design (e.g, tune), by means of Equation (6.13), the localization properties of the eigenfunctions of multilayer random networks. For instance, we anticipate the following cases: (i) Due to the banded nature of the adjacency matrices of the network models considered here, $b_{\text {eff }}<L$, it is unlikely to observe fully delocalized eigenfunctions unless the value of $x^{*}$ is driven to large values, for example, by increasing the size of the subnetworks $N$ and/or their sparsity $\tilde{\alpha}$ for a fixed value of $M$; and (ii) For a fixed subnetwork size $N$ and sparsity $\tilde{\alpha}$, the eigenfunctions of the multilayer network become more localized when increasing the number of subnetworks $M$.

Finally, in our last results section, we have shown a different interpretation for the matricial approach, evaluating the block matrices with the cost of increasing the power of the characteristic polynomial. Based on this approach we were able to extract bound for the spectra and the derivative of the eigenvalues, both as a function of the coupling parameter $p$ (which plays the same role as $\frac{\eta}{\lambda}=p$ in the first section and previous chapter). More specifically, we have shown some results for the supra Laplacian and adjacency matrices. To the best of our knowledge, this is a new approach for the spectral analysis of multilayer networks. We believe that this approach can be further explored, for instance in the analysis of dynamical processes, the evaluation of the eigenvectors and its localization properties, among many other possibilities. 

CHAPTER

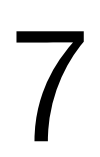

\section{CONCLUSION AND FUTURE WORKS}

In this thesis, we have studied epidemic-like processes on the top of single and multilayer networks, aiming to have a better understanding of these processes, especially in the interplay between structure and dynamics, and the improvement of their modeling techniques. In order to achieve such a goal, we divided our research into four connected chapters: (i) Chapter 3, where we define the dynamical processes and briefly review the literature, (ii) Chapter 4, where we study epidemic-like processes on single-layer networks, (iii) Chapter 5, where we extend the basics of epidemics to multilayer networks, as motivated by previous chapters, and (iv) Chapter 6, where we explore the spectral properties of multilayer networks, addressing the questions which arose during the development of Chapter 5 , on disease spreading in multilayer networks.

In more details, we began, in Chapter 3, with an analysis of the fundamentals of spreading processes on networks, presenting the main modeling approaches. This analysis ranged from the formal definition of these processes to the mean-field approximations. We also presented a classification of the literature methods, dividing the methods according to their mathematical nature: (i) continuous-time and (ii) cellular automata approach. Furthermore, we also associated each of those mathematical frameworks with their respective simulation algorithms. To round up this chapter, we performed an accuracy analysis comparing the mathematical and computational aspects of the methods discussed. During the development of this chapter, we identified some small literature gaps. Thus, our main goal was to properly and formally address them. We remark that in that chapter we have defined the main concepts for the development of this thesis. Consequently, the other contributions are a direct or indirect consequence of the analysis performed in Chapter 3.

Next, in Chapter 4, we have discussed some contributions for the single-layer theory. Firstly, in Section 4.1, we started tackling the modeling problem, proposing a unifying Markov chain, which covers many literature models. It is worth mentioning that the incorporated transitions present a physical/sociological meaning. For instance, forgetting and 
loss of interest mechanisms, such as recovery of interest on a given piece of information. Additionally, in this section we also focused on the applied/practical aspects of modeling, proposing a framework with a low computational cost and high accuracy. Secondly, in Section 4.2, we shifted our attention to the role of centrality on the identification of influential spreaders. In this part of our work, we extensively evaluated the correlations between absorbing state dynamics and centrality measures. One of our main results lies in the observation that spatial and non-spatial networks may present different dynamical outcomes, which also depends on the process under consideration. As an additional contribution, we also proposed a new centrality measure based on random walks (see also Section 2.1.2.3 for its definition and Appendix A for more details). More specifically, based on the concept of the accessible random walk, which is a well-known process in stochastic processes theory, but was not well explored in the context of complex networks. Furthermore, this measure proved to be correlated with SIR and MT dynamics, especially in spatial networks. On the contrary, in non-spatial networks, the best correlations between a centrality metric and the dynamical outcome depends on the process. Thus, the degree and coreness (as given by the $k$-core) are the ones more suited when it comes to analyzing epidemic spreading, confirming the results in (KITSAK et al., 2010). However, these measures are not the best when a rumor model is considered. Indeed, for the latter case, the average neighborhood degree, the closeness centrality and accessibility gives higher correlations. Complementary to the aforementioned results, our experiments also have emphasized the key role of spectral properties on spreading processes.

Moving from the single to the multilayer networks, we aimed to study more realistic models. This study was shown in Chapter 5. We began our analysis, in Section 5.1, focusing precisely on the localization of the leading eigenvector and identifying the layerlocalization phenomena. Furthermore, we also showed the interplay between the leading eigenvector layer-localization and SIS spreading processes as a function of the spreading ratio $\frac{\eta}{\lambda}$ for two and three layer systems. In the 2-Layer case, we showed the emergence and vanishing of a second susceptibility peak as a function of the spreading ratio. On the other hand, regarding the 3-Layer case, we observed a similar phenomenon, but another behavior, the barrier effect. Such phenomena appear as a consequence of the increased complexity level due to the network of layers configuration. In this case, we showed how an intermediate layer (which have a higher individual eigenvalue in comparison with the other layers) can act as a barrier, reducing the effects of a sustained epidemic process on other layers. Moreover, in Section 5.2, we evaluated the effects of assortative/disassortative multilayer structures on disease spreading. In order to do so, we proposed an extension of the traditional assortativity coefficient, allowing the new coefficient to measures different levels of correlations. We observed that assortative networks show a smaller epidemic threshold and that diseases have a faster initial growth rate in these networks, but a shorter duration. On the contrary, the fraction of infected individuals is larger in 
disassortative networks. Finally, we also showed that degree-degree correlations have a larger impact on the spreading dynamics when the coupled networks have similar levels of heterogeneity. It is also important to remark that the findings of this chapter motivated us to proceed our analysis focusing mainly on the spectral properties of our systems.

In fact, as observed and concluded in the previous chapters, the spectral properties of our systems play a major role in the dynamical outcome of our system. Indeed, it was already observed for many single-layer systems and also for many different dynamical processes, not only spreading dynamics (PASTOR-SATORRAS; VESPIGNANI, 2001b; PASTOR-SATORRAS; VESPIGNANI, 2001a; WANG et al., 2003; CHUNG; LU; VU, 2003; BOCCALETTI et al., 2006; BARRAT; BARTHLEMY; VESPIGNANI, 2008; GOLTSEV et al., 2012; KIVELÄ et al., 2014; BOCCALETTI et al., 2014). Generally, on spreading processes, the main mathematical object is the adjacency, or supra-adjacency, matrix. Thus, we devoted Chapter 6 to studying and numerically evaluating the spectral and eigenfunction properties of such matrix. Firstly, in Section 6.1 we extend the analysis we began in Chapter 5, evaluating the problems such as eigenvalue crossings and near crossings and the inverse participation ratio for identical systems and statistically equivalent systems. Followed by, still in the same section, a three-layer spectral analysis, where we showed the transition from the union of individual layer eigenvalues to the spectral distribution centered in the network of layers (a weighted version). Next, motivated by the observed dependency of dynamical processes and the spectral properties (eigenvalues and eigenvectors), we proceeded our analysis seeking for universal properties on the eigenfunctions of multilayers. This study was presented in Section 6.2, where we were able to demonstrate that the normalized localization length $\beta$ of the eigenfunctions of multilayer random networks scales as $x^{*} /\left(1+x^{*}\right)$. These findings are of special interest since we might be able to extend them for dynamical properties in future research. Finally, contrasting with the previous section of this chapter, where we proceeded a numerical evaluation, in Section 6.3 we focused on the analytical aspects of the spectral properties of multilayers. More specifically, we provided a new analytical interpretation for the 2-Layer multiplexes, exploring its block matricial nature. The results presented in Section 6.2 are still in the early phases, but they already present some new interesting insights for the interplay between different levels of complexity in the multilayers (layer structure and the network of layers).

The research presented in this thesis is not an end, but just the beginning of new prospects for single and multilayer theory, especially multilayer network since this theory is still in its infancy, as pointed out in 2014 in (KITSAK et al., 2010). First of all, regarding modeling, we open up new possibilities, allowing researchers to model information/disease spreading processes considering a series of new transitions, covering different types of human interactions. Note that we can also use the proposed model in order to infer the effects of a given transition on the population. These features would be of special interest in 
market campaign designs, for instance. On the other hand, regarding the interplay between structure and dynamics, most of our contributions lies on multilayers. Considering this structure, one of the main results of this thesis is the disease localization phenomena in multilayer networks. We showed some new interesting behavior in these kinds of systems, but also emphasizing the importance of different levels of complexity introduced in this structure (for instance, the network of layers and how it changes the spreading - the barrier effect). The observations made in this thesis show the importance of properly dealing with the multilayer structures, without neglecting them. Note that similar findings were already made in a series of papers (COZZO et al., 2013; KIVELÄ et al., 2014; BOCCALETTI et al., 2014; ARRUDA et al., 2016; ARRUDA et al., 2017) and here we corroborate that claim. However, we have proposed some new interesting ideas for this area, such as the layer-localization phenomena, a universality analysis for eigenfunctions and a new analytic tool on multilayers. These contributions might be applied to other dynamical processes and also further applied on spreading processes. As open questions, we might consider a better understanding on the second susceptibility peak, the applications of the barrier effects on immunization techniques, the consideration of sparse and different numbers of individuals on each layer aiming for more precise and realistic models. Those, among other possibilities, are possible future explorations of the theory presented here.

Among our expectations, we hope to motivate other researchers to further study this field, which we believe is mathematically and computationally challenging, but also socially fundamental for modern mathematical epidemiology. Hence, it is also a socially relevant issue, which provided us with a source of motivation. 
ADAMIC, L. A.; GLANCE, N. The political blogosphere and the 2004 US election: divided they blog. In: ACM. Proceedings of the 3rd international workshop on Link discovery. [S.l.], 2005. p. 36-43. Citation on page 175.

AKEMANN, G.; BAIK, J.; Di Francesco, P. The Oxford Handbook of Random Matrix Theory. [S.1.]: Oxford University Press, 2015. (Oxford Handbooks in Mathematics Series). ISBN 9780198744191. Citation on page 227.

ANDERSSON, H.; BRITTON, T. Stochastic epidemic models and their statistical analysis. New York: Springer, 2000. (Lecture notes in statistics). Citations on pages 84, 85,95 e 96.

ANGEL, O.; FRIEDMAN, J.; HOORY, S. The Non-Backtracking Spectrum of the Universal Cover of a Graph. arXiv:0712.0192, 2007. Citation on page 72.

ARENAS, A.; DÍAZ-GUILERA, A.; KURTHS, J.; MORENO, Y.; ZHOU, C. Synchronization in complex networks. Physics Reports, v. 469, n. 3, p. 93-153, 2008. ISSN 0370-1573. Citation on page 68.

ARRUDA, G. F.; BARBIERI, A. L.; RODRÍGUEZ, P. M.; RODRIGUES, F. A.; MORENO, Y.; COSTA, L. da F. Role of centrality for the identification of influential spreaders in complex networks. Physical Review E, APS, v. 90, n. 3, p. 032812, 2014. Citations on pages 51, 60, 70, 71, 117, 127 e 162.

ARRUDA, G. F. de; COZZO, E.; MORENO, Y.; RODRIGUES, F. A. On degree-degree correlations in multilayer networks. Physica D: Nonlinear Phenomena, v. 323-324, p. 5-11, 2016. ISSN 0167-2789. Nonlinear Dynamics on Interconnected Networks. Citations on pages $51,115,200$ e 258.

ARRUDA, G. F. de; COZZO, E.; PEIXOTO, T. P.; RODRIGUES, F. A.; MOREnO, Y. Disease Localization in Multilayer Networks. Phys. Rev. X, American Physical Society, v. 7, p. 011014, Feb 2017. Citations on pages 51, 68, 71, 78, 104, 105, 228 e 258.

ARRUDA, G. F. de; LEBEnSZTAYN, E.; RODRIGUES, F. A.; RODRÍGUEZ, P. M. A process of rumour scotching on finite populations. Royal Society Open Science, The Royal Society, v. 2, n. 9, 2015. Citation on page 51.

ARRUDA, G. F. de; RODRIGUES, F. A.; RODRIGUEZ, P. M.; COZZO, E.; MORENO, Y. A general Markov chain approach for disease and rumour spreading in complex networks . Journal of Complex Networks, 2017. Citation on page 51.

BANDYOPADHYAY, J. N.; JALAN, S. Universality in complex networks: Random matrix analysis. Phys. Rev. E, American Physical Society, v. 76, p. 026109, Aug 2007. Citation on page 227. 
BARABÁSI, A.-L.; ALBERT, R. Emergence of scaling in random networks. Science, American Association for the Advancement of Science, v. 286, n. 5439, p. 509-512, 1999. Citations on pages 64 e 65 .

BARRAT, A.; BARTHLEMY, M.; VESPIGNANI, A. Dynamical processes on complex networks. [S.l.]: Cambridge University Press New York, NY, USA, 2008. Citations on pages 45, 56, 63, 66, 67, 84, 85, 89, 95, 96, 98, 99, 100, 145, 190 e 257.

BARTHÉLEMY, M. Crossover from scale-free to spatial networks. EPL (Europhysics Letters), v. 63, n. 6, p. 915, 2003. Citation on page 66.

. Spatial networks. Physics Reports, Elsevier, v. 499, n. 1, p. 1-101, 2011. Citations on pages 66 e 174.

BASS, H. THE IHARA-SELBERG ZETA FUNCTION OF A TREE LATTICE. International Journal of Mathematics, v. 03, n. 06, p. 717-797, 1992. Citation on page 72 .

BHATIA, R. Matrix analysis. [S.1.]: Springer Verlag, 1997. Citation on page 59.

BOCCALETTI, S.; BIANCONI, G.; CRIADO, R.; Del Genio, C. I.; GÓMEZGARDEÑES, J.; ROMANCE, M.; SENDINA-NADAL, I.; WANG, Z.; ZANIN, M. The structure and dynamics of multilayer networks. Physics Reports, Elsevier, v. 544, n. 1, p. 1-122, 2014. Citations on pages 45, 46, 47, 257 e 258.

BOCCALETTI, S.; LATORA, V.; MOREnO, Y.; CHAVEZ, M.; HWANG, D. Complex networks: Structure and dynamics. Physics Reports, Elsevier, v. 424, n. 4, p. 175-308, 2006. Citations on pages 45, 54, 55, 56, 63, 66, 67, 173 e 257.

BOEING, G. OSMnx: New methods for acquiring, constructing, analyzing, and visualizing complex street networks. Computers, Environment and Urban Systems, v. 65, p. 126-139, 2017. Citations on pages 15 e 58.

BOGUÑÁ, M.; CASTELlAnO, C.; PASTOR-SATORRAS, R. Nature of the Epidemic Threshold for the Susceptible-Infected-Susceptible Dynamics in Networks. Phys. Rev. Lett., American Physical Society, v. 111, p. 068701, Aug 2013. Citations on pages 141 e 142 .

BOGUÑÁ, M.; PASTOR-SATORRAS, R. Epidemic spreading in correlated complex networks. Phys. Rev. E, American Physical Society, v. 66, p. 047104, Oct 2002. Citations on pages 98 e 99 .

BOGUÑÁ, M.; PASTOR-SATORRAS, R.; VESPIGNANI, A. Absence of Epidemic Threshold in Scale-Free Networks with Degree Correlations. Phys. Rev. Lett., APS, v. 90, n. 2, p. 028701, Jan 2003. Citation on page 98.

BOLLOBÁS, B. Random graphs. [S.1.]: Cambridge Univ Pr, 2001. Citations on pages 63 e 64.

BORGE-HOLTHOEFER, J.; MELONI, S.; GONCALVES, B.; MORENO, Y. Emergence of Influential Spreaders in Modified Rumor Models. Journal of Statistical Physics, v. 151, n. 1-2, p. 383-393, Sep. 2012. Citations on pages 87, 88, 89, 90, 146, 148, 162, 169, 171 e 172 . 
BORGE-HOLTHOEFER, J.; MORENO, Y. Absence of influential spreaders in rumor dynamics. Physical Review E, APS, v. 85, n. 2, p. 026116, 2012. Citations on pages $87,88,117,146,173,174,178,180,183$ e 185.

BORGE-HOLTHOEFER, J.; RIVERO, A.; GARCÍA, I.; CAUHÉ, E.; FERRER, A.; FERRER, D.; FRANCOS, D.; IÑIGUEZ, D.; PÉREZ, M.; RUIZ, G.; SANZ, F.; SERRANO, F.; VIÑAS, C.; TARANCÓN, A.; MORENO, Y. Structural and dynamical patterns on online social networks: the Spanish May 15th movement as a case study. PloS one, v. 6, n. 8, p. e23883, 2011. Citations on pages 169 e 171.

BORGE-HOLTHOEFER, J.; RIVERO, A.; MORENO, Y. Locating privileged spreaders on an online social network. Phys. Rev. E, American Physical Society, v. 85, p. 066123, Jun 2012. Citation on page 169.

BRIN, S.; PAGE, L. The Anatomy of a Large-Scale Hypertextual Web Search Engine. In: Computer Networks and ISDN Systems. [S.l.]: Elsevier Science Publishers B. V., 1998. p. 107-117. Citation on page 58.

C. Stark, B.-J. Breitkreutz, T. Reguly, L. Boucher, A. Breitkreutz, and M. Tyers. Biogrid: a general repository for interaction datasets. Nucleic Acids Research, v. 34, p. 535-539, 2006. Citation on page 236.

CARDILlO, A.; GÓMEZ-GARDENES, J.; ZANIN, M.; ROMANCE, M.; PAPO, D.; POZO, F. del; BOCCALETTI, S. Emergence of network features from multiplexity. Scientific Reports, Nature Publishing Group, v. 3, 2013. Citations on pages 46, 213, 214 e 216.

CATANZARO, M.; BOGUÑÁ, M.; PASTOR-SATORRAS, R. Generation of uncorrelated random scale-free networks. Phys. Rev. E, American Physical Society, v. 71, p. 027103, Feb 2005. Citation on page 67.

CATOR, E.; Van Mieghem, P. Second-order mean-field susceptible-infected-susceptible epidemic threshold. Phys. Rev. E, American Physical Society, v. 85, p. 056111, May 2012. Citations on pages 106, 107 e 109.

Nodal infection in Markovian susceptible-infected-susceptible and susceptibleinfected-removed epidemics on networks are non-negatively correlated. Phys. Rev. E, American Physical Society, v. 89, p. 052802, May 2014. Citation on page 101.

CHATTERJEE, S.; DURRETT, R. Contact processes on random graphs with power law degree distributions have critical value 0. Ann. Probab., The Institute of Mathematical Statistics, v. 37, n. 6, p. 2332-2356, 11 2009. Available: <https://doi.org/10.1214/ 09-AOP471>. Citation on page 142.

CHEN, L.; SUN, J. Global stability and optimal control of an \{SIRS $\}$ epidemic model on heterogeneous networks. Physica A: Statistical Mechanics and its Applications, v. 410, n. 0, p. 196-204, 2014. Citation on page 161.

CHUNG, F. R. K.; LU, L.; VU, V. H. The Spectra of Random Graphs with Given Expected Degrees. Proceedings of the National Academy of Sciences, v. 100, n. 11, p. 6313-6318, 2003. Citations on pages 46, 69, 89, 103 e 257. 
COLIZZA, V.; FLAMMINI, A.; SERRANO, M. A.; VESPIGNANI, A. Detecting richclub ordering in complex networks. 2006. Citations on pages 213 e 214.

CORNFORTH, D.; GREEN, D. G.; NEWTH, D. Ordered asynchronous processes in multi-agent systems. Physica D: Nonlinear Phenomena, v. 204, n. 1, p. 70-82, 2005. ISSN 0167-2789. Citation on page 130.

COSTA, L.; RODRIGUES, F.; TRAVIESO, G.; BOAS, P. Characterization of complex networks: A survey of measurements. Advances in Physics, Taylor \& Francis, v. 56, n. 1, p. 167-242, 2007. ISSN 0001-8732. Citations on pages 45, 55, 63, 66 e 67.

COSTA, L. d. F.; Cesar Jr, R. Shape analysis and classification: theory and practice. [S.l.]: CRC Press, Inc., 2000. Citation on page 175.

COSTA, L. d. F.; Oliveira Jr, O. N.; TRAVIESO, G.; RODRIGUES, F. A.; Villas Boas, P. R.; ANTIQUEIRA, L.; VIANA, M. P.; Correa Rocha, L. E. Analyzing and modeling real-world phenomena with complex networks: a survey of applications. Advances in Physics, Taylor \& Francis, v. 60, n. 3, p. 329-412, 2011. Citations on pages 45 e 145.

COTA, W.; FERREIRA, S. C. Optimized Gillespie algorithms for the simulation of Markovian epidemic processes on large and heterogeneous networks. Computer Physics Communications, v. 219, n. Supplement C, p. 303-312, 2017. ISSN 0010-4655. Citation on page 121 .

COZZO, E.; ARRUDA, G. F.; RODRIGUES, F. A.; MORENO, Y. Interconnected Networks. In: _. [S.l.]: Springer International Publishing, 2016. chap. Multilayer Networks: Metrics and Spectral Properties, p. 17-35. Citations on pages 51, 74, 76, 77, 79, $116,221,227$ e 281.

COZZO, E.; BAÑOS, R. A.; MELONI, S.; MORENO, Y. Contact-based social contagion in multiplex networks. Phys. Rev. E, APS, v. 88, n. 5, p. 050801, Nov 2013. Citations on pages 47, 79, 104, 105, 115, 189, 195, 209, 217 e 258.

COZZO, E.; MORENO, Y. Characterization of multiple topological scales in multiplex networks through supra-Laplacian eigengaps. Phys. Rev. E, American Physical Society, v. 94 , p. 052318 , Nov 2016. Citation on page 228.

DALEY, D. J.; KENDALL, D. G. Epidemics and Rumours. Nature, v. 204, p. 1118, 1964. Citations on pages 86, 87, 88 e 149.

Stochastic rumours. J. Inst. Math. Appl., v. 1, p. 42-55, 1965. Citations on pages 86,87 e 88 .

Darabi Sahneh, F.; SCOGLIO, C.; Van Mieghem, P. Exact coupling threshold for structural transition reveals diversified behaviors in interconnected networks. Phys. Rev. E, American Physical Society, v. 92, p. 040801, Oct 2015. Citations on pages 243 e 244.

De Domenico, M.; PORTER, M. A.; ARENAS, A. MuxViz: a tool for multilayer analysis and visualization of networks. Journal of Complex Networks, Oxford University Press, p. cnu038, 2014. Citations on pages 24 e 212. 
De Domenico, M.; SOlÉ-RIBALTA, A.; COZZO, E.; KIVELÄ, M.; MORENO, Y.; PORTER, M. A.; GÓMEZ, S.; ARENAS, A. Mathematical Formulation of Multilayer Networks. Phys. Rev. X, American Physical Society, v. 3, p. 041022, Dec 2013. Citations on pages $21,47,77,78,79,105,162,170,171,189,209,210$ e 218.

DICKMAN, R.; VIDIGAL, R. Quasi-stationary distributions for stochastic processes with an absorbing state. Journal of Physics A: Mathematical and General, v. 35, n. 5, p. 1147, 2002. Citations on pages 125 e 126.

DORMAND, J.; PRINCE, P. A family of embedded Runge-Kutta formulae. Journal of Computational and Applied Mathematics, v. 6, n. 1, p. 19-26, 1980. ISSN 0377-0427. Citation on page 193.

DOUGHERTY, E. R. Book. An introduction to morphological image processing. [S.l.]: SPIE Optical Engineering Press Bellingham, Wash., USA, 1992. xii, 161 p. : p. ISBN 081940845. Citation on page 175.

EDEN, M. A Two-dimensional Growth Process. In: Proceedings of the Fourth Berkeley Symposium on Mathematical Statistics and Probability, Volume 4: Contributions to Biology and Problems of Medicine. Berkeley, Calif.: University of California Press, 1961. p. 223-239. Citation on page 89.

ERDÖS, P.; RÉNYI, A. On random graphs, I. Publicationes Mathematicae (Debrecen), v. 6, p. 290-297, 1959. Citation on page 63.

ESTRADA, E.; HATANO, N. Communicability in complex networks. Physical Review E, APS, v. 77, n. 3, p. 036111, 2008. Citation on page 60.

ESTRADA, E.; HATANO, N.; BENZI, M. The physics of communicability in complex networks. Physics Reports, Elsevier, v. 514, n. 3, p. 89-119, 2012. Citations on pages 60 e 61.

EVANGELOU, S. N.; ECONOMOU, E. N. Eigenvector statistics and multifractal scaling of band random matrices. Phys. Lett. A, v. 151, 1990. Citation on page 229.

FENNELL, P. G.; MELNIK, S.; GLEESON, J. P. Limitations of discrete-time approaches to continuous-time contagion dynamics. Phys. Rev. E, American Physical Society, v. 94, p. 052125, Nov 2016. Citation on page 120.

FERREIRA, S. C.; CASTELLANO, C.; PASTOR-SATORRAS, R. Epidemic thresholds of the susceptible-infected-susceptible model on networks: A comparison of numerical and theoretical results. Phys. Rev. E, American Physical Society, v. 86, p. 041125, Oct 2012. Citations on pages 47, 101, 121, 125, 126, 127, 129, 131, 137, 138, 139, 140, 141 e 192.

FERREIRA, S. C.; SANDER, R. S.; PASTOR-SATORRAS, R. Collective versus hub activation of epidemic phases on networks. Phys. Rev. E, American Physical Society, v. 93, p. 032314, Mar 2016. Citation on page 142.

FORTUNATO, S. Community detection in graphs. Physics Reports, v. 486, n. 3, p. 75-174, 2010. ISSN 0370-1573. Citations on pages 68 e 70.

FYODOROV, Y. F.; MIRLIN, A. D. Scaling properties of localization in random band matrices: A $\sigma$-model approach. Phys. Rev. Lett., v. 67, 1991. Citation on page 229. 
Analytical derivation of the scaling law for the inverse participation ratio in quasione-dimensional disordered systems. Phys. Rev. Lett., v. 69, 1992. Citations on pages 229 e 231.

. Level-to-level fluctuations of the inverse participation ratio in finite quasi 1D disordered systems. Phys. Rev. Lett., v. 71, 1993. Citation on page 229.

Statistical properties of eigenfunctions of random quasi 1D one-particle Hamiltonians. Int. J. Mod. Phys. B, v. 8, 1994. Citation on page 229.

G. Casati, F. M. I.; MOLINARI, L. Scaling properties of the eigenvalue spacing distribution for band random matrices. J. Phys. A: Math. Gen., v. 24, 1991. Citation on page 229 .

G. Casati, I. G.; MASPERO, G. Landauer and Thouless conductance: A band random matrix approach. J. Phys. I (France), v. 7, 1997. Citation on page 229.

G. Casati, L. M.; IZRAILEV, F. M. Scaling properties of band random matrices. Phys. Rev. Lett., v. 64, 1990. Citations on pages 229 e 231.

G. Menichetti, D. R. P. P. R. J. M.; BIANCONI, G. Weighted multiplex networks. PLoS ONE, v. 9, 2014. Citation on page 230.

G. Zhu, H. Y. C. Y.; LI, B. Localizations on complex networks. Phys. Rev. E, v. 77, 2008. Citation on page 230.

GILLESPIE, D. T. Exact stochastic simulation of coupled chemical reactions. The Journal of Physical Chemistry, v. 81, n. 25, p. 2340-2361, 1977. Citation on page 121.

GIRVAN, M.; NEWMAN, M. Community structure in social and biological networks. Proceedings of the National Academy of Sciences, National Acad Sciences, v. 99, n. 12, p. 7821, 2002. Citation on page 56.

GLEESON, J. P.; MELNIK, S.; WARD, J. A.; PORTER, M. A.; MUCHA, P. J. Accuracy of mean-field theory for dynamics on real-world networks. Phys. Rev. E, American Physical Society, v. 85, p. 026106, Feb 2012. Citations on pages 56, 131, 167, 168 e 171.

GLEESON, J. P.; O’SUlLIVAN, K. P.; BAÑOS, R. A.; MORENO, Y. Effects of Network Structure, Competition and Memory Time on Social Spreading Phenomena. Phys. Rev. X, American Physical Society, v. 6, p. 021019, May 2016. Citation on page 83.

GOHBERG, I.; LANCASTER, P.; RODMAN, L. Matrix polynomials. [S.l.]: Siam, 1982. Citations on pages 222 e 240.

GOLTSEV, A. V.; DOROGOVTSEV, S. N.; OLIVEIRA, J. G.; MENDES, J. F. F. Localization and Spreading of Diseases in Complex Networks. Phys. Rev. Lett., American Physical Society, v. 109, p. 128702, Sep 2012. Citations on pages 47, 104, 105, 141, 142, 189, 190, 192, 193, 219, 228 e 257.

GOLUB, G. H.; LOAN, C. F. V. Matrix Computations. 3rd. ed. [S.l.]: The Johns Hopkins University Press, 1996. Citation on page 279.

GÓMEZ-GARDEÑES, J.; GÓMEZ, S.; ARENAS, A.; MORENO, Y. Explosive synchronization transitions in scale-free networks. Physical Review Letters, APS, v. 106, n. 12, p. 128701, 2011. Citation on page 273. 
GÓMEZ, S.; ARENAS, a.; BORGE-HOLTHOEFER, J.; MELONI, S.; MORENO, Y. Discrete-time Markov chain approach to contact-based disease spreading in complex networks. EPL (Europhysics Letters), IOP Publishing, v. 89, n. 3, p. 38009, Feb. 2010. ISSN 0295-5075. Citations on pages 68, 71, 113, 115, 160 e 161.

GOMEZ, S.; DIAZ-GUILERA, A.; GOMEZ-GARDEÑES, J.; PEREZ-VICENTE, C. J.; MOREnO, Y.; ARENAS, A. Diffusion Dynamics on Multiplex Networks. Phys. Rev. Lett., APS, v. 110, n. 2, p. 028701, Jan 2013. Citations on pages 47 e 228.

GONG, L.; TONG, P. von Neumann entropy and localization-delocalization transition of electron states in quantum small-world networks. Phys. Rev. E, v. 74, 2006. Citation on page 230 .

González-Bailón Sandra; Borge-Holthoefer Javier; Rivero Alejandro; Moreno Yamir. The Dynamics of Protest Recruitment through an Online Network. Sci. Rep., Macmillan Publishers Limited. All rights reserved, v. 1, dec 2011. Citations on pages 87, 88, 162 e 169.

GUIMERA, R.; DANON, L.; DÍAZ-GUILERA, A.; GIRALT, F.; ARENAS, A. Selfsimilar community structure in a network of human interactions. Physical Review E, v. 68, p. 065103, 2003. Citations on pages 169 e 175.

HASHIMOTO, K. Zeta functions of finite graphs and representations of p-adic groups. Advanced Studies in Pure Mathematics, v. 15, p. 211-280, 1989. Citation on page 72 .

HETHCOTE, H. W. The mathematics of infectious diseases. SIAM Review, SIAM, v. 42 , n. 4 , p. 599-653, 2000. Citation on page 145.

HIGHAM, N. J. The Scaling and Squaring Method for the Matrix Exponential Revisited. SIAM J. Matrix Analysis Applications, v. 26, n. 4, p. 1179-1193, 2005. Citation on page 279 .

HILL, M. O. Diversity and evenness: a unifying notation and its consequences. Ecology, Eco Soc America, v. 54, n. 2, p. 427-432, 1973. Citations on pages 60, 61 e 187.

HOLME, P.; KIM, B. J. Growing scale-free networks with tunable clustering. Phys. Rev. E, American Physical Society, v. 65, p. 026107, Jan 2002. Citation on page 133.

IZRAILEV, F. M. Simple models of quantum chaos: Spectrum and eigenfunctions. Phys. Rep., v. 196, 1990. Citation on page 230.

. Scaling properties of spectra and eigenfunctions for quantum dynamical and disordered systems. v. 5, 1995. Citation on page 229.

J. A. Mendez-Bermudez, A. A.-L. A. J. M.-M. F. A. R.; PERON, T. K. D. Universality in the spectral and eigenfunction properties of random networks. Phys. Rev. E, v. 91, 2015. Citations on pages 227 e 230.

J. F. Padgett, C. K. A. Robust Action and the Rise of the Medici, 1400-1434. American Journal of Sociology, p. 1259-1319, 1993. Citation on page 236.

JOST, L. Entropy and diversity. Oikos, Wiley Online Library, v. 113, n. 2, p. 363-375, 2006. Citations on pages 60 e 61. 
KAPFERER, B. Strategy and transaction in an African factory. 1972. Citation on page 236.

KARRER, B.; NEWMAN, M. E. J. Message passing approach for general epidemic models. Phys. Rev. E, American Physical Society, v. 82, p. 016101, Jul 2010. Citation on page 111.

KAWACHI, K.; SEKI, M.; YOSHIDA, H.; OTAKE, Y.; WARASHINA, K.; UEDA, H. A rumor transmission model with various contact interactions. Journal of Theoretical Biology, v. 253, n. 1, p. 55-60, 2008. Citations on pages 89, 90 e 146.

KIRKPATRICK, S. Optimization by simulated annealing: Quantitative studies. Journal of statistical physics, Springer, v. 34, n. 5-6, p. 975-986, 1984. Citation on page 62.

KISS, I. Z.; GREEN, D. M.; KAO, R. R. The effect of network mixing patterns on epidemic dynamics and the efficacy of disease contact tracing. Journal of The Royal Society Interface, The Royal Society, v. 5, n. 24, p. 791-799, 2008. Citation on page 218.

KITSAK, M.; GALLOS, L.; HAVLIN, S.; LILJEROS, F.; MUCHNIK, L.; STANLEY, H.; MAKSE, H. Identification of influential spreaders in complex networks. Nature Physics, Nature Publishing Group, v. 6, n. 11, p. 888-893, 2010. Citations on pages 57, 87, 146, $173,174,178,180,183,256$ e 257.

KIVELÄ, M.; ARENAS, A.; BARThELEMY, M.; GLEESON, J. P.; MORENO, Y.; PORTER, M. A. Multilayer networks. Journal of Complex Networks, Oxford University Press, v. 2, n. 3, p. 203-271, 2014. Citations on pages 45, 46, 47, 50, 73, 74, 75, 78, 189, 190, 212, 227, 257 e 258.

KONECT. Advogato network dataset. 2014. Available: <http://konect.uni-koblenz. de/networks/advogato $>$. Citation on page 175 .

Google+ network dataset. 2014. Available: <http://konect.uni-koblenz.de/ networks/ego-gplus>. Citation on page 175 .

KRACKHARDT, D. Cognitive social structures. Social Networks, v. 9, p. 104-134, 1987. Citation on page 236.

KRZAKAlA, F.; MOORE, C.; MOSSEL, E.; NEEMAN, J.; SLY, A.; ZDEBOROVÁ, L.; ZHANG, P. Spectral redemption in clustering sparse networks. Proceedings of the National Academy of Sciences, v. 110, n. 52, p. 20935-20940, 2013. Citations on pages 68 e 72 .

LANCASTER, P. Lambda-Matrices and Vibrating Systems. [S.l.]: Pergamon Press Inc., 1966. Citations on pages 222, 240 e 241.

LAZEGA. Oxford University Press, 2001. The Collegial Phenomenon: The Social Mechanisms of Cooperation Among Peers in a Corporate Law Partnership. Citation on page 236.

LEBENSZTAYN, E.; MACHADO, F.; RODRÍGUEZ, P. Limit Theorems for a General Stochastic Rumour Model. SIAM Journal on Applied Mathematics, v. 71, n. 4, p. 1476-1486, 2011. Citations on pages 87, 88 e 97. 
LEE, H. K.; SHIM, P.-S.; NOH, J. D. Epidemic threshold of the susceptible-infectedsusceptible model on complex networks. Phys. Rev. E, American Physical Society, v. 87, p. 062812, Jun 2013. Citations on pages 141 e 142.

LEUNG, C. C.; CHAU, H. F. Weighted assortative and disassortative networks model. Physica A: Statistical Mechanics and its Applications, v. 378, n. 2, p. 591-602, May 2007. Citations on pages 190, 210 e 213.

LEVEQUE, R. J. Finite difference methods for ordinary and partial differential equations - steady-state and time-dependent problems. [S.l.]: SIAM, 2007. I-XV, 1-341 p. ISBN 978-0-89871-629-0. Citation on page 276.

LI, W.; LIU, C.-C.; ZHANG, T.; LI, H.; WATERMAN, M. S.; ZHOU, X. J. Integrative analysis of many weighted co-expression networks using tensor computation. PLoS computational biology, Public Library of Science, v. 7, n. 6, p. e1001106, 2011. Citation on page 46 .

LITVAK, N.; HOFSTAD, R. van der. Uncovering disassortativity in large scale-free networks. Phys. Rev. E, American Physical Society, v. 87, p. 022801, Feb 2013. Citations on pages $62,190,210$ e 212 .

M. De Domenico, M. A. P.; ARENAS, A. MuxViz: A Tool for Multilayer Analysis and Visualization of Networks. J. Complex Netw., v. 3, p. 159-176, 2015. Citation on page 236.

MAKI, D. P.; THOMPSON, M. Mathematical models and applications. Englewood Cliffs, N.J.: Prentice-Hall Inc., 1973. Citations on pages 86, 87, 88, 97 e 149.

MASSA, P.; SALVETTI, M.; TOMASONI, D. Bowling Alone and Trust Decline in Social Network Sites. In: Proc. Int. Conf. Dependable, Autonomic and Secure Computing. [S.l.: s.n.], 2009. p. 658-663. Citation on page 175.

MATA, A. S.; FERREIRA, S. C. Multiple transitions of the susceptible-infectedsusceptible epidemic model on complex networks. Phys. Rev. E, American Physical Society, v. 91, p. 012816, Jan 2015. Citations on pages 121, 125, 126, 127, 138, 139, 140, 141, 192 e 198.

Mata, Angélica S.; Ferreira, Silvio C. Pair quenched mean-field theory for the susceptibleinfected-susceptible model on complex networks. EPL, v. 103, n. 4, p. 48003, 2013. Citations on pages $67,106,107,109,110,128,129,139,140$ e 141.

MCAUlEY, J.; LESKOVEC, J. Learning to Discover Social Circles in Ego Networks. In: Advances in Neural Information Processing Systems. [S.1.: s.n.], 2012. p. 548-556. Citation on page 175 .

MEILA, M.; SHI, J. Learning Segmentation by Random Walks. In: In Advances in Neural Information Processing Systems. [S.l.]: MIT Press, 2001. p. 873-879. Citations on pages 70 e 71 .

MENDEZ-BERMUDEZ, J. A.; ARRUDA, G. F. de; RODRIGUES, F. A.; MORENO, Y. Diluted banded random matrices: Scaling behavior of eigenfunction and spectral properties. Journal of Physics A: Mathematical and Theoretical, 2017. Citation on page 52. 
MÉNDEZ-BERMÚDEZ, J. A.; ARRUDA, G. F. de; RODRIGUES, F. A.; MORENO, Y. Scaling properties of multilayer random networks. Phys. Rev. E, American Physical Society, v. 96, p. 012307, Jul 2017. Citations on pages 51 e 52.

METHA, L. Random matrices. [S.l.]: Elsevier, Amsterdam, 2004. Citation on page 229.

MIEGHEM, P. V. Graph Spectra for Complex Networks. New York, NY, USA: Cambridge University Press, 2011. ISBN 9780521194587. Citations on pages 158 e 278.

MIEGHEM, P. V.; OMIC, J.; KOOIJ, R. Virus spread in networks. IEEE/ACM Trans. Netw., v. 17, n. 1, p. 1-14, 2009. Citations on pages 93, 101, 102, 103, 119, 133 e 189.

MIRLIN, A. D.; FYODOROV, Y. F. The statistics of eigenvector components of random band matrices: Analytical results. J. Phys. A: Math. Gen., v. 26, 1993. Citation on page 229 .

MORENO, Y.; GÓMEZ, J. B.; PACHECO, A. F. Epidemic incidence in correlated complex networks. Phys. Rev. E, American Physical Society, v. 68, p. 035103, Sep 2003. Citation on page 117 .

MOREnO, Y.; NEKOVEe, M.; PACHECO, A. F. Dynamics of Rumor Spreading in Complex Networks. Phys. Rev. E, v. 69, p. 066130, 2004. Citations on pages 88 e 117.

NEKOVEE, M.; MORENO, Y.; BIANCONI, G.; MARSILI, M. Theory of rumour spreading in complex social networks. Physica A: Statistical Mechanics and its Applications, v. 374, n. 1, p. 457-470, Jan. 2007. Citations on pages 88, 146, 159 e 162.

NEWMAN, M. Networks: an introduction. [S.1.]: Oxford University Press, Inc., 2010. Citations on pages 45, 56, 57, 63, 66, 69, 70, 84, 89, 145, 173 e 190.

NEWMAN, M. E. J. Assortative Mixing in Networks. Phys. Rev. Lett., APS, v. 89, n. 20, p. 208701, Oct 2002. ISSN 1079-7114. Citations on pages 45, 62, 66, 190, 210, 212 e 213 .

. Random Graphs with Clustering. Phys. Rev. Lett., American Physical Society, v. 103, p. 058701, Jul 2009. Citation on page 67.

NEWMAN, M. E. J.; PARK, J. Why social networks are different from other types of networks. Physical Review E, American Physical Society, v. 68, p. 036122, 2003. Citations on pages 45, 63 e 64 .

NICOSIA, V.; LATORA, V. Measuring and modeling correlations in multiplex networks. Phys. Rev. E, American Physical Society, v. 92, p. 032805, Sep 2015. Citations on pages 190 e 212 .

PASSERINI, F.; SEVERINI, S. The von Neumann entropy of networks. 2012. Citation on page 230 .

PASTOR-SATORRAS, R.; CASTEllanO, C.; Van Mieghem, P.; VESPIGNANi, A. Epidemic processes in complex networks. Rev. Mod. Phys., v. 87, n. 3, p. 925-979, Aug. 2015. Citations on pages 48, 83, 84, 85, 95, 96, 98, 100, 102, 103, 143, 145, 152 e 200 . 
PASTOR-SATORRAS, R.; VESPIGNANI, A. Epidemic dynamics and endemic states in complex networks. Phys. Rev. E, American Physical Society, v. 63, p. 066117, May 2001. Citations on pages 46, 100, 117, 156 e 257.

Epidemic Spreading in Scale-Free Networks. Phys. Rev. Lett., APS, v. 86, n. 14, p. 3200-3203, Apr 2001. Citations on pages 46, 98, 99, 100, 117 e 257.

PERON, T. K. D.; RODRIGUES, F. A. Explosive synchronization enhanced by timedelayed coupling. Physical Review E, APS, v. 86, n. 1, p. 016102, 2012. Citation on page 273 .

PINTO, P. C.; THIRAN, P.; VETTERLI, M. Locating the source of diffusion in largescale networks. Physical Review Letters, APS, v. 109, n. 6, p. 068702, 2012. Citation on page 187.

RADICCHI, F.; ARENAS, A. Abrupt transition in the structural formation of interconnected networks. Nature Physics, Nature Publishing Group, n. 11, p. 717-720, nov 2013. Citations on pages 27, 47, 227, 242, 243 e 244.

RADICCHI, F.; CASTELLANO, C. Leveraging percolation theory to single out influential spreaders in networks. Phys. Rev. E, American Physical Society, v. 93, p. 062314, Jun 2016. Citations on pages 111 e 141.

S. Jalan, N. S. G. V.; LI, B. Random matrix analysis of localization properties of gene coexpression network. Phys. Rev. E, v. 81, 2010. Citation on page 230.

SÁNCHEZ-GARCÍA, R. J.; COZZO, E.; MORENO, Y. Dimensionality reduction and spectral properties of multilayer networks. Phys. Rev. E, American Physical Society, v. 89, p. 052815, May 2014. Citations on pages 76, 77, 78, 79, 80, 116, 228 e 281.

SANDER, R. S.; COSTA, G. S.; FERREIRA, S. C. Sampling methods for the quasistationary regime of epidemic processes on regular and complex networks. Phys. Rev. E, American Physical Society, v. 94, p. 042308, Oct 2016. Citation on page 121.

Sampling methods for the quasistationary regime of epidemic processes on regular and complex networks. Phys. Rev. E, American Physical Society, v. 94, p. 042308, Oct 2016. Citation on page 139.

SEIDMAN, S. Network structure and minimum degree. Social networks, Elsevier, v. 5, n. 3, p. 269-287, 1983. Citations on pages 57 e 173.

SHI, J.; MALIK, J. Normalized Cuts and Image Segmentation. IEEE Trans. Pattern Anal. Mach. Intell., IEEE Computer Society, Washington, DC, USA, v. 22, n. 8, p. 888-905, Aug. 2000. ISSN 0162-8828. Citations on pages 70 e 71.

SHRESTHA, M.; SCARPINO, S. V.; MOORE, C. Message-passing approach for recurrent-state epidemic models on networks. Phys. Rev. E, American Physical Society, v. 92, p. 022821, Aug 2015. Citations on pages 68, 107, 109, 111 e 112.

SILVA, R. A. P. da; VIANA, M. P.; COSTA, L. da F. Predicting epidemic outbreak from individual features of the spreaders. Journal of Statistical Mechanics: Theory and Experiment, IOP Publishing, v. 2012, n. 07, p. P07005, 2012. Citations on pages 173 e 174 . 
SILVESTROV, P. G. Summing graphs for random band matrices. Phys. Rev. E, v. 55, 1997. Citation on page 229.

STANOEV, A.; DANIEL, T.; LJUPCO, K. Modeling the Spread of Multiple Concurrent Contagions on Networks. PLoS ONE, Public Library of Science, v. 9, n. 6, p. e95669, 06 2014. Citation on page 152 .

T. Kottos, A. P.; IZRAILEV, F. M. Finite-size corrections to Lyapunov spectra for band random matrices. J. Phys.: Condens. Matter, v. 10, 1998. Citation on page 229.

T. Kottos, A. P. F. M. I.; RUFFO, S. Scaling properties of Lyapunov spectra for the band random matrix model. Phys. Rev. E, v. 53, 1996. Citation on page 229.

T. Kottos, F. M. I.; POLITI, A. Finite-length Lyapunov exponents and conductance for quasi-1D disordered solids. Physica D, v. 131, 1999. Citation on page 229.

TELlER, S.; CLARA, G.; MANLIO, D. D.; JORDI, S.; SERGIO, G.; ALEX, A. Emergence of Assortative Mixing between Clusters of Cultured Neurons. PLOS Computational Biology, Public Library of Science, v. 10, n. 9, p. 1-17, 09 2014. Citation on page 210 .

TISSEUR, F.; MEERBERGEN, K. The Quadratic Eigenvalue Problem. SIAM Review, v. 43, n. 2, p. 235-286, 2001. Citations on pages 222 e 240.

TRAVENCOLO, B.; VIANA, M.; COSTA, L. d. F. Border detection in complex networks. New Journal of Physics, IOP Publishing, v. 11, p. 063019, 2009. Citations on pages 59, 70, 71 e 182 .

TRAVEnCOLO, B. A. N.; F. Costa, L. da. Accessibility in complex networks. Physics Letters A, Elsevier, v. 373, n. 1, p. 89-95, 2008. Citations on pages 59, 70, 71 e 187.

Van Mieghem, P. Epidemic phase transition of the SIS type in networks. EPL, v. 97, n. 4, p. 48004, 2012. Citations on pages 103, 104, 192, 193 e 195.

Van Mieghem, P. Performance Analysis of Complex Networks and Systems. [S.1.]: Cambridge University Press, 2014. Citations on pages 90, 91, 93, 94 e 119.

- Approximate formula and bounds for the time-varying susceptible-infectedsusceptible prevalence in networks. Phys. Rev. E, American Physical Society, v. 93, p. 052312, May 2016. Citations on pages 91 e 92.

Van Mieghem, P.; CATOR, E. Epidemics in networks with nodal self-infection and the epidemic threshold. Phys. Rev. E, American Physical Society, v. 86, p. 016116, Jul 2012. Citation on page 201.

VERBRUGGE, L. M. Multiplexity in adult friendships. Social Forces, Oxford University Press, v. 57, n. 4, p. 1286-1309, 1979. Citation on page 45.

VICKERS, M.; CHAN, S. Representing Classroom Social Structure. Melbourne: Victoria Institute of Secondary Education. 1981. Citation on page 236.

VIGER, F.; LATAPY, M. Efficient and Simple Generation of Random Simple Connected Graphs with Prescribed Degree Sequence. In: Proceedings of the 11th Annual International Conference on Computing and Combinatorics. Berlin, Heidelberg: Springer-Verlag, 2005. (COCOON'05), p. 440-449. Citations on pages 194 e 217. 
VOJTA, T. Rare region effects at classical, quantum and nonequilibrium phase transitions. Journal of Physics A: Mathematical and General, v. 39, n. 22, p. R143, 2006. Citation on page 141.

WANG, W. Localization in band random matrix models with and without increasing diagonal elements. Phys. Rev. E, v. 65, 2002. Citation on page 229.

WANG, W.; TANG, M.; STANLEY, H. E.; BRAUNSTEIN, L. A. Unification of theoretical approaches for epidemic spreading on complex networks. Reports on Progress in Physics, v. 80, n. 3, p. 036603, 2017. Citations on pages 104, 111, 112 e 190.

Wang Wei; Liu Quan-Hui; Zhong Lin-Feng; Tang Ming; Gao Hui; Stanley H. Eugene. Predicting the epidemic threshold of the susceptible-infected-recovered model. The Author(s), v. 6, p. 24676, apr 2016. Citation on page 111.

WANG, Y.; CHAKRABARTI, D.; WANG, C.; FALOUTSOS, C. Epidemic Spreading in Real Networks: An Eigenvalue Viewpoint. In: In SRDS. [S.l.: s.n.], 2003. p. 25-34. Citations on pages 46 e 257.

WATTS, D. Small Worlds: The Dynamics of Networks Between Order and Randomness. [S.l.]: Princeton University Press, 1999. Citations on pages 45 e 66.

WAXMAN, B. Routing of multipoint connections. Selected Areas in Communications, IEEE Journal on, IEEE, v. 6, n. 9, p. 1617-1622, 1988. Citation on page 65.

WHO. Ebola virus disease - Democratic Republic of the Congo. 2017. Available: <http://www.who.int/csr/don/13-may-2017-ebola-drc/en/>. Citation on page 48.

External review of pandemic response. 2017. Available: $<$ http://www.who. int/ihr/review_committee/en/>. Citation on page 48.

WIGNER, E. P. On the Distribution of the Roots of Certain Symmetric Matrices. Annals of Mathematics, Annals of Mathematics, v. 67, n. 2, p. 325-327, 1958. Citation on page 69.

WOLFE, D. A.; HOLLANDER, M. Nonparametric statistical methods. Nonparametric statistical methods, John Wiley New York, 1973. Citation on page 179.

ZACHARY, W. An Information Flow Modelfor Conflict and Fission in Small Groups1. Journal of anthropological research, v. 33, n. 4, p. 452-473, 1977. Citations on pages 15,60 e 61. 

APPENDIX

\section{A}

GENERALIZED RANDOM WALK ACCESSIBILITY: ADDITIONAL COMMENTS

Let us provide in what follows some exact expression for the metric just introduced. Although the graphs considered below are not representatives of real world networks, the analysis helps understanding what can be learned from the new metric. In addition, there are structures that already capture some important features of real networks, such as the star graph, which is an extreme example of an heterogeneous configuration but that have provided insightful hints about the dynamics under study in other cases (GÓMEZGARDEÑES et al., 2011; PERON; RODRIGUES, 2012).

\section{A.1 Accessibility in star graphs}

\section{A.1.1 First analytical approach}

For a star graph, the probability of transition between the central node $i$ and any of the $k$ leaves considering an ARW is given by (see Eqs. 2.10 and 2.11)

$$
\mathbf{P}(i, j)=\frac{1}{e k} \sum_{n=0}^{\infty} \frac{1}{(2 n+1) !}=\frac{1}{e k} \sinh (1), \quad i \neq j
$$

and between the leaves and central node $i$,

$$
\mathbf{P}(j, i)=\frac{\sinh (1)}{e} .
$$

In addition,

$$
\mathbf{P}(i, i)=\frac{\cosh (1)}{e}
$$

The probability of transition between leaves $j$ and $l$ is given by

$$
\mathbf{P}(j, l)=\frac{1}{e k}(\cosh (1)-1),
$$


and for $l=j$,

$$
\mathbf{P}(j, j)=\frac{1}{e}+\frac{1}{e k}(\cosh (1)-1)
$$

Therefore, the general form of the exponential matrix, considering the node number one as the hub of the star graph, is given as

$$
\mathbf{P}=\frac{1}{e}\left[\begin{array}{ccccc}
\cosh (1) & \frac{1}{k} \sinh (1) & \ldots & \ldots & \frac{1}{k} \sinh (1) \\
\sinh (1) & 1+\frac{1}{k}(\cosh (1)-1) & \frac{1}{k}(\cosh (1)-1) & \ldots & \frac{1}{k}(\cosh (1)-1) \\
\vdots & \frac{1}{k}(\cosh (1)-1) & \ddots & & \vdots \\
\vdots & \vdots & & \ddots & \frac{1}{k}(\cosh (1)-1) \\
\sinh (1) & \frac{1}{k}(\cosh (1)-1) & \ldots & \frac{1}{k}(\cosh (1)-1) & 1+\frac{1}{k}(\cosh (1)-1)
\end{array}\right]
$$

In this way, since $k=N-1$, the accessibility of the hub $i$ is

$$
\alpha(i)=\exp \left\{-x \log (x)-y \log \left(\frac{y}{N-1}\right)\right\},
$$

where $x=\frac{\cosh (1)}{e}$ and $y=\frac{\sinh (1)}{e}$. For any leaf $j$ connected with $i$,

$$
\begin{array}{r}
\alpha(j)=\exp \{-x \log (x)+(N-2) y \log (y)+ \\
(1 / e+y) \log (1 / e+y)\}
\end{array}
$$

where $x=\frac{\sinh (1)}{e}, y=\frac{(\cosh (1)-1)}{e(N-1)}$.

We show in Fig. 80 the results obtained for the accessibility on top of different networks and configurations. As it can be seen, Eq. A.7 can be considered to be a good predictor of the accessibility of the hubs in scale-free networks. However, as expected from the fact that the star graph does not capture any topological aspect of homogeneous networks, the star-graph approximation is not accurate for random Erdös-Rényi networks.

\section{A.1.2 Eigendecomposition analysis}

The exact values of accessibility in star graphs can also be calculated by the eigendecomposition analysis of $P$. The exponential matrix, Eq. 2.10, can be obtained as

$$
\mathbf{W}=e^{P}=\mathscr{V} \mathscr{D} \mathscr{V}^{-1}
$$

where $\mathscr{V}$ is a matrix whose columns are the eigenvectors of the matrix $\mathbf{W}$ and $\mathscr{D}$ is a matrix whose diagonal presents the exponential of each eigenvalue of $P$,

$$
(P-\lambda \mathbf{I}) v=0
$$

where $P$ is the transition matrix, $\lambda$ is its eigenvalue and $v$ is the associated eigenvector. 
Figure 80 - (Color online) Accessibility calculated in star (from Eqs. A.7 and A.8), complete, ring and line graphs (extreme nodes) compared to the maximum value obtained in Erdös-Rényi (ER) random graphs, scale-free networks of Barabási-Albert (BA), and spatial scale-free networks (SSF). $N$ is the network size. For complex networks, each point is an average over 50 networks with $\langle k\rangle \approx 4$.

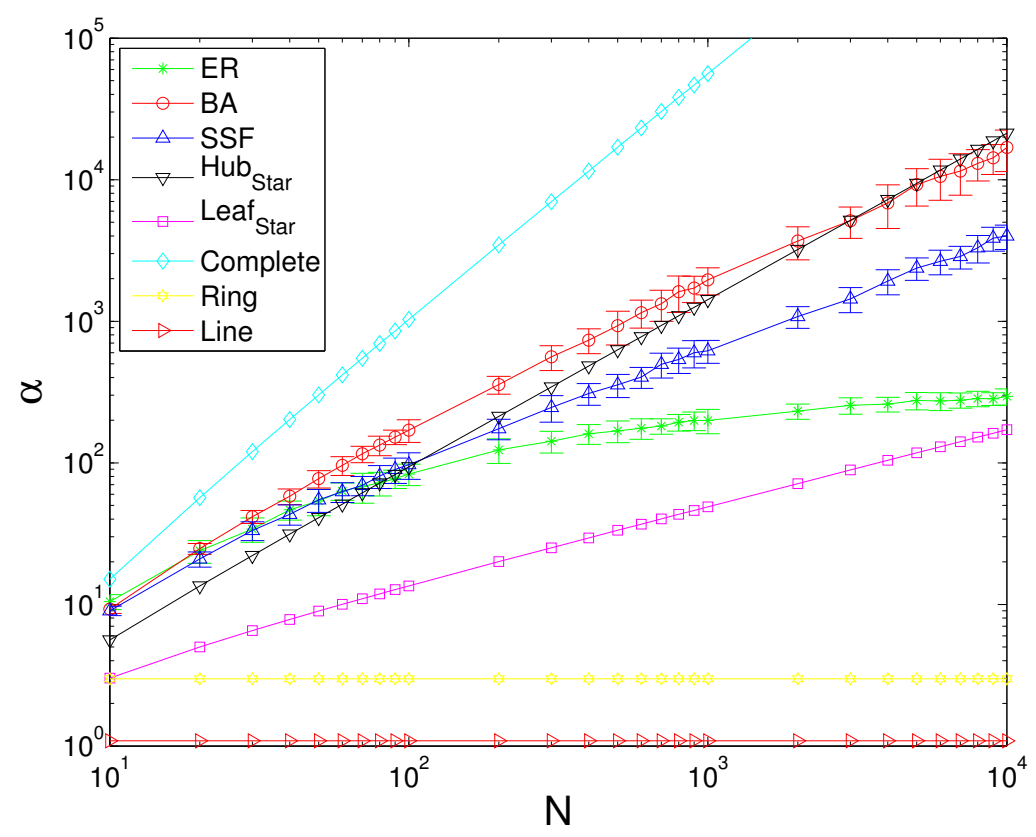

Source: Developed by the author.

In this way, for the star graph, the transition matrix is sparse and its characteristic polynomial, $\operatorname{det}(P-\lambda I)=0$, is calculated by the Laplace rule as

$$
\begin{array}{r}
\operatorname{det}(P-\lambda I)=(-\lambda)^{N}-(-\lambda)^{(N-2)}= \\
(-\lambda)^{(N-2)}\left((-\lambda)^{2}-1\right)=0,
\end{array}
$$

whose solutions are $\lambda_{1}=-1, \lambda_{2}=1$ and $\lambda_{i}=0, \forall i=2,3, \ldots, N$. Therefore, using the definition of an eigenvalue and eigenvector problem, it is possible to obtain the following equations for the eigenvectors. For $\lambda_{1}=-1$,

$$
\left\{\begin{array}{l}
v_{11}=-\frac{1}{N-1} \sum_{j=2}^{N} v_{1 j} \\
v_{1 j}=-v_{11} \quad j=2,3, \ldots, N
\end{array}\right.
$$

where $v_{p j}$ is the $j$-th element of the eigenvector $v_{p}$ associated with the eigenvalue $\lambda_{p}$. For $\lambda=1$,

$$
\left\{\begin{array}{l}
v_{21}=\frac{1}{N-1} \sum_{j=2}^{N} v_{2 j} \\
v_{2 j}=v_{21} \quad j=2,3, \ldots, N ;
\end{array}\right.
$$

finally, for $\lambda_{p}=0$ where $p=3, \ldots, N$, which has multiplicity $(N-2)$,

$$
\begin{cases}0= & \frac{1}{N-1} \sum_{j=2}^{N} v_{p j} \\ v_{p 1}= & 0\end{cases}
$$


which yields the matrix

$$
\mathscr{V}=\left[\begin{array}{cccccc}
-1 & 1 & 0 & \cdots & \cdots & 0 \\
1 & 1 & -1 & \cdots & \cdots & -1 \\
\vdots & \vdots & 1 & 0 & \cdots & 0 \\
\vdots & \vdots & 0 & \ddots & & \vdots \\
\vdots & \vdots & \vdots & & \ddots & 0 \\
1 & 1 & 0 & \cdots & 0 & 1
\end{array}\right]
$$

whose inverse is

$$
\mathscr{V}^{-1}=\left[\begin{array}{ccccc}
\frac{-1}{2} & \frac{1}{2(N-1)} & \frac{1}{2(N-1)} & \cdots & \frac{1}{2(N-1)} \\
\frac{1}{2} & \frac{1}{2(N-1)} & \frac{1}{2(N-1)} & \cdots & \frac{1}{2(N-1)} \\
0 & \frac{-1}{N-1} & \frac{N-2}{N-1} & & \frac{-1}{N-1} \\
\vdots & \vdots & & \ddots & \vdots \\
0 & \frac{-1}{N-1} & \cdots & & \frac{N-2}{N-1}
\end{array}\right] .
$$

Note that we used non-unit vectors to construct the matrices $\mathscr{V}$. This is not necessary since $\mathscr{D}$ is also multiplied by $\mathscr{V}^{-1}$ and the non-unit norms are compensated. Substituting in matrices A.15 and A.16 in Eq. A.9, after some algebra, we recover Eq. A.6. The accessibilty of hubs and leaves are calculated by Eqs. A.7 and A.8, respectively.

\section{A.2 Accessibility in ring graphs}

The generalized random walk accessibility can also be calculated exactly in rings, that are a special case of K-regular graphs, where $K=2$. The probability transition matrix has the form

$$
P=\left[\begin{array}{cccccc}
0 & \frac{1}{2} & 0 & \cdots & 0 & \frac{1}{2} \\
\frac{1}{2} & \ddots & \ddots & & & 0 \\
0 & \ddots & \ddots & \ddots & & \vdots \\
\vdots & & \ddots & \ddots & \ddots & \vdots \\
0 & & & \ddots & \ddots & \frac{1}{2} \\
\frac{1}{2} & 0 & \cdots & 0 & \frac{1}{2} & 0
\end{array}\right] .
$$

Such matrix has a well known spectra and is widely used in finite difference methods (LEVEQUE, 2007). As exposed in (LEVEQUE, 2007), the eigenvalues of $P$ are

$$
\lambda_{p}=\frac{1}{2}\left(\exp \left(\frac{2 \pi i p}{N}\right)+\exp \left(\frac{-2 \pi i p}{N}\right)\right)=\cos \left(\frac{2 \pi p}{N}\right)
$$

where $i=\sqrt{-1}$ and the associated elements of the eigenvector can be expressed as

$$
u_{p j}=\frac{\exp \left(\frac{2 \pi i p j}{N}\right)}{\sqrt{N}},
$$


where $\sqrt{N}$ is just a normalization factor. This set of eigenvectors diagonalizes the matrix $P$ as $P=U \Lambda U^{H}$, where $\Lambda$ is the diagonal matrix with the eigenvalues of $P$ (Eq. A.18), $U$ is the matrix whose columns are the eigenvector of $P$ and $U^{H}$ is the conjugate transpose of $U$. We can write the closed expression for $\mathbf{P}$ as

$$
\mathbf{P}(j, k)=\frac{1}{e} \sum_{p} \exp \left(\lambda_{p}\right) u_{p j} u_{p k}^{*}
$$

where $u_{p j}^{*}$ is the conjugate transpose of $u_{p j}$. Note that we used the complex domain to solve the problem, however the solution is on the real domain. Using Eqs. A.18 and A.19, we obtain

$$
\mathbf{P}(j, k)=\frac{1}{e N} \sum_{p=1}^{N} \exp \left(\cos \left(\frac{2 \pi p}{N}\right)\right) \exp \left(\frac{2 \pi i p(j-k)}{N}\right),
$$

which is a closed form for the evaluation of $\mathbf{P}$ in ring graphs. Furthermore we can use some graph spectra properties to separate the first eigenvalue from the summation

$$
\begin{aligned}
& \mathbf{P}(j, k)=\frac{1}{e}\left(\frac{k_{j}}{2 M}\right) \exp (1)+ \\
& \frac{1}{N} \sum_{p=2}^{N} \exp \left(\cos \left(\frac{2 \pi p}{N}\right)+\left(\frac{2 \pi i p(j-k)}{N}\right)\right) .
\end{aligned}
$$

Figure 80 shows the comparison between network models and the analytical solutions for the regular structures. Note that the solution for the ring does not depend on the network size. The results for the line graph, which again do not depend on the network size, are also presented in this figure. We also remark that the extremes of the line present the lowest values of accessibility, whereas the nodes in the center have the highest values.

\section{A.3 Accessibility in complete graphs}

The generalized random walk accessibility can also be calculated exactly for a complete graph, in which every pair of nodes is connected without self-connections. In this way, the probability of transition between any pair of nodes is $P(i, j)=\frac{1}{N-1}$ and the exponential matrix (see Eq. 2.10) is given by

$$
\begin{aligned}
\mathbf{P}(i, j) & =\frac{1}{e N} \sum_{n=0}^{\infty} \frac{(N-1)^{n}+(-1)^{n}(N-1)}{(N-1)^{n} n !}= \\
& \frac{\exp (1)+(N-1) \exp \left(\frac{-1}{N-1}\right)}{e N}, \quad i \neq j .
\end{aligned}
$$

The main diagonal of $\mathbf{P}$, which considers the paths starting and ending at the same node, is expressed as

$$
\mathbf{P}(i, i)=\frac{1}{e N} \sum_{n=0}^{\infty} \frac{(N-1)^{n}+(-1)^{n+1}}{(N-1)^{n} n !}=\frac{\exp (1)-\exp \left(\frac{-1}{N-1}\right)}{e N} .
$$


Therefore, the general form of the exponential matrix is given as

$$
\mathbf{P}=\frac{1}{e}\left[\begin{array}{cccc}
\frac{\exp (1)+(N-1) \exp \left(\frac{-1}{N-1}\right)}{N} & \frac{\exp (1)-\exp \left(\frac{-1}{N-1}\right)}{N} & \cdots & \frac{\exp (1)-\exp \left(\frac{-1}{N-1}\right)}{N} \\
\frac{\exp (1)-\exp \left(\frac{-1}{N-1}\right)}{N} & \ddots & & \vdots \\
\vdots & & \ddots & \vdots \\
\frac{\exp (1)-\exp \left(\frac{-1}{N-1}\right)}{N} & \cdots & \cdots & \frac{\exp (1)+(N-1) \exp \left(\frac{-1}{N-1}\right)}{N}
\end{array}\right]
$$

The accessibility of each node is

$$
\begin{array}{r}
\alpha(i)=\exp \{-\mathbf{P}(i, i) \log (\mathbf{P}(i, i))+ \\
-(N-1) \mathbf{P}(i, j) \log (\mathbf{P}(i, j))\}= \\
e\left(a^{-(N-1) a / e} b^{-b / e}\right),
\end{array}
$$

where

$$
a=\frac{1}{e N}\left(\exp (1)-\exp \left(\frac{-1}{N-1}\right)\right)
$$

and

$$
b=\frac{1}{e N}\left(\exp (1)+(N-1) \exp \left(\frac{-1}{N-1}\right)\right) .
$$

In the complete graph all nodes present the same value of accessibility and, since a random walker needs just one step to reach any other node, this value is the upper bound of the maximum value of accessibility for a network with $N$ nodes. Figure 80 shows the variation of the accessibility in complete graphs as a function of the network size.

\section{A.3.1 Eigendecomposition analysis}

The exact values of accessibility in complete graphs can also be obtained by the eigen-decomposition analysis, the graph spectra and its eigenvectors, as performed for the star graph. In this way, we get the following system (from Eq. A.10)

$$
\left[\begin{array}{cccc}
-\lambda & \frac{1}{N-1} & \cdots & \frac{1}{N-1} \\
\frac{1}{N-1} & \ddots & & \vdots \\
\vdots & & \ddots & \vdots \\
\frac{1}{N-1} & \cdots & \cdots & -\lambda
\end{array}\right] v=0
$$

which yields

$$
\sum_{i, i \neq j}^{N} v_{p i}\left(\frac{1}{N-1}\right)-\lambda_{p} v_{p j}=0, \quad \forall i, j ; i \neq j,
$$

where $v_{p j}$ is the $j$-th element of the eigenvector $v_{p}$ associated with the eigenvalue $\lambda_{p}$.

The eigenvalues of $P$ for a complete graph is the spectrum of the adjacency matrix multiplied by $\frac{1}{N-1}$, i.e., $\lambda_{1}=1, \lambda_{2}=\lambda_{3}=\ldots=\lambda_{N}=\frac{1}{N-1}$ (MIEGHEM, 2011). Therefore, for $\lambda_{1}=1$ we have

$$
(N-1) v_{1 j}=\sum_{i, i \neq j}^{N} v_{1 i}, \quad \forall i, j ; i \neq j .
$$


The solution is $v_{1 i}=v_{1 j}$. On the other hand, for $\lambda_{p}=\frac{-1}{N-1}$, where $p=2,3, \ldots, N$,

$$
\sum_{i, i \neq j}^{N} v_{p i}\left(\frac{1}{N-1}\right)+\frac{1}{N-1} v_{p j}=0
$$

i.e.,

$$
\left(\frac{1}{N-1}\right) \sum_{i}^{N} v_{p i}=0, \quad \forall i, j ; i \neq j
$$

The respective solution is $\sum_{i}^{N} v_{p i}=0$. Note that both solutions are not unique, whereas the Eq. A.9 has a unique solution. Without loss generality, we assume

$$
\mathscr{V}=\left[\begin{array}{ccccc}
1 & -1 & -1 & \cdots & -1 \\
1 & 1 & 0 & \cdots & 0 \\
\vdots & 0 & \ddots & & \vdots \\
\vdots & \vdots & & \ddots & \vdots \\
1 & 0 & \cdots & & 1
\end{array}\right]
$$

whose inverse is

$$
\mathscr{V}^{-1}=\left[\begin{array}{ccccc}
\frac{1}{N} & \frac{1}{N} & \frac{1}{N} & \cdots & \frac{1}{N} \\
\frac{-1}{N} & \frac{(N-1)}{N} & \frac{-1}{N} & \cdots & \frac{-1}{N} \\
\vdots & \frac{-1}{N} & \ddots & & \vdots \\
\vdots & \vdots & & \ddots & \vdots \\
\frac{-1}{N} & \frac{-1}{N} & \cdots & & \frac{(N-1)}{N}
\end{array}\right] .
$$

Substituting matrices $\mathscr{V}$ and $\mathscr{V}^{-1}$ in Eq. A.9 and using the information about the eigenvalues, we obtain the matrix in Eq. A.25 and the same expression for the accessibility (Eq. A.26).

As a practical comment about the matrix exponential, it is important to mention that it should be computed by the Padé approximation (GOLUB; LOAN, 1996; HIGHAM, 2005) and not by the truncated Taylor series or by Eqs. A.9. The former method is more precise and has a lower computational cost. However, Eq. A.9 is important for theoretical analysis, since it transforms the calculus of accessibility into a eigenvector and eigenvalue problem, which is well studied in the literature. 

APPENDIX

B

\section{INTERLACING PROPERTIES: PROOF OF EQUATION 2.50}

In this appendix we show the equivalence of the results presented in (SÁNCHEZGARCÍA; COZZO; MORENO, 2014; COZZO et al., 2016) (see also Chapter 2) and our normalized network of layers, Equation 2.50. Considering the matricial representation of a multilayer network, given by

$$
A=\oplus_{\alpha} A^{\alpha}+C=\left[\begin{array}{cccc}
A_{1} & C_{12} & \cdots & C_{1 m} \\
C_{21} & A_{2} & \cdots & C_{2 m} \\
\vdots & \vdots & \ddots & \vdots \\
C_{m 1} & C_{m 2} & \cdots & A_{m}
\end{array}\right]
$$

where $A \in \mathbb{R}^{n m \times n m}, A^{\alpha} \in \mathbb{R}^{n \times n}$ is the adjacency matrix of the layer $\alpha \in\{1,2, \ldots m\}$ and $C$ is a coupling matrix (see Chapter 2). Since we assume multilayer networks in which the layers have the same number of nodes we have $C_{i j}=I$. Assuming a partition of such network, represented by $S \in \mathbb{R}^{n m \times m}$, which is the characteristic matrix of such partition, where $S_{i j}=1$ if $i \in V_{j}$ and zero otherwise, where $V_{j}$ is the network of layers partition.

In order to use the results of (SÁNCHEZ-GARCÍA; COZZO; MORENO, 2014; COZZO et al., 2016) we have to prove that the network of layers matrix $\bar{R}$ (SÁNCHEZGARCÍA; COZZO; MORENO, 2014; COZZO et al., 2016) is an unfolding of our tensor $\Phi_{\tilde{\delta}}^{\tilde{\gamma}}(\lambda, \eta)$, formally given by

$$
\bar{R}=\Gamma^{-1} S^{T} A S,
$$

where $\Gamma$ is a diagonal matrix with normalizing constants (for more, see references (SÁNCHEZGARCÍA; COZZO; MORENO, 2014; COZZO et al., 2016)). In words, the product AS is a summation over the blocks of the matrix $A$, resulting in a matrix with the degree of each node. The subsequent left product with $S^{T}$ impose another summation, whose result is a matrix composed by the sum of all elements of the blocks. Finally, the product by 
$\Gamma^{-1}$ normalize the result by $\frac{1}{n}$. Formally we have,

$$
A S=\left[\begin{array}{cccc}
k^{11} & k^{12} & \cdots & k^{1 m} \\
k^{21} & k^{22} & \cdots & k^{2 m} \\
\vdots & \vdots & \ddots & \vdots \\
k^{m 1} & k^{m 2} & \cdots & k^{m m}
\end{array}\right]
$$

where $k^{i j} \in \mathbb{R}^{n \times 1}$ is a vector with the number of edges emanating from each node on layer $i$ to layer $j$ and $A S \in \mathbb{R}^{n m \times m}$. Then,

$$
S^{T} A S=\left[\begin{array}{cccc}
\sum k^{11} & \sum k^{12} & \cdots & \sum k^{1 m} \\
\sum k^{21} & \sum k^{22} & \cdots & \sum k^{2 m} \\
\vdots & \vdots & \ddots & \vdots \\
\sum k^{m 1} & \sum k^{m 2} & \cdots & \sum k^{m m}
\end{array}\right]
$$

where $\sum k^{i j} \in \mathbb{R}$ are scalars with the number of edges that connect a node on layer $i$ to a node on layer $j$. Finally, the product by $\Gamma^{-1}$ introduce the average degree instead of the summation, producing the same results as Equation 2.50. 
APPENDIX

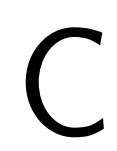

\section{FUNDAMENTALS OF SPREADING PROCESSES: COMPLEMENTARY COMMENTS}

\section{C.1 Formulation}

In tables 9 and 10 we present a summary of the mean field approximations discussed in Chapter 3. Here we focus on the approximations and its numerical approximations. We also focused on uncorrelated networks, assuming that the dynamical correlations are neglectable. Obviously, each mean field approach follows a different approximation. We might emphasize that, in Table 9 for the HMF equations one should consider $\mathbb{P}\left(k^{\prime} \mid k\right)=\frac{k^{\prime} \mathbb{P}\left(k^{\prime}\right)}{\mathbb{E}(k)}$ for uncorrelated networks. In order to solve them one might use a numerical approach, such as Runge-Kutta. For the sake of an example, see Figure 12 on the main text, where we compare the QMF and PQMF approaches with Monte Carlo approximations. 
Table 9 - Part I of the summary of mean field approximations for the SIS, SIR and MT processes.

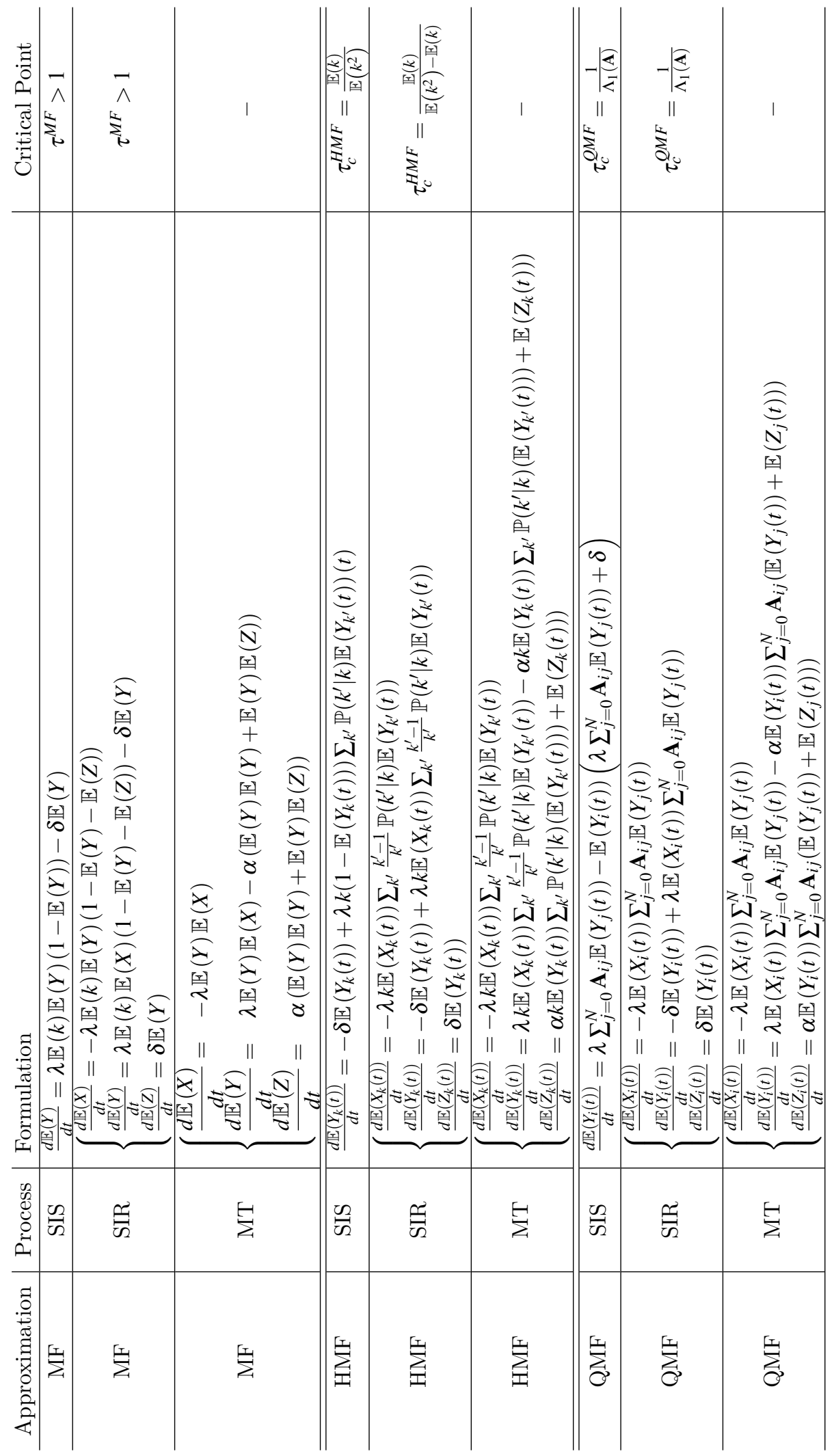


Table 10 - Part II of the summary of mean field approximations for the SIS, SIR and MT processes.

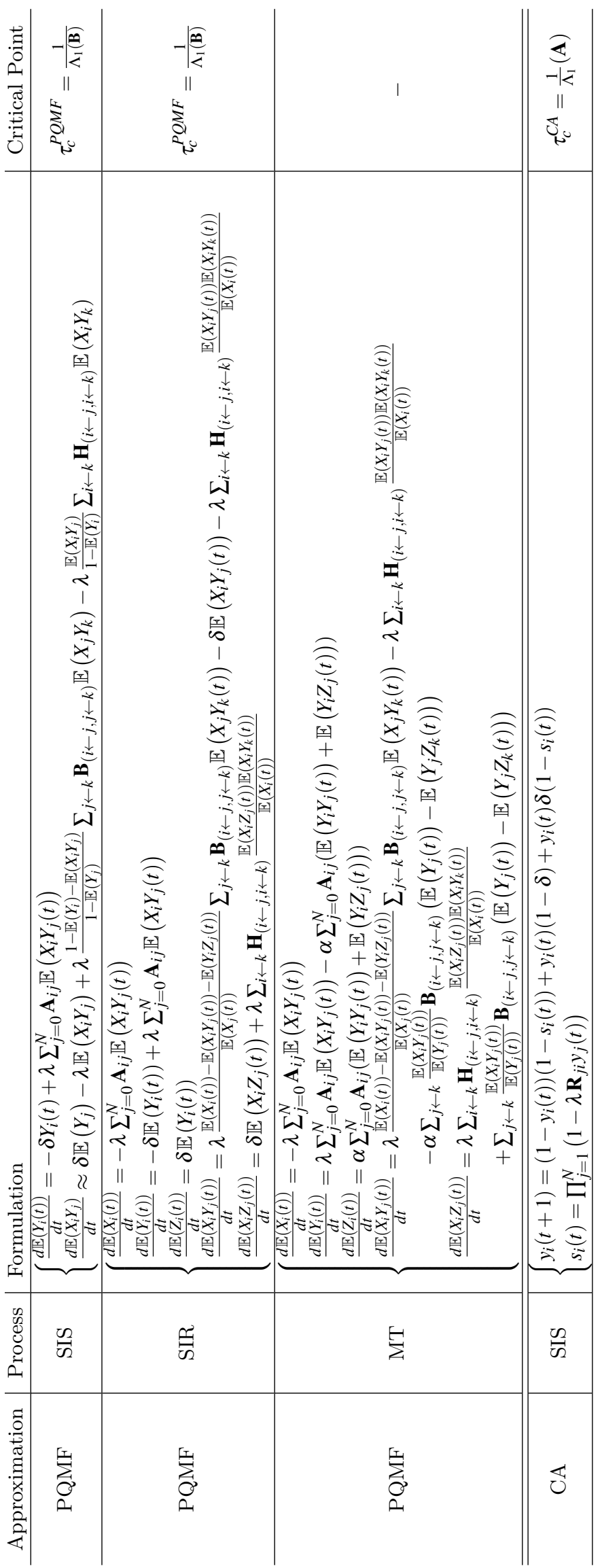



APPENDIX

$\mathrm{D}$

D

\section{LOCALIZATION IN INTERCONNECTED}

NETWORKS

\section{D.1 Localization on interconnected networks}

Complementary to the results presented in Section 5.1.4, here we present results for the lines $(2.3+2.6+2.6)$ and $(2.6+2.3+2.6)$. Similarly, the experiments here are conducted in terms of the inverse participation ratio, as it was done for the 2-Layer multiplex case.

Figure 81 shows the 10th larger eigenvalues of the 3-layer multiplex case. The dashed lines represent the leading eigenvalue of each layer. Note that the leading eigenvalue of the layer with $P(k) \sim k^{-2.9}$ is the 7 th larger on the network spectrum when $\frac{\eta}{\lambda}=0$. We observe that there is no crossings on the observed eigenvalues, which is an expected result, since the layers have different structures. Furthermore, it is important to remark that all networks of layers evaluated also show similar qualitative behaviors. The topology of the network of layers does not lead to qualitative differences on the dependence of $\Lambda_{i}$ on $\frac{\eta}{\lambda}$ for the first ten eigenvalues. We also notice that although it is only an approximation, the perturbation theory would be valid roughly up to $\frac{\eta}{\lambda} \lesssim 10$.

Figures 57 and 82 shows the $\operatorname{IPR}\left(\Lambda_{1}\right)$. On the main panel we present the individual contribution of each layer, while on the insets we have the total $\operatorname{IPR}\left(\Lambda_{1}\right)$. As mentioned on the main text, the first eigenvalue is usually enough to analyze the localization as a first order approximation. Here we observe that the layer with the largest eigenvalue dominates the dynamics. In addition, note the similarities between the multiplex and the line configuration $(2.6+2.3+2.6)$, where the non-dominant layers behave similarly. This is because for small values of $\frac{\eta}{\lambda}$, the effect of the extra edge in the network of layers (closing the triangle) is of order $\eta^{2}$ and so the similar behavior observed comparing the panel (b) of Figures 57 and 82 for the two configurations. As $\frac{\eta}{\lambda}$ grows, the symmetry in 
Figure 81 - Evaluation of the 8 first eigenvalues of $\mathscr{R}(\lambda, \eta)$ for the multiplex configuration as a function of of the ratio $\frac{\eta}{\lambda}$. It is noteworthy that such plot is visually equivalent for all the layer topologies composed by 3 layers. The dashed lines represents the individual layer leading eigenvalues.

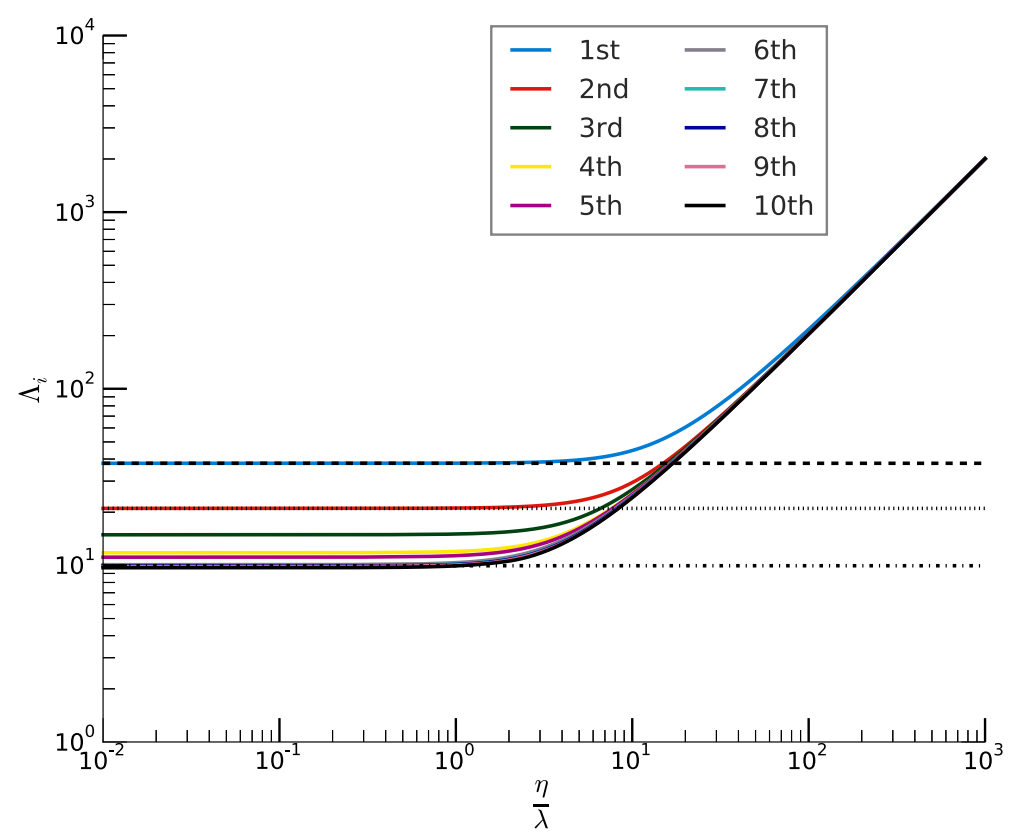

Source: Developed by the author.

the node-aligned multiplex dominates the eigenvector structure and the contributions of all layers are comparable. As we next show, the different contributions of the layers to the total $\operatorname{IPR}\left(\Lambda_{1}\right)$ are at the root of the multiple susceptibility peaks observed.

Complementing and reinforcing the analysis of Section 5.1.4, comparing the different line configurations of the network of layers, observe that the largest eigenvalue of the whole system, $\Lambda_{1}$, has its associated eigenvector localized in the dominant layer, that is, in the layer generated using $\gamma=2.3$. Depending on the position of that layer in the whole system - i.e., central or peripheral layer - , the contribution of the non-dominant layers to $\operatorname{IPR}\left(\Lambda_{1}\right)$ varies. In particular, when the dominant layer corresponds to an extreme node of the network of layers, the contribution of the other two layers will ordered according to the distance to the dominant one. Consequently, when the dominant layer is in the center of the network of layers, the contributions of the non-dominant ones are comparable -note that in panel (b) of Fig. 82, there is no difference in the contribution to $\operatorname{IPR}\left(\Lambda_{1}\right)$ of layers generated using $\gamma=2.6$ and $\gamma=2.9$. 
Figure 82 - Spectral properties of the tensor $\mathscr{R}(\lambda, \eta)$ as a function of the ratio $\frac{\eta}{\lambda}$ for a multiplex with two layers with the same degree distribution (different random realizations of the configuration model) and connected to its counterpart on the other layer. In the left panel we present the inverse participation ratio $(\operatorname{IPR}(\Lambda))$ of the two larger eigenvalues and the individual layer contributions, while in the right panel we show the leading eigenvalues. Every curve is composed by $10^{3} \log$ spaced points, in order to have enough resolution.
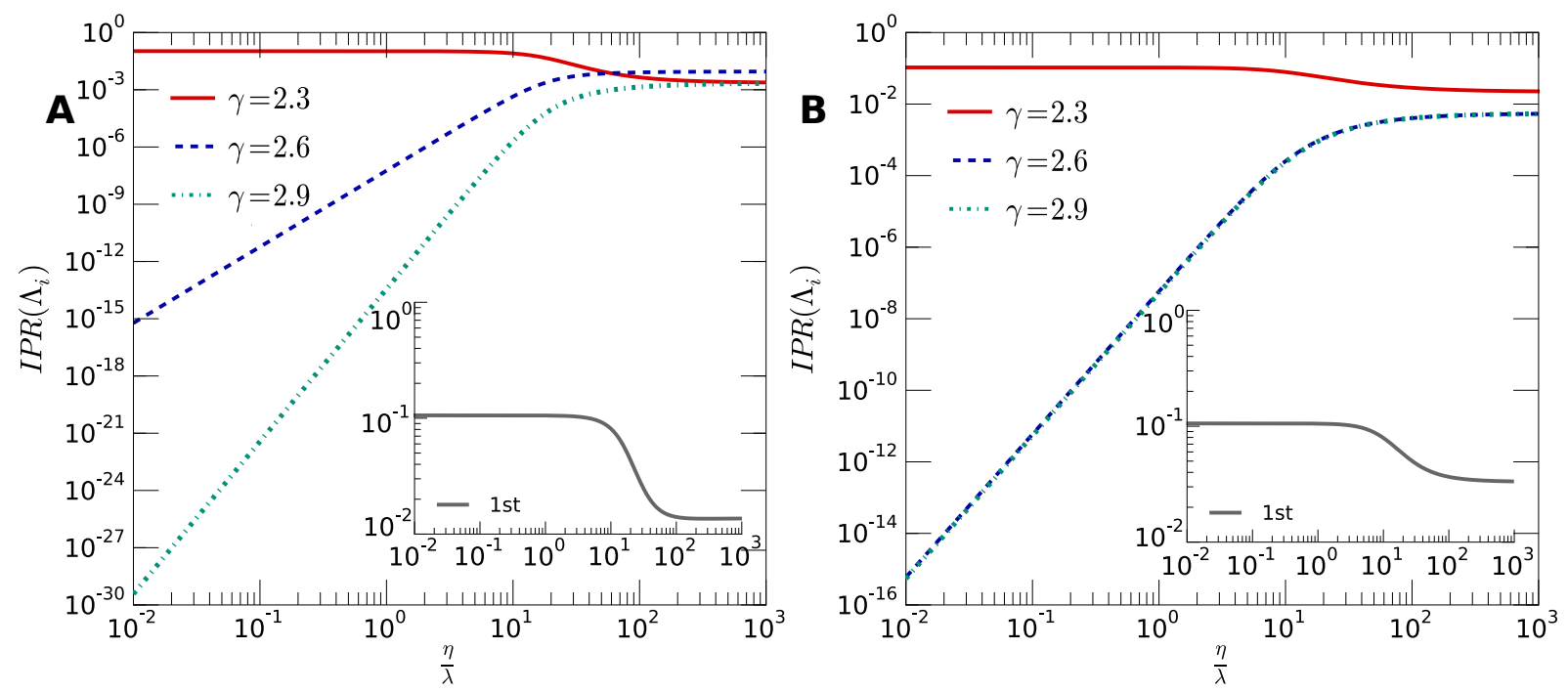

Source: Developed by the author.

\section{D.2 Multiple susceptibility peaks: additional results}

Figure 83 shows the susceptibility as a function of $\lambda$ for different ratios of $\frac{\eta}{\lambda}$. As observed in the main text, we also have three well-defined peaks in these curves when the ratio $\frac{\eta}{\lambda}$ is small. In addition, similar to the 2-layer case, such peaks tend to become less defined and vanish as the ratio $\frac{\eta}{\lambda}$ increases.

Regarding the third peak, note that it is less defined than the others because the average number of infected nodes is larger in this case. Consequently the susceptibility tends to be lower, since it measures the variance in relation to the average. The comparison of Figures 58 (b) and 83 shows that there is no difference in the position of the susceptibility peaks. As mentioned in the main text, the only observed difference is the barrier effect, shown in Fig. 58 (a). We also remark the similarities between the line $(2.6+2.3+2.6)$ and the multiplex case, which emphasize the role of the central node. In that line configuration, the layer with $\gamma=2.3$ spreads its influence to both layers, being this similar to the multiplex case, however with less intra-edges. 
Figure 83 - Susceptibility $\chi$ as a function of $\lambda$ considering all three layer configurations and many different ratios $\frac{\eta}{\lambda}$, which is represented by the color of the lines. The recovering rate is $\mu=1$. The simulated values are $\frac{\eta}{\lambda}=0.05,0.06,0.07,0.08,0.09,0.1,0.2,0.3$, $0.4,0.5,0.6,0.7,0.8,0.9,1.0,2,3,4,5,6,7,8,9,10,20$.
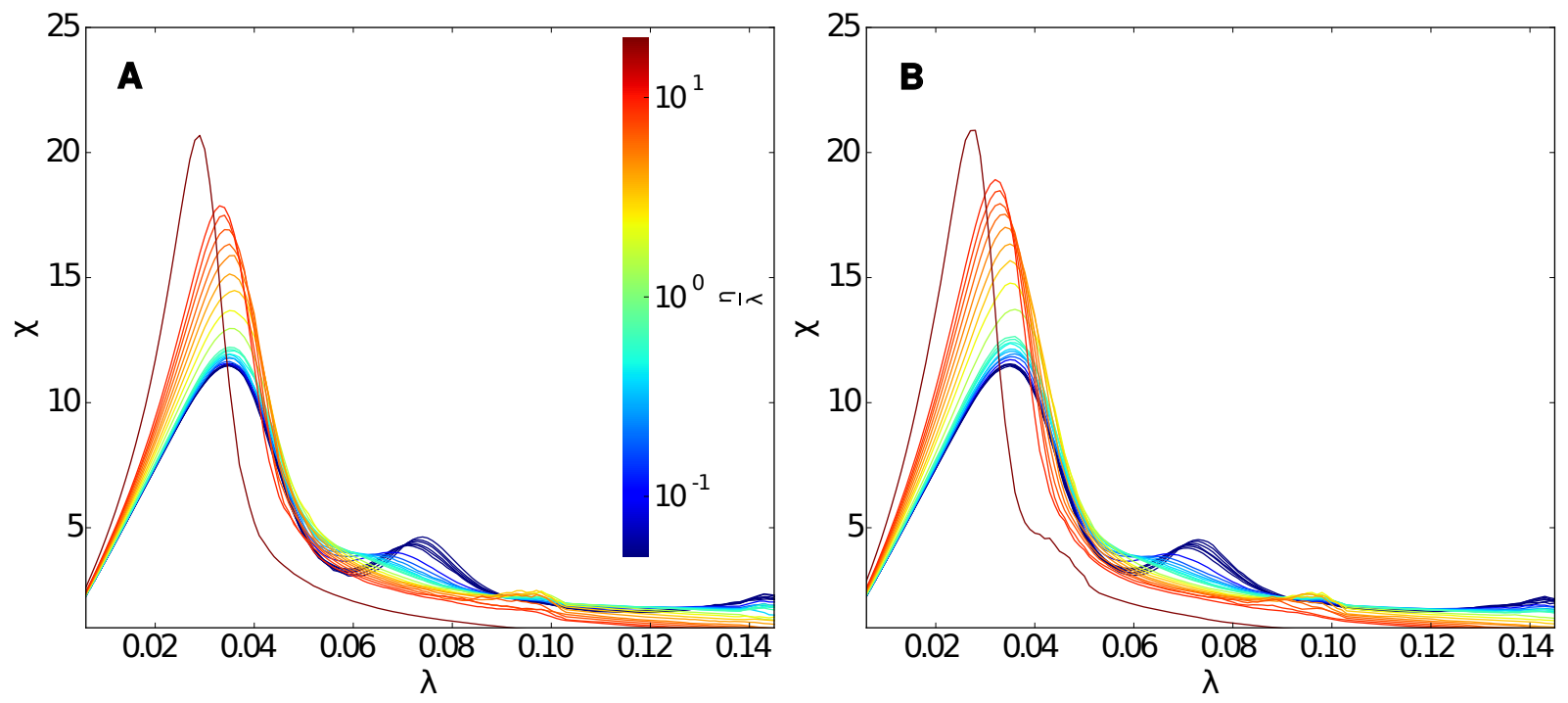

Source: Developed by the author. 
APPENDIX

E

\section{QUADRATIC EIGENVALUE PROBLEM: SPECTRAL PROPERTIES}

\section{E.1 Bounds}

Aiming to find bounds to equation 6.32 we study the scalar polynomial defined by $x^{T} \mathbf{Q}(\lambda) x=0$, where $x$ is an eigenvector (left or right), which guarantee that the polynomial is equal to zero. In order to simplify the problem we multiply $\mathbf{Q}(\lambda)$ by $\mathbf{D}$, obtaining a monic polynomial matrix, than we must bound the terms $b\left(x^{T}, x\right)$ and $\Delta\left(x^{T}, x\right)$, which allow us to bound both solutions. Those terms can be bounded by the numerical range of the matrices in which they are related. The numerical range is formally defined for any matrix $\mathbf{X}$ as $F(\mathbf{X})=\left\{x^{T} \mathbf{X} x: x \in \mathbb{C}\right.$ and $\left.x^{T} x=1\right\}$. Additionally, $\sigma(\mathbf{X}) \subseteq F(\mathbf{X})$, where $\boldsymbol{\sigma}(\mathbf{X})$ is in the set of eigenvalues of $\mathbf{X}$. Moreover, if $\mathbf{X}$ is an Hermitian matrix $x^{T} \mathbf{X} x$ is the Rayleigh quotient of $\mathbf{X}$, which implies $\lambda_{1}(\mathbf{X}) \leq x^{T} \mathbf{X} x \leq \lambda_{N}(\mathbf{X})$. Finally, to bound a non-Hermitian matrices we use the relation of the spectral norm and the numerical range, given as $\frac{1}{2}\|\mathbf{X}\|_{2} \leq r(\mathbf{X}) \leq\|\mathbf{X}\|_{2}$, where $r(\mathbf{X})$ is its numerical radius, defined as $r(\mathbf{X})=\max _{\|x\|_{2}=1}\left|x^{*} \mathbf{X} x\right|=\max \{|z|: z \in F(\mathbf{X})\}$. Firstly, consider the term $b\left(x^{T}, x\right)$, which is bounded by

$$
-\|\mathbf{B}\|_{2} \leq b\left(x^{T}, x\right) \leq\|\mathbf{B}\|_{2}
$$

however in many cases $\mathbf{B}$ is an Hermitian matrix, allowing us to improve this bound to

$$
\lambda_{\min }(\mathbf{B}) \leq b\left(x^{T}, x\right) \leq \lambda_{\max }(\mathbf{B})
$$

More precisely, observe that $\mathbf{B}$ is often related to the aggregated network enlightening the connection between both scales.

Next, evaluating $\Delta\left(x^{T}, x\right)=\left(x^{T} \mathbf{B} x\right)^{2}-4 x^{T} \mathbf{C} x$. Firstly, we can analyze the term $\left(x^{T} \mathbf{B} x\right)^{2}$, by observing that $\min \left\{\mu_{i}\right\} \leq x^{T} \mathbf{B} x \leq \max \left\{\mu_{i}\right\}$, (b) $\min \left\{\mu_{i}^{2}\right\} \leq x \mathbf{B}^{2} x \leq \max \left\{\mu_{i}^{2}\right\}$ and (c) $\min \left\{\left|\mu_{i}\right|\right\}^{2} \leq\left(x^{T} \mathbf{B} x\right)^{2} \leq \max \left\{\left|\mu_{i}\right|\right\}^{2}$, since $\min \left\{\mu_{i}^{2}\right\}=\min \left\{\left|\mu_{i}\right|\right\}^{2}$, hence, from (b) 
and (c), bounding $\left(x^{T} \mathbf{B} x\right)^{2}$ is equivalent to bounding $x \mathbf{B}^{2} x$. Secondly, we can factorize the $\Delta\left(x^{T}, x\right)=x^{T}\left(\mathbf{B}^{2}-4 \mathbf{C}\right) x$ and defining the matrix $\Delta=\mathbf{B}^{2}-4 \mathbf{C}$, we can focus on the problem $x^{T} \Delta x$ instead of the initial definition of $\Delta\left(x^{T}, x\right)$, since both have the same bounds. Besides, since in most of the problems on networks we area dealing with symmetric matrices, we might also impose that $\Delta\left(x^{T}, x\right) \geq 0$ because the spectra is real. In summary we have

$$
0 \leq \Delta\left(x^{T}, x\right) \leq\|\Delta\|_{2}
$$

The bounds can be further improved when applied on the analysis of particular matrices (adjacency, Laplacian, probability transition, ...), since their particularities also impose constraints on the solutions and can be explored to improve those results.

\section{E.2 Comments on symmetric problems: HQEP}

The matrix polynomial defined by the matrix coefficients 6.28 is not symmetric in most cases, however, a class of problems that arise naturally is defined by $\mathbf{M}_{12}=p \mathbf{I}$ and in this case the matrices $\mathbf{A}$ and $\mathbf{B}$ are Hermitian, however, $\mathbf{C}$ is not. However we can use the Toeplitz decomposition in order to analyze a simplified problem. Such states that any square matrix can be uniquely written as the sum an Hermitian $\left(\mathbf{X}=\mathbf{X}^{*}\right)$ and a skew Hermitian matrix $\left(\mathbf{X}=-\mathbf{X}^{*}\right)$ as $\mathbf{X}=\frac{1}{2}\left(\mathbf{X}_{\mathbf{1}}+\mathbf{X}_{\mathbf{2}}{ }^{*}\right)+\frac{1}{2}\left(\mathbf{X}_{\mathbf{1}}-\mathbf{X}_{\mathbf{2}}{ }^{*}\right)$. It allow us to decompose $p \mathbf{C}=\frac{1}{2}\left(\mathbf{M}_{11} \mathbf{M}_{22}+\mathbf{M}_{22} \mathbf{M}_{11}\right)+\frac{1}{2}\left(\mathbf{M}_{11} \mathbf{M}_{22}-\mathbf{M}_{22} \mathbf{M}_{11}\right)+p^{2} \mathbf{I}$. In this manner we can re-write our QEP into to parts, one composed by Hermitian matrices, which is called Hyperbolic Quadratic Eigenvalue Problem (HQEP), and a skew Hermitian matrix, that can be interpreted as a perturbation if the layers are similar. The advantage of such approach is that HQEP present interesting features, for instance the left and right eigenvalues coincide. The natural consequence of the perturbation theory is that the matrix $p \mathbf{C}$ of the HQEP is perturbed by $\frac{1}{2}\left(\mathbf{M}_{11} \mathbf{M}_{22}-\mathbf{M}_{22} \mathbf{M}_{11}\right)$ and such matrix norm goes to zero as the layers are more similar. From the Bauer and Fike theorem we can state a quality function for the approximation of the perturbed matrix $\mathbf{C}$ as

$$
|\lambda-\hat{\lambda}| \leq \kappa(\mathbf{U})|| \frac{1}{2}\left(\mathbf{M}_{11} \mathbf{M}_{22}-\mathbf{M}_{22} \mathbf{M}_{11}\right)|| \mid
$$

where $\hat{\lambda}$ is the eigenvalue of $\mathbf{C}=\mathbf{C}_{\mathbf{H}}+\mathbf{C}_{\mathbf{S}}, \mathbf{C}_{\mathbf{H}}=\mathbf{U} \Lambda \mathbf{U}^{-1}$ and $\kappa(\cdot)$ is the condition number with respect with respect to the matrix norm $\|\cdot \mid\| \cdot$. Considering the spectral norm $\|\cdot \cdot\|_{2}$ we have $\kappa(\mathbf{X})=\left|\frac{\sigma_{\max }(\mathbf{X})}{\sigma_{\min }(\mathbf{X})}\right|$. If $\kappa(\mathbf{U})$ is near 1 , small perturbations imply in small changes on the eigenvalues. On the other hand, large values of $\kappa(\mathbf{U})$ suggest a poor approximation. Observe that such analysis concerns only the matrix $\mathbf{C}$ and not the whole QEP, however it can be an estimate to the quality of the approximation and show that the general solution interpolates between a HQEP to a general QEP. 


\section{E.3 Limits for sparse intrer-layer coupling: singular $\mathbf{D}=$ $\mathbf{M}_{12}=\mathbf{M}_{21}$}

So far we assumed a node-aligned multiplex, fulfilling the invertibility condition of $\mathbf{M}_{12}$, which is necessary to formally define the problem, however we can use the limit of $\mathbf{D}_{i i}=\varepsilon \rightarrow 0$ to obtain an approximation of the sparse coupling. Observe that equation 6.16 can be analyzed in two different steps, first calculating the limit of decoupled edges and secondly the rest of the system. The first limit is analyzed as follows. From 6.16 the absent edges are factorized as

$$
\begin{aligned}
& p^{-1} \varepsilon^{-1} \tilde{\mathbf{D}}(i) \lambda^{2}-p^{-1} \varepsilon^{-1}\left(\mathbf{M}_{11} \tilde{\mathbf{D}}(i)+\tilde{\mathbf{D}}(i) \mathbf{M}_{22}\right) \lambda+ \\
& p^{-1} \varepsilon^{-1} \mathbf{M}_{11} \tilde{\mathbf{D}}(i) \mathbf{M}_{22}-p \mathbf{D}=0
\end{aligned}
$$

where $\tilde{\mathbf{D}}(i)=\boldsymbol{\varepsilon} \mathbf{D}^{-1}$. Multiplying equation E.5 by $p \varepsilon$ and using the following limit

$$
\lim _{\varepsilon \rightarrow 0}\left[\varepsilon \mathbf{D}^{-1}\right]_{j j}= \begin{cases}1 & \text { if }\left[\mathbf{D}^{-1}\right]_{j j} \in O(\varepsilon), \\ 0 & \text { otherwise. }\end{cases}
$$

we have

$$
\tilde{\mathbf{D}} \lambda^{2}-\left(\mathbf{M}_{11} \tilde{\mathbf{D}}+\tilde{\mathbf{D}} \mathbf{M}_{22}\right) \lambda+\mathbf{M}_{11} \tilde{\mathbf{D}} \mathbf{M}_{22}=0
$$

where the term of order $p \boldsymbol{\varepsilon} \mathbf{D}$ vanish in the limit. Observe that $\tilde{\mathbf{D}}=\lim _{\boldsymbol{\varepsilon} \rightarrow 0}\left[\varepsilon \mathbf{D}^{-1}\right]=\mathbf{I}$ if both layers are decoupled and the polynomial equation can be factorized as $\left(\mathbf{M}_{11}-\lambda \mathbf{I}\right)\left(\mathbf{M}_{22}-\lambda \mathbf{I}\right)=$ 0 , whose solutions are the union of the solution of the standard eigenvalue problem of each layer. An important observation is that the number nodes that are not connected to the other layer is also the number of eigenvalues that does not change as a function of $p$.

Note that equation E.7 present the solution of the nodes that do not have any counterpart on the other layer. In order to calculate the remaining solutions we have to redefine the original problem in terms of the Moore - Penrose pseudoinverse, denoted by $\mathbf{X}^{\dagger}$, for a matrix $\mathbf{X}$. Denoting by $\overline{\mathbf{D}}=p^{-1} \mathbf{D}^{\dagger}$ we have $\overline{\mathbf{D}}_{j j}=p^{-1} \mathbf{D}_{j j}^{-1}$ if $\mathbf{D}_{j j} \neq 0$ and $\overline{\mathbf{D}}_{j j}=0$ otherwise. Observe that the zeros of $\overline{\mathbf{D}}_{j j}$ are ones in $\tilde{\mathbf{D}}_{j j}$. For the sake of simplicity, in the following we assume that $\mathbf{M}_{12}$ is invertible, however the strategy mentioned above can be applied if it is not the case. From the computational point of view, we can reduce the cost to calculate the whole spectra as a function of a closed range of $p$ by separating it into two components, where a subset is constant and the remaining subset varies. 

ACCURACY OF NUMERICAL APPROXIMATIONS AND MONTE CARLO SIMULATIONS 
Figure 84 - Phase diagrams comparing the accuracy of QMF and PQMF mean field approaches considering different levels of inter-layer coupling and the AAA (Assortative+Assortative - Assortativelly coupled) network. For more see Section 3.7.6.2.
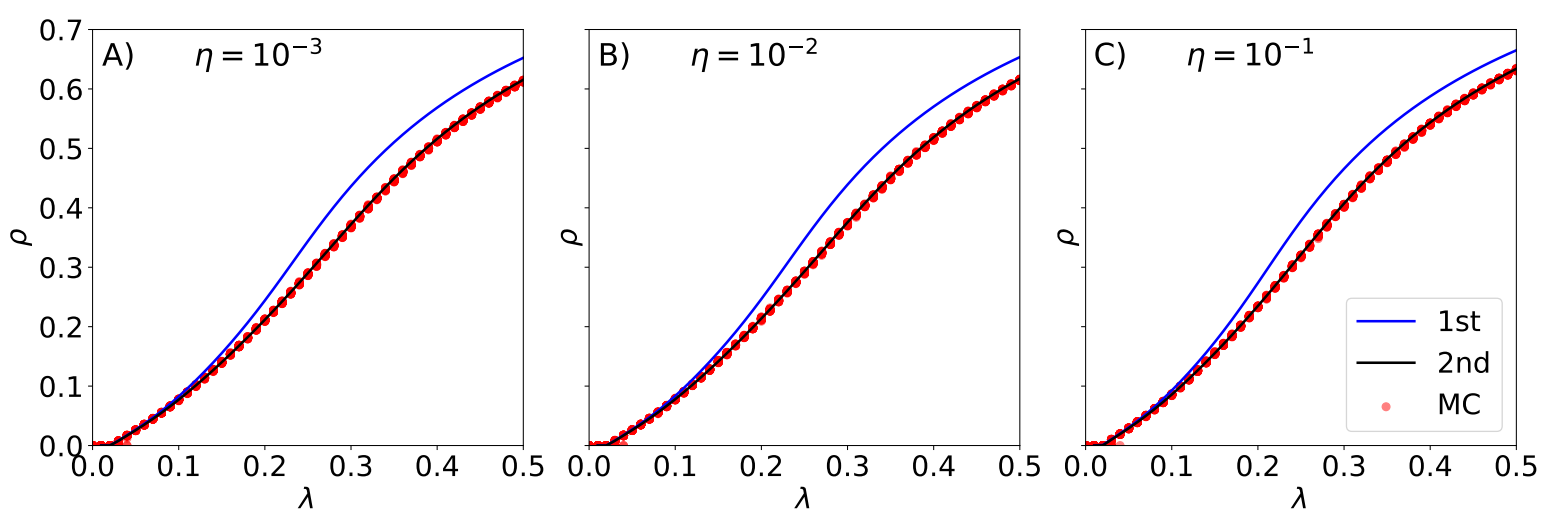

(a)
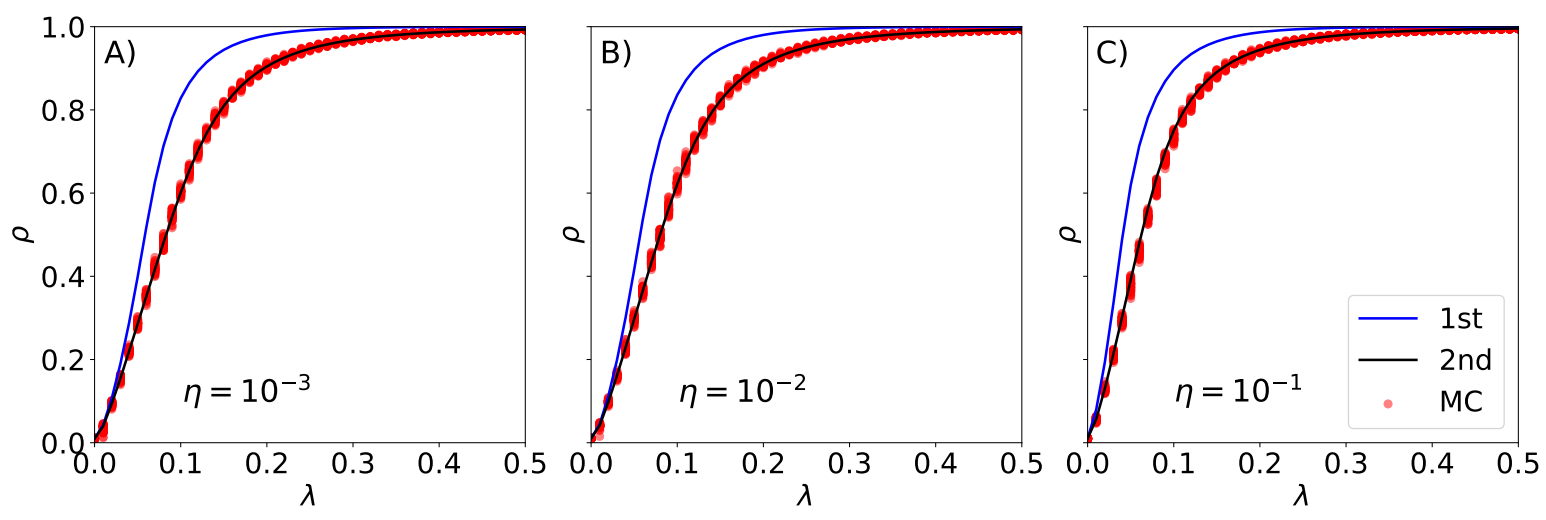

(b)
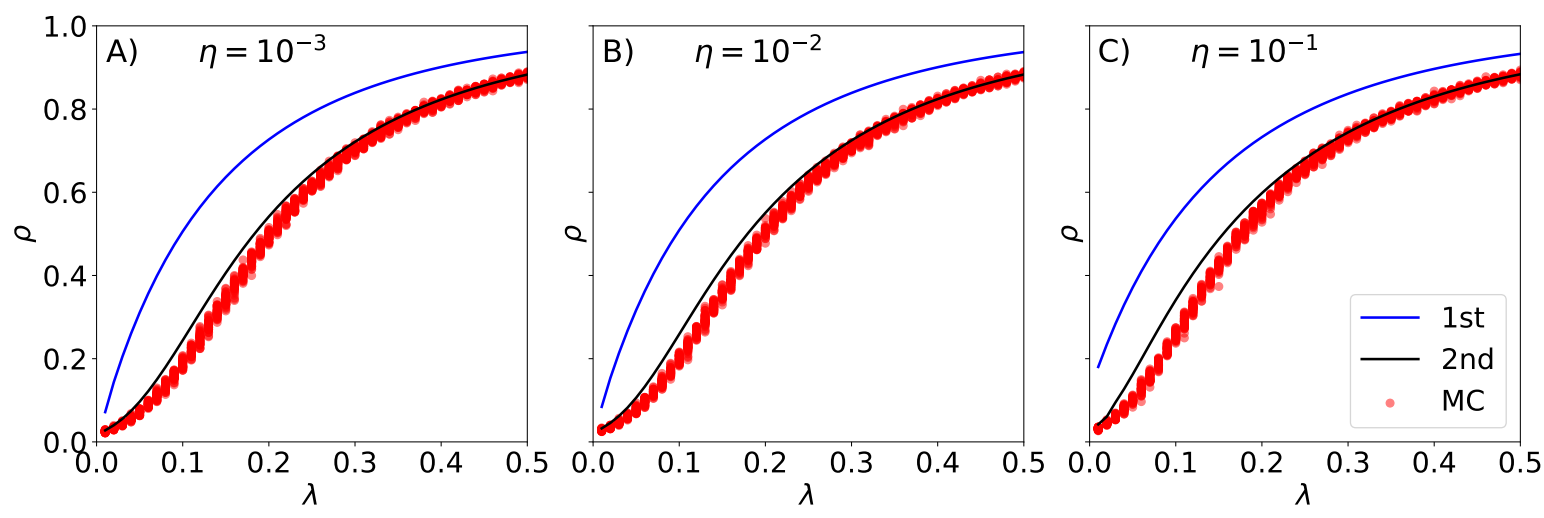

(c)

Source: Developed by the author. 
Figure 85 - Phase diagrams comparing the accuracy of QMF and PQMF mean field approaches considering different levels of inter-layer coupling and the ACA (Assortative+Clustered - Assortativelly coupled) network. For more see Section 3.7.6.2.
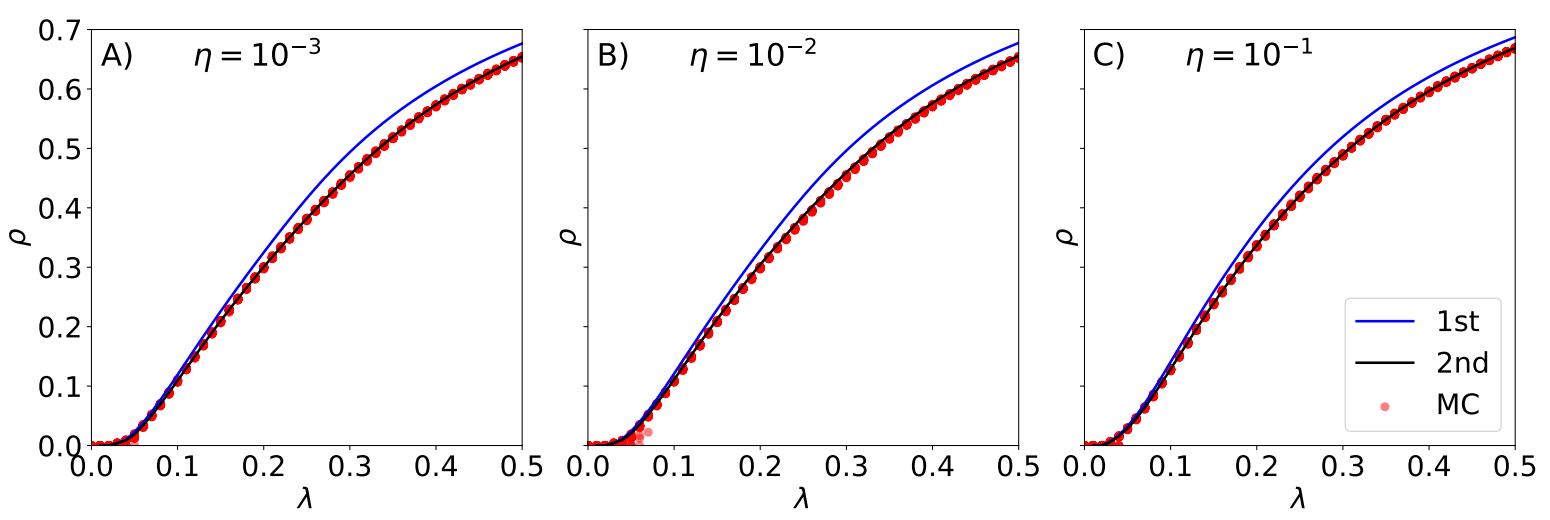

(a)
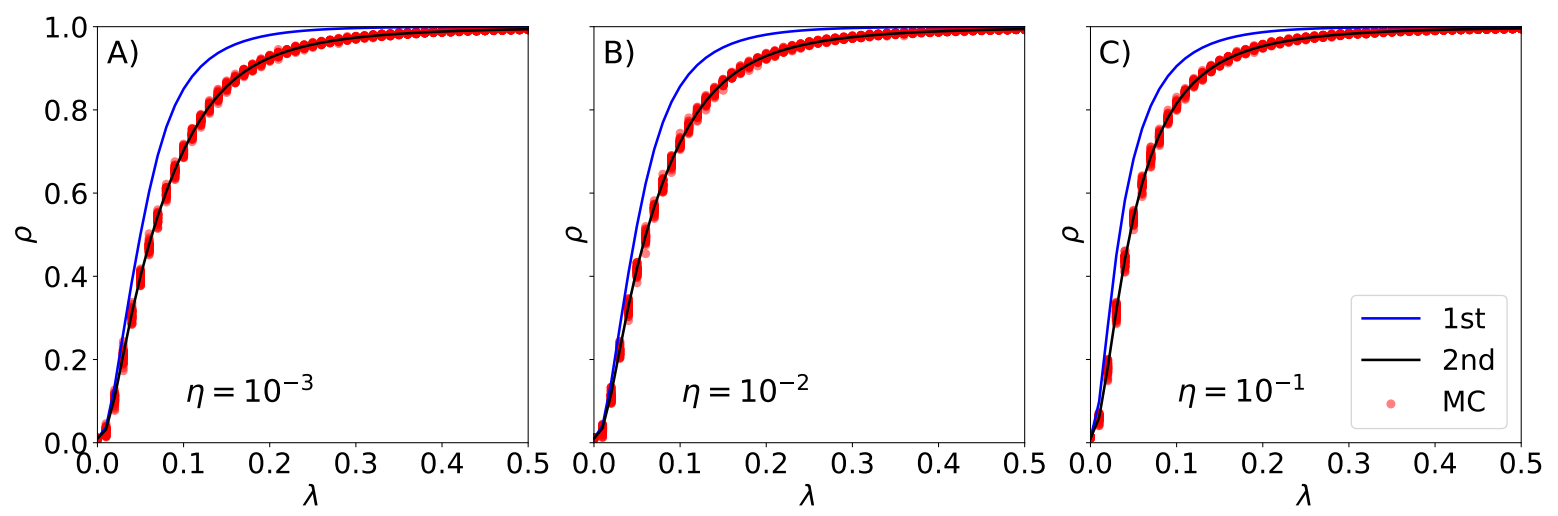

(b)
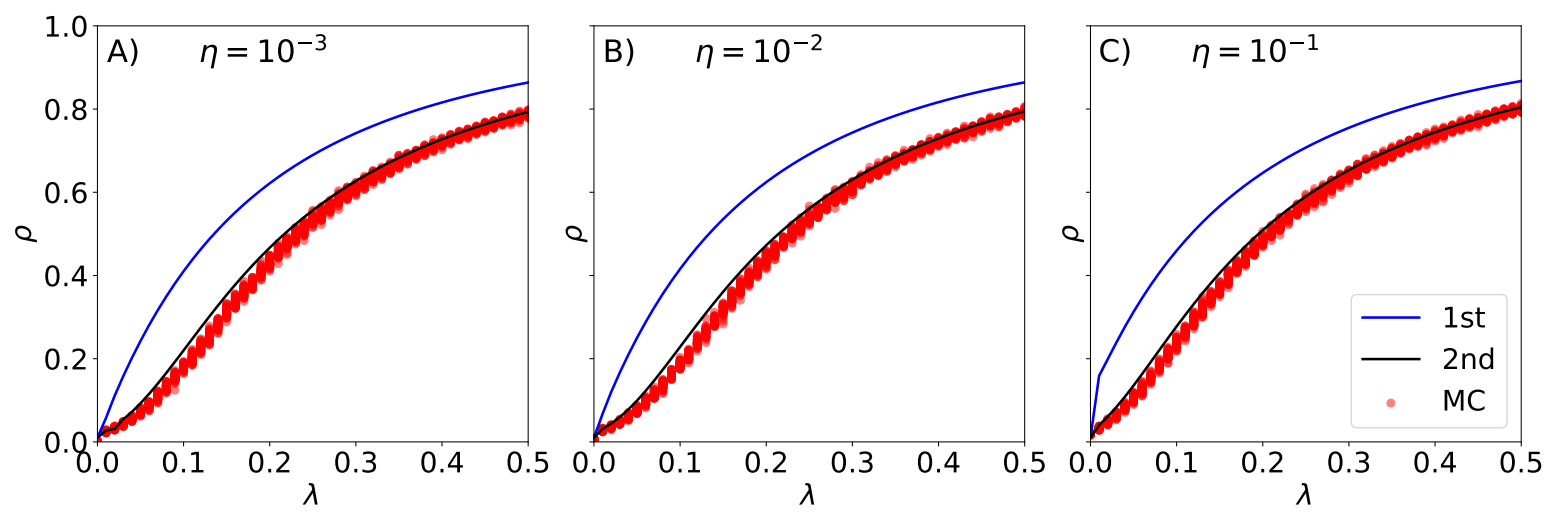

(c)

Source: Developed by the author. 
Figure 86 - Phase diagrams comparing the accuracy of QMF and PQMF mean field approaches considering different levels of inter-layer coupling and the ACR (Assortative+Clustered - randomly coupled) network. For more see Section 3.7.6.2.
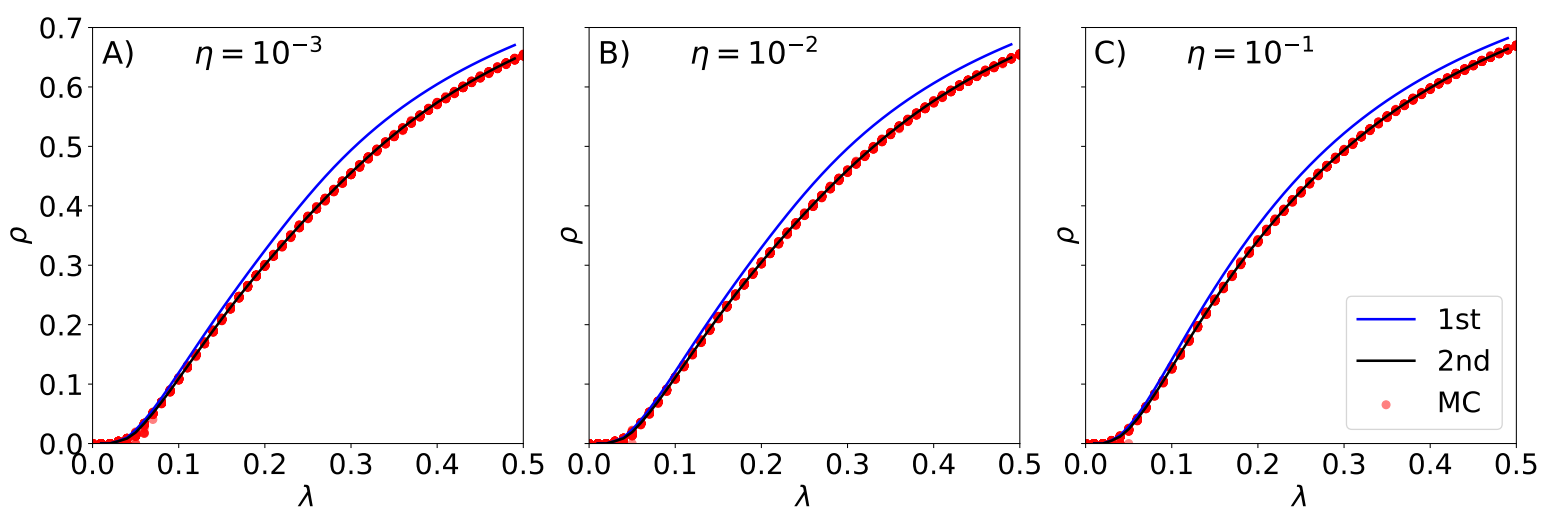

(a)
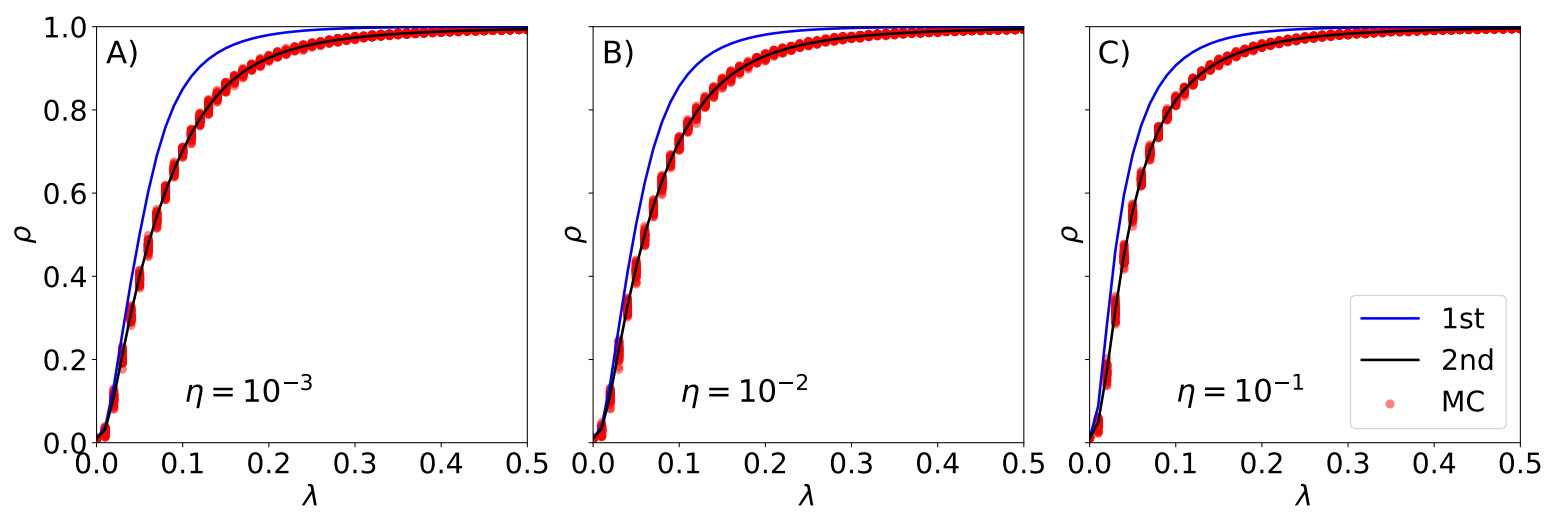

(b)
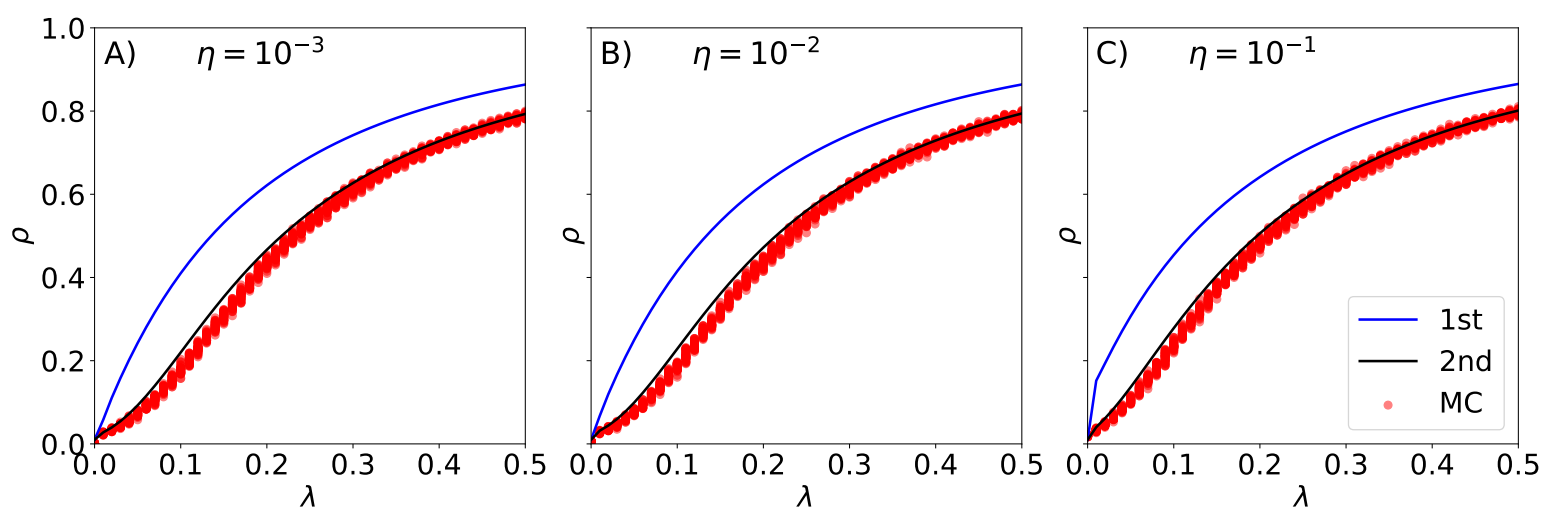

(c)

Source: Developed by the author. 
Figure 87 - Phase diagrams comparing the accuracy of QMF and PQMF mean field approaches considering different levels of inter-layer coupling and the ARR (Assortative+RRN - randomly coupled) network. For more see Section 3.7.6.2.
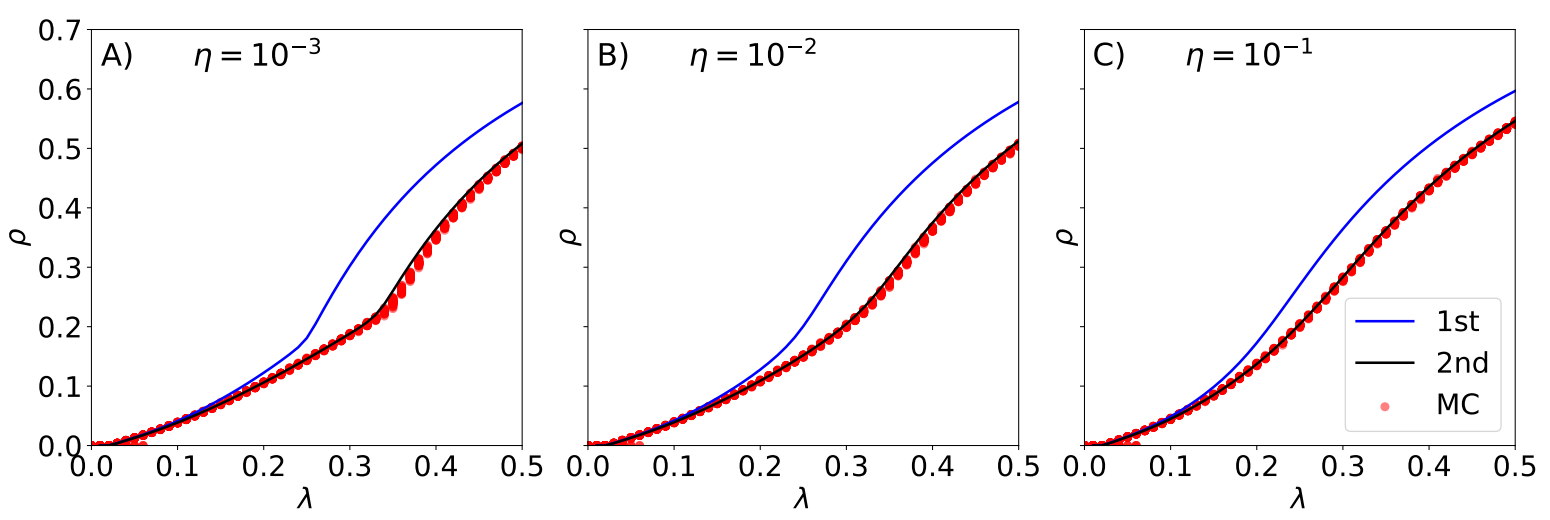

(a)
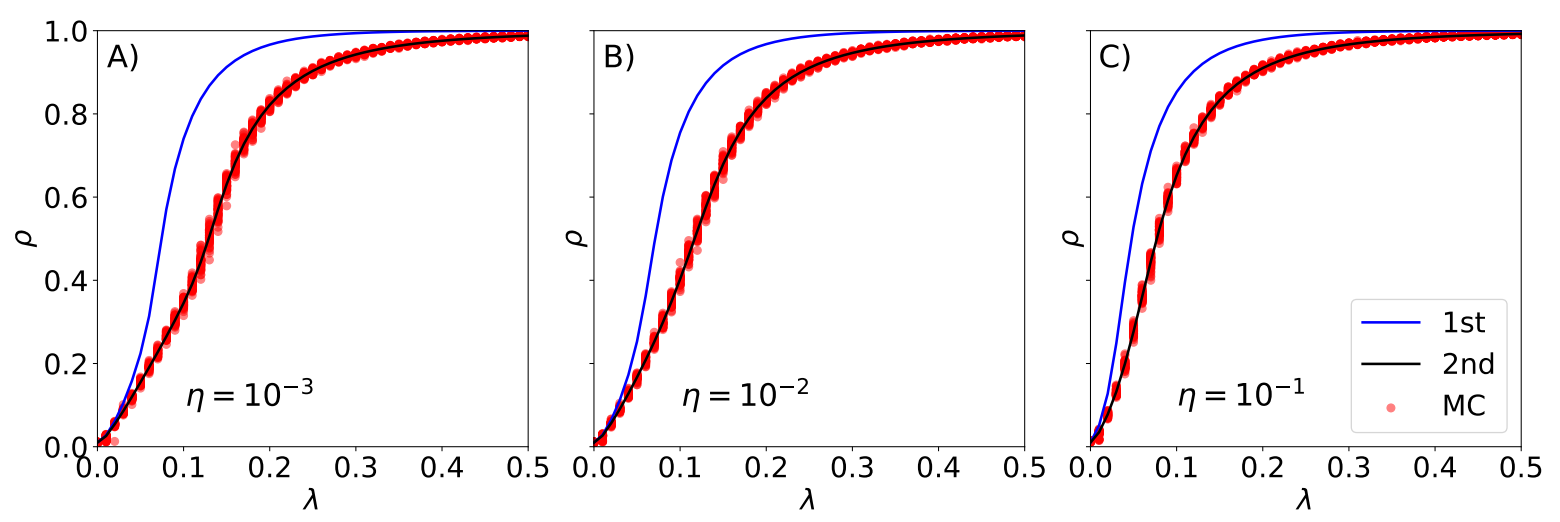

(b)
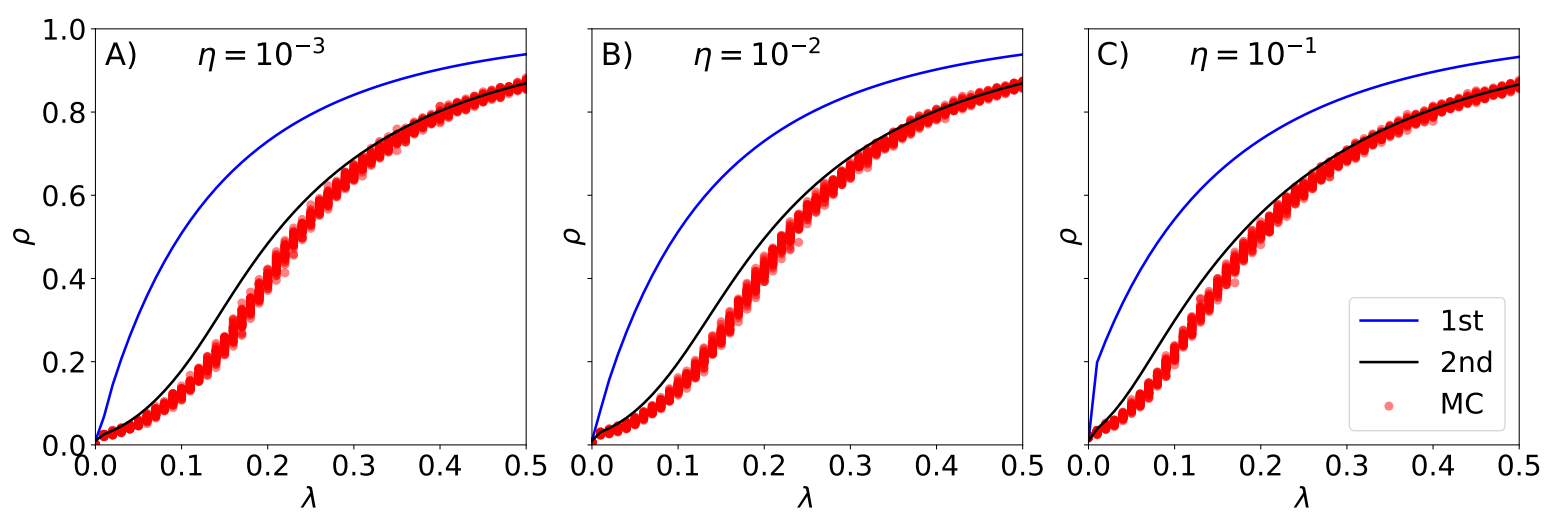

(c)

Source: Developed by the author. 


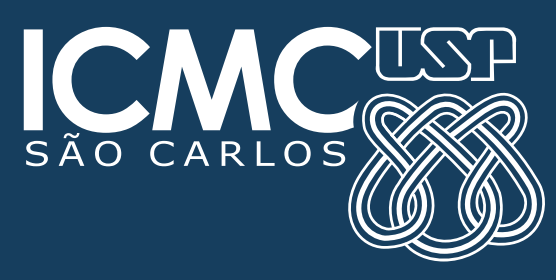

